\title{
Spin dynamics in semiconductors
}

\author{
M. W. Wu*, J. H. Jiang, M. Q. Weng \\ Hefei National Laboratory for Physical Sciences at Microscale, University of Science and Technology of China, Hefei, Anhui, 230026, China \\ Department of Physics, University of Science and Technology of China, Hefei, Anhui, 230026, China
}

\begin{abstract}
This article reviews the current status of spin dynamics in semiconductors which has achieved a lot of progress in the past years due to the fast growing field of semiconductor spintronics. The primary focus is the theoretical and experimental developments of spin relaxation and dephasing in both spin precession in time domain and spin diffusion and transport in spacial domain. A fully microscopic many-body investigation on spin dynamics based on the kinetic spin Bloch equation approach is reviewed comprehensively.
\end{abstract}

Keywords: spin dynamics; spin-orbit coupling; spin relaxation; spin dephasing; spin diffusion; spin transport; electron-electron Coulomb scattering; magnetic semiconductors; magnetization dynamics; ultrafast phenomena; quantum well; quantum wire; quantum dot; many-body effect; high field effect.

\section{Introduction}

Since the pioneering works by Lampel [1] and Parsons [2] and the following extensive experimental and theoretical works at Ioffe Institute in St. Petersburg and Ecole Polytechnique in Paris in 1970s and early 1980s, a lot of understanding on spin dynamics in semiconductors has been achieved. Some basic spin relaxation and dephasing mechanisms have also been proposed at that time. A nice review on these findings can be found in the book "Optical Orientation" [3].

Starting from the late 1990s, there is a big revival of research interest in the spin dynamics of semiconductors, jump-started by some nice experimental works by Awschalom and co-workers [4, 5]. An extensive number of experimental and theoretical investigations have been carried out on all aspects of spin properties. Properties of spin relaxation and dephasing in both the time domain (in the spacial uniform system) and the spacial domain (in spin diffusion and transport) have been fully explored in different materials at various conditions (such as temperature, external field, doping density/material and strain) and dimensions (including bulk materials, quantum wells, quantum wires and quantum dots). These materials include III-V and II-VI zinc-blende and wurtzite semiconductors and silicon/germanium, as well as diluted magnetic semiconductors. The spin relaxation and dephasing mechanisms have been rechecked and reinvestigated at different conditions. The spin-related material properties have also achieved much progress along with these investigations which facilitate the better understanding of the observed phenomena as well as the manipulation of the spin coherence. Many novel spin-related properties, such as the spin Hall effect [6-12], spin Coulomb drag effect [13-15], spin photogalvanic effect [16, 17] and persistent spin helix effect [18-20], have been discovered. For the sake of realizing the spintronic devices such as spin transistors, there have been extensive investigations on spin injection and detection. Much progress has been achieved on the spin injection from ferromagnetic materials into semiconductors. Nevertheless, a satisfactory realization of spin transistor which is crucial to the application of semiconductor spintronics is yet to come.

Despite decades of studies, theoretical understanding of the spin relaxation and dephasing which is one of the prerequisites of the realization of spintronics, in both spacial uniform and nonuniform systems is still not fully achieved.

\footnotetext{
${ }^{*}$ Corresponding author.

Email address: mwwu@ustc.edu.cn (M. W. Wu)
} 
Many new experimental findings go far beyond the previous theoretical understandings, thanks to the fast development of experimental techniques including the sample preparations and ultrafast optical techniques. Some of the physics requires a many-body and/or non-equilibrium (even far away from the equilibrium) theory. However, the previous theories [3] are all based on the single-particle approach and for systems near the equilibrium which thus have their strict limits in applying to the nowadays experiments. Some even give totally incorrect qualitative predictions. Wu et al. developed a fully microscopic many-body kinetic spin Bloch equation approach to study the spin dynamics in semiconductors and their nanostructures. Unlike other approaches widely used in the literature which treat scattering using the relaxation time approximation, the kinetic spin Bloch equation approach treats all scattering explicitly and self-consistently. Especially, the carrier-carrier Coulomb scattering is explicitly included in the theory. This allowed them to study spin dynamics not only near but also far away from the equilibrium, for example, the spin dynamics in the presence of high electric field (hot-electron condition) and/or with large initial spin polarization. They applied this approach to study spin relaxation/dephasing and spin diffusion/transport in various kinds of semiconductors and their nanostructures under diversified conditions and have offered a complete and systematic understanding of spin dynamics in semiconductors. Many novel effects were predicted and some of them have been verified experimentally.

In this review, we try to provide a full coverage of the latest developments of spin dynamics in semiconductors. Both experimental and theoretical developments are summarized. In theory, we first review the studies based on the single-particle approach. Then we provide a comprehensive review of the results based on the kinetic spin Bloch equation approach. We organize the paper as follows: We first review the spin interactions in semiconductors in Section 2. Then we briefly discuss the physics of spin dynamics in semiconductors in Section 3. Here we review the previous understanding on spin relaxation and dephasing mechanisms. Differing from the existing nice reviews on these topics [21-23], we try to provide a full and up-to-date picture of spin dynamics of itinerant carriers in semiconductors. We then review the latest experimental developments on spin relaxation and dephasing in time domain as well as the latest theoretical investigations based on the single-particle approach in Section 4. The theoretical investigations of the spin relaxation and dephasing in the spacial uniform systems based on the kinetic spin Bloch equation approach together with the experimental verifications are reviewed in Section 5. In Section 6 we review the latest experimental and theoretical (single-particle theory) results on spin diffusion and transport. Then in Section 7 we review the investigation on spin diffusion and transport using the kinetic spin Bloch equation approach and the related experimental results. We summarize in Section 8.

\section{Spin interactions in semiconductors}

\subsection{A short introduction of spin interactions}

Before introducing the spin interactions, it should be mentioned that the "spins" in semiconductors are not pure spins, or in other words, they are angular momentums which consist of both spin and orbital angular momentums. A direct consequence is that the $g$-factor is no longer $g_{0}=2.0023$ of pure electron spins.

There are various spin interactions in semiconductors. Besides the Zeeman interaction, there are spin-orbit coupling due to the space inversion asymmetry or strain, $s(p)$ - $d$ exchange interaction with magnetic impurities, hyperfine interaction with nuclear spins, spin-phonon interaction, electron-hole and electron-electron exchange interactions. Among these interactions, spin-orbit coupling usually plays the most important role in spin dynamics. For electrons in conduction band, spin-orbit coupling consists of the Dresselhaus, Rashba and strain-induced terms. For valence band electrons, besides these terms, there is an important spin-orbit coupling from the spin-dependent terms in the Luttinger Hamiltonian in cubic semiconductors. These spin-orbit couplings make the motion of carrier spin couple with the orbital motion and give rise to many novel effects in coherent (ballistic) and dissipative (diffusive) electron spin/charge dynamics. A direct consequence is that the spin polarization of a wave packet oscillates during its propagation [24-31]. Recent studies indicate that as the precession during the electron propagation correlates the spin polarization with real-space trajectory, the spin lifetime is enhanced at certain spacial inhomogeneous spin polarization states, such as, some spin grating states $[18,20,32-37]$. Another important spin interaction is the exchange interaction between carriers due to the permutative antisymmetry of the many-body fermion wavefunctions. It has been demonstrated to be important for spin dynamics of electrons in quantum dots [38] and localized electrons bound to impurities [39, 40]. The effect of the exchange interactions between free electrons on spin dynamics was only observed in experiments very recently and has attracted interest [41-43], though it has been predicted much earlier [44]. 
Spin interactions together with the induced spin structures are the physical foundations of the vital topic of coherent spin manipulation which is crucial for spin-based quantum information processing [45-52] and spintronic device operation [22,53-56]. For example, spin-orbit couplings enable coherent manipulation of spin by electric field [53, 57-76]. As electric field is more easily accessible and controllable in genuine electronic devices at nanoscale, the electrical manipulation of spins is important for semiconductor spintronics. The idea has been extended to a large variety of schemes of electrical or optical manipulation of spin coherence: coherent control via electrical modulation of $g$-tensor in nanostructures with spacial-dependent $g$-tensor [77-81]; and similar scheme based on spatially varying magnetic field [82, 83], or hyperfine field [70, 84, 85]; coherent manipulation based on tuning the exchange interaction between electrons in a double quantum dot by gate-voltage [38, 86]; coherent spin manipulation via detuned optical pulses which act as effective magnetic pulses as the Stark shift is spin-dependent due to spin-dependent virtual transition of the circularly polarized light [87-95]. The $p-d$ or $s-d$ exchange interactions and the induced spin structures further enable the above schemes to control $\mathrm{Mn}$ ion spins in paramagnetic phase [96-98] or magnetization in ferromagnetic phase [99, 100] in magnetic semiconductors via manipulating carrier spins.

One of the key obstacles for spintronic device operation and spin-based quantum information processing is the spin relaxation and spin dephasing (i.e., the decay of the longitudinal and transverse spin components, respectively). Spin relaxation and spin dephasing, in principle, also originate from the spin interactions: the fluctuation or inhomogeneity in spin interactions leads to spin relaxation and spin dephasing 1 For example, the spin-orbit coupling is the origin of one of the most efficient spin relaxation mechanisms-the D'yakonov-Perel' mechanism [101, 102]. Spin mixing due to conduction-valence band mixing together with momentum scattering give rise to the Elliott-Yafet spin relaxation mechanisms [103, 104]. Other spin relaxation mechanisms are the $s(p)-d$ exchange mechanism, the Bir-Aronov-Pikus mechanism [105, 106] due to the electron-hole exchange interaction [107], the hyperfine interaction mechanism and the anisotropic exchange interactions between localized electrons [39, 40]. Spin relaxation and dephasing in genuine semiconductor structures are the consequence of the coaction of these mechanisms. Hence it is important to determine the dominant spin relaxation mechanism under various conditions [3, 23, 39, 108-112].

Finally, as the focus of this review is spin dynamics in semiconductors, we only provide an overview for various spin interactions and explain the related physics using intuitive and qualitative pictures. For details, we suggest the salient works in the existing literature: for spin-orbit coupling in semiconductors, the readers could refer to the book by Winkler [113]; for knowledge of the $s(p)$ - $d$ exchange interactions, we suggest the review article by Jungwirth et al. [114] and that by Furdyna [115] and references therein; the hyperfine interaction in semiconductors has been reviewed recently by Fischer et al. [116]; details of the spin-phonon interaction can be found in Ref. [117]; the electron-hole exchange interaction has been discussed in detail in Refs. [107, 118, 119].

\subsection{Spin-orbit interactions in semiconductors and their nanostructures}

A consequence of the relativistic effect in atomic and condensed matter physics is that an electron moving in the atomic potential $V(\mathbf{r})$ feels an effective magnetic field acting on its spin

$$
H_{\mathrm{SO}}=\frac{1}{4 m_{0}^{2} c^{2}} \mathbf{p} \cdot[\boldsymbol{\sigma} \times(\boldsymbol{\nabla} V(\mathbf{r}))],
$$

where $m_{0}$ and $c$ represent the free electron mass and the velocity of light in vacuum, respectively, $\sigma$ is the Pauli matrices, and $\mathbf{p}$ stands for the canonical momentum. $V(\mathbf{r})$ is the total potential generated by other charges. In semiconductors, $V(\mathbf{r})$ includes the periodic potentials generated by the ion-core, the deviation of the periodic potential due to defects and phonons, and the external potential. This is the origin of various spin-orbit interactions in semiconductors.

\subsubsection{Electron spin-orbit coupling term in semiconductors due to space inversion asymmetry}

In semiconductors with space inversion symmetry, the electron spectrum satisfies the following relation, $\varepsilon_{\mathbf{k} \uparrow}=\varepsilon_{\mathbf{k} \downarrow}$. This is the consequence of the coaction of the time-reversal symmetry and the space-inversion symmetry. In the absence of space inversion symmetry, $\varepsilon_{\mathbf{k} \uparrow} \neq \varepsilon_{\mathbf{k} \downarrow}$. In this case, there should be some term which breaks the spin

\footnotetext{
${ }^{1}$ A practical operating scheme should both control the spin interactions and avoid their fluctuation and inhomogeneity carefully. Optimization of the ability of coherent control and spin lifetime is often needed.
} 
degeneracy of the same $\mathbf{k}$ states. The term should be an odd function of both $\mathbf{k}$ and $\boldsymbol{\sigma}$ as it breaks the space inversion symmetry whereas keeps the time reversal symmetry. This term is the so-called the spin-orbit coupling term. For electron with spin $S=1 / 2$, as $\sigma_{i} \sigma_{j}=\delta_{i, j}+\mathrm{i} \epsilon_{i j k} \sigma_{k}\left(i, j, k=x, y, z\right.$ and $\epsilon_{i j k}$ is Levi-Civita tensor), only the linear form of $\sigma_{i}$ can appear in the spin-orbit coupling term. Hence, the only possible form of the spin-orbit coupling term is

$$
H_{\mathrm{SO}}=\frac{1}{2} \mathbf{\Omega}(\mathbf{k}) \cdot \boldsymbol{\sigma}
$$

where $\boldsymbol{\Omega}(\mathbf{k})$ is an odd function of $\mathbf{k}$, which acts as an effective magnetic field. In a reversed logic, any electron spinorbit coupling which can be written in the form $H_{\mathrm{SO}}=\sum_{n, m, i, j} C_{n m}^{i j} \sigma_{i}^{n} k_{j}^{m}$, can be reduced to the form of Eq. (2). Hence the breaking of the space inversion symmetry is a prerequisite 2 for the appearance of electron spin-orbit coupling term in semiconductors 3

In semiconductors, there are several kinds of space inversion asymmetry: (i) the bulk inversion asymmetry due to structures lacking inversion center [123], such as zinc-blende III-V or II-VI semiconductors; (ii) the structure inversion asymmetry resulting from the inversion asymmetry of the potentials in nanostructures including external gate-voltage and/or built-in electric field [124, 125]; and (iii) interface inversion asymmetry associated with the chemical bonding within interfaces [126, 127]. Besides, strain can also induce inversion asymmetry and leads to spin-orbit coupling. The explicit form of the spin-orbit couplings due to these space inversion asymmetries can be obtained, e.g., by the $\mathbf{k} \cdot \mathbf{p}$ theory [113].

\subsection{Explicit form of the spin-orbit coupling terms in semiconductors}

The explicit form of the spin-orbit coupling in semiconductors can be derived from the $\mathbf{k} \cdot \mathbf{p}$ theory. For common III-V and II-VI semiconductors, such as GaAs, InAs, CdTe and ZnSe, the minimum $\mathbf{k} \cdot \mathbf{p}$ theory to describe carrier and spin dynamics is the eight-band Kane model [113, 128]. In the following, we introduce the spin-orbit coupling in the framework of such model.

\subsubsection{Kane Hamiltonian and block-diagonalization}

In zinc-blende structures, the Kane model is of the following form [117]

$$
H_{\text {Kane }}=\left[\begin{array}{ccc}
H_{c} & H_{c, v} & H_{c, s v} \\
H_{c, v}^{\dagger} & H_{v} & H_{v, s v} \\
H_{c, s v}^{\dagger} & H_{v, s v}^{\dagger} & H_{s v}
\end{array}\right] .
$$

The subscript $c, v, s v$ denote the conduction, valence and the split-off valence bands respectively. As $H_{c, v}, H_{v, s v}$ and $H_{c, s v}$ are nonzero, the electron motions in these bands are coupled together, which are obviously too complex. Fortunately, the couplings between these bands are much smaller than their separations. By a perturbative blockdiagonalization, one can decouple the motions in these bands. The block-diagonalization is achieved by the Löwdin partition method [129] which is actually a unitary transformation. For example, the eigen-energy $E_{n}(\mathbf{k})$ and eigen-state $\Psi_{n}(\mathbf{k})$ of the original Kane Hamiltonian satisfy the following eigen-equation

$$
H_{\text {Kane }} \Psi_{n}(\mathbf{k})=E_{n}(\mathbf{k}) \Psi_{n}(\mathbf{k})
$$

After a unitary transformation,

$$
\tilde{H}_{\text {Kane }} \tilde{\Psi}_{n}(\mathbf{k})=E_{n}(\mathbf{k}) \tilde{\Psi}_{n}(\mathbf{k}),
$$

where $\tilde{H}_{\mathrm{Kane}}=U H_{\mathrm{Kane}} U^{\dagger}$ is block-diagonal. As the couplings between bands $H_{c, v}, H_{v, s v}$ and $H_{c, s v}$ are small near the center of the concerned valley (For most of the III-V and II-VI semiconductors, the concerned valley is $\Gamma$ valley,

\footnotetext{
${ }^{2}$ Note that, it was found that there is intersubband spin-orbit coupling even in symmetric quantum wells [120, 121], which seems to contradict the above conclusion. However, even in symmetric quantum wells, the space inversion symmetry is broken at the boundary of the quantum well [120, 121].

${ }^{3}$ Differently, hole has spin $J=3 / 2$, where $J_{i}^{2} \neq 1$. Hence, terms which are quadratic in $\mathbf{J}$ can appear in the spin-orbit coupling. These terms should be even in $\mathbf{k}$ in order to keep the time-reversal symmetry. Hence, hole can have spin-orbit coupling terms which do not break space inversion symmetry. Actually, the Luttinger Hamiltonian [122] only contains spin-dependent terms quadratic in $\mathbf{k}$.
} 
where $\mathbf{k}=0$ is the valley center.), the results can be given in a perturbative expansion series. Below, we give the results with only the lowest order nontrivial terms kept, which are enough for the discussion of spin dynamics in most cases. Besides the transformation of the Hamiltonian, the wavefunction also changes slightly, $\tilde{\Psi}_{n}(\mathbf{k})=U \Psi_{n}(\mathbf{k})$. This modification of the wavefunction always leads to the mixing of different spin states. In the presence of spin-mixing, any momentum scattering can lead to spin flip and spin relaxation, which gives the Elliott-Yafet spin relaxation mechanism [103, 104].

In the following, we present the conduction (electron) and valence (hole) band parts of the block-diagonal Hamiltonian $\tilde{H}_{\text {Kane }}$.

\subsubsection{Electron Hamiltonian and spin-orbit coupling in bulk system}

By choosing $x, y, z$ axes along the crystal axes of [100], [010] and [001] respectively, the electron Hamiltonian can be written as

$$
H_{e}=\frac{k^{2}}{2 m_{e}}+\frac{1}{2} \boldsymbol{\Omega}_{D} \cdot \sigma+\frac{1}{2} \mathbf{\Omega}_{\mathrm{S}} \cdot \boldsymbol{\sigma}
$$

where $m_{e}$ is the electron (conduction band) effective mass. The second and third terms in the right hand side of the above equation describe the Dresselhaus spin-orbit coupling [123] and strain-induced spin-orbit coupling [117] respectively.

$$
\Omega_{D x}=2 \gamma_{D} k_{x}\left(k_{y}^{2}-k_{z}^{2}\right), \quad \Omega_{S x}=2 C_{3}\left(\epsilon_{x y} k_{y}-\epsilon_{x z} k_{z}\right)+2 D k_{x}\left(\epsilon_{y y}-\epsilon_{z z}\right),
$$

with other components obtained by cyclic permutation of indices. Here $\gamma_{D}$ is the Dresselhaus coefficient, which can be expressed formally as $\gamma_{D}=2 \eta /\left[3 m_{c v} \sqrt{2 m_{e} E_{g}(1-\eta / 3)}\right][117]$, where $\eta=\Delta_{\mathrm{SO}} /\left(E_{g}+\Delta_{\mathrm{SO}}\right)$ with $E_{g}$ and $\Delta_{\mathrm{SO}}$ being the bandgap and the spin-orbit splitting of the valence band respectively. $m_{c v}$ is a parameter with mass dimension and is related to the interaction between the conduction and valence bands [117]. The coefficients $\gamma_{D}, C_{3}$ and $D$ are crucial for spin dynamics. There have been a lot of studies on these quantities, especially $\gamma_{D}$, in common III-V semiconductors (such as GaAs, InAs, GaSb and InSb) both theoretically and experimentally [117, 130-141]. Interestingly, these studies give quite different values of $\gamma_{D}$ in GaAs from 7.6 to $36 \mathrm{eV} \cdot \AA^{3}$. Detailed lists of $\gamma_{D}$ in GaAs in the literature are presented in Refs. [139-141]. Latest theoretical advancements include the first principle calculations [139, 141] and full-Brillion zone investigations [141, 142]. Recently, from fitting the magnetotransport properties in chaotic GaAs quantum dots [140] and from fitting the spin relaxation in bulk GaAs via the fully microscopic kinetic spin Bloch equation approach [110], $\gamma_{D}$ in GaAs was found to be 9 and $8.2 \mathrm{eV} \cdot \AA^{3}$ respectively, which are close to the value $\gamma_{D}=8.5 \mathrm{eV} \cdot \AA^{3}$ from $a b$ initio calculations with $\mathrm{GW}$ approximation [139] 4$]$ The coefficient for strain-induced spin-orbit coupling is $C_{3}=2 c_{2} \eta /\left[3 \sqrt{2 m_{e} E_{g}(1-\eta / 3)}\right]$, where $c_{2}$ is the interband deformation-potential constant. $D$ comes from higher order corrections which is usually smaller than $C_{3}$. The strain-induced spin-orbit coupling was studied experimentally in Refs. [77, 144], indicating that the strain induced spin-orbit coupling can be more important than the Dresselhaus spin-orbit coupling and that both the $C_{3}$ and $D$ terms can be dominant. Recently, the coefficients $C_{3}$ and $D$ from ab initio calculations showed good agreement with the experimental results [145], where $C_{3}=6.8 \mathrm{eV} \cdot \AA$ and $D=2.1 \mathrm{eV} \cdot \AA$.

\subsubsection{Electron spin-orbit coupling in nanostructures}

Electric field can also break the space inversion symmetry and lead to additional spin-orbit coupling [124, 125]. In III-V and II-VI heterostructures the induced spin-orbit coupling, named the Rashba spin-orbit coupling, can be as important as (or even more important than) the Dresselhaus spin-orbit coupling due to bulk inversion asymmetry [146, 147]. The effect is caused by the total electric field, including the external electric field due to gate-voltage, the electric field due to built-in electrostatic potential and the electric field due to interfaces [113]. The leading effect of interfaces is to produce a spacial variation of band edge, which thus leads to an effective electric field. Via Löwdin partition method, one obtains

$$
H_{R}=\alpha_{0} \sigma \cdot\left(\mathbf{k} \times \mathcal{E}_{v}(\mathbf{r})\right) .
$$

Here $\alpha_{0}=e \eta(2-\eta) P^{2} /\left(3 m_{0}^{2} E_{g}^{2}\right)$ with $P$ being the interband momentum matrix [113], $\mathcal{E}_{v}(\mathbf{r})=\nabla V_{v}(\mathbf{r}) /|e|$ where $V_{v}(\mathbf{r})$ is the potential felt by valence electron. Averaging $\mathcal{E}_{v}(\mathbf{r})$ over, e.g., the subband wavefunction of a quantum well, one

\footnotetext{
${ }^{4}$ There are other recent papers [141, 143] with $\gamma_{D}$ close to $8.5 \mathrm{eV} \cdot \AA^{3}$.
} 
has

$$
H_{R}=\alpha_{R} \boldsymbol{\sigma} \cdot(\mathbf{k} \times \hat{\mathbf{n}}),
$$

where $\alpha_{R}=\alpha_{0}\left\langle\mathcal{E}_{v}(\mathbf{r})\right\rangle$ is the Rashba parameter and $\hat{\mathbf{n}}$ is a unit vector along the growth direction of the quantum well.

Historically, there is a paradox about the Rashba parameter. According to the Ehrenfest Theorem, the average of the force acting on a bound state is zero. Therefore, the Rashba parameter should be very small. This paradox is resolved by Lassnig [148], who pointed out that for the Rashba spin-orbit coupling in conduction band, $\alpha_{R}$ is related with the average of the electric field in valence band over the conduction band (subband) wavefunction. As pointed out above, the electric potential on the valence or conduction band comprises three parts,

$$
V_{v}(\mathbf{r})=V_{\text {ext }}(\mathbf{r})+V_{\text {built }}(\mathbf{r})+E_{v}(\mathbf{r}), \quad V_{c}(\mathbf{r})=V_{\text {ext }}(\mathbf{r})+V_{\text {built }}(\mathbf{r})+E_{c}(\mathbf{r}),
$$

where $E_{c}(\mathbf{r})$ and $E_{v}(\mathbf{r})$ are the position dependent conduction and valence band edges. $V_{\text {built }}(\mathbf{r})$ is the built-in electrostatic potential and $V_{\text {ext }}(\mathbf{r})$ is the external electrostatic potential. Let $V_{\text {Coul }}(\mathbf{r})=V_{\text {ext }}(\mathbf{r})+V_{\text {built }}(\mathbf{r})$ [total electrostatic (Coulomb) potential]. According to the Ehrenfest theorem, one has $\left\langle\nabla V_{\text {Coul }}(\mathbf{r})\right\rangle=-\left\langle\boldsymbol{\nabla} E_{c}(\mathbf{r})\right\rangle$, as the average over the (conduction) electron wavefunction (denoted as $\langle\ldots\rangle)$ of the net force felt by electron is zero. If the ratio of the valence band offset to the conduction band one is $r_{v c}$, then

$$
\left\langle\boldsymbol{\nabla} V_{v}(\mathbf{r})\right\rangle=\left\langle\boldsymbol{\nabla}\left(V_{\text {Coul }}(\mathbf{r})+r_{v c} E_{c}(\mathbf{r})\right)\right\rangle=\left(1-r_{v c}\right)\left\langle\nabla V_{\text {Coul }}(\mathbf{r})\right\rangle .
$$

For example, in GaAs/ $\mathrm{Al}_{x} \mathrm{Ga}_{1-x}$ As quantum wells $r_{v c} \simeq-0.5$, hence $1-r_{v c} \simeq 1.5$. Therefore, the Rashba spin-orbit coupling is proportional to the average of the sum of the external and built-in electric field. The above discussion is just a simple illustration on the existence of the Rashba spin-orbit coupling, where a few factors have been ignored. Nevertheless, the indication that the Rashba spin-orbit coupling can be tuned electrically inspired the community. Many proposals of spintronic devices based on electrical manipulation of spin-orbit coupling, such as, the spin fieldeffect transistors [54], were proposed $5_{5}^{5}$ In this background, the Rashba spin-orbit couplings in various heterostructures have been studied extensively. Theoretical investigations on the Rashba spin-orbit coupling in quantum wells were performed in Refs. [151-157]. Specifically, it was found that even in symmetric quantum wells, the built-in electric field contributes an intersubband Rashba spin-orbit coupling, though it does not contribute to the intrasubband spinorbit coupling [120, 121].

Experimental methods to determine the spin-orbit coupling coefficients consist of magnetotransport (including both the Shubnikov-de Haas oscillation [158-168] and weak (anti-)localization [137, 169-176]), optically probed spin dynamics (spin relaxation [177-179] and spin precession [60]), electron spin resonance [180] and spin-flip Raman scattering [133, 181], etc. Recently, it was proposed that the radiation-induced oscillatory magnetoresistance can be used as a sensitive probe of the zero-field spin-splitting [182]. The experimental results indicated that the largest Rashba parameter $\alpha_{R}$ can be $30 \times 10^{-12} \mathrm{eV} \cdot \mathrm{m}$ [161, 163] or even $40 \times 10^{-12} \mathrm{eV} \cdot \mathrm{m}$ [165] for InAs and $14 \times 10^{-12} \mathrm{eV} \cdot \mathrm{m}$ for GaSb [180] quantum wells, while the smallest Rashba parameter can be negligible [178, 183] 6

In asymmetric quantum wells, both the Rashba and Dresselhaus spin-orbit couplings exist. After averaging over the lowest subband wavefunction in (001) quantum well, the Dresselhaus spin-orbit coupling becomes,

$$
H_{D}=\beta_{D}\left(-k_{x} \sigma_{x}+k_{y} \sigma_{y}\right)+\gamma_{D}\left(k_{x} k_{y}^{2} \sigma_{x}-k_{y} k_{x}^{2} \sigma_{y}\right),
$$

where $\beta_{D}=\gamma_{D}\left\langle\hat{k}_{z}^{2}\right\rangle=\int d z \phi_{1}(z)^{*}\left(-\partial_{z}^{2}\right) \phi_{1}(z)$ with $\phi_{1}(z)$ being the lowest subband wavefunction. In narrow quantum wells, the linear-k term dominates. The ratio of the strength of the Dresselhaus spin-orbit coupling to the Rashba one $\beta_{D} / \alpha_{R}$ can be tuned by the well width or the electric field along the growth direction [37, 113]. The electric field dependence of the Dresselhaus and Rashba spin-orbit couplings in $\mathrm{GaAs} / \mathrm{Al}_{x} \mathrm{Ga}_{1-x}$ As quantum wells for varies well widths was studied in the work by Lau and Flatté [146], indicating that under high bias the Rashba spin-orbit coupling can exceed the Dresselhaus one 7 The interference of the two spin-orbit couplings leads to anisotropic spin splitting,

\footnotetext{
${ }^{5}$ For recent advancement in experiment on the spin field-effect transistors, see, e.g., Refs. [55, 149, 150].

${ }^{6}$ The channel width dependence [167], density dependence [159-162, 165, 166, 169-173], gate-voltage dependence [158, 159, 163, 164, 173, [178], temperature dependence [177] and interface effect [162, 165, 172, 174, 184] were investigated in various materials from InAs, InGaAs and GaAs to GaSb. Rashba spin-orbit coupling in quantum wire was studied in Refs. [185-191].

${ }^{7}$ The effects of structure inversion asymmetry, heterointerface and gate-voltage on the Dresselhaus and Rashba spin-orbit couplings in quantum wells were studied experimentally in Refs. [37, 55, 137, 162, 175, 178, 179, 183, 192, 193].
} 
which hence results in anisotropic spin precession [64-67], spin relaxation [194-199], spin diffusion [29, 32-34, 37] and spin photocurrent [200-202]. Reversely, the ratio of $\beta_{D} / \alpha_{\mathrm{R}}$ can be inferred from the anisotropy of spin precession [64, 203], spin relaxation [198, 199], spin diffusion [29, 33] and spin photocurrent [200, 201].

\subsubsection{Hole Hamiltonian and hole spin-orbit coupling in bulk system}

The hole Hamiltonian can be written as [113]

$$
H_{h}=H_{\mathrm{L}}+H_{\mathrm{SO}}^{h}+H_{\mathrm{hsp}}
$$

where $H_{\mathrm{L}}$ is the Luttinger Hamiltonian, $H_{\mathrm{SO}}^{h}$ is the spin-orbit coupling due to the space inversion asymmetry and $H_{\mathrm{hsp}}$ describes the hole-strain and hole-phonon interactions which can be found in Ref. [117]. The Luttinger Hamiltonian $H_{\mathrm{L}}$ is given by [122] (in the order of hole spin state $J_{z}=-\frac{3}{2},-\frac{1}{2}, \frac{1}{2}, \frac{3}{2}$ with $z$ axis along [001] direction)

$$
H_{\mathrm{L}}=\left[\begin{array}{cccc}
P+Q & L & M & 0 \\
L^{*} & P-Q & 0 & M \\
M^{*} & 0 & P-Q & -L \\
0 & M^{*} & -L^{*} & P+Q
\end{array}\right],
$$

where

$$
P=\frac{\gamma_{1}}{2 m_{0}} k^{2}, \quad Q=\frac{\gamma_{2}}{2 m_{0}}\left(k_{x}^{2}+k_{y}^{2}-2 k_{z}^{2}\right), \quad L=-\frac{2 \sqrt{3} \gamma_{3}}{2 m_{0}}\left(k_{x}-\mathrm{i} k_{y}\right) k_{z}, \quad M=-\frac{\sqrt{3}}{2 m_{0}}\left[\gamma_{2}\left(k_{x}^{2}-k_{y}^{2}\right)-2 \mathrm{i} \gamma_{3} k_{x} k_{y}\right]
$$

with $\gamma_{1}, \gamma_{2}$ and $\gamma_{3}$ being the Luttinger parameters.

$$
H_{\mathrm{SO}}^{h}=\frac{1}{\eta}\left(\mathbf{\Omega}_{D}+\mathbf{\Omega}_{S}\right) \cdot \mathbf{J}
$$

which is similar to the spin-orbit coupling in the conduction band except differed by a factor of $1 / \eta$. Here $\mathbf{J}=$ $\left(J_{x}, J_{y}, J_{z}\right)$ is the hole spin operator with $J=3 / 2$.

Under spherical approximation, the Luttinger Hamiltonian can be written in a compact form,

$$
H_{L}^{\mathrm{sp}}=\frac{\gamma_{1}}{2 m_{0}} k^{2}+\frac{\bar{\gamma}_{2}}{2 m_{0}}\left[\frac{5}{2} k^{2}-2(\mathbf{k} \cdot \mathbf{J})^{2}\right]
$$

where $\bar{\gamma}_{2}=\left(2 \gamma_{2}+3 \gamma_{3}\right) / 5$. Under this approximation, the spectrum of hole becomes parabolic,

$$
E_{\lambda_{1} / \lambda_{2}}(\mathbf{k})=\frac{k^{2}}{2 m_{0}}\left(\gamma_{1} \pm 2 \bar{\gamma}_{2}\right)
$$

The indices $\lambda_{1}= \pm \frac{1}{2}, \lambda_{2}= \pm \frac{3}{2}$ denote the hole spin. The four branches with different effective mass $m_{0} /\left(\gamma_{1} \pm 2 \bar{\gamma}_{2}\right)$ are the light hole and heavy hole branches respectively. The two fold degeneracy of the spectrum is a consequence of the coexistence of the time-reversal symmetry and the space inversion symmetry of the Luttinger Hamiltonian.

In zinc-blende III-V or II-VI semiconductors, $\gamma_{1}$ and $\bar{\gamma}_{2}$ are usually of the same order of magnitude (e.g., in GaAs, $\gamma_{1}=6.85$ and $\bar{\gamma}_{2}=2.5$ [204]). Therefore the spin-dependent term in Eq. (17) (the spin-orbit coupling term) is comparable with the spin-independent ones. This indicates that the spin-orbit coupling is very strong in bulk hole system, which is one of the main differences between bulk electron and hole systems. The main consequences are that: change in orbit motion (states) can influence strongly on spin dynamics [23, 113, 205, 206]; the spin relaxation/dephasing is usually much faster in hole system ( $100 \mathrm{fs})$ than in electron system [207-210].

\subsubsection{Hole spin-orbit coupling in nanostructures}

As mentioned above the hole spin-orbit coupling is important in determining hole spectrum and its spin state. In nanostructures, it is always necessary to diagonalize the hole Hamiltonian Eq. (13) including the confining potential [113, 143]. The dispersion and spin states can vary markedly with the confinement geometry [113]. It was 
demonstrated that in $p$-GaAs quantum wells the in-plane and out-of-plane effective masses and $g$-factors of the lowest heavy-hole subband can be quite different for different growth directions [113].

Similar to the electron case, the electric field can also induce the Rashba spin-orbit coupling in hole system, which is written as [113]

$$
H_{R}^{h}=\alpha_{0}^{h} \mathbf{J} \cdot\left(\mathbf{k} \times \mathcal{E}_{c}\right) .
$$

In [001] grown quantum wells, it is reduced to

$$
H_{R}^{h}=\alpha_{0}^{h}\left\langle\mathcal{E}_{c}\right\rangle\left(k_{y} J_{x}-k_{x} J_{y}\right),
$$

where $\alpha_{0}^{h}$ is a material dependent parameter [113] and $\left\langle\mathcal{E}_{c}\right\rangle$ is the average of the electric field (along the growth direction) acting on conduction band over the hole subband envelope function. In practice, to obtain the Rashba spinorbit coupling in, e.g., the lowest heavy-hole subband, one must further block-diagonalize the subband Hamiltonian via the Löwdin partition method [113]. Quite different from the electron case, the subband block-diagonalization gives another Rashba spin-orbit coupling which originates from the electric field on other hole subband rather than the conduction band. Practical calculation indicated that such kind of Rashba spin-orbit coupling is more important than that due to the valence-conduction band coupling [Eq. [20)] [113]. The expression of such kind of Rashba spin-orbit coupling in the lowest heavy-hole subband is given by [113],

$$
H_{R}^{\mathrm{HH}}=\alpha^{h h}\left\langle\mathcal{E}_{z}\right\rangle \mathrm{i}\left(k_{+}^{3} \sigma_{-}-k_{-}^{3} \sigma_{+}\right),
$$

where $k_{ \pm}=k_{x} \pm \mathrm{i} k_{y}, \sigma_{ \pm}=\sigma_{x} \pm \mathrm{i} \sigma_{y}$ with $\sigma$ being the Pauli spin matrices in the manifold of hole spin $J_{z}=-3 / 2,3 / 2$. $\left\langle\mathcal{E}_{z}\right\rangle$ is the electric field acting on the valence band averaged over the lowest hole subband envelope function. For an infinite depth rectangular quantum well [113],

$$
\alpha^{h h}=\frac{e}{m_{0}^{2}} \frac{64}{9 \pi^{2}} \gamma_{3}\left(\gamma_{2}+\gamma_{3}\right)\left[\frac{1}{\Delta_{11}^{h l}}\left(\frac{1}{\Delta_{12}^{h l}}-\frac{1}{\Delta_{12}^{h h}}\right)+\frac{1}{\Delta_{12}^{h l} \Delta_{12}^{h h}}\right],
$$

where $\Delta_{i j}^{h l}(i, j=1,2 \ldots)$ is the splitting between the $i$-th heavy-hole subband and the $j$-th light-hole subband. Similar to the case in electron system, the hole Rashba spin-orbit coupling can also be tuned by gate-voltage and structure asymmetry but now $\alpha^{h h}$ is also electric field dependent [113, 211-214].

\subsection{Spin-orbit coupling in nanostructures due to interface inversion asymmetry}

The interface inversion asymmetry is a kind of inversion asymmetry caused by the inversion asymmetric bonding of atoms at the interfaces of nanostructures 8 It was first pointed out by Aleiner and Ivchenko [215] that the symmetry at the interface may be different from the symmetry away from the interface, and hence may introduce a new spinorbit coupling [127, 216, 217]. The interface-induced spin-orbit coupling was usually studied in quantum wells. The underlying physics is similar for other nanostructures.

Let us start by examining the case of GaAs/AlAs quantum wells. Without the interface effect, the quantum wells possess a $D_{2 d}$ symmetry. It is easy to find that due to the difference of $\mathrm{Al}$ and $\mathrm{Ga}$ atoms, the symmetry at the interface is reduced to $C_{2 v}$ [113]. For quantum wells which possess a mirror symmetry, the asymmetry induced by the two interfaces cancels out [113]. However, in the situation that the materials in the well and barrier do not share a common atom, the interface effect at the two interfaces can not be canceled out as they are different interfaces [126]. For example, in InAs/GaSb quantum wells, the two interfaces are Sb-In-As and In-As-Ga. The symmetry of the quantum well is then reduced to $C_{2 v}$ and results in new spin-orbit coupling terms for holes and electrons. It induces a term $\sim\left(J_{x} J_{y}+J_{y} J_{x}\right)$ and independent of $\mathbf{k}$ in the hole Hamiltonian [113], which mixes the heavy-hole and light-hole spins effectively in narrow quantum wells [216, 217] and largely suppresses the hole spin lifetime in these structures [216]. The induced electron spin-orbit coupling is of the linear-Dresselhaus-type $\sim\left(k_{x} \sigma_{x}-k_{y} \sigma_{y}\right)$ [218].

The experimental test of the importance of the interface-induced electron spin-orbit coupling was performed by comparing the spin dynamics in InGaAs-AlInAs quantum wells (which share one common atom) with that in InGaAsInP quantum wells (which have no common atom) with similar parameters and overall quality [219]. The observed

\footnotetext{
${ }^{8}$ The interface inversion asymmetry induced spin-orbit coupling is also reviewed in Ref. [113].
} 
huge difference in spin relaxation times between these two samples indicates that the interface inversion asymmetry induced spin-orbit coupling is important in narrow quantum wells without common atom in well and barrier.

It should be pointed out that in [110] grown quantum wells, even in the case without common atom in well and barrier, the two interfaces can be symmetric 9 Hence the interface inversion asymmetry does not contribute. By comparing the spin relaxation in InAs/GaSb quantum wells with different growth directions, with the help of theoretical calculation, it was found that the interface inversion asymmetry plays an important role in narrow InAs/GaSb quantum wells [221, 222].

In genuine nanostructures, as interfaces can not be perfect, the interface inversion asymmetry exists in most samples. A quantitative analysis of such effect requires the knowledge of specific interfaces, which is usually very difficult to acquire. In general, since it is an interface effect, the interface inversion asymmetry should be important to spin-orbit coupling in narrow quantum wells with no common atom in the barrier and well, whereas unimportant in other cases.

\subsection{Zeeman interaction with external magnetic field}

The Zeeman interaction for electron is written as

$$
H_{\mathrm{Z}}=\mu_{\mathrm{B}} \mathbf{S} \cdot \hat{g} \cdot \mathbf{B}
$$

where $\hat{g}$ is the $g$-tensor and $\mu_{\mathrm{B}}$ is the Bohr magneton. The $g$-tensor determines the spin precession (both the frequency and the direction) in the presence of an external magnetic field. It is hence an important quantity for spin dynamics. For typical III-V semiconductors, such as GaAs, the conduction band $g$-tensor is isotropic $\hat{g}_{e}=g_{e} \hat{1}$. In GaAs, at conduction band edge, $g_{e}=-0.44$, whereas for higher energy $g$ factor increases [223-225]. An empirical relation for the energy $\varepsilon$ dependence of the $g$ factor in GaAs was found from experiments as $g_{e}(\varepsilon)=-0.44+6.3 \varepsilon$ ( $\varepsilon$ in unit of $\mathrm{eV}$ ) [225-227]. Experiments indicated that the $g$ factor varies from -0.45 to -0.3 in the temperature range of $4 \sim 300 \mathrm{~K}$ [228-232]. Recent calculations [232] indicated that the temperature dependence of $g$-factor is mainly due to the combined effects of (i) the energy-dependent $g$ factor and (ii) the thermal dilatation of lattice.

In quantum wells the $g$ factors of the barrier material and well material are usually different or even of opposite signs. The $g$ factor can then be tuned by well width. For example, in GaAs/AlAs quantum wells: at large well width the electronic property is GaAs-like and $g_{e}<0$; at small well width the subband wavefunction is largely penetrated into the AlAs barrier and the electronic property is AlAs-like, hence $g_{e}>0$. This phenomenon has been observed in experiment [233]. Moreover, due to the confinement in quantum well, which reduces the symmetry of the system, the electron $g$-factor usually becomes anisotropic: the in-plane $g$-factor $g_{\|}$is different from the out-of-plane one $g_{\perp}$ [113, 234-236].

In parabolic GaAs/AlGaAs quantum wells, as the $\mathrm{Al}$ concentration is tuned almost continuously with position in the growth direction, the $g$-tensor becomes position dependent $\hat{g}_{e}(z)$. The effective $g$-tensor is an average over position $\hat{g}^{*}=\int d z \hat{g}_{e}(z)\left|\psi_{e}(z)\right|^{2}$ where $\psi_{e}(z)$ is the electron subband wavefunction. This fact enables efficient tuning of electron $g$-tensor by modulating the subband wavefunction via, e.g., gate-voltage [67, 78, 237]. A time-dependent gate-voltage can induce a modification to the Zeeman interaction $\delta H_{Z}=\mu_{\mathrm{B}} \mathbf{S} \cdot \delta \hat{g} \cdot \mathbf{B}(\delta \hat{g}$ is the change of $g$-tensor due to gate-voltage). In proper geometry, the time-dependent gate-voltage induces a spin precession perpendicular to the unperturbed spin precession. In such a way, electron spin resonance is achieved with time-dependent gate voltage. Such a spin resonance, which is called $g$-tensor modulation resonance, was first proposed and realized by Kato et al. [77].

The hole Zeeman interaction is complicated. In bulk system there are two terms which contribute to the hole Zeeman interaction,

$$
H_{\mathrm{Z}}^{h}=2 \kappa \mu_{\mathrm{B}} \mathbf{B} \cdot \mathbf{J}+2 q \mu_{\mathrm{B}} \mathbf{B} \cdot \mathcal{T} .
$$

Here $\kappa$ is the isotropic valence band $g$ factor, whereas $q$ is the anisotropic one. $\mathcal{T}=\left(J_{x}^{3}, J_{y}^{3}, J_{z}^{3}\right)$. In GaAs $\kappa=1.2$, whereas $q=0.01$ is much smaller and can always be neglected. It is noted that there is no direct coupling between spin-up and -down heavy holes $\left(J_{z}=\frac{3}{2},-\frac{3}{2}\right)$ in the Zeeman interaction unless in the much smaller anisotropic term [the second term in Eq. (24)].

\footnotetext{
${ }^{9}$ The study on the quantum well growth direction dependence of the interface induced spin-orbit coupling is presented in Ref. [220].
} 
In (001) quantum wells, the lowest subband is the heavy-hole subband if no strain is applied. According to the above analysis, for an in-plane magnetic field, the coupling of spin-up and -down states is rather small. Within the subband quantization, Löwdin partition method gives the dominant terms of the Zeeman interaction in the lowest heavy-hole subband as

$$
H_{\mathrm{Z}}^{\mathrm{HH}}=\frac{3 \kappa \mu_{\mathrm{B}}}{m_{0} \Delta_{11}^{h l}}\left\{\sigma_{x}\left[B_{x} \gamma_{2}\left(k_{x}^{2}-k_{y}^{2}\right)-2 B_{y} \gamma_{3} k_{x} k_{y}\right]+\sigma_{y}\left[2 B_{x} \gamma_{3} k_{x} k_{y}+B_{y} \gamma_{2}\left(k_{x}^{2}-k_{y}^{2}\right)\right]\right\},
$$

where $\Delta_{11}^{h l}$ denotes the splitting between the lowest heavy-hole subband and the lowest light-hole subband. Note that the in-plane $g$ factor of the lowest heavy-hole subband is k-dependent and the average of the $g$ factor is zero. The in-plane $g$-factor of the lowest heavy-hole subband varies significantly with the growth direction of the quantum well as pointed out by Winkler et al. [113, 238].

Zeeman interaction of holes in quantum wires has been investigated only recently. Experimentally, Danneau et al. found that the $g$-factor of holes in quantum wires along [233] direction is largely anisotropic [239]. Theoretically, Csontos and Zülicke reported that the hole $g$-factor in quantum wire can vary largely with subband [240] and can be tuned effectively by confinement [241]. These predictions were partly confirmed recently in the experiment by Chen et al. [242]. Similar results were found in hole quantum dots [243, 244]. The large anisotropy and variation of the hole $g$-factor in these quantum confined systems are not surprising, as (i) the symmetry of these structures is reduced and (ii) the hole spin-orbit coupling is very strong.

\subsection{Spin-orbit coupling in Wurtzite semiconductors and other materials}

In previous discussions, the spin-orbit coupling and $g$-factor in bulk and nanostructures of the zinc-blende III-V and II-VI compounds are reviewed. Besides these two important kinds of materials, there are several other kinds of materials with different structures which have also attracted much attention of the semiconductor spintronic community.

One of these materials is the wurtzite structure semiconductors, such as $\mathrm{GaN}, \mathrm{AlN}$ and $\mathrm{ZnO} 10$ In contrast to the zinc-blende semiconductors such as GaAs, the existence of hexagonal $c$-axis in wurtzite semiconductors leads to an intrinsic wurtzite structure inversion asymmetry in addition to the bulk inversion asymmetry [246, 247]. Therefore, the electron spin splittings include both the Dresselhaus effect [245, 248] (cubic in $k$ ) and Rashba effect (linear in k) [124, 125, 249-254]. In a recent work by $\mathrm{Fu}$ and $\mathrm{Wu}$ [245], a Kane-type Hamiltonian was constructed and the spin-orbit coupling for electron and hole bands were investigated in the full Brillion zone in bulk $\mathrm{ZnO}$ and $\mathrm{GaN}$.

For the states near the $\Gamma$ point $(\mathbf{k}=0)$, by choosing the $z$ axis along the $c$ axis of the crystal, the electron spin-orbit coupling in the conduction band reads [245]

$$
H_{\mathrm{SO}}^{e}=\left[\mathbf{\Omega}_{e}^{R}(\mathbf{k})+\mathbf{\Omega}_{e}^{D}(\mathbf{k})\right] \cdot \boldsymbol{\sigma} / 2
$$

with

$$
\mathbf{\Omega}_{e}^{R}(\mathbf{k})=2 \alpha_{e}\left(k_{y},-k_{x}, 0\right), \quad \mathbf{\Omega}_{e}^{D}(\mathbf{k})=2 \gamma_{e}\left(b k_{z}^{2}-k_{\|}^{2}\right)\left(k_{y},-k_{x}, 0\right) .
$$

Here $\boldsymbol{\Omega}_{e}^{R}(\mathbf{k})$ and $\boldsymbol{\Omega}_{e}^{D}(\mathbf{k})$ are the Rashba-type and Dresselhaus terms, respectively. $\alpha_{e}$ and $\gamma_{e}$ are the corresponding spin-orbit coupling coefficients. $b$ is a coefficient originating from the anisotropy induced by the lattice structure. It was found that in $\mathrm{ZnO}$ and $\mathrm{GaN}, b=3.855$ and 3.959 respectively [245]. The value of $\alpha_{e}$ and $\gamma_{e}$ are listed in Table 1 [245].

In wurtzite semiconductors, the valence bands consist of three separated bands: $\Gamma_{9 v}$ (heavy-hole, spin $\pm 3 / 2$ ), $\Gamma_{7 v}$ (light hole, spin $\pm 1 / 2$ ) and $\Gamma_{7^{\prime} v}$ (split-off hole, spin $\pm 1 / 2$ ). The spin-orbit couplings in these bands at small $k$ can also be written in the general form of $\frac{1}{2}\left[\boldsymbol{\Omega}_{i}^{R}(\mathbf{k})+\boldsymbol{\Omega}_{i}^{D}(\mathbf{k})\right] \cdot \boldsymbol{\sigma}$, which includes both the linear Rashba-type term $\frac{1}{2} \boldsymbol{\Omega}_{i}^{R}(\mathbf{k})$, and the cubic Dresselhaus term $\frac{1}{2} \boldsymbol{\Omega}_{i}^{D}(\mathbf{k})$, with $i=e, 9,7,7^{\prime}$ being the band index. For heavy-hole band $\Gamma_{9 v}$, the spin-orbit coupling fields are written as [245]

$$
\mathbf{\Omega}_{9}^{R}(\mathbf{k})=0, \quad \mathbf{\Omega}_{9}^{D}(\mathbf{k})=2 \gamma_{9}\left(k_{y}\left(k_{y}^{2}-3 k_{x}^{2}\right), k_{x}\left(k_{x}^{2}-3 k_{y}^{2}\right), 0\right) .
$$

\footnotetext{
${ }^{10} \mathrm{GaN}$ also has zinc-blende structure phase. The spin-orbit coupling in zinc-blende GaN was studied in Ref. [245]
} 
For valence bands $\Gamma_{7 v}$ (light-hole) and $\Gamma_{7^{\prime} v}$ (split-off hole), the spin-orbit coupling fields are the same as that given in Eq. (27) with only the coefficients $\alpha_{e}$ and $\gamma_{e}$ being replaced by $\alpha_{i}$ and $\gamma_{i}\left(i=7,7^{\prime}\right)$ (the coefficients for $\Gamma_{7 v}$ and $\Gamma_{7^{\prime} v}$ bands) respectively [245]. The coefficients $\alpha_{i}$ and $\gamma_{i}\left(i=e, 9,7,7^{\prime}\right)$ are listed in Table 1 [245] 11

Table 1: Rashba-type $\left(\alpha_{i}\right)$ (in meV·Å) and Dresselhaus $\left(\gamma_{i}\right)\left(\right.$ in $\mathrm{eV} \cdot \AA^{3}$ ) spin-orbit coupling coefficients in wurtzite $\mathrm{ZnO}$ and GaN at small momentum with $i=e, 9,7,7^{\prime}$. From Fu and Wu [245]. ( ${ }^{\mathrm{a}}$ from Ref. [249]; ${ }^{\mathrm{b}}$ from Ref. [250])

\begin{tabular}{lllllllll}
\hline \hline & $\alpha_{e}$ & $\alpha_{9}$ & $\alpha_{7}$ & $\alpha_{7^{\prime}}$ & $\gamma_{e}$ & $\gamma_{9}$ & $\gamma_{7}$ & $\gamma_{7^{\prime}}$ \\
\hline $\mathrm{ZnO}:$ & $1.1^{\mathrm{a}}$ & 0 & $35^{\mathrm{a}}\left(21^{\mathrm{b}}\right)$ & $51^{\mathrm{a}}$ & 0.33 & 0.09 & 6.3 & 6.1 \\
$\mathrm{GaN}:$ & $9.0^{\mathrm{b}}$ & 0 & $45^{\mathrm{b}}$ & $32^{\mathrm{b}}$ & 0.32 & 0.07 & 15.3 & 15.0 \\
\hline \hline
\end{tabular}

Interestingly, in two-dimensional electron system (quantum wells or heterojunctions) where the growth direction is along the $c$-axis, the linear-k spin-orbit coupling is of the same form for both the Dresselhaus and Rashba-type terms due to the crystal field. Additional contribution to the spin-orbit coupling comes from the electric field across the two-dimensional electron system, which is just the Rashba spin-orbit coupling [124, 125]. Therefore, all the linear in $\mathbf{k}$ terms are of the Rashba-type 12 which makes them indistinguishable in experiments. Indeed, experiments revealed that the spin-orbit coupling is dominated by the Rashba-type spin-orbit coupling, where no effect that signals spin-orbit coupling of the form of $\left(k_{x} \sigma_{x}-k_{y} \sigma_{y}\right)$ was observed [247, 256-260]. The measured Rashba parameter lies in the range of 0.6 to $8 \times 10^{-12} \mathrm{eV} \cdot \mathrm{m}$ for various conditions [251-254, 261-268].

Another kind of material of special interest is the so-called gapless semiconductor, such as HgTe, partly because that in $\mathrm{HgTe} / \mathrm{CdTe}$ quantum wells the quantum spin Hall effect was observed [269]. HgTe, which is also a zinc-blende II-VI semiconductor, has the same symmetry as CdTe and GaAs. However, the $\Gamma_{8}$ band, which consists of heavy and light holes in GaAs, is higher than the conduction band $\Gamma_{6}$. Moreover, the heavy-hole band is inverted, i.e., the effective mass of electron in the heavy-hole band is positive. On the other hand, the conduction band $\Gamma_{6}$, becomes hole-like. In HgTe/CdTe quantum wells, the relative position of the $\Gamma_{6}$ and $\Gamma_{8}$ bands can be tuned by well width. At the crossing band point a massless Dirac spectrum is realized [270]. The spin-orbit coupling in these bands was investigated both theoretically [271] and experimentally [147, 272-278], where the Rashba spin-orbit coupling was found to be very large $\left(\simeq 4 \times 10^{-11} \mathrm{eV} \cdot \mathrm{m}\right)$ in two-dimensional electron system.

Spin dynamics in silicon has attracted renewed interest recently due to the experimental advancement in silicon spintronic devices [149, 150, 279-282] and also due to recent development in spin qubits based on silicon quantum dots [283-285] or donor bound electrons [286-289]. Silicon has a diamond structure with space inversion symmetry, hence bulk silicon does not have the Dresselhaus spin-orbit coupling. In two-dimensional or other nanostructures, the Rashba spin-orbit coupling due to structure inversion asymmetry emerges [124, 125, 290]. Moreover, the interface inversion asymmetry also contributes to new spin-orbit coupling, which can be either the Rashba-type or the linearDresselhaus-type or their combinations depending on the symmetry of the interface [291, 292]. The effect of the electric field on electron spin-orbit coupling in $\mathrm{Si} / \mathrm{SiGe}$ quantum well was discussed in Ref. [293]. In practice, the doping inhomogeneity can induce a random (in-plane position dependent) electric field along the growth direction even in nominally symmetrically doped quantum wells. This random electric field can induce a random Rashba spin-orbit coupling [294]. Similarly, the surface roughness can also induces a random spin-orbit coupling [291]. Experimentally, the electron spin-orbit coupling in silicon quantum wells was determined via the anisotropy of the electron spin resonance frequency and line-width [295-297]. Hole spin-orbit coupling in silicon quantum wells was studied in Refs. [298, 299].

\footnotetext{
${ }^{11}$ Using these coefficients, Buß et al. found reasonable agreement between calculated spin relaxation times and the experimentally measured ones recently [255].

${ }^{12}$ The symmetry of the cubic term is also the same as the linear one.
} 


\subsection{Hyperfine interaction}

The origin of the hyperfine interaction is that the nuclear spins feel the magnetic field generated by carriers due to their spins and orbital angular momentums. For conduction band electron, which has $s$-wave symmetry, the magnetic field generated by electron orbital motion is negligible. The electron hyperfine interaction is of the form [3, 300]

$$
H_{\mathrm{hf}}=\sum_{i, v} \frac{2 \mu_{0}}{3} \beta_{v} \gamma_{e} \gamma_{v} \delta\left(\mathbf{r}-\mathbf{R}_{i, v}\right) \mathbf{S} \cdot \mathbf{I}_{i, v} .
$$

Here $\mu_{0}$ is vacuum permeability, $\gamma_{e}=2 \mu_{\mathrm{B}}$ and $i$ is the index of the Wigner-Seitz cell. For III-V and II-VI compounds (such as GaAs), there are two atoms (such as Ga and As) in each Wigner-Seitz cell. And the nuclei of certain atoms have their isotopes (such as ${ }^{69} \mathrm{Ga}$ and ${ }^{71} \mathrm{Ga}$ ). The index $v$ labels both the atomic site in the Wigner-Seitz cell and the isotopes. $\beta_{v}$ is the abundance and $\sum_{v} \beta_{v}=N_{\text {atm }}$ with $N_{\text {atm }}$ being the number of atoms within a Wigner-Seitz cell. $N_{\text {atm }}=2$ for zinc-blende structures. $\gamma_{v}=g_{v} \mu_{N}$ with $g_{v}$ and $\mu_{N}$ representing the $g$ factor of the $v$ nucleus and the nuclear magneton respectively. $\mathbf{R}_{i, v}$ and $\mathbf{I}_{i, v}$ represent the position and spin of the $v$ nucleus in $i$-th Wigner-Seitz cell. In practical calculation it is assumed that the isotopes are distributed uniformly. Within the envelope function approximation, the electron hyperfine interaction is written as

$$
H_{\mathrm{hf}}=\sum_{i, v} A_{\nu} v_{0}\left|\psi\left(\mathbf{R}_{i}\right)\right|^{2} \mathbf{S} \cdot \mathbf{I}_{i, v}=\mathbf{h} \cdot \mathbf{S},
$$

where $A_{v}=\frac{2 \mu_{0}}{3} \gamma_{e} \beta_{v} \gamma_{v}\left|u_{c}\left(\mathbf{R}_{v}\right)\right|^{2} N_{0}$ and $\mathbf{h}=\sum_{i, v} A_{v} v_{0}\left|\psi\left(\mathbf{R}_{i}\right)\right|^{2} \mathbf{I}_{i, v}$. Here $v_{0}$ is the volume of the Wigner-Seitz cell and $N_{0}=1 / v_{0}, u_{c}$ is Bloch wave amplitude. $A=\sum_{v} A_{v}$ measures the strength of the hyperfine interaction. In GaAs, possible isotopes are ${ }^{69} \mathrm{Ga},{ }^{71} \mathrm{Ga}$ and ${ }^{75} \mathrm{As}$, which all have spin $I=3 / 2$. Their natural abundances are $\beta_{69} \mathrm{Ga}=0.6$, $\beta_{\urcorner_{1} \mathrm{Ga}}=0.4$ and $\beta_{{ }^{71} \mathrm{As}}=1$. The average strength of the hyperfine interaction is $A \simeq 90 \mu \mathrm{eV}$. In silicon, the possible isotopes are ${ }^{28} \mathrm{Si}$ and ${ }^{29} \mathrm{Si}$ with natural abundances $\beta_{28} \mathrm{Si}=0.9533$ and $\beta_{29} \mathrm{Si}=0.0467$. In silicon, the main isotope ${ }^{28} \mathrm{Si}$ has no nuclear spin and only the minor isotope ${ }^{29} \mathrm{Si}$ has spin $I=1 / 2$. Hence the average hyperfine interaction in silicon $A \sim 0.2 \mu \mathrm{eV}$ is much smaller than that in GaAs.

For holes, as the valence band Bloch wavefunction is very small at the nuclei position (which is a property of $p$-wave symmetry), the contact hyperfine interaction is negligible. However, the long range dipole-dipole interaction and the interaction between electron orbital angular momentum and nuclear spin do not vanish for holes 13 Within the envelope function approximation, Fischer et al. showed that the hyperfine interaction for heavy-holes in quantum well is of the Ising form [301]

$$
H_{\mathrm{hf}}^{h}=\sum_{i, v} A_{v}^{h} v_{0}\left|\psi\left(\mathbf{R}_{i}\right)\right|^{2} S^{z} I_{i, v}^{z}
$$

where $S^{z}$ is the $z$-component of the heavy-hole spin and $A_{v}^{h}$ is the hole hyperfine coupling constant. Fischer et al. estimated that the hole hyperfine coupling constant $A_{v}^{h}$ is about $-10 \mu \mathrm{eV}$ in GaAs [301], about one order of magnitude smaller than the electron one.

Besides inducing spin relaxation, the hyperfine interaction can also transfer the nonequilibrium carrier spin polarization to nuclear spin system, which is called the dynamic nuclear polarization [3, 302-310]. Via the hyperfine interaction, periodic optical pumping of electron spin polarization acts as a periodic magnetic field on nuclear spin and induces a nuclear spin resonance [311]. As nuclear spin relaxes much slower than electron spin, nuclear spin was proposed as long-lived "quantum memory" for spin qubits [312, 313]. Such proposal was realized in experiment in ${ }^{31} \mathrm{P}$ donors in isotopically pure ${ }^{28} \mathrm{Si}$ crystal [314].

\subsection{Exchange interaction with magnetic impurities}

The exchange interaction between carrier and magnetic impurities, such as Mn, is very important in diluted magnetic semiconductors [315], as it is the physical origin of various magnetic orders in these materials. For example, the $d$ orbital electrons of Mn impurities mix with $s$ (conduction band) or $p$ (valence band) electrons in the semiconductor

\footnotetext{
${ }^{13}$ In contrast, these interactions are zero for (conduction band) electrons due to $s$-wave symmetry.
} 
matrix, which leads to the exchange interactions between carriers and Mn impurities [315, 316]. These interactions, known as the $s-d$ and $p-d$ exchange interactions, can be written as

$$
H_{\mathrm{sd}}=-\sum_{i} J_{\mathrm{sd}} \mathbf{S} \cdot \mathbf{S}_{d}^{(i)} \delta\left(\mathbf{r}-\mathbf{R}_{i}\right), \quad H_{\mathrm{pd}}=-\sum_{i} J_{\mathrm{pd}} \mathbf{J} \cdot \mathbf{S}_{d}^{(i)} \delta\left(\mathbf{r}-\mathbf{R}_{i}\right) .
$$

Here $\mathbf{S}_{d}^{(i)}$ is the $i$ th Mn spin at $\mathbf{R}_{i} . \mathbf{S}$ and $\mathbf{J}$ represent the electron and hole spins respectively. $J_{\mathrm{sd}}$ and $J_{\mathrm{pd}}$ stand for the $s-d$ and $p-d$ exchange constants respectively. The exchange constants, which are basic parameters of magnetic semiconductors, have been extensively studied both theoretically and experimentally [for review in (III,Mn)V, see Ref. [114] and references therein; for (II,Mn)VI, see Ref. [115]]. To illustrate the strength of the $s(p)-d$ exchange interaction, we give two examples: in GaMnAs, $N_{0} J_{\mathrm{sd}} \approx-0.1 \mathrm{eV}$ [233, 317], $N_{0} J_{\mathrm{pd}} \approx-1 \mathrm{eV}$ [114]; in CdMnTe, $N_{0} J_{\mathrm{sd}} \approx 0.22 \mathrm{eV}, N_{0} J_{\mathrm{pd}} \approx-0.88 \mathrm{eV}[115]$ where $N_{0}=1 / v_{0}$ with $v_{0}$ being the volume of the unit cell.

\subsection{Exchange interaction between carriers}

The exchange interaction between carriers originates from the combination of the carrier-carrier Coulomb interaction and the permutative antisymmetry of the wavefunction of the fermionic carrier system. The exchange interaction usually increases with the overlap between the wavefunction of the carriers. Therefore, it is usually strong in localized carrier system, such as carriers in quantum dots or those bound to ionized impurities. Experiments have shown that the exchange interaction between electrons in quantum dots can be tuned via the magnetic field14 [319, 320] or by the gate voltage [38, 86, 319], varying from several meV to very small. The exchange interaction between electrons bound to adjacent donors is believed to be responsible for the spin relaxation of the donor bound electrons [39, 40, 321- 324, 324, 325]. The underlying physics is that due to the spin-orbit coupling, the exchange interaction becomes anisotropic which does not conserve the total spin of the electron system any more and hence leads to spin relaxation.

Besides, the exchange interaction between extended carriers also shows up in spin dynamics. For example, the exchange interaction between electrons and hole 15 leads to electron spin relaxation, which is called the Bir-AronovPikus mechanism. Specifically, this exchange interaction consists of two parts: the short-range part and the long-range part. The short-range part is written as [118]

$$
H_{\mathrm{SR}}=-\frac{1}{V} \frac{1}{2} \frac{\Delta E_{\mathrm{SR}}}{\left|\phi_{3 \mathrm{D}}(0)\right|^{2}} \hat{\mathbf{J}} \cdot \hat{\mathbf{S}} \delta_{\mathbf{K}, \mathbf{K}^{\prime}},
$$

where $\Delta E_{\mathrm{SR}}$ is the exchange splitting of the exciton ground state and $\left|\phi_{\mathrm{BD}}(0)\right|^{2}=1 /\left(\pi a_{0}^{3}\right)$ with $a_{0}$ being the exciton Bohr radius. $\mathbf{K}=\mathbf{k}_{e}+\mathbf{k}_{h}$ is the sum of the electron $\mathbf{k}_{e}$ and hole $\mathbf{k}_{h}$ wave-vectors of the interacting electron-hole pair. $V$ is the volume of the sample. The long-range part reads [118]

$$
H_{\mathrm{LR}}=\frac{1}{V} \frac{3}{8} \delta_{\mathbf{K}, \mathbf{K}^{\prime}} \frac{\Delta E_{\mathrm{LT}}}{\left|\phi_{3 \mathrm{D}}(0)\right|^{2}}\left(\hat{M}_{z} \hat{S}_{z}+\frac{1}{2} \hat{M}_{-} \hat{S}_{+}+\frac{1}{2} \hat{M}_{+} \hat{S}_{-}\right),
$$

where $\Delta E_{\mathrm{LT}}$ is the longitudinal-transverse splitting; $\hat{M}_{z}, \hat{M}_{-}$and $\hat{M}_{+}\left(=\hat{M}_{-}^{\dagger}\right)$ are operators in hole spin space. $\hat{S}_{ \pm}=$ $\hat{S}_{x} \pm i \hat{S}_{y}$ are the electron spin ladder operators. The expressions for $\hat{M}_{z}$ and $\hat{M}_{-}$are given below (in the order of hole spin $\left.J_{z}=\frac{3}{2}, \frac{1}{2},-\frac{1}{2},-\frac{3}{2}\right)$,

$$
\hat{M}_{z}=\frac{1}{K^{2}}\left[\begin{array}{cccc}
-K_{\|}^{2} & \frac{2}{\sqrt{3}} K_{z} K_{-} & \frac{1}{\sqrt{3}} K_{-}^{2} & 0 \\
\frac{2}{\sqrt{3}} K_{z} K_{+} & \left(\frac{1}{3} K_{\|}^{2}-\frac{4}{3} K_{z}^{2}\right) & -\frac{4}{3} K_{z} K_{-} & -\frac{1}{\sqrt{3}} K_{-}^{2} \\
\frac{1}{\sqrt{3}} K_{+}^{2} & -\frac{4}{3} K_{z} K_{+} & \left(\frac{4}{3} K_{z}^{2}-\frac{1}{3} K_{\|}^{2}\right) & \frac{2}{\sqrt{3}} K_{z} K_{-} \\
0 & -\frac{1}{\sqrt{3}} K_{+}^{2} & \frac{2}{\sqrt{3}} K_{z} K_{+} & K_{\|}^{2}
\end{array}\right],
$$

\footnotetext{
${ }^{14}$ This was first predicted by Burkard et al. [318].

${ }^{15}$ The electron-hole exchange interaction is in fact from the conduction-valence band mixing [107].
} 


$$
\hat{M}_{-}=\frac{1}{K^{2}}\left[\begin{array}{cccc}
0 & 0 & 0 & 0 \\
-\frac{2}{\sqrt{3}} K_{\|}^{2} & \frac{4}{3} K_{z} K_{-} & \frac{2}{3} K_{-}^{2} & 0 \\
\frac{4}{\sqrt{3}} K_{z} K_{+} & -\frac{8}{3} K_{z}^{2} & -\frac{4}{3} K_{z} K_{-} & 0 \\
2 K_{+}^{2} & -\frac{4}{\sqrt{3}} K_{z} K_{+} & -\frac{2}{\sqrt{3}} K_{\|}^{2} & 0
\end{array}\right] .
$$

Here $K_{ \pm}=K_{x} \pm i K_{y}$ and $K_{\|}^{2}=K_{x}^{2}+K_{y}^{2}$. The short-range and long-range parts of the electron-hole exchange interaction can be written in a compact form $(i=z,+,-)$,

$$
H_{\mathrm{ex}}=\frac{1}{V} \delta_{\mathbf{K}, \mathbf{K}^{\prime}}\left(\hat{\mathcal{J}}_{z}(\mathbf{K}) \hat{S}_{z}+\frac{1}{2} \hat{\mathcal{J}}_{-}(\mathbf{K}) \hat{S}_{+}+\frac{1}{2} \hat{\mathcal{J}}_{+}(\mathbf{K}) \hat{S}_{-}\right) \quad \text { with } \quad \mathcal{J}_{i}(\mathbf{K})=\frac{1}{\left|\phi_{3 D}(0)\right|^{2}}\left[\frac{3}{8} \Delta E_{\mathrm{LT}} \hat{M}_{i}(\mathbf{K})-\frac{1}{2} \Delta E_{\mathrm{SR}} \hat{J}_{i}\right] .
$$

In semiconductor nanostructures, e.g., in quantum wells, the above electron-hole exchange interaction should be written in the subband bases. Such expressions have been derived in Ref. [118]. Besides, it is shown that the quantum confinement can enhance the electron-hole exchange interaction in quantum wells [119].

Unlike the electron-hole exchange interaction, the exchange interaction between free electrons has almost been ignored by the community until several years ago. It was first pointed out by Weng and $\mathrm{Wu}$ that the exchange interaction between free electrons (the Coulomb Hartree-Fock term), which acts as an effective magnetic field along the spin polarization direction, plays important role in spin dynamics [44]. For example, if the spin polarization is along the $z$ axis, the effective magnetic field from the Hartree-Fock term reads [44, 110],

$$
B_{\mathrm{HF}}(\mathbf{k})=\sum_{\mathbf{q}} V_{\mathbf{q}}\left(f_{\mathbf{k}-\mathbf{q} \uparrow}-f_{\mathbf{k}-\mathbf{q} \downarrow}\right) / g_{e} \mu_{\mathrm{B}},
$$

where $f_{\mathbf{k}-\mathbf{q} \uparrow}$ and $f_{\mathbf{k}-\mathbf{q} \downarrow}$ are the distributions on the spin-up and spin-down bands respectively. $g_{e}$ is electron $g$-factor and $V_{\mathbf{q}}=e^{2} /\left[\epsilon_{0} \kappa_{0} q^{2} \epsilon(\mathbf{q})\right]$ in bulk with $\epsilon(\mathbf{q})$ being the dielectric function. At large spin polarization, $B_{\mathrm{HF}}$ can be as large as a few tens of Tesla for an electron density of $4 \times 10^{11} \mathrm{~cm}^{-2}$ at $120 \mathrm{~K}$ in GaAs quantum well [44]. Recently, the effect of the exchange interaction between electrons was observed in experiments in high mobility two-dimensional electron system [41, 42, 326]. The Hartree-Fock effective magnetic field has also been observed in other experiments recently [327]. Detailed review on the Hartree-Fock effective magnetic field on spin dynamics is given in Sec. 5.4.3.

\section{Spin relaxation and spin dephasing in semiconductors}

After briefly introducing various spin interactions, we now move to one of the central issues in this review: spin relaxation and dephasing, i.e., the dissipative part of the spin dynamics. As stated before, any fluctuation or inhomogeneity in spin interactions can lead to spin relaxation and spin dephasing. From the spin interactions, one can identify the possible spin relaxation/dephasing mechanisms. In what follows, we first introduce the concepts of spin relaxation and dephasing in time domain as well as in spacial domain. We then introduce the relevant spin relaxation mechanisms in semiconductors and their nanostructures.

\subsection{Spin relaxation and spin dephasing}

Simply speaking, spin relaxation is related to the nonequilibrium population decay of the spin-resolved energy eigenstates, whereas spin dephasing is related to the destruction of phase coherence of these eigenstates. For single spin system with isotropic $g$-factor, spin relaxation is related to the decay of spin polarization parallel to the external magnetic field, whereas spin dephasing is related to the decay of spin polarization transverse to the magnetic field. Spin relaxation time (denoted as $T_{1}$ ) and spin dephasing time $\left(T_{2}\right)$ are quantities to characterize the time scale of spin relaxation and spin dephasing 16 Microscopically, spin relaxation and spin dephasing are usually related to different processes. Although spin relaxation also inevitably leads to spin dephasing, there are processes which only contribute to spin dephasing, called pure dephasing processes [328]. Spin relaxation and spin dephasing in single spin system are caused by the unavoidable coupling with the fluctuating environment, such as the phonon system, nuclear spin system and other electrons nearby. These processes are always irreversible.

\footnotetext{
${ }^{16}$ Spin decay does not have to be exponential. However, in most cases, a characteristic decay time can be identified.
} 
Often there are many electron spins forming a spin ensemble. For an ensemble of spins, spin polarization can decay without coupling to any environment: when spin precession frequencies or directions vary from part to part within the ensemble, the total spin polarization gets a free-induction-decay due to destructive interference. This variation is called the inhomogeneous broadening [329, 330]. For a finite ensemble with independent spins, the decay of total spin polarization can be reversible: after some time, spins in all parts of the ensemble rotate to the same direction 17 However, quite often, the ensemble is very large, e.g., there are $\sim 10^{16} \mathrm{~cm}^{-3}$ electrons in $n$-doped GaAs. In this regime, the spin decay due to inhomogeneous broadening can not be reversed automatically. However, the inhomogeneous broadening induced spin decay can still be removed by the technique of spin echo. Standard spin echo is achieved by a $\pi$-pulse magnetic field which is perpendicular to the inhomogeneous broadened (effective) magnetic field, to invert the sign of the phase gained through spin precession so that the gained phase can be gradually canceled out in the subsequent evolution. The ensemble spin relaxation and spin dephasing times including the inhomogeneous broadening are usually denoted as $T_{1}^{*}$ and $T_{2}^{*}$ respectively, whereas the irreversible (echoed) spin relaxation and spin dephasing times are denoted as $T_{1}$ and $T_{2}$ respectively. In the following, when we need not to discriminate the two, we simply denote the spin relaxation time and dephasing time as $\tau_{s}$ and called it spin lifetime. There are a lot of factors which contribute to inhomogeneous broadening, such as, the k-dependent spin-orbit coupling [332] or the energy(or k)-dependent $g$-factor [333, 334]. In quantum dots, due to the spin-orbit coupling, electron or hole $g$-factor is size and geometry dependent [335]. The variations of the size and geometry of quantum dots in a quantum dot ensemble also lead to the inhomogeneous broadening [331]. Finally, the time and/or space variations of the nuclear hyperfine field also lead to the inhomogeneous broadening.

Spin diffusion and transport also suffers spin relaxation. In the absence of the inhomogeneous broadening, spin diffusion length $L_{s}$ is related to spin lifetime $\tau_{s}$ as $L_{s}=\sqrt{D_{s} \tau_{s}}$, where $D_{s}$ is the spin diffusion constant. However, in semiconductors it has been shown that the inhomogeneous broadening usually dominates spin diffusion [25, 28, 336], which breaks down the above relation [32, 35]. The inhomogeneous broadening in spin diffusion and transport is different from that in spin relaxation in time domain: electron at different $\mathbf{k}$ state has different velocity and/or spin precession frequency $\mathbf{\Omega}(\mathbf{k})$ (e.g., due to the spin-orbit coupling), making the spin propagation frequency along the spin diffusion direction $(\hat{\mathbf{n}}) \mathbf{k}$-dependent $\sim \boldsymbol{\Omega}(\mathbf{k}) /(\mathbf{k} \cdot \hat{\mathbf{n}})$ [25, 28, 29, 336, 337]. This inhomogeneous broadening can not be removed by traditional spin-echo. However, it can be tuned via controlling spin-orbit coupling [18, 29, 32, 34, 337, 338]. For example, when both the Rashba and Dresselhaus spin-orbit couplings exist, by tuning the two to be comparable, spin diffusion length can be largely increased [18, 29, 32, 34, 337-340]. Such enhancement of spin diffusion length has been achieved in a recent experiment, where the control over both the doping asymmetry and well width was shown to be effective to manipulate the relative strength of the Rashba and Dresselhaus spin-orbit couplings and hence the spin diffusion length [37]. Studies on spin diffusion in the literature are reviewed in Section 6 and 7. Below we concentrate on spin relaxation and spin dephasing in time domain, where the basic physics and the mechanisms responsible for spin relaxation and spin dephasing are reviewed.

\subsubsection{Single spin relaxation and spin dephasing due to fluctuating magnetic field}

As stated above, fluctuations in spin interaction due to the coupling with the environment are the origin of spin relaxation and spin dephasing in single spin system. In this subsection, we discuss generally the spin relaxation and spin dephasing in single spin system, where the fluctuations in spin interaction are simply characterized as fluctuating magnetic fields. These fluctuating magnetic fields are assumed to have a correlation time $\tau_{c}$ which is much shorter than the spin relaxation/dephasing time, so that the Markovian approximation is justified [341]. We also assume that the fluctuations are weak so that they can be treated within the Born approximation. The spin dynamics is then solved by the standard Born-Markov method [341] 18

The Hamiltonian is simply given by

$$
H=\frac{1}{2}\left[\omega_{0} \hat{\mathbf{n}}_{z}+\omega(t)\right] \cdot \sigma
$$

where $\hat{\mathbf{n}}_{z}$ is a unit vector along $z$ direction. $\omega_{0}$ and $\omega(t)$ are spin precession frequencies due to the static and fluctuating magnetic fields respectively. The correlation of the fluctuating magnetic fields is assumed to be (for $i, j=x, y, z$ )

\footnotetext{
${ }^{17}$ This phenomenon was recently observed in experiments in spin dephasing in an ensemble of self-assemble quantum dots [331]

${ }^{18}$ It is noted that discussions in this subsection are similar to those in Ref. [22] in Sec. IV B2.
} 
$\overline{\omega_{i}(t) \omega_{j}\left(t^{\prime}\right)}=\delta_{i, j} \overline{\omega_{i}^{2}} e^{-\left|t-t^{\prime}\right| / \tau_{c}}$, with the overline denoting the ensemble average. The requirement of the shortness of the correlation time $\tau_{c}$ and the weakness of the fluctuating magnetic fields is equivalent to $\sqrt{\sum_{i} \overline{\omega_{i}^{2}}} \tau_{c} \ll 1$. Spin relaxation time and spin dephasing time are obtained by solving the Born-Markov equation of motion. After standard derivation one obtains that [22],

$$
\frac{1}{T_{1}}=\frac{\left(\overline{\omega_{x}^{2}}+\overline{\omega_{y}^{2}}\right) \tau_{c}}{1+\omega_{0}^{2} \tau_{c}^{2}}, \quad \frac{1}{T_{2}}=\overline{\omega_{z}^{2}} \tau_{c}+\frac{\left.\overline{\left(\omega_{x}^{2}\right.}+\overline{\omega_{y}^{2}}\right) \tau_{c}}{2\left(1+\omega_{0}^{2} \tau_{c}^{2}\right)} .
$$

From these results, one may find several features of spin relaxation/dephasing. First, a trivial result, only the noise magnetic fields perpendicular to the spin polarization lead to spin decay as only these fields can rotate spin. Second, in the absence of static magnetic field, spin relaxation/dephasing rate is proportional to the noise correlation time $\tau_{c}$. That is to say that the more fluctuating the noise (the shorter correlation time) is, the more ineffective it is. This counter-intuitive result is known as motional narrowing [329]. Third, a static magnetic field suppresses spin relaxation/dephasing caused by the fluctuating magnetic field perpendicular to it. The underlying physics is better understood in the fashion of what was presented in the review article by Fabian et al. [22]: In the presence of magnetic field, the spin-flip scattering terms acquire a dynamic phase. However, the scattering terms which conserve the spin along the magnetic field do not acquire such dynamic phase. The spin-flip term is then proportional to

$$
\int_{0}^{t \gg \tau_{c}} d t^{\prime} \overline{\omega_{i}(0) \omega_{i}\left(t^{\prime}\right)} \exp \left(-\mathrm{i} \omega_{0} t^{\prime}\right)+\text { H.c. } \propto \overline{\omega_{i}^{2}} \frac{\tau_{c}}{\omega_{0}^{2} \tau_{c}^{2}+1}
$$

Therefore the effect of fluctuating magnetic field perpendicular to the static magnetic field is suppressed.

In the following, we illustrate several limits which are frequently encountered in spin relaxation in semiconductors. Eq. (40) can be written as

$$
1 / T_{2}=1 / T_{2}^{\prime}+1 /\left(2 T_{1}\right)
$$

where $1 / T_{2}^{\prime}=\overline{\omega_{z}^{2}} \tau_{c}$. Therefore, in general case, $T_{2} \leq 2 T_{1}$. In the isotropic noise case, $\overline{\omega_{x}^{2}}=\overline{\omega_{v}^{2}}=\overline{\omega_{z}^{2}}$, at very low static magnetic field $\omega_{0} \tau_{c} \ll 1$, spin relaxation time is equal to spin dephasing time, $T_{1}=T_{2}$ [342]. At very large static magnetic field, spin relaxation vanishes $1 / T_{1} \rightarrow 0$, whereas the spin dephasing rate is finite, $1 / T_{2}=1 / T_{2}^{\prime}$. This is a pure dephasing case. The noise can be anisotropic. For example, when there are only transverse noises, $\omega_{x} \neq 0$, $\omega_{y} \neq 0$ and $\omega_{z}=0, T_{2}=2 T_{1} 19$ Another example, there are only longitudinal noises, $\omega_{x}=\omega_{y}=0$ and $\omega_{z} \neq 0$. In this case, there is no spin relaxation $1 / T_{1}=0$ but spin dephasing rate is still finite, i.e., $1 / T_{2}=\overline{\omega_{z}^{2}} \tau_{c}$. This is another pure dephasing case. If $\overline{\omega_{x}^{2}}+\overline{\omega_{y}^{2}} \ll \overline{\omega_{z}^{2}}$, then $T_{2} \ll T_{1}$.

\subsubsection{Ensemble spin relaxation and spin dephasing: a simple model}

To illustrate the role of the inhomogeneous broadening in the ensemble spin relaxation and spin dephasing, here we introduce a simple model which actually has been well studied in the context of semiconductor optics. Denote the conduction band as "spin up", the valence band as "spin down" and their distributions as $f_{\mathbf{k}}^{\uparrow}$ and $f_{\mathbf{k}}^{\downarrow}$. The interband coherence, like spin coherence, is a complex variable $\rho_{\mathbf{k}}=\rho_{c v}(\mathbf{k}) e^{-\mathrm{i} \omega t}$ ( $\omega$ is the laser light frequency). Hence the model system is equivalent to a spin ensemble. The optical relaxation and dephasing is described by the Bloch equation [345, 346]

$$
\partial_{t} \mathbf{S}_{\mathbf{k}}=\omega_{\mathbf{k}} \times \mathbf{S}_{\mathbf{k}}-\left[\begin{array}{ccc}
\frac{1}{T_{2}} & 0 & 0 \\
0 & \frac{1}{T_{2}} & 0 \\
0 & 0 & \frac{1}{T_{1}}
\end{array}\right]\left(\mathbf{S}_{\mathbf{k}}-\mathbf{S}_{\mathbf{k}}^{0}\right) .
$$

In semiconductor optics, $\omega_{\mathbf{k}}=\left(-d_{c v} \mathcal{E}, 0, \delta_{\mathbf{k}}\right)$ where $d_{c v}$ is the interband optical dipole, $\mathcal{E}$ is the laser electric field and $\delta_{\mathbf{k}}=\varepsilon_{c, k}-\varepsilon_{v, k}-\omega=k^{2} / 2 m_{R}+E_{g}-\omega$ is the detuning with the frequency of light. For simplicity, take $\omega=E_{g}$ and

\footnotetext{
${ }^{19} \mathrm{An}$ example is the spin relaxation in GaAs quantum dots due to the spin-orbit coupling and phonon scattering as demonstrated by Golovach et al. [343]. This conclusion was later generalized to spin relaxation due to arbitrary phonon scattering at low temperature when only the lowest two Zeeman levels are relevant by Jiang et al. [344].
} 
hence $\delta_{\mathbf{k}}=k^{2} /\left(2 m_{R}\right) \cdot m_{R}$ is the reduced effective mass of electron-hole pair. $\mathbf{S}_{\mathbf{k}}=\left(\operatorname{Re} \rho_{\mathbf{k}},-\operatorname{Im} \rho_{\mathbf{k}},\left[f_{\mathbf{k}}^{\uparrow}-f_{\mathbf{k}}^{\downarrow}\right] / 2\right)$ and the equilibrium polarization is $\mathbf{S}_{\mathbf{k}}^{0}=(0,0,-1 / 2) . T_{1}$ and $T_{2}$ represent the irreversible decay due to fluctuations. In the absence of laser field $\mathcal{E}=0$, the solution of the equation gives the transverse polarization as

$$
\left[\begin{array}{l}
S_{\mathbf{k}}^{x}(t) \\
S_{\mathbf{k}}^{y}(t)
\end{array}\right]=\left[\begin{array}{cc}
\cos \left(\delta_{\mathbf{k}} t\right) & -\sin \left(\delta_{\mathbf{k}} t\right) \\
\sin \left(\delta_{\mathbf{k}} t\right) & \cos \left(\delta_{\mathbf{k}} t\right)
\end{array}\right]\left[\begin{array}{c}
S_{\mathbf{k}}^{x}(0) \\
S_{\mathbf{k}}^{y}(0)
\end{array}\right] e^{-t / T_{2}} .
$$

As the oscillation frequency $\delta_{\mathbf{k}}$ varies with $\mathbf{k}$, the total transverse polarizations $S_{x}(t)=\sum_{\mathbf{k}} S_{\mathbf{k}}^{x}(t)$ and $S_{y}(t)=\sum_{\mathbf{k}} S_{\mathbf{k}}^{y}(t)$ get an additional decay due to inhomogeneous broadening besides $e^{-t / T_{2}}$. In two-dimensional system, assuming a simplified initial condition: $S_{\mathbf{k}}^{x}(0)=0, S_{\mathbf{k}}^{y}(0)=-1$ for $k \leq k_{m}$ and $S_{\mathbf{k}}^{y}(0)=0$ for $k>k_{m}$, one obtains

$$
S_{x}(t)=e^{-t / T_{2}} \sum_{\mathbf{k}} \sin \left(\delta_{\mathbf{k}} t\right)=e^{-t / T_{2}} \frac{k_{m}^{2}}{4 \pi} \frac{1-\cos \left(\delta_{k_{m}} t\right)}{t / T_{2}^{\prime \prime}}
$$

The inhomogeneous broadening induces a power law decay with a characteristic time $T_{2}^{\prime \prime}=2 m_{R} / k_{m}^{2}$. Now the optical dephasing rate is

$$
1 / T_{2}^{*}=1 / T_{2}+1 / T_{2}^{\prime \prime}
$$

The inhomogeneous broadening induced dephasing $1 / T_{2}^{\prime \prime}$ can be removed by photon echo. Exerting a $\pi$-pulse along the $y$-axis at time $T$, the transverse polarization after the pulse is

$$
\left[\begin{array}{c}
S_{\mathbf{k}}^{x}(T) \\
S_{\mathbf{k}}^{y}(T)
\end{array}\right]=\left[\begin{array}{cc}
\cos \left(\delta_{\mathbf{k}} T\right) & \sin \left(\delta_{\mathbf{k}} T\right) \\
-\sin \left(\delta_{\mathbf{k}} T\right) & \cos \left(\delta_{\mathbf{k}} T\right)
\end{array}\right]\left[\begin{array}{c}
S_{\mathbf{k}}^{x}(0) \\
S_{\mathbf{k}}^{y}(0)
\end{array}\right] e^{-T / T_{2}}
$$

The transverse polarization at time $t=2 T$ is given by

$$
\left[\begin{array}{c}
S_{\mathbf{k}}^{x}(2 T) \\
S_{\mathbf{k}}^{y}(2 T)
\end{array}\right]=\left[\begin{array}{cc}
\cos \left(\delta_{\mathbf{k}} T\right) & -\sin \left(\delta_{\mathbf{k}} T\right) \\
\sin \left(\delta_{\mathbf{k}} T\right) & \cos \left(\delta_{\mathbf{k}} T\right)
\end{array}\right]\left[\begin{array}{c}
S_{\mathbf{k}}^{x}(T) \\
S_{\mathbf{k}}^{y}(T)
\end{array}\right] e^{-T / T_{2}}=\left[\begin{array}{c}
S_{\mathbf{k}}^{x}(0) \\
S_{\mathbf{k}}^{y}(0)
\end{array}\right] e^{-2 T / T_{2}} .
$$

Now the inhomogeneous broadening induced dephasing is removed and only the irreversible dephasing $1 / T_{2}$ remains. Theoretically, the decay rate of the incoherently summed optical coherence $P(t)=\sum_{\mathbf{k}}\left|\rho_{\mathbf{k}}(t)\right|=\sum_{\mathbf{k}}\left|S_{\mathbf{k}}^{x}(t)+i S_{\mathbf{k}}^{y}(t)\right|$, can be used to obtain the irreversible optical dephasing rate $1 / T_{2}$ [347], as the phase of $\rho_{\mathbf{k}}(t)$ is removed.

Similar to the case of optical dephasing, the inhomogeneous broadening of the spin precession induces spin dephasing in spin ensemble [334]. This inhomogeneous broadening can be removed by spin echo [348]. As pointed out by $\mathrm{Wu}$ and co-workers, the irreversible spin dephasing can be obtained from the decay of the incoherently summed spin coherence [334, 336, 349, 350], similar to that in optical dephasing.

In system with isotropic $g$-tensor, the spin polarization parallel to the magnetic field does not precess. However, in general, the $g$-tensor can be anisotropic and spin polarization parallel to the magnetic field can also precess. In this case, spin polarization along the magnetic field may also suffer the inhomogeneous broadening induced decay. We then have both $T_{1}^{*}$ and $T_{2}^{*}$ in such situation 20 An example of such ensemble is the spins in self-assembled quantum dots [352-354].

\subsection{Spin relaxation mechanisms}

In this subsection, we introduce spin relaxation mechanisms in semiconductors. Generally speaking, any fluctuation or inhomogeneity of spin interaction can induce spin relaxation and dephasing. However, there are several mechanisms which are more efficient than others. For the materials widely used in spintronics, such as III-V and II-VI semiconductors, there are only a few relevant spin relaxation/dephasing mechanisms, such as the Elliott-Yafet mechanism [103, 104], the D'yakonov-Perel' mechanism [101, 102], the Bir-Aronov-Pikus mechanism [105, 106] and the $g$-tensor inhomogeneity mechanism [333, 334]. These mechanisms dominate spin relaxation and dephasing in metallic regime. In the insulating regime, other mechanisms such as the anisotropic exchange interaction [40, 322] and the hyperfine interaction [3] prevail. In semiconductor quantum dots, spin relaxation/dephasing is dominated by the electron-phonon scattering and the hyperfine interaction as well as their combinations. In magnetically doped semiconductors, the exchange interaction with magnetic impurities can also play an important role in spin relaxation and dephasing. Below we give a brief introduction to these mechanisms.

\footnotetext{
${ }^{20}$ A simple example demonstrating the same physics is presented in Ref. [351].
} 


\subsubsection{Elliott-Yafet mechanism}

The Elliott-Yafet mechanism was first proposed by Elliott [104] and Yafet [103] during their study on spin relaxation in silicon and alkali metals. It was pointed out by Elliott that due to the spin-orbit interaction the electronic eigenstates (Bloch states) mix spin-up and spin-down states. For example [21, 104],

$$
\begin{aligned}
& \Psi_{\mathbf{k} n \uparrow}(\mathbf{r})=\left[a_{\mathbf{k} n}(\mathbf{r})|\uparrow\rangle+b_{\mathbf{k} n}(\mathbf{r})|\downarrow\rangle\right] e^{i \mathbf{k} \cdot \mathbf{r}}, \\
& \Psi_{\mathbf{k} n \downarrow}(\mathbf{r})=\left[a_{-\mathbf{k} n}^{*}(\mathbf{r})|\downarrow\rangle-b_{-\mathbf{k} n}^{*}(\mathbf{r})|\uparrow\rangle\right] e^{i \mathbf{k} \cdot \mathbf{r}},
\end{aligned}
$$

where $a_{\mathbf{k} n}(\mathbf{r})$ and $b_{\mathbf{k} n}(\mathbf{r})$ possess the lattice periodicity. These two Bloch states are connected by time reversal and space inversion operators $[21,104]$. Usually the spin mixing is very small, i.e., $|b| \ll 1$ and $|a| \approx 1$. When the spinorbit interaction is much smaller than the band splitting, an estimation of $|b|$ from perturbation theory gives [21, 355] $|b| \sim \max \left\{L_{\mathrm{SO}} / \Delta E\right\}$, where $L_{\mathrm{SO}}$ is the spin-orbit interactions with other bands and $\Delta E$ is the band distances. In the presence of such spin mixing, any spin-independent scattering can cause spin flip and hence spin relaxation. It should be emphasized that without scattering the spin-mixing alone can not lead to any spin relaxation. Besides, there is another process leading to spin relaxation: the phonon modulation of the spin-orbit interaction. As the spin-orbit interaction is induced by the periodic lattice ions, the lattice vibration can then directly couple to spin and lead to spin-flip. Such kind of process was first considered by Overhauser within jullium model for metal [356] and then by Yafet with specific band structure [103]. This process is called the Yafet process, whereas the previous one due to the spin mixing is called the Elliott process.

Elliott observed a relation, the "Elliott relation", between spin lifetime $\tau_{s}$ and the deviation of the electron $g$-factor from that of the free electron $g_{0}=2.0023[104]$ : $1 / \tau_{s} \approx(\Delta g)^{2} / \tau_{p}$ where $\Delta g=g-g_{0}$ and $\tau_{p}$ is the average momentum scattering time. The relation is based on the observation that $\Delta g \sim|b|$ by perturbation theory. The Elliott relation was verified experimentally by Monod and Beuneu, who observed an empirical factor of 10 , i.e., $1 / \tau_{s} \simeq 10(\Delta g)^{2} / \tau_{p}$ [357]. Of course these relations are rough, the specific ratio of $\tau_{s} / \tau_{p}$ depends on specific scattering and band structure. Systematic theoretical study was given by Yafet [103], where the microscopic band structure and electron-phonon scattering were considered to obtain the spin lifetime. Yafet gave a relation between the spin lifetime $\tau_{s}$ and the resistivity $\rho: 1 / \tau_{s} \sim\left\langle b^{2}\right\rangle \rho$ [103]. The Yafet relation was tested experimentally by Monod and Beuneu [358]. It was found that in many materials the Yafet relation agrees well with experiments [21, 358]. However, it is not consistent with the experimental results in $\mathrm{MgB}_{2}$ where the spin relaxation rate is not proportional to the resistivity above $150 \mathrm{~K}$. The problem was solved by Simon et al. [355] recently via generalizing the Elliott-Yafet theory to the regime where the scattering-induced spectral broadening of the quasi-particle $1 / \tau_{p}$ is comparable with the band gap. This condition is satisfied in $\mathrm{MgB}_{2}$ because one of the band across the Fermi surface is very close to the nearest band $(\simeq 0.2 \mathrm{eV})$ and the electron-phonon interaction is strong. In this regime, the spin lifetime is given by,

$$
\tau_{s}^{-1}=\frac{L_{\mathrm{eff}}^{2} \tau_{p}}{1+\Delta \omega_{\mathrm{eff}}^{2} \tau_{p}^{2}},
$$

where $\Delta \omega_{\text {eff }}$ is the average band gap and $L_{\text {eff }}$ is the interband spin-orbit interaction. In the weak scattering or large band-gap regime, $\Delta \omega_{\mathrm{eff}} \tau_{p} \gg 1$, the above equation returns to the Yafet relation, $1 / \tau_{s}=\left(L_{\mathrm{eff}} / \Delta \omega_{\mathrm{eff}}\right)^{2} / \tau_{p}$. Spin relaxation due to the Elliott-Yafet mechanism in polyvalent metals (such as aluminum) was studied by Fabian and Das Sarma [359, 360]. Through realistic calculation, they found that the electron spin relaxation is significantly enhanced at the Brillouin zone boundaries, special symmetry points and lines of accidental degeneracy. The total spin relaxation rate is then determined by the spin relaxation rates at these "spin-hot-spots" [359, 360].

In III-V or II-VI semiconductors zinc-blende structure, for realistic calculation of the Elliott-Yafet spin relaxation, one should start from the Kane Hamiltonian. By Löwdin partitioning (block-diagonalization), the conduction band wavefunction is transformed to $\tilde{\Psi}_{c}(\mathbf{k})=U \Psi_{n}(\mathbf{k})$ where $U$ is the unitary matrix for Löwdin partitioning. This transformation mixes spin-up and -down states a little, which enables spin flip by spin-independent fluctuation. After the transformation $U$, the conduction band matrix element of a spin-independent fluctuation of the lattice potential (including the electron-electron interaction) $V_{c \mathbf{k}, c \mathbf{k}^{\prime}}=\left\langle c \mathbf{k}|V| c \mathbf{k}^{\prime}\right\rangle$, changes into $\tilde{V}_{c \mathbf{k}, c \mathbf{k}^{\prime}}=\left\langle c \mathbf{k}\left|U^{\dagger} V U\right| c \mathbf{k}^{\prime}\right\rangle$, which may contain spin-flip part. The spin-flip interaction consists of long-range and short-range parts. The long-range part comes from the first order perturbation of the wavefunction (both in $U$ and $U^{\dagger}$ ) and the intraband spin-independent fluctuation. The short-range part comes from the combination of $U$ (or $U^{\dagger}$ ) and the interband electron-phonon interactions. For example, for $U=e^{S_{\mathbf{k}}}$, the lowest order long-range part is given by $\left\langle c \mathbf{k}\left|\frac{1}{2}\left(S_{\mathbf{k}}^{\dagger} S_{\mathbf{k}}^{\dagger} V+2 S_{\mathbf{k}}^{\dagger} V S_{\mathbf{k}^{\prime}}+V S_{\mathbf{k}^{\prime}} S_{\mathbf{k}^{\prime}}\right)\right| c \mathbf{k}^{\prime}\right\rangle$, 
whereas the lowest order short-range part is given by $\left\langle c \mathbf{k}\left|S_{\mathbf{k}}^{\dagger} V+V S_{\mathbf{k}^{\prime}}\right| c \mathbf{k}^{\prime}\right\rangle$. The long-range interaction is the Elliott process, whereas the short-range interaction is the Yafet process.

After the transformation, the leading term of the long-range part is given by [117]

$$
\tilde{V}_{c \mathbf{k}, c \mathbf{k}^{\prime}}=V_{c \mathbf{k}, c \mathbf{k}^{\prime}}\left[1-\mathrm{i} \lambda_{c}\left(\mathbf{k} \times \mathbf{k}^{\prime}\right) \cdot \sigma\right],
$$

where $\lambda_{c}=\eta(1-\eta / 2) /\left[3 m_{e} E_{g}(1-\eta / 3)\right]$ with $\eta=\Delta_{\mathrm{SO}} /\left(\Delta_{\mathrm{SO}}+E_{g}\right)$. The spin lifetime due to long-range part of the Elliott-Yafet mechanism is

$$
\tau_{s}^{-1}=A \frac{\left\langle\varepsilon_{\mathbf{k}}\right\rangle^{2}}{E_{g}^{2}} \eta^{2}\left(\frac{1-\eta / 2}{1-\eta / 3}\right)^{2} \tau_{p}^{-1},
$$

where the numerical factor $A \sim 1$ depending on the specific scattering. The short-range interaction due to the interband electron-optical-phonon interaction is given by

$$
\tilde{V}_{\mathbf{k}, \mathbf{k}^{\prime}}^{\mathrm{OP}}=-\frac{1}{3} \frac{\eta d_{2}\left[\mathbf{U} \times\left(\mathbf{k}+\mathbf{k}^{\prime}\right)\right] \cdot \boldsymbol{\sigma}}{\left.2 m_{e} E_{g}(1-\eta / 3)\right]^{1 / 2}} .
$$

Here $\mathbf{U}$ is the relative displacement of the two atoms in a unit cell which can be expressed as combinations of phonon creation and annihilation operators [117]. Assuming that the longitudinal and transversal optical phonons have the same frequency $\omega_{\mathrm{LO}}=\omega_{\mathrm{TO}}$, the spin relaxation due to the short-range interaction reads [117]

$$
\tau_{s}^{-1}=A_{\mathrm{sr}} \frac{\left\langle\varepsilon_{\mathbf{k}}\right\rangle^{2}}{E_{g} E_{0}} \frac{\eta^{2}}{1-\eta / 3} \tau_{p}^{-1}
$$

Here $E_{0}=C^{2} /\left(4 d_{2} m_{e}\right)$ with $C=e \omega_{\mathrm{LO}} \sqrt{4 \pi D\left(\kappa_{\infty}^{-1}-\kappa_{0}^{-1}\right)}\left(D\right.$ is the volume density, $\kappa_{0}$ and $\kappa_{\infty}$ are the static and high frequency dielectric constants) and $A_{\mathrm{sr}} \sim 1$. The short-range interaction due to the interband electron-acoustic-phonon interaction can be derived similarly. The explicit form is given in Ref. [117]. Usually the long-range interaction is more important than the short-range one in III-V semiconductors [21, 117].

The Elliott-Yafet spin relaxation in in bulk InSb at low temperature was first studied by Chazalviel [361], where only the electron-impurity scattering is considered. Very recently, Jiang and Wu studied the Elliott-Yafet spin relaxation in bulk III-V semiconductors with all relevant scatterings (i.e., electron-impurity, electron-phonon, electronelectron Coulomb and electron-hole Coulomb scatterings) included in a fully microscopic fashion [110]. The ElliottYafet spin relaxation in bulk silicon was studied systematically by Cheng et al. [362], where the Elliott and the Yafet processes interfere destructively and the spin relaxation is largely suppressed [362].

Similarly, both the admixture of different spin states due to the Löwdin partitioning and the phonon modulation of spin-orbit interaction lead to the Elliott-Yafet spin relaxation for holes and split-off holes.

\subsubsection{D'yakonov-Perel' mechanism}

In III-V and II-VI semiconductors, due to the bulk inversion asymmetry in lattice structure, spin-orbit coupling emerges in conduction band. In semiconductor nanostructures, the structure and interface inversion asymmetry further contributes additional spin-orbit coupling. Spin-orbit coupling is equivalent to a $\mathbf{k}$-dependent effective magnetic field $H_{\text {SO }}=\frac{1}{2} \boldsymbol{\Omega}(\mathbf{k}) \cdot \boldsymbol{\sigma}$, where $\boldsymbol{\Omega}(\mathbf{k})$ is the spin precession frequency. In the presence of momentum scattering, electron changes its momentum $\mathbf{k}$ randomly, hence spin precesses randomly between adjacent scattering events. This randomwalk-like evolution of spin phase leads to spin relaxation. This spin relaxation mechanism is called the D'yakonovPerel' mechanism [101, 102]. There are two regimes for the D'yakonov-Perel' spin relaxation: (i) strong scattering regime where $\langle\Omega\rangle \tau_{p} \ll 1(\langle\ldots\rangle$ stands for the average over the electron ensemble), and (ii) weak scattering regime where $\langle\Omega\rangle \tau_{p} \gtrsim 1$. Here $\tau_{p}$ is the momentum scattering time. The spin lifetime in regime (i) can be estimated as $1 / \tau_{s}=\left\langle\Omega^{2}\right\rangle \tau_{p}$ according to the random walk theory. This spin relaxation has the salient feature of motional narrowing, i.e., stronger momentum scattering leads to longer spin lifetime. In regime (ii) the momentum scattering no longer impedes spin relaxation. In contrast, via the spin-orbit coupling, momentum scattering provides a spin relaxation channel [334, 363]. In this regime, stronger momentum scattering leads to shorter spin lifetime [363]. Analytic results with only the electron-impurity scattering give that the irreversible spin lifetime is $\tau_{s}=2 \tau_{p}[363-$ 365]. As $\langle\Omega\rangle \tau_{p} \gtrsim 1$, the spin precession due to the spin-orbit coupling is not inhibited by the scattering. As a 
consequence, the ensemble spin polarization oscillates at zero magnetic field [363, 364]. Besides, such prominent spin precession leads to the free induction decay. The ensemble spin lifetime is then limited by both the irreversible decay and the free induction decay $\tau_{s}^{-1} \simeq \sqrt{\left\langle\Omega^{2}\right\rangle}[21,363,365]$. Both regime (i) and regime (ii) have been realized experimentally in GaAs quantum wells [366, 367]. Especially, the crossover from regime (ii) to regime (i) has been observed by Brand et al. [368].

The characteristic of the D'yakonov-Perel' spin relaxation is that during adjacent momentum scatterings spins precess coherently. Hence a spin-echo at a time scale comparable to or smaller than the momentum scattering time $\tau_{p}$ can efficiently suppress the D'yakonov-Perel' spin relaxation. Such a proposal was given in a recent work by Pershin [348].

In most cases the system is in the strong scattering regime. In this regime, some analytical results about spin lifetime can be obtained for isotropic band, if one assumes that (1) the carrier-carrier scattering can be neglected 21 (2) the system is near equilibrium 22 and (3) the electron-phonon scattering can be treated in the elastic scattering approximation. Within these assumptions and approximations, for three-dimensional case, following Pikus and Titkov, the evolution of spin polarization along $z$ direction is [117]

$$
\partial_{t} S_{z}=-\tilde{\tau}_{l}\left[S_{z}\left\langle\Omega_{x}^{l} \Omega_{x}^{l}+\Omega_{y}^{l} \Omega_{y}^{l}\right\rangle-S_{x}\left\langle\Omega_{x}^{l} \Omega_{z}^{l}\right\rangle-S_{y}\left\langle\Omega_{y}^{l} \Omega_{z}^{l}\right\rangle\right],
$$

where $\langle\ldots\rangle$ represents the average over the direction of $\mathbf{k}$. The equations for the evolution of $S_{x}$ and $S_{y}$ can be obtained by index permutation. The momentum scattering time $\tilde{\tau}_{l}$ is given by

$$
\tilde{\tau}_{l}^{-1}=\int_{-1}^{1} W(\theta)\left[1-P_{l}(\cos \theta)\right] d \cos \theta .
$$

The integer $l$ is determined by the angular dependence of the spin-orbit field. The above results assume that the spinorbit coupling Hamiltonian satisfies $\hat{H}_{\mathrm{SO}}=\frac{1}{2} \mathbf{\Omega}^{l} \cdot \boldsymbol{\sigma}=\sum_{m} \hat{C}_{l m} Y_{m}^{l}\left(\theta_{\mathbf{k}}, \phi_{\mathbf{k}}\right)$, where $\theta_{\mathbf{k}}$ and $\phi_{\mathbf{k}}$ are the angular coordinates of $\mathbf{k}$ in spherical coordinates, $Y_{m}^{l}$ stands for the spherical hamonics and the coefficients $\hat{C}_{l m}$ are $2 \times 2$ matrices. Here the summation is taken over $m$, whereas $l$ is fixed. For example, $l=1$ for linear spin-orbit coupling due to strain and $l=3$ for Dresselhaus cubic spin-orbit coupling. If there are both linear and cubic spin-orbit couplings, the summation over $l$ should also be added into the above equations. According to Eq. (56), with the summation over $l$, the spin relaxation tensor is given by [3, 21, 22]

$$
\left(\tau_{s}^{-1}\right)_{i j}=\sum_{l} \frac{\tilde{\tau}_{p}}{\gamma_{l}}\left[\left\langle\left|\mathbf{\Omega}^{l}\right|^{2}\right\rangle \delta_{i j}-\left\langle\Omega_{i}^{l} \Omega_{j}^{l}\right\rangle\right],
$$

where $\gamma_{l}=\tilde{\tau}_{p} / \tilde{\tau}_{l}$ and $\tilde{\tau}_{p}=\tilde{\tau}_{1}$. For two-dimensional case, expanding the spin-orbit coupling as $\hat{H}_{\mathrm{SO}}=\sum_{l} \frac{1}{2} \mathbf{\Omega}^{l} e^{\mathrm{i} l \theta_{\mathbf{k}}} \cdot \boldsymbol{\sigma}$, one obtains a similar result [369, 370]

$$
\left(\tau_{s}^{-1}\right)_{i j}=\sum_{l} \frac{\tilde{\tau}_{p}}{\gamma_{l}}\left[\left|\mathbf{\Omega}^{l}\right|^{2} \delta_{i j}-\Omega_{i}^{l} \Omega_{j}^{-l}\right]
$$

with $\tilde{\tau}_{l}^{-1}=\int_{0}^{2 \pi} W(\theta)(1-\cos l \theta) d \theta$. To give an example, consider electron spin relaxation due to the D'yakonov-Perel' mechanism in bulk III-V semiconductors. In the absence of strain, the electron spin-orbit coupling comes solely from the Dresselhaus term,

$$
\boldsymbol{\Omega}(\mathbf{k})=2 \gamma_{D}\left[k_{x}\left(k_{y}^{2}-k_{z}^{2}\right), k_{y}\left(k_{z}^{2}-k_{x}^{2}\right), k_{z}\left(k_{x}^{2}-k_{y}^{2}\right)\right] .
$$

In this case spin relaxation is isotropic

$$
\left(\tau_{s}^{-1}\right)_{i j}=\delta_{i j} \frac{\tilde{\tau}_{p}}{\gamma_{3}} \frac{8}{105}\left(2 \gamma_{D}\right)^{2} k^{6}
$$

Here $\gamma_{3}$ depends on relevant scattering: for ionized-impurity scattering $\gamma_{3} \simeq 6$; for acoustic-phonon scattering $\gamma_{3} \simeq 1$; for optical-phonon scattering $\gamma_{3} \simeq 41 / 6$. After the average over electron distribution, the spin relaxation rate is given by [21],

$$
\tau_{s}^{-1} \simeq Q^{\prime} \tau_{m} \alpha^{2}\left\langle\varepsilon_{\mathbf{k}}^{3}\right\rangle / E_{g} .
$$

\footnotetext{
${ }^{21}$ It was first pointed out by Wu and Ning [334] that although normal (not umklapp) carrier-carrier scattering does not contribute to the mobility, it contributes to the D'yakonov-Perel' spin relaxation as it randomizes the momentum.

${ }^{22}$ That is, electron distribution is close to the equilibrium distribution and the spin polarization is very small.
} 
$\alpha=2 \gamma_{D} \sqrt{2 m_{e}^{3} E_{g}}$ is a dimensionless parameter. $\tau_{m}=\left\langle\tilde{\tau}_{p}\left(\varepsilon_{\mathbf{k}}\right) \varepsilon_{\mathbf{k}}\right\rangle /\left\langle\varepsilon_{\mathbf{k}}\right\rangle \underline{23} Q^{\prime} \sim 0.1$ is a numerical constant depending on the relevant momentum scattering [21] $Q^{\prime}=\frac{128}{3675} \gamma_{3}^{-1}\left(v+\frac{7}{2}\right)\left(v+\frac{5}{2}\right)$, where the power law $\tilde{\tau}_{p} \sim \varepsilon_{\mathbf{k}}^{v}$ is assumed for each kind of momentum scattering. For nondegenerate electron system, one obtains $\left(Q=\frac{105}{8} Q^{\prime}\right)$

$$
\tau_{s}^{-1} \simeq Q \tau_{m} \alpha^{2}\left(k_{\mathrm{B}} T\right)^{3} / E_{g},
$$

where $Q \sim 1$ depending on relevant scattering: $Q \simeq 1.5$ for ionized impurity scattering, $Q \simeq 3$ for longitudinal optical phonon scattering [371], $Q \simeq 0.8$ for piezoelectric acoustic phonon scattering, and $Q \simeq 2.7$ for acoustic phonon scattering due to deformation potential [3, 21]].

Finally, it should be noted that caution must be taken on the assumptions and approximations used in the above results. For example, in intrinsic bulk GaAs at temperature below $100 \mathrm{~K}$, the electron-electron and electron-hole scatterings dominate the momentum scattering. The assumption that the carrier-carrier scattering can be neglected does not hold. Therefore, the above results fail [110]. It has been shown that the electron-electron scattering is important for spin relaxation in high mobility two-dimensional system where the above results also fail [366, 372].

The influence of the magnetic field on the D'yakonov-Perel' spin relaxation comes from two factors: the Zeeman interaction and the orbital effect 24 The Zeeman interaction leads to the Larmor spin precession and induces a slowdown of the relaxation for spin component parallel to the Larmor spin precession direction [117],

$$
\tau_{s}(B) \simeq \tau_{s}(0)\left[1+\left(\omega_{\mathrm{L}} \tau_{c}\right)^{2}\right],
$$

where $\omega_{\mathrm{L}}$ is the Larmor frequency. $\tau_{c}=\gamma_{l}^{-1} \tilde{\tau}_{p}$ is the correlation time of the random spin precession due to spin-orbit coupling. This result is similar to Eq. (40). The Larmor spin precession also mixes the relaxation rates of the spin components perpendicular to the Larmor spin precession direction. This may lead to considerable effect on the spin relaxation in semiconductor nanostructures where the spin relaxation tensor is usually anisotropic [199, 377, 388]. In non-quantizing magnetic field, the orbital effect induces the cyclotron motion where the electron velocity (hence its k) rotates under the Lorentz force. As the spin-orbit effective magnetic field $\mathbf{\Omega}(\mathbf{k})$ is $\mathbf{k}$ dependent, the rotation in $\mathbf{k}$ leads to the rotation in the spin-orbit field. This leads to the reduction of the D'yakonov-Perel' spin relaxation which is related to the rotating component of $\boldsymbol{\Omega}(\mathbf{k})[117,376]$

$$
\tau_{s}(B) \simeq \tau_{s}(0)\left[1+\left(\omega_{c} \tau_{c}\right)^{2}\right],
$$

where $\omega_{c}=e B / m_{e}$ is the cyclotron frequency. Note that the ratio of the Larmor frequency and the cyclotron frequency is $\omega_{\mathrm{L}} / \omega_{c}=\left|g_{e}\right| m_{e} /\left(2 m_{0}\right)$. In semiconductors, as $m_{e}$ is usually smaller than $m_{0}$ and often $\left|g_{e}\right|<2$, the ratio is always much smaller than 1 (in GaAs, it is $\simeq 0.015$ ). Hence the cyclotron effect is stronger than the Zeeman interaction [117]. The reduction of spin relaxation rate is saturated at high magnetic field, where Landau level quantization dominates. At such high magnetic field, electron spin relaxes similar to that in localized states or that in quantum dot [382-387].

The hole D'yakonov-Perel' spin relaxation is different from the electron one mainly in two aspects: (1) the hole spin-orbit coupling is usually much stronger, and (2) hole has spin $J=3 / 2$. Due to the strong spin-orbit coupling, the D'yakonov-Perel' mechanism is very efficient in hole system and the spin lifetime is usually very short. For example,

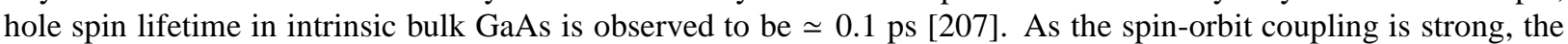
hole system is usually in the weak scattering regime of the D'yakonov-Perel' spin relaxation [206, 208, 363]. Lü et al. found that in two-dimensional hole system the spin polarization oscillates without an exponential decay in the weak scattering regime [363]. In this case, the spin lifetime is not easily characterized. However, the incoherently summed spin coherence $P(t)=\sum_{\mathbf{k}}\left|\rho_{\mathbf{k}}(t)\right|\left(\rho_{\mathbf{k}}\right.$ denotes the spin coherence) shows good exponential decay and from its decay rate the irreversible spin dephasing rate is obtained [363]. Another salient feature of hole spin system is that hole has spin $J=3 / 2$. As a consequence the spin density matrix of hole system is $4 \times 4$ which contains spin coherence that does not correspond to any spin polarization. Also, the hole spin-orbit coupling may not be equivalent to an effective magnetic field as it can be high powers of the hole spin operator $\mathbf{J}$ (such as $(\mathbf{k} \cdot \mathbf{J})^{2}$ in the Luttinger Hamiltonian

\footnotetext{
${ }^{23}$ In the remaining part of the paper, we denote all these quantities $\left(\tilde{\tau}_{l}, \tilde{\tau}_{p}\right.$ and $\left.\tau_{m}\right)$ as $\tau_{p}$ in qualitative discussions, if we can.

${ }^{24}$ Effect of magnetic field on the D'yakonov-Perel' spin relaxation was studied in the semiclassical limit in Refs. [117, 333, 373-381] as well as in the quantum limit in Refs. 382-387.
} 
[Eq. [17]]). In the work of Winkler [389], the hole spin density matrix is decomposed by the spin multipole series to provide a more systematic understanding on the spin-dependent phenomena in hole system. Winkler found that the hole spin-orbit coupling can induce transfer between different spin multipoles, while the magnetic field can only induce spin precession of the spin dipole (i.e., the spin polarization) in bulk hole system [389]. The transfer of the hole spin multipoles under the hole spin-orbit coupling was found to influence the hole spin polarization relaxation due to the D'yakonov-Perel' mechanism [208].

\subsubsection{Bir-Aronov-Pikus mechanism}

It was proposed by Bir, Aronov and Pikus that the electron-hole exchange scattering can lead to efficient electron spin relaxation in $p$-type semiconductors [105, 106]. According to the electron-hole exchange interaction given in Eq. (37), within elastic scattering approximation, spin lifetime limited by the Bir-Aronov-Pikus mechanism is given by the Fermi Golden rule,

$$
\frac{1}{\tau_{s}(\mathbf{k})}=4 \pi \sum_{\substack{\mathbf{q}, \mathbf{k}^{\prime} \\ m, m^{\prime}}} \delta\left(\varepsilon_{\mathbf{k}}+\varepsilon_{\mathbf{k}^{\prime} m^{\prime}}^{h}-\varepsilon_{\mathbf{k}-\mathbf{q}}-\varepsilon_{\mathbf{k}^{\prime}+\mathbf{q} m}^{h}\right)\left|\mathcal{J}_{\mathbf{k}^{\prime}+\mathbf{q} m}^{(+) \mathbf{k}^{\prime} m^{\prime}}\right|^{2} f_{\mathbf{k}^{\prime} m^{\prime}}^{h}\left(1-f_{\mathbf{k}^{\prime}+\mathbf{q} m}^{h}\right) .
$$

Here $\varepsilon_{\mathbf{k} m}^{h}$ is hole energy with spin index $m, \mathcal{J}$ is the electron-hole exchange interaction matrix element [see Eq. (37) in Sec. 2.9] and $f_{h}$ is hole distribution function. As hole spin and momentum relax very fast, $f_{h}$ is taken as hole equilibrium distribution. In bulk semiconductors, there are several facts which could help to reduce the above complicated equation: (i) the heavy-hole density of states is much larger than the light-hole one (thanks to the much larger heavy-hole effective mass), which makes the contribution to the spin relaxation rate mainly from the heavy hole; (ii) the heavy-hole effective mass is much larger than the electron one, which enables the elastic scattering approximation. Using these approximations and including only the short-range exchange interaction, one obtains a simple result for nondegenerate hole system [117]

$$
\tau_{s}^{-1}=\frac{2}{\tau_{0}} n_{h} a_{\mathrm{B}}^{3} \frac{\left\langle v_{\mathbf{k}}\right\rangle}{v_{\mathrm{B}}},
$$

where $a_{\mathrm{B}}$ is the exciton Bohr radius, $1 / \tau_{0}=(3 \pi / 64) \Delta E_{\mathrm{SR}}^{2} /\left(E_{\mathrm{B}}\right)$ with $E_{\mathrm{B}}$ being the exciton Bohr energy, $n_{h}$ is the hole density, $\left\langle v_{\mathbf{k}}\right\rangle=\left\langle k / m_{e}\right\rangle$ is the average electron velocity, and $v_{\mathrm{B}}=1 /\left(m_{R} a_{\mathrm{B}}\right)$ with $m_{R} \approx m_{e}$ being the reduced mass of the interacting electron-hole pair. In the presence of localized holes, the equation is improved to be [117]

$$
\tau_{s}^{-1}=\frac{2}{\tau_{0}} N_{A} a_{\mathrm{B}}^{3} \frac{\left\langle v_{\mathbf{k}}\right\rangle}{v_{\mathrm{B}}}\left(\frac{n_{h}}{N_{A}}+\frac{5}{3} \frac{N_{A}-n_{h}}{N_{A}}\right)
$$

where $N_{A}$ is the acceptor density. For degenerate hole system [117],

$$
\tau_{s}^{-1} \simeq \frac{3}{\tau_{0}} n_{h} a_{\mathrm{B}}^{3} \frac{\left\langle v_{\mathbf{k}}\right\rangle}{v_{\mathrm{B}}} \frac{k_{\mathrm{B}} T}{E_{\mathrm{F}}^{h}}
$$

with $E_{\mathrm{F}}^{h}$ denoting the hole Fermi energy. In an interacting electron-hole plasma, electron and hole attract each other and there is an enhancement of the electron-hole exchange interaction due to this attraction. This enhancement is described by the Sommerfeld factor. For unscreened Coulomb potential, the Sommerfeld factor is $|\psi(0)|^{2}=\frac{2 \pi}{\varepsilon_{\mathbf{k}} / E_{\mathrm{B}}} /[1-$ $\left.\exp \left(-\frac{2 \pi}{\varepsilon_{\mathrm{k}} / E_{\mathrm{B}}}\right)\right]$. With this factor, spin relaxation is enhanced, $1 / \tau_{s}^{\prime}=|\psi(0)|^{4} / \tau_{s}$. However, for a completely screened Coulomb potential, there is no enhancement, $|\psi(0)|^{2}=1$. The effect of the Sommerfeld factor was discussed in Refs. [117, 390]. It should be noted that the long-range electron-hole exchange interaction can not be neglected, often (such as in GaAs) it is more important than the short-range one [110]. Hence the above analytical formulae is quite limited, unless $E_{\mathrm{SR}}$ is substituted by some proper average of the whole (both the short-range and the longrange) electron-hole exchange interaction. The analytical expressions for the Bir-Aronov-Pikus spin relaxation in bulk materials and in quantum wells with both short-range and long-range interactions are given in Ref. [390].

Spin relaxation rate due to the Bir-Aronov-Pikus mechanism is usually calculated via Eq. (66), which actually implies the elastic scattering approximation [118, 390, 390, 391]. Recently Zhou and Wu [109] reinvestigated the Bir-Aronov-Pikus spin relaxation without such approximation from the fully microscopic kinetic spin Bloch equation approach [44, 334, 350]. They found that spin relaxation rate was largely overestimated at low temperature in the 
previous theories. The underlying physics is that at low temperature the Pauli blocking impedes spin-flip in fully occupied states [109]. The spin-flip is only allowed around the chemical potential, which suppresses the Bir-AronovPikus spin relaxation at low temperature. Amo et al. found similar arguments and results [392].

Finally, holes also suffer the Bir-Aronov-Pikus spin relaxation. However, as hole spin-orbit coupling is strong, the D'yakonov-Perel' mechanism is very efficient and the Bir-Aronov-Pikus mechanism rarely shows up. The BirAronov-Pikus mechanism may become important for holes in heavily $n$-doped quantum wells, where the D'yakonovPerel' mechanism is suppressed by the hole-electron and hole-impurity scatterings and the spin lifetime can be rather long ( $\gtrsim 500 \mathrm{ps})[393-395]$.

\subsection{4. g-tensor inhomogeneity}

Under a given magnetic field, spin precession (both direction and frequency) is determined by the $g$-tensor. Margulis and Margulis first proposed a spin relaxation mechanism in the presence of magnetic field due to the momentumdependent $g$-factor [333]. Later Wu and Ning also pointed out that energy-dependent $g$-factor gives rise to an inhomogeneous broadening [334] in spin precession and hence results in spin relaxation. Without scattering the inhomogeneous broadening only leads to a reversible spin relaxation. Any scattering, including the electron-electron scattering, which randomizes spin precession, results in irreversible spin relaxation [334]. In the irreversible regime, under a magnetic field $\mathbf{B}$ along $j$ direction, the relaxation rate of the spin component along the $i$ direction $[i, j=x, y, z]$ can be obtained in analogy with Eq. (40),

$$
\frac{1}{\tau_{s, i i}}=\left(\mu_{\mathrm{B}} B\right)^{2} \sum_{l \neq i} \frac{\left(\overline{g_{l j}^{2}}-{\overline{g_{l j}}}^{2}\right) \tau_{c}}{1+\left(\mu_{\mathrm{B}} B\right)^{2} \sum_{n \neq l}{\overline{g_{n j}}}^{2} \tau_{c}^{2}} .
$$

Here $l, n=x, y, z, g_{i j}$ is the $g$-tensor and $\tau_{c}$ is the correlation time of spin precession limited by scattering. $\overline{g_{l j}}$ denotes the ensemble average of $g_{l j}$. The above equation holds only in the motional narrowing regime, i.e.,

$\left(\mu_{\mathrm{B}} B\right) \sqrt{\sum_{l \neq i}\left(\overline{g_{l j}^{2}}-{\overline{g_{l j}}}^{2}\right)} \tau_{c} \ll 1$ for any $i$. In III-V or II-VI semiconductors and their nanostructures, the electron $g$-tensor is k-dependent [223, 224], hence $\tau_{c}$ is limited by momentum scattering $\left.\left(\tau_{c} \simeq \tau_{p}\right)[333,374], 25\right]$ In the case of isotropic $g$-tensor, the $g$-tensor inhomogeneity only leads to spin relaxation transverse to the magnetic field. For example, in III-V or II-VI bulk semiconductors, the $\mathbf{k}$-dependent $g$-factor limited electron spin dephasing time is

$$
T_{2}^{-1} \simeq\left(\mu_{\mathrm{B}} B\right)^{2}\left(\overline{g^{2}}-\bar{g}^{2}\right) \tau_{p} .
$$

It is noted that the induced spin dephasing rate increases with increasing magnetic field. The $g$-tensor inhomogeneity mechanism usually dominates at high magnetic field [374].

Another limit is that there is no scattering or the scattering is very weak. In this case, the spin lifetime is limited by free induction decay 26 In this regime, if the inhomogeneity of the $g$-tensor can be characterized by a Gaussian distribution, the coherent spin precession leads to a Gaussian decay $\sim \exp \left(-t^{2} / \tau_{s}^{2}\right)$ with the spin lifetime being

$$
\frac{1}{\tau_{s, i i}} \simeq \frac{\mu_{\mathrm{B}} B}{\sqrt{2}} \sqrt{\sum_{l \neq i}\left(\overline{g_{l j}^{2}}-{\overline{g_{l j}}}^{2}\right)}
$$

In self-assembled quantum dot ensemble, the $g$-tensor inhomogeneity mechanism leads to efficient spin relaxation, masking the intrinsic irreversible one [331, 396, 397]. However, such spin relaxation can be removed by spin echo or mode-locking techniques in experiments [331]. The $g$-tensor inhomogeneity mechanism is also important for the spin relaxation of localized electrons in $n$-type quantum wells [398] and localized holes in $p$-type quantum wells [399, 400], 27

\footnotetext{
${ }^{25}$ If the $g$-tensor is only energy dependent, then $\tau_{c}$ is only limited by inelastic scattering.

${ }^{26}$ Out of the strong scattering and no scattering limits, the spin lifetime is limited by both the free induction decay time and the irreversible spin relaxation time.

${ }^{27}$ The $\mathbf{k}$-dependent hole $g$-factor in [001] quantum wells is given by Eq. 25.
} 


\subsubsection{Hyperfine interaction}

Recalling that the hyperfine interaction between electron spin and nuclear spins is

$$
H_{\mathrm{hf}}=\sum_{i, v} A_{\nu} v_{0}\left|\psi\left(\mathbf{R}_{i}\right)\right|^{2} \mathbf{S} \cdot \mathbf{I}_{i, v}=\mathbf{h} \cdot \mathbf{S} .
$$

Here $v$ labels both the atomic site in the Wigner-Seitz cell and the isotopes, while $i$ is the index of the cell. $A_{v}$ is the coupling constant of the hyperfine interaction. $v_{0}$ is the volume of the Wigner-Seitz cell and $N_{0}=1 / v_{0} . \psi(\mathbf{r})$ is the envelope function of the electron wavefunction. $\mathbf{h}$ is the effective hyperfine field acting on electron spin, which is also called the Overhauser field. The hyperfine interaction exchanges electron spin with nuclear spin and hence leads to electron spin relaxation.

There are different scenarios of the hyperfine interaction induced electron spin relaxation acting in several regimes. There are four regimes: (i) the non-interacting spin ensemble without spin echo [38]; (ii) the non-interacting spin ensemble with spin echo [38]; (iii) the hopping regime where electrons at different local sites are weakly connected; and (iv) the metallic regime where most electrons are in extended states.

In regime (i), spins are separated in space or time and do not interact with each other, i.e., they evolve independently. Examples are spins in a number of singly charged self-assembled quantum dots [401] and single spin in a quantum dot (or two spins in a double quantum dot) measured at different times [38, 402]. In this regime, the spin relaxation is determined by the free induction decay due to the random local Overhauser field. For the quantum dots ensemble, each electron spin in a quantum dot interacts with about $10^{3} \sim 10^{6}$ nuclear spins. According to central limit theorem, the distribution of the Overhauser field is Gaussian

$$
P(\mathbf{h})=\frac{1}{\left(2 \pi \sigma_{h}^{2}\right)^{3 / 2}} \exp \left(-\mathbf{h}^{2} / 2 \sigma_{h}^{2}\right) .
$$

The variance $\sigma_{h}$ is given by

$$
\sigma_{h}=\sqrt{\frac{1}{3} \sum_{v} A_{v}^{2} I_{v}\left(I_{v}+1\right) v_{0} \int d^{3} \mathbf{r}|\psi(\mathbf{r})|^{4}}=h_{1} / \sqrt{N_{L}}
$$

where $h_{1}=\sqrt{\frac{2}{3} \sum_{v} A_{v}^{2} I_{v}\left(I_{v}+1\right)}$ and $N_{L}=2 /\left[v_{0} \int d \mathbf{r}|\psi(\mathbf{r})|^{4}\right]$ is the effective number of the nuclei. Assume that the Overhauser field is quasi-static, i.e., the Overhauser field fluctuates at the time scale much longer than the electron spin lifetime [403]. Under such an approximation, the electron spin polarization decays as $\exp \left(-t^{2} / \tau_{s}^{2}\right)$, where the spin lifetime is

$$
\tau_{s}^{-1}=\sqrt{2} \sigma_{h} .
$$

For $N_{L} \sim 10^{5}$, calculation gives a short spin lifetime on the time scale of $10 \mathrm{~ns}(1 \mu \mathrm{s})$ in GaAs (silicon) quantum dots ${ }^{28}$ Electron spin relaxation under external (static or time-dependent) magnetic field can be different from the zero field case, but is still well described by averaging over spin precession under the coaction of the external and Overhauser field with the distribution $P(\mathbf{h})[301,403,405] 229$ It was proposed that high nuclear spin polarization [406] or state-narrowing of the nuclear distribution [407, 408] can markedly narrow the distribution of the Overhauser field and suppress the spin relaxation. Recent experiment in double quantum dot demonstrated the enhancement of the electron spin lifetime by a factor of $\sim 70$ through polarizing the nuclear spins [409].

In regime (ii), the inhomogeneous broadening of the Overhauser field is removed by spin-echo. The spin relaxation is then caused by the temporal fluctuation of the Overhauser field. The fluctuation is caused by the nuclear spin dynamics due to the hyperfine interaction and nuclear spin dipole-dipole interaction. As nuclear spin relaxes much slower than electron spin, the electron spin dynamics due to the hyperfine interaction is non-Markovian. Furthermore, the fluctuation in nuclear spin system is a many-body problem, where the collective excitation is not obvious.

\footnotetext{
${ }^{28}$ This timescale is much shorter than both the time scale of the nuclear spin precession under the hyperfine field generated by electron $\sim 1 \mu \mathrm{s}$ $(\sim 100 \mu \mathrm{s})$ and nuclear spin-flip time due to the nuclear spin dipole-dipole interaction $\sim 100 \mu \mathrm{s}(\sim 6000 \mu \mathrm{s})$ in GaAs (silicon) [303, 404], which justifies the quasi-static treatment of the Overhauser field [403].

${ }^{29}$ The magnetic field may also change the electronic wavefunction and hence the spin relaxation as $N_{L}$ depends on the wavefunction [301].
} 
These factors make it difficult to calculate the spin lifetime limited by the hyperfine interaction. Nevertheless, recent developments attacked the problem via the equation of motion approach [344, 410-413], Green function approach [410, 414, 415] and the quantum cluster expansion approach [289, 416-422]. It was predicted theoretically that via designed magnetic pulses, the electron spin coherence lost through the hyperfine interaction can be restored [423431]. Recent experiment confirmed the theoretical prediction [432]. Other experimental investigations in regime (ii) involve: the study of the decay of spin echo of a single spin in a quantum dot [402], two spins in a double quantum dot [38, 433] and spins of donor bound electrons in silicon [288]; the development of an optical mode-locking method to study the irreversible electron spin dephasing in self-assembled quantum dots [331, 434].

Regime (iii) is the hopping regime where electrons at different local sites are weakly connected. The hopping between the local sites induces the exchange interaction between electrons. The exchange interaction interrupts the spin precession induced by the random local Overhauser field. If the exchange interaction is weak, the electron spin ensemble suffers both the free induction decay and the irreversible spin decay due to the randomization caused by the exchange interaction. If the exchange interaction is strong, the spin precession around the random local Overhauser field is motional narrowed [39, 40, 323, 435]. An example of regime (iii) is the localized electron spin ensemble in the insulating phase of a $n$-doped semiconductor.

The exchange interaction between electrons limits the correlation time $\tau_{c}$ of the spin precession due to the random Overhauser field. In the following, we focus on the motional narrowing limit, which is also the focus in the literature. In this limit, $\left\langle\omega_{\mathrm{hf}} \tau_{c}\right\rangle \ll 1\left(\langle\ldots\rangle\right.$ denoting the electron ensemble average) with $\omega_{\mathrm{hf}}$ being the spin precession frequency due to the hyperfine interaction. In this regime, the spin lifetime is given by [3, 435]

$$
\tau_{s}^{-1}=\frac{2}{3}\left\langle\omega_{\mathrm{hf}}^{2}\right\rangle \tau_{c}
$$

Here the prefactor $\frac{2}{3}$ is due to the facts that only transverse fluctuation can lead to spin relaxation and that the angular distribution of the fluctuation field is uniform. Roughly $\tau_{c} \simeq 1 /\left\langle J_{i j}\right\rangle$, where $\left\langle J_{i j}\right\rangle$ is the averaged exchange coupling strength with $i$ and $j$ being the site indices [39, 40].

In regime (iv), the metallic regime, most of the electrons are extended and $\mathbf{k}$ is a good quantum number. In this regime, the spin relaxation due to the hyperfine interaction is described as a spin-flip scattering. For example, in bulk semiconductors, the spin lifetime due to the hyperfine interaction is given by [436],

$$
\tau_{s}^{-1}=\left\langle\frac{1}{\tau_{s}\left(\varepsilon_{\mathbf{k}}\right)}\right\rangle=\frac{m_{e}\langle k\rangle}{3 \pi} v_{0} \sum_{v} \beta_{v} A_{v}^{2} I_{v}\left(I_{v}+1\right) .
$$

Here $\langle k\rangle=\sum_{\mathbf{k}} k f_{\mathbf{k}}\left(1-f_{\mathbf{k}}\right) / \sum_{\mathbf{k}} f_{\mathbf{k}}\left(1-f_{\mathbf{k}}\right)$ with $f_{\mathbf{k}}$ the Fermi distribution. Calculation by Fishman and Lampel [436] in bulk GaAs shows that the spin lifetime is on the order of $10^{3} \sim 10^{4} \mathrm{~ns}$ in the metallic regime, whereas the measured spin lifetime is less than $300 \mathrm{~ns}$ [21, 39]. Therefore the hyperfine interaction mechanism is irrelevant to the spin relaxation in the metallic regime in bulk GaAs.

Hole spin relaxation due to the hyperfine interaction attracted a lot of interest recently [116, 301, 437-440]. For a long time, it was believed that holes interact weakly with nuclear spin and the hyperfine interaction is negligible to hole spin relaxation [3]. However, recent calculations indicated that the hole-nucleus hyperfine interaction is only one order of magnitude smaller than the electron one [301], which still provides considerable effects on hole spin relaxation [301]. In principle, the above discussions on electron spin relaxation due to the hyperfine interaction are also applicable to hole system. Differently, the heavy-hole hyperfine interaction in quantum wells is of the Ising form $H_{\mathrm{hf}}^{h}=\sum_{i, v} A_{v}^{h} v_{0}\left|\psi\left(\mathbf{R}_{i}\right)\right|^{2} S^{z} I_{i, v}^{z}$ ( $z$ axis is along the growth direction). Consequently, the hole spin relaxation due to the hyperfine interaction is different from the electron one: it is anisotropic [301]. Further studies on the hole spin relaxation due to the hyperfine interaction, especially in quantum dots where hole spin is a promising candidate for qubits [441, 442], are required.

\subsubsection{Anisotropic exchange interaction}

The anisotropic exchange interaction is an efficient spin relaxation mechanism in the insulating phase of doped semiconductors [39, 40, 322-324, 324, 325, 443, 444]. The interaction comes from a correction to the Heisenberg exchange interaction between carriers bound to adjacent dopants due to the spin-orbit coupling. It can be written as 
[40, 322],

$$
H_{\text {anex }} \approx-J_{i j}\left[\sin \left(\gamma_{i j}\right) \frac{\vec{\gamma}_{i j}}{\gamma_{i j}} \cdot\left(\mathbf{S}_{i} \times \mathbf{S}_{j}\right)+\left(1-\cos \gamma_{i j}\right)\left(\frac{\vec{\gamma}_{i j}}{\gamma_{i j}} \cdot \mathbf{S}_{i}\right)\left(\frac{\vec{\gamma}_{i j}}{\gamma_{i j}} \cdot \mathbf{S}_{j}\right)\right],
$$

in which $J_{i j}$ is the isotropic exchange constant. $\vec{\gamma}_{i j}$ is a vector due to spin-orbit coupling. It is estimated as $\vec{\gamma}_{i j} \approx$ $m_{e} \boldsymbol{\Omega}_{\mathrm{SO}}\left(\sqrt{2 m_{e} E_{B}} \mathbf{r}_{r_{i j}}\right) r_{i j} / \sqrt{2 m_{e} E_{B}}$, where $\boldsymbol{\Omega}_{\mathrm{SO}}(\mathbf{k})$ is the spin precession frequency, $E_{B}>0$ is the electron binding energy and $\mathbf{r}_{i j}$ is the vector connecting the positions of site $i$ and $j$. The first term is the Dzhyaloshinskii-Moriya interaction and the second one is the scalar exchange interaction. The scalar exchange interaction does not conserve the spin perpendicular to $\vec{\gamma}_{i j}$, whereas the Dzhyaloshinskii-Moriya interaction does not conserve spin along any direction. Consequently the anisotropic exchange interaction leads to decay of total carrier spin polarization [322]. As $\gamma_{i j}$ is usually very small in insulating phase, $\sin \left(\gamma_{i j}\right) \approx \gamma_{i j}$ and $1-\cos \left(\gamma_{i j}\right) \approx \frac{1}{2} \gamma_{i j}^{2}$. One should note that $\gamma_{i j}$ is proportional to the strength of the spin-orbit coupling.

In insulating phase, electron spin precesses around the anisotropic exchange field produced by a randomly oriented adjacent electron spin, which results in a random-walk-like spin precession. The correlation time of spin precession, is limited by hopping time as well as the isotropic exchange interaction with adjacent electrons. The latter perturbs the spin precession of individual electron but conserves the total spin polarization. Studies indicate that the latter is much more efficient than the former in GaAs [40]. Hence an estimation of the correlation time is $\tau_{c} \approx 1 /\left\langle J_{i j}\right\rangle$, and the spin precession frequency is $\Omega \approx\left\langle J_{i j} \gamma_{i j}\right\rangle$. Therefore spin relaxation time reads [40]

$$
\tau_{s}^{-1} \approx \frac{2}{3}\left\langle J_{i j}\right\rangle\left\langle\gamma_{i j}\right\rangle^{2}
$$

Here the prefactor $\frac{2}{3}$ is due to the facts that only transverse fluctuation can lead to spin relaxation and that the angular distribution of the random exchange field is uniform (as the adjacent electron spins orient randomly). It should be noted that the spin relaxation rate, which $\propto\left\langle\gamma_{i j}\right\rangle^{2}$, is proportional to the square of the strength of the spin-orbit coupling.

The anisotropic exchange interaction mechanism has also been reviewed by Kavokin in Ref. [40], where many aspects of spin relaxation of localized electrons were discussed. However, we note that there was no theoretical study on the anisotropic exchange interaction for holes in insulating phase up till now. As the spin-orbit coupling is stronger, one would expect that the anisotropic exchange interaction is more efficient in hole system. Recently there are a few experimental studies [400, 445, 446] on hole spin relaxation in the insulating phase in two-dimensional hole system. Theoretical investigation on the role of the anisotropic exchange interaction in hole spin relaxation under experimental conditions is desired.

\subsubsection{Exchange interaction with magnetic impurities}

In magnetic semiconductors, the exchange interaction with magnetic impurities can be an important source of the spin relaxation. In (II,Mn)VI magnetic semiconductors, the $s-d$ exchange interaction has been recognized to be responsible for electron spin relaxation [447-450]. Recently, it was found that in $p$-type paramagnetic GaMnAs quantum wells, the $s$ - $d$ exchange interaction dominates electron spin relaxation at low temperature [111]. In general, the spin relaxation due to the $s-d(p-d)$ exchange interaction is similar to that due to the hyperfine interaction. There can be several regimes, where different scenarios take place. However, there are only two regimes which are commonly encountered: the insulating regime where most carriers are localized and the metallic regime where most carriers are extended. In the insulating regime, the exchange interaction with randomly oriented magnetic impurity spins induces random spin precessions. There are several processes interrupt such random spin precessions: (i) exchange interaction between carriers; (ii) carrier hopping; (iii) fluctuation or diffusion of the magnetic impurity spins. These processes limit the correlation time $\tau_{c}$ of the random spin precession. If $\tau_{c}$ is small, so that $\left\langle\boldsymbol{\Omega}_{s(p)-d}\right\rangle \tau_{c} \ll 1\left(\boldsymbol{\Omega}_{s(p)-d}\right.$ is the spin precession frequency due to the $s(p)-d$ exchange interaction and $\langle\ldots\rangle\rangle$ represents the ensemble average). In this regime, the spin relaxation rate reads

$$
\tau_{s}^{-1}=\frac{2}{3}\left\langle\Omega_{s(p)-d}^{2}\right\rangle \tau_{c} .
$$

In metallic regime, spin relaxation rate is given by the Fermi Golden rule. For example, in bulk system, the electron spin relaxation rate is [451, 452]

$$
\tau_{s}^{-1}=\frac{m_{e}\langle k\rangle}{3 \pi} J_{\mathrm{sd}}^{2} N_{M} S_{M}\left(S_{M}+1\right),
$$


where $J_{\text {sd }}$ is the $s$ - $d$ exchange constant, $N_{M}$ is the density of magnetic ions with spin $S_{M},\langle k\rangle=\sum_{\mathbf{k}} k f_{\mathbf{k}}\left(1-f_{\mathbf{k}}\right) / \sum_{\mathbf{k}} f_{\mathbf{k}}(1-$ $f_{\mathbf{k}}$ ) with $f_{\mathbf{k}}$ being the Fermi distribution. Hole spin relaxation rate due to the $p$ - $d$ exchange interaction can also be calculated via the Fermi Golden rule, only that the spectrum and the eigen-spinor are more complicated. In principle, the same scheme is applicable to electrons (holes) in nanostructures [448].

\subsubsection{Other spin relaxation mechanisms}

The main remaining spin relaxation mechanisms are various spin-phonon interactions (except those which have been grouped into the Elliott-Yafet mechanism). Generally the origin of the spin-phonon interactions is that when spin interactions depend on the positions of atomic ions (both host and dopant), lattice vibration couples directly with spin. There are several such kinds of spin interactions, such as the strain-induced spin-orbit coupling and the hyperfine interaction. The strain also modifies $g$-tensor [453]. All such lattice dependent spin interactions can be the origin of the spin-phonon interactions [453-458]. In nanostructure, the gate-voltage and structure induced modification of spin-orbit coupling [113] and $g$-tensor [81, 113] further induce more spin-phonon interactions [344, 457]. Besides, spin mixing associated with spin-independent phonon scattering also leads to the spin-flip phonon scattering. For example, for localized electrons which are bound to impurities or confined in quantum dots, spin-orbit coupling and hyperfine interaction can induce spin mixing and enables spin-flip electron-phonon scattering [344, 459].

In existing literature, there are a lot of studies on the spin-phonon interaction induced spin relaxation. Most of them focus on spin relaxation of donor-bound electrons [324, 453, 460-463] and electrons/holes in quantum dots [343, 344, 454-460, 464-492]. A comprehensive study of electron spin relaxation/dephasing due to various spin-phonon interactions in GaAs quantum dots is presented in Ref. [344]. The spin-phonon scatterings are usually limited by the electron-phonon scattering rate and the effect of the spin-orbit interaction. It was believed that they are unimportant in metallic regime. The relative importance of different spin-phonon interactions are compared for various conditions in GaAs quantum dots [344] and in $\mathrm{Si} / \mathrm{Ge}$ quantum dots [486]. Among those spin-phonon interactions, the phonon induced $g$-tensor fluctuation was found to be irrelevant in GaAs quantum dots [344], whereas it is important in $\mathrm{Si} / \mathrm{Ge}$ quantum dots [486]. Finally, the gate noise can also lead to spin relaxation in gated quantum dots in the presence of the hyperfine interaction, spin-orbit interaction or electron-electron exchange interaction [493, 494].

\subsubsection{Relative efficiency of spin relaxation mechanisms in bulk semiconductors}

In this subsection, we briefly discuss and review the relative efficiency of various spin relaxation mechanisms. We give some rough discussions based on the analytical formulae which are based on some approximations 30 For simplicity, we restrict the discussion here on the bulk III-V semiconductors. The situation in III-V semiconductor nanostructures or II-VI semiconductors and their nanostructures may be different. Nevertheless, some insight can be gained from these discussions. We also comment on hole spin relaxation and carrier spin relaxation in centrosymmetric semiconductors such as silicon and germanium.

For spin relaxation in bulk III-V semiconductors in the insulating regime, as stated before, the anisotropic exchange interaction dominates at high doping density, whereas the hyperfine interaction dominates at low doping density. In metallic regime, the spin relaxation mechanisms are the D'yakonov-Perel', the Elliott-Yafet and the BirAronov-Pikus mechanisms. In $n$-doped semiconductors the Bir-Aronov-Pikus mechanism is ineffective due to lack of holes. Hence the relevant mechanisms are the Elliott-Yafet and the D'yakonov-Perel' mechanisms. From Eqs. (53) and (62), the ratio of the spin lifetimes due to the Elliott-Yafet and the D'yakonov-Perel' mechanisms in bulk III-V semiconductors is given by [110, 117],

$$
\frac{\tau_{\mathrm{EY}}}{\tau_{\mathrm{DP}}} \approx A_{1}\left(\frac{m_{e}}{m_{c v}}\right)^{2}\left\langle\varepsilon_{\mathbf{k}}\right\rangle \tau_{p}^{2} E_{g} \frac{(1-\eta / 3)}{(1-\eta / 2)^{2}},
$$

where the prefactor $A_{1} \sim 1$. It is inferred from the above expression that the Elliott-Yafet spin relaxation is more important in semiconductors with smaller $E_{g}$, such as InAs and InSb. The factor $\left\langle\varepsilon_{\mathbf{k}}\right\rangle \tau_{p}^{2}$ indicates that the Elliott-Yafet mechanism is more important at lower temperature with higher impurity density.

\footnotetext{
${ }^{30}$ Besides competition, there are also some cooperation between different spin relaxation mechanisms. For example, spin-flip momentum scatterings due to the Elliott-Yafet, the Bir-Aronov-Pikus, the $s(p)$ - $d$ exchange interaction and other mechanisms also lead to the randomization of momentum, they thus contribute to the D'yakonov-Perel' spin relaxation as well. However, as these spin-flip momentum scatterings are usually much weaker than the spin-conserving ones, they only lead to a small modification of the D'yakonov-Perel' spin relaxation [109, 111].
} 
In $p$-type and intrinsic semiconductors the relative efficiencies of the Bir-Aronov-Pikus and the D'yakonov-Perel' mechanisms are always compared. The Bir-Aronov-Pikus mechanism is believed to be important for high hole density at low temperature [21, 117]. In such case the hole system is degenerate, thus it is meaningful to compare the D'yakonov-Perel' and the Bir-Aronov-Pikus mechanisms for degenerate holes. From Eqs. (62) and (69) one finds

$$
\frac{\tau_{\mathrm{BAP}}}{\tau_{\mathrm{DP}}} \approx A_{2} \tau_{p} \tau_{0} \frac{\left\langle\varepsilon_{\mathbf{k}}\right\rangle^{5 / 2} E_{\mathrm{B}}^{1 / 2}}{E_{g}}\left(\frac{m_{e}}{m_{c v}}\right)^{2} \frac{\eta^{2}}{1-\eta / 3} \frac{1}{n_{h} a_{\mathrm{B}}^{3}} \frac{E_{\mathrm{F}}^{h}}{k_{\mathrm{B}} T},
$$

where the prefactor $A_{2} \sim 1$ and $E_{\mathrm{F}}^{h}$ is hole Fermi energy. It is seen from the above formula that the Bir-AronovPikus mechanism is more important in semiconductors with larger band gap $E_{g}$, smaller spin-orbit interaction (i.e., smaller $\eta$ ) and stronger electron-hole exchange interaction (i.e., smaller $\tau_{0}$ ). From the above equation, one finds four controlling factors: $\tau_{p},\left\langle\varepsilon_{\mathbf{k}}\right\rangle, n_{h}$ and $T$ (Note that $E_{\mathrm{F}}^{h} \sim n_{h}^{2 / 3}$ ). The Bir-Aronov-Pikus mechanism is more important at higher hole/impurity density and lower electron density. For nondegenerate electron, as $\left\langle\varepsilon_{\mathbf{k}}\right\rangle \sim k_{\mathrm{B}} T$, the Bir-AronovPikus mechanism is more important at low temperature. However, for degenerate electron, the Bir-Aronov-Pikus mechanism is more important at high temperature, as $\left\langle\varepsilon_{\mathbf{k}}\right\rangle \sim E_{\mathrm{F}}$. Such qualitative different behavior for nondegenerate and degenerate electron was illustrated in a recent study [110] (see also Sec. 5.6.).

The Elliott-Yafet mechanism can be comparable to the Bir-Aronov-Pikus mechanism in $p$-type semiconductors. The ratio of the two spin lifetimes for degenerate hole is

$$
\frac{\tau_{\mathrm{EY}}}{\tau_{\mathrm{BAP}}} \approx A_{3} \frac{\tau_{p}}{\tau_{0}} n_{h} a_{\mathrm{B}}^{3} \frac{E_{g}^{2}}{E_{\mathrm{B}}^{1 / 2}\left\langle\varepsilon_{\mathbf{k}}\right\rangle^{3 / 2}} \frac{1}{\eta^{2}}\left(\frac{1-\eta / 3}{1-\eta / 2}\right)^{2} \frac{k_{\mathrm{B}} T}{E_{\mathrm{F}}^{h}},
$$

where $A_{3} \sim 1$. According to the above equation, the Elliott-Yafet mechanism is more important in semiconductors with larger $\tau_{0}$ (i.e., weaker electron-hole exchange interaction), smaller band-gap $E_{g}$ and larger $\eta$ (i.e., larger spin-orbit interaction). Hence the Elliott-Yafet mechanism may exceed the Bir-Aronov-Pikus mechanism in semiconductors with small band-gap and large spin-orbit interaction, such as InSb and InAs. For a given material, the Elliott-Yafet mechanism is more important at stronger momentum scattering (i.e., smaller $\tau_{p}$ ) and lower hole density. For degenerate electrons, the Elliott-Yafet mechanism is more important at larger electron density and lower temperature, as $\left\langle\varepsilon_{\mathbf{k}}\right\rangle \sim E_{\mathrm{F}}$. However, for nondegenerate electrons, the Elliott-Yafet mechanism is more important at higher temperature, as $\left\langle\varepsilon_{\mathbf{k}}\right\rangle \sim k_{\mathrm{B}} T$.

In brief, some understanding of the topic can be obtained from the above discussions. Nevertheless, as there are a lot of approximations in these formulae, they can not give a picture of whole parameter range. In some case the manybody effects which are absent in the above analysis plays an important role. Moreover, the relative efficiency relies strongly on the genuine material parameters. Only a realistic calculation can fully resolve the problem. A systematic calculation from the fully microscopic kinetic spin Bloch equation approach by Jiang and Wu [110] indicated that the Elliott-Yafet mechanism is less important than the D'yakonov-Perel' mechanism in all parameter regime in $n$-type bulk III-V semiconductors in metallic regime. In the same work, the Bir-Aronov-Pikus mechanism was found to be unimportant in intrinsic samples, whereas it dominates spin relaxation in $p$-type samples at low temperature and high hole density when the electron density is low. However, the Bir-Aronov-Pikus mechanism is ineffective when the electron density is high [110]. It was also found via the same approach that in intrinsic or $p$-type GaAs quantum wells when electron density is comparable with hole density, the Bir-Aronov-Pikus mechanism is unimportant [109]. In a recent work [112], the relative importance of the Bir-Aronov-Pikus mechanism and the D'yakonov-Perel' mechanism in $p$-type GaAs quantum wells was compared comprehensively. Systematic comparisons of various spin relaxation mechanisms in paramagnetic GaMnAs quantum wells was performed in Ref. [111] also via the kinetic spin Bloch equation approach.

Finally, in centrosymmetric semiconductors, such as silicon and germanium, the situation is totally different. In bulk system, as the spin-orbit coupling term in conduction band vanishes, the D'yakonov-Perel' mechanism is absent in electron spin relaxation. Hence in $n$-type bulk silicon and germanium, the Elliott-Yafet mechanism dominates electron spin relaxation in metallic regime [21, 22, 362]. In insulating regime, electron spin relaxation is dominated by other mechanisms such as the hyperfine interaction and the spin-phonon interaction [289, 453, 461, 463]. In nanostructures, which breaks the centro-inversion symmetry, spin-orbit coupling term shows up [291, 294, 295]. Experiment indicated that in silicon quantum wells the D'yakonov-Perel' mechanism is dominant in high mobility 
regime, whereas the Elliott-Yafet mechanism is more important in low mobility regime [297]. Due to the nature of indirect band, optical spin orientation is not easily accesible. Hence the electron spin dynamics was studied only in $n$-type system, whereas the hole spin dynamics in $p$-type system. In $p$-type system, like the situation in III-V semiconductors, hole spin-orbit coupling in the Luttinger Hamiltonian is large and the D'yakonov-Perel' mechanism is believed to be dominant. The D'yakonov-Perel' mechanism also dominates hole spin relaxation in nanostructures of silicon and germanium [299].

\section{Spin relaxation: experimental studies and single-particle theories}

The study of spin lifetime and spin diffusion length is one of the central focuses in spintronics. A large quantity of papers can be found in the past decades. Recent development of spin grating measurement gives new insight into spin diffusion [13, 33, 495], while spin noise spectroscopy enables measurement of the intrinsic carrier spin lifetime with negligible disturbance on the carrier system [496-499]. Also the latest technique of tomographic Kerr rotation achieves spin state tomography [500]. We expect that these new experimental techniques will inspire and enable more important findings.

In this section we review experimental studies and single-particle theories on spin relaxation and dephasing times. Here, the term "single-particle theory" refers to the theory where the carrier-carrier scattering is not considered. It will be shown in next section that the carrier-carrier scattering plays an important role in spin relaxation. We focus on spin relaxation in III-V and II-VI semiconductors where the bulk inversion asymmetry induced spin-orbit coupling plays an important role. Spin relaxation in centrosymmetric semiconductors, such as silicon and germanium, is also reviewed. The discussions in the previous section will be used frequently.

\subsection{Carrier spin relaxation at low temperature in insulating regime}

Carriers in semiconductors are mostly ionized from dopants. At low temperature, dopants can trap those carriers. When doping density is low, carriers are bound to isolated dopants and the system behaves as an insulator. At elevated doping density, carrier system becomes metallic after the metal-insulator transition at some critical density $n_{c}$. In metallic regime carrier transport is band-like, whereas in insulating regime it is dominated by carrier hopping. It is then not difficult to understand that the relevant carrier spin relaxation mechanisms in insulating regime are different from those in the metallic regime. In this subsection, we review spin relaxation at low temperature in insulating regime. Remarkably, the longest spin lifetimes in GaAs was reported as $\tau_{s} \simeq 300 \mathrm{~ns}$ [501, 502] at zero magnetic field. At high magnetic field in lightly doped GaAs, the spin lifetime can reach $\tau_{s} \simeq 19 \mu$ s [503]. In high purity GaAs, the spin relaxation time of donor-bound electrons can be as long as several milliseconds [504] 31

It has been identified that spin relaxation in insulating regime is dominated by anisotropic exchange interaction mechanism at high doping density whereas by the hyperfine interaction mechanism at low doping density, at zero magnetic field [39, 40, 323]. If $\sum_{f} \sqrt{\left\langle\omega_{f}^{2}\right\rangle} \tau_{c} \ll 1$, spin relaxation rate is given by [3, 435]

$$
\tau_{s}^{-1} \simeq \sum_{f} \frac{2}{3}\left\langle\omega_{f}^{2}\right\rangle \tau_{c},
$$

where $\omega_{f}$ is the electron spin precession frequency due to the random effective magnetic field. The index $f$ denotes the mechanism leading to the random fields: it can be the hyperfine interaction with the nuclei and the anisotropic exchange interaction with adjacent electrons. $\langle\ldots\rangle$ represents ensemble average. $\tau_{c}$ is the correlation time of the random spin precessions. $\tau_{c}$ can be limited by disturbance of electron spin state or the random field $\omega_{f}$. Realistic calculation indicated that in $\mathrm{GaAs} \tau_{c}$ is mainly limited by the former due to the isotropic exchange interaction [39, 40] 32 Therefore $\tau_{c} \simeq 1 /\left\langle J_{i j}\right\rangle$ where $J_{i j}$ is the isotropic exchange coupling constant between $i$ and $j$ donor-bound

\footnotetext{
${ }^{31}$ The ensemble spin dephasing time $\left(T_{2}^{*} \simeq 5 \mathrm{~ns}[505]\right)$ and irreversible spin dephasing time $\left(T_{2}=7 \mu \mathrm{s}[506]\right)$ are much shorter under the same condition.

${ }^{32}$ In fact such disturbance of electron spin state can be viewed as spin diffusion in a network coupled by the isotropic exchange interaction [40]. From the random-walk theory, $\tau_{c}$ is related to the spin diffusion constant $D_{s}$ as $\tau_{c} \simeq\left(3 n_{D}^{2 / 3} D_{s}\right)^{-1}[40]$.
} 
electrons. The average of $J_{i j}$ is taken over the donor configuration. Spin relaxation rate due to the anisotropic exchange interaction is then given by [see also Eq. (80)]

$$
\tau_{s}^{-1} \approx \frac{2}{3}\left\langle J_{i j}\right\rangle\left\langle\gamma_{i j}\right\rangle^{2}
$$

where $\gamma_{i j}$ is the spin precession angle during the tunnel hoping from $i$ to $j$ donor site (see Sec. 3.2.6). The hyperfine interaction-limited spin lifetime is

$$
\tau_{s}^{-1} \simeq \frac{2}{3} \sigma_{h}^{2} \frac{1}{\left\langle J_{i j}\right\rangle}
$$

where $\sigma_{h}$ [given by Eq. (75)] is the variance of the Overhauser field. It is noted that the spin relaxation rates due to the two mechanisms have different dependences on $\left\langle J_{i j}\right\rangle$. Naively, it is expected that $\left\langle J_{i j}\right\rangle$ increases with decreasing interdonor distance (i.e., increasing doping density). It is then easy to understand that the anisotropic exchange interaction dominates spin relaxation at high doping density, whereas the hyperfine interaction dominates at low doping density [39, 40, 323]. For hydrogen-like centers, the exchange coupling constant is given by [325]

$$
J_{i j} \simeq 0.82 \frac{e^{2}}{4 \pi \epsilon_{0} \kappa_{0} a}\left(\frac{r_{i j}}{a}\right)^{5 / 2} \exp \left(-\frac{2 r_{i j}}{a}\right)
$$

where $a$ is the Bohr radius of the hydrogen-like wavefunction. It is noted that the dependence on inter-donor distance $r_{i j}$ is exponential 33

Usually both localized and free (extended) electrons coexist and they are also coupled by exchange interactions. It was found that the exchange coupling between the localized and free electrons is so efficient that the two share a common spin lifetime [40, 324, 508]. The spin relaxation rate of the whole electron system is then given by [324],

$$
\tau_{s}^{-1}=\tau_{l s}^{-1} n_{l} /\left(n_{l}+n_{f}\right)+\tau_{f s}^{-1} n_{f} /\left(n_{l}+n_{f}\right),
$$

where $n_{l}$ and $n_{f}$ are the densities of localized and free electrons, respectively. $\tau_{l s}$ and $\tau_{f s}$ are the spin lifetimes of localized and free electrons, separately. In many cases spin relaxation is dominated by localized electrons as they have much faster spin relaxation rate [40,324]. Besides, the exchange interaction between free and localized electrons can also limit $\tau_{c}$ and elongate the spin lifetime [501, 502]. The effect of electron localization on spin relaxation in $n$-doped quantum wells, especially in symmetric (110) quantum wells, was discussed in a recent theoretical work [509].

In the presence of magnetic field, the situation becomes much more complicated [40, 510]. First, magnetic field reduces the isotropic exchange coupling (as the wavefunction becomes more localized) and hence increases $\tau_{c}$ [40]. The anisotropic exchange interaction is also reduced. Second, spin relaxation due to the random spin precession is slowed down by the magnetic field parallel to spin polarization [see Eq. (40)]. Third, spin relaxation due to $g$-tensor inhomogeneity increases with the magnetic field. Fourth, at high magnetic field, spin-flip electron-phonon scattering may come into play [383]. All these factors complicate the magnetic field dependence of the spin relaxation. Up till now, there is little discussion on the problem [40, 510]. Experimental results on magnetic field dependence of spin lifetime are available in Ref. [511]. Yet the observed magnetic field dependence is to be explained by theory [511]. Before turning to experimental studies, it should be mentioned that until now there are only a few theoretical works on spin relaxation in insulating regime [39, 40, 322-324, 324, 325, 443, 507, 510, 512, 513], more studies are needed. Especially hole spin relaxation in insulating regime was discussed only in Ref. [399] where only the $g$-factor fluctuation mechanism was studied.

We now review the experimental studies on carrier spin relaxation at low temperature in insulating regime. Many efforts have been devoted to the spin relaxation in $n$-GaAs at low temperature [4, 39, 144, 321, 497, 501-503, 511,

\footnotetext{
${ }^{33}$ As stated in the previous footnote, $\tau_{c}$ is actually limited by spin diffusion due to exchange coupling in the network of randomly distributed donors. In some clusters where the inter-donor distance is shorter than the average one, spin diffusion becomes much faster due to the exponential dependence. Therefore this kind of cluster serves as easy passages of spin diffusion. At low doping density spin diffusion is dominated by such kind of small cluster. In this case the spin diffusion should be treated with percolation theory [40]. Such kind of studies were performed in Refs. [40, 507].
} 
514-521]. In one of the seminal work by Kikkawa and Awschalom [4], a surprisingly long spin lifetime (130 ns) was observed in $n$-GaAs, which sheds light on the possibility of the semiconductor spintronics. This, together with the observation of a very long spin diffusion length $(100 \mu \mathrm{m})$ in GaAs [5] as well as the progress on spin injection into semiconductors [522, 523], excited the society and led to the rapid rising of the field of semiconductor spintronics. Interestingly, most of these works were done in bulk $n$-GaAs with a doping density of $10^{16} \mathrm{~cm}^{-3}$.

Dzhioev et al. [39] measured, in a wide range $\left(10^{14} \sim 10^{19} \mathrm{~cm}^{-3}\right)$, the doping density dependence of spin lifetime at low temperature $T \leq 5 \mathrm{~K} 34$ The results are presented in Fig. 1. Spin lifetime first increases before reaching its first maximum at doping density $n_{D} \simeq 3 \times 10^{15} \mathrm{~cm}^{-3}$; it then decreases with doping density; around the metalinsulator transition point at doping density $n_{D}=2 \times 10^{16} \mathrm{~cm}^{-3}$, spin lifetime increases rapidly and reaches its second maximum; after that (i.e., in metallic regime) spin lifetime decreases monotonically with doping density. This intricate behavior is due to the different spin relaxation mechanisms in metallic and insulating regimes. In metallic regime, spin relaxation is dominated by the D'yakonov-Perel' mechanism where $\tau_{s}^{-1} \sim\left\langle\varepsilon_{\mathbf{k}}^{3}\right\rangle \tau_{p}$. At such low temperature, electron system is degenerate. Hence $\tau_{p} \sim n_{D} / k_{F}^{3} \sim n_{D}^{0}$ (according to Brooks-Herring formula) and $\left\langle\varepsilon_{\mathbf{k}}^{3}\right\rangle \sim n_{D}^{2}$. Therefore $\tau_{s}$ decreases with doping density $n_{D}$ as $\tau_{s} \sim n_{D}^{-2}$. In insulating regime, the D'yakonov-Perel' mechanism is irrelevant. The relevant spin relaxation mechanisms are the anisotropic exchange interaction (at high doping density) and the hyperfine interaction (at low doping density). As indicated by Eqs. (87) and (88), spin relaxation due to these two mechanisms have different doping density dependences (as $\left\langle J_{i j}\right\rangle$ increases with increasing doping density). Therefore, their competition leads to the nonmonotonic doping density dependence: spin lifetime first increases then decreases with increasing doping density. It is noted in the figure that the measured $\tau_{c}$ decreases with increasing doping density, which agrees with the theory as $\tau_{c} \simeq 1 /\left\langle J_{i j}\right\rangle$.

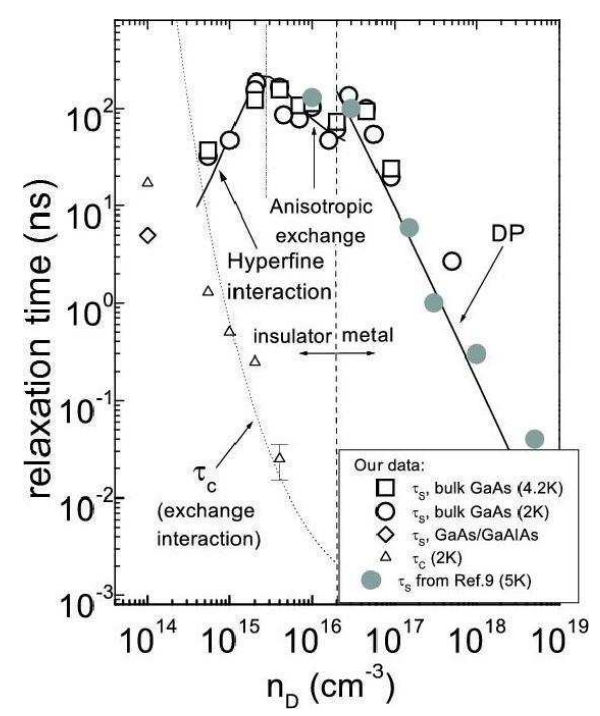

Figure 1: Spin lifetime $\tau_{s}\left(\square, \bigcirc, \diamond\right.$ and $\bullet$ ) and spin correlation time $\tau_{c}(\Delta)$ as function of donor concentration $n_{\mathrm{D}}$ in $n$-GaAs. Solid curves: theoretical calculation of $\tau_{s}$. Dotted curve: theoretical calculation of $\tau_{c}$ due to exchange interaction between adjacent electrons. The vertical dashed lines indicate the positions of the peaks of the spin lifetime. Especially, the dashed line at $n_{\mathrm{D}}=2 \times 10^{16} \mathrm{~cm}^{-3}$ also denotes the metalinsulator transition. The hyperfine interaction, the anisotropic exchange interaction and the D'yakonov-Perel' mechanisms dominate the spin relaxation in the low, medium, high doping density regimes respectively, as indicated in the figure. From Dzhioev et al. [39].

It was demonstrated by Dzhioev et al. that different spin relaxation scenarios can be invoked by optical pumping [501, 502]. At low concentration spin relaxation is limited by the hyperfine interaction mechanism, where the spin lifetime is $\tau_{s}=5 \mathrm{~ns}$. The authors showed that by injecting additional electrons, the exchange interaction between those electrons and the donor-bound electrons motionally narrows the random spin precession caused by the hyperfine interaction. The spin lifetime is then elongated to $\tau_{s}=300 \mathrm{~ns} 35$

\footnotetext{
${ }^{34}$ Similar investigation in CdTe was performed in a recent work [524].

${ }^{35}$ As the photo-excited electrons may affect the spin relaxation significantly at low temperature in insulating regime [40, 501, 502], it is important
} 
Recently, Schreiber et al. studied energy resolved electron spin relaxation in $n$-GaAs near the metal-insulator transition [519]. They found that there are three components in the time-resolved Kerr rotation under magnetic field parallel to the surface by examining the spin precession frequency as well as spin lifetime. The different precession frequencies indicate that those components should be attributed to electrons at different energy states. The sequential emergence of the three components confirms such hypothesis. The authors attributed these three states as: delocalized states in donor band, localized states in the tail of donor band and free electron states in the conduction band. The authors also found that absolute value of the $g$-factor in localized states in the donor band tails is smaller than those in the other two states.

The magnetic field and temperature dependences of spin relaxation time in lightly doped $n$-GaAs were measured systematically by Colton et al. in Refs. [503, 511]. The main results are shown in Fig. [2] Surprisingly a complicated nonmonotonic magnetic field dependence was observed: spin lifetime first increases $(B<2-2.5 \mathrm{~T})$ then decreases $(2-2.5<B<3-4 \mathrm{~T})$ and further increases $(B>3-4 \mathrm{~T})$ with increasing magnetic field [511]. Moreover, spin lifetime decreases rapidly with increasing temperature. For $1<T<20 \mathrm{~K}$ and $0.5 \leq B \leq 2.5 \mathrm{~T}$, it exhibits a power law $\tau_{s} \sim T^{-1.57}$ [511]. The underlying physics is still unclear [40, 511]. Recently, the temperature dependence of spin lifetime in quantum wells was studied carefully in Refs. [445, 525, 526]. In these works, differently, it was found that the temperature dependence of electron (hole) spin lifetime is quite weak for $T<5 \mathrm{~K}(T<2 \mathrm{~K})$. At higher temperature, the spin lifetime decreases dramatically with increasing temperature. It was proposed that the excited localized states from thermal activation, which have much shorter spin lifetimes than the ground states, is responsible for the observed phenomena [526]. The temperature dependence of spin lifetime in insulating regime was also studied theoretically in Refs. [40, 507].
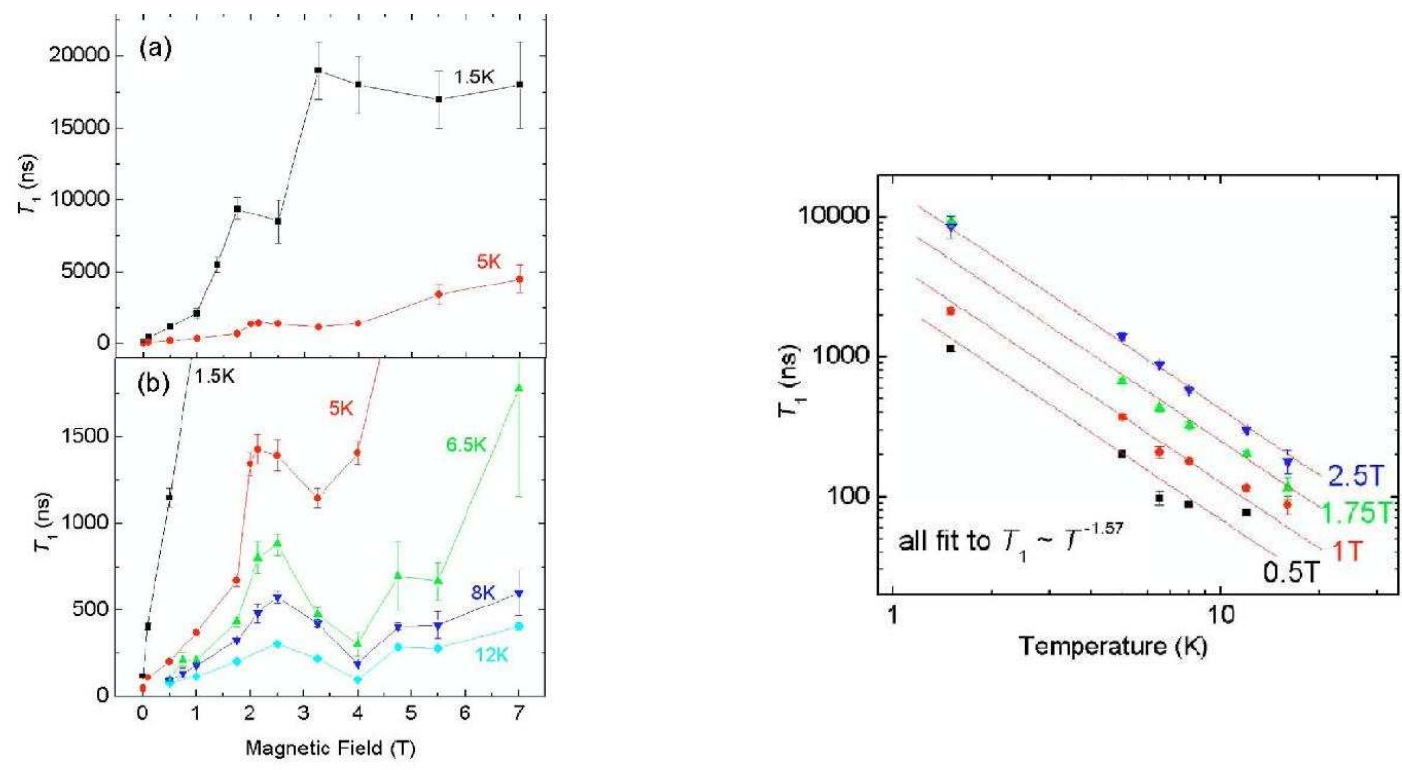

Figure 2: Left: Spin lifetime $T_{1}$ as function of magnetic field at various temperatures (a) $T=1.5$ and $5 \mathrm{~K}$; (b) $T=1.5,5,6.5,8$ and $12 \mathrm{~K}$. Right: Spin lifetime $T_{1}$ as function of temperature for various magnetic field. All temperature dependences can be fitted to $T_{1} \sim T^{-1.57}$, as indicated by the thin solid lines. Doping density is $n_{D}=10^{15} \mathrm{~cm}^{-3}$. From Colton et al. [511].

The electric field dependence of the spin lifetime in insulating regime also reflects characteristics of electron localization. Furis et al. measured such dependence [527]. Their results showed that the spin lifetime varies slowly with electric field in the linear transport regime. However, after the threshold field of impact ionization, spin lifetime drops rapidly with electric field. At such threshold, the free electrons get enough energy from the electric field to

to reexamine the spin relaxation times obtained by Hanle, or Faraday/Kerr measurements via the spin noise spectroscopy method. Such study has been done by Römer et al. in Ref. [520], where the spin relaxation times measured by different methods are compared in very dilute doped $\left(n_{D}=10^{14} \mathrm{~cm}^{-3}\right)$, low doped $\left(n_{D}=2.7 \times 10^{15} \mathrm{~cm}^{-3}\right)$, and doping close to metal-insulator transition $\left(n_{D}=1.8 \times 10^{16} \mathrm{~cm}^{-3}\right)$ samples. 
ionize the localized electrons [527]. After the localized electrons become free and the electron system acquires enough energy to become a hot electron system, the D'yakonov-Perel' spin relaxation is accelerated. The spin lifetime hence drops rapidly.

Density dependence of spin lifetime in two-dimensional electron system was studied by Sandhu et al. [528] and Chen et al. [398] (see Fig. 3), where metal-insulator transition is involved. In the work of Sandhu et al., metalinsulator transition was demarcated by conductivity [528]. Differently Chen et al. revealed metal-insulator transition by determining the density of states at band edge via $g$-factor measurement [398]. In GaAs $g$-factor has an energy dependence $g(\varepsilon)=g_{c}+\beta \varepsilon$. Therefore at zero (low) temperature the density of states $D(\varepsilon)$ is written as

$$
D(\varepsilon)=\beta\left(\frac{d g^{*}}{d n}\right)^{-1},
$$

where $g^{*}$ is the measured (ensemble averaged) $g$-factor and $n$ is electron density. By measuring the density dependence of $g^{*}$, Chen et al. obtained the density of states near band edge (see Fig. 3) where the tail reveals the existence of localized states. Electron localization also manifests itself in the magnetic field dependence of spin lifetime: spin dephasing rate in insulating regime was found to follow

$$
\frac{1}{T_{2}^{*}(B)}=\frac{1}{T_{2}^{*}(0)}+\frac{\Delta g \mu_{\mathrm{B}} B}{\sqrt{2}},
$$

due to the $g$-factor inhomogeneity and localization 36 Similar dependence was also found in other two-dimensional structures at low temperature [529-531], two-dimensional hole system [400, 531] and in ZnSe epilayers close to $\mathrm{GaAs} / \mathrm{ZnSe}$ interface [532]. In localized regime, the electron (hole) spin lifetime in two-dimensional system is very long. Usually electron spin lifetime is of the order of nanoseconds to microseconds [445, 531, 533, 534], whereas hole spin lifetime can reach nanoseconds [445, 446]. As temperature increases, spin lifetime decreases due to delocalization and enhancement of the D'yakonov-Perel' spin relaxation which increases with electron kinetic energy [400, 445, 525, 535, 536]. Pump density dependence of spin lifetime was studied in Refs. [534, 535] where spin lifetime was found to decrease with pump density at $T<2 \mathrm{~K}$, similar to that in bulk materials in insulating regime [4, 39, 518]. The underlying physics is similar to the temperature dependence: the increasing pump density leads to the increase of electron density as well as the thermalization of the electron system, and enhances spin relaxation.

Spin relaxation in type-II quantum wells was studied in both GaAs and ZnSe quantum wells at very low temperature [537, 538]. As electrons and holes are spatially separated, the electron-hole exchange interaction is weakened and spin relaxation time is elongated. Especially, in GaAs type-II quantum well, electron spin lifetime can be as long as $7 \mu$ s at $1.7 \mathrm{~K}[537]$.

Recently, Korn et al. observed extremely long hole spin lifetime, $\simeq 70 \mathrm{ns,} \mathrm{in} \mathrm{two-dimensional} \mathrm{hole} \mathrm{system} \mathrm{in}$ (001) quantum wells at $0.4 \mathrm{~K}$ [446]. They also observed the decrease of hole spin lifetime with increasing magnetic field roughly following Eq. (92). The low-field $(\sim 0.2 \mathrm{~T})$ spin lifetime was observed to decrease by about one order of magnitude when temperature increases from $0.4 \mathrm{~K}$ to $4.5 \mathrm{~K}$. Interestingly, spin relaxation of localized holes due to the hyperfine interaction can be inhibited by a very small in-plane magnetic field $(\sim 10 \mathrm{mT})$ due to the Ising form of the hole hyperfine interaction [301]. Also, the g-tensor inhomogeneity mechanism is not dominant at small (but still much larger than $10 \mathrm{mT}$ ) magnetic field [446]. Hence the most likely spin relaxation mechanism at such magnetic field is the anisotropic exchange interaction mechanism.

Finally, it should be mentioned that dynamic nuclear polarization is more efficient in insulating regime than in metallic regime because the hyperfine interaction plays a more important role in electron spin relaxation in insulating regime. The dynamic nuclear spin polarization, in turn, affects the electron spin dynamics. Experimental studies involve continuous [3, 302] and time-resolved [311, 539-541] measurements of electron spin dynamics to reveal the nuclear spin dynamics.

\subsection{Carrier spin relaxation in metallic regime}

At high temperature and/or high density, carrier system is in metallic regime where most of the carriers are in extended states in the conduction/valence band with $\mathbf{k}$ being a good quantum number. The relevant spin relaxation

\footnotetext{
${ }^{36}$ In metallic regime spin relaxation rate either decreases with increasing magnetic field or varies as $\left[1 / T_{2}^{*}(B)-1 / T_{2}^{*}(0)\right] \sim B^{2}$ due to the $g$-factor inhomogeneity.
} 

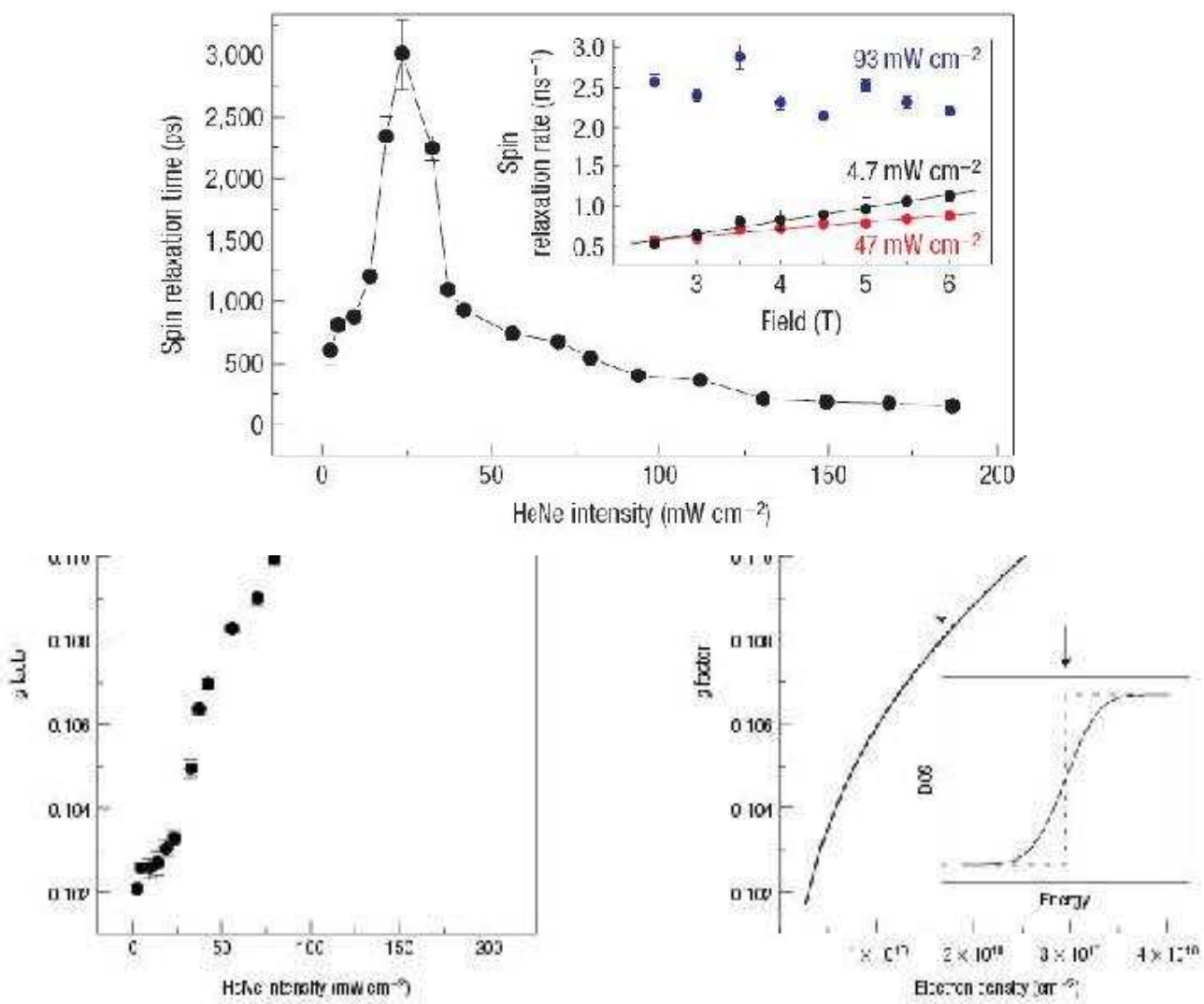

Figure 3: Up: Spin relaxation time as function of HeNe laser excitation intensity (HeNe intensity). Inset indicates magnetic field dependence of spin relaxation rate for different HeNe laser intensities [solid lines denote the fittings according to Eq. [92]]. Down: The (a) measured and (b) calculated $g$-factor as function of $\mathrm{HeNe}$ laser intensity (electron density). Inset of (b) shows the calculated density of states (DOS) of the two-dimensional electron gas with a localization tail (solid curve) and that of the unperturbed two-dimensional electron gas (dashed curve). The temperature is $4 \mathrm{~K}$. From Chen et al. [398].

mechanisms in the metallic regime are the Elliott-Yafet, the D'yakonov-Perel' and the Bir-Aronov-Pikus mechanisms. In the presence of magnetic field, the $g$-tensor inhomogeneity mechanism can also be important [374]. Their relative efficiencies differ in various materials and structures. The different temperature, mobility and carrier density dependences of these mechanisms together with their competition result in various behaviors observed in experiments. In this subsection, we review electron/hole spin relaxation in metallic regime in both bulk semiconductors and semiconductor nanostructures 37 We focus on the experiments and single-particle theories, whereas the many-body theory is also mentioned partly when it is necessary to understand the experimental results. Review of the many-body theory and its salient predictions and results are presented in the next section.

\footnotetext{
${ }^{37}$ The experiments and the corresponding theory of electron spin relaxation in $p$-type bulk semiconductors are summarized in the seminal book "Optical Orientation" [3]. We will not review this topic here.
} 


\subsubsection{Electron spin relaxation in n-type bulk III-V and II-VI semiconductors}

As there are few holes, the Bir-Aronov-Pikus mechanism is irrelevant in $n$-type III-V and II-VI semiconductors. Relevant mechanisms are the D'yakonov-Perel' and Elliott-Yafet mechanisms. As has been shown in recent work [110], the Elliott-Yafet mechanism is less important than the D'yakonov-Perel' mechanism in $n$-type III-V semiconductors, even in the narrow band-gap semiconductors such as InAs and InSb. We neglect the Elliott-Yafet mechanism in the subsequent discussions. A simple theory of the D'yakonov-Perel' spin relaxation in bulk system has been presented in Sec. 3.2.2 [21, 117]. The main result is

$$
\tau_{\mathrm{DP}}^{-1} \simeq Q \tau_{p} \alpha^{2}\left\langle\varepsilon_{\mathbf{k}}^{3}\right\rangle / E_{g}
$$

$Q \sim 1$. In the presence of magnetic field, the $g$-tensor inhomogeneity mechanism also works [374],

$$
\tau_{s}^{-1} \simeq\left(\mu_{\mathrm{B}} B\right)^{2}\left(\overline{g^{2}}-\bar{g}^{2}\right) \tau_{p}
$$

The $g$-tensor is assumed to be isotropic. The above equations should be taken only qualitatively, as they are based on a series of approximations and assumptions which are to be justified. From these equations the qualitative behavior of spin relaxation can be understood. To achieve such understanding, the knowledge of momentum scattering time $\tau_{p}$ is needed. In $n$-type III-V and II-VI semiconductors, momentum scattering mainly consists of the impurity, phonon, electron-electron scatterings. In earlier theories based on single particle approach, the electronelectron scattering is considered irrelevant to spin relaxation 38 Moreover, the acoustic phonon and transverse-optical phonon scatterings are negligible. Therefore, the important scattering mechanisms are the ionized-impurity scattering and the longitudinal-optical-phonon scattering. According to the Brooks-Herring formula, the temperature and density dependence of ionized-impurity scattering time is [21] $\tau_{p}^{\mathrm{ei}} \sim T^{3 / 2} n_{D}^{-1}\left(n_{D}\right.$ is doping density) for $T \gg T_{F}$ (nondegenerate regime) and $\tau_{p}^{\mathrm{ei}} \sim T^{0} n_{D}^{0}$ for $T \ll T_{F}$ (degenerate regime). Based on these dependences, for the D'yakonov-Perel' spin relaxation due to the ionized-impurity scattering, $\tau_{s} \sim T^{-9 / 2} n_{D}$ in nondegenerate regime and $\tau_{s} \sim T^{0} n_{D}^{-2}$ in degenerate regime. For spin relaxation due to the $g$-tensor inhomogeneity mechanism associated with the ionized-impurity scattering, roughly, $\tau_{s} \sim B^{-2} T^{-7 / 2} n_{D}$ in nondegenerate regime and $\tau_{s} \sim B^{-2} T^{0} n_{D}^{-4 / 3}$ in degenerate regime. The longitudinal-optical-phonon scattering is only important at high temperature $k_{B} T \gtrsim \omega_{\mathrm{LO}} / 4\left(\omega_{\mathrm{LO}}\right.$ is longitudinal-optical-phonon frequency). For example, in GaAs the longitudinal-optical-phonon scattering is important only at $T \gtrsim 100 \mathrm{~K}$. The temperature and density dependences of longitudinal-optical-phonon scattering are complex. Roughly, the longitudinal-optical-phonon scattering rate increases with temperature but varies weakly with density 39

We first review experimental works. Let us start with temperature dependence of spin lifetime. Spin lifetime was measured as function of temperature in GaAs [4, 371, 520, 543], GaSb [544], GaN [545-548], InAs [549-552], InSb [551, 553], InP [554], ZnSe [555], $\mathrm{ZnO}$ [556], CdTe [524] and $\mathrm{HgCdTe}$ [557, 558]. It was found to decrease with increasing temperature at high temperature (nondegenerate regime) in all these works [e.g., see Fig. 4] 40 This is consistent with the discussion in above paragraph: $\tau_{s} \sim T^{-9 / 2}$ for ionized-impurity scattering. For longitudinaloptical-phonon scattering, as $\tau_{p}^{-1}$ varies slower than $1 /\left\langle\varepsilon_{\mathbf{k}}^{3}\right\rangle \sim T^{-3}, \tau_{s}$ still decreases with temperature. It was observed that at high density in degenerate regime the spin lifetime varies slowly with temperature [544, 555, 560]. This is understood as that in degenerate regime the ionized-impurity scattering and longitudinal-optical-phonon scattering times as well as $\left\langle\varepsilon_{\mathbf{k}}\right\rangle$ vary slowly with temperature [see Eq. (93)].

The magnetic field dependence of spin lifetime was studied in GaAs [4, 517, 561] and GaN [255, 545]. As indicated in Sec. 3.2.2, spin lifetime due to the D'yakonov-Perel' mechanism increases with increasing magnetic field. On the other hand, spin lifetime due to the $g$-tensor inhomogeneity mechanism decreases with increasing magnetic field. These two mechanisms compete with each other, hence spin lifetime first increases then decreases with increasing magnetic field [374]. The peak magnetic field $B_{p}$ roughly satisfies $\alpha^{2}\left\langle\varepsilon_{\mathbf{k}}^{3}\right\rangle / E_{g} \simeq\left(\mu_{\mathrm{B}} B_{p}\right)^{2}\left(\overline{g^{2}}-\bar{g}^{2}\right)$. In

\footnotetext{
${ }^{38}$ In fact, electron-electron scattering plays important role in the D'yakonov-Perel' spin relaxation in $n$-type bulk III-V semiconductors, as was shown by Jiang and Wu in Ref. [110].

${ }^{39}$ Dyson and Ridley calculated the longitudinal-optical-phonon scattering time for nondegenerate electron system. They found that in nondegenerate regime the longitudinal-optical-phonon scattering rate increases with electron energy and temperature [542].

${ }^{40}$ At low temperature the system may be insulating where spin relaxation is governed by localized electrons [324, 559].
} 


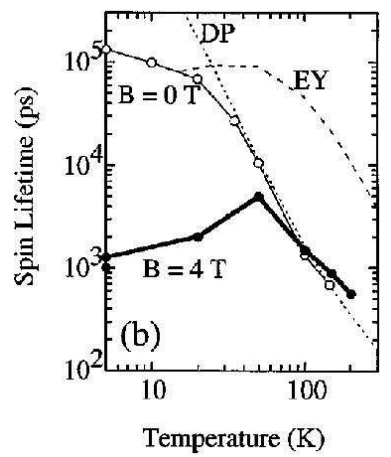

Figure 4: Temperature dependence of spin lifetime for bulk $n$-GaAs at $B=0$ and $B=4 \mathrm{~T}$ with doping density $n_{\mathrm{D}}=10^{16} \mathrm{~cm}^{-3}$. The dotted and dashed curves represent the calculated D'yakonov-Perel' (DP) and ElliottYafet (EY) spin lifetimes respectively. From Kikkawa and Awschalom [4].

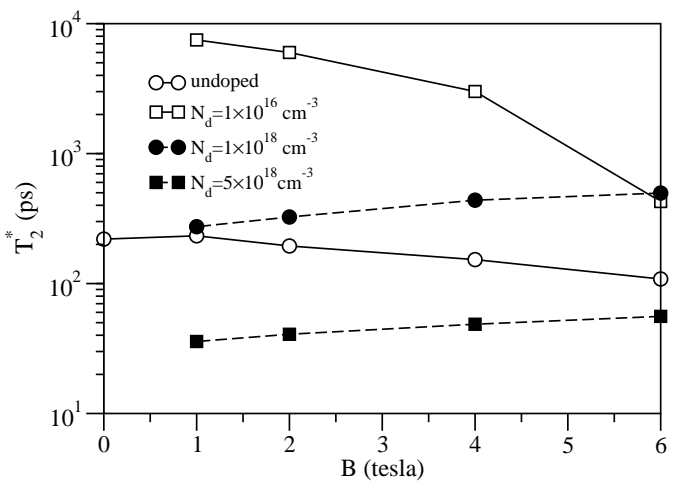

Figure 5: Magnetic field $B$ dependence of ensemble spin dephasing time $T_{2}^{*}$ for bulk GaAs at various doping density at temperature $T=5 \mathrm{~K}$. Experimental data from Kikkawa and Awschalom [4](re-plotted by Žutić et al. [21]).

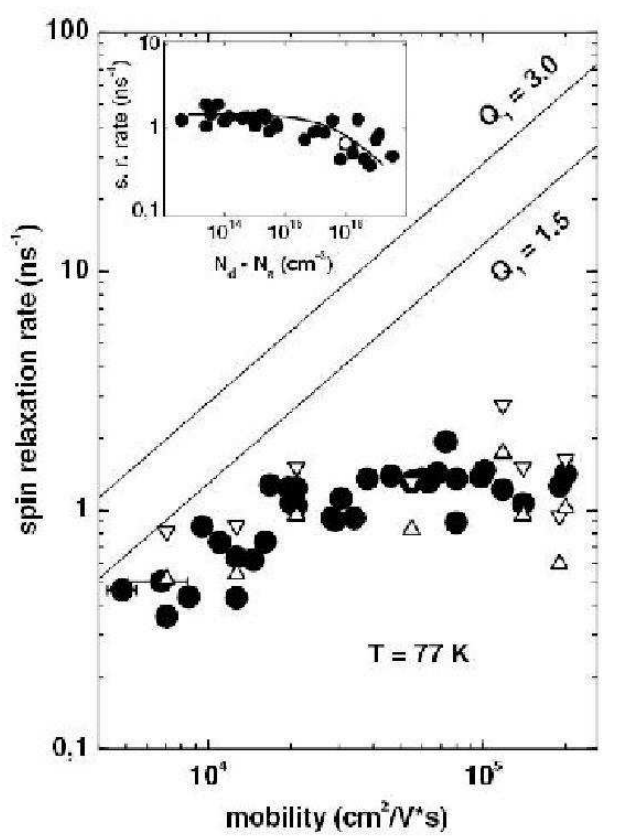

Figure 6: Mobility dependence of spin relaxation rate in bulk $n$-GaAs at temperature $T=77 \mathrm{~K}$. Experimental results are represented by dots. Solid lines: calculation from $1 / \tau_{s}=Q_{1} \tau_{p} \alpha^{2}\left(k_{B} T\right)^{3} / E_{g}$ with $Q_{1}=3$ (scattering by phonons, upper straight line) and $Q_{1}=1.5$ (scattering by ionized impurities, lower straight line). Triangles: calculation with $1 / \tau_{s}=Q_{1} \tau_{p} \alpha^{2}\left(k_{B} T\right)^{3} / E_{g}$ using $\tau_{p}$ measured from spin-relaxation suppression in Faraday geometry, assuming scattering by phonons $(\nabla)$, and ionized impurities $(\triangle)$. Inset: Density dependence of the spin relaxation rate (s. r. rate). $N_{d}$ and $N_{a}$ denote the donor and acceptor densities respectively. Data point (o) is taken from Ref. [4] at $T=100 \mathrm{~K}$. Solid curve is a guide to the eyes. From Dzhioev et al. 371].

III-V and II-VI bulk semiconductors the electron $g$-factor is energy-dependent $g \simeq g_{c}+\beta \varepsilon_{\mathbf{k}}$, hence $\left(\overline{g^{2}}-\bar{g}^{2}\right) \sim\left\langle\varepsilon_{\mathbf{k}}^{2}\right\rangle$. Therefore, the peak magnetic field $B_{p} \sim\left\langle\varepsilon_{\mathbf{k}}\right\rangle$ which increases with the average energy. Typical experimental results are shown in Fig. 5 It is seen that for the undoped sample the spin lifetime first increases and then decreases with increasing magnetic field. For $B>1 \mathrm{~T}$, at low density the spin lifetime decreases with magnetic field whereas at high density it increases with magnetic field. This is easily understood since $\left\langle\varepsilon_{\mathbf{k}}\right\rangle$ is larger at high density, the peak $B_{p}$ moves to higher magnetic field [374].

The variation of spin lifetime with mobility has been investigated in GaAs [371] and InAs [550] at zero magnetic field 41 In GaAs, the spin relaxation rate was found to increase with mobility, which is consistent with the D'yakonovPerel' mechanism. However, the simple formula, e.g., Eq. (63) in Sec. 3.2.2, can not explain the results qualitatively [371] (see Fig. 6). Momentum scattering that does not contribute to mobility was suggested to be responsible for the smaller spin relaxation rate at high mobility regime [371].

The photo-excitation density dependence of spin lifetime was investigated in $n$-type GaAs [4, 562, 563], GaSb [544] and InAs [552]. In all these measurements, the spin lifetime was found to decrease with excitation density in $n$ doped samples. The decrease was unexplained in some works [4, 562] or was assigned to the Elliott-Yafet mechanism

\footnotetext{
${ }^{41}$ The magnetic field is always zero in this subsection unless otherwise specified.
} 
[552]. Other works explained the decrease as a result of thermalization of the electron system via photo-excitation [544]. The decrease of spin lifetime may be due to the increase of $\left\langle\varepsilon_{\mathbf{k}}^{3}\right\rangle$ as the electron density increases. However, the thermalization effect may also play an important role 42

The doping density dependence was studied in GaAs [4, 39, 371] and InAs [549]. In all these works spin relaxation time was found to decrease with doping density in degenerate regime. There is few data in the nondegenerate regime up till now [4, 549]. The decrease of spin lifetime in degenerate regime is understood as following: in degenerate regime the ionized-impurity scattering time and longitudinal-optical-phonon scattering time vary slowly with density, whereas $\left\langle\varepsilon_{\mathbf{k}}^{3}\right\rangle \sim n_{D}^{2}$ increases with density. Therefore the spin lifetime $\tau_{s} \sim 1 /\left(\left\langle\varepsilon_{\mathbf{k}}^{3}\right\rangle \tau_{p}\right)$ decreases with density.

Electric field dependence was studied in GaAs [527, 567], $\mathrm{ZnO}$ [568] and GaN [548]. Usually, spin lifetime decreases with increasing electric field in metallic regime in GaAs [527, 567]. This is because the electric field induces electron drift and the hot-electron effect which increase the average electron energy and then enhance the D'yakonov-Perel' spin relaxation [110]. However, the hot-electron effect also gives rise to the enhancement of momentum scattering [569] which leads to the suppression of the D'yakonov-Perel' spin relaxation. These two effects compete with each other [569]. It was found that in the case of cubic-k spin-orbit coupling, the former effect dominates because the spin lifetime varies rapidly with the electron energy [110, 570]. The spin lifetime hence decreases with increasing electric field [110, 570]. This is the case for bulk GaAs. The situation is more complicated in the case of linear-k spin-orbit coupling: the spin lifetime first increases due to the enhancement of momentum scattering then decreases due to the increases of electron energy [569]. This is the case for bulk wurzite $\mathrm{ZnO}$ where Ghosh et al. observed that the spin lifetime first increases then decreases with increasing electric field [568]. Usually the effect of electric field on spin relaxation is more pronounced at low temperature [110, 372, 527, 548, 568]. The spin lifetime can also be reduced by applying strain which induces additional spin-orbit coupling [144, 145, 571, 572]. It was found that $1 / \tau_{s} \sim \epsilon^{2}$, where $\epsilon$ is the applied stress [572] when the strain-induced spin-orbit coupling dominates. Under strain field the temperature and density dependence of spin relaxation becomes weaker as the strain-induced spin-orbit coupling is linear in $\mathbf{k}$ [see Eq. (7)]. It is also found that the spin relaxation can be anisotropic under strain [572].

In wurzite $\mathrm{GaN}$ and $\mathrm{ZnO}$, the spin-orbit coupling is different from that in zinc-blende semiconductors. The spinorbit coupling in conduction band reads

$$
H_{\mathrm{SO}}^{e}=\left[\mathbf{\Omega}_{e}^{R}(\mathbf{k})+\mathbf{\Omega}_{e}^{D}(\mathbf{k})\right] \cdot \boldsymbol{\sigma} / 2
$$

with

$$
\mathbf{\Omega}_{e}^{R}(\mathbf{k})=2 \alpha_{e}\left(k_{y},-k_{x}, 0\right), \quad \mathbf{\Omega}_{e}^{D}(\mathbf{k})=2 \gamma_{e}\left(b k_{z}^{2}-k_{\|}^{2}\right)\left(k_{y},-k_{x}, 0\right) .
$$

It is seen that the symmetry of the spin-orbit coupling is quite different from that of the Dresselhaus one. Unlike in bulk GaAs, electron spin relaxation in bulk $\mathrm{GaN}$ is anisotropic. Lifetime of spins along [0001] direction is smaller than those along other directions. The anisotropy of spin relaxation was demonstrated in recent experiment [255].

Spin lifetime $\tau_{s}$ at room temperature is also of concern due to interest in possible device application. It was measured in GaAs [543, 573] with $\tau_{s}$ in the range of 15-110 ps, in InAs [552, 574, 575] with $\tau_{s}$ in the range of 2-24 ps and in $\mathrm{InSb}\left[551,553\right.$, 558] with $\tau_{s}$ in the range of 2-300 ps depending on the doping density.

We now review the single-particle theory on electron spin relaxation in $n$-type bulk III-V and II-VI semiconductors. Besides the early works in 1970s and early 1980s, which have been summerized in the book "Optical Orientation" [3], there are also a lot of theoretical studies in the past decade after the rising of the semiconductor spintronics [576]. These studies try to go beyond the widely used formulae for the D'yakonov-Perel' [Eqs. (62) and (63)] and ElliottYafet [Eq. (53)] spin relaxation [117]. These developments go in the same paradigm: calculate spin-orbit coupling and scattering via microscopic theory and then average the spin relaxation rate at different $\mathbf{k}$ as

$$
\tau_{s}^{-1}=\frac{\int d \mathbf{k}\left(f_{\mathbf{k}}^{\uparrow}-f_{\mathbf{k}}^{\downarrow}\right) \tau_{s}^{-1}(\mathbf{k})}{\int d \mathbf{k}\left(f_{\mathbf{k}}^{\uparrow}-f_{\mathbf{k}}^{\downarrow}\right)} .
$$

\footnotetext{
${ }^{42}$ The thermalization effect was shown to be crucial in a recent fully microscopic calculation [564]. This is also supported by the experimental results that the spin lifetime was observed to decrease with photon energy of the pump laser [543, 565, 566].
} 
The spin relaxation rate at different $k, \tau_{s}^{-1}(k)$, due to the D'yakonov-Perel' mechanism is given in Eq. (58), while that due to the Elliott-Yafet mechanism is given by

$$
\tau_{s}^{-1}(\mathbf{k})=\sum_{\mathbf{k}^{\prime}}\left[\Gamma\left(\mathbf{k} \uparrow, \mathbf{k}^{\prime} \downarrow\right)+\Gamma\left(\mathbf{k} \downarrow, \mathbf{k}^{\prime} \uparrow\right)\right],
$$

with $\Gamma\left(\mathbf{k} \uparrow, \mathbf{k}^{\prime} \downarrow\right)$ and $\Gamma\left(\mathbf{k} \downarrow, \mathbf{k}^{\prime} \uparrow\right)$ denoting the spin-flip scattering rates. Such a paradigm was first applied to $n$ type bulk GaAs, InAs and GaSb by Lau et al. where the spin-orbit coupling was calculated via the fourteen-band $\mathbf{k} \cdot \mathbf{p}$ theory [577]. The momentum scattering rates due to the electron-impurity and electron-phonon scattering were calculated via standard methods in transport theory. Similar schemes were then applied to bulk GaAs and GaN by Krishnamurthy et al. [578]. Later the scheme was developed to the case of arbitrary band structure by Yu et al. [209] ${ }^{43}$ The role of scattering by dislocations in both the D'yakonov-Perel' and Elliott-Yafet spin relaxation was studied by Jena within such scheme [579]. A systematic study on spin relaxation in III-V semiconductors based on such paradigm was given by Song and Kim [108]. However, in their work the Boltzmann statistics was assumed which makes the discussion at low temperature and/or high density meaningless. The Elliott-Yafet spin relaxation due to the electron-electron scattering was studied in Refs. [580, 581] within such paradigm. Recently spin relaxation in $\mathrm{ZnO}$ due to the D'yakonov-Perel' and Elliott-Yafet mechanisms was also studied in such paradigm [559]. An improvement of the paradigm was given in Ref. [582]. In that work, it was argued that the strong scattering condition $\langle\Omega(\mathbf{k})\rangle \tau_{p} \ll 1$ may not be satisfied for large $k$ as both $\langle\Omega(\mathbf{k})\rangle$ and $\tau_{p}$ increase with $k$. Hence the spin relaxation rate should be modified to include the free induction decay as [582]

$$
\frac{1}{\tau_{s}^{\prime}(k)}=\left(\tau_{s}\left(\varepsilon_{k}\right)+\frac{\sqrt{128 E_{g}}}{\alpha \varepsilon_{k}^{3 / 2}}\right)^{-1} .
$$

Spin relaxation rate of the electron ensemble is then obtained by averaging over $\mathbf{k}$ via Eq. 97). A closer examination on the D'yakonov-Perel' spin relaxation due to the electron-longitudinal-optical-phonon scattering from the equation of motion was given in Ref. [542], where it was shown that the elastic scattering approximation can have problem in treating the longitudinal-optical-phonon scattering to spin relaxation. The effect of the electron-longitudinal-opticalphonon scattering on the D'yakonov-Perel' spin relaxation may not be characterized by a single momentum scattering time used in the paradigm. Spin relaxation due to the D'yakonov-Perel' mechanism under electric field was studied via the Monte Carlo method in Ref. [583], where spin lifetime was found to decrease with the electric field.

\subsubsection{Electron spin relaxation in intrinsic bulk III-V and II-VI semiconductors}

In intrinsic semiconductors electrons are generated together with equall number of holes by photo excitation. Due to the large number of holes, the Bir-Aronov-Pikus mechanism is now relevant to the spin relaxation. As have been pointed out in Ref. [110] that the Elliott-Yafet mechanism is unimportant in intrinsic III-V semiconductors. Similar conclusion should also hold for II-VI semiconductors. Hence, the relevant spin relaxation mechanisms are the BirAronov-Pikus and the D'yakonov-Perel' mechanisms. It was debated about the relative importance of these two mechanisms in intrinsic III-V and II-VI semiconductors [21, 390, 392, 584-586]. Single-particle theory based on the elastic scattering approximation comes to the conclusion that spin relaxation due to the Bir-Aronov-Pikus mechanism can be more important at low temperature and high hole density [390]. However, as pointed out by $\mathrm{Zhou}$ and Wu [109], the Pauli blocking of electrons at low temperature and/or high hole density largely reduces the Bir-AronovPikus spin relaxation from a fully microscopic kinetic spin Bloch equation approach [44, 334, 350]. After that Jiang and $\mathrm{Wu}$ showed that in intrinsic bulk III-V semiconductors the Bir-Aronov-Pikus mechanism is unimportant via the same approach [110], where similar conclusion should also hold for II-VI semiconductors. Therefore, the relevant spin relaxation mechanism is the D'yakonov-Perel' mechanism. Spin relaxation in intrinsic bulk III-V and II-VI semiconductors can then be understood easily.

Experimental studies on spin relaxation in intrinsic semiconductors involve temperature dependence [229, 582, 587, 588] and density dependence [566, 582, 585]. The temperature dependence coincides with what predicted by

\footnotetext{
${ }^{43}$ In this work, the approach that the authors used to calculate hole spin relaxation rate seems to have some problem, as they concluded that the hole spin relaxation is dominated by the Elliott-Yafet mechanism which is not true.
} 
theory: spin lifetime varies slowly in degenerate regime and then decreases rapidly in nondegenerate regime with increasing temperature. The density dependence, however, indicates an interesting behavior: spin lifetime increases with electron density [582]. This is totally different from what observed in $n$-type semiconductors where spin lifetime decreases with electron density [4, 39, 371]. However, in intrinsic semiconductors, the main scattering mechanisms are the carrier-carrier scattering and the longitudinal-optical-phonon scattering, which makes spin relaxation difficult to understand via the single-particle theory. From the many-body kinetic spin Bloch equation approach [44, 334, 350], Jiang and $\mathrm{Wu}$ found a nonmonotonic density dependence of spin lifetime due to the nonmonotonic density dependence of the carrier-carrier scattering rate: in nondegenerate regime the carrier-carrier scattering rate increases with increasing density whereas in degenerate regime it decreases with increasing density [110]. After their prediction Teng et al. observed in intrinsic bulk GaAs that at high excitation density $\left(n_{e}>10^{17} \mathrm{~cm}^{-3}\right)$ spin lifetime decreases with density [585]. However, Teng et al. assigned the decrease of spin lifetime to the Bir-Aronov-Pikus mechanism which is wrong (see comment [589]). Later Ma et al. observed such a nonmonotonic density dependence of spin lifetime in bulk CdTe [566] 44

\subsubsection{Electron spin relaxation in n-type and intrinsic III-V and II-VI semiconductor two-dimensional structures}

As was found in $n$-type and intrinsic bulk III-V and II-VI semiconductors that spin relaxation is dominated by the D'yakonov-Perel' mechanism, it is reasonable to believe that the same conclusion holds for the two-dimensional case. Indeed, calculations showed that the Elliott-Yafet mechanism is unimportant in GaAs and InGaAs quantum wells [111, 591, 592] 45 Fully microscopic many-body kinetic spin Bloch equation calculation indicated that the BirAronov-Pikus mechanism is ineffective in intrinsic GaAs quantum wells [109] 46 These evidences enable us to focus on spin relaxation due to the D'yakonov-Perel' mechanism.

\section{Anisotropic spin relaxation}

One of the salient features of the D'yakonov-Perel' spin relaxation in two-dimensional structures (quantum wells and heterostructures) is that it varies significantly with the growth direction and the structure. The former originates from tailoring the symmetry of the Dresselhaus spin-orbit coupling by the growth direction. The latter partly comes from the fact that the strength of the linear Dresselhaus term can be tuned by quantum confinement. Moreover, the Rashba spin-orbit coupling also appears in asymmetric two-dimensional structures. The joint effect of the Dresselhaus and the Rashba spin-orbit couplings leads to anisotropic spin relaxation. The strength of the Rashba spin-orbit coupling can be controlled by the gate voltage. The tunability of spin relaxation opens route to a variety of new phenomena and functionalities [22, 596].

Let us start with the spin-orbit coupling which is crucial to the D'yakonov-Perel' spin relaxation. Assuming that the confinement is strong enough so that only the lowest subband is involved. Consider a simple case: (001) quantum well, where the Dresselhaus spin-orbit coupling is

$$
H_{D}=\beta_{D}\left(-k_{x} \sigma_{x}+k_{y} \sigma_{y}\right)+\gamma_{D}\left(k_{x} k_{y}^{2} \sigma_{x}-k_{y} k_{x}^{2} \sigma_{y}\right)
$$

$\beta_{D}=\gamma_{D}\left\langle\psi_{1}\left|\hat{k}_{z}^{2}\right| \psi_{1}\right\rangle$ where $\psi_{1}$ is the envelope function of the lowest subband and $\hat{k}_{z}=-i \partial_{z} \cdot \beta_{D} \sim \gamma_{D}(\pi / a)^{2}$ with $a$ being the well width. The Rashba spin-orbit coupling reads

$$
H_{R}=\alpha_{R}\left(k_{y} \sigma_{x}-k_{x} \sigma_{y}\right) \text {. }
$$

For narrow quantum well when $\mathbf{k}^{2} \ll(\pi / a)^{2}\left[\mathbf{k}=\left(k_{x}, k_{y}\right)\right.$ is the in-plane wavevector], the linear term dominates

$$
H_{\mathrm{SOC}}=\left(\alpha_{R} k_{y}-\beta_{D} k_{x}\right) \sigma_{x}+\left(-\alpha_{R} k_{x}+\beta_{D} k_{y}\right) \sigma_{y} .
$$

\footnotetext{
${ }^{44}$ They, however, assigned the decrease of the spin lifetime with increasing density to the Elliott-Yafet mechanism, which is incorrect (see comment [590]).

${ }^{45}$ In narrow bandgap semiconductor InSb quantum wells, experiment gives some evidences that the Elliott-Yafet spin relaxation can be more important than the D'yakonov-Perel' one at low mobility samples as revealed by Litvinenko et al. in Ref. [593]. Nevertheless, the two mechanisms are still comparable in their experiment.

${ }^{46}$ However, in some wide bandgap semiconductors which have weak spin-orbit coupling and strong electron-hole exchange interaction, the Bir-Aronov-Pikus mechanism can be important in intrinsic samples, especially when excitons are formed. Such as the situation in ZnSe [594]. Also in some special cases where the D'yakonov-Perel' mechanism is suppressed, the Bir-Aronov-Pikus or the Elliott-Yafet mechanisms may be important, such as in (110) quantum wells [595]. We will return to these special cases after the discussion in common cases.
} 
It was first pointed out by Averkiev and Golub [194] that the spin relaxation is anisotropic when the Rashba and Dresselhaus spin-orbit couplings compete with each other. The spin relaxation tensor is then

$$
\tau_{z z}^{-1}=2 \tau_{x x}^{-1}=2 \tau_{y y}^{-1}=8 m\left\langle\tau_{p} \varepsilon_{\mathbf{k}}\right\rangle\left(\alpha_{R}^{2}+\beta_{D}^{2}\right), \quad \tau_{x y}^{-1}=-8 m\left\langle\tau_{p} \varepsilon_{\mathbf{k}}\right\rangle \alpha_{R} \beta_{D}
$$

Diagonalizing the spin relaxation tensor, one obtains

$$
\tau_{z z}^{-1}=8 m\left\langle\tau_{p} \varepsilon_{\mathbf{k}}\right\rangle\left(\alpha_{R}^{2}+\beta_{D}^{2}\right), \quad \tau_{ \pm \pm}^{-1}=4 m\left\langle\tau_{p} \varepsilon_{\mathbf{k}}\right\rangle\left(\alpha_{R} \mp \beta_{D}\right)^{2},
$$

where the principal axes are $\mathbf{n}_{ \pm}=\frac{1}{\sqrt{2}}(1, \pm 1,0)$ and $z$-axis. It is meaningful to rewrite the spin-orbit coupling in these principal axis,

$$
H_{\mathrm{SOC}}=\left(\alpha_{R}-\beta_{D}\right) k_{+} \sigma_{-}-\left(\alpha_{R}+\beta_{D}\right) k_{-} \sigma_{+} .
$$

Recall that $\tau_{i j}^{-1} \sim\left[\overline{\Omega^{2}} \delta_{i j}-\overline{\Omega_{i} \Omega_{j}}\right]$. As averaging over angle $\overline{k_{+} k_{-}}=0$ and $\overline{k_{+}^{2}}=\overline{k_{-}^{2}}$, one readily finds that $1 / \tau_{++} \sim$ $\left(\alpha_{R}-\beta_{D}\right)^{2}, 1 / \tau_{--} \sim\left(\alpha_{R}+\beta_{D}\right)^{2}, 1 / \tau_{+-}=0$ and $1 / \tau_{z z} \sim 2\left(\alpha_{R}^{2}+\beta_{D}^{2}\right)$. If $\alpha_{R}=\beta_{D}\left(\alpha_{R}=-\beta_{D}\right), 1 / \tau_{++}=0\left(1 / \tau_{--}=0\right)$, i.e., spin lifetime is infinite along $\mathbf{n}_{+}\left(\mathbf{n}_{-}\right)$. However, taking into account of the cubic Dresselhaus term, spin lifetime along this particular direction is finite but still much larger than that along other directions [597]. Therefore spin relaxation is highly anisotropy when $\alpha_{R} \simeq \pm \beta_{D}[196,370]$.

Experimentally as only the spin polarization along the quantum well growth direction can be measured 47 spin relaxation anisotropy is revealed indirectly by comparing spin relaxation rates along $z$ direction in the two cases with magnetic field along [110] and [1 10$]$ directions. When a moderate magnetic field is along [110] direction, spin polarization along the growth direction evolves

$$
S_{z}(t)=S_{z}(0) \exp \left[-\frac{1}{2}\left(\tau_{z z}^{-1}+\tau_{--}^{-1}\right) t\right] \cos \left(\omega_{0} t+\phi_{0}\right)
$$

The observed envelope decay rate is $\frac{1}{2}\left(\tau_{z z}^{-1}+\tau_{--}^{-1}\right)$. Applying magnetic field along [1 10$]$ direction, one obtains a decay rate of $\frac{1}{2}\left(\tau_{z z}^{-1}+\tau_{++}^{-1}\right)$. Comparing these two, one can obtain spin lifetimes $\tau_{++}$and $\tau_{--}$and the ratio $\left|\alpha_{R} / \beta_{D}\right|$ can be extracted 48 The spin relaxation anisotropy in (001) quantum wells was first observed by Averkiev et al. [198] via the Hanle measurements, and then by several groups via time-resolved measurements [66, 199, 598, 599]. Among these works, the spin relaxation anisotropy was demonstrated to be controlled/affected by density [598], magnetic field [199], gate-voltage (see Fig. 7] [599, 600] and temperature [66, 598]. The symmetry of the spin-orbit coupling in other two-dimensional structures with different growth directions also has marked effect on spin relaxation. In some particular cases, such as (111) and (110) quantum wells, spin relaxation can be largely suppressed. As there are also a lot of papers on those topics, we will discuss them in detail later while below we only focus on (001) quantum wells.

\section{Spin relaxation in $(001)$ quantum wells}

Widely concerned topics are the temperature, density, mobility, excitation density, magnetic field, gate-voltage and quantum subband quantization energy dependences of spin relaxation. These dependences reveal intriguing underlying physics and provide important knowledges for spintronic device design. Below we first review experimental studies and then the single-particle theories. To benefite the understanding, we first present some simple analytical results which assume only elastic scattering in the strong scattering regime [196]

$$
\tau_{z}^{-1}=4\left\langle\left[\tilde{\tau}_{1}\left(\alpha_{R}^{2}+\tilde{\beta}_{D}^{2}\right) k^{2}+\tilde{\tau}_{3} \gamma_{D}^{2} k^{6} / 16\right]\right\rangle, \quad \tau_{ \pm}^{-1}=2\left\langle\left[\tilde{\tau}_{1}\left(\alpha_{R} \mp \tilde{\beta}_{D}\right)^{2} k^{2}+\tilde{\tau}_{3} \gamma_{D}^{2} k^{6} / 16\right]\right\rangle,
$$

Here $\tau_{i}(i=z,+,-)$ denotes the relaxation time of the spin component along $i$ direction and $\langle\ldots\rangle$ represents the average defined by Eq. (97). $\tilde{\beta}_{D}=\beta_{D}-\frac{1}{4} \gamma_{D} k^{2}$. It is noted that when the linear spin-orbit coupling dominates, the spin lifetime varies as $1 /\left\langle\tau_{p} \varepsilon_{\mathbf{k}}\right\rangle$ which changes with temperature and density slowly. However, when the cubic term becomes important, the spin lifetime can vary as $1 /\left\langle\tau_{p} \varepsilon_{\mathbf{k}}^{2}\right\rangle$ or $1 /\left\langle\tau_{p} \varepsilon_{\mathbf{k}}^{3}\right\rangle$. In the latter case, spin relaxation varies rapidly with density and temperature.

- Temperature dependence. In experiments, the temperature dependence was measured in metallic regime in Refs. [66, 367, 591, 593, 601-604]. In heavily-doped (low mobility) quantum wells, spin lifetime decreases with

\footnotetext{
${ }^{47}$ Note that a recently developed technique of tomographic Kerr rotation can measure spin polarization in other directions [500].

${ }^{48}$ Actually, via such method, one can determine the ratio but not sure whether it is $\left|\alpha_{R} / \beta_{D}\right|$ or $\left|\beta_{D} / \alpha_{R}\right|[198]$.
} 

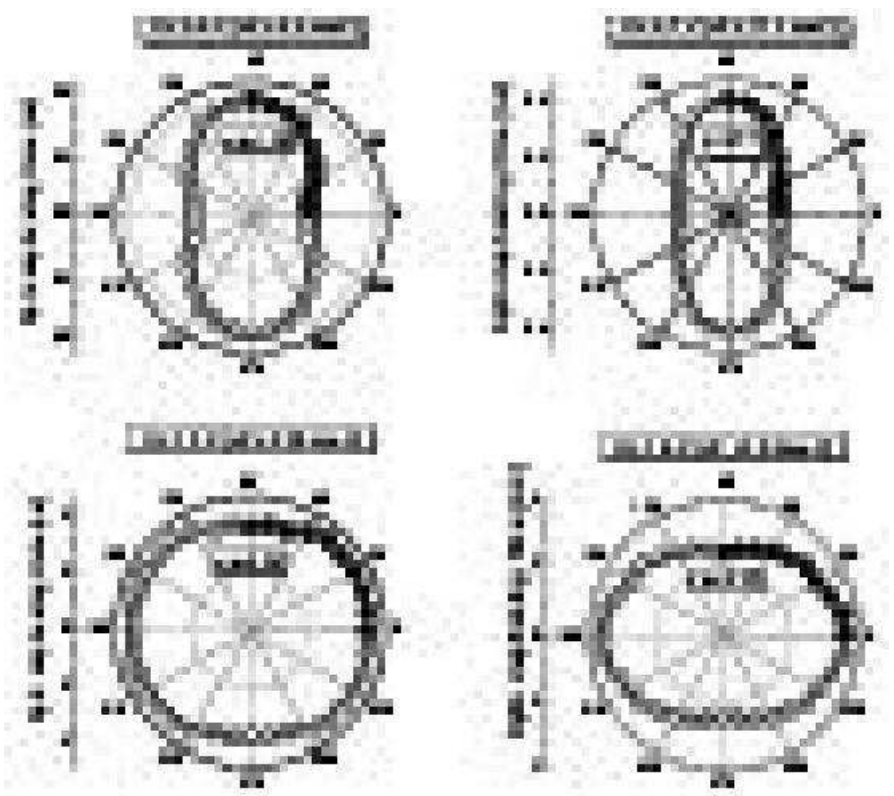

Figure 7: Polar plot of the spin dephasing time measured as a function of the angle (in degree) between the magnetic field and the [110] axis at four applied biases $U$ in coupled double quantum wells. The spin dephasing time at each angle is represented by the distance to the center of the polar coordinates. Experimental data are shown by points; theoretical values are presented by solid curves. The ratio of the Rashba spin-orbit coupling parameter $\alpha$ to the Dresselhaus one $\beta, \alpha / \beta$, extracted from the anisotropy of spin dephasing time is also presented in each figure. The values $\Delta E$ represent the exciton Stark shifts for the given biases $U$. Temperature is $T=2 \mathrm{~K}$. From Larionov and Golub [599].

increasing temperature. A good example is the experiment of Ohno et al. [602] [Fig. 8]. In heavily-doped quantum wells, the momentum scattering is dominated by the electron-impurity scattering except that at high temperature the electron-longitudinal-optical-phonon scattering may become more important. When the electron-impurity scattering dominates, as both $\tau_{p}$ and $\left\langle\varepsilon_{\mathbf{k}}\right\rangle$ increase with temperature, spin lifetime decreases with increasing temperature rapidly. At high temperature the rise of electron-longitudinal-optical-phonon scattering slows down the decrease or even leads to an increase of the spin lifetime with increasing temperature.

In undoped quantum wells, Malinowski et al. 603] presented a systematic study on electron spin relaxation. The main results are shown in Fig. 9 It is seen that the temperature dependence of spin relaxation rate varies largely with quantum well width. At small well width, spin relaxation rate changes slowly with temperature, whereas at large well width, it increases rapidly with temperature. The temperature dependence can be fitted roughly as $\sim T^{0}$ for narrow quantum well and $\sim T^{2}$ for wide quantum well. The authors explained that as the longitudinal-opticalphonon scattering dominates at high temperature for undoped quantum wells, roughly $\tau_{p} \sim T^{-1}$ [178]. For narrow quantum wells, spin relaxation rate $\tau_{s}^{-1} \sim \tau_{p}\left\langle\varepsilon_{\mathbf{k}}\right\rangle \sim T^{0}$, whereas for wide quantum wells $\tau_{s}^{-1} \sim \tau_{p}\left\langle\varepsilon_{\mathbf{k}}^{3}\right\rangle \sim T^{2}$. It is noted that in the wide quantum well, the increase of spin relaxation rate is still slower than that in bulk samples at low temperature, which indicates the crossover of the leading spin-orbit coupling from linear term to cubic term with increasing temperature.

In high mobility two-dimensional electron system at sufficiently low temperature the momentum scattering can be very weak. In this situation, the D'yakonov-Perel' spin relaxation is in the weak scattering regime where spin polarization shows precessional decay [367, 368]. Brand et al. observed the transition from the precessional decay regime to the motional-narrowing regime [368]. As spin lifetime changes from $\tau_{s} \sim \tau_{p}^{-1}$ in motional-narrowing regime to $\tau_{s} \sim \tau_{p}$ in precessional decay regime, the temperature dependence of spin lifetime has a turning point around the transition 49 This was observed in experiment by Leyland et al. (see Fig. 10) [367]. Several works on temperature dependence of spin relaxation in high mobility two-dimensional electron system revealed the importance of the electron-electron scattering to spin relaxation [367, 591, 604, 605]. This important issue will be reviewed in the

\footnotetext{
${ }^{49}$ Such behavior also exists in hole spin relaxation in Si/Ge quantum wells as reported by Zhang and Wu in Ref. [299].
} 


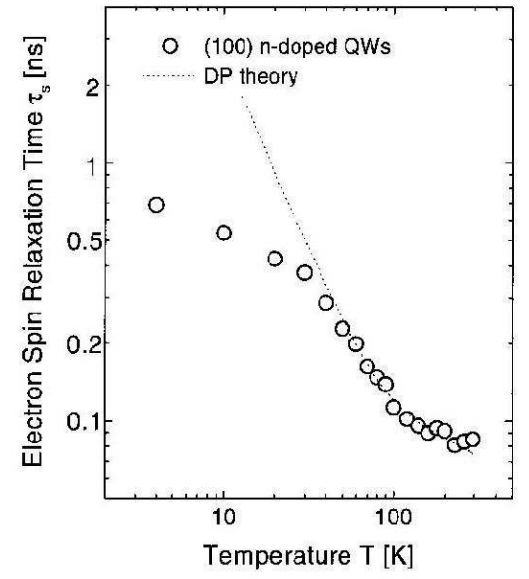

Figure 8: Temperature $T$ dependence of electron spin relaxation time $\tau_{s}$ for $n$-doped (001) GaAs/AlGaAs quantum wells with well width $7.5 \mathrm{~nm}$. Electron density is $4 \times 10^{10} \mathrm{~cm}^{-2}$ in each quantum well. Dotted curve is the calculated result of spin lifetime based on the D'yakonov-Perel' theory. From Ohno et al. [602].

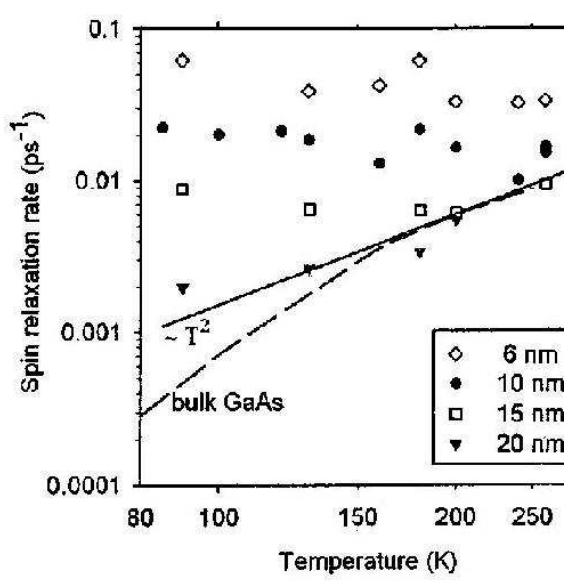

Figure 9: Temperature dependence of spin relaxation rate for undoped (001) GaAs/AlGaAs quantum wells with various well widths. $\diamond$ : well width $6 \mathrm{~nm}$; ๑: well width $10 \mathrm{~nm}$; $\square$ : well width $15 \mathrm{~nm} ; \boldsymbol{\nabla}$ : well width $20 \mathrm{~nm}$. Solid curve is a fit with $1 / \tau_{s} \sim T^{2}$ dependence. Dashed curve represents the data in intrinsic bulk GaAs by Maruschak et al. (as reproduced in Ref. [3]). From Malinowski et al. 603].

framework of the many-body theory in Section 5.

- Excitation density dependence. Excitation density dependence of spin relaxation was investigated in Refs. [66, 514, 606-612]. Interestingly, it was discovered that the spin lifetime decreases with excitation density at low temperature in $n$-doped quantum wells [66, 608, 610]. At room temperature in undoped quantum wells, Aleksiejunas et al. found that at low density spin lifetime increases with excitation density whereas at high density it decreases (see Fig. 11] [607]. Moreover, Teng et al. observed a peak in density dependence at room temperature in undoped quantum well [609]. As in intrinsic quantum wells the dominant scatterings are the many-body electron-electron and electron-hole Coulomb scatterings, the results can not be explained in the framework of single-particle approach. Nevertheless, many-body theories have revealed the underlying physics: the electron-electron and electron-hole Coulomb scatterings increase with density in nondegenerate (low density) regime, whereas decrease in degenerate (high density) regime [110, 111, 299, 613]. The nonmonotonic density dependence of these many-body carrier-carrier Coulomb scattering results in the nonmonotonic density dependence of spin lifetime [110, 111, 299, 613].

- Mobility dependence. Spin lifetime as function of mobility $\mu\left(\propto \tau_{p}\right)$ was studied in Refs. [607, 614] at room temperature where the qualitative relation $\tau_{s} \sim \mu^{-1}$ was observed which signals the D'yakonov-Perel' spin relaxation mechanism. However, Brand et al. found that in high mobility two-dimensional electron system at low temperature $(T<100 \mathrm{~K})$, spin lifetime deviates from $\tau_{s} \sim \mu^{-1}$ largely [368]. Electron-electron scattering is proved to be the key to understand the observed results 50 In high mobility two-dimensional electron system at low temperature, the electron-electron scattering is the dominant scattering as other scatterings (the electron-impurity and electronphonon scatterings) are weak. As it randomizes the momentum, the electron-electron scattering also contributes to the D'yakonov-Perel' spin relaxation. However, since the electron-electron scattering does not contribute to mobility, $\tau_{s} \sim \mu^{-1}$ does not hold anymore. The total momentum scattering time with the electron-electron scattering included, $\tau_{p}^{*}$, is then much smaller than that deduced from mobility, $\tau_{p}$ (see Fig. 12). At room temperature, the electronlongitudinal-optical-phonon scattering becomes the strongest momentum scattering which also limits the mobility, hence the relation $\tau_{s} \sim \mu^{-1}$ is recovered.

- Quantum well width dependence. The quantum well width dependence of spin relaxation was investigated in Refs. [592, 602, 603, 614, 616-619]. All these studies were performed at room temperature in nondegenerate regime. In this regime, by neglecting the cubic Dresselhaus term and the Rashba term and assuming infinite well

\footnotetext{
${ }^{50}$ This was first predicted theoretically by Wu and Ning [332, 334] and later by Glazov and Ivchenko [615].
} 


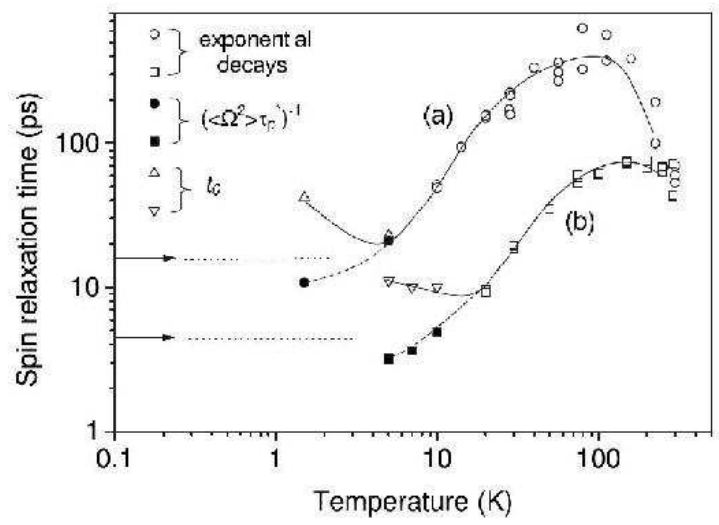

Figure 10: Temperature dependence of spin lifetime for sample (a) (well width $20 \mathrm{~nm}$, electron density $1.8 \times 10^{11} \mathrm{~cm}^{-2}$ ) and (b) (well width $10 \mathrm{~nm}$, electron density $\left.3.1 \times 10^{11} \mathrm{~cm}^{-2}\right)$. The curves are guides for the eyes. Open circles and squares are spin-lifetimes measured in the high temperature regime where spin evolution is exponential. Solid symbols are values of $\left(\left|\Omega\left(k_{F}\right)\right|^{2} \tau_{p}^{*}\right)^{-1}\left(\right.$ denoted in the figure as $\left.\left(\left\langle\Omega^{2}\right\rangle \tau_{p}^{*}\right)^{-1}\right)\left(\Omega\left(k_{F}\right)\right.$ is obtained from analysis of the spin evolution in the low-temperature oscillatory regime. $\tau_{p}^{*}$ is the momentum scattering time including the electronelectron scattering.). Open triangles are the decay time constant of the oscillatory spin evolution, $t_{0}$, obtained by fitting the experimental data with oscillatory exponential decay $S_{z}(t)=S_{0} \exp \left(-t / t_{0}\right) \cos (\omega t+\phi)$. Arrows indicate the value of $\left|\Omega\left(k_{F}\right)\right|^{-1}$ for each sample, corresponding to the condition $\left|\Omega\left(k_{F}\right)\right| \tau_{p} \simeq 1$. From Leyland et al. [367].

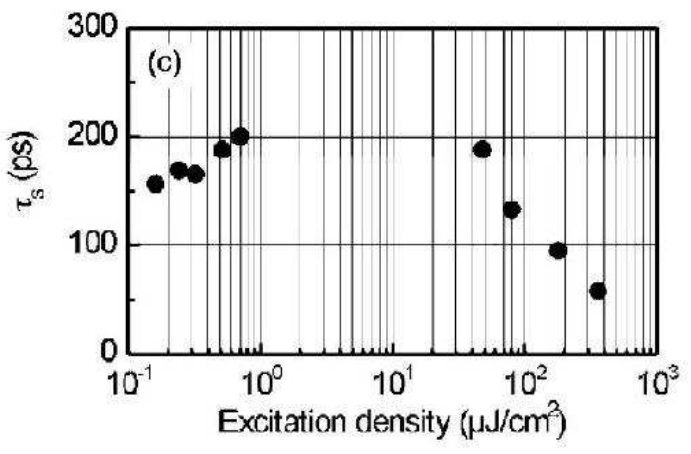

Figure 11: Excitation density dependence of spin lifetime $\tau_{s}$ in undoped (001) InGaAs multiple quantum wells at room temperature. From Aleksiejunas et al. [607].

depth, spin lifetime is given by [369] $\tau_{s}=E_{g} /\left(2 \alpha^{2} E_{1 e}^{2} k_{B} T \tau_{p}\right)$ with $\alpha=2 \gamma_{D} \sqrt{2 m_{e}^{3} E_{g}}$ and $E_{1 e}$ being the electron subband quantization energy. It hence gives $\tau_{s} \sim E_{1 e}^{-2}$. Most experimental data roughly agree with the relation. For example, Tackeuchi et al. fitted $\tau_{s} \sim E_{1 e}^{-2.2}$ for multiple GaAs/AlGaAs quantum well [616]. However, there are some situations where results deviate largely from the above relation [614]. Britton et al. reported that for small $E_{1 e}$, the relation is largely deviated whereas for large $E_{1 e}$, the spin lifetime generally shows the quadratic behavior [618]. A similar result was obtained by Malinowski et al. [603] which is shown in Fig. 13. It is seen that for medium $E_{1 e}$, the relation agrees well with experimental result. At small $E_{1 e}$ as the cubic Dresselhaus spin-orbit coupling plays important role, the relation deviates from the data. At large $E_{1 e}$ as the wavefunction penetration is unnegligible and the infinite-depth-well assumption no longer holds, the relation also deviates from experimental result.

- Magnetic field dependence. Spin lifetime has also been measured as function of magnetic field in quantum wells in metallic regime in Refs. [66, 199, 326, 525, 620, 621]. There are two special configurations of magnetic field: one that the magnetic field lies in the quantum well plane (the Voigt configuration) and the other that magnetic field is perpendicular to the well plane (the Faraday configuration). In the Voigt configuration, the orbital effect of magnetic field is negligible. In this case the magnetic field has two consequences on spin relaxation: first it mixes 


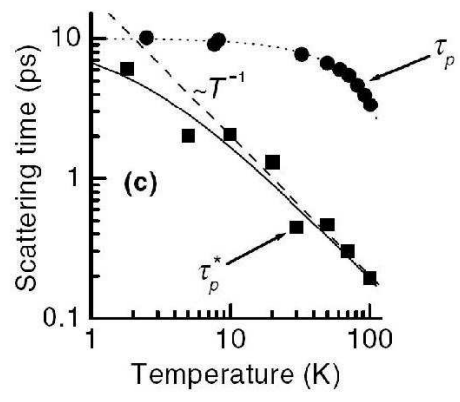

Figure 12: Momentum scattering time $\tau_{p}^{*}$ (the total momentum scattering including the electron-electron scattering) and $\tau_{p}$ (the momentum scattering time extracted from mobility) as function of temperature. The electron density is $1.9 \times 10^{11} \mathrm{~cm}^{-2}$. Dotted curve is a guide to the eyes for $\tau_{p}$. Dashed curve represents an empirical fit of electron-electron scattering time as $\sim T^{-1}$ at high temperature. Solid curve sums these two contributions $\left(1 / \tau_{p}\right.$ and the electron-electron scattering rate) as a guide curve to $\tau_{p}^{*}$. The data points of $\tau_{p}^{*}$ is obtained from Monte Carlo simulation of spin evolution. From Brand et al. [368].

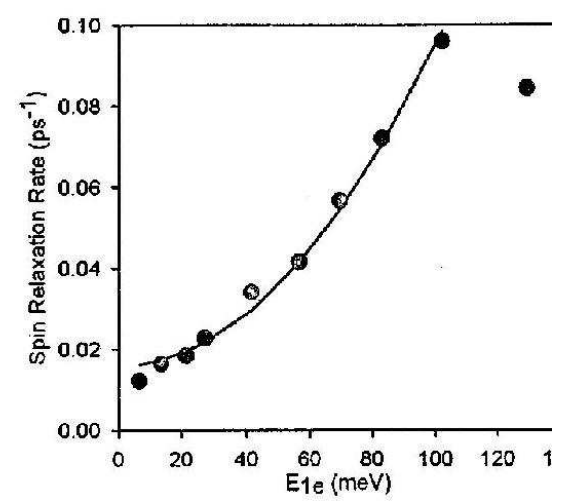

Figure 13: Spin relaxation rate as function of the subband quantization energy $E_{1 e}$ in $\mathrm{GaAs} / \mathrm{Al}_{x} \mathrm{Ga}_{1-x}$ As quantum wells at room temperature. The solid curve is a fit to the data points of the form $\tau_{s}^{-1}=a+b E_{1 e}^{2}$. From Malinowski et al. [603].

the in-plane and out-of-plane spin relaxations due to the Larmor precession; second the Larmor spin precession slows down spin relaxation by a factor of $\left(1+\omega_{L}^{2} \tau_{p}^{2}\right)$. In usual condition $\omega_{L} \tau_{p} \lesssim 0.1$ (e.g., $B=2 \mathrm{~T}$ and $\tau_{p}=1 \mathrm{ps}$ in GaAs yield $\left.\omega_{L} \tau_{p}=0.05\right)$, thus the second effect is weak. The first effect is usually more important as the in-plane and out-of-plane spin lifetimes differ largely. The effect of mixing saturates soon at a low magnetic field around $0.1 \mathrm{~T}$. This is consistent with experiments: the magnetic field dependence is usually more pronounced at low field [66, 199, 620], whereas at high field the dependence becomes weak [525]. In asymmetric quantum wells the in-plane spin relaxation can be quite anisotropic. In this case the effect of magnetic field depends largely on its direction [66, 198, 199, 598, 599]. Interestingly, Stich et al. found that the magnetic field dependence of spin lifetime for $B \|[110]$ exhibits a minimum whereas the magnetic dependence for $B \|$ [110] shows a maximum for an asymmetric (001) quantum well [199]. In the Faraday configuration with magnetic field perpendicular to quantum well plane, the orbital effect induces cyclotron motion which has an important effect on spin relaxation. As the cyclotron frequency is much larger than the Larmor frequency (in GaAs $\omega_{c} \simeq 70 \omega_{L}$ ), the cyclotron motion effectively suppresses the spin relaxation. This phenomena was observed by Sih et al. in InGaAs/GaAs quantum well (See Fig. 14] [621] 51] At higher magnetic field and low temperature, spin lifetime oscillates with magnetic field, as the Landau level filling affects both spin precession and momentum scattering. Finally in materials with strong energy dependence of the $g$-factor (e.g., in narrow band-gap semiconductors) at high magnetic field, the $g$-tensor inhomogeneity mechanism can be important. In InGaAs quantum wells, spin relaxation was observed to first increase and then decrease with increasing magnetic field for an in-plane magnetic field [623]. The increase is due to the mixing of out-of-plane and in-plane spin relaxations, whereas the decrease can be attributed to the $g$-factor inhomogeneity spin relaxation mechanism.

- Gate-voltage dependence. The spin relaxation can also be tuned by the gate-voltage [525, 599]. The gatevoltage may have several consequences on spin relaxation. First it changes electron density. Second it changes the Rashba spin-orbit coupling. The modification of the envelope function along the growth direction further changes the linear Dresselhaus spin-orbit coupling. Third it may change the mobility. Hence the underlying mechanism for the gate-voltage dependence of the spin lifetime is complex.

- Initial spin polarization dependence. Initial spin polarization dependence was studied by Stich et al. [41, 42, 326] where spin relaxation rate decreases with initial spin polarization as predicted by Weng and Wu [44]. The underlying physics will be reviewed in the next section on the kinetic spin Bloch equation approach [44, 334, 350] where the Coulomb Hartree-Fock term acts as an effective magnetic field in spin precession [44]. This effective magnetic field is always along spin polarization direction. Realistic calculations from the kinetic spin Bloch equations

\footnotetext{
${ }^{51}$ Also observed in $\mathrm{Si} / \mathrm{Ge}$ quantum well by Wilamowski and Jantsch [622].
} 


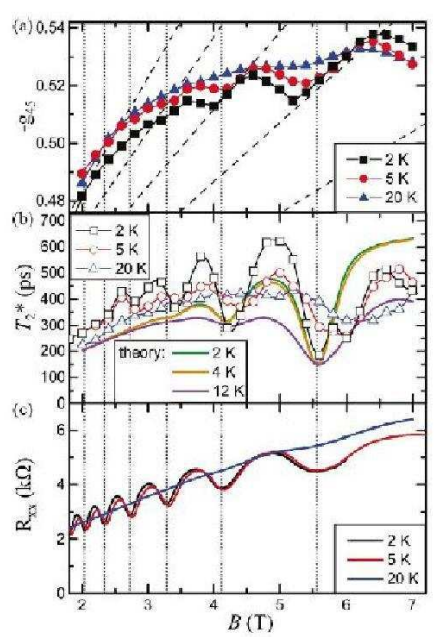

Figure 14: Up panel: Spin dephasing time $T_{2}^{*}$ measured (symbols) as a function of magnetic field $B$ at $T=2 \mathrm{~K}, 5 \mathrm{~K}$, and $20 \mathrm{~K}$ and calculated (curves) from a spin relaxation model at $T=2 \mathrm{~K}, 4 \mathrm{~K}$, and $12 \mathrm{~K}$. Down panel: the longitudinal resistivity $R_{x x}$ as a function of B at $T=2 \mathrm{~K}, 5$ $\mathrm{K}$, and $20 \mathrm{~K}$. Measurement is performed in single $n$-modulation doped InGaAs/GaAs quantum well. Also shown are dotted curves indicating the position of $B_{n}$ for Landau-level index $n=16,14,12,10,8$ and 6 ( $n$ labels the filled Landau levels). From Sih et al. [621].

indicated that the induced magnetic field can be as large as tens of Tesla [44]. Such large longitudinal effective magnetic field largely suppresses the spin relaxation. Besides spin relaxation, experimentalists also found evidence of the effective magnetic field in the sign change of the Kerr rotation [327]. Recently the Coulomb Hartree-Fock effective magnetic field was again discussed in the issue of spin accumulation [43].

- Excitation photon energy dependence and others. The excitation photon energy dependence of spin relaxation was investigated in Ref. [605] where the energy-dependent momentum scattering time due to the electron-electron scattering was revealed. Spin dynamics in higher subband was studied in Refs. [624-626], indicating the important role of inter-subband momentum scattering. Spin dynamics under microwave driving field was discussed in Ref. [627].

- Interface inversion asymmetry induced spin relaxation. The interface inversion asymmetry induced spinorbit coupling and its consequence to spin relaxation were studied in Refs. [219, 221, 222] 52 As was reviewed in Section 2.4, in the case where the quantum well and the barrier materials share no common atom, the interface inversion asymmetry exhibits even in symmetric quantum wells. The interface inversion asymmetry induced spinorbit coupling is important in narrow quantum wells. By comparing with the case with common atom of the same well width, it was discovered that spin relaxation in quantum wells without common atom is always shorter [219, 221, 617]. This effect is especially marked in narrow quantum wells. It can not be explained by the D'yakonov-Perel' theory taking into account only the Dresselhaus spin-orbit coupling [617]. As in [110]-grown quantum well, the two interfaces could be symmetric even in the case without common atom, which gives a special case without the interface-induced spin-orbit coupling [222]. By comparing spin relaxation in [110]-grown quantum well and [001]grown quantum well with same well width, one can also estimate the value of interface-induced spin-orbit coupling [222].

- Spin relaxation in diluted nitride materials. Spin relaxation in diluted nitride materials such as GaAsN and InGaAsN was studied in Refs. [629-631]. Interestingly, the spin lifetime increases with increasing temperature up to room temperature for $T>40 \mathrm{~K}$, but decreases with increasing temperature below $40 \mathrm{~K}$. It was found that less than $1 \%$ doping of nitride in GaAs and InGaAs makes the spin lifetime at room temperature increase by more than one order of magnitude in as-grown samples before annealing [629]. After annealing the spin lifetime is drastically droped [629]. The above unusual behavior indicates that localized electrons bound to nitride dopants play an important role. Even for delocalized electrons, scattering with nitride dopants greatly reduces $\tau_{p}$ and hence increases the spin lifetime.

\footnotetext{
${ }^{52}$ The well width dependence of spin lifetime in narrow quantum wells where the well and barrier materials do not share any common atom (e.g., in InGaAs/InP quantum well) may also contain the information of the interface inversion asymmetry [617, 628].
} 
Annealing improves the crystal quality of diluted nitride material which hence increases the mobility and reduces the spin lifetime. Spin lifetime at room temperature as function of subband quantization energy $E_{1 e}$ gives $\tau_{s} \sim E_{1 e}^{-1}$ in unannealed InGaAsN/GaAs multiple quantum wells [631].

\section{Single-particle theories for spin relaxation in (001) quantum wells}

We now turn to review the single-particle theory of electron spin relaxation in $n$-type and intrinsic two-dimensional electron system. As in bulk system, the single-particle theory assumes that for all $\mathbf{k}$ the strong scattering criteria $\Omega_{\mathbf{k}} \tau_{p} \ll 1$ is fulfilled. It is also assumed that the carrier-carrier scattering is irrelevant. 53 Within the elastic scattering approximation 54 spin lifetime for system near the equilibrium (also implying that the spin polarization is very small) can be calculated via Eq. (107). Such paradigm has been widely applied to study spin relaxation. Often the spinorbit coupling in the two-dimensional structure is calculated via the $\mathbf{k} \cdot \mathbf{p}$ method within the multiband envelopefunction approximation together with the Schrödinger-Poisson equation of the heterostructure (see, e.g., Ref. [633]). The momentum scattering times are calculated via the formulae developed for calculating the mobility due to the electron-impurity and electron-phonon scatterings. It should be mentioned that in heterostructures many factors such as structure, doping and gate voltage can affect the spin-orbit coupling. To quantitatively determine the spin lifetime, one has to quantitatively determine the spin-orbit coupling. In theoretical calculation, the spin-orbit coupling is determined by the multiband envelope-function calculation with realistic structure parameters. In experiments, the Rashba and linear Dresselhaus spin-orbit couplings can be determined quantitatively via several methods, such as electric-field-induced spin precession [64, 66] and current-induced modification of $g$-factor [634].

A good success of such paradigm is that it well reproduces the subband quantization energy $E_{1 e}$ dependence of the electron spin lifetime [577] which can not be explained by the simple relation of $\tau_{s} \sim E_{1 e}^{-2}$ from the D'yakonovKachorovskii theory [see Fig.[15(b)]. This is due to the fact that this paradigm takes full account of spin-orbit coupling via diagonalizing the multiband envelope-function equation. In the same work Lau et al. also demonstrated that their calculation achieved better agreement with experiments in the mobility dependence of the spin lifetime compared with the D'yakonov-Kachorovskii theory (see Fig. 15). They also demonstrated the role of the electron-longitudinaloptical-phonon scattering to spin relaxation as function of temperature compared with that of the impurity scattering as depicted in Fig. 15. Systematic calculation via such paradigm of various dependences of electron spin lifetime can be found in Ref. [633].

- Temperature dependence. The temperature dependence of the D'yakonov-Perel' spin relaxation was calculated within such paradigm in Refs. [577, 635, 636]. Kainz et al. attempted to perform a microscopic calculations of the temperature-dependent spin-relaxation rates with realistic system parameters [636]. By using the parameters from experiments, they calculated the temperature dependence of spin relaxation time and compared it with the experimental data by Ohno et al. 602] (see Fig. 16). In their calculation the momentum scattering time was not calculated microscopically but inferred from the Hall mobility. They classified three types of scatterings and assumed that the Hall mobility is solely limited by each type of scattering. By doing so they obtained three spin lifetimes. Finding that the experimental measured spin lifetime falls into the region determined by the three calculated ones, they concluded that the calculation agrees with the experiments. By close examination, they found that the electron-ionized-impurity scattering dominates at low temperature $(T<100 \mathrm{~K})$, whereas the electron-longitudinal-optical-phonon scattering dominates at high temperature [636]. The temperature dependence was found to be more pronounced at low electron density [635, 636].

- Electron density dependence. The electron density dependence of spin relaxation was calculated in Refs. 195 , 197, 635-638]. At zero temperature (degenerate regime), Golub et al. found that spin relaxation rate increases monotonically with electron density [195]. Interestingly, Averkiev et al. found that the ratio of spin lifetime $\tau_{-} / \tau_{+}\left[\tau_{+}\right.$ $\left(\tau_{-}\right)$denotes spin lifetime for spin along [110] ([1ㅣㅣ]) direction) has a peak in density dependence: at low density the spin relaxation is dominated by $1 / \tau_{-}$whereas at high density $1 / \tau_{+}$becomes more and more important for $\alpha_{R}, \beta_{D}>0$ [195, 635]. From Eq. (107), one can understand the above results by noting that $\tilde{\beta}_{D}=\beta_{D}-\frac{1}{4} \gamma_{D} k^{2}$ can become

\footnotetext{
${ }^{53}$ However, it has been found that in high mobility two-dimensional system, electron-electron scattering is the dominant momentum scattering to the D'yakonov-Perel' spin relaxation [44, 368, 372].

${ }^{54}$ Although most of the single-particle theory is based on the elastic scattering approximation, some works go beyond that. Dyson and Ridley developed a method to calculate the momentum scattering time due to electron-longitudinal-optical-phonon scattering beyond the elastic scattering approximation. In bulk system, they showed that the elastic scattering approximation may have problem in treating the longitudinal-optical-phonon scattering as it is essentially inelastic [542]. They further applied their method to study the D'yakonov-Perel' spin relaxation associated with the electron-longitudinal-optical-phonon scattering in quantum wells and wires in Ref. [632].
} 


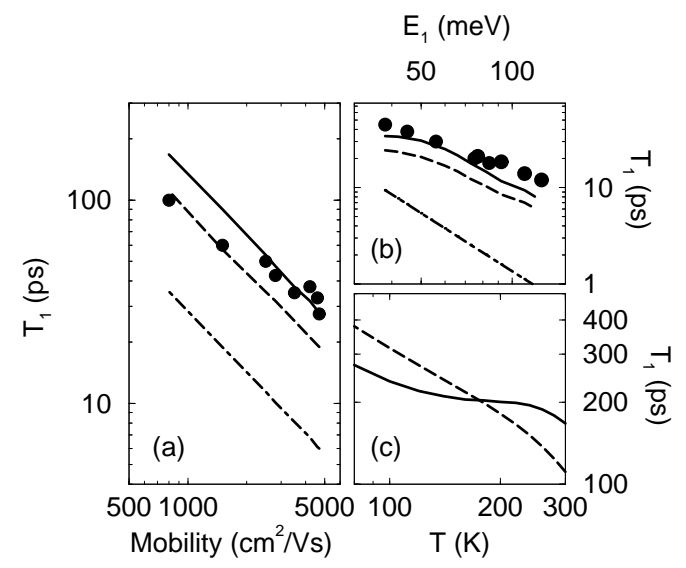

Figure 15: Electron-spin lifetime $T_{1}$ as a function of (a) mobility, (b) subband quantization energy $E_{1}$, and (c) temperature, for 75$\AA \mathrm{GaAs} / \mathrm{Al}_{0.4} \mathrm{Ga}_{0.6} \mathrm{As}$ multiple quantum wells at room temperature. Dots represent the results of experiment (Ref. [614]). The theoretical results with electron-optical-phonon scattering (solid curves) and electron-neutral-impurity scattering (dashed curves) are shown, as well as the results from the D'yakonov-Kachorovskii theory (dot-dashed curves). From Lau et al. [577].

negative at large $k$. Similar results have been obtained in temperature dependence in nondegenerate regime [635]. In heterojunction, both the Rashba spin-orbit coupling and the linear Dresselhaus term depend on the electron density as the built-in electric field and the wave-function across the junction vary with electron density. $\alpha_{R}$ and $\beta_{D}$ in heterojunction can be estimated as $\alpha_{R} \simeq \alpha_{0} n_{e} e^{2} /\left(2 \kappa_{0} \epsilon_{0}\right)$ and $\beta_{D} \simeq \gamma_{D}\left[16.5 \pi n_{e} e^{2} m^{*} /\left(8 \kappa_{0} \epsilon_{0}\right)\right]^{\frac{2}{3}}$, where $\alpha_{0}$ represents the Rashba coefficient, $\kappa_{0}$ is static dielectric constant and $\epsilon_{0}$ stands for the vacuum dielectric constant [635]. Averkiev et al. found that the spin relaxation rate $1 / \tau_{+}$has a minimum in the density dependence due to the cancellation of the Rashba and linear Dresselhaus spin-orbit coupling, whereas $1 / \tau_{-}$and $1 / \tau_{z}$ increase with density monotonically (see Fig. 17] [635]. At the electron density where $1 / \tau_{+}$has a minimum, the spin relaxation is highly anisotropic. A more careful consideration of the same problem within the multiband envelope-function approach was given by Kainz et al. for various well width in Refs. [197, 636] where the spin-orbit coupling was treated more carefully. Via similar approach the Rashba and Dresselhaus spin-orbit couplings in $\delta$-doped $\mathrm{InSb} / \mathrm{Al}_{x} \mathrm{In}_{1-x} \mathrm{Sb}$ asymmetric quantum well was calculated in a range of carrier densities [637] 55 Based on these results, the density dependence of spin lifetime was calculated where a minimum in the density dependence of the in-plane spin relaxation rate was also observed [637]. The spin-orbit coupling and the density dependence of spin relaxation time in such quantum well were also studied by $\mathrm{Li}$ et al. [638], where the calculation was compared with the experimental results in Ref. [593]. The authors also showed from the eight-band $\mathbf{k} \cdot \mathbf{p}$ model that the spin-orbit coupling deviates strongly from the linear-k Rashba/Dresselhaus model [638].

- Gate-voltage dependence. The gate-voltage dependence of spin lifetime for a triangular quantum well defined by a structure with an infinite height barrier at left boundary and a constant electric field at right boundary was studied by Averkiev et al. [635]. They found that the in-plane spin lifetime $\tau_{+}$has a maximum when the Rashba and linear Dresselhaus spin-orbit couplings cancel each other. The gate-voltage dependence of the spin-orbit coupling in $\delta$-doped $\mathrm{InSb} / \mathrm{In}_{1-x} \mathrm{Al}_{x} \mathrm{Sb}$ asymmetric quantum well was calculated in Ref. [637]. It was also shown in Ref. [640] that the spin lifetimes can be tuned effectively via the electric field along the growth direction. Systematic study on the dependence of spin lifetime on the electric field across the GaAs/AlGaAs quantum well at room temperature was performed by Lau and Flatté [146]. From a 14-band envelope-function approach they calculated the Rashba and Dresselhaus spinorbit couplings as function of electric field for various well widths (see Fig. 18). They further calculated the spin lifetime as function of electric field and reported that the electric field effect is important at wide quantum well where the subband wavefunction is easily affected by the electric field. Again the spin lifetime along the [110] direction has a maximum as function of electric field. For a $7.5 \mathrm{~nm}$ quantum well the contribution of the Rashba spin-orbit coupling in spin relaxation exceeds that of the Dresselhaus one at $150 \mathrm{kV} / \mathrm{cm}$. For such narrow quantum well, the Dresselhaus

\footnotetext{
${ }^{55}$ Density dependence of spin splitting in such structure was measured and compared with calculation in Ref. [639].
} 


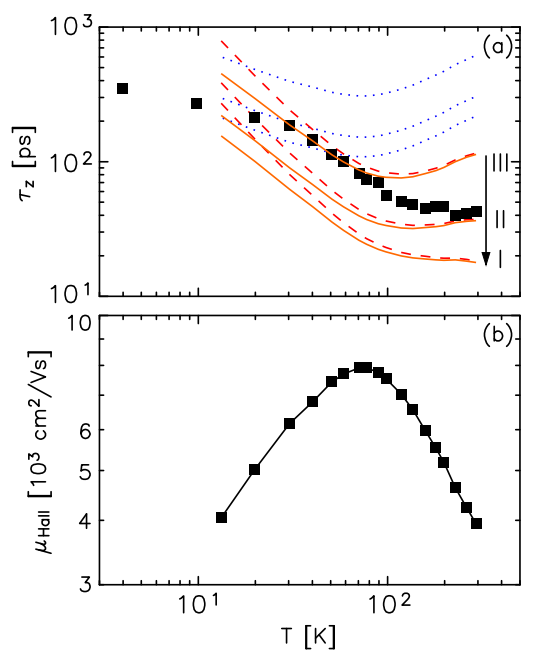

Figure 16: (a) Spin-lifetime $\tau_{z}$ and (b) measured Hall mobility $\mu_{\text {Hall }}$ in experiments as a function of temperature $T$. In (a) three solid curves denote calculation with the mobility attributed to the three types of scattering (labeled as III, II and I in the figure.) The first type of scattering (I): phonon scatterings due to the deformation potential, scattering by screened ionized impurities, neutral impurities, alloy and surface roughness; The second type of scattering (II): other kinds of phonon scatterings; The third type of scattering (III): scattering by weakly screened ionized impurities. The dotted curves refer to the calculation in the degenerate limit (zero temperature) whereas the dashed curves denote the calculation in the nondegenerate limite (the Boltzmann statistics). From Kainz et al. [636].

spin-orbit coupling varies little with electric field (up to $200 \mathrm{kV} / \mathrm{cm}$ ), whereas the Rashba spin-orbit coupling varies linearly with electric field. Similar results were obtained by Yang and Chang [156].

- Nonlinear Rashba spin-orbit coupling to spin relaxation. Usually the multi-band envelope-function calculation includes the nonparabolic effect in the spin-orbit coupling, e.g., at high energy the Rashba spin-orbit coupling deviates from linearity [146, 156]. The nonlinear effect in the Rashba spin-orbit coupling was studied comprehensively by Yang and Chang [156]. They proposed a model to characterize such nonlinear effect where the Rashba parameter is substituted by $\tilde{\alpha}_{R}=\frac{\alpha_{R}}{1+\zeta k^{2}}$, where $\zeta$ is a parameter depending on quantum well structure and material. The model fits well with the calculation. The nonlinearity is more pronounced in narrow band-gap semiconductors. Within such model the spin relaxation as function of electric field, well width, density and the ratio $\alpha_{R} / \beta_{D}$ was investigated comprehensively [156].

- Magnetic field dependence. The magnetic field dependence of the spin relaxation in two-dimensional system with both the Rashba and Dresselhaus spin-orbit couplings was discussed by Glazov [377] 56 The main results are that the spin relaxation tensor given by Eq. (107) is extended to the case with magnetic field along arbitrary direction. A special case is that when the magnetic field is along the growth direction of the two-dimensional structure, where Glazov gave

$$
\frac{1}{\tau_{z}}=4 \tilde{\tau}_{1} k^{2}\left[\frac{\alpha_{R}^{2}}{1+\left(\omega_{L}-\omega_{c}\right)^{2} \tilde{\tau}_{1}^{2}}+\frac{\beta_{D}^{2}}{1+\left(\omega_{L}+\omega_{c}\right)^{2} \tilde{\tau}_{1}^{2}}\right]+\frac{\tilde{\tau}_{3} k^{6} \gamma_{D}^{2}}{4} \frac{1}{1+\left(\omega_{L}-3 \omega_{c}\right)^{2} \tilde{\tau}_{3}^{2}} .
$$

Spin lifetime as function of the direction of magnetic field was discussed and compared with experimental data [377]. Magnetic field effect on spin relaxation in the weak scattering regime where the scattering frequency is comparable with the spin precession frequency was studied by Glazov [380]. In such regime, spin polarization shows zero-field oscillations due to the spin-orbit field induced spin precession. Glazov found that the magnetic-field-induced cyclotron rotation (which also rotates the k-dependent spin-orbit field) leads to fast oscillations of spin polarization around a non-zero value and a strong suppression of spin relaxation [380]. Recent experiment confirmed such prediction in (001) quantum wells and showed that the effects are absent in (110) quantum wells as the spin-orbit field is along the growth direction which does not lead to any spin precession [641]. Spin relaxation in the presence of both electric and magnetic fields was discussed theoretically by Bleibaum [378, 379] 57 In the presence of spin-orbit coupling

\footnotetext{
${ }^{56}$ Similar study for spin relaxation in $\mathrm{Si} / \mathrm{Ge}$ quantum well with only the Rashba spin-orbit coupling was given by Tahan et al. [290].

${ }^{57}$ This has been reported earlier by Weng et al. [569] from the kinetic spin Bloch equation approach (see next section).
} 


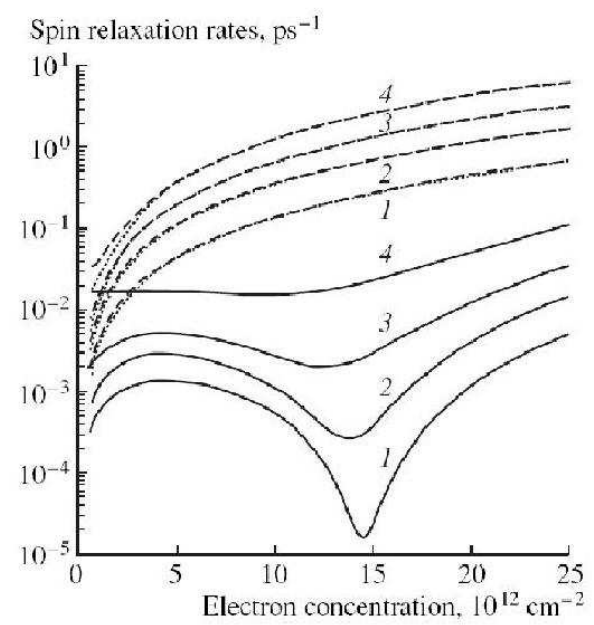

Figure 17: Spin-relaxation rates $1 / \tau_{+}$(solid curves), $1 / \tau_{z}$ (dashed curves) and $1 / \tau_{-}$(dotted curves) as function of electron concentration for Boltzmann electron gas in GaAs/AlAs heterostructure at temperature $T=(1) 30$, (2) 77, (3) 150 and (4) $300 \mathrm{~K}$. From Averkiev et al. [635].

the electric field can induce an effective magnetic field due to electron drifting [569]. Spin relaxation is modified by such effective magnetic field and the real magnetic field due to the induced spin precession. The above effects are in the Markovian limit. Besides, there could emerge non-Markovian spin dynamics under magnetic field. Glazov and Sherman considered the case that the spin information can be stored in the closed orbits of cyclotron motion and transferred to the open orbits [381] 58 As the closed orbit averaged out the spin-orbit coupling, the electron spin lifetime in the closed orbits can be much longer than that in the open orbits. In such a system, the fraction of spins in the open orbits soon decays, whereas the fraction of spins in the closed orbits decays slowly exhibiting a long-lived tail [381] 59 The decay of the tail is governed by the scattering processes which transfer electrons between closed and open orbits [381]. Based on a semiclassical model, the spin dynamics under weak/strong scattering and weak/strong magnetic-field was discussed [381]. Particularly, they showed that at strong magnetic field $B$ along the growth direction, the tail disappears and the spin lifetime is elongated as $\sim B^{3}$ [381]. Under high magnetic field in the quantum Hall regime, spin relaxation was studied in Refs. [382-387] 60 It was shown that at zero temperature with filling factor $v=1$, the spin relaxes asymptotically with a power law rather than exponential [384]. The spin relaxation is sensitive to the filling factor and temperature. Experiments have shown that via tuning the filling factor the effect of the hyperfine interaction can be manipulated [643]. As the magnetic field is high, the $g$-tensor inhomogeneity mechanism can be important [387]. In general, spin relaxation in quantum Hall regime is quite different from that in the classical regime. The spin-flip electron-phonon scattering plays important role. Besides, the electron-electron interaction is crucial to the ground state as well as to spin dynamics [384].

- Weak localization effect and others. As in the D'yakonov-Perel' mechanism $\tau_{s} \sim 1 / \tau_{p}$, the weak localization correction to mobility will lead to correction in spin relaxation as well. The weak localization correction to spin relaxation was studied in Refs. [644-646]. The classical memory effect, which exists when the characteristic scale of the disorder are comparable with the mean free path, leads to a non-Markovian spin dynamics with nonexponential tail $\sim 1 / t^{2}$ for quantum wells with equal Dresselhaus and Rashba spin-orbit coupling strengths [647]. Pershin and Privman proposed that the D'yakonov-Perel' spin relaxation in two-dimensional system can be suppressed by a lattice of antidots [648].

- Spin relaxation in rolled-up two-dimensional electron gas. Spin dynamics in rolled-up two-dimensional electron gas was investigated by Trushin and Schliemann [649]. It was shown that the symmetry of spin-orbit coupling varies with the radius rolled-up structure [649]. At certain radius, spin precession and relaxation of a special spin

\footnotetext{
${ }^{58}$ The non-Markovian spin dynamics can also emerge in insulating regime, where the nuclear spins stored the historical electron spin information.

${ }^{59}$ As spins in the closed orbits oscillate due to spin-orbit coupling, the tail also oscillates.

${ }^{60}$ Experimental studies can be found in Refs. [642, 643].
} 

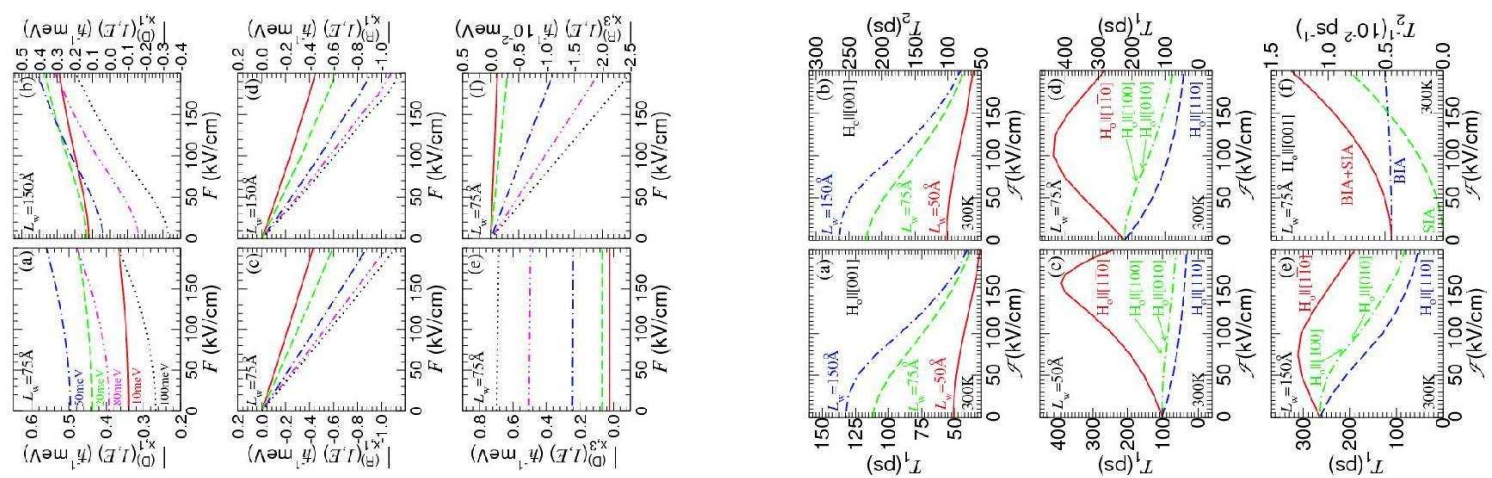

Figure 18: Left figure: Electric field $\mathcal{F}$ (along the growth direction) dependence of electron spin precession vector for a $75 \AA$ and a $150 \AA \mathrm{GaAs} / \mathrm{Ga}_{0.6} \mathrm{Al}_{0.4} \mathrm{As}$ quantum well at $300 \mathrm{~K}$ for different energies $E=10 \mathrm{meV}$ (red solid curve), $20 \mathrm{meV}$ (green dashed curve), $50 \mathrm{meV}$ (blue dot-dashed curve), $80 \mathrm{meV}$ (purple double-dot-dashed curve) and $100 \mathrm{meV}$ (black dotted curve). $x$-component of the spin precession vector induced by the linear Dresselhaus term $\Omega_{x, 1}^{(\mathrm{D})}(1, E)$ for quantum wells with well width (a) $L_{w}=75 \AA$ and (b) $L_{w}=150 \AA$. $x$-component of the spin precession vector induced by the Rashba term $\Omega_{x, 1}^{(\mathrm{R})}(1, E)$ for (c) $L_{w}=75 \AA$ and (d) $L_{w}=150 \AA$ quantum wells. $x$-component of the spin precession vector induced by the cubic Dresselhaus term $\Omega_{x, 3}^{(\mathrm{D})}(1, E)$ for (e) $L_{w}=75 \AA$. $x$-component of the spin precession vector induced by higher order (cubic) Rashba term $\Omega_{x, 3}^{(\mathrm{R})}(1, E)$ for (f) $L_{w}=75 \AA$ (Note that the scale is two orders of magnitude smaller). Right figure: Electric field $\mathcal{F}$ (along the growth direction) dependence of electron spin relaxation time $T_{1}$, dephasing time $T_{2}$ and dephasing rate $1 / T_{2}$ for $L_{w}=50 \AA$, $L_{w}=75$ Ånd $L_{w}=150 \AA \mathrm{GaAs} / \mathrm{Ga}_{0.6} \mathrm{Al}_{0.4}$ As quantum wells at $300 \mathrm{~K}$ with mobility $\mu=800 \mathrm{~cm}^{2} / \mathrm{V}$ s. Note that the relative importance of the Dresselhaus (BIA) term and Rashba (SIA) term to spin relaxation as function of the electric field along the growth direction is depicted in (f). From Lau and Flatté [146].

component is completely quenched, very similar to that in quantum wells with $\alpha_{R}=\beta_{D}$ (consider only the linear-k spin-orbit coupling) [649].

- Crossover from two-dimension to one-dimension. An interesting problem is that how the spin relaxation varies with the channel width of the two-dimensional structure. This problem was investigated theoretically first by Mal'shukov and Chao [650] and later by Kiselev and Kim [651, 652], showing that spin relaxation for some nonuniform distributed spin polarization and the uniform spin polarization along certain direction (direction of the effective magnetic of the linear spin-orbit coupling with $\mathbf{k}$ along the unconstrained direction) is suppressed when the channel width is smaller than the spin precession length [650-652]. Originally, these studies considered only the Rashba spin-orbit coupling. Recently Kettemann extended the theory to include the Dresselhaus spin-orbit coupling (both linear and cubic terms) [653]. After that experimental studies on submicron InGaAs wires observed that spin lifetime first increases and then decreases with decreasing channel width (see Fig. 19] [654, 655]. The suppression of spin relaxation was first explained by the theory of Mal'shukov and Chao, which, however, is doubtful as the spin polarization distribution created by optical excitation is not of the type pointed out in their work. Detailed theoretical examination indicated different explanations [656].

- Non-uniform system: Random spin-orbit coupling to spin relaxation. All the above studies are devoted to uniform system. For example, the Rashba spin-orbit coupling is considered as $H_{R}=\alpha_{R}\left(\sigma_{x} k_{y}-\sigma_{y} k_{x}\right)$ where $\alpha_{R}=\alpha_{0} \mathcal{E}_{z}$ is proportional to a uniform electric field along thegrowth direction. However, in genuine system, such electric field can not be uniform due to imperfection. Such imperfection can arise from the the fluctuation during the growth, making $\mathcal{E}_{z}$ position-dependent: $\mathcal{E}_{z} \rightarrow \mathcal{E}_{z}\left(\mathbf{r}_{\|}\right)$with $\mathbf{r}_{\|}=(x, y)$. Spin relaxation due to this random Rashba spin-orbit coupling can be important when other spin-relaxation sources are ineffective [294, 657]. This was studied comprehensively by Sherman and co-workers in Refs. [294, 657-659]. A particular case is the symmetric (110) quantum wells, where the average Rashba spin-orbit coupling is zero and the Dresselhaus spin-orbit coupling does not lead to any spin relaxation due to symmetry. Glazov and Sherman obtained that under weak and moderate magnetic field along the growth direction at $r_{c} \gg l_{d}\left(r_{c}\right.$ is the cyclotron radius and $l_{d}$ is characteristic length of random dopant distribution) and $\alpha_{R} k \tau_{p} \ll 1$ [658],

$$
\tau_{z}^{-1}=4\left\langle\left(\delta \alpha_{R}\right)^{2}\right\rangle k^{2} \tau_{d}+\frac{4\left\langle\alpha_{R}\right\rangle^{2} k^{2} \tau_{p}}{\left(1+\omega_{c}^{2} \tau_{p}^{2}\right)}
$$



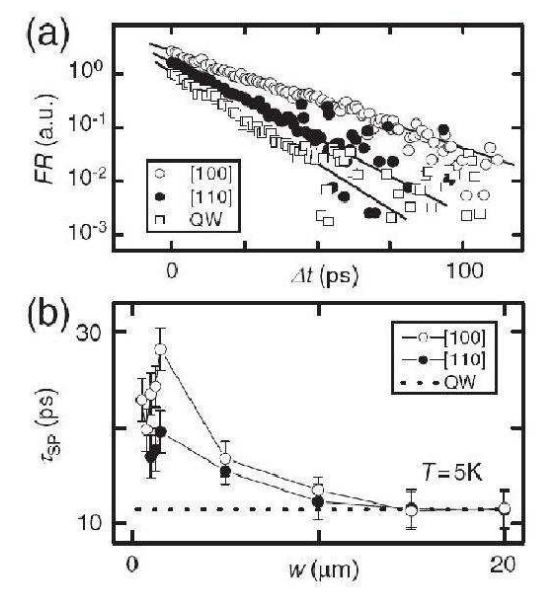

Figure 19: (a) Faraday rotation (FR) at $5 \mathrm{~K}$ for quantum well (open squares) and $750 \mathrm{~nm}$ wires patterned along [100] (open circles) and [110] (filled circles) as function of delay time $\Delta t$. Black curves are guides to the eyes, and the data are offset for clarity. (b) Width dependence $w$ of spin lifetime $\tau_{\mathrm{SP}}$ for quantum wires patterned along [100] (open circles) and [110] (filled circles). The dotted line depicts the spin lifetime of the unpatterned quantum well. Measurements were performed at $B=0$. From Holleitner et al. [654].

where $\delta \alpha_{R}=\alpha_{R}-\left\langle\alpha_{R}\right\rangle$ is the random Rashba coefficient with $\left\langle\alpha_{R}\right\rangle$ representing the average Rashba coefficient. $\tau_{d}=l_{d} / v_{\mathbf{k}}$. The Zeeman interaction is ignored as $\omega_{L} \ll \omega_{c}$. It is seen that both the random and the average spin-orbit couplings lead to spin relaxation similar to the D'yakonov-Perel' mechanism. As $\omega_{c} \tau_{d} \ll 1$ (as $r_{c} \gg l_{d}$ ), the magnetic field does not suppress the spin relaxation due to the random Rashba spin-orbit coupling. A simple estimation indicates that in asymmetrically doped quantum well, the random spin-orbit coupling leads to a spin relaxation rate two orders of magnitude smaller than that due to the averaged one, leading to a spin lifetime of several tens of nanoseconds in GaAs quantum wells [294]. The spin relaxation due to the random Rashba spin-orbit coupling may be an important source for (110) quantum wells [660]. At high magnetic field, $r_{c} \simeq l_{d}$, the spin relaxation exhibits nonexponential tail due to the memory effect as spin in closed orbits decays slowly [658].

- Decay of non-uniformly distributed spin polarization. The decay of a standing wave of spin polarization in a two-dimensional system with only Rashba spin-orbit coupling was studied by Pershin [36]. It was found that the spin relaxation depends on the period of the standing wave. The coherent spin precession of electrons moving in the same direction was shown be responsible for such phenomena. In experiments such standing wave can be generated by spin-grating technique [495]. In a series of theoretical [18, 20, 32, 34, 338] and experimental [33, 37, 661] works, spin relaxation in spin-grating system was studied, where the spin relaxation in such system is essentially related to the spin diffusion limited by the D'yakonov-Perel' mechanism [18, 32]. The spin relaxation in spin-grating system or other nonuniform spin distributions will be reviewed in Section 6 and Sec. 7.

Spin relaxation in (110) quantum wells: experiments and theories

We now turn to spin relaxation in two-dimensional structures grown along the [110] direction. The bulk Dresselhaus spin-orbit coupling becomes

$$
H_{D}=\gamma_{D}\left[\left(-k_{x}^{2}-2 k_{y}^{2}+k_{z}^{2}\right) k_{z}, 4 k_{x} k_{y} k_{z}, k_{x}\left(k_{x}^{2}-2 k_{y}^{2}-k_{z}^{2}\right)\right] \cdot \sigma / 2
$$

where the three axises are $\mathbf{e}_{x}=\frac{1}{\sqrt{2}}(1,-1,0), \mathbf{e}_{y}=(0,0,-1)$ and $\mathbf{e}_{z}=\frac{1}{\sqrt{2}}(1,1,0)$. When only the lowest subband is considered, the effective spin-orbit coupling is

$$
H_{\text {SOC }}=\gamma_{D}\left[0,0, k_{x}\left(k_{x}^{2}-2 k_{y}^{2}-\left\langle\hat{k}_{z}^{2}\right\rangle\right)\right] \cdot \sigma / 2+H_{R}
$$

in which $\left\langle\hat{k}_{z}^{2}\right\rangle$ denotes the average over the lowest subband and $H_{R}=\alpha_{R}\left(\sigma_{x} k_{y}-\sigma_{y} k_{x}\right)$ is the Rashba spin-orbit coupling. In symmetric two-dimensional structures $H_{R}=0$. It is noted that the effective magnetic field is then along the [110] direction for all $\mathbf{k}$. The D'yakonov-Perel' spin relaxation for spin polarization along the [110] direction is then absent. The question arises that what kind of mechanism is now responsible for spin relaxation along the [110] direction. To 
explore the problem, Ohno et al. performed a systematic study of the dependence of spin lifetime on the characteristic parameters such as the subband quantization energy, electron mobility and the temperature (see Fig. 20) [595, 662]. After a careful speculation on the observed dependences of spin relaxation time, they concluded that the spin relaxation in undoped (110) quantum wells is dominated by the Bir-Aronov-Pikus mechanism [595]. However, the temperature dependence of spin lifetime is anomalous and seems contradictory to the Bir-Aronov-Pikus mechanism: the spin lifetime increases with increasing temperature [595, 602]. The anomalous increase of spin lifetime with temperature can be understood as following: the dissociation of excitons increases with temperature rapidly which reduces the electron-hole exchange interaction markedly and suppresses the Bir-Aronov-Pikus mechanism [595] 61] Similar effect was also found in ZnSe quantum wells [594]. However, the situation in $n$-doped quantum wells is relatively obscure [595], although the observed mobility dependence suggests that the Elliott-Yafet mechanism may dominate the spin relaxation at room temperature (see Fig. 201 [595].
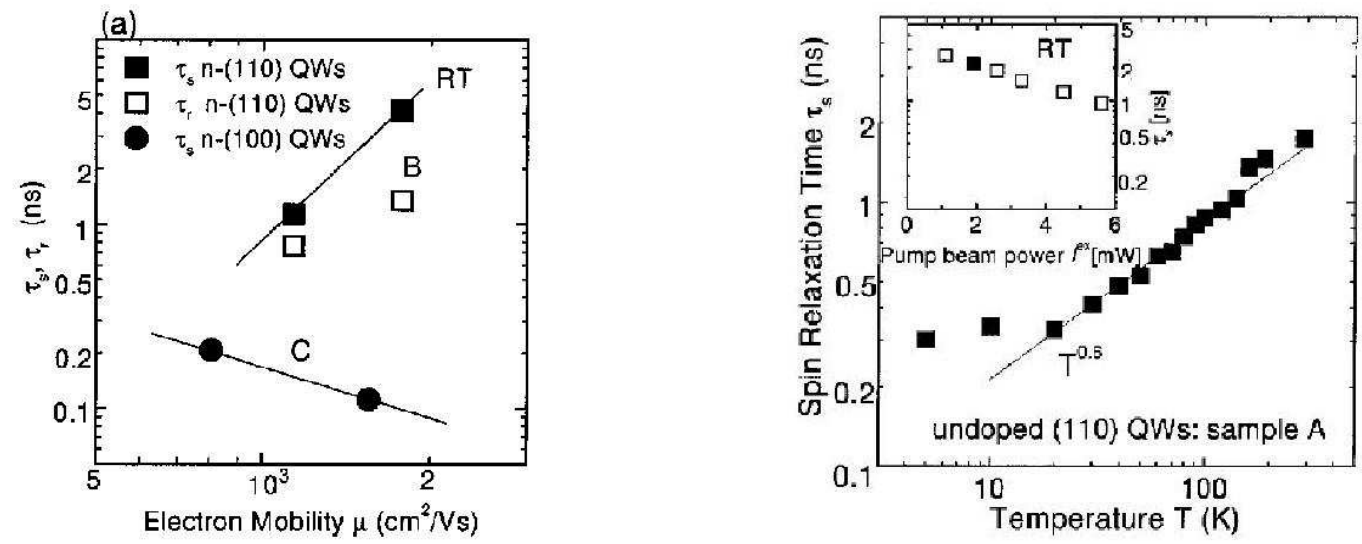

Figure 20: Left: Spin lifetime $\tau_{s}$ as well as carrier recombination time $\tau_{r}$ in $n$-doped GaAs (110) and (100) quantum wells as function of electron mobility $\mu$ at room temperature. Right: Temperature $T$ dependence of spin relaxation time $\tau_{s}$ in undoped (110) quantum wells. The inset shows the excitation intensity $I^{\mathrm{ex}}$ dependence of $\tau_{s}$ at room temperature. From Ohno et al. [595].

We now focus on the electron spin relaxation in $n$-doped symmetric (110) quantum wells. Let us reexamine the three spin relaxation mechanisms in symmetric (110) quantum wells more carefully. The D'yakonov-Perel' mechanism leads to a spin relaxation tensor,

$$
\tau_{z}^{-1}=0, \quad \tau_{x}^{-1}=\tau_{y}^{-1}=\left\langle\tau_{p} \beta_{D}^{2} k^{2}\right\rangle / 2
$$

when only the linear spin-orbit coupling term is considered. For the Elliott-Yafet mechanism, usually the in-plane spin relaxation rate is larger than the out-of-plane one [111], but the two are generally comparable [111, 370]. However, the Bir-Aronov-Pikus mechanism is isotropic [111]. Therefore the spin relaxation anisotropy will reflect the relevance of the Bir-Aronov-Pikus mechanism. The experimental investigation was first performed by Döhrmann et al. by measuring spin decay time in the presence of an in-plane magnetic field [388]. With an in-plane magnetic field, e.g., $\mathbf{B} \| \mathbf{e}_{y}$, spin dynamics is governed by the following equations,

$$
\partial_{t} S_{x}=-S_{x} / \tau_{x}+\omega_{L} S_{z}, \quad \partial_{t} S_{z}=-S_{z} / \tau_{z}-\omega_{L} S_{x}
$$

The solution is

$$
S_{z}(t)=S(0) e^{-\frac{1}{2}\left(\tau_{x}^{-1}+\tau_{z}^{-1}\right) t} \cos (\omega t-\phi) / \cos (\phi)
$$

for $2 \omega_{L}>\left|\tau_{z}^{-1}-\tau_{x}^{-1}\right|$. Here $\tan \phi=\frac{\tau_{x}^{-1}-\tau_{z}^{-1}}{2 \omega}$ and $\omega=\sqrt{\omega_{L}^{2}-\left(\tau_{x}^{-1}-\tau_{z}^{-1}\right)^{2} / 4}$. The observed magnetic field dependence is a step-function-like: for a moderate magnetic field $B>0.5 \mathrm{~T}$, spin lifetime changes from $\tau_{z}$ to $2 /\left(\tau_{x}^{-1}+\tau_{z}^{-1}\right)$. The

\footnotetext{
${ }^{61}$ This is also confirmed by the increase of photo-carrier lifetime with temperature [663].
} 
measured spin lifetimes at $B=0$ and $B=0.6 \mathrm{~T}$ in GaAs (110) modulation $n$-doped quantum wells as function of temperature are shown in Fig. 21 62 It is seen that at low temperature the anisotropy is quite weak which indicates that the spin relaxation is dominated by the Bir-Aronov-Pikus mechanism. With increasing temperature, spin relaxation anisotropy increases as the Bir-Aronov-Pikus mechanism becomes weaker and the D'yakonov-Perel' mechanism for in-plane spin relaxation grows stronger 63 The decrease of $\tau_{z}$ at high temperature was explained by the intersubband spin relaxation mechanism [388, 667]. The intersubband spin relaxation mechanism can be understood as following: when higher subbands are involved, the spin-orbit coupling

$$
H_{D}=\gamma_{D}\left[\left(-k_{x}^{2}-2 k_{y}^{2}+k_{z}^{2}\right) k_{z}, 4 k_{x} k_{y} k_{z}, k_{x}\left(k_{x}^{2}-2 k_{y}^{2}-k_{z}^{2}\right)\right] \cdot \sigma / 2
$$

can enable intersubband spin-flip scattering as the first two terms couple states with different spin and parity. At high temperature when the higher subbands are populated, the intersubband spin relaxation mechanism can be important [388]. This mechanism also explains the mobility dependence of spin lifetime in Fig. 20. Recently, Zhou and Wu proposed a virtual intersubband spin relaxation, where the higher subbands need not to be populated [668]. However, calculation indicated that both the real and virtual intersubband spin relaxation mechanisms are ineffective at low temperature for modulation $n$-doped quantum wells as the scattering is suppressed [388, 668].

The photo excitation of holes in the photoluminescence or Faraday/Kerr rotation measurement makes the BirAronov-Pikus mechanism become involved. This masks the intrinsic electron spin relaxation in $n$-doped (110) quantum wells at low temperature [388]. Recent advancement in spin noise spectroscopy method [497, 498, 669] enables probing spin relaxation without photo-carrier excitation. The Bir-Aronov-Pikus mechanism is then removed, and the intrinsic spin lifetime can be approached. This method was applied to modulation $n$-doped GaAs (110) quantum wells by Müller et al., where a much longer spin lifetime of $\tau_{z}=24 \mathrm{~ns}$ at low temperature $(20 \mathrm{~K})$ was obtained by careful analysis of the data [660]. The authors attributed the spin relaxation at such low temperature to the random Rashba spin-orbit coupling mechanism [294, 670]. Later, theoretical calculation of the spin relaxation time limited by the random Rashba spin-orbit coupling by Zhou and Wu via the fully microscopic kinetic spin Bloch equation approach [670] agrees well with the experimental results by Müller et al. [660].

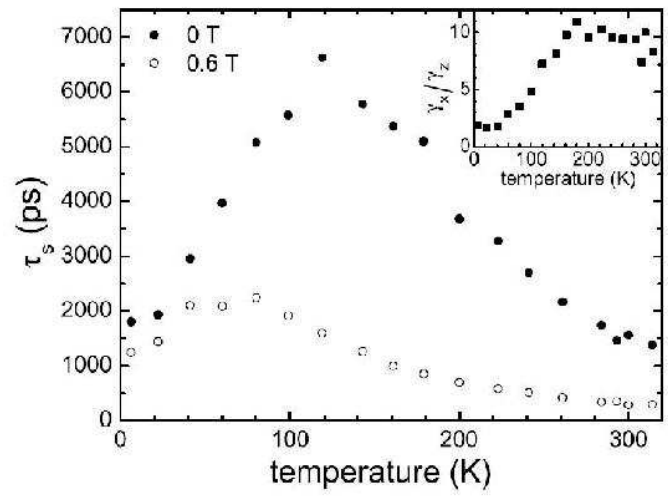

Figure 21: Temperature dependence of spin lifetime $\tau_{s}$ for $B=0$ (filled circles) and $B=0.6 \mathrm{~T}$ (open circles) in (110) GaAs quantum wells. Inset: Corresponding temperature dependence of spin relaxation anisotropy $\gamma_{x} / \gamma_{z}=\tau_{z} / \tau_{x}$. From Döhrmann et al. [595].

Other investigations include the achievement of high temperature gate control of spin lifetime [193, 671, 672], which can be useful in spin switch devices or spin field-effect transistors. The typical results of spin lifetime as function of the electric field $\mathcal{E}$ along the growth direction are shown in Fig. 22. The low field deviation of $\tau_{z} \sim$ $\mathcal{E}^{2}$ indicates the relevance of spin relaxation mechanism other than the D'yakonov-Perel' one associated with the Rashba spin-orbit coupling. Bel'kov et al. studied the relation of symmetry to spin relaxation in (110) quantum

\footnotetext{
${ }^{62}$ Similar temperature dependence of spin lifetime in (110) InGaAs quantum wells was studied in Ref. [664].

${ }^{63}$ In InGaAs quantum wells larger spin relaxation anisotropy was found [665, 666], which is due to the much stronger Dresselhaus spin-orbit coupling in InGaAs.
} 
wells [183, 663]. The symmetry of the quantum well was probed by the magnetic field induced photo-galvanic effect. At certain configuration, the photocurrent is proportional to the Rashba spin-orbit coupling coefficient. The authors observed that photocurrent indeed vanishes for symmetric quantum wells. It was also observed that the spin lifetime is longest in symmetric quantum wells. Therefore the experiment demonstrated that the structure inversion asymmetry can be tuned down to zero. As only the Rashba spin-orbit coupling contributes to the D'yakonov-Perel' spin relaxation, the (110) quantum wells can be used as a good platform to investigate the Rashba spin-orbit coupling. Eldridge et al. presented an all-optical measurements of the Rashba spin-orbit coupling: by measuring the spin lifetime via polarized pump-probe reflection technique and measuring the diffusion constant from spin-grating method (from which $\tau_{p}$ is extracted), they extracted the Rashba coefficient $\alpha_{0}=\left(2 m k_{B} T \tau_{s} \tau_{p}\right)^{-1 / 2} /(e \mathcal{E})$ from the D'yakonov-Perel' theory [179]. At low temperature, they found good quantitative agreement with the $\mathbf{k} \cdot \mathbf{p}$ calculation and an unexpected temperature dependence. In undoped (110) quantum wells, Eldridge et al. discovered that the asymmetry of the band edge profile does not contribute to the Rashba spin-orbit coupling when the electrostatic potential is absent [178]. These findings confirm the theory of Lassnig [148] (see Sec. 2.3.3). Spin relaxation in (110) quantum wells under surface acoustic waves was studied in Refs. [673, 674].

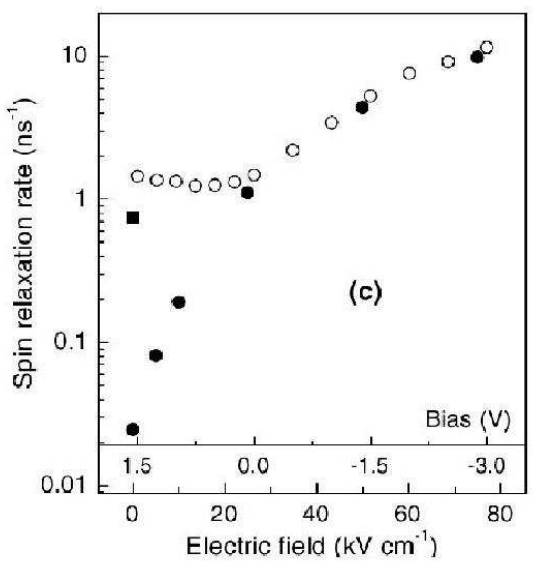

Figure 22: Measured spin relaxation rate vs. bias voltage and corresponding electric field (open circles) compared with calculation for a symmetrical quantum well (filled circles). $T=170 \mathrm{~K}$. From Karimov et al. [193].

Theoretical investigation on spin relaxation in (110) quantum wells is few up to now [640, 668, 670, 675-678]. Among these works, Wu and Gonokami first proposed that the D'yakonov-Perel' mechanism can be effective for relaxation of spin pointing along growth direction when an in-plane magnetic field is exerted [675]. Later it was shown that the in-plane spin relaxation rate can be tuned by strain which can be induced by the mole fraction of gallium in an InGaAs/InP quantum well [676]. Effect of electric field across quantum well on spin relaxation was studied in Ref. [640]. Recently, Zhou and Wu proposed a virtual intrasubband spin-flip electron-phonon and electronimpurity scattering due to the intersubband spin-orbit coupling [668]. Tarasenko gave the spin relaxation tensor in asymmetric (110) quantum wells with a finite Rashba spin-orbit coupling [677]. He found that in the presence of the Rashba spin-orbit coupling, the decay of electron spin initially oriented along the growth direction is characterized by two spin lifetimes. Glazov et al. proposed a symmetric multiple (110) GaAs quantum well structure to suppress the spin relaxation due to the random Rashba effect [678]. In such structure, the donor Coulomb potentials seen by electrons in the central quantum well is largely screened by the electrons in other quantum wells, which hence strongly suppresses the random Rashba spin-orbit coupling and spin relaxation [678]. In this structure, spin-flip scattering between electrons in different quantum wells, however, leads to an additional spin relaxation. The spin-flip inter-well electron-electron scattering is comparable with the random Rashba spin-orbit coupling mechanism in the non-degenerate regime, but is suppressed in the degenerate regime [678].

\section{Spin relaxation in (111) quantum wells}

Besides (110) quantum wells, spin relaxation in (111) quantum wells also attracted much attention due to its particular symmetry. In (111) quantum wells the linear Dresselhaus spin-orbit coupling is of the same form of the 
Rashba one. The linear-k spin-orbit coupling term is then

$$
H_{\mathrm{SO}, 1}=\alpha_{\mathrm{IA}}\left(\sigma_{x} k_{y}-\sigma_{y} k_{x}\right),
$$

where $\alpha_{\mathrm{IA}}=\alpha_{R}+2 \gamma_{D}\left\langle\hat{k}_{z}^{2}\right\rangle / \sqrt{3}$. The cubic-k term reads

$$
H_{\mathrm{SO}, 3}=\frac{\gamma_{D}}{2 \sqrt{3}}\left[k^{2}\left(-k_{y} \sigma_{x}+k_{x} \sigma_{y}\right)+\sqrt{2}\left(3 k_{x}^{2}-k_{y}^{2}\right) k_{y} \sigma_{z}\right] .
$$

The spin lifetimes are then

$$
\begin{aligned}
\tau_{x}^{-1}=\tau_{y}^{-1} & =\left\langle k^{2} \tilde{\tau}_{1}\left[12 \alpha_{\mathrm{IA}}^{2}-4 \sqrt{3} \gamma_{D} \alpha_{\mathrm{IA}} k^{2}+\left(1+2 \tilde{\tau}_{3} / \tilde{\tau}_{1}\right) \gamma_{D}^{2} k^{4}\right] / 6\right\rangle \\
\tau_{z}^{-1} & =\left\langle k^{2} \tilde{\tau}_{1}\left(\gamma_{D} k^{2}-2 \sqrt{3} \alpha_{\mathrm{IA}}\right)^{2} / 3\right\rangle .
\end{aligned}
$$

It was first proposed by Cartoixà et al. [340, 679] that by tuning the gate-voltage, the condition $\gamma_{D}\left\langle k^{2}\right\rangle=2 \sqrt{3} \alpha_{\text {IA }}$ can be achieved, where spin relaxation is suppressed for all spin components (see Fig. 23] : $\tau_{z}=\infty$ and $\tau_{x}=\tau_{y}=$ $3 /\left(\gamma_{D}^{2}\left\langle k^{6}\right\rangle \tilde{\tau}_{3}\right)$. Microscopic calculation using eight-band $\mathbf{k} \cdot \mathbf{p}$ method indicated the feasibility of such scheme in an InAlAs/InGaAs/InP quantum well [680]. Similar scheme based on tuning spin-orbit coupling via strain was also proposed [676].

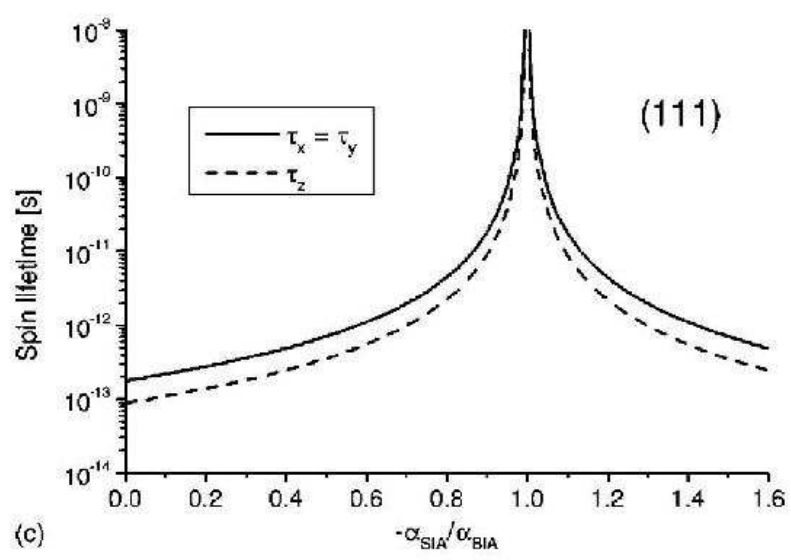

Figure 23: Calculated spin lifetime for (111) quantum wells as function of $-\alpha_{\mathrm{SIA}} / \alpha_{\mathrm{BIA}}\left(\alpha_{\mathrm{SIA}}=\alpha_{R}, \alpha_{\mathrm{BIA}}=2 \gamma_{D}\left\langle\hat{k}_{z}^{2}\right\rangle / \sqrt{3}\right)$. From Cartoixà et al. [679].

\section{Spin relaxation in arbitrarily oriented quantum wells}

Accounting only the linear-k Dresselhaus spin-orbit coupling, spin relaxation tensor for an arbitrarily oriented quantum well reads

$$
\tau_{i j}^{-1}=\left(\delta_{i j} \operatorname{Tr} \hat{v}-v_{i j}\right) / \tau_{s}^{0}\left(\varepsilon_{\mathbf{k}}\right)
$$

Here $\tau_{s}^{0}$ is the in-plane spin lifetime for (001) quantum well. The tensor $\hat{v}$ depends on the orientation of the quantum well $\mathbf{n}=\left(n_{x}, n_{y}, n_{z}\right)$ as [369]

$$
v_{x x}=4 n_{x}^{2}\left(n_{y}^{2}+n_{z}^{2}\right)-\left(n_{y}^{2}-n_{z}^{2}\right)^{2}\left(9 n_{x}^{2}-1\right), \quad v_{x y}=n_{x} n_{y}\left[9\left(n_{x}^{2}-n_{z}^{2}\right)\left(n_{y}^{2}-n_{z}^{2}\right)-2\left(1-n_{z}^{2}\right)\right],
$$

with other components obtained by cyclic permutation of the $x, y$ and $z$ indexes.

Spin relaxation in (sub)-monolayers: experiments

Spin relaxation in undoped sub-monolayer and monolayer InAs structures grown in GaAs matrix was studied in

\footnotetext{
${ }^{64}$ The $x, y$ and $z$ axis are taking along the [100], [010] and [001] directions respectively.
} 
Refs. [681, 682]. The monolayer structure on (001) surface can be regarded as an ideal two-dimensional system. However, the (311) oriented monolayer forms wire-like or disk-like microstructures on GaAs steps and facets. In submonolayer InAs structures, such as 1/3 monolayer and 1/2 monolayer, InAs was found to be organized as disk-like islands with lateral size of tens of nanometers. However, the carrier system is still of two-dimensional nature but with lower density of states and smaller mobility. It was then found that the spin relaxation is suppressed by reducing the layer thickness (see Fig. 24) [681]. The boundaries and deformation potentials are enhanced with decreased coverage, which leads to the decrease of momentum scattering time and suppresses spin relaxation [681]. For monolayer structures, the (311) structure has a much longer spin lifetime than the (001) one as the surface roughness is much larger in the former [681]. The $g$-factor and spin dephasing time were also measured as function of excitation density for these structures [681]. The spin dephasing time decreases with excitation density for all the 1/3,1/2 and 1 monolayer structures [681]. The decrease of spin lifetime may be due to the enhancement of the D'yakonov-Perel' mechanism as $\left\langle k^{6}\right\rangle$ increases or due to the enhancement of the Bir-Aronov-Pikus mechanism as the hole density increases. However, via examination of the temperature dependence of spin lifetime, Yang et al. [681] found that the Bir-Aronov-Pikus mechanism can only be important at low excitation density in 1/3 monolayer structure due to the strong electron-hole exchange interaction between the spatially confined electrons and holes [682]. The temperature dependence of spin lifetime at higher excitation density exhibits a peak [681, 682], which signals the D'yakonov-Perel' spin relaxation associated with the electron-electron scattering [110, 372]. Finally, the density dependence of $g$-factor was used to analysis the electronic density of states in these structures [681].

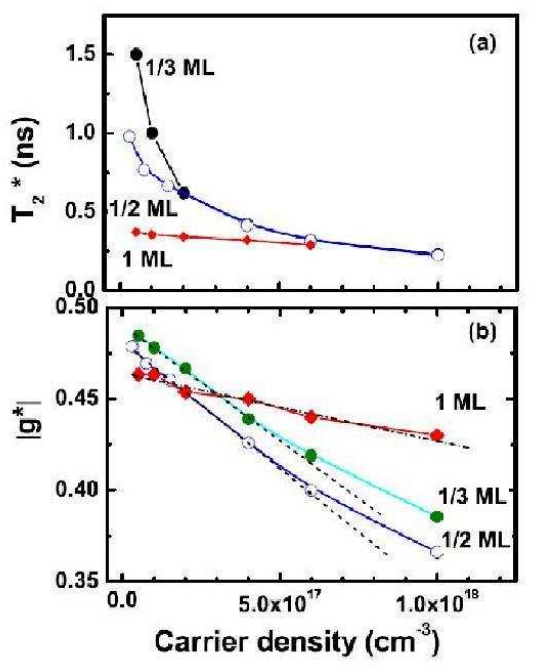

Figure 24: (a) Electron-spin dephasing time $T_{2}^{*}$ and (b) effective $g$-factor $\left|g^{*}\right|$ for $1 / 3$ monolayer (ML), $1 / 2 \mathrm{ML}$ and $1 \mathrm{ML}$ InAs on (100) GaAs substrates with various carrier densities. The temperature is $77 \mathrm{~K}$. From Yang et al. [681].

\section{Spin relaxation in parabolic quantum wells: experiments}

A good candidate for spintronic device structure is the parabolic quantum wells, where the $g$-factor and spin lifetime can be tuned efficiently by electrical means [67, 78, 683]. Recently, the Rashba and linear Dresselhaus spinorbit couplings in parabolic quantum wells were measured by monitoring the spin precession frequency of drifting electrons via time-resolved Kerr rotation [67]. It was found that the Rashba spin splitting can be tuned significantly by the gate biases, whereas the Dresselhaus spin-orbit coupling varies only weakly. The spin relaxation was then tuned by gate-voltage (see Fig. 25). It was observed that the anisotropy of spin relaxation vanishes when the Rashba spin-orbit coupling is tuned to zero.

\section{Spin relaxation in II-VI semiconductor two-dimensional structures: experiments}

Spin relaxation in II-VI semiconductor two-dimensional structures has also been widely studied. The first remarkable advancement is that the spin lifetime can be increased by several orders of magnitude via $n$-type doping in $\mathrm{ZnSe}$ quantum wells [584]. It was observed that the spin lifetime is on the order of nanoseconds and is only weakly temperature dependent [584]. The spin lifetime in undoped $\mathrm{ZnSe}$ quantum wells is on the order of $10 \mathrm{ps}$ at low temperature 

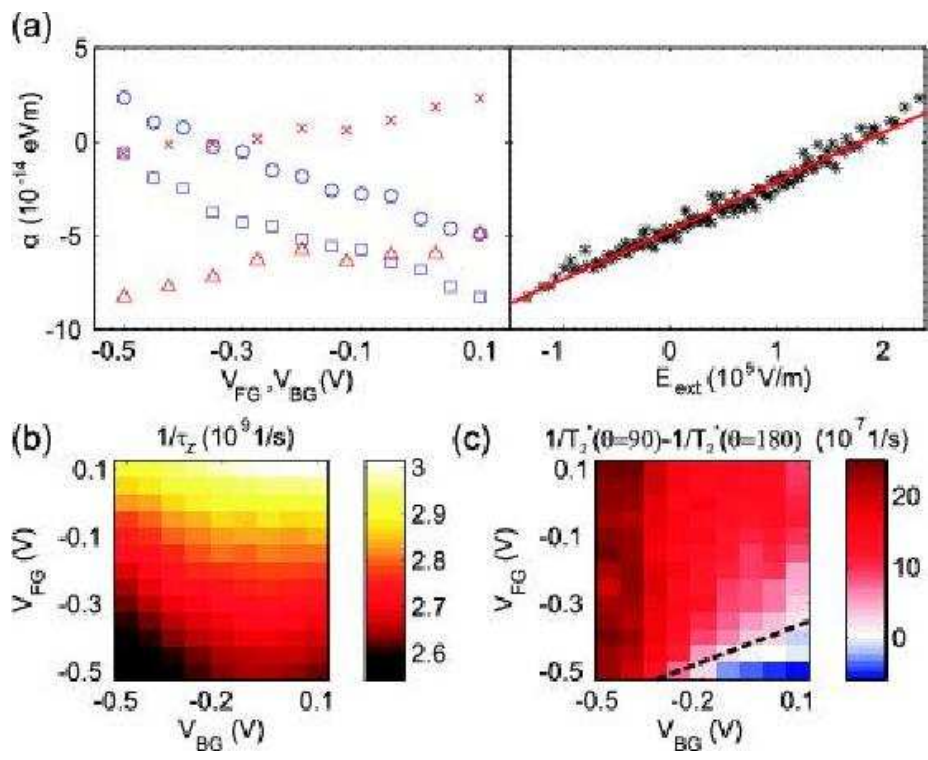

Figure 25: (001) parabolic quantum well. (a) Left panel: the Rashba spin-orbit coupling coefficient $\alpha$ as a function of the front-gate: FG (back-gate: $\mathrm{BG})$ voltage with fixed BG (FG) voltage $\left(\triangle: V_{\mathrm{FG}}=0.1 \mathrm{~V} ; \times: V_{\mathrm{FG}}=-0.5 \mathrm{~V} ; \circ: V_{\mathrm{BG}}=0.1 \mathrm{~V} ; \square: V_{\mathrm{BG}}=-0.5 \mathrm{~V}\right)$. Right panel: $\alpha$ as a function of the external electric field $E_{\text {ext }}$. The red line represents a least-squares fit. (b) Out-of-plane spin relaxation rate $1 / \tau_{z}=1 / T_{2}^{*}(\theta=90)+1 / T_{2}^{*}(\theta=180)$. (c) In-plane spin-dephasing asymmetry $1 / T_{2}^{*}(\theta=90)-1 / T_{2}^{*}(\theta=180) . \theta=90(\theta=180)$ denotes that the external magnetic field is along [110] ([110]) direction. The dashed line marks the gate voltage where $\alpha=0$. From Studer et al. [67].

[584, 594]. In undoped quantum wells the spin lifetime was observed to increase from less than $10 \mathrm{ps}$ at $20 \mathrm{~K}$ to $500 \mathrm{ps}$ at $200 \mathrm{~K}$ [594]. As the exciton binding energy is large $(19 \mathrm{meV})$, at low temperature spin dynamics is governed by the exciton behavior. Experimental examination on the relation between spin lifetime and momentum lifetime reveals a motional narrowing nature $\tau_{s} \sim \tau_{p}^{-1}$, which coincides with the picture of Maialle et al. [118]: the electron-hole exchange interaction serves as an effective magnetic field depending on exciton momentum. The momentum dependent spin precession leads to a spin relaxation similar to the D'yakonov-Perel' one for electron spin. At high temperature the spin relaxation is further suppressed with increasing temperature due to the ionization of excitons which weakens the electron-hole exchange interaction [594]. The spin relaxations of electron, exciton and trion were studied in $n$-doped single CdTe quantum wells with different doping density [684]. It was found that the exciton spin lifetime (18-36 ps) is much shorter than the electron one $(\sim 180 \mathrm{ps})$ and the trion spin relaxation is governed by the fast hole spin relaxation [684]. Spin relaxation in (110) ZnSe quantum wells was studied in Ref. [685] where behaviors quite different from that in GaAs (110) quantum wells were found. Spin lifetime was reported to decrease monotonically with increasing temperature in CdTe quantum wells [535]. The electron density dependence of spin lifetime in CdTe quantum wells was found to be nonmotonic: it exhibits a peak at $8 \times 10^{10} \mathrm{~cm}^{-2}$ for $T=5 \mathrm{~K}[686]$.

\subsubsection{Electron spin relaxation in p-type III-V and II-VI semiconductor two-dimensional structures}

The two main electron spin relaxation mechanisms in $p$-type two-dimensional structures are the D'yakonov-Perel' and the Bir-Aronov-Pikus mechanisms. It is believed that at low temperature and/or high hole density the Bir-AronovPikus mechanism dominates. The Elliott-Yafet mechanism may also dominate spin relaxation in heavily $p$-doped narrow bandgap semiconductors at very low temperature where the Bir-Aronov-Pikus mechanism is suppressed by Pauli blocking [117]. The D'yakonov-Perel' mechanism determines spin relaxation in other regimes.

Experimental investigations on electron spin relaxation in $p$-type quantum wells are scarce. In a 6 nm $p$-modulationdoped GaAs multiple quantum well with hole density $n_{h}=4 \times 10^{11} \mathrm{~cm}^{-2}$, Damen et al. measured an electron spin lifetime of $150 \mathrm{ps}$ at $10 \mathrm{~K}$ [687]. In $\delta$-doped double heterostructures with a doping density $8 \times 10^{12} \mathrm{~cm}^{-2}$, extremely long spin lifetimes up to $20 \mathrm{~ns}$ was observed at $6 \mathrm{~K}$ and a decrease of spin lifetime with temperature $\tau_{s} \sim T^{-0.6}$ was found for $T=6-60 \mathrm{~K}$ (see Fig. 26] [688]. The extremely long spin lifetime was explained as suppression of the electron-hole exchange interaction by spatially separated electrons and holes in the $\delta$-doped double heterostructures. 
However, the D'yakonov-Perel' spin relaxation should also be suppressed to achieve such long spin lifetime, i.e., the momentum scattering should be strong in the structure 65 Later Gotoh et al. measured spin relaxation in a device where the spacial separation between electrons and holes can be tuned by a gate-voltage at room temperature [689]. They showed that the spin relaxation is enhanced when the spacial electron-hole separation is shortened. From the observed results, they concluded that electron spin relaxation is dominated by the Bir-Aronov-Pikus mechanism in their structures. However, the gate-voltage also modifies the Rashba spin-orbit coupling, theses effects should also be taken into account for a close examination. The temperature dependence of spin relaxation at low temperature $(T \leq 60 \mathrm{~K})$ in $p$-modulation-doped GaAs quantum wells was investigated in Refs. [690-692] where a decrease of spin lifetime was found and the spin relaxation varies from 600 ps to 40 ps. Energy-resolved spin dynamics at the sufaces of $p$-GaAs was studied by Schneider et al. using time- and spin- resolved two-photon photoemission [693]. The spin lifetime was found to increase with decreasing electron kinetic energy in agreement with the corresponding theory [390]. They also observed a suppression of spin relaxation in (001) surfaces where hole density is reduced by about an order of magnitude due to the band-bending effect.
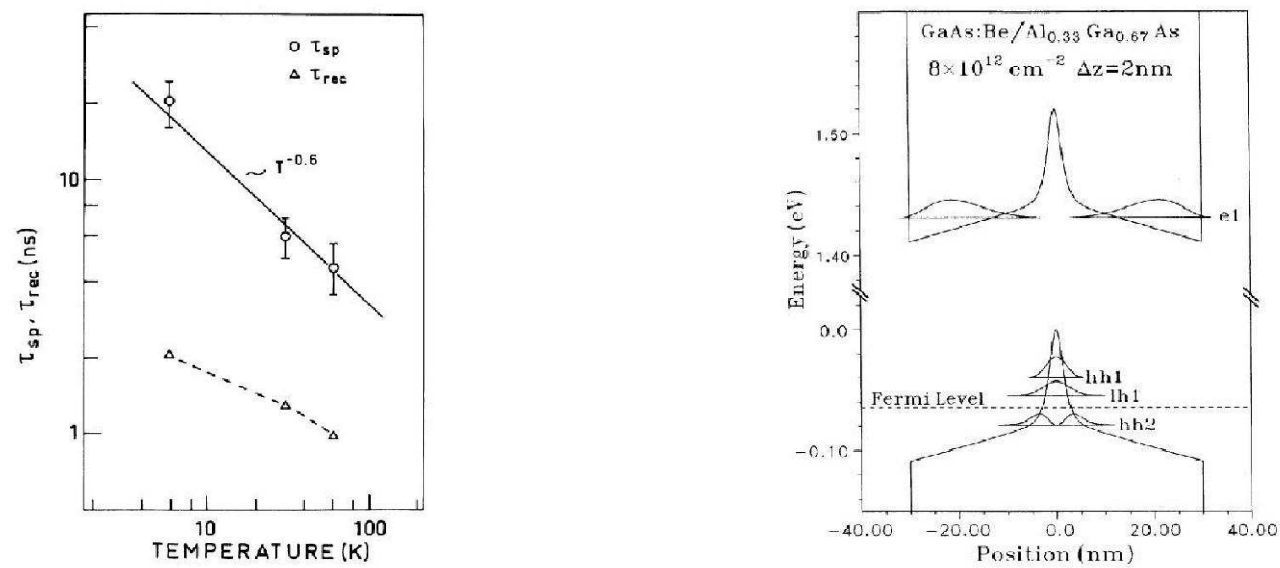

Figure 26: Left: Temperature dependence of the electron-spin lifetime $\tau_{\text {sp }}$ and luminescence recombination time $\tau_{\text {rec }}$. The solid line indicates a $T^{-0.6}$ power law, the dashed line is drawn as a guide to the eyes. Right: Self-consistent potential profile of the structure investigated. Subband energies and probability densities at $k_{\|}=0$ are also shown. "hh" and "lh" denote heavy-hole and light-hole respectively; "e1" denotes the first electron subband. The original structure is a $60 \mathrm{~nm}$ wide $\mathrm{GaAs} / \mathrm{Al}_{0.33} \mathrm{Ga}_{0.67} \mathrm{As}$ quantum well, where it is $\delta$-doped in its center with Be ( $p$-type). From Wagner et al. [688].

Theoretically, the role of electron and hole spin relaxation dynamics in time-resolved luminescence spectral was studied by Uenoyama and Sham [694,695]. The ultrafast carrier and spin dynamics was then investigated from a fully microscopic kinetic spin Bloch equation approach [44, 334, 350] including also the carrier-carrier Coulomb scattering by $\mathrm{Wu}$ and Metiu [350]. Calculation of the Bir-Aronov-Pikus spin relaxation in excitonic system and later in electronhole plasma was performed by Maialle et al. [118, 390, 391] where the energy-dependent spin relaxation time and the effects of the valence-band spin mixing as well as the electric field along the growth direction were studied. These studies found that (i) spin lifetime decreases with electron kinetic energy (see Fig. 27); (ii) the valence-band spin mixing can lead to a factor of two correction; and (iii) spin relaxation due to the Bir-Aronov-Pikus mechanism can be tuned substantially by gate-voltage. The relative importance of the Bir-Aronov-Pikus, Elliott-Yafet and D'yakonovPerel' mechanisms was also compared in GaAs quantum wells [390]. Maialle suggested that the Bir-Aronov-Pikus mechanism is more important than the D'yakonov-Perel' mechanism at medium energy in contrast to that the BirAronov-Pikus mechanism is more important at low energy in bulk GaAs from the same calculation (see Fig. 27).

\footnotetext{
${ }^{65}$ The electron-impurity scattering is the only possible candidate to suppress the D'yakonov-Perel' spin relaxation in the experimental condition, as other scatterings are limited at such low temperature and low electron density.
} 

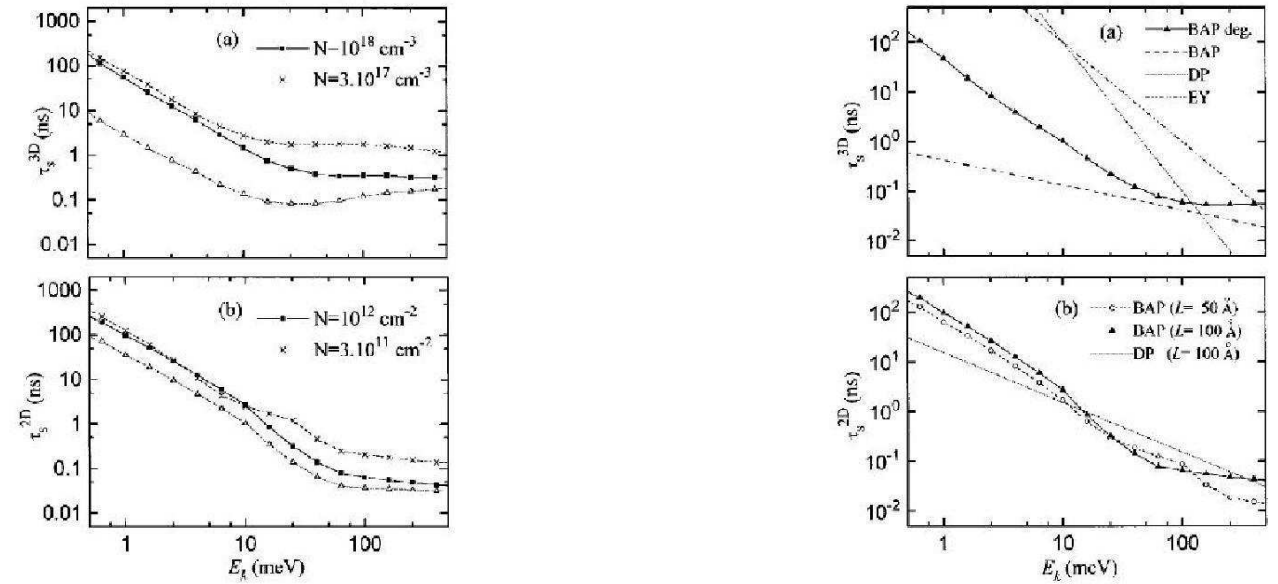

Figure 27: Left: Electron spin lifetime $\tau_{s}$ due to the Bir-Aronov-Pikus mechanism in bulk GaAs and GaAs quantum wells as function of the electron kinetic energy for different hole densities $N$ as labeled in the figure. The dotted curves with open triangles are the same as the solid curves but including the Sommerfeld factor. Right: Calculated electron spin lifetimes $\tau_{s}$ due to the D'yakonov-Perel' (DP), Elliott-Yafet (EY) and Bir-Aronov-Pikus (BAP) mechanisms as functions of the electron kinetic energy. The spin lifetimes due to the D'yakonov-Perel' and Elliott-Yafet processes are taken from Ref. [436], whereas the Bir-Aronov-Pikus (BAP) spin lifetimes are calculated by assuming both nondegenerate holes (label in the figure as "BAP") and degenerate holes (label in the figure as "BAP deg.") with (a) hole densities $N=4 \times 10^{18} \mathrm{~cm}^{-3}$ in bulk GaAs and (b) $N=10^{12} \mathrm{~cm}^{-2}$ in GaAs quantum wells with different well widths L. From Maialle [390].

\subsubsection{Electron spin relaxation in III-V and II-VI semiconductor one-dimensional structures}

There are much less works on electron spin relaxation in one-dimensional structures compared with the electron spin relaxation in two-dimensional structures. It is believed that the D'yakonov-Perel' mechanism is the most efficient one, unless it is suppressed in certain geometry. Due to the additional constrain, the spin-orbit couplings in onedimensional system is quite different from those in the two-dimensional case. By denoting the unconstrained direction as $\hat{z}$, the Rashba spin-orbit coupling for the lowest subband is then

$$
H_{R}=\alpha_{0}|e|^{-1}\left[\sigma_{x}\left\langle\partial_{y} V(x, y)\right\rangle-\sigma_{y}\left\langle\partial_{x} V(x, y)\right\rangle\right] k_{z},
$$

where $V(x, y)$ is the electrostatic potential and $\langle\ldots\rangle$ stands for the average over the lowest subband wavefunction. The Dresselhaus spin-orbit coupling depends on the growth direction of the quantum wire. If $\hat{z} \|[001]$, then

$$
H_{D}=\gamma_{D}\left\langle\left(\hat{k}_{x}^{2}-\hat{k}_{y}^{2}\right)\right\rangle \sigma_{z} k_{z} .
$$

One notices that spin precession direction is the same for all $k_{z}$. Such symmetry leads to an infinite spin lifetimes for spin polarization along the spin precession direction. However, spin lifetime in other directions are still finite. By proper arrangement of the growth direction and the confinement, the Rashba and Dresselhaus spin-orbit couplings can be cancelled, where relaxation for all the spin components due to the D'yakonov-Perel' mechanism can be inhibited [696, 697].

Experimentally, spin relaxation in undoped rectangular GaAs/AlAs quantum wires with small $(<20 \mathrm{~nm})$ lateral sizes was studied via time-resolved photoluminescence [698]. The measured spin lifetime is shown in Fig. 28]together with the spin lifetime in quantum wells for comparison. One finds that spin lifetime in the narrower $(12 \mathrm{~nm} \times 12 \mathrm{~nm})$ quantum wire can be larger than that in the wider one $(19 \mathrm{~nm} \times 13 \mathrm{~nm})$. This is in contrast to the fact that both the D'yakonov-Perel' and Bir-Aronov-Pikus mechanisms increase with the confinement, as also indicated by the spin lifetime in quantum wells for different well widths. The temperature dependence of the wide quantum wire is similar to that in quantum wells, which indicates the multi-subband effect [696]. In narrow quantum wire, the temperature dependence is nonmonotonic, possibly indicating the effect of the electron-hole Coulomb scattering [109, 110]. Wire width dependence of the spin relaxation in wide quantum wires was studied in Refs. [654, 655], where the crossover from two dimension to one dimension was discussed. Transport studies of spin lifetime was reported in Ref. [699].

Theoretically, the study of spin relaxation in quantum wires focused on the D'yakonov-Perel' mechanism. Pramanik et al. studied spin relaxation in a wide quantum wire $(4 \mathrm{~nm} \times 30 \mathrm{~nm})$ using a semiclassical approach (via 


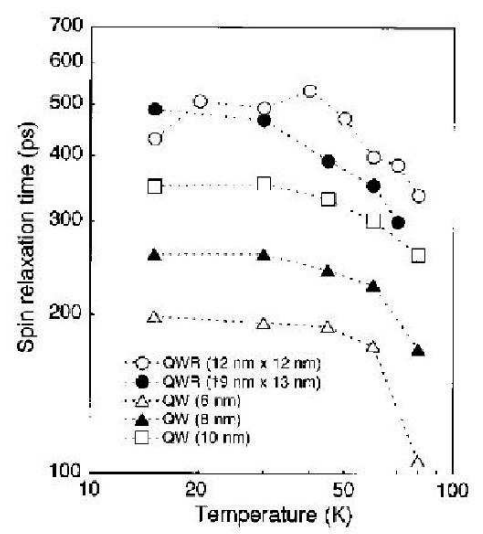

Figure 28: Temperature dependence of the spin relaxation time in quantum wires and quantum wells with various confinements (as labeled in the figure). From Sogawa et al. [698].

the Monte Carlo simulation) [701]. In their work spin relaxation was found to be anisotropic as analyzed above. They showed that electric fields which drive electrons to high $k$ states lead to faster spin relaxation [701]. Later Dyson and Ridley studied electron spin relaxation in quantum wires due to the electron-longitudinal-optical-phonon scattering [632]. Unlike previous studies concerning the electron-longitudinal-optical-phonon scattering, no elastic scattering approximation was made. They gave analytical expressions for the energy dependent scattering time where the inelastic nature of the collision was fully taken into account. They found that the spin relaxation rate increases with confinement asymmetry as indicated by Eq. (123) where the Dresselhaus spin-orbit coupling is proportional to $\left\langle\left(\hat{k}_{x}^{2}-\hat{k}_{y}^{2}\right)\right\rangle$. Spin lifetime due to the electron-longitudinal-optical-phonon scattering was also found to increase with temperature [632]. In the presence of both the Rashba and Dresselhaus spin orbit couplings, the spin relaxation becomes anisotropic. Consider a special case, where the strength of the Rashba spin-orbit coupling is equal to that of the linear Dresselhaus spin-orbit coupling: In two-dimensional case, this leads to a zero spin-splitting for $\mathbf{k}$ along [110] direction, whereas a maximum spin-splitting for $\mathbf{k}$ along [110] direction. By supperimposing additional constrainment to form quantum wires with the growth direction along [110] direction and keeping the condition that the strengths of the Rashba and linear Dresselhaus spin-orbit couplings are equal, it is easy to understand that the spin-orbit field is zero and spin relaxation is inhibited. Such condition holds for narrow quantum wires where only the lowest subband is relevant. Recently, Liu et al. showed that it also holds for quasi-one-dimensional quantum wires where the wire width is comparable to the spin precession length or mean free path (see Fig. 29] [697]. The underlying physics is that electrons with a large transverse momentum component to the wire orientation almost do not contribute to the spin-dephasing because of motional narrowing as they suffer strong boundary scattering. Only electrons with a large momentum component parallel to wire orientation contribute significantly to spin relaxation. Hence spin relaxation depends largely on the wire orientation for equal Rashba and Dresselhaus spin-orbit coupling strengths when the cubic Dresselhaus term is neglected66 (see Fig. 29] [697]. The authors also considered the realistic conditions of imperfect orientation along [110] direction, the cubic Dresselhaus term and inequality of the Rashba and linear Dresselhaus spin-orbit coupling strengths [697]. Recent experimental results confirmed the above theoretical predictions (see Fig. 30] [700]. In the experiment, the strength of the Rashba and linear Dresselhaus spin-orbit coupling is not equal. From the wire orientation dependence, the authors estimated that the ratio of the strengths of the Rashba and linear Dresselhaus spin-orbit coupling is about 2.

\subsubsection{Hole spin relaxation in metallic regime}

Because of the complexity of the valence bands, hole spin relaxation is quite different from the electron spin relaxation. In bulk III-V and II-VI semiconductors, due to the strong spin-orbit coupling and the heavy-light-hole mixing,

\footnotetext{
${ }^{66}$ Note that the dependence of the spin relaxation on quantum wire orientation was also shown by Holleitner et al. 654, 655] where a shorter spin lifetime in wires along [110] direction than that in wires along [100] direction was found. This may be because of an opposite sign of the Rashba spin-orbit coupling in those wires according to the theory by Liu et al. [697].
} 


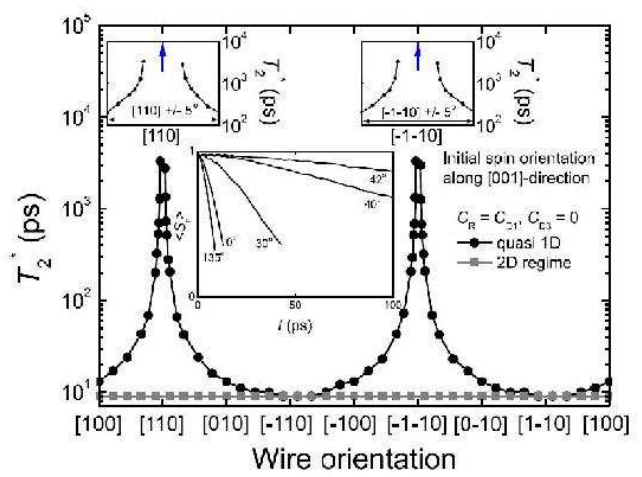

Figure 29: The spin-dephasing time $T_{2}^{*}$ of a spin ensemble is plotted as a function of the wire orientation with respect to the [100] lattice direction. The spin polarization is initially oriented along the [001] direction. $T_{2}^{*}$ is strongly enhanced for a quasione-dimensional wire oriented in the [110] direction (black) as compared to an ensemble in a two-dimensional system (gray). In the calculation, the Rashba and Dresselhaus spin-orbit coupling strengths are taken to be equal, and the cubic Dresselhaus term is ignored. The two upper insets show the results near the two peaks. The lower inset shows the evolution of the spin polarization for various wire orientation (with respect to the [100] direction). From Liu et al. [697].

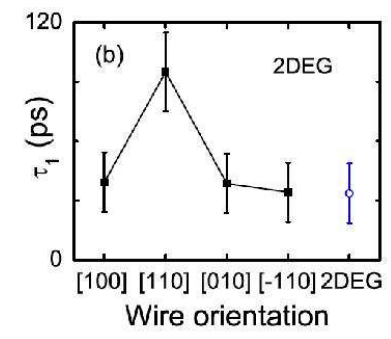

Figure 30: Spin-dephasing time $\tau_{1}$ measured via Kerr rotation in quasi-one-dimensional structures with different wire orientation together with spin relaxation in two-dimensional electron gas (2DEG). From Denega et al. [700].

the D'yakonov-Perel' spin relaxation is very efficient, yielding very short spin lifetime $\tau_{s} \sim 100 \mathrm{fs}$ [207, 210]. In nanostructures, the heavy-light-hole degeneracy at $\mathbf{k}=0$ is lifted and the effect of the spin-orbit coupling is reduced. Spin relaxation is hence slowed down. In quantum wells, hole spin relaxation is in the picosecond regime, usually longer at low temperature [400, 445, 446]. Hole spin relaxation in nanostructures is more complicated, as hole spin-orbit coupling and hole subband structure can vary largely with the geometry (e.g., size and growth direction) of the nanostructures [113]. Besides the D'yakonov-Perel' mechanism, other mechanisms may also be important. For example, consider a unstrained symmetric $p$-type quantum well with only the lowest heavy-hole subband being relevant. The Rashba spin-orbit coupling vanishes as the structure is symmetric. The Luttinger Hamiltonian only induces hole-spin-mixing but no zero-field spin splitting in the lowest heavy-hole subband due to its space-inversion symmetry. The zero-field spin splitting can only originate from the Dresselhaus spin-orbit coupling, which is much weaker than the spin-orbit coupling in bulk system due to the Luttinger Hamiltonian. The D'yakonov-Perel' mechanism is then largely suppressed. On the other hand, the spin-mixing induced by the Luttinger Hamiltonian leads to an effective "spin"-flip scattering associated with any momentum scattering (the Elliott-Yafet-type mechanism), such as hole-phonon scattering [694, 695, 702-704]. Such "spin"-flip processes can be very efficient, especially near a subband anti-crossing point [705]. In general both the D'yakonov-Perel' and Elliott-Yafet-type mechanisms should be considered. Ferreira and Bastard compared the two spin relaxation mechanisms and found that the D'yakonov-Perel' mechanism is usually more important in asymmetric quantum wells [706]. Finally, in heavily $n$-doped samples, the electron-hole exchange interaction (the Bir-Aronov-Pikus mechanism) may also be relevant.

Below we first review hole spin relaxation in bulk materials. Experiments in undoped GaAs revealed that hole spin lifetime is about $100 \mathrm{fs}$ at room temperature [207] 67 Theretical calculation of the ultrafast nonequilibrium dynamics under optical excitation including the spin-orbit coupling as well as hole-phonon and hole-hole scatterings repeated the observed results [210]. Near the equilibrium, calculation reported a spin lifetime about 200 fs for heavy-hole and less than $100 \mathrm{fs}$ for light-hole at room temperature [209]. Hole spin relaxation was also considered in the framework of non-Markovian stochastic theory [708]. Spin lifetime of the $\Gamma_{7}$ band hole in wurtzite GaN was measured to be 120 ps [709], much longer than that in GaAs. This is because unlike the zinc-blende structures, valence bands in

\footnotetext{
${ }^{67}$ Hole spin relaxation in split-off bands was studied by Kauschke et al. [707].
} 


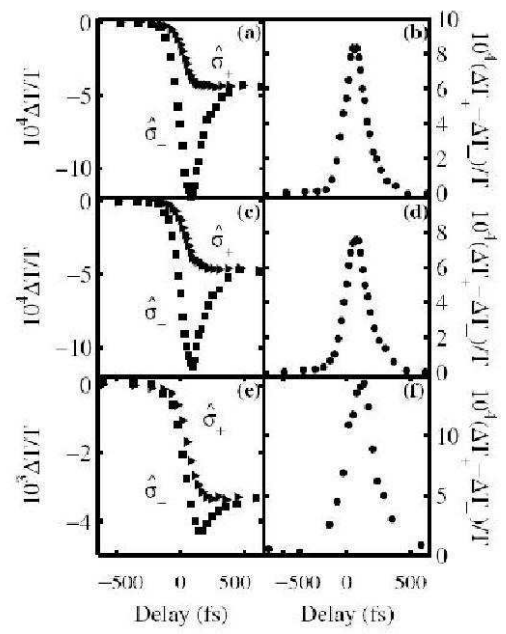

Figure 31: Pump-probe measurements of hole spin dynamics in bulk undoped GaAs at different probe wavelengths $3200 \mathrm{~nm}$ (top), $3000 \mathrm{~nm}$ (middle) and $3800 \mathrm{~nm}$ (bottom). $T$ : transmision, $\Delta T$ : differential transmision. Difference in differential transmision probed by right ( $\left.\Delta T_{+}\right)$and left $\left(\Delta T_{-}\right)$circular polarizations $\left(\Delta T_{+}-\Delta T_{-}\right) / T$ is related to hole spin polarization. From Hilton and Tang [207].

wurtzite semiconductors are splitted into three separated bands, $\Gamma_{9}, \Gamma_{7}$ and $\Gamma_{7^{\prime}}$. The band separation reduces the effect of the spin-orbit coupling and suppresses the D'yakonov-Perel spin relaxation.

Hole spin relaxation in bulk semiconductor under strain was studied by D'yakonov and Perel' [710]. It was found that the hole spin along the strain axis relaxes much slower than the unstrained samples. This is because strain lifts the heavy-hole and light-hole degeneracy at $\Gamma$ point and largely reduces the spin-orbit effect in both heavy-and light-hole bands.

More studies were devoted to hole spin relaxation in two-dimensional structures. Experimentally, Ganichev et al. measured hole spin relaxation by spin-sensitive bleaching of intersubband hole spin orientation in a $p$-type quantum well with well width $L_{w}=15 \mathrm{~nm}$. At low temperature $(T<50 \mathrm{~K})$ hole spin lifetime varies as $\sim T^{-1 / 2}$ for hole density $=2 \times 10^{11} \mathrm{~cm}^{-2}$ [711]. Subsequent studies via the same method extended the data to $T<140 \mathrm{~K}$ and to different well widths (see Fig. 33) [712]. Strikingly, spin relaxation is more efficient in wider quantum wells, in contrast to the inverse tendency in electron case. This is a specific feature of two-dimensional hole systems where the spin-orbit coupling is determined by heavy-light hole mixing, which is stronger in wider quantum wells [see Eq. (22), the Rashba coefficient is proportional to the inverse of the hole subband splitting, i.e., the Rashba coefficient decreases with increasing well width] [206]. Minkov et al. found that the hole spin lifetime (deduced from the weak antilocalization measurements) decreases rapidly with hole density at very low temperature $T=0.44 \mathrm{~K}$ for $3<n_{h}<10 \times 10^{11} \mathrm{~cm}^{-2}$ but varies slowly with temperature for $T<5 \mathrm{~K}$ at $n_{h} \simeq 8 \times 10^{11} \mathrm{~cm}^{-2}$ in $p$-InGaAs/GaAs quantum wells [713]. This is consitent with the D'yakonov-Perel' spin relaxation in degenerate regime, where temperature (hole density) changes both hole distribution and hole-momentum scattering marginally (markedly). In undoped 1.5 monolayer InAs/GaAs quantum well at $10 \mathrm{~K}$, Li et al. observed long hole spin lifetime $\left(\tau_{s}^{h} \simeq 200 \mathrm{ps}\right)$ which can be comparable to the electron spin lifetime $\tau_{s}^{e} \simeq 350 \mathrm{ps}$ in the same structure [714]. This may be because that the hole spin-orbit coupling is weak in such ultrathin layers, as hole spin-orbit coupling decreases with quantum well width [206]. Interestingly, it was found that the hole spin lifetime has a peak in the temperature dependence [715], which resembles the temperature dependence of electron spin lifetime in similar structure [681, 682]. This is consistent with the D'yakonov-Perel' spin relaxation due to the hole-hole Coulomb scattering [299]. Baylac et al. observed that the hole spin lifetime decreases with photo-excitation energy at very low temperature $(1.7 \mathrm{~K})[716]$. The result was explained by the fact that hole spin relaxation rate increases with hole kinetic energy [716]. Both the D'yakonov-Perel' and Elliott-Yafet mechanisms give such dependence [363, 702, 706]. In a designed double quantum well structure, Lu et al. demonstrated that hole spin relaxation in a narrow well with width $L_{w}=4.5 \mathrm{~nm}$ at room temperature can be suppressed $\left(\tau_{s}^{h} \simeq 100 \mathrm{ps}\right)$ if electrons tunnel out to an adjacent quantum well rapidly [611]. This gives an evidence that the Bir-Aronov-Pikus 
mechanism plays important role in hole spin relaxation. Hole spin relaxation time in $n$-doped quantum wells was first measured by Damen et al., finding that $\tau_{s}^{h} \simeq 4$ ps at $10 \mathrm{~K} \mathrm{[687].} \mathrm{Long} \mathrm{hole} \mathrm{spin} \mathrm{lifetime} \mathrm{up} \mathrm{to} \mathrm{nanoseconds}$ was observed in later experiments in $n$-doped quantum wells at low temperature [393, 394, 445, 717, 718] which is assigned to localized holes. Hole spin relaxation in $n$-type quantum wells was reported to decrease with magnetic field [717] which is because that the $g$-tensor inhomogeneity mechanism is important in two-dimensional hole system [399]. Also hole spin lifetime decreases drastically with increasing temperature in $n$-type quantum wells [393, 445] (see Fig. 32) as spin relaxation rate increases with increasing hole kinetic energy. Hole spin relaxation in type-II quantum wells was studied by Kawazoe et al. [719] where two spin decay components were observed: a faster one with $\tau_{s}^{h} \sim 20-100 \mathrm{ps}$ and a slower one with $\tau_{s}^{h} \sim 20 \mathrm{~ns}$. The slower decay was attributed to the localized holes [719]. In II-VI semiconductor quantum wells, such as CdTe quantum wells, hole spin lifetime is on the order of tens of ps [531, 684, 720].

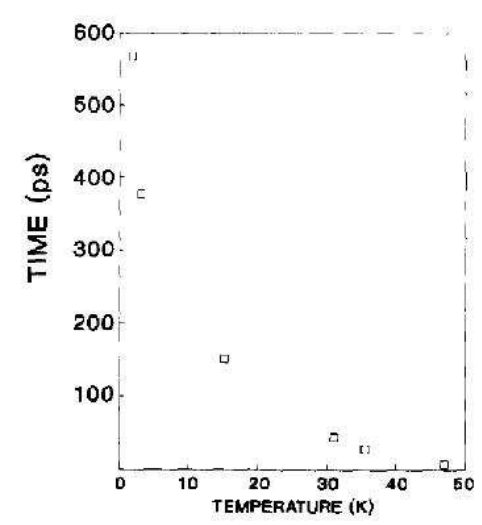

Figure 32: Temperature dependence of hole-spin lifetime in $n$-doped GaAs/AlGaAs quantum wells. Reproduced from Baylac et al. [393].

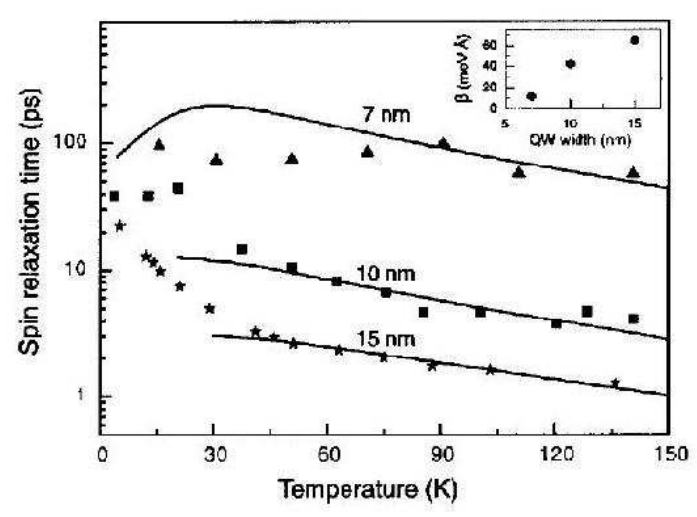

Figure 33: Temperature dependence of hole-spin relaxation time in $p$ doped GaAs/AlGaAs quantum wells. The curves are the calculated results. The inset shows the hole spin-orbit coupling constant $\beta$ (in meV $\AA$ ) as function of quantum well width. From Schneider et al. [712].

Theoretically, hole spin relaxation and its relation to photoluminescence spectra were studied by Uenoyama and Sham in $n$-doped, undoped and $p$-doped quantum wells [694, 695], where the hole-phonon scattering was examined. Specific calculations of "spin"-flip scattering, which leads to the Elliott-Yafet-like spin relaxation, were performed by Ferreira and Bastard [702, 706] and by Vervoort et al. [216], taking into account of the interface inversion asymmetry. The D'yakonov-Perel' spin relaxation due to the "spin"-conserving scattering via standard single particle paradigm was given by Ferreira and Bastard [706]. The role of the Bir-Aronov-Pikus mechanism in hole spin relaxation in quantum wells was discussed by Maialle [395]. It was found that in narrow quantum wells, the Bir-Aronov-Pikus mechanism is comparable with other mechanisms.

Finally, it should be mentioned that up till now the hole spin relaxation in one-dimensional structures was only investigated by Lü et al. theoretically [705]. They studied the topic comprehensively via the kinetic spin Bloch equation approach [44, 334, 350]. Their work will be introduced in Section 5.

\subsection{Carrier spin relaxation in III-V and II-VI paramagnetic diluted magnetic semiconductors}

Semiconductors dilutedly doped with magnetic impurities, such as Mn, are interesting materials because the magnetism and electrics can be incorporated together. Understanding carrier spin dynamics in such kind of materials is important for the application. Also the spin dynamics of the magnetic impurity or the magnetization dynamics is closely related to the carrier spin dynamics. Most of the works in the literature focus on Mn doped III-V and II-VI diluted magnetic semiconductors. In this subsection we review carrier spin dynamics in these materials. We restrict our review on spin dynamics in the paramagnetic phase, where the description is much easier.

A direct consequence of the $s(p)-d$ exchange interaction is that it contributes to the carrier spin-flip scattering and hence carrier spin relaxation. The $s(p)-d$ exchange interaction is believed to dominate spin relaxation in (II,Mn)-VI 
semiconductors [449]. Besides spin relaxation, the $s(p)-d$ exchange interaction also leads to spin precession: carrier spin precesses in the mean field of the $s(p)-d$ exchange interaction. Under external magnetic field, carrier spins precess in the coaction of the external field and the mean field of $\mathrm{Mn}$ spins. For example, $\mathbf{B} \| \hat{\mathbf{x}}$, electron spin Larmor precession frequency is then

$$
\omega_{L}=E_{Z}^{e}=\left|g_{e} \mu_{B} B-x N_{0} J_{\mathrm{sd}}\left\langle S_{x}^{d}\right\rangle\right| .
$$

$\left\langle S_{x}^{d}\right\rangle=-\frac{5}{2} B_{5 / 2}\left[5 g_{\mathrm{Mn}} \mu_{B} B /\left(2 k_{B} T\right)\right]$ is the average Mn spin polarization at equilibrium where $B_{5 / 2}(x)$ is the spin-5/2 Brillouin function and $g_{\mathrm{Mn}}$ is the Mn spin $g$-factor. When the Mn density is high, the exchange interaction can be much larger than the Zeeman interaction $g_{e} \mu_{B} B$, leading to the giant Zeeman splitting. In $\mathrm{ZnSe} / \mathrm{MnSe}$ heterostructures, a large $g$-factor of 400 was observed by Crooker et al. [447]. The contribution from the exchange interaction to spin precession leads to nonlinear magnetic field dependence of Larmor frequency, which can be used to determine the strength of the exchange coupling in experiment [233, 317, 721, 722]. The exchange mean field is largely reduced at elevated temperature. This leads to a drastic decrease in spin precession frequency with increasing temperature [233, 317, 450]. The precession of carrier spins and Mn spins can be strongly coupled when their frequencies match (see Fig. 34) [721, 723]. Besides, nonequilibrium carrier spin polarization can be transferred to the Mn spin system via exchange interaction [98, 724, 725] and vice versa [726-731]. Mn spin beats were observed by Crooker et al. (see Fig. 35] [724, 725] and theoretically studied by Linder and Sham [732].

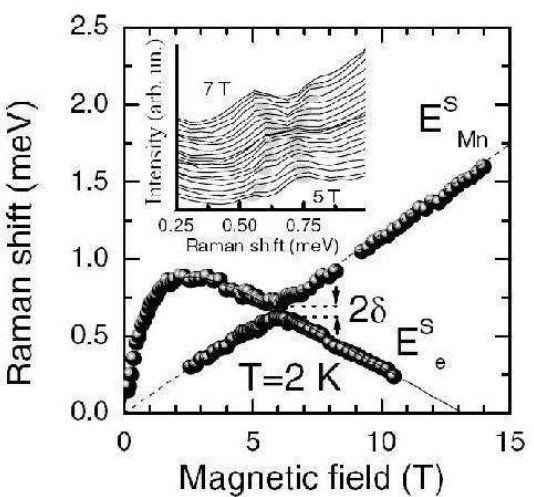

Figure 34: Raman shift for the spin-flip transitions for electron and $\mathrm{Mn}$ ion as function of magnetic field. The expected behavior for noninteracting subsystems calculated is indicated by the solid and dashed curves. $E_{\mathrm{Mn}}^{S}$ and $E_{e}^{S}$ denote Mn and electron spin splitting respectively. The anti-crossing splitting is $2 \delta$. The temperature is $T=2 \mathrm{~K}$. Inset shows the Raman spectra (every $0.1 \mathrm{~T}$ ) in the region of the anti-crossing. From Teran et al. [721].

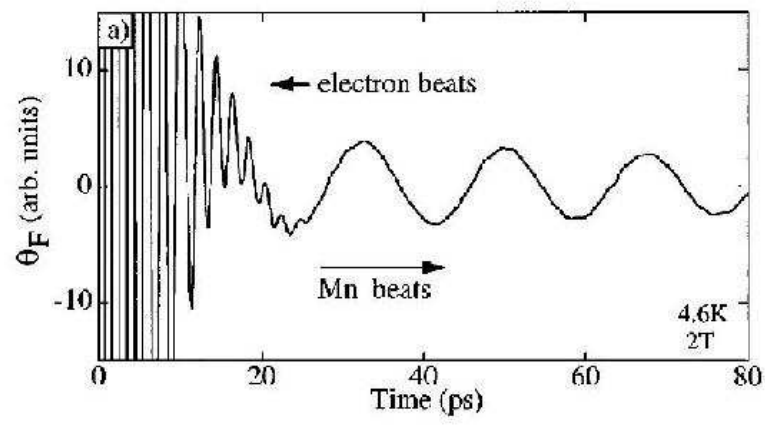

Figure 35: Induced Faraday rotation $\Theta_{\mathrm{F}}$, showing the final oscillations of the electron spins superimposed on an induced precession of the Mn spins. From Crooker et al. [724].

Historically, carrier spin dynamics was first studied in (II,Mn)VI diluted magnetic semiconductors. In these materials no charge doping is invoked, as $\mathrm{Mn} \Rightarrow \mathrm{Mn}^{2+}+2 e^{-}$. At high doping density and low temperature the Mn-Mn interaction leads to a spin-glass order. However, at high temperature or low doping density it is paramagnetic. The bandgap also changes with $\mathrm{Mn}$ doping. For example in $\mathrm{CdMnTe}$, the bandgap increases with Mn doping density. A widely studied system is $\mathrm{Cd}_{1-x} \mathrm{Mn}_{x} \mathrm{Te} / \mathrm{Cd}_{1-y} \mathrm{Mn}_{y} \mathrm{Te}$ quantum well, where the layer with higher Mn density serves as barrier. A particular case is $x=0$, the $s(p)$ - $d$ exchange interaction is then incorporated through the wavefunction penetrated into the barrier layers. The $s(p)-d$ exchange interaction can then be engineered via tuning the structure [733]. Another example of such structure is to insert MnSe layers into $\mathrm{ZnCdSe}$ quantum wells [447]. Crooker et al. found that the $s(p)-d$ exchange interaction changes significantly with the number of MnSe layers [in their experiment, they used $1(\times 3$ atomic layer), $3(\times 1$ atomic layer) and $24(\times 1 / 8$ atomic layer) layers of MnSe] in $\mathrm{ZnCdSe}$ quantum well while keeping the overall Mn doping density unchanged [447]. The modification of the $s(p)-d$ exchange interaction comes from tuning the overlap between the confined electrons/holes and $\mathrm{Mn}$ ions by structure engineering. Another reason is that the Mn-Mn antiferromagnetic coupling between neighboring Mn spins which "locks" the Mn spins is largely suppressed in 1/8 MnSe sub-monolayers as the average distance between Mn spins increases. Interestingly 
Smyth et al. found that the spin subband of the $\mathrm{ZnMnSe} / \mathrm{ZnSe}$ superlattice can be significantly modified by external magnetic field [734]: Due to the giant Zeeman splitting, the spin-down subband is lowered and spin-up subband is raised. At the threshold magnetic field $B_{c}$, spin-up subband energy is as high as the barrier energy. Above $B_{c}$ spin-up electrons switch into the ZnSe layers. The spin and electronic structure is then tuned by the external magnetic field. In parabolic $\mathrm{ZnSe} / \mathrm{ZnCdSe}$ quantum well with MnSe monolayers inserted, Myers et al. demonstrated that the $s$ - $d$ exchange interaction can be manipulated by gate voltage [735]. As the $s(p)$ - $d$ exchange interaction is tuned, the carrier spin dynamics is also manipulated [447, 734-736].

Femtosecond pump-probe measurements were first performed by Freeman et al. [733], revealing a very short spin relaxation time of $\sim 3 \mathrm{ps}$ in $\mathrm{Cd}_{1-x} \mathrm{Mn}_{x} \mathrm{Te} / \mathrm{Cd}_{1-y} \mathrm{Mn}_{y} \mathrm{Te}$ quantum wells [733, 737]. After that, Bastard and Chang [448], Bastard and Ferreira [703] presented theoretical studies on spin relaxation due to the $s-d$ exchange interaction in such kind of quantum wells. They obtained comparable spin lifetime with experiments. They also predicted well width dependence of spin lifetime and proposed a bias double-quantum-well structure to manipulate the spin lifetime. Interesting behavior was observed under magnetic field. As the neighbor Mn ions are coupled antiferromagnetically, Mn ions can link together to form clusters which have small spin momentums and become ineffective in spin-flip scattering. Hence, only isolated Mn ions count. Bastard and Chang took an effective Mn density as $n_{\mathrm{eff}}=x(1-x)^{12} N_{0}$ to warrant only Mn ions without any nearest neighbors. Akimoto et al. [736] found that electron spin relaxation rate in $\mathrm{CdTe} / \mathrm{CdMnTe}$ quantum wells is proportional to the probability of finding electron in the barrier layer of CdMnTe, which is consistent with the theory of Bastard and Chang [448]. As stated in previous paragraph, spin-up subband is raised higher than the spin-down one. Hence spin-flip scattering from up-spin to down-spin is more favorable than the opposite one at low temperature. Smyth et al. reported that spin-flip time of spin-up electron decreases with increasing magnetic field whereas that of spin-down electron increases: the spin-flip times vary with magnetic field as two branches [734]. Similar behavior was observed in other diluted magnetic heterostructures (see inset of Fig. 36 [447, 738-742]. Such two-branch behavior is weakened with increasing excess photo-carrier energy or decreasing exchange interaction [738, 741]. Both electron and hole spin lifetimes were found to be smaller in diluted magnetic heterostructures compared to the nonmagnetic ones [724], which indicates the relevance of the $s(p)$ $d$ exchange interaction to the spin relaxation.

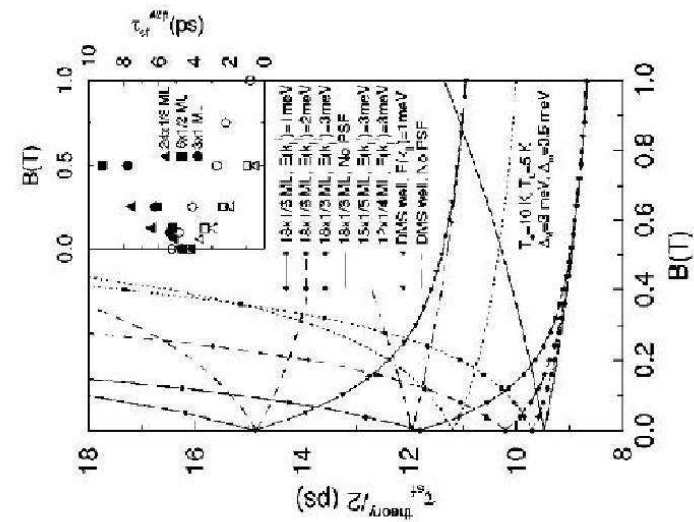

Figure 36: Calculated electron spin-flip times $\tau_{\mathrm{sf}}^{\text {theory }} / 2$ as a function of the magnetic field $B$. The inclusion of phase-space-filling (PSF) enhances the two-branch feature of the $\tau_{s f}$ vs. $B$ curves. Different structures are considered: heterostructures with $18 \times 1 / 6$ monolayer $(\mathrm{ML}), 15 \times 1 / 5$ ML, $12 \times 1 / 4 \mathrm{ML}$ and diluted magnetic semiconductor (DMS) quantum well. Inset: experimental results by Crooker et al. [447] with various heterostructures: $24 \times 1 / 8 \mathrm{ML}$ (triangle), $6 \times 1 / 2 \mathrm{ML}$ (square) and $3 \times 1 \mathrm{ML}$ (circle). The two branches of spin-flip time correspond to $\tau_{\uparrow \rightarrow \downarrow}$ and $\tau_{\downarrow \rightarrow \uparrow}$. The calculation is not intended to compare quantitatively with the experimental results. From Egues and Wilkins [743].

Theoretically, spin relaxation rate can be calculated from the Fermi Golden rule [451, 452],

$$
\Gamma_{\mathbf{k} \uparrow, \mathbf{k}^{\prime} \downarrow}=2 \pi\left|\left\langle\mathbf{k} \uparrow\left|H_{s-d}\right| \mathbf{k}^{\prime} \downarrow\right\rangle\right|^{2} \delta\left(\varepsilon_{\mathbf{k} \uparrow}-\varepsilon_{\mathbf{k}^{\prime} \downarrow}\right) .
$$

The spin-flip rates are

$$
\tau_{\uparrow \rightarrow \downarrow}^{-1}=\left\langle\Gamma_{\mathbf{k} \uparrow, \mathbf{k}^{\prime} \downarrow}\left(1-f_{\mathbf{k}^{\prime} \downarrow}\right)\right\rangle, \quad \tau_{\downarrow \rightarrow \uparrow}^{-1}=\left\langle\Gamma_{\mathbf{k}_{\downarrow}, \mathbf{k}^{\prime} \uparrow}\left(1-f_{\mathbf{k}^{\prime} \uparrow}\right)\right\rangle .
$$


Here $\uparrow \rightarrow \downarrow(\downarrow \rightarrow \uparrow)$ denotes the spin-up to spin-down (spin-down to spin-up) transition. $\langle\ldots\rangle$ means average over the electron ensemble. The total spin relaxation rate is $\tau_{s}^{-1}=\tau_{\uparrow \rightarrow \downarrow}^{-1}+\tau_{\downarrow \rightarrow \uparrow}^{-1}$. For example in quantum wells at zero magnetic field [111, 744, 745],

$$
\tau_{s}^{-1}=I_{s} x N_{0} J_{\text {sd }}^{2} m_{e}\left\langle\left\langle S_{-}^{d} S_{+}^{d}+S_{+}^{d} S_{-}^{d}\right\rangle\right\rangle /\left(4 L_{w}\right),
$$

where $J_{\mathrm{sd}}$ is the $s$ - $d$ exchange constant, $x$ stands for the mole fraction of Mn and $N_{0}$ denotes the density of the unit cells. $\mathbf{S}^{d}=\left(S_{x}^{d}, S_{y}^{d}, S_{z}^{d}\right)$ is Mn spin operator and $S_{ \pm}^{d}=S_{x}^{d} \pm i S_{y}^{d} .\langle\langle\ldots\rangle\rangle$ stands for average over Mn spin distribution. Here $I_{s}=L_{w} \int\left|\psi_{e}^{\uparrow}(z)\right|^{2}\left|\psi_{e}^{\downarrow}(z)\right|^{2} \mathrm{~d} z$ with $\psi_{e}^{\uparrow(\downarrow)}$ being the spin-resolved electron subband wavefunction and $L_{w}$ denoting the well width. At equilibrium state, $\left\langle\left\langle S_{-}^{d} S_{+}^{d}+S_{+}^{d} S_{-}^{d}\right\rangle\right\rangle=\frac{4}{3} S_{d}\left(S_{d}+1\right)$. As to the two spin-flip transition rates, $\tau_{\uparrow \rightarrow \downarrow}^{-1} \propto$ $\left\langle\left\langle S_{-}^{d} S_{+}^{d}\right\rangle\right\rangle=S_{d}\left(S_{d}+1\right)-\left\langle\left\langle\left(S_{z}^{d}\right)^{2}\right\rangle\right\rangle-\left\langle\left\langle S_{z}^{d}\right\rangle\right\rangle$ whereas $\tau_{\uparrow \rightarrow \downarrow}^{-1} \propto\left\langle\left\langle S_{+}^{d} S_{-}^{d}\right\rangle\right\rangle=S_{d}\left(S_{d}+1\right)-\left\langle\left\langle\left(S_{z}^{d}\right)^{2}\right\rangle\right\rangle+\left\langle\left\langle S_{z}^{d}\right\rangle\right\rangle$. In the presence of a magnetic field along the $z$ axis, the Mn spin polarization $\left\langle\left\langle S_{z}^{d}\right\rangle\right\rangle\langle 0$. This enhances the spin-up to spin-down transition but suppresses the inverse process. The calculation of Egues and Wilkins indicated that the phase-spacefilling effect (Pauli blocking factor $\left(1-f_{\mathbf{k}^{\prime} \uparrow(\downarrow)}\right)$ ) further enhances the difference between the two spin-flip scattering rates (see Fig. 36) [743]. It is noted that the total spin relaxation rate is proportional to $S_{d}\left(S_{d}+1\right)-\left\langle\left\langle\left(S_{z}^{d}\right)^{2}\right\rangle\right\rangle$ which decreases with increasing magnetic field. The underlying physics is that the magnetic field pins the Mn spin and then suppresses the spin-flip processes and electron spin relaxation [746, 747]. Magnetic field effect on electron/hole/exciton spin-flip scattering rates at low temperature in CdMnTe quantum wells was investigated comprehensively by Tsitsishvili and Kalt [745]. In most cases the magnetic field dependence of spin relaxation rate is weak. Unlike longitudinal magnetic field, a transverse magnetic field always leads to faster spin relaxation (for both electrons and holes) (see Fig. 37) [449, 724, 725, 748-750]. This is because that the magnetic field pinning of Mn spins along transverse direction increases the factor $S_{d}\left(S_{d}+1\right)-\left\langle\left\langle\left(S_{z}^{d}\right)^{2}\right\rangle\right\rangle=\left\langle\left\langle\left(S_{x}^{d}\right)^{2}+\left(S_{y}^{d}\right)^{2}\right\rangle\right\rangle[111,744]$. Electron spin relaxation is enhanced for exciton bound electrons as the center-of-mass effective mass is larger for exciton. This was first predicted by Bastard and Ferreira [703] and then confirmed experimentally by Smits et al. [749]. Temperature dependence of electron, hole and exciton spin relaxation has been studied in Refs. [450, 749, 751, 752]. Smits et al. presented a systematic experimental study on excitonic enhancement of electron/hole spin relaxation in CdMnTe based quantum well structures [749].
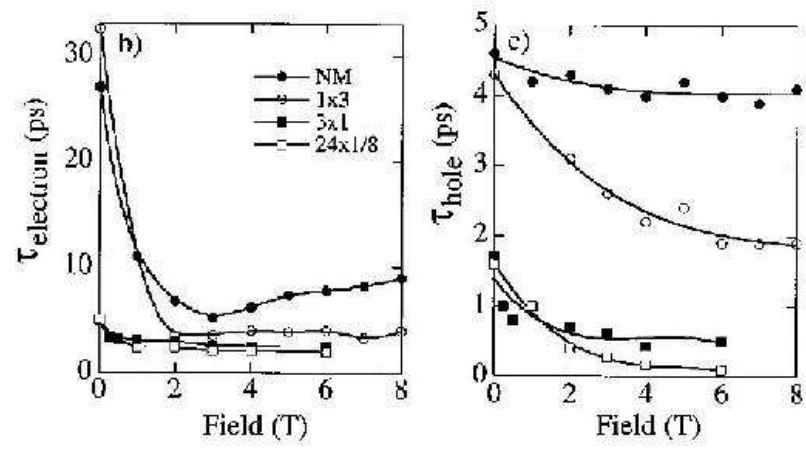

Figure 37: Electron (left) and hole (right) spin lifetime as function of transverse magnetic field for nonmagnetic $(\mathrm{NM})\left(\mathrm{Zn}_{0.77} \mathrm{Cd} \mathrm{d}_{0.23} \mathrm{Se} / \mathrm{ZnSe}\right)$ quantum well as well as that for quantum well with $1 \times 3,3 \times 1$ and $24 \times 1 / 8 \mathrm{MnSe}$ monolayers inserted (for the details of the heterostructures see Ref. [724]). $T=4.6$ K. From Crooker et al. [724].

Recently, Rönnburg et al. presented a systematic experimental investigation on electron spin relaxation in bulk CdMnTe. Their major results are shown in Figs. 38 and 39. In the experiment, the photon energy was chosen to be centered on the $1 \mathrm{~s}$ exciton absorption lines and the photo-excitation intensity was kept low in order to minimize sample heating and carrier-density-dependent effects. A salient feature is that electron spin lifetime increases with temperature, which signals a motional-narrowing spin relaxation mechanism. This is quite different from the previously considered $s-d$ exchange scattering mechanism where electron spin relaxation rate in bulk system is proportional to $\langle k\rangle$, which increases with temperature. Rönnburg et al. then presented a new theory of spin relaxation based on the 
thermal and spacial fluctuation of the $s-d$ mean field. The transverse spin relaxation rate is given by

$$
\frac{1}{T_{2}}=\gamma^{2} \tau_{0}\left[\left\langle\left(\delta M_{z}\right)^{2}\right\rangle+\frac{\left(\left\langle\left(\delta M_{x}\right)^{2}\right\rangle+\left\langle\left(\delta M_{y}\right)^{2}\right\rangle\right) / 2}{1+\left(g_{e} \mu_{B} B+\gamma\left\langle\delta M_{z}\right\rangle\right)^{2} \tau_{0}^{2}}\right]
$$

where $\gamma=J_{\mathrm{sd}} /\left(g_{\mathrm{Mn}} \mu_{B}\right), \mathbf{M}=\left(M_{x}, M_{y}, M_{z}\right)$ is the (Mn spin) magnetization and $\delta M_{i}(i=x, y, z)$ denotes the fluctuation of magnetization felt by optically excited electrons. $\tau_{0}$ stands for the correlation time of the fluctuations. $g_{e}$ is the intrinsic $g$-factor of the itinerant electrons. By working out the thermal and spacial fluctuations, the spin relaxation rate is given explicitly as [450]

$$
\frac{1}{T_{2}}=\frac{\gamma^{2} \tau_{0}}{N_{\mathrm{ex}}}\left[n_{0} x k_{B} T \chi(B, T)+\left\langle M_{z}\right\rangle^{2}+\frac{n_{0} x k_{B} T[3 \chi(0, T)-\chi(B, T)]-\left\langle M_{z}\right\rangle^{2}}{2+2\left(g_{e} \mu_{B} B+\gamma\left\langle\delta M_{z}\right\rangle\right)^{2} \tau_{0}^{2}}\right] .
$$

Here $\chi(B, T)=\frac{\partial\left\langle M_{z}(B, T)\right\rangle}{\partial B}$ is the susceptibility. $\langle\ldots\rangle$ denotes the thermal average of Mn spins. By assuming the spacial fluctuation to be a Poisson distribution, it gives the amplitude of the spacial fluctuation $\left\langle\left(\delta M_{i}\right)^{2}\right\rangle_{\mathrm{sf}}=\left\langle M_{i}\right\rangle^{2}(i=x, y, z)$. According to the central limit theorem, the fluctuation is reduced by the number of Mn spins felt by the electron wavepacket $N_{\text {ex }}$. In intrinsic bulk CdMnTe at low temperature electrons and holes are bound together to form excitons. The spacial extension of exciton-bound electron wave-packet is controlled by two factors: the exciton Bohr radius and the thermal length $L_{\mathrm{th}}$ of the center-of-mass motion. The latter is estimated as the inverse of the thermal fluctuation of the center-of-mass wave-vector, $L_{\mathrm{th}}=0.37 / \sqrt{m k_{B} T}$ with $m=m_{e}+m_{h}$. Taking into account these two factors, one obtains the volume of the wave-packet $V_{\mathrm{ex}}$ and then the number of Mn spins within the volume $N_{\mathrm{ex}}=x n_{0} V_{\mathrm{ex}}$. The correlation time also consists of two contributions, $1 / \tau_{0}=1 / \tau_{\mathrm{prop}}+1 / \tau_{\mathrm{sf}}^{\mathrm{Mn}} \cdot \tau_{\mathrm{sf}}^{\mathrm{Mn}}$ is the Mn spin-flip time which is at least several hundreds of picoseconds [725] and hence ineffective. $\tau_{\text {prop }}$ denotes the exciton propagation time, which is the time for an exciton to see a new environment of Mn spins. It is approximated as the time for an exciton to propagate a distance equal to its spacial extension with a thermal average velocity. In such a way $\tau_{0}$ is determined. By taking parameters in the literature, Rönnburg et al. calculated the transverse spin lifetime and found that the calculated results agree remarkably well with experimental data both qualitatively and quantitatively (see Fig. 39). The Mn doping $x$ dependence of $T_{2}$ at small $x$ shows $1 / x$ behavior as $M_{z}, \chi$ and $N_{\text {ex }}$ are proportional to $x$. At high Mn doping, the antiferromagnetic interaction between neighboring Mn ions should be taken into account with a correction $x_{\text {eff }}=x(1-x)^{12}\left[743\right.$ ]. This leads to the observed increase of $T_{2}$ at large $x$ in Fig. 39] The magnetic field dependence also agrees well with the calculation (not shown). The intial decrease of $T_{2}$ with magnetic field is mainly due to the increase of $\left\langle M_{z}\right\rangle^{2}$. At high magnetic field, $\chi(B, T)$ and $\left\langle M_{z}\right\rangle$ saturate. The magnetic field dependence then mainly comes from the factor $1 /\left[2+2\left(g_{e} \mu_{B} B+\gamma\left\langle\delta M_{z}\right\rangle\right)^{2} \tau_{0}^{2}\right]$ which makes $T_{2}$ increase with $B$ (Similar behavior was also observed in the experiment of Cronenberger et al. [753]). The magnetic field dependence is more pronounced at low temperature where $\tau_{0}$ is larger and the magnetic field dependence of $\left\langle M_{z}\right\rangle$ is stronger.

The Mn density dependence is informative and reveals the underlying spin relaxation mechanisms. In CdMnTe quantum wells, spin relaxation rate was found to be proportional to $x$ for $x>4 \times 10^{-3}$, indicating spin relaxation due to the $s$ - $d$ exchange scattering (see Fig. 40) [449]. However, in GaMnAs quantum wells Poggio et al. found that spin lifetime first increases and then decreases with increasing Mn density. A peak appears around $x \sim 10^{-4}$ for well width from 3 to $10 \mathrm{~nm}$ (see Fig. 41] [233]. It was speculated that the dominant spin relaxation mechanisms for Mn densities below and above the peak density are different. However, calculation based on realistic parameters is needed to determine the relevance of various mechanisms. Such calculation has been done by Jiang et al. recently [111].

Relaxation dynamics of spin-polarized electrons excited at higher energy subband was revealed by probe-energy dependence of the spin lifetime in Ref. [754]. The probe-energy dependence is understood by the intraband electron energy relaxation and subsequent spin relaxation. Energy-resolved spin relaxation in wurtzite magnetic semiconductor CdMnSe was studied in Ref. [755].

At low temperature, $\mathrm{Mn}$ ion can bind a hole to form a neutral center. Hence optically excited electrons interact with the neutral centers rather than the $\mathrm{Mn}$ ions and holes separately. The ground state of the neutral center is a twofold degenerate state with angular momentum $m_{J}= \pm 1$ as the $p$ - $d$ exchange interaction is anti-ferromagnetic 68 The

\footnotetext{
${ }^{68}$ Such a spin structure can be utilized to establish optical initialization and readout of Mn spin [98] as circularly polarized light (with spin $\sigma_{J}= \pm 1$ ) selectively excites the $m_{J}= \pm 1$ state due to the optical selection rules.
} 


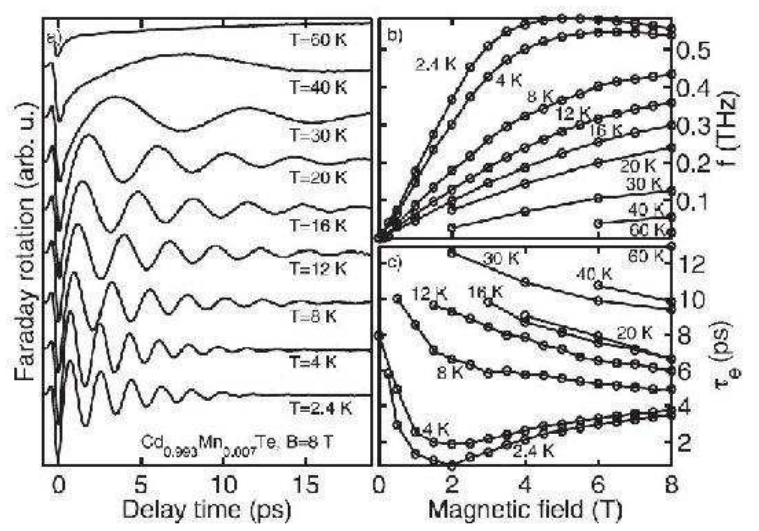

Figure 38: Results of time-resolved Faraday rotation experiments on $\mathrm{Cd}_{1-x} \mathrm{Mn}_{x} \mathrm{Te}(\mathrm{x}=0.0073)$ at $8 \mathrm{~T}$ and for various temperatures: (a) Temperature dependence of the Faraday-rotation transients; (b) temperature and magnetic-field dependence of the electron spin precession frequency extracted from the transients; (c) fitted electron spin dephasing time $\tau_{e}$ as a function of temperature and magnetic field. From Rönnburg et al. [450].
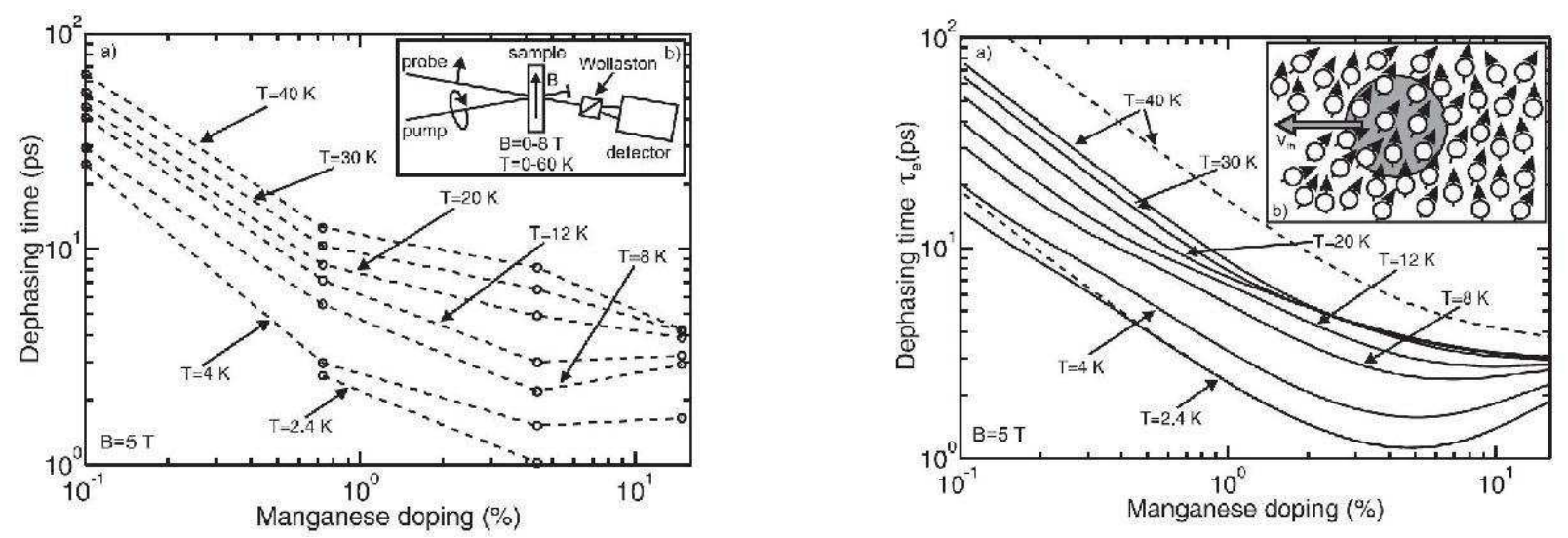

Figure 39: Left: experiment. Right: theory. Doping density and temperature dependence of electron spin dephasing time. Magnetic field is fixed at $5 \mathrm{~T}$. Inset in right figure: the picture of exciton moving through the local moments of magnetic dopants. Inset in the left figure: experimental set-up. From Rönnburg et al. [450].

exchange interaction between electron and the neutral center consists of two origins, the $s$ - $d$ exchange interaction and the electron-hole exchange interaction. Interestingly, both interactions are ferromagnetic. Due to opposite Mn and hole spin orientations, the whole exchange interaction is weakened 69 The cancellation of the two exchange interaction leads to suppression of spin relaxation. As GaMnAs is partially compensated semiconductors, i.e., interstitial Mn's act as donors whereas substitutional Mn's act as acceptors, hole density is smaller than the Mn acceptor density. Hence there are still some charged Mn acceptors. Recently, Astakhov et al. found that the exchange interaction can be further reduced by generating more holes via optical pumping. They found that below some threshold excitation power, spin relaxation is suppressed by increasing the optical excitation power (see Fig. 42) [757]. A picture of localized electrons was presented in their paper to explain the results at low temperature. In such picture, the spin relaxation is due to random spin precession when electrons pass by the neutral centers. The spin relaxation rate is $1 / \tau_{s}=\frac{2}{3}\left\langle\omega^{2}\right\rangle \tau_{c}$ where $\tau_{c}$ is the correlation time. In the presence of a longitudinal magnetic field, spin relaxation is suppressed by a factor of $1 /\left[1+\left(\omega_{L} \tau_{c}\right)^{2}\right]$. By measuring the longitudinal magnetic field dependence, Akimov et al. found that $\tau_{c}$ in GaMnAs is much longer than that in GaAs:Ge with similar acceptor concentration. They explained that the strong exchange coupling between $\mathrm{Mn}$ acceptor and hole protects hole spin from dissipation and the correlation time of random electron

\footnotetext{
${ }^{69}$ This leads to a deviation of the $s$ - $d$ exchange constant deduced from the measured electron spin precession frequency from the genuine $s$ - $d$ exchange constant [756].
} 


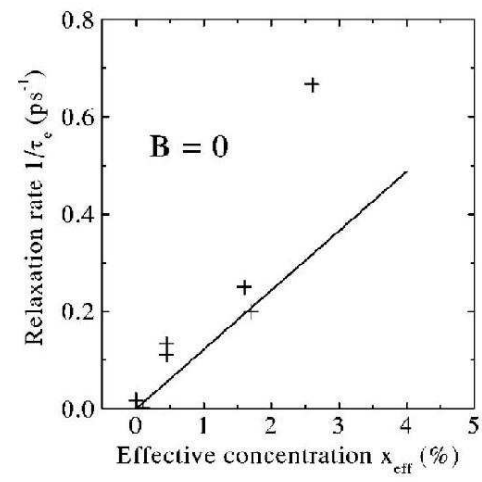

Figure 40: Electron spin relaxation rates vs. Mn effective content $x_{\text {eff }}$ for a QW width $L_{w}=8 \mathrm{~nm}$ : experimental data (crosses), calculated values (solid line). From Camilleri et al. [449].

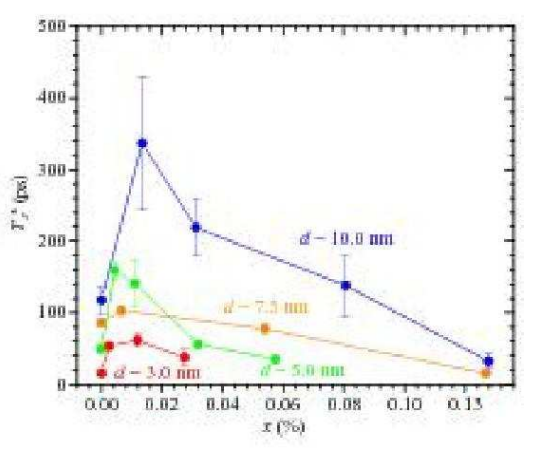

Figure 41: The transverse electron spin lifetime $T_{2}^{*}$ at $T=5 \mathrm{~K}$ versus the percentage of Mn $x$ for four quantum well sample sets of varies widths. The plotted values of $T_{2}^{*}$ are the mean values from magnetic field $B=0$ to $8 \mathrm{~T}$, and the error bars represent the standard deviations. From Poggio et al. [233].

spin precession is only limited by electron hopping, leading to a large $\tau_{c}$ [758].
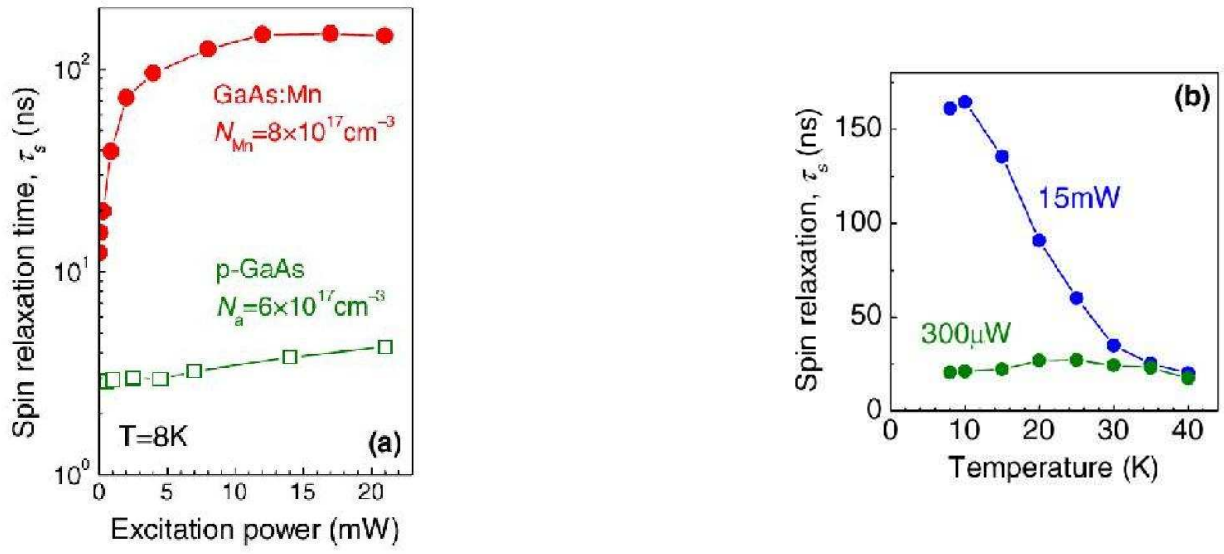

Figure 42: (a) Spin relaxation time $\tau_{s}$ vs. excitation power for GaAs:Mn $\left(N_{\mathrm{Mn}}=8 \times 10^{17} \mathrm{~cm}^{-3}\right)$ (dots) and for p-GaAs containing nonmagnetic acceptors $\left(N_{a}=6 \times 10^{17} \mathrm{~cm}^{-3}\right)$ (open squares). (b) Spin lifetimes vs. temperature for different excitation powers in GaAs:Mn. Curves are to guide the eyes. From Astakhov et al. [757].

Hole spin relaxation in diluted magnetic quantum wells and heterostructures was studied in Refs. [449, 724, 725, 749]. For heavy-holes, the $p$ - $d$ exchange interaction is not able to flip hole spin unless facilitated by heavy-light hole mixing [702]. It was reported that spins of exciton-bound holes relax faster than free holes due to enhanced heavylight hole mixing in excitons [749]. Also the mixing is increased by the mean field of the $p$ - $d$ interaction. Camilleri observed a relation for hole spin relaxation rate $1 / \tau_{s}^{h} \simeq a\left(x_{\text {eff }}\langle M\rangle\right)^{2}+b$ where $x_{\text {eff }}$ is the effective (isolated) Mn mole fraction, $\langle M\rangle$ denotes the magnetization and $b$ stands for the heavy-light hole mixing independent of $\langle M\rangle$ and other spin relaxation sources [449]. Hole spin relaxation always decreases with increasing magnetic field [449, 724, 725, 749]. Theoretical investigation of hole spin relaxation is presented in Refs. [702, 745].

\subsection{Spin relaxation in Silicon and Germanium in metallic regime}

Spin relaxation in silicon and germanium is an old topic. Spin relaxation of donor bound electrons has been studied intensively since the middle of last century [453, 759-764] and revisited in recent decades in the context of 
quantum computation $[289,416,417,460,765,766]$. In this subsection, we focus on spin relaxation in silicon and germanium in the metallic regime.

Let us first start with bulk silicon 70 As silicon possesses space inversion symmetry, the spin-orbit coupling term of the conduction band is zero. Hence the D'yakonov-Perel' mechanism is absent. In $n$-doped silicon, the only relevant spin relaxation mechanism is the Elliott-Yafet mechanism. Experimentally, electron spin relaxation in bulk silicon was mostly studied via electron spin resonance as the optical orientation and detection method is forbidden due to its indirect band gap nature. Most of the investigations focused on the low temperature regime [767-770], whereas only a few covered the high temperature regime [763, 771]. Among these works, a systematic investigation was performed by Lépine [763]. Analysis indicated that the spin relaxation scenario varies with temperature71 At high temperature $(T>150 \mathrm{~K})$ most of electrons are in extended states and spin relaxation is dominated by the band electrons other than donors. Theoretically, conduction band electron spin relaxation in silicon was first studied by Elliott [104] and Yafet [103] separately. The Elliott-Yafet spin relaxation consists of both the Elliott process where spin-flip is due to the spin-mixing of conduction band and the Yafet process where spin-flip is caused by direct spinphonon coupling. By taking into account of the Yafet process due to intravalley electron-acoustic phonon scattering, Yafet gave a qualitative relation of $\tau_{s} \sim T^{-\frac{5}{2}}$ [103]. Recently, Cheng et al. found that the Elliott process and the Yafet process interfere destructively in silicon. The total spin relaxation rate is much slower than that predicted by the individual Yafet or Elliott process [362]. They gave new qualitative relation of $\tau_{s} \sim T^{-3}$, which agrees quite well with experiments [362] [see Fig. 43].

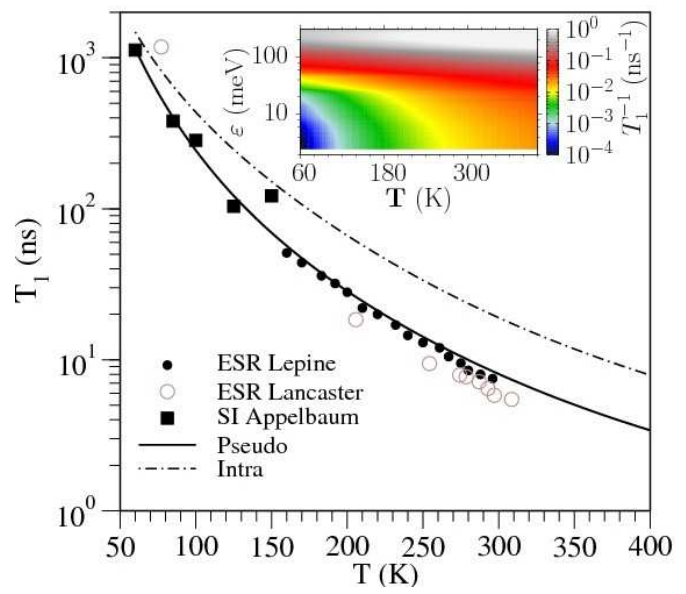

Figure 43: Spin relaxation time $T_{1}$ in bulk silicon as a function of temperature $T$. The solid curve is the calculation (Pseudo), the symbols are the spin injection (SI Appelbaum) [150] and the electron spin resonance (ESR Lepine [763] and ESR Lancaster [771]) data (see Ref. [22]). The dashed-dotted curve is the spin lifetime calculated with only the intravalley scattering (Intra). The inset shows the contour plot of the spin relaxation rate $T_{1}^{-1}$ of hot electrons, as a function of the electron energy $\varepsilon$ and lattice temperature $T$. From Cheng et al. [362].

Two-dimensional structure can introduce structure and/or interface inversion asymmetry which induces the spinorbit coupling in conduction band. Spin-orbit coupling in silicon or germanium quantum wells was studied in Refs. [290- 293, 772]. Correspondingly, the D'yakonov-Perel' spin relaxation in two-dimensional structure was investigated in Ref. [290]. Experimentally, Tyryshkin et al. measured spin echoes and deduced a spin coherence time up to $3 \mu$ s for a high mobility two-dimensional electron system formed in Si/SiGe quantum wells [773]. The angular dependence of spin relaxation indicated that the D'yakonov-Perel' mechanism due to the Rashba spin-orbit coupling is the relevant one in high mobility samples [295, 296, 773]. Like that in bulk silicon, spin relaxation in two-dimensional silicon structures was also studied mostly via electron spin resonance [295-297, 622, 774-780]. Recently, electrically detected spin resonance has also been developed and applied to silicon two-dimensional structures [287, 781, 782].

The dependence of spin lifetime on the orientation of external magnetic field was studied in Refs. [296, 622, 776], revealing the relevance of the D'yakonov-Perel' spin relaxation mechanism in high mobility samples. Examination

\footnotetext{
${ }^{70}$ There is few study in bulk germanium in metallic regime.

${ }^{71}$ The analysis has been summerized in Ref. [21].
} 
of the relation between the spin lifetime and the momentum scattering rate indicated that in low mobility samples the spin relaxation is dominated by the Elliott-Yafet mechanism 297] 72 Temperature and density dependences of spin relaxation rate were studied in Refs. [295, 622, 774] and [295] respectively. Spin relaxation can be suppressed by cyclotron motion as demonstrated by Wilamowski et al. [622]. Spin relaxation in quantum Hall regime was studied by Matsunami et al. [782].

Theoretically, Sherman pointed out that even in nominally symmetric silicon quantum wells, the doping inhomogeneity can cause a random spin-orbit coupling [294]. Such effect may play an important role in spin relaxation in symmetric silicon quantum wells [294, 657]. Similarly, the surface roughness can also induces a random spin-orbit coupling due to interface inversion asymmetry [291]. Hole spin-orbit coupling in silicon quantum wells was discussed in Refs. [298, 299]. The effect of carrier-carrier scattering on the D'yakonov-Perel' spin relaxation in $n$-type silicon quantum wells [783] and $p$-type silicon (germanium) quantum wells [299] has also been reported in the recent literature.

\subsection{Magnetization dynamics in magnetic semiconductors}

Magnetic semiconductors, especially the Mn doped III-V magnetic semiconductors, have attracted much attention for decades. The invention of ferromagnetic III-V semiconductors opens the perspective of integrating the magnetic recording and electronic circuit on one chip and has inspired a lot of studies [114, 784-787]. As the ferromagnetic order in III(Mn)-V magnetic semiconductors originates from the carrier mediated Mn spin-spin interactions via the strong $s(p)$ - $d$ exchange interactions [114], the manipulation of carriers would lead to efficient control over the magnetization. The efficient manipulation of magnetization via optical [788-808] and electrical [809-812] means have been realized.

In this subsection we review recent studies on optically induced magnetization dynamics in III(Mn)-V ferromagnetic semiconductors. Historically, the optically induced magnetization dynamics was first discovered in ferromagnetic metal (nickel) [813]. The studies in metal have some relation with those in ferromagnetic semiconductors, which hence should also be mentioned. After more than a decade study, the picture of the underlying physics has been discovered and some usefull models have been raised. However, studies which can quantitatively relate the microscopic carrier dynamics with the observed macroscopic magnetization dynamics are still absent.

We first review optically induced magnetization dynamics in ferromagnetic metals. Let us start from the observed phenomena. In experiments, strong femtosecond laser pulses (usually $\sim 1 \mathrm{~mJ} \mathrm{~cm}^{-2}$ ) are used to pump the ferromagnetic materials. The magnetization is then monitored by probe pulses via magneto-optical Kerr effect. The relative magnetization $M(t) / M(0)$ is assumed to be proportional to the relative Kerr rotation $\theta(t) / \theta(0)$ where $\theta(0)[M(0)]$ is the Kerr rotation (magnetization) before pumping. A typical curve is shown in Fig. 44 The magnetization dynamics is characterized by three stages: (i) an ultrafast demagnetization within $1 \mathrm{ps}$; (ii) a recovery of magnetization via equilibration processes such as electron-phonon scattering from 1 ps to $\sim 20$ ps; (iii) after $\sim 20$ ps a damped precession of the magnetization due to the effective exchange field [814, 815]. The characteristic time scale of these stages are the ultrafast demagnetization time $\tau_{M}$, the energy lifetime $\tau_{E}$ and the Landau-Lifschitz-Gilbert damping time $\tau_{\text {LLG }}$. In Nickel, $\tau_{M} \sim 100 \mathrm{fs}, \tau_{E} \sim 0.5 \mathrm{ps}$, and $\tau_{\mathrm{LLG}} \sim 700 \mathrm{ps}$ [815]. Many works have been attributed to study the magnetization dynamics by various methods to reveal the underlying mechanism and the dependences of the dynamics on the external conditions [813-820]. It was found that the magnetization in stage (ii) is governed by the hot-electron temperature, which agrees well with the static $M(T)$ curve [816]. The damped precession of the magnetization in stage (iii) was found to be due to the deviation of the magnetization direction from the equilibrium one caused by the laser pumping [815]. Among these processes, the ultrafast demagnetization and the magnetization precession have attracted a lot of attention to uncover the underlying microscopic mechanisms.

The ultrafast demagnetization in ferromagnetic metals is the first story which closely grasped the attention of the society. It was first discovered by Beaurepaire et al. in nickel in 1996 [813]. The magnetization decreases by $50 \%$ within $\lesssim 100$ femtoseconds, which is the fastest magnetization dynamics ever found. The large "instantaneous" demagnetization has been puzzling researchers, as previous measurement yielded a spin-lattice lifetime of $\sim 100$ ps [821]. Purely electronic mechanisms such as electron-magnon scattering was also suggested to explain the ultrafast demagnetization. However, although the pure electronic processes can flip individual electron spins rapidly [822], they

\footnotetext{
${ }^{72}$ The relation between spin lifetime and momentum scattering time was also studied in Refs. [295, 622].
} 


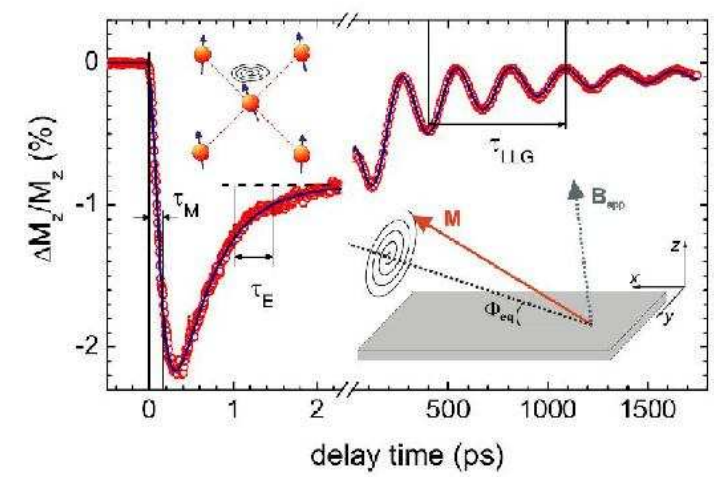

Figure 44: Main: experimental development of the induced perpendicular component of $\mathbf{M}, \Delta \mathrm{M}_{\mathrm{z}} / \mathrm{M}_{\mathrm{z}}$, after laser heating a nickel thin film at $t=0$. Insets: precession of an individual spin in the exchange field (left) and of $\mathbf{M}$ in the effective field (right). $\tau_{\mathrm{M}}, \tau_{\mathrm{E}}$ and $\tau_{\mathrm{LLG}}$ denote the demagnetization time, the energy relaxation time and the Landau-Lifschitz-Gilbert damping time, respectively. The three stages of the magnetization dynamics is clearly seen. From Koopmans et al. [815].

conserve the total spin of the electron system and hence can not lead to demagnetization [814, 817]. Koopmans et al. first suggested that the measured optical signal at the first picosecond does not reflect the magnetization dynamics, but only reflects the optical dynamics [814]. They found that the relative change in the Kerr rotation does not coincide with that in Kerr ellipticity within the first $0.5 \mathrm{ps,} \mathrm{which} \mathrm{distincts} \mathrm{with} \mathrm{the} \mathrm{magnetization} \mathrm{induced} \mathrm{Kerr} \mathrm{effect.} \mathrm{Hence}$ the "instantaneous" decrease of the Kerr rotation or ellipticity can not be directly related to the demagnetization [814]. A close examination indicated that the photo-excited electrons thermalize also within the first picosecond [814]. A more direct and reliable measurement of the spin dynamics via X-ray magnetic circular dichroism observed a demagnetization time of $\sim 120 \mathrm{fs}$ [818]. In the same experiment, the photo-electron thermalization time was identified as $\sim 640 \mathrm{fs}$. At this stage, the time scale of the magnetization dynamics is faithfully characterized. However, the demagnetization mechanism is still unclear. Although several mechanisms and models have been proposed [813, 815, 823 -825], they have not yet been confirmed by experiments unambiguously.

The focus of this subsection is the photo-induced magnetization dynamics in ferromagnetic III(Mn)-V semiconductors. One of the significant difference is that the magnetization is provided by the Mn spin, hence the magnetization and carrier degrees of freedom are separated. Furthermore, unlike the complex energy bands in metals, the energy bands in ferromagnetic III(Mn)-V semiconductors are well described by the Kane model with the $s(p)-d$ exchange interaction [114], which greatly simplifies the theoretical study. Theoretical studies can then help to identify the underlying mechanisms of the photo-induced magnetization dynamics [99, 826-828].

Experimentally, laser-induced demagnetization in (III,Mn)V ferromagnetic semiconductors was first studied by Kojima et al. [807] in GaMnAs. The magnetization dynamics was monitored via the magneto-optical Kerr rotation. They observed a slow demagnetization time of $\sim 500 \mathrm{ps}$, which was attributed to the possible thermal isolation between the charge and spin system in GaMnAs [807]. The sub-picosecond ultrafast demagnetization in ferromagnetic semiconductors was first demonstrated by Wang et al. [788] in InMnAs with much higher intensity $\gtrsim 1 \mathrm{~mJ} / \mathrm{cm}^{-2} \mathrm{per}$ pump pulse than the one in the experiment of Kojima et al. $\left(45 \mu \mathrm{J} / \mathrm{cm}^{-2}\right)$. A typical trace of the time-resolved Kerr rotation is illustrated in Fig. 45. The phenomena are similar to that in ferromagnetic metals: a sub-picosecond $(\sim 200 \mathrm{fs})$ demagnetization and a long time recovery. However, it is noted that the magnetization further decreases after the first $1 \mathrm{ps}$, in contrast with the rapid recovery in ferromagnetic metals [815]. In InMnAs, this slow demagnetization process can last for several hundreds of picosecond at low pump fluence. With increased pump fluence the duration of the slow demagnetization process decreases whereas the decay rate increases. Meanwhile the degree of demagnetization due to the fast process increases. At high pump fluence $\gtrsim 10 \mathrm{~mJ} / \mathrm{cm}^{2}$, the magnetization is completely quenched after the fast process and the slow demagnetization diminishes. The slow demagnetization process is assigned to the Mn spin-lattice relaxation in the existing literature [788]. The arguments are as follows: In III(Mn)-V ferromagnetic semiconductors the large hole spin polarization blocks the carrier-Mn spin-flip scattering, hence the Mn spin system is thermally isolated [807]. On the other hand, the carrier system is efficiently coupled to phonon bath via the carrierphonon interaction at a time scale of $\lesssim 1 \mathrm{ps}$. The thermalized phonon bath then leads to a Mn spin-lattice relaxation 

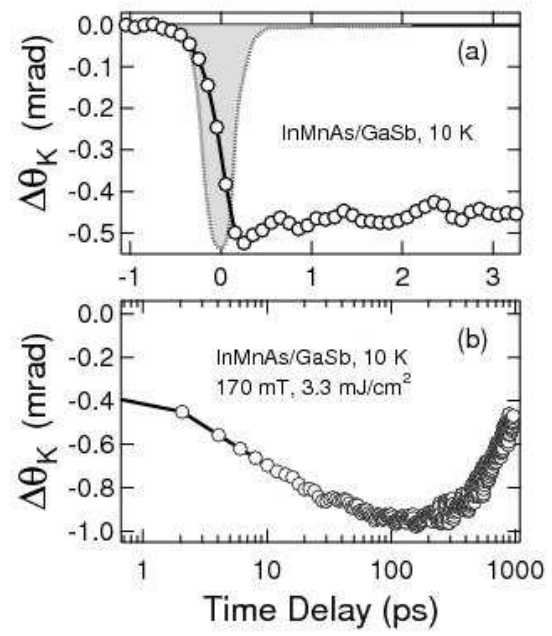

Figure 45: (a) The first 3 ps of demagnetization dynamics in InMnAs. The shadowed region denotes the cross correlation of the pump and probe pulses. (b) Demagnetization dynamics covering the entire time range of the experiment. $\Delta \theta_{\mathrm{K}}$ is the derived difference Kerr rotation which is supposed to be proportional to the induced magnetization change along the growth direction. From Wang et al. [788].

at the time scale of $100 \mathrm{ps}$, which results in the slow demagnetization process [807].

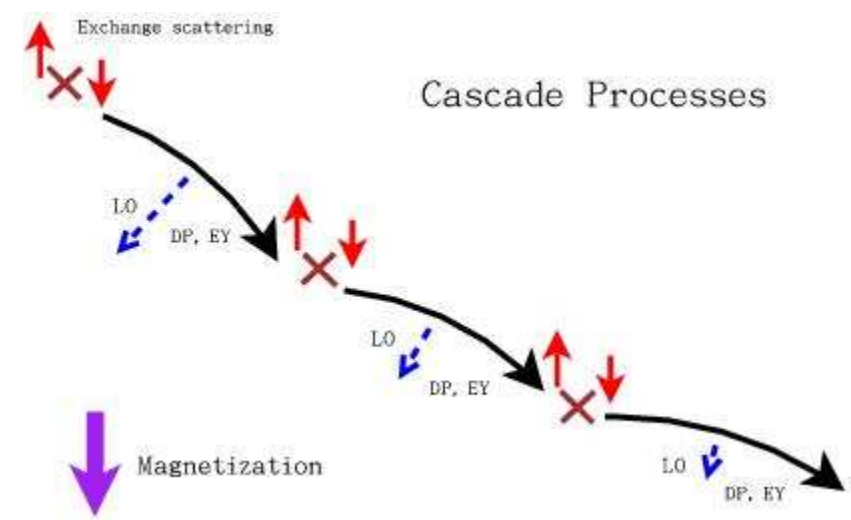

Figure 46: Illustration of the microscopic process of ultrafast demagnetization. $\times$ denotes the $p$ - $d$ exchange scattering. Thin arrow denotes the spin polarization direction of photo-excited holes. Bold arrow represents the initial magnetization direction. Each $p$ - $d$ exchange scattering reduces the magnetization and transfers the spin polarization into the hole system. Holes lose their spin polarization quickly through the D'yakonov-Perel' (DP) and Elliott-Yafet (EY) mechanisms. During these processes, the efficient hole-longitudinal-optical-phonon scattering [denoted as blue arrow (LO) in the figure] relaxes the excess energy of the photo-excited holes. After the energy relaxation, hole spin relaxation slows down (the holelongitudinal-optical-phonon scattering also slows down) and becomes less efficient than the $p$ - $d$ exchange scattering. Hole spin polarization then saturates and the cascade processes terminate.

We now focus on the fast demagnetization process. The underlying physics of the ultrafast demagnetization is illustrated in Fig. 46 The key process is that via the strong $p$ - $d$ coupling the Mn spin polarization is efficiently transferred to the hole spin polarization, which is similar to the inverse Overhauser effect [826]. However, such processes is suppressed if the hole spin relaxation rate is smaller than the rate at which the spin polarization is injected from the Mn spin system via the $p$ - $d$ exchange scattering. Efficient hole spin relaxation is required for a ultrafast demagnetization. It is known that hole spin relaxation is very fast in bulk $p$-GaAs, at a time scale of $100 \mathrm{fs}$, due to the strong spin-orbit coupling [207, 210]. However, in III(Mn)-V ferromagnetic semiconductors, the large spin splitting due to the mean $p$ - $d$ exchange field removes the $\Gamma$ point degeneracy and suppresses the effect of the spin-orbit coupling at low kinetic energy [3, 21]. Fortunately for photo-excited holes, of which the kinetic energy is high [788], the spinorbit splitting is larger than the exchange splitting. The hole spin relaxation can again be very efficient. The fast spin 
relaxation of photo-excited holes facilitates the "inverse Overhauser effect" and leads to the ultrafast demagnetization. Due to the hole-phonon and hole-hole scattering, the photo-excited holes soon lose their energy and thermalize within 1 ps. After the energy relaxation process, the ultrafast demagnetization is terminated. Such scenario has been laid out in recent studies [790, 826$] 73$

In Ref. [826], Cywinski et al. developed a mean field theory to describe the above scenario of ultrafast demagnetization. In their theory, the magnetization was described by the mean $\mathrm{Mn}$ spin polarization which was then characterized by a single Mn spin density matrix. The Mn-Mn correlations beyond the mean field level were argued to be averaged out in such a strong carrier excitation regime. The instantaneous Mn and hole spin splitting was provided by the mean field of the $p$ - $d$ exchange interaction. The hole dynamics was then described by the spin-resolved distribution function $f_{n \mathbf{k}}$ with $n$ denoting the (spin) band index. The magnetization dynamics was obtained, in principle, by solving the coupled kinetic equations of holes and Mn spin. The "inverse Overhauser effect", i.e., the transfer of spin polarization from Mn spin system to hole spin system due to the nonequilibrium hole distribution triggered by the photo-excitation, was then included in the kinetic equation. Within such a model, the Mn spin-flip rate due to the "inverse Overhauser effect" (from $m$ state to $m \pm 1$ state with $-5 / 2 \leq m \leq 5 / 2$ being the Mn spin index) can be written as [826]

$$
W_{m, m \pm 1}=\frac{\beta^{2}}{4} 2 \pi\left|\left\langle m\left|S^{\mp}\right| m+1\right\rangle\right|^{2} \sum_{n n^{\prime}} \int \frac{d \mathbf{k}}{(2 \pi)^{3}} \frac{d \mathbf{k}^{\prime}}{(2 \pi)^{3}}\left|\left\langle n^{\prime} \mathbf{k}^{\prime}\left|s^{ \pm}\right| n \mathbf{k}\right\rangle\right|^{2} f_{n \mathbf{k}}\left(1-f_{n^{\prime} \mathbf{k}^{\prime}}\right) \delta\left(\tilde{\varepsilon}_{n \mathbf{k}}-\tilde{\varepsilon}_{n^{\prime} \mathbf{k}^{\prime}} \pm \Delta_{M}\right),
$$

where $\beta$ is the $p$ - $d$ exchange constant and $S^{ \pm}\left(s^{ \pm}\right)$are the Mn (hole) spin ladder operators. $\Delta_{M}$ represents the Mn instantaneous mean field spin splitting produced by the holes. $\tilde{\varepsilon}_{n \mathbf{k}}$ is the instantaneous band energy with the mean field exchange interaction included. By considering the hole energy- and spin-relaxation in a phenomenological way, the magnetization dynamics was solved within a simplified two band hole approximation [826]. The results reproduced the main features of the ultrafast demagnetization: (i) the process is terminated at the time scale of hole energy equilibration time $\tau_{E}$ [826]; (ii) the demagnetization rate and the degree of the magnetization change increase with the pump fluence and hole spin relaxation rate [826]. The obtained demagnetization time is on the order of $1 \mathrm{ps}$ [826]. The feature of the magnetization dynamics also qualitatively agrees with the observation in experiments [790, 826]. Further calculations based on the realistic band structure and microscopic interactions, such as what has been done in paramagnetic phase in Ref. [111], is needed to elucidate the underlying physics unambiguously and to compare with the experimental results.

At low pump fluence, such as $\sim 10 \mu \mathrm{J} \mathrm{cm}^{-2}$ (compared to the fluence $\gtrsim 1 \mathrm{~mJ} \mathrm{~cm}^{-2}$ used in demagnetization measurements), rich magnetization dynamics is triggered by photo illumination. Damped precession of the magnetization was observed, where the driving force was attributed to the photo-induced change in the magnetic anisotropy energy [751, 797, 800-802, 804, 805, 829, 830]. It was also discovered that the magnetization precession can be coherently manipulated by photo pulses [798, 799]. Besides, with circularly polarized light, the magnetization is rotated due to the $p$ - $d$ exchange mean field produced by the photo-injected hole spin polarization [751, 797, 803]. The photoinduced change in the magnetic anisotropy energy has been argued to originate from: (i) the change of the temperature of the hole gas due to hot photo-excited holes [800]; (ii) the change of the hole density [829]; (iii) the nonthermal effect [805]. The dependence of the precession frequency (closely related to the magnetic anisotropy energy) and damping constant (the Gilbert constant) were hence studied for various photo intensity, temperature and hole density, and compared with theoretical results $[827,828,831]$. It should be mentioned that there is other mechanism which can also trigger such magnetization precession, such as the photo-induced spin precession due to the interband polarization proposed by Chovan and Perakis [99]. Very recently Kapetanakis et al. [832] developed a nonequilibrium theory based on the original idea of Ref. [99] and well explained the experimental results of magnetization precession [791]. In fact, within the first picosecond, the hot photo-excited holes can also modify the magnetization direction besides changing the magnetization amplitude (demagnetization) due to the fact that in the presence of hole spin-orbit coupling the total (hole $+\mathrm{Mn}$ ) spin polarization is not conserved. Once the magnetization direction deviates from the equilibrium one, a damped precession is motivated by the effective magnetic field due to the external and internal (anisotropy) magnetic fields [814].

\footnotetext{
${ }^{73}$ Note that a similar picture of $\mathrm{Mn}$ ion spin relaxation in $\mathrm{ZnMnSe} / \mathrm{ZnBeSe}$ paramagnetic quantum well was also depicted in Ref. [730].
} 


\section{Spin relaxation and dephasing based on kinetic spin Bloch equation approach}

In this section, we introduce a fully microscopic many-body approach named kinetic spin Bloch equation approach developed by $\mathrm{Wu}$ et al. [44, 332, 349, 350]. Unlike the approaches reviewed in the previous sections which treat scatterings using the relaxation time approximation, the kinetic spin Bloch equation approach treats all scatterings explicitly and self-consistently to all orders. Especially, the carrier-carrier Coulomb scattering is explicitly included in the theory. This allows them to study spin dynamics not only near but also far away from the equilibrium, for example, the spin dynamics in the presence of high electric field (hot-electron condition) [569] and with large initial spin polarizations [41, 42, 44]. This section only focuses on the results in spacial uniform systems while those in the spacial non-uniform systems are given in Section 7. We organize this section as follows: In Section 5.1 we first introduce the kinetic spin Bloch equation approach. Then we address the effect of electron-electron Coulomb scattering on the spin dynamics in Section 5.2. The general kinetic spin Bloch equations in $n$-or $p$-doped confined structures are given in Section 5.3 where we even include the case of spacial gradient 74 The results of spin relaxation and dephasing in quantum wells, quantum wires, and bulk samples are reviewed in Sections 5.4, 5.5 and 5.6, respectively.

\subsection{Kinetic spin Bloch equation approach}

\subsubsection{Model and Hamiltonian}

We first use a four-spin-band model to demonstrate the establishment of the kinetic spin Bloch equations and apply these equations to investigate the spin precession and relaxation/dephasing. By considering a (001) zinc blende quantum well with its growth axis in the $z$-direction and a moderate magnetic field along the $x$-direction (Voigt configuration), we write the Hamiltonian of electrons in the conduction and valence bands as

$$
H=\sum_{\mu \mathbf{k} \sigma} \varepsilon_{\mu \mathbf{k}} c_{\mu \mathbf{k} \sigma}^{\dagger} c_{\mu \mathbf{k} \sigma}+\sum_{\mu \mathbf{k} \sigma \sigma^{\prime}} g \mu_{B}\left[\mathbf{B}+\mathbf{\Omega}_{\mu}(\mathbf{k})\right] \cdot \mathbf{S}_{\mu \sigma \sigma^{\prime}} c_{\mu \mathbf{k} \sigma}^{\dagger} c_{\mu \mathbf{k} \sigma^{\prime}}+H_{E}+H_{I},
$$

with $\mu=c$ and $v$ standing for the conduction and valence bands, respectively. Here we only include the lowest subbands of the conduction and valence bands. This is valid for quantum wells with sufficiently small well widths. $\varepsilon_{v \mathbf{k}}=-E_{g} / 2-k^{2} / 2 m_{h}=-E_{g} / 2-\varepsilon_{h k}$ and $\varepsilon_{c \mathbf{k}}=E_{g} / 2+k^{2} / 2 m_{e}=E_{g} / 2+\varepsilon_{e k}$ with $m_{h}$ and $m_{e}$ denoting effective masses of the hole and electron, respectively. $E_{g}$ is the band gap. For quantum well without strain, the heavy hole band is above the light hole one and hence the valence band to be considered is the heavy-hole band with $\mathbf{S}_{v \sigma \sigma^{\prime}}$ representing spin $3 / 2$ matrices and $\sigma= \pm 3 / 2$. $\mathbf{S}_{c \sigma \sigma^{\prime}}$ stands for the spin $1 / 2$ matrices for conduction electrons. $\boldsymbol{\Omega}_{\mu}(\mathbf{k})$ represents the effective magnetic field from the D'yakonov-Perel' term of the $\mu$-band.

$H_{E}$ in Eq. (131) denotes the dipole coupling with the light field $E_{-\sigma}(t)$ with $\sigma= \pm$ representing the circularly polarized light. Due to the selection rule

$$
H_{E}=-d \sum_{\mathbf{k}}\left[E_{-}(t) c_{c \mathbf{k} \frac{1}{2}}^{\dagger} c_{v \mathbf{k} \frac{3}{2}}+\text { H.c. }\right]-d \sum_{\mathbf{k}}\left[E_{+}(t) c_{c \mathbf{k}-\frac{1}{2}}^{\dagger} c_{v \mathbf{k}-\frac{3}{2}}+\text { H.c. }\right]
$$

in which $d$ denotes the optical-dipole matrix element and $E_{\sigma}(t)=E_{\sigma}^{(0)}(t) \cos \omega t$ with $\omega$ being the central frequency of the light pulse. $E_{\sigma}^{(0)}(t)$ denotes a Gaussian pulse with pulse width $\delta t . H_{I}$ stands for the interaction Hamiltonian. It contains both the spin-conserving scattering, such as the electron-electron Coulomb, electron-phonon and electronimpurity scatterings, and the spin-flip scattering, such as the scattering due to the Bir-Aronov-Pikus and Elliott-Yafet mechanisms.

It can be seen from $H_{E}$ that the laser pulse introduces the optical coherence between the conduction and valence bands $P_{\mathbf{k} \frac{1}{2} \frac{3}{2}} \equiv e^{i \omega t}\left\langle c_{v \mathbf{k} \frac{3}{2}}^{\dagger} c_{\mathbf{c} \frac{1}{2}}\right\rangle$ and $P_{\mathbf{k}-\frac{1}{2}-\frac{3}{2}} \equiv e^{i \omega t}\left\langle c_{v \mathbf{k}-\frac{3}{2}}^{\dagger} c_{c \mathbf{k}-\frac{1}{2}}\right\rangle$. In the meantime, due to the presence of the magnetic field in the Voigt configuration, these optical coherences may further transfer coherence to $P_{\mathbf{k}-\frac{1}{2} \frac{3}{2}} \equiv e^{i \omega t}\left\langle c_{v \mathbf{k} \frac{3}{2}}^{\dagger} c_{c \mathbf{k}-\frac{1}{2}}\right\rangle$ and $P_{\mathbf{k} \frac{1}{2}-\frac{3}{2}} \equiv e^{i \omega t}\left\langle c_{v \mathbf{k}-\frac{3}{2}}^{\dagger} c_{\mathbf{c k} \frac{1}{2}}\right\rangle$, of which the direct optical transition is forbidden. Due to the presence of the magnetic field and/or effective magnetic field, if there is any spin polarization, i.e., $f_{\mu \mathbf{k} \sigma} \equiv\left\langle c_{\mu \mathbf{k} \sigma}^{\dagger} c_{\mu \mathbf{k} \sigma}\right\rangle \neq f_{\mu \mathbf{k}-\sigma}$, electrons can flip from

\footnotetext{
${ }^{74}$ For the case of spin diffusion and transport, see Section 7.
} 
$\sigma$-band to $-\sigma$-band, and hence induce the correlation between two spin bands, i.e., $\rho_{\mu \mathbf{k} \sigma-\sigma} \equiv\left\langle c_{\mu \mathbf{k}-\sigma}^{\dagger} c_{\mu \mathbf{k} \sigma}\right\rangle \equiv \rho_{\mu \mathbf{k}-\sigma \sigma}^{*}$. Similar to the optical coherence which is represented by $P_{\mathbf{k} \sigma \sigma^{\prime}}$, the spin coherence can be well represented by $\rho_{\mu \mathbf{k} \sigma-\sigma}$. In the following we call it spin coherence. These coherences as well as electron/hole distributions illustrated in Fig. 47 are the quantities to be determined self-consistently through the kinetic spin Bloch equations.

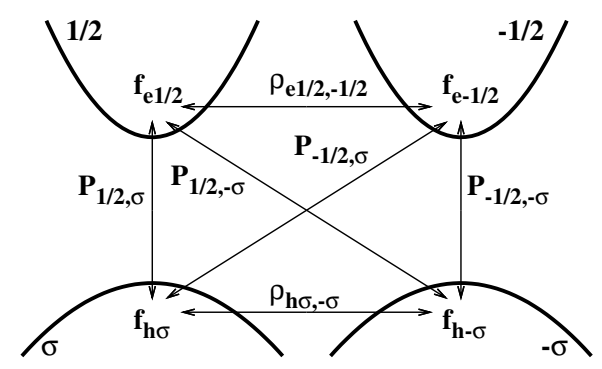

Figure 47: Illustration of the four-spin-band model: two conduction bands with spin $\pm 1 / 2$ and two valence bands with spin $\pm \sigma$. The spin coherence $\rho$, optical and forbidden optical coherences $P$ as well as carrier distributions of the four bands are also illustrated.

\subsubsection{Kinetic spin Bloch equations} [350]

We construct the kinetic spin Bloch equations using the nonequilibrium Green function method [833] as follows

$$
\dot{\rho}_{\mu v, \mathbf{k}, \sigma \sigma^{\prime}}=\left.\dot{\rho}_{\mu v, \mathbf{k}, \sigma \sigma^{\prime}}\right|_{\mathrm{coh}}+\left.\dot{\rho}_{\mu v, \mathbf{k}, \sigma \sigma^{\prime}}\right|_{\text {scat }} .
$$

Here $\rho_{\mu v, \mathbf{k}, \sigma \sigma^{\prime}}$ represents the single-particle density matrix with $\mu$ and $v=c$ or $v$. ( $\mu$ and $v$ can be subband indices and/or valley indices also.) The diagonal elements describe the carrier distribution functions $\rho_{\mu \mu, \mathbf{k}, \sigma \sigma}=f_{\mu \mathbf{k} \sigma}$ and the off-diagonal elements either represent the optical coherence $\rho_{c v, \mathbf{k}, \sigma \sigma^{\prime}}=P_{\mathbf{k} \sigma \sigma^{\prime}} e^{-i \omega t}$ for the inter conduction band and valence band polarization, or the spin coherence $\rho_{\mu \mu, \mathbf{k}, \sigma-\sigma}$ for the inter spin-band correlation. The coherent terms $\left.\dot{\rho}_{\mu \nu, \mathbf{k}, \sigma \sigma^{\prime}}\right|_{\text {coh }}$ are composed of the contributions from the energy spectrum, electric pumping field, magnetic and/or effective magnetic field as well as the Coulomb Hartree-Fock term. The scattering terms $\left.\dot{\rho}_{\mu \nu, \mathbf{k}, \sigma \sigma^{\prime}}\right|_{\text {scat }}$ consist of the spin-conserving scatterings such as the electron-electron Coulomb, electron-phonon and electron-non-magneticimpurity scatterings and/or the spin-flip scatterings such as the scatterings due to the Bir-Aronov-Pikus and/or ElliottYafet mechanisms and the hyperfine interaction. The detailed expressions of these terms will be given in the following subsections for different specific problems.

By solving the kinetic spin Bloch equations (133) with the initial conditions:

$$
\rho_{\mu \nu, \mathbf{k}, \sigma \sigma^{\prime}}(0)=\left\{\begin{array}{ll}
f_{\mu \mathbf{k} \sigma}(0) & \text { with } \mu=v \text { and } \sigma=\sigma^{\prime} \\
0 & \text { with } \mu \neq v \text { and } / \text { or } \sigma \neq \sigma^{\prime}
\end{array},\right.
$$

one obtains the time evolution of the density matrix.

\subsubsection{Faraday rotation angle, spin dephasing and spin relaxation}

The Faraday rotation angle can be calculated for two degenerate Gaussian pulses with a delay time $\tau$. The first pulse (pump) is circularly polarized, e.g., $E_{\text {pump }}^{0}(t)=E_{ \pm}^{0}(t)$, and travels in the $\mathbf{k}_{1}$-direction. The second pulse (probe) is linear polarized and is much weaker than the first one, e.g., $E_{\text {probe }}^{0}(t) \equiv \chi\left[E_{-}^{0}(t-\tau)+E_{+}^{0}(t-\tau)\right]$ with $\chi \ll 1$. The probe pulse travels in the $\mathbf{k}_{2}$ direction. The Faraday rotation angle is defined as [732, 834]

$$
\Theta_{\mathrm{F}}(\tau)=C \sum_{\mathbf{k}} \int \operatorname{Re}\left[\bar{P}_{\mathbf{k} \frac{1}{2} \frac{3}{2}}(t) E_{-}^{0^{*}}(t-\tau)-\bar{P}_{\mathbf{k}-\frac{1}{2}-\frac{3}{2}}(t) E_{+}^{0^{*}}(t-\tau)\right] d t,
$$

with $\bar{P}_{\mathbf{k} \sigma \sigma^{\prime}}$ standing for the optical transition in the probe direction. $C$ is a constant. The spin relaxation/dephasing can be determined from the slope of the envelope of the Faraday rotation angle $\Theta_{\mathrm{F}}(\tau)$. However, calculation of this 
quantity is quite time consuming. In reality, we use the slope of the envelope of

$$
\Delta N_{\mu}=\sum_{\mathbf{k}}\left(f_{\mu \mathbf{k}|\sigma|}-f_{\mu \mathbf{k}-|\sigma|}\right), \quad \rho_{\mu}=\sum_{\mathbf{k}}\left|\rho_{\mu \mu, \mathbf{k},|\sigma|-|\sigma|}\right|, \quad \rho_{\mu}^{*}=\left|\sum_{\mathbf{k}} \rho_{\mu \mu, \mathbf{k},|\sigma|-|\sigma|}\right|,
$$

to substract the spin relaxation time $T_{1}$, spin dephasing time $T_{2}$ and ensemble spin dephasing time $T_{2}^{*}$, respectively [350, 835]. Similarly, the optical dephasing is described by the incoherently summed polarization [346, 833],

$$
P_{\sigma \sigma}(t)=\sum_{\mathbf{k}}\left|P_{\mathbf{k}, \sigma \sigma}(t)\right| .
$$

The later was first introduced by Kuhn and Rossi [347]. It is understood that both true dissipation and the interference among the $\mathbf{k}$ states may contribute to the decay. The incoherent summation is therefore used to isolate the irreversible decay from the decay caused by interference.

\subsection{Electron-electron Coulomb scattering to spin dynamics}

It has long been believed that the spin-conserving electron-electron Coulomb scattering does not contribute to the spin relaxation/dephasing [836]. It was first pointed out by Wu and Ning [334] that in the presence of inhomogeneous broadening in spin precession, i.e., the spin precession frequencies are $\mathbf{k}$-dependent, any scattering, including the spin-conserving scattering, can cause irreversible spin dephasing. This inhomogeneous broadening can come from the energy-dependent $g$-factor [225, 226, 334, 837-840], the D'yakonov-Perel' term [332], the random spin-orbit coupling [294], and even the momentum dependence of the spin diffusion rate along the spacial gradient [336]. This can be illustrated from the kinetic spin Bloch equations of a four-spin band model in a quantum well with the lowest valence band being the heavy-hole band and $\rho_{c v, \mathbf{k}, \sigma \sigma^{\prime}}=P_{\mathbf{k} \sigma \sigma^{\prime}} e^{-i \omega t}$. When the D'yakonov-Perel' term $\boldsymbol{\Omega}(\mathbf{k})=0$, the coherent parts of the kinetic spin Bloch equations are given by [334]

$$
\begin{aligned}
\left.\frac{\partial f_{e \mathbf{k} \sigma}}{\partial t}\right|_{\text {coh }}= & 2 \sum_{\mathbf{q}} V_{\mathbf{q}} \operatorname{Im}\left(\sum_{\sigma^{\prime}} P_{\mathbf{k}+\mathbf{q} \sigma \sigma^{\prime}}^{*} P_{\mathbf{k} \sigma \sigma^{\prime}}+\rho_{c c, \mathbf{k}+\mathbf{q},-\sigma \sigma} \rho_{c c, \mathbf{k}, \sigma-\sigma}\right)-g \mu_{B} B \operatorname{Im} \rho_{c c, \mathbf{k}, \sigma-\sigma}, \\
\left.\frac{\partial \rho_{c c, \mathbf{k}, \sigma-\sigma}}{\partial t}\right|_{\mathrm{coh}}= & i \sum_{\mathbf{q}} V_{\mathbf{q}}\left[\left(f_{e \mathbf{k}+\mathbf{q} \sigma}-f_{e \mathbf{k}+\mathbf{q}-\sigma}\right) \rho_{c c, \mathbf{k}, \sigma-\sigma}-\left(f_{e \mathbf{k} \sigma}-f_{e \mathbf{k}-\sigma}\right) \rho_{c c, \mathbf{k}+\mathbf{q}, \sigma-\sigma}\right. \\
& \left.+P_{\mathbf{k}+\mathbf{q} \sigma \sigma_{1}} P_{\mathbf{k}-\sigma \sigma_{1}}^{*}-P_{\mathbf{k}+\mathbf{q}-\sigma \sigma_{1}}^{*} P_{\mathbf{k} \sigma \sigma_{1}}\right]+\frac{i}{2} g \mu_{B} B\left(f_{e \mathbf{k} \sigma}-f_{e \mathbf{k}-\sigma}\right), \\
\left.\frac{\partial P_{\mathbf{k} \sigma \sigma^{\prime}}}{\partial t}\right|_{\mathrm{coh}}= & -i \delta_{\sigma \sigma^{\prime}}(\mathbf{k}) P_{\mathbf{k} \sigma \sigma^{\prime}}-\frac{i}{2} g \mu_{B} B P_{\mathbf{k}-\sigma \sigma^{\prime}}-i \sum_{\mathbf{q}} V_{\mathbf{q}}\left[P _ { \mathbf { k } + \mathbf { q } \sigma \sigma ^ { \prime } } \left(1-f_{h \mathbf{k} \sigma^{\prime}}\right.\right. \\
& \left.\left.-f_{e \mathbf{k} \sigma}\right)-P_{\mathbf{k}+\mathbf{q}-\sigma \sigma^{\prime}} \rho_{c c, \mathbf{k}, \sigma-\sigma}+P_{\mathbf{k}-\sigma \sigma^{\prime}} \rho_{c c, \mathbf{k}+\mathbf{q}, \sigma-\sigma}\right],
\end{aligned}
$$

for electron distribution function $f_{\text {ek } \sigma} \equiv f_{c \mathbf{k} \sigma}$, spin coherence and optical coherence, respectively. $f_{h \mathbf{k} \sigma} \equiv 1-f_{v \mathbf{k} \sigma}$ is the hole distribution function. The first term on the right hand side of Eq. (138) is the Fock term from the Coulomb scattering with $V_{\mathbf{q}}=\frac{2 \pi e^{2}}{q}$ denoting the Coulomb matrix element. The first term of Eq. (140) gives the free evolution of the polarization components with the detuning

$$
\delta_{\sigma \sigma^{\prime}}(\mathbf{k})=\varepsilon_{e h \mathbf{k}}-\Delta_{0}-\sum_{\mathbf{q}} V_{\mathbf{q}}\left(f_{e \mathbf{k}+\mathbf{q} \sigma}+f_{h \mathbf{k}+\mathbf{q} \sigma^{\prime}}\right),
$$

in which $\varepsilon_{e h \mathbf{k}}=\varepsilon_{e \mathbf{k}}+\varepsilon_{h \mathbf{k}}$ and $\Delta_{0}=\omega-E_{g} . \Delta_{0}$ is the detuning of the center frequency of the light pulses with respect to the unrenormalized band gap. The last term in Eq. (140) describes the excitonic correlations whereas the first term in Eq. (139) describes the Hartree-Fock contributions to the spin coherence.

For spin-conserving scattering,

$$
\left.\sum_{\mathbf{k}} \frac{\partial \rho_{\mu \nu}, \mathbf{k}, \sigma \sigma^{\prime}}{\partial t}\right|_{\text {scat }}=0
$$


By performing the summation over $\mathbf{k}$ from both sides of the kinetic spin Bloch equations and further noticing that the Hartree-Fock contributions from the Coulomb interaction in the coherent parts of the kinetic spin Bloch equations satisfy

$$
\left.\sum_{\mathbf{k}} \frac{\partial \rho_{\mu \nu}, \mathbf{k}, \sigma \sigma^{\prime}}{\partial t}\right|_{\text {coh }} ^{\mathrm{HF}}=0
$$

one has

$$
\begin{aligned}
\frac{\partial^{2}}{\partial t^{2}} \operatorname{Im} \sum_{\mathbf{k}} \rho_{c c, \mathbf{k}, \sigma-\sigma} & =-g^{2} \mu_{B}^{2} B^{2} \operatorname{Im} \sum_{\mathbf{k}} \rho_{c c, \mathbf{k}, \sigma-\sigma}, \\
\frac{\partial}{\partial t} \operatorname{Re} \sum_{\mathbf{k}} \rho_{c c, \mathbf{k}, \sigma-\sigma} & =0 .
\end{aligned}
$$

This shows that there is no spin dephasing in the absence of inhomogeneous broadening and spin-flip scattering. For optical dephasing, $\delta_{\sigma \sigma^{\prime}}(\mathbf{k})$ in Eq. (140) is k-dependent, i.e., here exists the inhomogeneous broadening. Therefore any optical-dipole conserving scattering can lead to irreversible optical dephasing [833]. Similarly, if there is inhomogeneous broadening in the spin precession, any spin-conserving scattering can lead to irreversible spin dephasing. As electron-electron Coulomb scattering is a spin-conserving scattering, of course it contributes to the spin dephasing in the presence of the inhomogeneous broadening [334].

Wu and Ning first showed that with the energy-dependent $g$-factor as an inhomogeneous broadening, the Coulomb scattering can lead to irreversible spin dephasing [334]. In [001]-grown $n$-doped quantum wells, the importance of the Coulomb scattering for spin relaxation/dephasing was proved by Glazov and Ivchenko [615] by using perturbation theory and by Weng and $\mathrm{Wu}$ [44] from the fully microscopic kinetic spin Bloch equation approach. In a temperaturedependent study of the spin dephasing in [001]-oriented $n$-doped quantum wells, Leyland et al. experimentally verified the effects of the electron-electron Coulomb scattering [368, 591]. Later Zhou et al. even predicted a peak from the Coulomb scattering in the temperature dependence of the spin relaxation time in a high-mobility low-density $n$-doped [001] quantum well [372]. This was later demonstrated by Ruan et al. experimentally [604].

\subsection{Kinetic spin Bloch equations in $n$ - or p-doped confined structures}

In this section we present the spin dynamics in $n$ - or $p$-type confined semiconductor structures. In this case, we only need to consider a simplified single-particle density matrix $\rho_{\mathbf{k}}$ which consists only the electron/hole distribution functions and the inter-spin-band polarization (spin coherence). In the effective-mass approximation, the Hamiltonian of electrons in the confined system reads

$$
\begin{aligned}
H & =\sum_{i}\left[H_{e}\left(\mathbf{R}_{i}\right)+e \mathbf{E} \cdot \mathbf{r}_{i}\right]+H_{I}, \\
H_{e}(\mathbf{R}) & =\frac{\mathbf{P}^{2}}{2 m^{*}}+H_{\mathrm{so}}(\mathbf{P})+V\left(\mathbf{r}_{c}\right)+\frac{1}{2} g^{*} \mu_{B} \mathbf{B} \cdot \sigma,
\end{aligned}
$$

in which $\mathbf{r}_{c}$ represents the coordinate along the confinement and $\mathbf{R}=\left(\mathbf{r}, \mathbf{r}_{c}\right) . \mathbf{P}=-i \hbar \nabla-e \mathbf{A}(\mathbf{R})$ is the momentum with $\mathbf{B}=\nabla \times \mathbf{A} . H_{\text {so }}$ in Eq. (147) is the Hamiltonian of the spin-orbit coupling which consists the Dresselhaus/Rashba term as well as the strain-induced spin-orbit coupling. $V\left(\mathbf{r}_{c}\right)$ represents the confinement potential. The energy spectrum of single-particle Hamiltonian (147) reads

$$
H_{e}|\mathbf{k}, n\rangle=\varepsilon_{n}(\mathbf{k})|\mathbf{k}, n\rangle
$$

with $n$ denoting the index for both subband [subjected to the confinement $V\left(\mathbf{r}_{c}\right)$ ] and spin. Hence $|\mathbf{k}, n\rangle=|\mathbf{k}\rangle|n\rangle_{\mathbf{k}}$.

Due to the spin-orbit coupling, ${ }_{\mathbf{k}}\left\langle n \mid n^{\prime}\right\rangle_{\mathbf{k}^{\prime}}$ usually is not diagonal if $\mathbf{k}^{\prime} \neq \mathbf{k}$. The Hilbert space spanned by $|\mathbf{k}, n\rangle$ is helix space [597, 841]. The kinetic spin Bloch equations in this space are very complicated. Usually we use the wavefunctions $|n\rangle_{\mathbf{k}}$ at a fixed $\mathbf{k}_{0}$ as a complete basis, i.e., $\left\{|n\rangle_{0}\right\}$. Therefore, the Hilbert space becomes $\left\{|\mathbf{k}\rangle|n\rangle_{0}\right\}$ which is complete and orthogonal. We call this space collinear space. The eigenfunction $|\mathbf{k}, n\rangle$ can be expanded in this space as

$$
|\mathbf{k}, n\rangle=\sum_{m}\langle m|\langle\mathbf{k} \mid \mathbf{k}, n\rangle| \mathbf{k}\rangle|m\rangle_{0}
$$


The matrix element of the Hamiltonian $H_{e}$ in the collinear space reads

$$
E_{\mathbf{k}}^{n n^{\prime}}={ }_{0}\left\langle n\left|\left\langle\mathbf{k}\left|H_{e}\right| \mathbf{k}\right\rangle\right| n^{\prime}\right\rangle_{0}
$$

As $|\mathbf{k}\rangle|n\rangle_{0}$ in general is not the eigenfunction of $H_{e}, E_{\mathbf{k}}^{n n^{\prime}}$ is not diagonal. $e \mathbf{E} \cdot \mathbf{r}$ in Eq. (146) is the driving term of the electric field along the confinement-free directions. $H_{I}$ is the interaction Hamiltonian.

In the collinear space, the density operator can be written as

$$
\rho(\mathbf{Q})=\sum_{\mathbf{k}, n_{1} n_{2}} I_{n_{1} n_{2}}\left(\mathbf{q}_{c}\right) c_{\mathbf{k}+\mathbf{q} n_{1}}^{\dagger} c_{\mathbf{k} n_{2}}
$$

in which $I_{n_{1} n_{2}}\left(\mathbf{q}_{c}\right)={ }_{0}\left\langle n_{1}\left|e^{i \mathbf{q}_{c} \cdot \mathbf{r}_{c}}\right| n_{2}\right\rangle_{0}$ is the form factor. The interaction Hamiltonian $H_{I}$ reads

$$
\begin{aligned}
& H_{\mathrm{ee}}=\sum_{\mathbf{Q} \mathbf{k}^{\prime}, n_{1} n_{2} n_{3} n_{4}} V(\mathbf{Q}) I_{n_{1} n_{2}}\left(\mathbf{q}_{c}\right) I_{n_{3} n_{4}}\left(\mathbf{q}_{c}\right) c_{\mathbf{k}^{\prime}+\mathbf{q} n_{3}}^{\dagger} c_{\mathbf{k}-\mathbf{q} n_{1}}^{\dagger} c_{\mathbf{k} n_{2}} c_{\mathbf{k}^{\prime} n_{4}}, \\
& H_{\mathrm{ep}}=\sum_{\mathbf{Q} \mathbf{k}, n_{1} n_{2}, \lambda} M_{\lambda}(\mathbf{Q}) I_{n_{1} n_{2}}\left(\mathbf{q}_{c}\right) c_{\mathbf{k}+\mathbf{q} n_{1}}^{\dagger} c_{\mathbf{k}, n_{2}}\left(a_{\mathbf{Q} \lambda}+a_{-\mathbf{Q} \lambda}^{\dagger}\right), \\
& H_{\mathrm{ei}}=\sum_{\mathbf{Q} \mathbf{k}, n_{1} n_{2}} V_{i}(\mathbf{Q}) I_{n_{1} n_{2}}\left(\mathbf{q}_{c}\right) \rho_{i}(\mathbf{Q}) c_{\mathbf{k}+\mathbf{q} n_{1}}^{\dagger} c_{\mathbf{k} n_{2}},
\end{aligned}
$$

for electron-electron, electron-phonon and electron-impurity interactions, respectively.

By solving the non-equilibrium Green function with generalized Kadanoff-Baym Ansatz and the gradient expansion [833], the kinetic spin Bloch equations read

$$
\frac{\partial \rho_{\mathbf{k}}(\mathbf{r}, t)}{\partial t}=\left.\frac{\partial}{\partial t} \rho_{\mathbf{k}}(\mathbf{r}, t)\right|_{\mathrm{dr}}+\left.\frac{\partial}{\partial t} \rho_{\mathbf{k}}(\mathbf{r}, t)\right|_{\mathrm{dif}}+\left.\frac{\partial}{\partial t} \rho_{\mathbf{k}}(\mathbf{r}, t)\right|_{\mathrm{coh}}+\left.\frac{\partial}{\partial t} \rho_{\mathbf{k}}(\mathbf{r}, t)\right|_{\mathrm{scat}} .
$$

The first term on the right hand side of the above equation is

$$
\left.\frac{\partial}{\partial t} \rho_{\mathbf{k}}(\mathbf{r}, t)\right|_{\mathrm{dr}}=\frac{1}{2}\left\{\nabla_{\mathbf{r}} \bar{\varepsilon}_{\mathbf{k}}(\mathbf{r}, t), \nabla_{\mathbf{k}} \rho_{\mathbf{k}}(\mathbf{r}, t)\right\}
$$

with $\{A, B\}=A B+B A$ representing the anti-commutator. $\left(\bar{\varepsilon}_{\mathbf{k}}(\mathbf{r}, t)\right)_{n_{1} n_{2}}=E_{\mathbf{k}}^{n_{1} n_{2}}+e \mathbf{E} \cdot \mathbf{r} \delta_{n_{1} n_{2}}+\Sigma_{\mathrm{HF}}^{n_{1} n_{2}}(\mathbf{k}, \mathbf{r}, t)$, in which $\sum_{\mathrm{HF}}^{n_{1} n_{2}}(\mathbf{k}, \mathbf{r}, t)=-\sum_{\mathbf{Q}} I\left(\mathbf{q}_{c}\right) \rho_{\mathbf{k}-\mathbf{q}}(\mathbf{r}, t) V(\mathbf{Q}) I\left(-\mathbf{q}_{c}\right)$ is the Hartree-Fock term. The second term describes the diffusion

$$
\left.\frac{\partial}{\partial t} \rho_{\mathbf{k}}(\mathbf{r}, t)\right|_{\text {dif }}=-\frac{1}{2}\left\{\nabla_{\mathbf{k}} \bar{\varepsilon}_{\mathbf{k}}(\mathbf{r}, t), \nabla_{\mathbf{r}} \rho_{\mathbf{k}}(\mathbf{r}, t)\right\}
$$

The coherent term is given by

$$
\left.\frac{\partial}{\partial t} \rho_{\mathbf{k}}(\mathbf{r}, t)\right|_{\mathrm{coh}}=-i\left[\bar{\varepsilon}_{\mathbf{k}}(\mathbf{r}, t), \rho_{\mathbf{k}}(\mathbf{r}, t)\right]
$$

where $[A, B]=A B-B A$ is the commutator. The scattering term in Eq. 155) reads

$$
\left.\frac{\partial}{\partial t} \rho_{\mathbf{k}}(\mathbf{r}, t)\right|_{\text {scat }}=-\left\{S_{\mathbf{k}}(>,<)-S_{\mathbf{k}}(<,>)+S_{\mathbf{k}}(>,<)^{\dagger}-S_{\mathbf{k}}(<,>)^{\dagger}\right\},
$$

with

$$
\begin{aligned}
S_{\mathbf{k}}(>,<)= & \sum_{\mathbf{Q}} N_{i} \int_{-\infty}^{t} d \tau V_{i}(\mathbf{Q}) I\left(\mathbf{q}_{c}\right) e^{-i E_{\mathbf{k}-\mathbf{q}}(t-\tau)} \rho_{\mathbf{k}-\mathbf{q}}^{>}(\tau) V_{i}(-\mathbf{Q}) I\left(-\mathbf{q}_{c}\right) \rho_{\mathbf{k}}^{<}(\tau) e^{i E_{\mathbf{k}}(t-\tau)} \\
& +\sum_{\mathbf{Q}} \int_{-\infty}^{t} d \tau M_{\lambda}(\mathbf{Q}) I\left(\mathbf{q}_{c}\right) e^{-i E_{\mathbf{k}-\mathbf{q}}(t-\tau)} \rho_{\mathbf{k}-\mathbf{q}}^{>}(\tau) M_{\lambda}(-\mathbf{Q}) I\left(-\mathbf{q}_{c}\right) \rho_{\mathbf{k}}^{<}(\tau) e^{i E_{\mathbf{k}}(t-\tau)}\left[N^{<}(\mathbf{Q}) e^{i \omega_{\mathbf{Q}}(t-\tau)}+N^{>}(\mathbf{Q}) e^{-i \omega_{\mathbf{Q}}(t-\tau)}\right] \\
& +\sum_{\mathbf{Q} \mathbf{k}^{\prime} \mathbf{q}_{c}^{\prime}} \int_{-\infty}^{t} d \tau V(\mathbf{Q}) I\left(\mathbf{q}_{c}\right) e^{-i E_{\mathbf{k}-\mathbf{q}}(t-\tau)} \rho_{\mathbf{k}-\mathbf{q}}^{>}(\tau) V\left(-\mathbf{q}_{c}^{\prime}\right) I\left(-\mathbf{q}_{c}^{\prime}\right) \rho_{\mathbf{k}}^{<}(\tau) e^{i E_{\mathbf{k}}(t-\tau)} \operatorname{Tr}\left[I\left(-\mathbf{q}_{c}\right) e^{-i E_{\mathbf{k}}(t-\tau)} \rho_{\mathbf{k}^{\prime}}^{>}(\tau)\right. \\
& \left.\quad \times I\left(\mathbf{q}_{c}^{\prime}\right) \rho_{\mathbf{k}^{\prime}-\mathbf{q}}^{<}(\tau) e^{-i E_{\mathbf{k}^{\prime}-\mathbf{q}}(t-\tau)}\right] .
\end{aligned}
$$


Here $N^{<}(\mathbf{Q})=N(\mathbf{Q})$ is the phonon distribution, $N^{>}(\mathbf{Q})=N(\mathbf{Q})+1, \rho_{\mathbf{k}}^{>}=1-\rho_{\mathbf{k}}$, and $\rho_{\mathbf{k}}^{<}=\rho_{\mathbf{k}} . N_{i}$ is the impurity density. As $E_{\mathbf{k}}=\sum_{n} \varepsilon_{n}(\mathbf{k}) T_{\mathbf{k}, n}$ with $T_{\mathbf{k}, n}=|\mathbf{k}, n\rangle\langle\mathbf{k}, n|, e^{i E_{\mathbf{k}} t}=\sum_{n} e^{i \varepsilon_{n}(\mathbf{k}) t} T_{\mathbf{k}, n}$. By further assuming the spin precession period is much longer than the average momentum scattering time and applying the Markovian approximation, i.e., replacing $\rho_{\mathbf{k}}(\tau)$ in the integrand of Eq. (160) by

$$
\rho_{\mathbf{k}}(\tau) \approx e^{i E_{\mathbf{k}}(t-\tau)} \rho_{\mathbf{k}}(t) e^{-i E_{\mathbf{k}}(t-\tau)},
$$

the time integral can be carried out and one arrives at the energy conservation:

$$
\begin{aligned}
S_{\mathbf{k}}(>,<)= & \pi \sum_{\mathbf{Q}, n_{1} n_{2}} N_{i} V_{i}(\mathbf{Q}) I\left(\mathbf{q}_{c}\right) \rho_{\mathbf{k}-\mathbf{q}}^{>}(t) T_{\mathbf{k}-\mathbf{q}, n_{1}} V_{i}(-\mathbf{Q}) I\left(-\mathbf{q}_{c}\right) T_{\mathbf{k}, n_{2}} \rho_{\mathbf{k}}^{<}(t) \delta\left(\varepsilon_{n_{1}}(\mathbf{k}-\mathbf{q})-\varepsilon_{n_{2}}(\mathbf{k})\right) \\
+ & \pi \sum_{\mathbf{Q}, n_{1} n_{2}} M_{\lambda}(\mathbf{Q}) I\left(\mathbf{q}_{c}\right) \rho_{\mathbf{k}-\mathbf{q}}^{>}(t) T_{\mathbf{k}-\mathbf{q}, n_{1}} M_{\lambda}(-\mathbf{Q}) I\left(-\mathbf{q}_{c}\right) T_{\mathbf{k}, n_{2}} \rho_{\mathbf{k}}^{<}(t) \\
& \times\left[N^{<}(\mathbf{Q}) \delta\left(\varepsilon_{n_{1}}(\mathbf{k}-\mathbf{q})-\varepsilon_{n_{2}}(\mathbf{k})-\omega_{\mathbf{Q}}\right)+\mathbf{N}^{>}(\mathbf{Q}) \delta\left(\varepsilon_{\mathbf{n}_{1}}(\mathbf{k}-\mathbf{q})-\varepsilon_{\mathbf{n}_{2}}(\mathbf{k})+\omega_{\mathbf{Q}}\right)\right] \\
+ & \pi \sum_{\quad \mathbf{k}^{\prime} \mathbf{q}_{c}^{\prime}, n_{1} n_{2} n_{3} n_{4}} V(\mathbf{Q}) I\left(\mathbf{q}_{c}\right) \rho_{\mathbf{k}-\mathbf{q}}^{>}(t) T_{\mathbf{k}-\mathbf{q}, n_{1}} V(-\mathbf{Q}) I\left(-\mathbf{q}_{c}^{\prime}\right) T_{\mathbf{k}, n_{2}} \rho_{\mathbf{k}}^{<}(t) \\
& \times \operatorname{Tr}\left[I\left(-\mathbf{q}_{c}\right) \rho_{\mathbf{k}^{\prime}}^{>}(t) T_{\mathbf{k}^{\prime}, n_{3}} I\left(\mathbf{q}_{c}^{\prime}\right) T_{\mathbf{k}^{\prime}-\mathbf{q}, n_{4}} \rho_{\mathbf{k}^{\prime}-\mathbf{q}}^{<}(t)\right] \delta\left(\varepsilon_{n_{1}}(\mathbf{k}-\mathbf{q})-\varepsilon_{n_{2}}(\mathbf{k})+\varepsilon_{n_{3}}\left(\mathbf{k}^{\prime}\right)-\varepsilon_{n_{4}}\left(\mathbf{k}^{\prime}-\mathbf{q}\right)\right) .(16
\end{aligned}
$$

The expression of $S_{\mathbf{k}}(<,>)$ can be obtained by interchanging < and $>$ in Eqs. (161) and (162).

\subsection{Spin relaxation and dephasing in $n$ - or p-type quantum wells}

In this section, we review the spin dynamics in $n$ - or $p$-type quantum wells under various conditions. We first write the kinetic spin Bloch equations in both the helix and collinear spin spaces in quantum wells in Sec. 5.4.1. Then we review the spin dynamics near the equilibrium and far away from the equilibrium in Sec.5.4.2 and 5.4.3 respectively. We highlight the effect of the Coulomb scattering to the spin relaxation and dephasing in Sec. 5.4.4 After that we review the non-Markovian effect of hole spin dynamics in Sec.5.4.5. We review the electron spin relaxation due to the Bir-Aronov-Pikus mechanism in Sec.5.4.6. The spin dynamics in the presence of a strong THz laser field is reviewed in Sec. 5.4.7 Spin relaxation in paramagnetic GaMnAs quantum wells 75 GaAs (110) quantum wells, Si/SiGe and $\mathrm{Ge} / \mathrm{SiGe}$ quantum wells and wurtzite $\mathrm{ZnO}$ (001) quantum wells are reviewed in Secs. 5.4.8,5.4.11, respectively.

\subsubsection{Kinetic spin Bloch equations in (001) quantum wells}

We first consider a quantum well with a small well width so that only the lowest subband is needed. The electron Hamiltonian of Eq. (147) now reads

$$
H_{e}=\frac{\mathbf{k}^{2}}{2 m^{*}}+\mathbf{h}(\mathbf{k}) \cdot \boldsymbol{\sigma}+\varepsilon_{0} .
$$

Here $\varepsilon_{0}$ is the energy of the lowest subband. It can be calculated from the Schrödinger equation with confinement potential $V\left(\mathbf{r}_{c}\right)=V(z) . \mathbf{h}(\mathbf{k})=\frac{1}{2} g \mu_{B}\left[\mathbf{B}+\mathbf{\Omega}^{2 D}(\mathbf{k})\right]$ with $\mathbf{\Omega}^{2 D}(\mathbf{k})$ being the Dresselhaus and/or Rashba terms. The eigenenergy and eigenfunction of Eq. (163) are $\varepsilon_{\xi}(\mathbf{k})=\frac{k^{2}}{2 m^{*}}+\xi|\mathbf{h}(\mathbf{k})|+\varepsilon_{0}$ and $|\mathbf{k}, \xi= \pm\rangle=\frac{T_{\mathbf{k}, \xi}|\uparrow\rangle}{\left\langle\uparrow\left|T_{\mathbf{k}, \xi}\right| \uparrow\right\rangle}$, respectively. The projector operator reads $T_{\mathbf{k}, \xi}=\frac{1}{2}\left[1+\xi \frac{\mathbf{h}(\mathbf{k})}{\mathbf{h}(\mathbf{k}) \mid} \cdot \sigma\right]$. The space spanned by $\{|\mathbf{k}, \xi\rangle\}$ is the helix space. As $|\mathbf{k}, \xi\rangle$ is k-dependent, the kinetic spin Bloch equations are more complicated in this space. Meanwhile, one may use the space spanned by $\left\{|\mathbf{k}\rangle|n\rangle_{0}\right\}$. Here we choose $\mathbf{k}_{0}=0$, i.e., the $\Gamma$-point and then $|n\rangle_{0}$ is the eigenvector of $\sigma_{z},|\sigma\rangle=|\uparrow\rangle$ or $|\downarrow\rangle$, which is $\mathbf{k}$-independent. This space is the collinear space. In the collinear spin space, the density matrix is

$$
\rho_{\mathbf{k}}=\left(\begin{array}{cc}
f_{\mathbf{k} \uparrow} & \rho_{\mathbf{k} \uparrow \downarrow} \\
\rho_{\mathbf{k} \downarrow \uparrow} & f_{\mathbf{k} \downarrow}
\end{array}\right) .
$$

When an electric field $\mathbf{E}$ is applied along the quantum well, the kinetic spin Bloch equations are given by [28, 569]

$$
\frac{\partial \rho_{\mathbf{k}}(t)}{\partial t}=e \mathbf{E} \cdot \nabla_{\mathbf{k}} \rho_{\mathbf{k}}(t)-i\left[\mathbf{h}(\mathbf{k}) \cdot \boldsymbol{\sigma}+\Sigma_{\mathrm{HF}}(\mathbf{k}, t), \rho_{\mathbf{k}}(t)\right]+\left.\frac{\rho_{\mathbf{k}}(t)}{\partial t}\right|_{\text {scat }} .
$$

\footnotetext{
${ }^{75}$ Recently Shen et al. have further extended the kinetic spin Bloch equation theory to study the Gilbert damping in ferromagnetic semiconductors 842.
} 
Here $\Sigma_{\mathrm{HF}}(\mathbf{k}, t)=-\sum_{\mathbf{q}} V_{\mathbf{q}} \rho_{\mathbf{k}-\mathbf{q}}(t)$, and $S_{\mathbf{k}}(>,<)$ in the scattering terms [Eq. [162)] reads

$$
\begin{aligned}
S_{\mathbf{k}}(>,<)= & \pi N_{i} \sum_{\mathbf{q}, \eta_{1} \eta_{2}}\left|U_{\mathbf{q}}\right|^{2} \rho_{\mathbf{k}-\mathbf{q}}^{>}(t) T_{\mathbf{k}-\mathbf{q}, \eta_{1}} T_{\mathbf{k}, \eta_{2}} \rho_{\mathbf{k}}^{<}(t) \delta\left(\varepsilon_{\eta_{1}}(\mathbf{k}-\mathbf{q})-\varepsilon_{\eta_{2}}(\mathbf{k})\right) \\
+ & \pi \sum_{\mathbf{Q}, \eta_{1} \eta_{2}}\left|g_{\mathbf{Q}, \lambda}\right|^{2} \rho_{\mathbf{k}-\mathbf{q}}^{>}(t) T_{\mathbf{k}-\mathbf{q}, \eta_{1}} T_{\mathbf{k}, \eta_{2}} \rho_{\mathbf{k}}^{<}(t)\left[N^{<}(\mathbf{Q}) \delta\left(\varepsilon_{\eta_{1}}(\mathbf{k}-\mathbf{q})-\varepsilon_{\eta_{2}}(\mathbf{k})-\omega_{\mathbf{Q}}\right)\right. \\
& \left.+N^{>}(\mathbf{Q}) \delta\left(\varepsilon_{\eta_{1}}(\mathbf{k}-\mathbf{q})-\varepsilon_{\eta_{2}}(\mathbf{k})+\omega_{\mathbf{Q}}\right)\right] \\
+ & \pi \sum_{\mathbf{q} \mathbf{k}^{\prime}, \eta_{1} \eta_{2} \eta_{3} \eta_{4}} V_{\mathbf{q}}^{2} \rho_{\mathbf{k}-\mathbf{q}}^{>}(t) T_{\mathbf{k}-\mathbf{q}, \eta_{1}} T_{\mathbf{k}, \eta_{2}} \rho_{\mathbf{k}}^{<}(t) \operatorname{Tr}\left[\rho_{\mathbf{k}^{\prime}}^{>}(t) T_{\mathbf{k}^{\prime}, \eta_{3}} T_{\mathbf{k}^{\prime}-\mathbf{q}, \eta_{4}} \rho_{\mathbf{k}^{\prime}-\mathbf{q}}^{<}(t)\right] \\
& \times \delta\left(\varepsilon_{\eta_{1}}(\mathbf{k}-\mathbf{q})-\varepsilon_{\eta_{2}}(\mathbf{k})+\varepsilon_{\eta_{3}}\left(\mathbf{k}^{\prime}\right)-\varepsilon_{\eta_{4}}\left(\mathbf{k}^{\prime}-\mathbf{q}\right)\right),
\end{aligned}
$$

with $\left|U_{\mathbf{q}}\right|^{2}=\sum_{q_{z}} V_{i}(\mathbf{Q}) V_{i}(-\mathbf{Q}) I\left(q_{z}\right) I\left(-q_{z}\right)$ and $\left|g_{\mathbf{Q}, \lambda}\right|^{2}=M_{\lambda}(\mathbf{Q}) I\left(q_{z}\right) M_{\lambda}(-\mathbf{Q}) I\left(-q_{z}\right)$ and $V_{q}=\sum_{q_{z}} V(\mathbf{Q}) I\left(q_{z}\right) I\left(-q_{z}\right)$ being the matrix elements with the form factors. It is noted that $I_{\sigma_{1} \sigma_{2}}\left(q_{z}\right)$ is diagonal in the collinear spin space $|\sigma\rangle$. In the calculation, one either uses the static screening or the screening under the random-phase approximation [372] for $V_{\mathbf{q}}$ and $U_{\mathbf{q}}$ [843], according to the different conditions of investigation. In the collinear spin space, the screened Coulomb potential and electron-impurity interaction potential in the random-phase approximation read

$$
\begin{aligned}
V_{\mathbf{q}} & =\sum_{q_{z}} v_{\mathbf{Q}}\left|I\left(i q_{z}\right)\right|^{2} / \epsilon(\mathbf{q}), \\
\left|U_{\mathbf{q}}\right|^{2} & =\sum_{q_{z}} u_{\mathbf{Q}}^{2}\left|I\left(i q_{z}\right)\right|^{2} / \epsilon(\mathbf{q})^{2},
\end{aligned}
$$

where $v_{\mathbf{Q}}=\frac{4 \pi e^{2}}{\mathbf{Q}^{2}}$ is the bare Coulomb potential, $u_{\mathbf{Q}}^{2}=Z_{i}^{2} v_{\mathbf{Q}}^{2}$ with $Z_{i}$ the charge number of impurity, and

$$
\epsilon(\mathbf{q})=1-\sum_{q_{z}} v_{\mathbf{Q}}\left|I\left(i q_{z}\right)\right|^{2} \sum_{\mathbf{k} \sigma} \frac{f_{\mathbf{k}+\mathbf{q} \sigma}-f_{\mathbf{k} \sigma}}{\epsilon_{\mathbf{k}+\mathbf{q}}-\epsilon_{\mathbf{k}}} .
$$

Eq. (165) is valid in both the collinear and helix spin spaces. By performing a unitary transformation $\rho_{\mathbf{k}}^{h}=U_{\mathbf{k}}^{\dagger} \rho_{\mathbf{k}}^{c} U_{\mathbf{k}}$, one may transfer the density matrix from the collinear space $\rho_{\mathbf{k}}^{c}$ to the helix one $\rho_{\mathbf{k}}^{h}$, with

$$
U_{\mathbf{k}}=\left(\begin{array}{cc}
\langle\uparrow \mid \mathbf{k},+\rangle & \langle\uparrow \mid \mathbf{k},-\rangle \\
\langle\downarrow \mid \mathbf{k},+\rangle & \langle\downarrow \mid \mathbf{k},-\rangle
\end{array}\right) .
$$

Finally, we point out that the energy spectrum $\varepsilon_{\eta}(\mathbf{k})$ in the scattering Eq. (166) contains the spin-orbit coupling. When the coupling is much smaller than the Fermi energy, one may neglect the coupling and hence

$$
\delta\left(\varepsilon_{\eta}(\mathbf{q})-\varepsilon_{\eta^{\prime}}(\mathbf{k})\right) \approx \delta(\varepsilon(\mathbf{q})-\varepsilon(\mathbf{k}))
$$

By further utilizing the relation

$$
\sum_{\eta} T_{\mathbf{k}, \eta}=1
$$

the scattering becomes

$$
\begin{aligned}
S_{\mathbf{k}}(>,<)= & \pi N_{i} \sum_{\mathbf{q}}\left|U_{\mathbf{q}}\right|^{2} \rho_{\mathbf{k}-\mathbf{q}}^{>}(t) \rho_{\mathbf{k}}^{<}(t) \delta(\varepsilon(\mathbf{k}-\mathbf{q})-\varepsilon(\mathbf{k})) \\
& +\pi \sum_{\mathbf{Q}}\left|g_{\mathbf{Q}, \lambda}\right|^{2} \rho_{\mathbf{k}-\mathbf{q}}^{>}(t) \rho_{\mathbf{k}}^{<}(t)\left[N^{<}(\mathbf{Q}) \delta\left(\varepsilon(\mathbf{k}-\mathbf{q})-\varepsilon(\mathbf{k})-\omega_{\mathbf{Q}}\right)+\mathbf{N}^{>}(\mathbf{Q}) \delta\left(\varepsilon(\mathbf{k}-\mathbf{q})-\varepsilon(\mathbf{k})+\omega_{\mathbf{Q}}\right)\right] \\
& +\pi \sum_{\mathbf{q} \mathbf{k}^{\prime}} V_{\mathbf{q}}^{2} \rho_{\mathbf{k}-\mathbf{q}}^{>}(t) \rho_{\mathbf{k}}^{<}(t) \operatorname{Tr}\left[\rho_{\mathbf{k}^{\prime}}^{>}(t) \rho_{\mathbf{k}^{\prime}-\mathbf{q}}^{<}(t)\right] \delta\left(\varepsilon(\mathbf{k}-\mathbf{q})-\varepsilon(\mathbf{k})+\varepsilon\left(\mathbf{k}^{\prime}\right)-\varepsilon\left(\mathbf{k}^{\prime}-\mathbf{q}\right)\right)
\end{aligned}
$$

This is the form used in the electron systems $[25,27-29,32,41,42,44,69,111,199,336,372,569,570,609,835$, 844 846]. 
Before discussing the results from the full kinetic spin Bloch equations, we first show a simplest case by keeping only the electron-impurity scattering in the scattering term, where one can obtain an analytical solution in the spacial homogeneous system by further neglecting the Coulomb Hartree-Fock contribution [363]. The kinetic spin Bloch equations then read

$$
\frac{\partial}{\partial t} \rho_{\mathbf{k}}(t)=-i\left[\alpha\left(k_{y} \sigma_{x}-k_{x} \sigma_{y}\right), \rho_{\mathbf{k}}(t)\right]-2 \pi N_{i} \sum_{\mathbf{q}}\left|U_{\mathbf{q}}\right|^{2} \delta\left(\varepsilon_{\mathbf{k}-\mathbf{q}}-\varepsilon_{\mathbf{k}}\right)\left[\rho_{\mathbf{k}}(t)-\rho_{\mathbf{k}-\mathbf{q}}(t)\right] .
$$

By expanding $\rho_{\mathbf{k}}$ as $\rho_{\mathbf{k}}=\sum_{l} \rho_{l}(k) e^{i \theta_{k} l}$, one has

$$
\frac{\partial}{\partial t} \rho_{l}(k, t)=\alpha k\left\{\left[S^{+}, \rho_{l+1}(k, t)\right]-\left[S^{-}, \rho_{l-1}(k, t)\right]\right\}-\left|U_{l}(k)\right|^{2} \rho_{l}(k, t),
$$

in which $\left|U_{l}(k)\right|^{2}=\frac{m^{*} N_{i}}{2 \pi \hbar^{2}} \int_{0}^{2 \pi} d \theta\left|U\left(\sqrt{2 k^{2}(1-\cos \theta)}\right)\right|^{2}(1-\cos l \theta)$ with $\left|U_{0}(k)\right|^{2}=0$ and $\left|U_{-1}(k)\right|^{2}=\left|U_{1}(k)\right|^{2} \equiv \frac{1}{\tau_{p}(k)}$. By multiplying $\frac{1}{2} \sigma$ and then performing trace from both sides of Eq. (174), one comes to

$$
\frac{\partial}{\partial t} \mathbf{S}_{l}(k, t)=\alpha k\left[\mathcal{F}^{\dagger} \mathbf{S}_{l+1}(k, t)-\mathcal{F} \mathbf{S}_{l-1}(k, t)\right]-\left|U_{l}(k)\right|^{2} \mathbf{S}_{l}(k, t),
$$

with $\mathbf{S}_{l}(k, t)=\frac{1}{2} \operatorname{Tr}\left[\rho_{l}(k, t) \sigma\right]$ and $\mathcal{F}=\left(\begin{array}{ccc}0 & 0 & 1 \\ 0 & 0 & -i \\ -1 & i & 0\end{array}\right)$. In deriving Eq. (175), we have used the relation $\frac{1}{2} \operatorname{Tr}\left(\left[S^{+}, \rho_{l}\right] \sigma\right)=$ $\mathcal{F}^{\dagger} \mathbf{S}_{l}(k, t)$ and $\frac{1}{2} \operatorname{Tr}\left(\left[S^{-}, \rho_{l}\right] \sigma\right)=\mathcal{F} \mathbf{S}_{l}(k, t)$. By keeping only the lowest three orders of $\mathbf{S}_{l}$ in the strong scattering limit $\left(x_{k} \ll 1\right)$, i.e., $l=0, \pm 1$, one has

$$
\left[\frac{\partial}{\partial t}-\alpha k\left(\begin{array}{ccc}
-\frac{1}{\alpha k \tau_{p}(k)} & -\mathcal{F} & 0 \\
\mathcal{F}^{\dagger} & 0 & -\mathcal{F} \\
0 & \mathcal{F}^{\dagger} & -\frac{1}{\alpha k \tau_{p}(k)}
\end{array}\right)\right]\left(\begin{array}{c}
\mathbf{S}_{1} \\
\mathbf{S}_{0} \\
\mathbf{S}_{-1}
\end{array}\right)=0
$$

The solution of Eq. (176) reads

$$
\begin{aligned}
& \mathbf{S}_{1}(k, t)=-\frac{x_{k}}{2 \sqrt{1-x_{x}^{2}}} e^{-\frac{t}{2 \tau_{p}(k)}} \sinh \frac{t}{2 \tau_{p}(k) / \sqrt{1-x_{k}^{2}}}\left(\begin{array}{l}
1 \\
i \\
0
\end{array}\right) f\left(\varepsilon_{k}-\mu\right), \\
& \mathbf{S}_{0}(k, t)=e^{-\frac{t}{2 \tau_{p}(k)}}\left[\frac{\sinh \frac{t}{2 \tau_{p}(k) / \sqrt{1-x_{k}^{2}}}}{\sqrt{1-x_{k}^{2}}}+\cosh \frac{t}{2 \tau_{p}(k) / \sqrt{1-x_{k}^{2}}}\right]\left(\begin{array}{l}
0 \\
0 \\
1
\end{array}\right) f\left(\varepsilon_{k}-\mu\right), \\
& \mathbf{S}_{-1}(k, t)=-\frac{x_{k}}{2 \sqrt{1-x_{x}^{2}}} e^{-\frac{t}{2 \tau_{p}(k)}} \sinh \frac{t}{2 \tau_{p}(k) / \sqrt{1-x_{k}^{2}}}\left(\begin{array}{c}
1 \\
-i \\
0
\end{array}\right) f\left(\varepsilon_{k}-\mu\right),
\end{aligned}
$$

with the initial conditions being $\mathbf{S}_{1}(k, 0)=\mathbf{S}_{-1}(k, 0)=0$ and $\mathbf{S}_{0}(k, 0)=f\left(\varepsilon_{k}-\mu\right) \mathbf{e}_{z}$, i.e., the initial spin polarization being along the $z$-axis. $x_{k}$ in Eqs. (177,179) is $x_{k}=4 \alpha k \tau_{p}(k)$. From Eqs. (177, 179), electron spin at momentum $\mathbf{k}$ reads

$$
\mathbf{S}_{\mathbf{k}}(t)=\mathbf{S}_{0}(k, t)+\mathbf{S}_{1}(k, t) e^{i \theta_{k}}+\mathbf{S}_{-1}(k, t) e^{-i \theta_{k}}
$$

and hence the component along the $z$-axis is given by

$$
S_{\mathbf{k}}^{z}(t)=S_{0}^{z}(k, t)=\frac{1}{2} f\left(\varepsilon_{k}-\mu\right)\left[\left(1+1 / \sqrt{1-x_{k}^{2}}\right) e^{-\frac{t}{2 \tau p^{(k)}}\left(1-\sqrt{1-x_{k}^{2}}\right)}+\left(1-1 / \sqrt{1-x_{k}^{2}}\right) e^{-\frac{t}{2 \tau_{p}(k)}\left(1+\sqrt{1-x_{k}^{2}}\right)}\right] .
$$

From Eq. (181), one can see the different time evolutions of the spin polarization at different regimes. When $x_{k}>$ 1, i.e., $\alpha k \tau_{p}(k)>1 / 4$, the system is in the weak scattering regime and the terms $e^{ \pm \frac{t}{2 \tau_{p}(k)} \sqrt{1-x_{k}^{2}}}$ give just the spin oscillations. Hence the spin relaxation is given by

$$
1 / \tau_{s}(\mathbf{k})=1 /\left[2 \tau_{p}(k)\right] .
$$


However, the spin oscillation frequency is $\omega_{\mathbf{k}}=\sqrt{(2 \alpha k)^{2}-1 /\left[2 \tau_{p}(k)\right]^{2}}$. One can see from $\omega_{\mathbf{k}}$ that the scattering tends to suppress the spin oscillation frequency. This indicates the counter-effect of the scattering to the inhomogeneous broadening. When $x_{k}<1$, the spin polarization decays according to

$$
1 / \tau_{s, \pm}(\mathbf{k})=\left(1 \pm \sqrt{1-\left[4 \alpha k \tau_{p}(k)\right]^{2}}\right) /\left[2 \tau_{p}(k)\right] .
$$

In the strong scattering limit, i.e., $x_{k} \ll 1$,

$$
\begin{aligned}
1 / \tau_{s,+}(\mathbf{k}) & =1 / \tau_{p}(k) \\
1 / \tau_{s,-}(\mathbf{k}) & =(2 \alpha k)^{2} \tau_{p}(k) .
\end{aligned}
$$

As $x_{k} \ll 1, \tau_{s,-}(\mathbf{k}) \gg \tau_{s,+}(\mathbf{k})$ and the spin relaxation is determined by $\tau_{s,-}(\mathbf{k})$. It is noted that $\tau_{s,-}(\mathbf{k})$ is exactly the result in the literature [3, 101].

Finally, one can see from Eqs. (183) and (185) that in the weak scattering regime, a stronger scattering leads to a faster spin relaxation. Nevertheless, in the strong scattering regime, a stronger scattering leads to a weaker spin relaxation. This can be understood as following. In the presence of inhomogeneous broadening, the scattering has dual effects to the spin relaxation: (i) It gives a spin relaxation channel; (ii) It has a counter-effect to the inhomogeneous broadening by making the system more homogeneous. In the weak scattering limit, the counter effect is weak and hence adding a new scattering always leads to an additional relaxation channel and hence a fast spin relaxation. In the strong scattering limit, the counter effect becomes significant and hence adding a new scattering always leads to a longer spin relaxation time.

\subsubsection{Spin relaxation and dephasing in n-type (001) GaAs quantum wells near the equilibrium}

By numerically solving the kinetic spin Bloch equations with all the relevant scatterings included, Weng and $\mathrm{Wu}$ studied the spin dephasing in $n$-type GaAs quantum wells at high temperature ( $\geq 120 \mathrm{~K}$ ), where the electron-acoustic phonon scattering is unimportant, first for small well width [44] with only the lowest subband and then for large well width [844] with mutisubband effect considered. They further investigated the hot-electron effect in spin dephasing by applying a large in-plane electric field [569], where the hot-electron effect is investigated. By further increasing the in-plane electric field, electrons can populate higher subband and/or higher valleys. These effects were investigated by Weng and $\mathrm{Wu}$ [844] for mutisubband case and Zhang et al. [845] for multivalley case. The spin relaxation at low temperature was first investigated by Zhou et al. [372] from kinetic spin Bloch equation approach by including the electron-acoustic phonon scattering. Jiang and Wu studied the effect of strain on spin relaxation [570]. Spin relaxation for system with competing Dresselhaus and Rashba terms was investigated theoretically by Cheng and Wu [597] and both experimentally and theoretically by Stich et al. [199]. Spin relaxation with large initial spin polarization was first studied by Weng and Wu [44] and many predictions were verified experimentally with good agreement between the experimental data and theoretical calculations by Stich et al. [41, 42]. The density dependence of the spin relaxation was also investigated both theoretically and experimentally [609]. In this and next sections we review the main results of the above investigations.

It was revealed that the spin relaxation time based on the D'yakonov-Perel' mechanism has a rich temperature dependence depending on different impurity densities, carrier densities and well widths.

Figure 48 shows the temperature dependence of the spin relaxation time of a $7.5 \mathrm{~nm} \mathrm{GaAs} / \mathrm{Al}_{0.4} \mathrm{Ga}_{0.6} \mathrm{As}$ quantum well at different electron and impurity densities [372]. For this small well width, only the lowest subband is needed in the calculation. It is shown in the figure that when the electron-impurity scattering is dominant, the spin relaxation time decreases with increasing temperature monotonically. This is in good agreement with the experimental finding as shown in Fig. 49, where the dots are experimental data from Ohno et al. [602], and the theoretical calculation based on the kinetic spin Bloch equations well reproduces the experimental results from $20 \mathrm{~K}$ to $300 \mathrm{~K}$ [372]. However, it is shown that for sample with high mobility, i.e., low impurity density, when the electron density is low enough, there is a peak at low temperature. This peak, located around the Fermi temperature of electrons $T_{F}^{e}=E_{F} / k_{B}$, is identified to be solely due to the Coulomb scattering [372, 847]. It disappears when the Coulomb scattering is switched off, as shown by the dashed curves in the figure. This peak also disappears at high impurity densities. It is also noted in Fig. 48(c) that for electrons of high density so that $T_{F}^{e}$ is high enough and the contribution from the electron-longitudinal opticalphonon scattering becomes marked, the peak disappears even for sample with no impurity and the spin relaxation time 

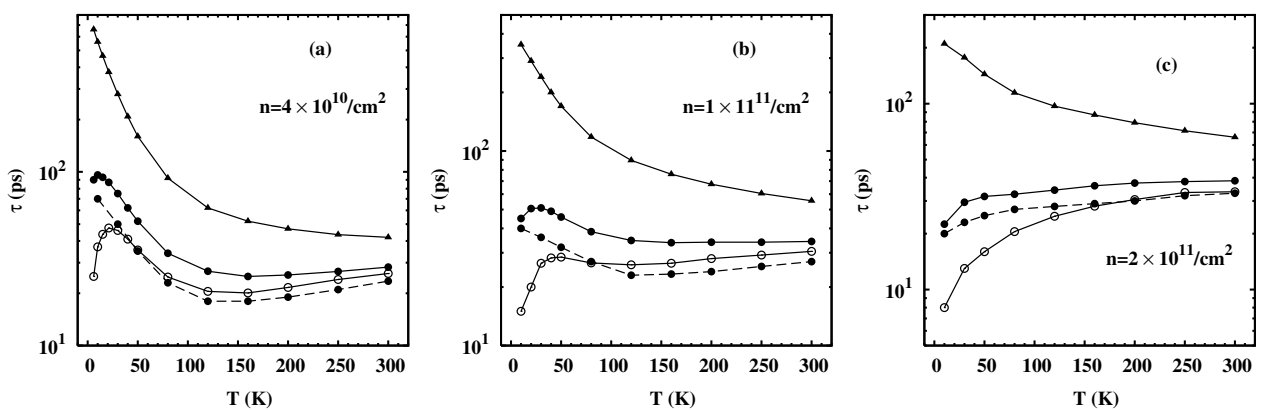

Figure 48: Spin relaxation time $\tau$ vs. the temperature $T$ in GaAs (001) quantum well with well width $a=7.5 \mathrm{~nm}$ and electron density $n$ being (a) $4 \times 10^{10} \mathrm{~cm}^{-2}$, (b) $1 \times 10^{11} \mathrm{~cm}^{-2}$, and (c) $2 \times 10^{11} \mathrm{~cm}^{-2}$, respectively. Solid curves with triangles: impurity density $n_{i}=n$; solid curves with dots: $n_{i}=0.1 n$; solid curves with circles: $n_{i}=0$; dashed curves with dots: $n_{i}=0.1 n$ and no Coulomb scattering. From Zhou et al. [372].
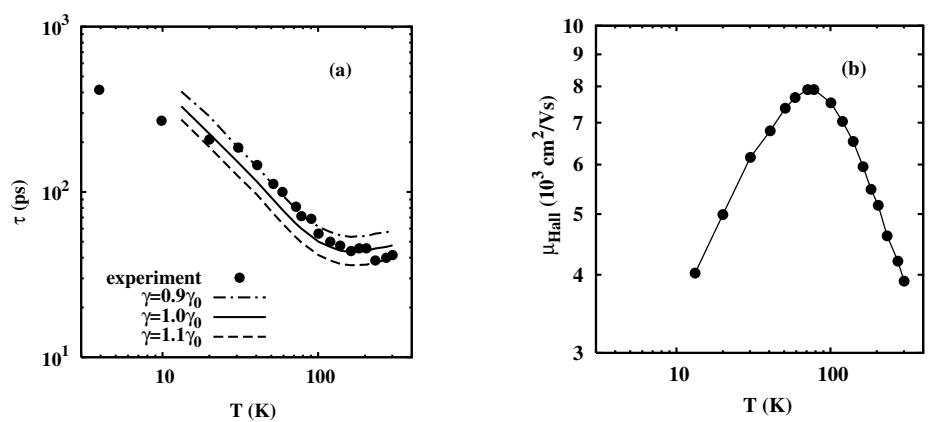

Figure 49: (a) Spin relaxation time $\tau$ vs. temperature $T$ for GaAs (001) quantum well with well width $a=7.5 \mathrm{~nm}$ and electron density $n=$ $4 \times 10^{10} \mathrm{~cm}^{-2}$ at three different spin-splitting parameters. Dots: experimental data; dot-dashed curve: $\gamma=0.9 \gamma_{0}$; solid curve: $\gamma=\gamma_{0}$; dashed curve: $\gamma=1.1 \gamma_{0} \cdot \gamma_{0}=0.0114 \mathrm{eV} \cdot \mathrm{nm}^{3}$. (b) Hall mobility $\mu_{\text {Hall }} V s$. temperature $T$ (Ref. [602]), from which the temperature dependence of the impurity density is determined. From Zhou et al. [372].

increases with temperature monotonically. The physics leading to the peak is due to the crossover of the Coulomb scattering from the degenerate to the non-degenerate limit. At $T<T_{F}^{e}$, electrons are in the degenerate limit and the electron-electron scattering rate $1 / \tau_{\mathrm{ee}} \propto T^{2}$. At $T>T_{F}^{e}, 1 / \tau_{\mathrm{ee}} \propto T^{-1}$ [843, 848]. Therefore, at low electron density so that $T_{F}^{e}$ is low enough and the electron-acoustic phonon scattering is very weak comparing with the electron-electron Coulomb scattering, the Coulomb scattering is the dominant scattering for high mobility sample. Hence the different temperature dependence of the Coulomb scattering leads to the peak. It is noted that the peak is just a feature of the crossover from the degenerate to the non-degenerate limit. The location of the peak also depends on the strength of the inhomogeneous broadening. When the inhomogeneous broadening depends on momentum linearly, the peak tends to appear at the Fermi temperature. A similar peak was predicted in the electron spin relaxation in $p$-type GaAs quantum well and the hole spin relaxation in (001) strained asymmetric $\mathrm{Si} / \mathrm{SiGe}$ quantum well, where the electron and hole spin relaxation times both show a peak at the hole Fermi temperature $T_{F}^{h}[112,299]$. When the inhomogeneous broadening depends on momentum cubically, the peak tends to shift to a lower temperature. It was predicted that a peak in the temperature dependence of the electron spin relaxation time appears at a temperature in the range of $\left(T_{F}^{e} / 4\right.$, $\left.T_{F}^{e} / 2\right)$ in the intrinsic bulk GaAs [110] and a peak in the temperature dependence of the hole spin relaxation time at $T_{F}^{h} / 2$ in $p$-type $\mathrm{Ge} / \mathrm{SiGe}$ quantum well [299]. Ruan et al. demonstrated the peak experimentally in a high-mobility low-density GaAs $/ \mathrm{Al}_{0.35} \mathrm{Ga}_{0.65} \mathrm{As}$ heterostructure [604] as shown in Fig. [50] where a peak appears at $T_{F}^{e} / 2$ in the spin relaxation time versus temperature curve.

It is also seen from Fig. 48 that at high temperature (> $100 \mathrm{~K})$, except for the case where the electron-impurity 


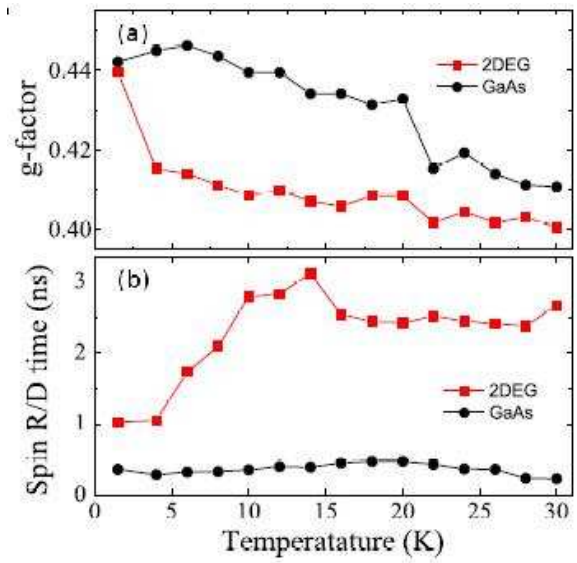

Figure 50: (a) Measured electron $g$-factor as a function of temperature for two-dimensional electron gas ( $\mathrm{squares}$ in $\mathrm{GaAs}_{\mathrm{A}} / \mathrm{Al}_{0.35} \mathrm{Ga}_{0.65} \mathrm{As}_{\mathrm{s}}$ heterostructure and bulk GaAs (circles). (b) Measured electron spin relaxation/dephasing time as a function of temperature for two-dimensional electron gas (squares) and bulk GaAs (circles). All of the data were taken at $B=0.5 \mathrm{~T}$ and powers of pump:probe $=200: 20 \mu \mathrm{W}$. From Ruan et al. 604].

scattering dominates the scattering, the spin relaxation time increases with increasing temperature. Weng and Wu also showed this in a $15 \mathrm{~nm}$ quantum well with a magnetic field $B=4 \mathrm{~T}$ in the Voigt configuration [44]. They even compared the spin relaxation times obtained from the kinetic spin Bloch equation approach and from the singleparticle model [196, 577] $1 / \tau=\int_{0}^{\infty} d E_{\mathbf{k}}\left(f_{\mathbf{k} \frac{1}{2}}-f_{\mathbf{k}-\frac{1}{2}}\right) \Gamma(\mathbf{k}) / \int_{0}^{\infty} d E_{\mathbf{k}}\left(f_{\mathbf{k} \frac{1}{2}}-f_{\mathbf{k}-\frac{1}{2}}\right)$, in which $\Gamma(\mathbf{k})$ is the spin relaxation rate. It is seen from the inset of Fig. 51 that the spin relaxation time based on the previous single-particle model decreases rather than increases with increasing temperature. As the impurity density is set to zero in both computations, the main difference comes from the Coulomb scattering which is missing in the single particle calculation.

However, even for sample without any impurity, the temperature dependence of the spin relaxation time can also decrease with increasing temperature at high temperatures. This was shown by Weng and Wu in GaAs (001) quantum well with wider well width [844]. In the calculation, they considered two well widths, i.e., $17.8 \mathrm{~nm}$ and $12.7 \mathrm{~nm}$, and showed the spin relaxation times as function of temperature with both the lowest subband and multisubband effects considered. One can see from the dashed curves (only the lowest subband is calculated) in Fig. 52 that for small well width, the spin relaxation time increases with increasing temperature but for larger well width, the spin relaxation time first increases and then decreases with temperature. It is also noted that when the multisubband effect is included (solid curves), similar results are also obtained.

The physics leading to above rich behaviors originates from the competition between the inhomogeneous broadening and the scattering. With the increase of temperature, electrons are distributed to higher momentum states. This leads to a larger inhomogeneous broadening from the D'yakonov-Perel' term and hence a shorter spin relaxation time. In the meantime, higher temperature also causes scattering (especially the electron-phonon scattering) stronger. The scattering tends to make electrons distribute more homogeneously and suppresses the inhomogeneous broadening. Therefore the scattering tends to cause a longer spin relaxation time with the increase of temperature. When the electron-impurity scattering dominates the whole scattering, the spin relaxation time decreases with increasing temperature monotonically, thanks to the increase of the inhomogeneous broadening together with the weak temperature dependence of the electron-impurity scattering. For samples with high mobility, at low temperature where the scattering is determined by the electron-electron Coulomb scattering, a peak appears due to the crossover from the degenerate to non-degenerate limits. At high temperature where electrons are in non-degenerate limit, the scattering is determined by the electron-electron and electron-phonon scatterings. The calculations show that for small well width when only the linear Dresselhaus term is dominant, the temperature dependence of the scattering is stronger and the spin relaxation time increases with temperature. However, for wide quantum well, at certain temperature the cubic Dresselhaus term becomes dominant. The fast increase of the inhomogeneous broadening from the cubic term overcomes the effect from the scattering and the spin relaxation time decreases with temperature. Jiang and Wu further 

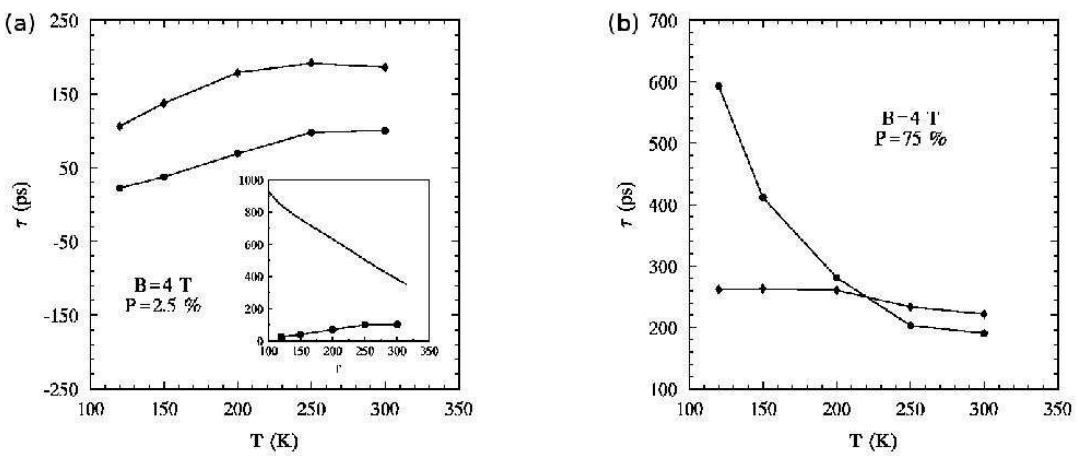

Figure 51: Spin dephasing time $\tau$ vs. temperature $T$ with spin polarization $P=2.5 \%$ (a) and $P=75 \%$ (b) under two different impurity levels in GaAs (001) quantum well. Curves with dots: $N_{i}=0$; curves with squares: $N_{i}=0.1 N_{e}$. The spin dephasing times predicted by the simplified treatment of D'yakonov-Perel' term (solid curve) and the kinetic spin Bloch equation approach (dots) for $N_{i}=0$ are plotted in the inset for comparison. From Weng and Wu [44].

introduced strain to change the relative importance of the linear and cubic D'yakonov-Perel' terms and showed the different temperature dependences of the spin relaxation time [570]. This prediction has been realized experimentally by Holleitner et al. in Ref. [655], where they showed that in $n$-type two-dimensional InGaAs channels, when the linear D'yakonov-Perel' term is suppressed, the spin relaxation time decreases with temperature monotonically, as shown in Fig. 53. It is noted for the unstructured quantum well in Fig. 53, where the linear term is important, the spin relaxation time increases with temperature. Similar findings were reported by Malinowski et al. [603], who measured the spin relaxation in intrinsic $\mathrm{GaAs} / \mathrm{Al}_{x} \mathrm{Ga}_{1-x}$ As quantum wells with different well widths, for temperature higher than $80 \mathrm{~K}$ as shown in Fig. 54 (a). One clearly sees an increase of the spin relaxation time with increasing temperature for small well width and a decrease of the spin relaxation time for large well width. Figure 54(b) shows the theoretical calculation based on the kinetic spin Bloch equations for three small well widths, where the lowest subband approximation is valid for the small well widths $(6$ and $10 \mathrm{~nm})$ and barely valid for the $15 \mathrm{~nm}$ well width. All the relevant scatterings, such as the electron-electron, electron-hole, electron-phonon and electron-impurity scatterings are included in the computation. The peaks in the cases of 10 and $15 \mathrm{~nm}$ well widths originate from the competition of the linear and cubic D'yakonov-Perel' terms addressed above.

Similar situation also happens in the density dependence of the spin relaxation time. A peak in the density dependence of the spin relaxation time was predicted theoretically and realized experimentally [609], as shown in Fig. 55 where the spin relaxation time is plotted against the photoexcited carrier density in a GaAs quantum well at room temperature. This peak can be easily understood from the relation $\tau_{s} \propto\left[\left\langle\Omega^{2}(\mathbf{k})\right\rangle \tau_{p}^{*}\right]^{-1}$, where $\tau_{p}^{*}$ should include the effect from the Coulomb scattering [334, 591, 615]. When the system is in the non-degenerate limit, the average of $\left\langle\Omega^{2}(\mathbf{k})\right\rangle$ is performed at the Boltzmann distribution and is therefore independent of the carrier density. Consequently $\tau_{s}$ increases with increasing carrier density as $\tau_{p}^{*}$ decreases with the density. However, when the carrier density is high enough and the average should be performed using the Fermi distribution, $\left\langle\Omega^{2}(\mathbf{k})\right\rangle$ becomes density dependent. Especially in the strong degenerate limit, $\langle k\rangle \sim k_{F} \propto \sqrt{N}$ and $\left\langle\Omega^{2}(\mathbf{k})\right\rangle$ becomes strongly density dependent. Moreover, the carrier-carrier Coulomb scattering decreases with increasing density at strong degenerate limit. As a result, the spin relaxation time decreases with increasing carrier density. The peak position depends on the competition between the inhomogeneous broadening and the scattering. Also the linear and cubic D'yakonov-Perel' terms influence the peak position.

By including the intersubband scattering, especially the intersubband Coulomb scattering, Weng and Wu studied the multisubband effect in spin relaxation [844]. It was discovered that although the higher subband has a much larger inhomogeneous broadening, the spin relaxation times of two subbands are identical thanks to the strong intersubband Coulomb scattering [844]. This prediction was later verified experimentally by Zhang et al. [625], who studied the spin dynamics in a single-barrier heterostructure by time-resolved Kerr rotation. By applying a gate voltage, they 


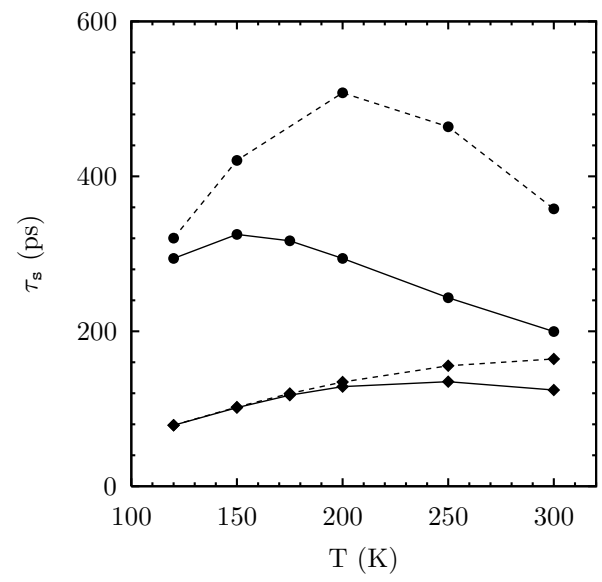

Figure 52: Spin dephasing time $\tau_{s} v s$. the background temperature $T$ for two GaAs (001) quantum wells with width $a=17.8 \mathrm{~nm}(\bullet)$ and $12.7 \mathrm{~nm}$ $(\checkmark)$. The solid curves are the spin dephasing time calculated with the lowest two subbands included and the dashed curves are those calculated with only the lowest subband. From Weng and Wu [844].

effectively manipulated the confinement of the second subband and the measured spin relaxation times of the first and second subbands are almost identical at large gate voltage as shown in Fig. 56 (c). The big deviations at small gate voltages are because that the wavefunctions of the second subband are extended due to the weak confinement and hence the intersubband Coulomb scattering becomes weaker.

Finally we address spin relaxation in systems with both the Dresselhaus and Rashba spin-orbit couplings. It has been first pointed out by Averkiev and Golub [194] that for (001) quantum well with identical Dresselhaus and Rashba spin-orbit coupling strengths, when the cubic Dresselhaus term is ignored, the effective magnetic field becomes fixed and is aligned along the (110) or (110) direction depending on the sign of the Rashba field. Therefore one obtains infinite spin lifetime if the spin polarization is parallel to the effective magnetic field direction. In reality, due to the presence of the cubic Dresselhaus term and/or the difference between the Dresselhaus and Rashba spin-orbit coupling strengths, the spin life time along the (110) or (110) direction is still finite. But there is strong anisotropy of the spin lifetime along different spin polarizations. Cheng and Wu studied this anisotropy under identical linear Dresselhaus and Rashba coupling strengths, but with the cubic Dresselhaus term included [597]. Stich et al. applied a magnetic field parallel to the (110) and ( $\overline{1} 10)$ directions and obtained large magnetoanisotropy of electron spin dephasing in a high mobility (001) GaAs quantum well [199]. The initial spin polarization was obtained by optical pumping and is therefore along the growth direction of the quantum well. The experimental setup is illustrated in Fig. 57(a). Due to the mixing of the different anisotropic spin orientations by the magnetic field, they observed different magnetic field dependences of the spin dephasing time along the (110) and (110) directions, as shown in Fig. 57(d). The maximum and minimum are determined by the relative strengths of the Dresselhaus and Rashba terms. It is also seen that calculation based on the fully microscopic kinetic spin Bloch equations can well reproduce the experimental findings.

\subsubsection{Spin relaxation and dephasing in n-type (001) GaAs quantum wells far away from the equilibrium}

Due to the full account of the Coulomb scattering, the kinetic spin Bloch equation approach can be applied to study spin system far away from the equilibrium. By so called far away from the equilibrium, one refers to the spin dynamics with large spin polarization and/or with large in-plane electric field where the hot-electron effect becomes significant.

Weng and Wu first studied the spin relaxation with large initial spin polarization [44] and discovered marked increase of the spin relaxation/dephasing time with increasing spin polarization as shown in Fig. 58 a). It is noted that the plotted spin dephasing time is the inverse of the spin dephasing rate. Therefore one expects a markedly increased total spin lifetime. This enhancement is more pronounced in samples with larger mobility, as shown in Fig. 58(b). The physics leading to such an increase was identified from the Coulomb Hartree-Fock term. In the presence of the 


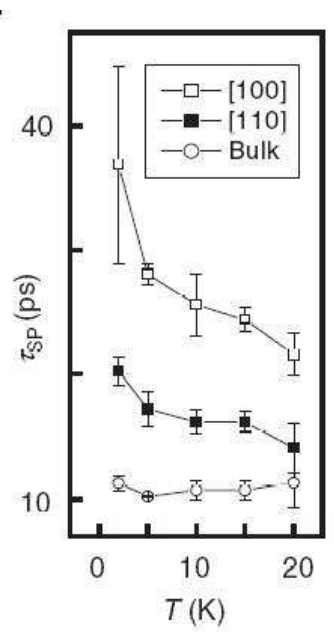

Figure 53: Measured temperature dependence of the spin relaxation time for two-dimensional $n$-InAsGa channels with a width of $1.5 \mu$ m, where the cubic in $\mathbf{k}$ terms of the Dresselhaus term dominate the spin relaxation. Open and filled squares represent data of channels along [100] and [110], while the open circles depict the spin relaxation time of the unstructured quantum well. From Holleitner et al. [655].

spin polarization, the Coulomb Hartree-Fock term provides an effective magnetic field along the $z$-axis:

$$
B_{z}^{\mathrm{HF}}=\frac{1}{g \mu_{B}} \sum_{\mathbf{q}} V_{\mathbf{q}}\left(f_{\mathbf{k}+\mathbf{q} \frac{1}{2}}-f_{\mathbf{k}+\mathbf{q}-\frac{1}{2}}\right) .
$$

This effective magnetic field can be as large as $20 \mathrm{~T}$ and effectively blocks the spin precession as the energies of the spin-up and -down electrons are no longer detuned. This can be seen clearly from Fig. 59 that by removing the longitudinal component of the Hartree-Fock term, the polarization dependence of the spin relaxation time becomes pretty mild. It is noted that unlike a real magnetic field which breaks the time-reversal symmetry, the Coulomb interaction does not. This can be seen from the fact that the Hartree-Fock term cancels each other after performing the summation over all the $\mathbf{k}$ states [Eq. (143)]. Therefore the spin relaxation time with a large effective magnetic field is still finite. As the effective magnetic field decreases with temperature, Weng and Wu pointed out that the spin relaxation time should decrease with increasing temperature at large spin polarization, in contrast to the case with small spin polarization [as shown in Fig. 51 (b)].

These predictions have been realized experimentally by Stich et al. [41, 42] and Zhang et al. [327]. By changing the intensity of the circularly polarized lasers, Stich et al. measured the spin dephasing time in a high mobility $n$-type GaAs quantum well as a function of initial spin polarization as shown in Fig. 60. (a). Indeed they observed an increase of the spin dephasing time with the increased spin polarization, and the theoretical calculation based on the kinetic spin Bloch equations nicely reproduced the experimental findings when the Hartree-Fock term was included [Fig. 60) (b)]. It was also shown that when the Hartree-Fock term is removed, one does not see any increase of the spin dephasing time [Fig. 60.c)]. Later, they further improved the experiment by replacing the circular-polarized laser pumping with the elliptic polarized laser pumping. By doing so, they were able to vary the spin polarization without changing the carrier density. Figure. 61 shows the measured spin dephasing times as function of initial spin polarization under two fixed pumping intensities, together with the theoretical calculations with and without the Coulomb Hartree-Fock term. Again the spin dephasing time increases with the initial spin polarization as predicted and the theoretical calculations with the Hartree-Fock term are in good agreement with the experimental data. Moreover, Stich et al. also confirmed the prediction of the temperature dependences of the spin dephasing time at low and high spin polarizations [44]. Figure 62 (a) shows the measured temperature dependences of the spin dephasing time at different initial spin polarizations. As predicted, the spin dephasing time increases with increasing temperature at small spin polarization but decreases at large spin polarization. The theoretical calculations also nicely reproduced the experimental data. The hot-electron temperatures in the calculation were taken from the experiment [Fig.662(b)]. The effective magnetic 

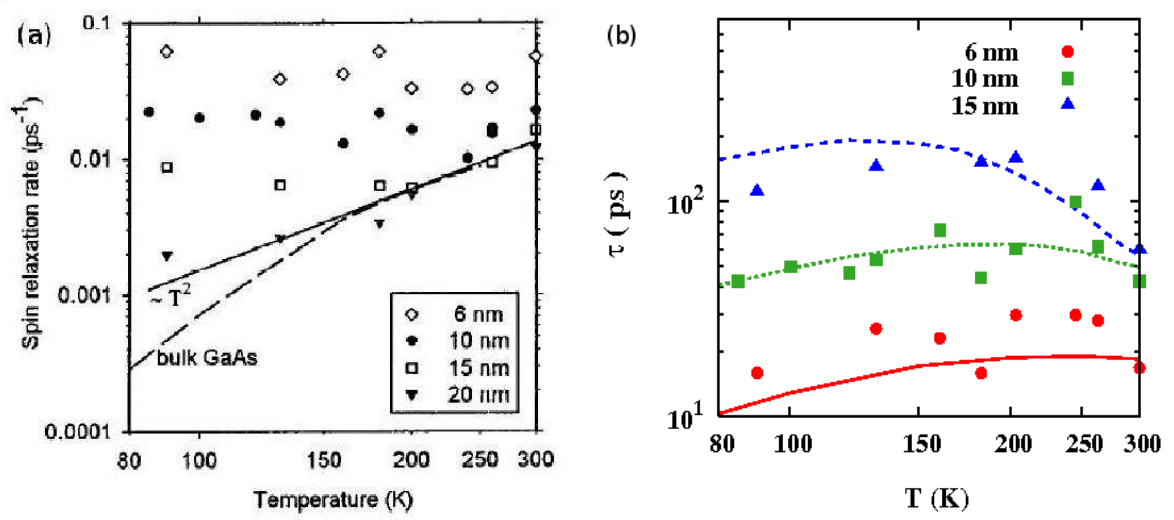

Figure 54: Electron spin relaxation in intrinsic $\mathrm{GaAs} / \mathrm{Al}_{0.35} \mathrm{Ga}_{0.65}$ As quantum wells of a variety of widths. (a) Experimental results from Malinowski et al. 603]. The solid line represents a quadratic dependence of relaxation rate on temperature. The dashed curve is data on electron spin relaxation in bulk GaAs from Maruschak et al. (as reproduced by Meier and Zakharchenya [3]). From Malinowski et al. [603]. (b) Theoretical calculation via the kinetic spin Bloch equation approach (curves). The dots are data from (a). The parameters are $N_{e}=N_{h}=4 \times 10^{11} \mathrm{~cm}^{-2}$, $N_{i}=0.1 N_{e}, \gamma_{\mathrm{D}}=0.0162 \mathrm{eV} \cdot \mathrm{nm}^{3}$. Here $N_{e}$ and $N_{h}$ are the electron and hole densities respectively, $N_{i}$ is the impurity density, and $\gamma_{\mathrm{D}}$ is the spin-orbit splitting parameter.

field from the Hartree-Fock term has been measured by Zhang et al. [327] from the sign switch of the Kerr signal and the phase reversal of Larmor precessions with a bias voltage in a GaAs heterostructure.

Korn et al. [326] also estimated the average effect by applying an external magnetic field in the Faraday configuration, as shown in Fig. 63) (a) for the same sample reported above [41, 42]. They compared the spin dephasing times of both large and small spin polarizations as function of external magnetic field. Due to the effective magnetic field from the Hartree-Fock term, the spin relaxation times are different under small external magnetic field but become identical when the magnetic field becomes large enough. From the merging point, they estimated the mean value of the effective magnetic field is below $0.4 \mathrm{~T}$. They further showed that this effective magnetic field from the Hartree-Fock term cannot be compensated by the external magnetic field, because it does not break the time-reversal symmetry and is therefore not a genuine magnetic field, as said above. This can be seen from Fig. 63 (b) that the spin relaxation time at large spin polarization shows identical external magnetic field dependences when the magnetic field is parallel or antiparallel to the growth direction.

The spin dynamics in the presence of a high in-plane electric field was first studied by Weng et al. [569] in GaAs quantum well with only the lowest subband by solving the kinetic spin Bloch equations. To avoid the "runaway" effect [849, 850], the electric field was calculated upto $1 \mathrm{kV} / \mathrm{cm}$. Then Weng and Wu further introduced the second subband into the model and the in-plane electric field was increased upto $3 \mathrm{kV} / \mathrm{cm}$ [844]. Zhang et al. included $L$ valley and the electric field was further increased upto $7 \mathrm{kV} / \mathrm{cm}$ [845].

The effect of in-plane electric field to the spin relaxation in system with strain was investigated by Jiang and Wu [570]. Zhou et al. also investigated the electric-field effect at low lattice temperatures [372].

The in-plane electric field leads to two effects. (i) It shifts the center-of-mass of electrons to $\mathbf{k}_{d}=m^{*} \mathbf{v}_{d}=m^{*} \mu \mathbf{E}$ with $\mu$ representing the mobility, which further induces an effective magnetic field via the D'yakonov-Perel' term [569]. The induced effective magnetic field can be estimated by

$$
\mathbf{B}_{\mathrm{tot}}=\mathbf{B}+\mathbf{B}^{*}=\mathbf{B}+\frac{1}{g \mu_{B}} \int d \mathbf{k}\left(f_{\mathbf{k} \frac{1}{2}}-f_{\mathbf{k}-\frac{1}{2}}\right) \boldsymbol{\Omega}(\mathbf{k}) / \int d \mathbf{k}\left(f_{\mathbf{k} \frac{1}{2}}-f_{\mathbf{k}-\frac{1}{2}}\right) .
$$

(ii) The in-plane electric field also leads to the hot-electron effect [851]. By taking the electron distribution function as the drifted Fermi function $f_{\mathbf{k} \sigma}=\left\{\exp \left[\left(\left(\mathbf{k}-m^{*} \mathbf{v}_{d}\right)^{2} / 2 m^{*}-\mu_{\sigma}\right) / k_{B} T_{e}\right]+1\right\}^{-1}$ in the case with only the lowest subband included, the effective magnetic field for small spin polarization can be roughly estimated as [569]

$$
B^{*}=\gamma_{\mathrm{D}} m^{* 2} v_{d}\left\{E_{f} /\left[2\left(1-e^{-E_{f} / k_{B} T_{e}}\right)\right]-E_{c}\right\} / g \mu_{B},
$$




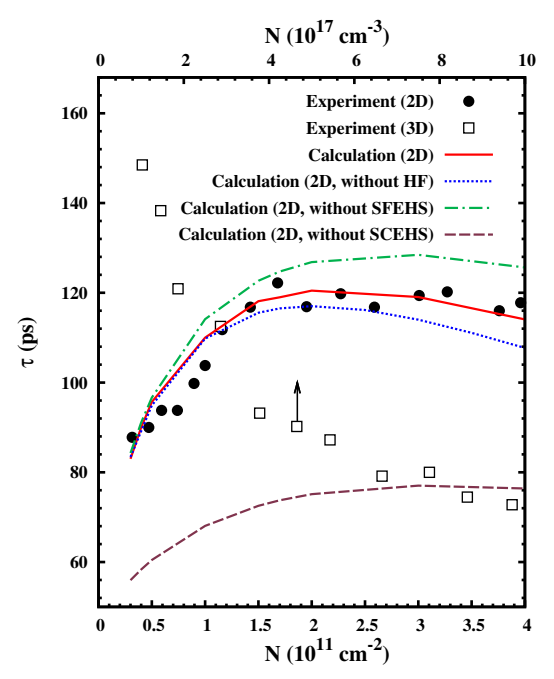

Figure 55: Carrier density dependence of electron spin relaxation time $\tau$ at room temperature. Dots: experimental data in a GaAs (001) quantum well (2D) with a width of $10 \mathrm{~nm}$; open squares: experimental data in bulk material (3D). Solid curve: full theoretical calculation; chain curve: theoretical calculation without the spin-flip electron-hole scattering (SFEHS); dashed curve: theoretical calculation without the spin-conserving electron-hole scattering (SCEHS); dotted curve: theoretical calculation without the Coulomb Hartree-Fock term. Note the scale of the bulk data is on the top frame of the figure. From Teng et al. [609].

with $E_{f}$ and $E_{c}$, the Fermi and confinement energies of the quantum well, respectively. $T_{e}$ is the hot-electron temperature. Figure 64 a) shows the spin precession in the presence of a high electric field in a $15 \mathrm{~nm}$ quantum well at $T=120 \mathrm{~K}$. One finds that the spin precesses even in the absence of any external magnetic field and the spin precession frequency changes with the direction of the electric field in the presence of an external magnetic field. The effective magnetic field deduced from the spin precessions in Fig. 64 a) is plotted in Fig. 64 b), which is in good agreement with the corresponding result from Eq. (188).

The spin relaxation time can be effectively manipulated by the in-plane electric field. When only the lowest subband is considered, the electric field influences the spin relaxation via concurrent effects of the increase of the inhomogeneous broadening by driving electrons to higher momentum states and the increase/decrease (depending on the type of scattering and also the degenerate/nondegenerate limit) of scattering from the hot-electron temperature. Weng et al. reported rich electric field dependence of the spin relaxation time under various conditions [569]. When the electric field is high enough so that higher subbands and/or valleys are involved, due to the different spin-orbit coupling strengths and/or effective masses in different subbands and valleys, the spin relaxation time can be manipulated more effectively [844, 845]. This can be seen from Fig. 65 (a) and (b) where spin relaxation times are plotted against the in-plane electric field in GaAs quantum wells, with the second subband and $L$ valley being populated at high electric field, respectively. The inhomogeneous broadening comes from the Dresselhaus terms. In $\Gamma$ valley, it reads

$$
\mathbf{h}_{\Gamma}\left(\mathbf{k}_{\Gamma}\right)=\frac{\gamma}{2}\left(k_{\Gamma x}\left(k_{\Gamma y}^{2}-\left\langle k_{\Gamma z}^{2}\right\rangle\right), k_{\Gamma y}\left(\left\langle k_{\Gamma z}^{2}\right\rangle-k_{\Gamma x}^{2}\right),\left\langle k_{\Gamma z}\right\rangle\left(k_{\Gamma x}^{2}-k_{\Gamma y}^{2}\right)\right),
$$

and in $L$-valley, it reads [106, 142 ]

$$
\mathbf{h}_{L_{i}}\left(\mathbf{k}_{L_{i}}\right)=\beta\left(k_{L_{i} x}, k_{L_{i} y},\left\langle k_{L_{i} z}\right\rangle\right) \times \hat{\mathbf{n}}_{i} .
$$

Here $\hat{\mathbf{n}}_{i}$ is the unit vector along the longitudinal principle axis of $L_{i}$ valley. Note the coordinate system of above equations has been given in Ref. [852]. $\mathbf{k}_{\Gamma}=\mathbf{k}$ and $\mathbf{k}_{L_{i}}=\mathbf{k}-\mathbf{K}_{L_{i}}^{0}$ with $\mathbf{K}_{L_{i}}^{0}=\frac{\pi}{a_{0}}(1, \pm 1, \pm 1) . a_{0}$ is the lattice constant. $\left\langle k_{\lambda z}\right\rangle\left(\left\langle k_{\lambda z}^{2}\right\rangle\right)$ represents the average of the operator $-i \partial / \partial z-K_{\lambda z}^{0}\left[\left(-i \partial / \partial z-K_{\lambda z}^{0}\right)^{2}\right]$ over the electron states in $\lambda$-valley. Under the infinite-depth assumption, $\left\langle n\left|k_{\lambda z}^{2}\right| n\right\rangle=\frac{n^{2} \pi^{2}}{a^{2}}$ with $n$, the subband index and $a$, the well width. Therefore, when the linear- $k$ terms are dominant, the inhomogeneous broadening of the second subband in $\mathbf{h}_{\Gamma}\left(\mathbf{k}_{\Gamma}\right)$ is four times as large as the first subband. Also a tight-binding calculation by Fu et al. [142] indicates $\beta=0.026 \mathrm{eV} \cdot \mathrm{nm}$, which is much larger than $\frac{1}{2} \gamma_{\mathrm{D}}\left\langle k_{\Gamma z}^{2}\right\rangle \approx 0.001 \mathrm{eV} \cdot \mathrm{nm}$ when only the lowest subband of the $\Gamma$-valley is considered and the well 


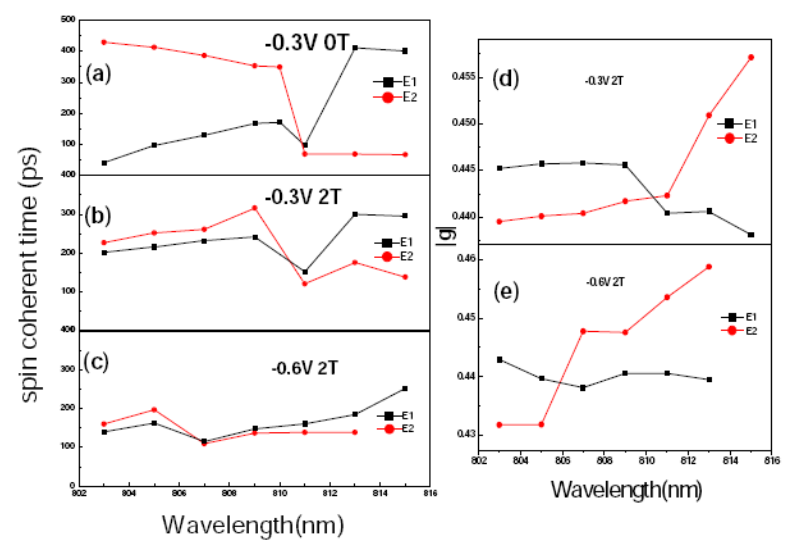

Figure 56: (a), (b) and (c) Measured spin coherence times for the 1st and 2nd subbands of a single-barrier GaAs/AlAs heterostructure, as the wavelength varies for three different conditions: $-0.3 \mathrm{~V}, 0 \mathrm{~T} ;-0.3 \mathrm{~V}, 2 \mathrm{~T}$ and $-0.6 \mathrm{~V}, 2 \mathrm{~T}$. The points are experimental results, and the curves are best fittings to the points. (d) and (e) Effective $g^{*}$ factors for the 1 st and 2 nd subbands plotted as a function of wavelength. From Zhang et al. [625].

width is set to be $7.5 \mathrm{~nm}[845]$. Therefore, the inhomogeneous broadenings of the higher subband and valley are much larger. It is therefore easy to understand that the spin relaxation time decreases with increasing electric field when more electrons are excited to higher subband and/or valley.

By simply looking at the inhomogeneous broadenings of $\Gamma$ and $L$ valleys, one may naively come to the conclusion that the spin relaxation rate of electrons in $L$ valley should be much faster than that in $\Gamma$ valley. Also by noticing the $g$-factor of $L$ valley $g_{L}=1.77$ [853], which is also much larger than that of $\Gamma$ valley, $g_{\Gamma}=-0.44$ [204], one may also speculate that the spin precession under a magnetic field in the Voigt configuration should be much faster in the $L$ valley than in the $\Gamma$ valley. Zhang et al. showed both are in fact incorrect [845]. They calculated the spin precessions of electrons in $\Gamma$ and $L$ valleys in GaAs quantum well with both high in-plane electric field and a magnetic filed in the Voigt configuration, and discovered that the spin precession frequencies as well as spin dephasing times of both valleys are identical to each other, as shown in Fig. 66, and the "effective" $g$-factor of the $L$ valley from the spin precession is 0.44 , which is in the same magnitude as that of the $\Gamma$ valley. The physics leading to these unexpected results was revealed due to the strong intervalley electron-phonon scattering. As shown in Fig. 67 where the spin precessions of an $n$-type (001) GaAs quantum well under electric field $E=2 \mathrm{kV} / \mathrm{cm}$ are plotted with all the scatterings included (a), without the intervalley electron-phonon scattering (b), without the intervalley electron-electron Coulomb scattering (c), and without intervalley scattering (d), respectively. It is seen that when the intervalley electron-phonon scattering is switched off, the spin polarization in the $L$ valleys decays pretty fast. This is in contrast to the multi-subband case addressed in the previous subsection where the inter-subband Coulomb scattering is the cause of the identical spin relaxation times of each subband.

\subsubsection{Effect of the Coulomb scattering to the spin dephasing and relaxation}

As addressed in the previous subsections, the Coulomb scattering can contribute to the spin dephasing and relaxation due to the D'yakonov-Perel' mechanism. A natural question would be how the Coulomb scattering changes the spin dephasing/relaxation time. Glazov and Ivchenko pointed out that the Coulomb scattering can prolong the spin dephasing/relaxation time limited by the D'yakonov-Perel' mechanism. Weng et al. compared the electron spin dephasing times in (001) GaAs quantum well with and without the Coulomb scattering in the presence of an in-plane electric field [569]. As shown in Fig. 68(a), in which the spin dephasing times with and without the Coulomb scattering are plotted against in-plane electric field. It is seen that adding Coulomb scattering always leads to a longer spin dephasing time both near (at $E \sim 0$ ) and far away from (with hot-electron effect) the equilibrium. This looks quite contradictory to the previous understanding of optical dephasing [833] where adding a Coulomb scattering always leads to a shorter optical dephasing time. Also as stated in Sec.5.2 that in the presence of the inhomogeneous broadening, adding a new scattering leads to a new dephasing channel. How can the additional "channel" leads to a longer spin dephasing time? To understand this, Lü et al. [363] put a scaling coefficient $\gamma$ in front of the D'yakonov-Perel' 

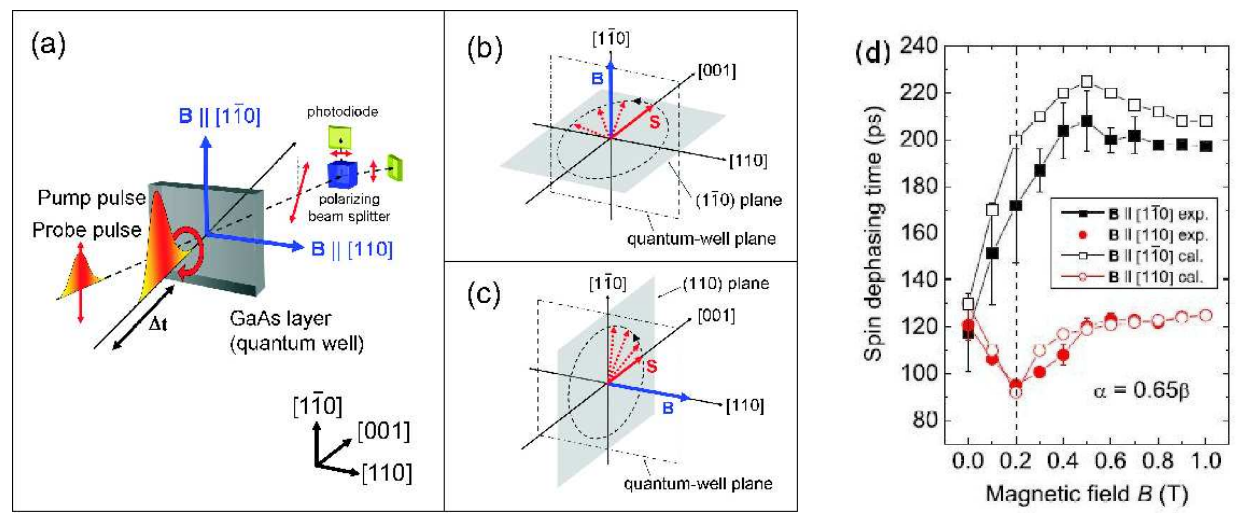

Figure 57: (a) Schematic of the time-resolved Faraday rotation experiment. In-plane magnetic fields are applied either in the [110] or [1]10] directions. (b) Sketch of the precession of optically oriented spins about a [110] in-plane magnetic field. (c) Sketch of the precession of optically oriented spins about a [110] in-plane magnetic field. (d) Comparison of the experimental (solid symbols) and theoretically calculated (open symbols) spin dephasing times for different in-plane magnetic-field directions in GaAs (001) quantum well. $\alpha$ and $\beta$ are the Rashba and Dresselhaus spin-orbit coefficients, respectively. From Stich et al. [199].

term and compared the spin dephasing times of electrons, heavy holes and light holes in (001) GaAs quantum well with and without the Coulomb scattering as function of $\gamma$. As shown in Fig. 68 b) and (c), for larger $\gamma$, i.e., in the weak scattering limit, adding Coulomb scattering always leads to a shorter spin dephasing time. However, for small $\gamma$, i.e., in the strong scattering limit, adding Coulomb scattering leads to a longer spin dephasing time. This can be understood as follows: In the weak scattering limit, the counter effect of the scattering to the inhomogeneous broadening is pretty weak, and the only effect of the scattering is an additional spin dephasing channel. However, in the strong scattering limit, the counter effect of the scattering is more pronounced and hence the spin dephasing time is increased by adding the Coulomb scattering. It is noted that $\gamma=1$ corresponds to the genuine case and from the figure one notices that electrons happen to be in the strong scatterign limit. Therefore adding the Coulomb scattering leads to a longer spin dephasing time. However, both heavy and light holes are in the weak scattering limit. Consequently adding the Coulomb scattering makes the spin dephasing time shorter. In fact, for optical dephasing problem, the inhomogeneous broadening in Eq. (140) happens to be in the weak scattering limit. Therefore, the Coulomb scattering always brings a shorter optical dephasing time [833].

The strong/weak scattering limit can be measured by $\xi \equiv\left|g^{*} \mu_{B} \boldsymbol{\Omega}(\mathbf{k})\right| \tau_{p}^{*}$. When $\xi \gg 1(\ll 1)$, the system is in the weak (strong) scattering limit. Here $\tau_{p}^{*}$ is the effective momentum scattering time from not only the carrier-phonon and carrier-impurity scatterings, but also from the Coulomb scattering [591, 615]. It is also noted that the regime of strong and weak scattering can be changed even for the same sample by temperature. It has been shown by Harley group [368, 591] and also later by Stich et al. [41, 199] that at low temperature electrons in high mobility (001) GaAs quantum well can be in the weak scattering limit. Also for holes in p-type (001) GaAs quantum wells, Lü et al. have shown that the system can be changed from the weak scattering limit to the strong one by impurity density 76 and temperature, etc. [363]. In fact, in the strong (weak) scattering limit, besides the Coulomb scattering, adding any scattering, can cause a longer (shorter) spin dephasing/relaxation time.

\subsubsection{Non-Markovian effect in the weak scattering limit}

As stated in Sec. 5.3, the scattering terms of the kinetic spin Bloch equations are given by Eqs. (159) and (160), which are time-integrals. By applying the Markovian approximation [Eq. [161)], one can carry out the time-integral and the scattering terms are simplified to Eq. (162), where one has the energy conservation [ $\delta$-functions in Eq. (161)] and the trace of the history disappears. This approximation is valid only in the strong scattering limit where the spin precession between two adjacent scatterings is negligible. However, in the weak scattering limit, electron spin

\footnotetext{
${ }^{76}$ It is noted that the impurity density should be $1 / 4$ of the value presented in Ref. [363].
} 

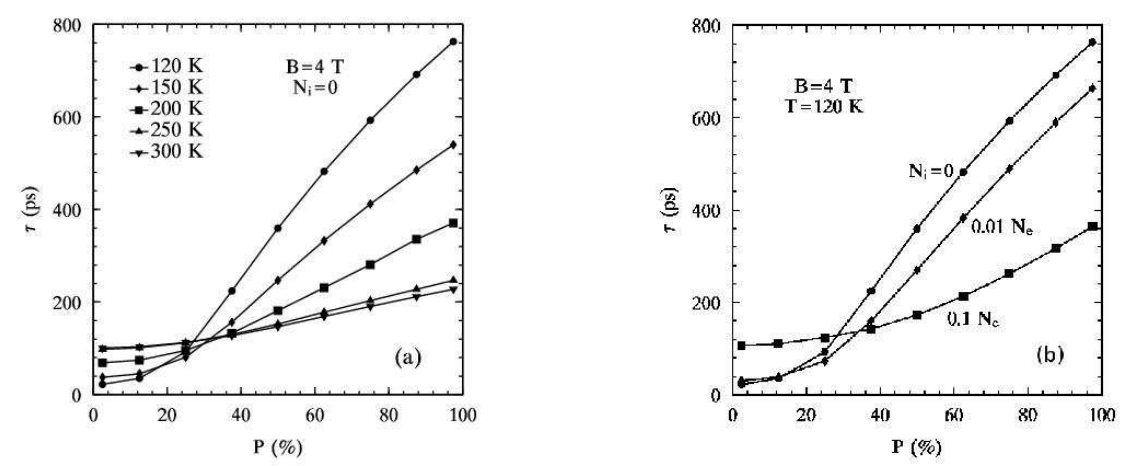

Figure 58: (a) Spin dephasing time $\tau$ vs. the initial spin polarization $P$ of electrons in GaAs (001) quantum well at different temperatures. The impurity density $N_{i}=0$. (b) Spin dephasing time $\tau$ vs. the initial spin polarization $P$ at $T=120 \mathrm{~K}$ for different impurity levels. Dots $(\bullet)$ : $N_{i}=0$; Diamonds $(\checkmark): N_{i}=0.01 N_{e}$; Squares ( $\left.\mathbf{\square}\right): N_{i}=0.1 N_{e}$. The curves are plotted for the aid of eyes. From Weng and Wu [44].

can experience many precessions between two scattering events, and the Markovian approximation is invalid. In this circumstance one has to trace back the history by carrying out the time integral. Hence the dynamics becomes non-Markovian.

Glazov and Sherman first investigated the electron spin relaxation in GaAs quantum wells under a strong magnetic field by the Monte Carlo simulation [381]. They reported a longer non-Markovian spin relaxation time [381]. Zhang and $\mathrm{Wu}$ studied the non-Markovian hole spin dynamics in $p$-type GaAs quantum wells using kinetic spin Bloch equation approach [854]. By performing time derivative of the time integral in Eq. (160), they transferred the integrodifferential kinetic spin Bloch equations in non-Markovian limit to a larger set of differential equations. They compared the time evolutions of the incoherently summed spin coherence of heavy holes in both the Markovian and non-Markovian limits at different Rashba strengths. From the decay of the incoherently summed spin coherence, one may extract $T_{2}{ }_{77}^{7}$ As shown in Fig.69 d), in the strong scattering limit, both approaches yield the same spin dephasing time. Nevertheless, in the weak scattering limit, the non-Markovian kinetics gives a longer hole spin dephasing time, which is in good agreement with the prediction of Glazov and Sherman [381]. The physics behind this phenomena is easily understood from the nature of non-Markovian scattering, which keeps some memory of the coherence during the scattering. What is most important is that they predicted quantum spin beats when the spin precession time is comparable to the momentum scattering time. The beats are solely from the non-Markovian effect (or memory effect), with the beating frequency being exactly the longitudinal optical-phonon frequency [see Fig. 69 b) and (c)]. The quantum spin beats are similar to the longitudinal optical-phonon quantum beats in optical four wave mixing signal [855]. However, a measurement of these quantum spin beats cannot be performed by the regular Faraday/Kerr rotation measurement. A possible way is through the spin echo measurement.

\subsubsection{Electron spin relaxation due to the Bir-Aronov-Pikus mechanism in intrinsic and p-type GaAs quantum wells}

It has long been believed in the literature that the Bir-Aronov-Pikus mechanism is dominant at low temperature in $p$-type samples and has important contribution to intrinsic sample with high photo-excitation [574, 687-689, 693, 856, 857]. These conclusions were made based on Eq. (66) from the Fermi Golden rule, which in (001) quantum well reads [109]

$$
\frac{1}{\tau_{\mathrm{BAP}}(\mathbf{k})}=4 \pi \sum_{\mathbf{k}^{\prime} \mathbf{q}} \delta\left(\varepsilon_{\mathbf{k}-\mathbf{q}}^{e}-\varepsilon_{\mathbf{k}}^{e}+\varepsilon_{\mathbf{k}^{\prime}}^{h}-\varepsilon_{\mathbf{k}^{\prime}-\mathbf{q}}^{h}\right)|M(\mathbf{K}-\mathbf{q})|^{2} f_{\mathbf{k}^{\prime}-\mathbf{q}}^{h}\left(1-f_{\mathbf{k}}^{h}\right)
$$

\footnotetext{
${ }^{77}$ Instead of $T_{2}^{*}$ which is measured from the Faraday/Kerr rotation experiment.
} 


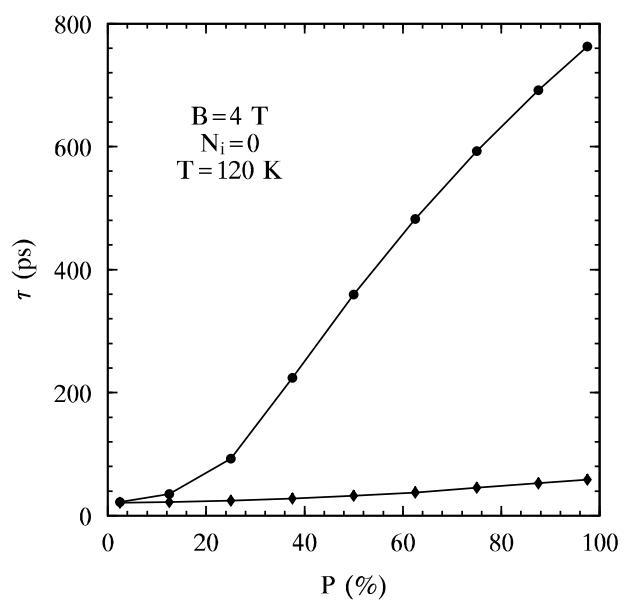

Figure 59: Spin dephasing time $\tau$ vs. the initial spin polarization $P$ for $T=120 \mathrm{~K}$ and $N_{i}=0$ in GaAs (001) quantum well. Dots $(\bullet)$ : With the longitudinal component of the Hartree-Fock term included; Diamonds $(\checkmark)$ : Without the longitudinal component of the Hartree-Fock term. The curves are plotted for the aid of eyes. From Weng and Wu [44].

with $\mathbf{K}=\mathbf{k}+\mathbf{k}^{\prime}$ and

$$
|M(\mathbf{k}-\mathbf{q})|^{2}=\frac{9 \Delta E_{L T}^{2}}{16\left|\phi_{3 D}(0)\right|^{4}}\left[\sum_{q_{z}} \frac{f_{e x}\left(q_{z}\right)(\mathbf{K}-\mathbf{q})^{2}}{q_{z}^{2}+(\mathbf{K}-\mathbf{q})^{2}}\right]^{2}
$$

Here $\Delta E_{L T}$ is the longitudinal-transverse splitting in bulk. $\left|\phi_{3 D}(0)\right|^{2}=1 /\left(\pi a_{0}^{3}\right)$ denotes the bulk exciton state at zero relative distance. $f_{e x}\left(q_{z}\right)$ is the form factor [109, 118]. In the meantime, the spin relaxation time from the D'yakonovPerel' mechanism used for comparison was calculated without the Coulomb scattering which has been demonstrated to be important in the previous sections.

Zhou and $\mathrm{Wu}$ reexamined the problem using the fully microscopic kinetic spin Bloch equation approach [109]. They constructed the kinetic spin Bloch equations in intrinsic and $p$-type (001) GaAs quantum wells with the electronphonon, electron-impurity, electron-electron Coulomb and electron-heavy hole Coulomb scatterings explicitly included. The electron-heavy hole Coulomb scattering includes the spin-conserving scattering and the spin-flip scattering (the Bir-Aronov-Pikus term). They also extended the screening under the random phase approximation Eq. (169) to include the contribution from heavy holes:

$$
\epsilon(\mathbf{q})=1-\sum_{q_{z}} v_{Q} f_{e}\left(q_{z}\right) \sum_{\mathbf{k}, \sigma} \frac{f_{\mathbf{k}+\mathbf{q}, \sigma}-f_{\mathbf{k}, \sigma}}{\varepsilon_{\mathbf{k}+\mathbf{q}}^{e}-\varepsilon_{\mathbf{k}}^{e}}-\sum_{q_{z}} v_{Q} f_{h}\left(q_{z}\right) \sum_{\mathbf{k}^{\prime}, \sigma} \frac{f_{\mathbf{k}^{\prime}+\mathbf{q}, \sigma}^{h}-f_{\mathbf{k}^{\prime}, \sigma}^{h}}{\varepsilon_{\mathbf{k}^{\prime}+\mathbf{q}}^{h}-\varepsilon_{\mathbf{k}^{\prime}}^{h}},
$$

where $f_{e}\left(q_{z}\right)$ and $f_{h}\left(q_{z}\right)$ are the form factors [109]. The scattering terms from the spin-flip electron-heavy hole exchange interaction (Bir-Aronov-Pikus term) read

$$
\begin{aligned}
\left.\frac{\partial f_{\mathbf{k}, \sigma}}{\partial t}\right|_{\mathrm{BAP}}=- & 2 \pi \sum_{\mathbf{k}^{\prime}, \mathbf{q}} \delta\left(\varepsilon_{\mathbf{k}-\mathbf{q}}^{e}-\varepsilon_{\mathbf{k}}^{e}+\varepsilon_{\mathbf{k}^{\prime}}^{h}-\varepsilon_{\mathbf{k}^{\prime}-\mathbf{q}}^{h}\right)|M(\mathbf{K}-\mathbf{q})|^{2} \\
& \times\left[\left(1-f_{\mathbf{k}^{\prime}, \sigma}^{h}\right) f_{\mathbf{k}^{\prime}-\mathbf{q},-\sigma}^{h} f_{\mathbf{k}, \sigma}\left(1-f_{\mathbf{k}-\mathbf{q},-\sigma}\right)-f_{\mathbf{k}^{\prime}, \sigma}^{h}\left(1-f_{\mathbf{k}^{\prime}-\mathbf{q},-\sigma}^{h}\right)\left(1-f_{\mathbf{k}, \sigma}\right) f_{\mathbf{k}-\mathbf{q},-\sigma}\right], \\
\left.\frac{\partial \rho_{\mathbf{k}}}{\partial t}\right|_{\mathrm{BAP}}=- & \pi \sum_{\mathbf{k}^{\prime}, \mathbf{q}, \sigma} \delta\left(\varepsilon_{\mathbf{k}-\mathbf{q}}^{e}-\varepsilon_{\mathbf{k}}^{e}+\varepsilon_{\mathbf{k}^{\prime}}^{h}-\varepsilon_{\mathbf{k}^{\prime}-\mathbf{q}}^{h}\right)|M(\mathbf{K}-\mathbf{q})|^{2} \\
& \times\left[\left(1-f_{\mathbf{k}^{\prime}, \sigma}^{h}\right) f_{\mathbf{k}^{\prime}-\mathbf{q},-\sigma}^{h}\left(1-f_{\mathbf{k}-\mathbf{q},-\sigma}\right) \rho_{\mathbf{k}}+f_{\mathbf{k}^{\prime}, \sigma}^{h}\left(1-f_{\mathbf{k}^{\prime}-\mathbf{q},-\sigma}^{h}\right) f_{\mathbf{k}-\mathbf{q}, \sigma} \rho_{\mathbf{k}}\right] .
\end{aligned}
$$



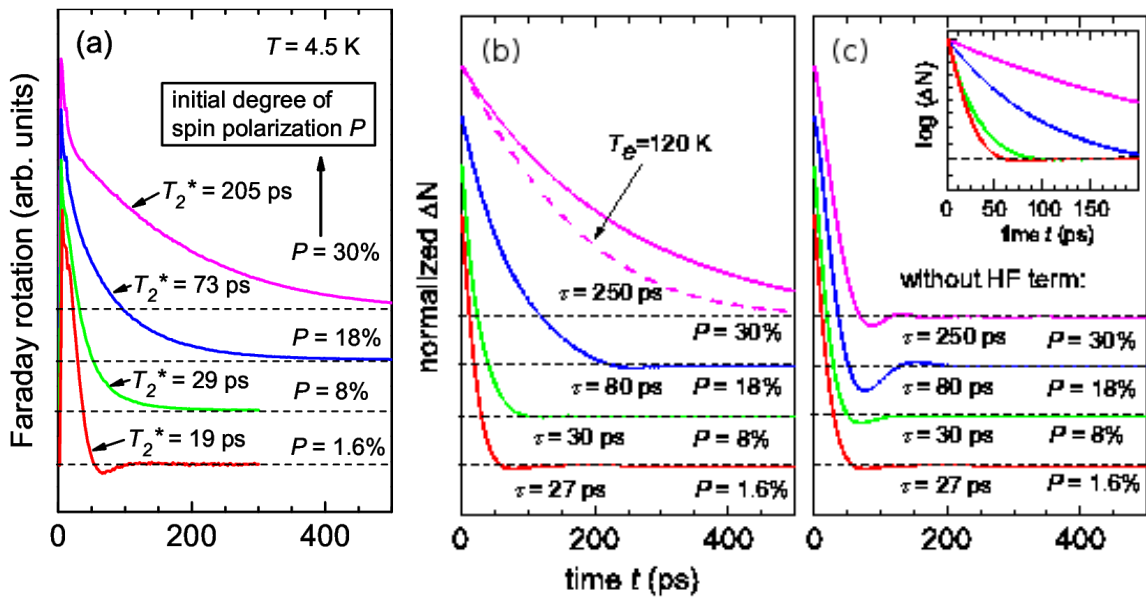

Figure 60: (a) Normalized time-resolved Faraday rotation traces for different degrees of initial electron spin polarization, $P$ in GaAs (001) quantum well. The total densities of electrons, $n_{t o t}=n_{e}+n_{p h}^{\text {tot }}$, are $2.19,2.66,3.83,8.39$ (in units of $10^{11} \mathrm{~cm}^{-2}$ ) for $P=1.6 \%, 8 \%, 18 \%, 30 \%$, respectively. (b) Calculated spin decay curves for the experimental parameters, like initial spin polarization, total electron densities, electron mobility, and temperature $T=4.5 \mathrm{~K}$ (solid lines). In the calculation, a phenomenological decay time is incorporated as a single fitting parameter $\tau$ (see text). The dashed curve for $P=30 \%$ is calculated for a hot electron temperature of $T_{e}=120 \mathrm{~K}$. (c) Same as (b) but calculated without the Hartree-Fock term. In particular for large $P$ the decay is much faster than in the experiments [cf. (a)]. The inset shows the data, displayed in (b), in a semilogarithmic plot. At low $P$ the zero-field oscillations are superimposed. From Stich et al. [41].

It is noted from Eq. (194) that there are quadratic terms in the sense of the electron distribution function $f_{\mathbf{k} \sigma}$. This immediately implies that one cannot recover Eq. (191) from the Fermi Golden rule. The only way to recover Eq. (191) is under the elastic scattering approximation: $\varepsilon_{\mathbf{k}-\mathbf{q}}^{e} \approx \varepsilon_{\mathbf{k}}^{e}$, and $\varepsilon_{\mathbf{k}^{\prime}}^{h} \approx \varepsilon_{\mathbf{k}^{\prime}-\mathbf{q}}^{h}$ where the quadratic terms exactly cancel each other and the spin relaxation time is given by Eq. (191). It is well known that the elastic scattering approximation is valid only in the non-degenerate regime at high temperatures. When the temperature is low enough so that electrons are degenerate, the elastic scattering approximation is not valid any more. Therefore the quadratic terms become unnegligible. In fact, the terms $\left(1-f_{\mathbf{k}-\mathbf{q},-\sigma}\right)$ and $\left(1-f_{\mathbf{k} \sigma}\right)$ in Eq. (194) come from the Pauli blocking, which reduces the spin relaxation rate. Therefore the Bir-Aronov-Pikus mechanism becomes less important at low temperatures when the Pauli blocking becomes important. This effect was missing in the literature [574, 687-689, 693, 856, 857]. In Fig. 70 (a), the spin relaxation times due to the Bir-Aronov-Pikus mechanism in intrinsic GaAs quantum well calculated from the full spin-flip electron-hole exchange scattering [Eq. (194)] are compared with those without the Pauli blocking at different pumping densities. It is seen that the previous calculations based on Eq. (191) always overestimate the importance of the Bir-Aronov-Pikus mechanism at low temperatures. In Fig. 70(b), the spin relaxation times of intrinsic GaAs quantum wells due to the Bir-Aronov-Pikus mechanism and the D'yakonov-Perel' mechanism are compared at different photo-excitations. It is seen that instead of being important at low temperature, the Bir-AronovPikus mechanism is even negligible at low temperature when the Pauli blocking becomes important. Two errors in the previous treatment may cause the incorrect conclusion that the Bir-Aronov-Pikus mechanism is dominant at low temperature: (i) The previous theory overlooked the Pauli blocking and hence overestimated the importance of the Bir-Aronov-Pikus mechanism; (ii) It overlooked the contribution of the Coulomb scattering to the D'yakonov-Perel' mechanism and thus underestimated the importance of the D'yakonov-Perel' mechanism. It is also noted that the temperature at which the Bir-Aronov-Pikus may have some contribution is around the hole Fermi temperature. Similar conclusions were also obtained in $p$-type GaAs quantum wells [109].

Later, Zhou et al. performed a thorough investigation of electron spin relaxation in $p$-type (001) GaAs quantum wells by varying impurity, hole and photo-excited electron densities over a wide range of values [112], under the idea that very high impurity density and very low photo-excited electron density may effectively suppress the importance of the D'yakonov-Perel' mechanism and the Pauli blocking. Then the relative importance of the Bir-Aronov-Pikus and D'yakonov-Perel' mechanisms may be reversed. This indeed happens as shown in the phase-diagram-like picture 

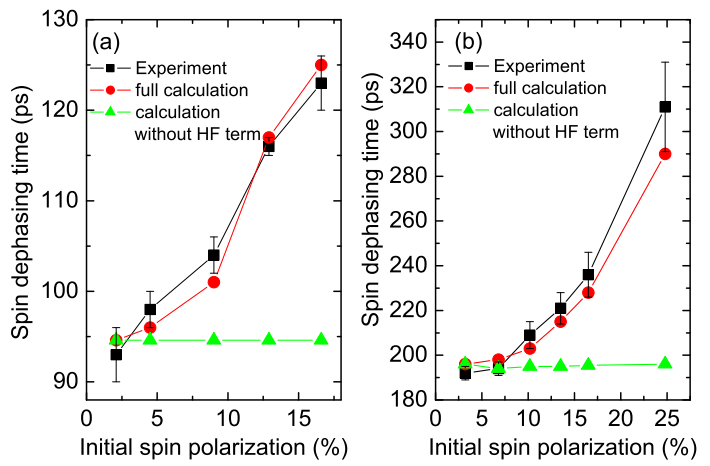

Figure 61: GaAs (001) quantum well: (a) The spin dephasing times as a function of initial spin polarization for constant, low excitation density and variable polarization degree of the pump beam. The measured spin dephasing times are compared to calculations with and without the HartreeFock (HF) term, showing its importance. (b) The spin dephasing times measured and calculated for constant, high excitation density and variable polarization degree. From Stich et al. [42].
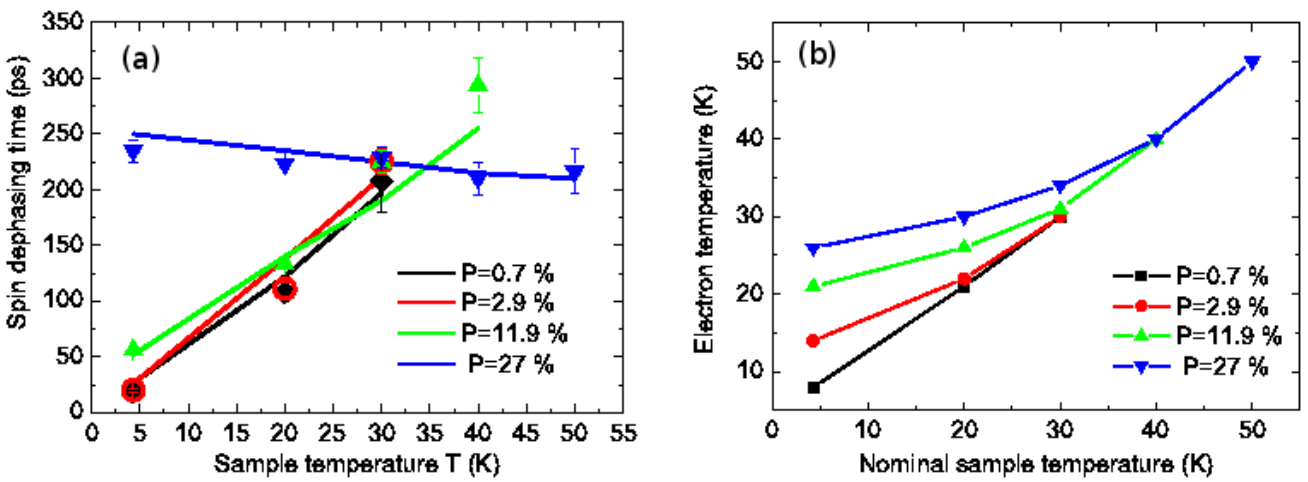

Figure 62: GaAs (001) quantum well: (a) Spin dephasing time as a function of sample temperature, for different initial spin polarizations. The measured data points are represented by solid points, while the calculated data are represented by lines of the same colour. (b) Electron temperature determined from intensity-dependent photoilluminance measurements as a function of the nominal sample temperature, for different pump beam fluence and initial spin polarization, under experimental conditions corresponding to the measurements shown in (a). The measured data points are represented by solid points, while the curves serve as guide to the eye. From Stich et al. [42].

in Fig. 71 where the relative importance of the Bir-Aronov-Pikus and D'yakonov-Perel' mechanisms is plotted as function of hole density and temperature at low and high impurity densities and photo-excitation densities. For the situation of high hole density they even included multi-hole subbands as well as the light hole band. It is interesting to see from the figures that at relatively high photo-excitations, the Bir-Aronov-Pikus mechanism becomes more important than the D'yakonov-Perel' mechanism only at high hole densities and high temperatures (around hole Fermi temperature) when the impurity is very low [zero in Fig. 71 (a)]. Impurities can suppress the D'yakonov-Perel' mechanism and hence enhance the relative importance of the Bir-Aronov-Pikus mechanism. As a result, the temperature regime is extended, ranging from the hole Fermi temperature to the electron Fermi temperature for high hole density. When the photo-excitation is weak so that the Pauli blocking is less important, the temperature regime where the Bir-Aronov-Pikus mechanism is important becomes wider compared to the high excitation case. In particular, if the impurity density is high enough and the photo-excitation is so low that the electron Fermi temperature is below the lowest temperature of the investigation, the Bir-Aronov-Pikus mechanism can dominate the whole temperature regime of the investigation at sufficiently high hole density, as shown in Fig. 71)(d). The corresponding spin relaxation times of each mechanism under high or low impurity and photo-excitation densities are demonstrated in Fig. 72, They also discussed the density dependences of spin relaxation with some intriguing properties related to the high hole subbands 

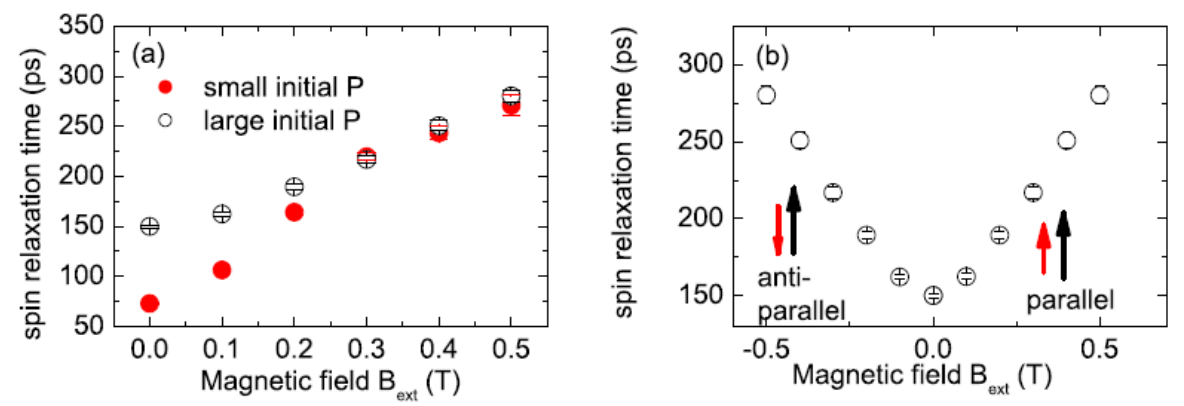

Figure 63: (a) Spin dephasing times as a function of an external magnetic field perpendicular to the quantum well plane for small and large initial spin polarization in GaAs (001) quantum well. (b) Same as (a) for large initial spin polarization and both polarities of the external magnetic field. From Korn et al. [326].
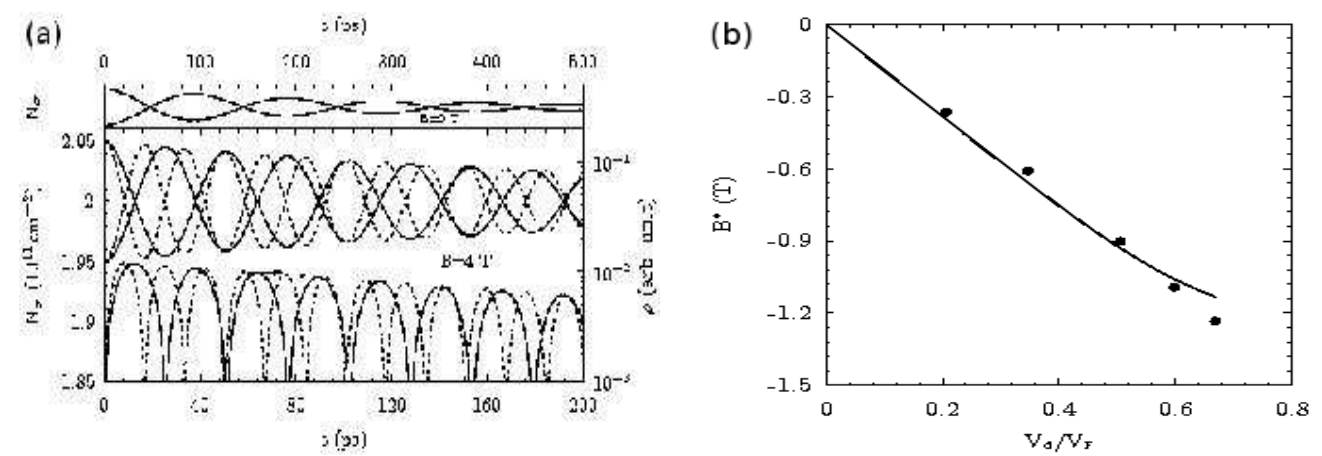

Figure 64: (a) Electron densities of spin-up and -down electrons and the incoherently summed spin coherence $\rho$ vs. time $t$ for GaAs (001) quantum well (the well width is $15 \mathrm{~nm}$ ) with initial spin polarization $P=2.5 \%$ under different electric fields $E=0.5 \mathrm{kV} / \mathrm{cm}$ (solid curves) and $E=-0.5 \mathrm{kV} / \mathrm{cm}$ (dashed curves). Top panel: $B=0 \mathrm{~T}$; bottom panel: $B=4 \mathrm{~T}$. Note the scale of the spin coherence is on the right side of the figure and the scale of the top panel is different from that of the bottom one. (b) Net effective magnetic field $B^{*}$ from the D'yakonov-Perel' term $v s$. the drift velocity $V_{d}$ at $T=120 \mathrm{~K}$ with impurity density $N_{i}=0$. The solid curve is the corresponding result from Eq. [188]. From Weng et al. [569].

[112].

The predicted Pauli-blocking effect in the Bir-Aronov-Pikus mechanism has been partially demonstrated experimentally by Yang et al. [681], as shown in Fig.73] They showed by increasing the pumping density, the temperature dependence of the spin dephasing time deviates from the one from the Bir-Aronov-Pikus mechanism and the peaks at high excitations agree well with those predicted by Zhou and Wu [109].

\subsubsection{Spin dynamics in the presence of a strong THz laser field in quantum wells with strong spin-orbit coupling}

The influence of a strong $\mathrm{THz}$ field, which can effectively change the electron orbital momentums and significantly modify the electron density of states, has been extensively studied in spin-unrelated problems such as the dynamic Franz-Keldysh effect and optical side-band effect [58, 59, 61, 82, 841, 858]. So far, the application of strong THz field to the spin systems is very limited and only in theory. Johnsen is the first one who investigated the optical sideband generation in systems with spin-orbit coupling and suggested the formation of odd sidebands which are otherwise absent without the spin-orbit coupling [859]. Cheng and Wu showed theoretically that in InAs quantum wells, a strong in-plane $\mathrm{THz}$ electric field can induce a large spin polarization oscillating at the same frequency of the $\mathrm{THz}$ driving field [841]. Later Jiang et al. and Zhou suggested similar effects in single charged InAs quantum dots [860] and in $p$-type GaAs (001) quantum wells [861]. These calculations in Refs. [841, 859-861] are performed without any dissipation. However, in reality, due to the scattering there is spin dephasing and relaxation. Whether the large spin 

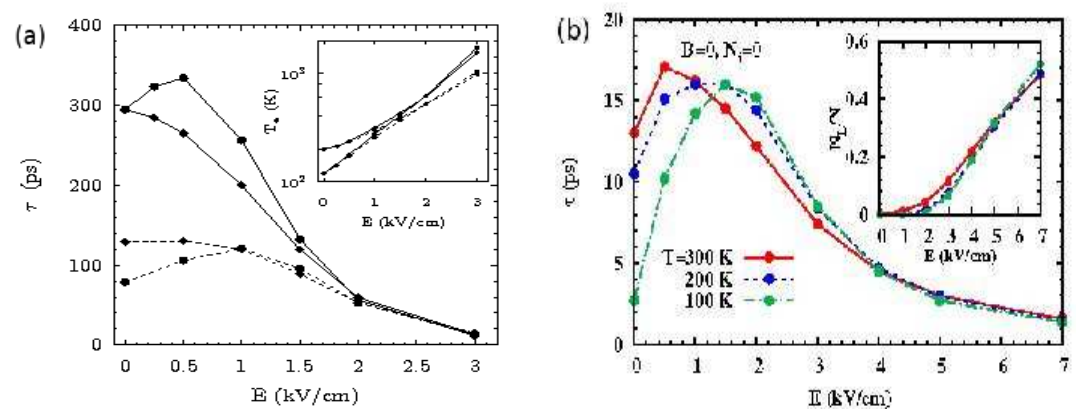

Figure 65: GaAs (001) quantum wells: (a) Spin dephasing time $v s$. the applied electric field $E$ at different temperatures and well widths: $\bullet$, $T=120 \mathrm{~K} ; \diamond, T=200 \mathrm{~K}$; Solid curves, $a=17.8 \mathrm{~nm}$; Dashed curves, $a=12.7 \mathrm{~nm}$. Inset: The corresponding electron temperature $T_{e}$ as a function of the electric field. From Weng and Wu [844]. (b) Spin relaxation time $\tau$ vs. electric filed $E$ when temperature $T=300$ (solid curves), 200 (dashed curves) and $100 \mathrm{~K}$ (chain curves) with $B=N_{i}=0$. Inset: fraction of electrons in $L$ valleys against electric field. From Zhang et al. [845].

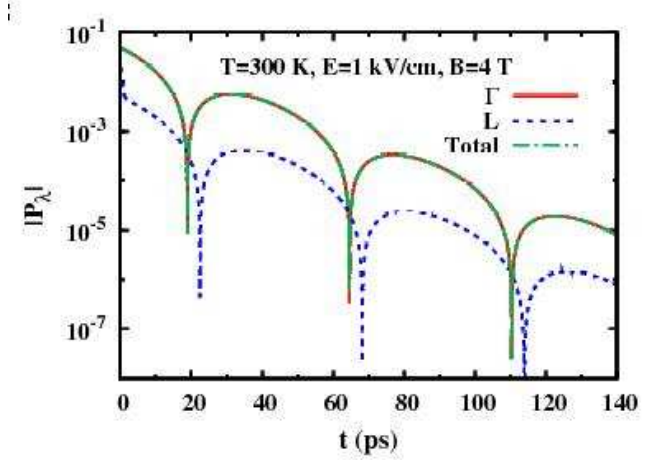

Figure 66: Temporal evolution of spin polarization $\left|P_{\lambda}\right|$ in $\lambda$ valley $(\lambda=\Gamma, L)$ of GaAs $(001)$ quantum well with electric field $E=1 \mathrm{kV} / \mathrm{cm}$ and magnetic field $B=4 \mathrm{~T}$. From Zhang et al. [845].

polarization oscillations are still kept in the presence of the dissipation is unanswered in Refs. [841, 860, 861]. Also the $\mathrm{THz}$ field can effectively affect the density of states, and also cause the hot-electron effect, which in turn should have pronounced effect on the spin relaxation/dephasing. To answer these questions, Jiang et al. extended the kinetic spin Bloch equations to study the spin kinetics in the presence of strong THz laser fields via the Floquet-Markov approach, first in quantum dots [482] then in quantum wells [69]. Their investigation suggested that the dissipation does not block large $\mathrm{THz}$ spin polarization oscillations and the spin relaxation and dephasing can be effectively manipulated by the THz field.

In the Coulomb gauge $\mathbf{A}(t)=\mathbf{E} \cos (\Omega t) / \Omega$ and the scalar potential $\Phi=0$, the electron Hamiltonian in a $(001)$ quantum well with small well width along the $z$-axis reads

$$
H_{e}=\sum_{\mathbf{k} \sigma \sigma^{\prime}} H_{0}^{\sigma \sigma^{\prime}}(\mathbf{k}, t) \hat{c}_{\mathbf{k} \sigma}^{\dagger} \hat{c}_{\mathbf{k} \sigma^{\prime}}
$$

with

$$
\begin{aligned}
\hat{H}_{0}(\mathbf{k}, t) & =\frac{[\mathbf{k}+e \mathbf{A}(t)]^{2}}{2 m^{*}} \hat{\mathbf{1}}+\alpha_{\mathrm{R}}\left\{\hat{\sigma}_{x} k_{y}-\hat{\sigma}_{y}\left[k_{x}+e A(t)\right]\right\} \\
& =\left\{\varepsilon_{\mathbf{k}}+\gamma_{E} k_{x} \Omega \cos (\Omega t)+E_{\mathrm{em}}[1+\cos (2 \Omega t)]\right\} \hat{\mathbf{1}}+\alpha_{\mathrm{R}}\left(\hat{\sigma}_{x} k_{y}-\hat{\sigma}_{y} k_{x}\right)-\alpha_{\mathrm{R}} \hat{\sigma}_{y} e E \cos (\Omega t) / \Omega
\end{aligned}
$$

Here $\varepsilon_{\mathbf{k}}=\frac{\mathbf{k}^{2}}{2 m^{*}}, \gamma_{E}=\frac{e E}{m^{*} \Omega^{2}}$ and $E_{\mathrm{em}}=\frac{e^{2} E^{2}}{4 m^{*} \Omega^{2}} . \Omega$ is the Thz frequency and $\alpha_{\mathrm{R}}$ is the Rashba coefficient. It is noted that 


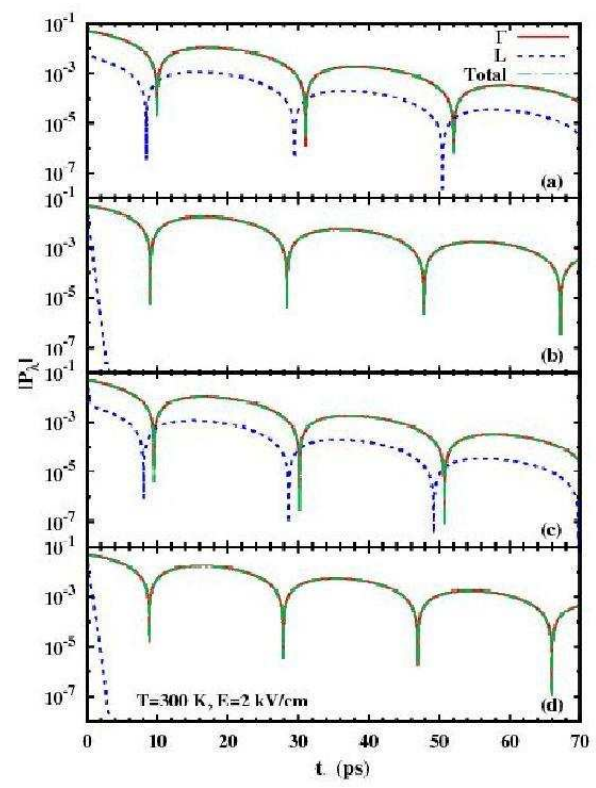

Figure 67: Spin polarization $\left|P_{\lambda}\right| v s$. $t$ under electric field $E=2 \mathrm{kV} / \mathrm{cm}$ in GaAs (001) quantum well. Solid curve: $\Gamma$ valley; dashed curve: $L$ valley; and chain curve: the total. (a): with both the intervalley electron-phonon scattering $\left(H_{\Gamma-\mathrm{L}}^{\mathrm{e}-\mathrm{p}}\right)$ and intervalley electron-electron Coulomb scattering $\left(H_{\Gamma-L}^{\mathrm{e}-\mathrm{e}}\right) ;(\mathrm{b})$ : without $H_{\Gamma-\mathrm{L}}^{\mathrm{e}-\mathrm{p}} ;(\mathrm{c})$ : without $H_{\Gamma-\mathrm{L}}^{\mathrm{e}-\mathrm{e}}$; and (d): without $H_{\Gamma-\mathrm{L}}^{\mathrm{e}-\mathrm{e}}$ and $H_{\Gamma-\mathrm{L}}^{\mathrm{e}-\mathrm{p}} . T=300 \mathrm{~K}$ and $N_{i}=B=0$. From Zhang et al. [845].

the last term manifests that the THz electric field acts as a THz magnetic field along the $y$ axis

$$
B_{\mathrm{eff}}=-2 \alpha_{\mathrm{R}} e E \cos (\Omega t) /\left(g \mu_{B} \Omega\right)
$$

where $g$ is the electron $g$-factor. We will show later that this THz-field-induced effective magnetic field has many important effects on spin kinetics. The term proportional to $E_{\mathrm{em}}$ is responsible for the dynamical Franz-Keldysh effect $[841,862,863]$. This term does not contain any dynamic variable of the electron system and thus has no effect on the kinetics of the electron system. Usually, the largest time-periodic term is the term $\gamma_{E} k_{x} \Omega \cos (\Omega t)$, where the sideband effect mainly comes from. Under an intense THz field, this term can be comparable to or larger than $\varepsilon_{\mathbf{k}}$. The Hamiltonian of the scattering remains all the same as before, e.g., Eqs. (152,154).

The Schrödinger equation for electron with momentum $\mathbf{k}$ reads

$$
i \partial_{t} \Psi_{\mathbf{k}}(t)=\hat{H}_{0}(\mathbf{k}, t) \Psi_{\mathbf{k}}(t)
$$

According to the Floquet theory [864], the solution to the above equation is

$$
\Psi_{\mathbf{k} \eta}(t)=e^{i \mathbf{k} \cdot \mathbf{r}-i \varepsilon_{\mathbf{k}} t} \phi_{1}(z) \xi_{\mathbf{k} \eta}(t) e^{-i\left[\gamma_{E} k_{x} \sin (\Omega t)+E_{\mathrm{em}} t+E_{\mathrm{em}} \frac{\sin (2 \Omega t)}{2 \Omega}\right]} \equiv e^{i \mathbf{k} \cdot \mathbf{r}} \Phi_{\eta}(z) e^{-i\left[\gamma_{E} k_{x} \sin (\Omega t)+E_{\mathrm{em}} t+E_{\mathrm{em}} \frac{\sin (2 \Omega t)}{2 \Omega}\right]},
$$

with $\eta= \pm$ denoting the spin branch and $\phi_{1}(z)$ being the wavefunction of the lowest subband. $\xi_{\mathbf{k} \eta}(t)=e^{-i y_{\mathbf{k} \eta} t} \sum_{n \sigma} v_{n \sigma}^{\mathbf{k} \eta} e^{i n \Omega t} \chi_{\sigma}$ where $y_{\mathbf{k} \eta}$ and $v_{n \sigma}^{\mathbf{k} \eta}$ are the eigen-values and eigen-vectors of the equation

$$
\left(y_{\mathbf{k} \eta}-n \Omega\right) v_{n \sigma}^{\mathbf{k} \eta}=\frac{i \sigma}{2 \Omega} \alpha_{\mathrm{R}} e E\left(v_{n-1,-\sigma}^{\mathbf{k} \eta}+v_{n+1,-\sigma}^{\mathbf{k} \eta}\right)+\alpha_{\mathrm{R}}\left(k_{y}+i \sigma k_{x}\right) v_{n,-\sigma}^{\mathbf{k} \eta}
$$

This equation is equivalent to Eq. (2) in Ref. [841]. For each $\mathbf{k}$, the spinors $\left\{\left|\xi_{\mathbf{k} \eta}(t)\right\rangle\right\}$ at any time $t$ form a completeorthogonal basis of the spin space [482, 865, 866]. The time evolution operator for state $\mathbf{k}$ can be written as

$$
\hat{U}_{0}^{e}(\mathbf{k}, t, 0)=\sum_{\eta}\left|\xi_{\mathbf{k} \eta}(t)\right\rangle\left\langle\xi_{\mathbf{k} \eta}(0)\right| e^{-i\left[\varepsilon_{\mathbf{k}} t+\gamma_{E} k_{x} \sin (\Omega t)\right]} e^{-i\left[E_{\mathrm{em}} t+E_{\mathrm{em}} \sin (2 \Omega t) /(2 \Omega)\right]} .
$$



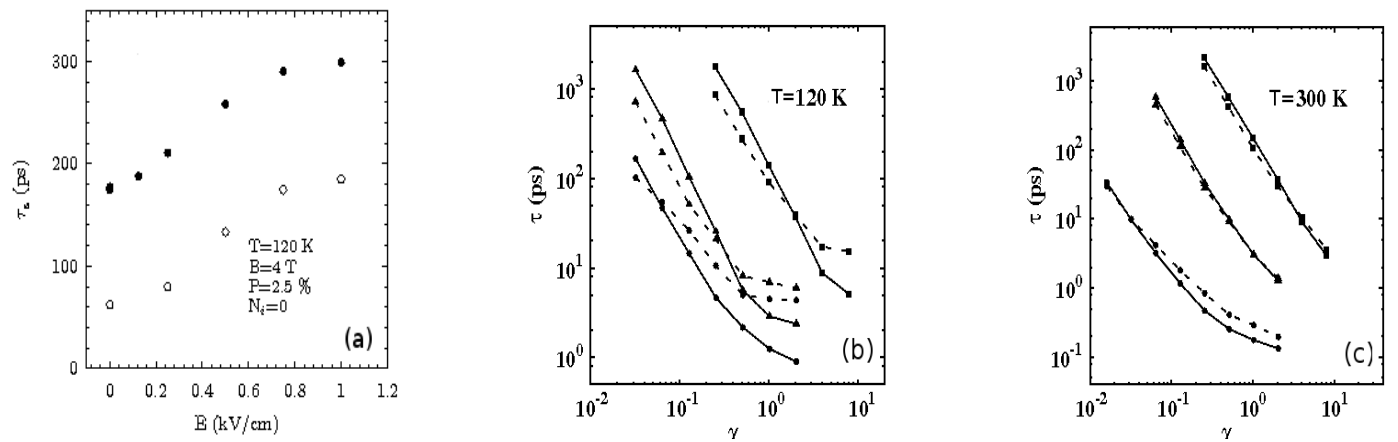

Figure 68: (a) Spin dephasing time in GaAs (001) quantum wells with (close circles) and without (open circles) the Coulomb scattering. From Weng et al. [569]. (b) and (c): Spin dephasing time vs. the scaling coefficient of the D'yakonov-Perel' term $\gamma$ for $T=120 \mathrm{~K}$ and $300 \mathrm{~K}$, respectively. The solid (dashed) curves are the results with (without) the Coulomb scattering. $\mathbf{\square}:$ Electrons; $\mathbf{\Delta}$ : light holes; $\bullet$ : heavy holes. The impurity density $N_{i}=0$ and magnetic field $B=0$. From Lü et al. [363].

At zero temperature and in the absence of any scattering, the spectral function is [841]

$$
A\left(\mathbf{k} ; t_{1}, t_{2}\right)=\sum_{\eta= \pm 1} \Phi_{\eta}\left(\mathbf{k}, t_{1}\right) \Phi_{\eta}^{\dagger}\left(\mathbf{k}, t_{2}\right)
$$

from which the density matrix reads

$$
\rho\left(t_{1}, t_{2}\right)=\frac{1}{(2 \pi)^{2}} \int d \mathbf{k} A\left(\mathbf{k} ; t_{1}, t_{2}\right) .
$$

Note $\rho\left(t_{1}, t_{2}\right)$ is a $2 \times 2$ matrix in the spin space. In the collinear spin space, $\rho_{\uparrow \uparrow}=\rho_{\downarrow \downarrow}$. The average magnetic moment from the THz field (along the $x$-axis) and the Rashba field is given by

$$
\mathbf{M}(T)=\left(0,-\frac{g \mu_{B}}{n_{\uparrow}+n_{\downarrow}} \int_{-\infty}^{E_{f}(T)} d \omega \operatorname{Im} \rho_{\uparrow, \downarrow}(\omega, T), 0\right),
$$

with $E_{f}(T)$ determined from $n_{\sigma}=\frac{1}{2 \pi} \int_{-\infty}^{E_{f}(T)} d \omega \rho_{\sigma, \sigma}(\omega, T)$. Here $T=\left(t_{1}+t_{2}\right) / 2$ and $\omega$ is Fourier transferred from $t=t_{1}-t_{2}$. Figure 74 shows the average magnetic moment $M_{y}$ versus the time $T$ at different electric fields and THz frequencies. It is seen that a $\mathrm{THz}$ magnetic signal is effectively induced by the $\mathrm{THz}$ electric one.

To extend the kinetic spin Bloch equations to the situation with an intense THz field, Jiang et al. pointed out [69] that it is insufficient to include this field only in the driving term as in the previous investigation of the hotelectron effect where a static electric field is applied [372, 569]. The correct way is to evaluate the collision integral with the Floquet wavefunctions, i.e., the solution of the time-dependent Schrödinger equation Eq. (200) [867]. Moreover, the Markovian approximation should be made with respect to the spectrum determined by the Floquet wavefunctions [867]. These improvements constitute the Floquet-Markov approach [865, 867], which works well when the driven system is in dynamically stable regime and the system-reservoir coupling can be treated perturbatively. With the Floquet-Markov approach and by projecting the density matrix in the Floquet picture $\hat{\rho}_{\mathbf{k}}^{F}(t)=$ $\hat{U}_{0}^{e \dagger}(\mathbf{k}, t, 0) \hat{\rho}_{\mathbf{k}}(t) \hat{U}_{0}^{e}(\mathbf{k}, t, 0)$, the kinetic spin Bloch equations read

$$
\partial_{t} \hat{\rho}_{\mathbf{k}}^{F}(t)=\left.\partial_{t} \hat{\rho}_{\mathbf{k}}^{F}(t)\right|_{\mathrm{coh}}+\left.\partial_{t} \hat{\rho}_{\mathbf{k}}^{F}(t)\right|_{\text {scat }},
$$

where $\left.\partial_{t} \hat{\rho}_{\mathbf{k}}^{F}(t)\right|_{\text {coh }}$ and $\left.\partial_{t} \hat{\rho}_{\mathbf{k}}^{F}(t)\right|_{\text {scat }}$ are the coherent and scattering terms, respectively. The coherent terms, which describe the coherent precession determined by the electron Hamiltonian $H_{e}$ and the Hartree-Fock contribution of the electron-electron Coulomb interaction, can be written as

$$
\left.\partial_{t} \hat{\rho}_{\mathbf{k}}^{F}(t)\right|_{\mathrm{coh}}=i \sum_{\mathbf{k}^{\prime}, q_{z}} V_{\mathbf{k}-\mathbf{k}^{\prime}, q_{z}}\left|I\left(i q_{z}\right)\right|^{2}\left[\hat{S}_{\mathbf{k}, \mathbf{k}^{\prime}}(t, 0) \hat{\rho}_{\mathbf{k}^{\prime}}^{F}(t) \hat{S}_{\mathbf{k}^{\prime}, \mathbf{k}}(t, 0), \hat{\rho}_{\mathbf{k}}^{F}(t)\right]
$$



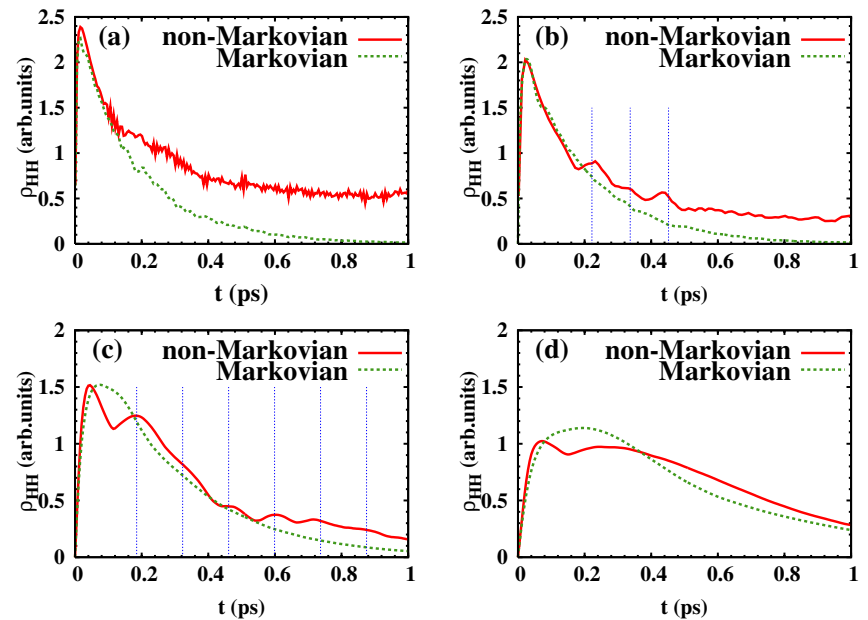

Figure 69: Time evolutions of the incoherently summed spin coherence $\rho_{\mathrm{HH}}$ of heavy holes in GaAs (001) quantum wells with Rashba spin-orbit coupling strength (a) $\gamma_{54}^{7 h 7 h} E_{z} m_{0} / \hbar^{2}=6.25 \mathrm{~nm}$, (b) $2.5 \mathrm{~nm}$, (c) $0.625 \mathrm{~nm}$ and (d) $0.25 \mathrm{~nm}$, respectively. $\gamma_{54}^{7 h 7 h}$ is the Rashba coefficient and $E_{z}$ is the electic field from the gate voltage [363]. Correspondingly, the mean spin precession time $\Omega^{-1}$ is (a) $0.046 \mathrm{ps,} \mathrm{(b)} 0.116 \mathrm{ps}$, (c) $0.463 \mathrm{ps}$ and (d) 1.160 ps. Solid curve: in non-Markovian limit; Dashed curve: in Markovian limit. The well width $a=5 \mathrm{~nm}$ and the temperature $T=300 \mathrm{~K}$. From Zhang and $\mathrm{Wu}$ [854].

Here $\hat{\Sigma}_{\mathrm{HF}}^{F}(\mathbf{k}, t)=-\sum_{\mathbf{k}^{\prime}, q_{z}} V_{\mathbf{k}-\mathbf{k}^{\prime}, q_{z}}\left|I\left(i q_{z}\right)\right|^{2} \hat{\rho}_{\mathbf{k}^{\prime}}^{F}(t)$ is the Coulomb Hartree-Fock self-energy. The scattering terms are composed of terms due to the electron-impurity $\left(\left.\partial_{t} \rho_{\mathbf{k}}^{F}\right|_{\mathrm{ei}}\right)$, electron-phonon $\left(\left.\partial_{t} \rho_{\mathbf{k}}^{F}\right|_{\mathrm{ep}}\right)$ and electron-electron $\left(\left.\partial_{t} \rho_{\mathbf{k}}^{F}\right|_{\mathrm{ee}}\right)$ scatterings, respectively. Under the generalized Kadanoff-Baym Ansatz [833], these scattering terms read [69]

$$
\begin{aligned}
& \left.\partial_{t} \rho_{\mathbf{k}}^{F\left(\eta \eta^{\prime}\right)}(t)\right|_{\mathrm{ei}}=-\sum_{\mathbf{k}^{\prime}, q_{z}, n}^{\eta_{1} \eta_{2} \eta_{3}} \pi n_{i} U_{\mathbf{k}-\mathbf{k}^{\prime}, q_{z}}^{2}\left|I\left(i q_{z}\right)\right|^{2}\left[\left\{S_{\mathbf{k}, \mathbf{k}^{\prime}}^{\left(\eta \eta_{1}\right)}(t, 0) S_{\mathbf{k}^{\prime}, \mathbf{k}}^{(n)\left(\eta_{2} \eta_{3}\right)} \delta\left(n \Omega+\bar{\varepsilon}_{\mathbf{k}^{\prime} \eta_{2}}-\bar{\varepsilon}_{\mathbf{k} \eta_{3}}\right)\right.\right. \\
& \left.\left.\times\left(\rho_{\mathbf{k}^{\prime}}^{>F\left(\eta_{1} \eta_{2}\right)}(t) \rho_{\mathbf{k}}^{<F\left(\eta_{3} \eta^{\prime}\right)}(t)-\rho_{\mathbf{k}^{\prime}}^{<F\left(\eta_{1} \eta_{2}\right)}(t) \rho_{\mathbf{k}}^{>F\left(\eta_{3} \eta^{\prime}\right)}(t)\right)\right\}+\left\{\eta \leftrightarrow \eta^{\prime}\right\}^{*}\right], \\
& \left.\partial_{t} \rho_{\mathbf{k}}^{F\left(\eta \eta^{\prime}\right)}(t)\right|_{\mathrm{ep}}=-\sum_{\mathbf{k}^{\prime}, q_{z}, n, \lambda, \pm}^{\eta_{1} \eta_{2} \eta_{3}} \pi\left|M_{\lambda, \mathbf{k}-\mathbf{k}^{\prime}, q_{z}}\right|^{2}\left|I\left(i q_{z}\right)\right|^{2}\left[\left\{S_{\mathbf{k}, \mathbf{k}^{\prime}}^{\left(\eta \eta_{1}\right)}(t, 0) S_{\mathbf{k}^{\prime}, \mathbf{k}}^{(n)\left(\eta_{2} \eta_{3}\right)} e^{\mp i t \omega_{\lambda, \mathbf{k}-\mathbf{k}^{\prime}, q_{z}}} \delta\left( \pm \omega_{\lambda, \mathbf{k}-\mathbf{k}^{\prime}, q_{z}}+n \Omega+\bar{\varepsilon}_{\mathbf{k}^{\prime} \eta_{2}}-\bar{\varepsilon}_{\mathbf{k} \eta_{3}}\right)\right.\right. \\
& \left.\left.\times\left(N_{\lambda, \mathbf{k}-\mathbf{k}^{\prime}, q_{z}}^{ \pm} \rho_{\mathbf{k}^{\prime}}^{>F\left(\eta_{1} \eta_{2}\right)}(t) \rho_{\mathbf{k}}^{<F\left(\eta_{3} \eta^{\prime}\right)}(t)-N_{\lambda, \mathbf{k}-\mathbf{k}^{\prime}, q_{z}}^{\mp} \rho_{\mathbf{k}^{\prime}}^{<F\left(\eta_{1} \eta_{2}\right)}(t) \rho_{\mathbf{k}}^{>F\left(\eta_{3} \eta^{\prime}\right)}(t)\right)\right\}+\left\{\eta \leftrightarrow \eta^{\prime}\right\}^{*}\right], \\
& \left.\partial_{t} \rho_{\mathbf{k}}^{F\left(\eta \eta^{\prime}\right)}(t)\right|_{\mathrm{ee}}=-\sum_{\mathbf{k}^{\prime}, \mathbf{k}^{\prime \prime}, n, n^{\prime}}^{\eta_{1} \ldots \eta_{7}} \pi\left[\left.\sum_{q_{z}} V_{\mathbf{k}-\mathbf{k}^{\prime}, q_{z}} I\left(i q_{z}\right)\right|^{2}\right]^{2}\left[\left\{T_{\mathbf{k}, \mathbf{k}^{\prime}}^{\left(\eta \eta_{1}\right)}(t, 0) T_{\mathbf{k}^{\prime}, \mathbf{k}}^{\left(n^{\prime}\right)\left(\eta_{2} \eta_{3}\right)} T_{\mathbf{k}^{\prime \prime}, \mathbf{k}^{\prime \prime}-\mathbf{k}+\mathbf{k}^{\prime}}^{\left(n-n^{\prime}\right)\left(\eta_{4} \eta_{5}\right)} T_{\mathbf{k}^{\prime \prime}-\mathbf{k}+\mathbf{k}^{\prime}, \mathbf{k}^{\prime \prime}}^{\left(\eta_{6} \eta_{7}\right)}(t, 0)\right.\right. \\
& \times \delta\left(n \Omega+\bar{\varepsilon}_{\mathbf{k}^{\prime} \eta_{2}}-\bar{\varepsilon}_{\mathbf{k} \eta_{3}}+\bar{\varepsilon}_{\mathbf{k}^{\prime \prime} \eta_{4}}-\bar{\varepsilon}_{\mathbf{k}^{\prime \prime}-\mathbf{k}+\mathbf{k}^{\prime} \eta_{5}}\right)\left(\rho_{\mathbf{k}^{\prime}}^{>F\left(\eta_{1} \eta_{2}\right)}(t) \rho_{\mathbf{k}}^{<F\left(\eta_{3} \eta^{\prime}\right)}(t) \rho_{\mathbf{k}^{\prime \prime}-\mathbf{k}+\mathbf{k}^{\prime}}^{<F\left(\eta_{5} \eta_{6}\right)}(t) \rho_{\mathbf{k}^{\prime \prime}}^{>F\left(\eta_{7} \eta_{4}\right)}(t)\right. \\
& \left.\left.\left.-\rho_{\mathbf{k}^{\prime}}^{<F\left(\eta_{1} \eta_{2}\right)}(t) \rho_{\mathbf{k}}^{>F\left(\eta_{3} \eta^{\prime}\right)}(t) \rho_{\mathbf{k}^{\prime \prime}-\mathbf{k}+\mathbf{k}^{\prime}}^{>F\left(\eta_{5} \eta_{6}\right)}(t) \rho_{\mathbf{k}^{\prime \prime}}^{<F\left(\eta_{7} \eta_{4}\right)}(t)\right)\right\}+\left\{\eta \leftrightarrow \eta^{\prime}\right\}^{*}\right]
\end{aligned}
$$

In these equations, $N_{\lambda, \mathbf{k}-\mathbf{k}^{\prime}, q_{z}}^{ \pm}=N_{\lambda, \mathbf{k}-\mathbf{k}^{\prime}, q_{z}}+\frac{1}{2}(1 \pm 1)$ stands for the phonon number, $n_{i}$ is the impurity density, $\hat{\rho}_{\mathbf{k}}^{>}=\hat{\mathbf{1}}-\hat{\rho}_{\mathbf{k}}$, $\hat{\rho}_{\mathbf{k}}^{<}=\hat{\rho}_{\mathbf{k}}$, and $\bar{\varepsilon}_{\mathbf{k} \eta}=\varepsilon_{\mathbf{k}}+y_{\mathbf{k} \eta}$.

$$
S_{\mathbf{k}^{\prime}, \mathbf{k}}^{\left(\eta_{1} \eta_{2}\right)}(t, 0)=\left\langle\xi_{\mathbf{k}^{\prime} \eta_{1}}(t) \mid \xi_{\mathbf{k} \eta_{2}}(t)\right\rangle e^{i\left[\left(\varepsilon_{\mathbf{k}^{\prime}}-\varepsilon_{\mathbf{k}}\right) t+\gamma_{E} \sin (\Omega t)\left(k_{x}^{\prime}-k_{x}\right)\right]}=\sum_{n} S_{\mathbf{k}^{\prime}, \mathbf{k}}^{(n)\left(\eta_{1} \eta_{2}\right)} e^{i t\left(n \Omega+\bar{\varepsilon}_{\mathbf{k}^{\prime} \eta_{1}}-\bar{\varepsilon}_{\mathbf{k} \eta_{2}}\right)},
$$

with

$$
S_{\mathbf{k}^{\prime}, \mathbf{k}}^{(n)\left(\eta_{1} \eta_{2}\right)}=\sum_{m \sigma} F_{m \sigma}^{\mathbf{k}^{\prime} \eta_{1} *} F_{n+m \sigma}^{\mathbf{k} \eta_{2}}
$$



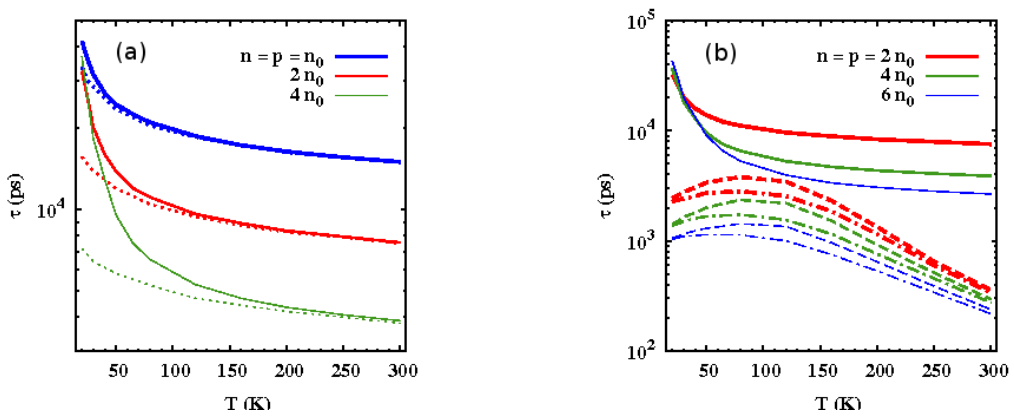

Figure 70: GaAs (001) quantum wells: (a) Spin relaxation time due to the Bir-Aronov-Pikus mechanism with full spin-flip electron-hole exchange scattering (solid curves) and with only the linear terms in the spin-flip electron-hole exchange scattering (dotted curves) at different electron densities against temperature $T . n_{0}=10^{11} \mathrm{~cm}^{-2}$. (b) Spin relaxation time due to the Bir-Aronov-Pikus (solid curves) and D'yakonov-Perel' (dashed curves) mechanisms and the total spin relaxation time (dash-dotted curves) vs. temperature $T$ in intrinsic quantum wells at different densities $(n=p=2$, $4,6 n_{0}$ ) when well width $a=20 \mathrm{~nm}$ and impurity density $n_{i}=n . n_{0}=10^{11} \mathrm{~cm}^{-2}$. From Zhou and Wu [109].

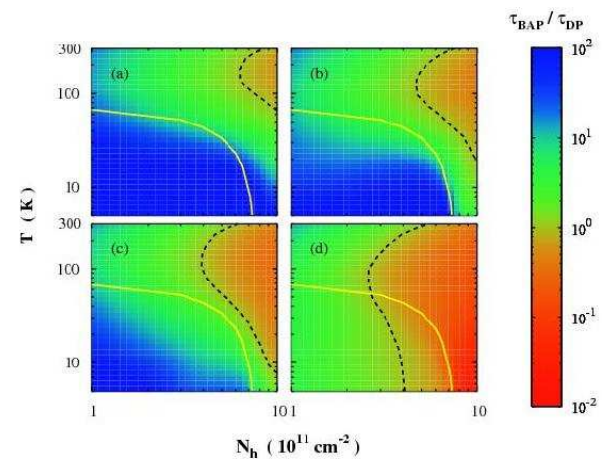

Figure 71: Ratio of the spin relaxation time due to the Bir-Aronov-Pikus mechanism to that due to the D'yakonov-Perel' mechanism, $\tau_{\mathrm{BAP}} / \tau_{\mathrm{DP}}$, in GaAs (001) quantum wells as function of temperature and hole density with (a) $N_{i}=0, N_{e x}=10^{11} \mathrm{~cm}^{-2}$; (b) $N_{i}=0, N_{e x}=10^{9} \mathrm{~cm}-2$; (c) $N_{i}=N_{h}, N_{e x}=10^{11} \mathrm{~cm}^{-2}$; (d) $N_{i}=N_{h}, N_{e x}=10^{9} \mathrm{~cm}^{-2}$. The black dashed curves indicate the cases satisfying $\tau_{\mathrm{BAP}} / \tau_{\mathrm{DP}}=1$. Note the smaller the ratio $\tau_{\mathrm{BAP}} / \tau_{\mathrm{DP}}$ is, the more important the Bir-Aronov-Pikus mechanism becomes. The yellow solid curves indicate the cases satisfying $\partial_{\mu_{h}}\left[N_{\mathrm{LH}^{(1)}}+N_{\mathrm{HH}^{(2)}}\right] / \partial_{\mu_{h}} N_{h}=0.1$. In the regime above the yellow curve the multi-hole-subband effect becomes significant. From Zhou et al. [112].

Here $F_{n \sigma}^{\mathbf{k} \eta}=\sum_{m} v_{n+m \sigma}^{\mathbf{k} \eta} J_{m}\left(\gamma_{E} k_{x}\right)$ with $J_{m}(x)$ standing for the $m$-th order Bessel function.

$$
T_{\mathbf{k}^{\prime}, \mathbf{k}}^{\left(\eta_{1} \eta_{2}\right)}(t, 0)=\left\langle\xi_{\mathbf{k}^{\prime} \eta_{1}}(t) \mid \xi_{\mathbf{k} \eta_{2}}(t)\right\rangle e^{i\left(\varepsilon_{\mathbf{k}^{\prime}}-\varepsilon_{\mathbf{k}}\right) t}=\sum_{n} T_{\mathbf{k}^{\prime}, \mathbf{k}}^{(n)\left(\eta_{1} \eta_{2}\right)} e^{i t\left(n \Omega+\bar{\varepsilon}_{\mathbf{k}^{\prime} \eta_{1}}-\bar{\varepsilon}_{\mathbf{k} \eta_{2}}\right)}
$$

with

$$
T_{\mathbf{k}^{\prime}, \mathbf{k}}^{(n)\left(\eta_{1} \eta_{2}\right)}=\sum_{m \sigma} v_{m \sigma}^{\mathbf{k}^{\prime} \eta_{1} *} v_{n+m \sigma}^{\mathbf{k} \eta_{2}}
$$

$\left\{\eta \leftrightarrow \eta^{\prime}\right\}$ stands for the same terms as in the previous \{\} but with the interchange $\eta \leftrightarrow \eta^{\prime}$. The term of the electronelectron scattering is quite different from those of the electron-impurity and electron-phonon scattering, as the momentum conservation eliminates the term of $e^{i \gamma_{E} \sin (\Omega t) k_{x}}$.

The above equations clearly show the sideband effects, i.e., $n \Omega$ in the $\delta$-functions. The extra energy, $n \Omega$, is provided by the THz field during each scattering process. This makes transitions from the low-energy states (small $k$ ) to high-energy ones (large $k$ ) become possible, even through the elastic electron-impurity scattering. These processes are the sideband-modulated scattering processes.

The kinetic spin Bloch equations are solved numerically. After that, $\rho_{\mathbf{k}}^{F\left(\eta \eta^{\prime}\right)}(t)$ for each $\mathbf{k}$ is obtained. From

$$
\rho_{\mathbf{k}}^{F\left(\eta \eta^{\prime}\right)}(t)=\left\langle\xi_{\mathbf{k} \eta}(0)\left|\hat{U}_{0}^{e \dagger}(\mathbf{k}, t, 0) \hat{\rho}_{\mathbf{k}}(t) \hat{U}_{0}^{e}(\mathbf{k}, t, 0)\right| \xi_{\mathbf{k} \eta^{\prime}}(0)\right\rangle=\left\langle\xi_{\mathbf{k} \eta}(t)\left|\hat{\rho}_{\mathbf{k}}(t)\right| \xi_{\mathbf{k} \eta^{\prime}}(t)\right\rangle,
$$




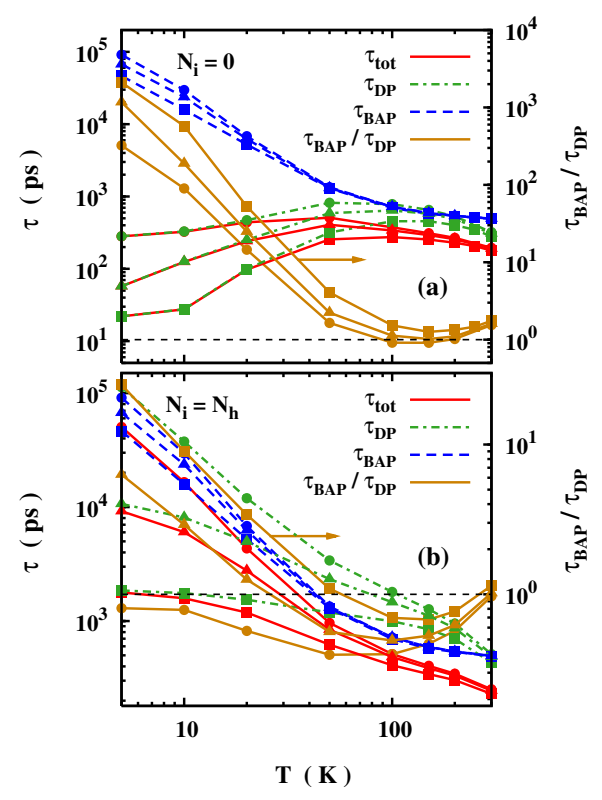

Figure 72: Spin relaxation times of electrons in GaAs (001) quantum wells due to the D'yakonov-Perel' and Bir-Aronov-Pikus mechanisms, the total spin relaxation time together with the ratio $\tau_{\mathrm{BAP}} / \tau_{\mathrm{DP}} v s$. temperature $T$ for $N_{\mathrm{ex}}=10^{9} \mathrm{~cm}^{-2}\left(\right.$ curves with $\bullet$ ), $3 \times 10^{10} \mathrm{~cm}^{-2}(\mathrm{curves}$ with $\mathbf{\Delta})$ and $10^{11} \mathrm{~cm}^{-2}$ (curves with $\mathbf{\square}$ ) with hole density $N_{h}=5 \times 10^{11} \mathrm{~cm}^{-2}$ and impurity densities (a) $N_{i}=0$ and (b) $N_{i}=N_{h}$. The electron Fermi temperatures for those excitation densities are $T_{\mathrm{F}}^{e}=0.41,12.4$ and $41.5 \mathrm{~K}$, respectively. The hole Fermi temperature is $T_{\mathrm{F}}^{h}=124 \mathrm{~K}$. Note the scale of $\tau_{\mathrm{BAP}} / \tau_{\mathrm{DP}}$ is on the right-hand side of the frame. From Zhou et al. [112].

by performing a unitary transformation, one comes to the single particle density matrix $\hat{\rho}_{\mathbf{k}}(t)$ in the collinear basis $\{|\sigma\rangle\}$ which is composed by the eigen-states of $\hat{\sigma}_{z}$. In this spin space, the spin polarization along any direction can be obtained readily, e.g., $S_{z}=\sum_{\mathbf{k}} \frac{1}{2}\left(\rho_{\mathbf{k}}^{\uparrow \uparrow}-\rho_{\mathbf{k}}^{\downarrow \downarrow}\right), S_{x}=\sum_{\mathbf{k}} \operatorname{Re}\left\{\rho_{\mathbf{k}}^{\uparrow \downarrow}\right\}$ and $S_{y}=-\sum_{\mathbf{k}} \operatorname{Im}\left\{\rho_{\mathbf{k}}^{\uparrow \downarrow}\right\}$. From the temporal evolution of $S_{z}$, the spin relaxation time is extracted.

By numerically solving the kinetic spin Bloch equations with all the scattering included, Jiang et al. showed that with dissipation, the THz field can still pump a large (several percent) spin polarization which oscillates at the same frequency with the $\mathrm{THz}$ field, as shown in Fig. 75. This feature coincides with what predicted by Cheng and $\mathrm{Wu}$ in the dissipation-free investigation [841]. What differs from the previous case is shown in Fig. 75 that there is a delay

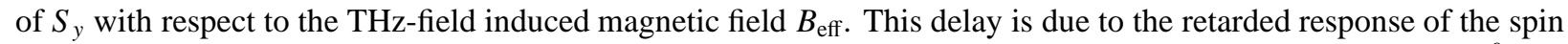
polarization to the spin pumping caused by the THz field. The amplitude of the steady-state spin polarization $S_{y}^{0}$ (the peak value of $S_{v}$ ) depends on the THz field strength and the THz frequency. These features have been addressed in detail in Ref. [69].

Apart from pumping $\mathrm{THz}$ spin polarizations, the $\mathrm{THz}$ field can effectively manipulate the electron density of states and cause the hot-electron effect. Both in turn can lead to the manipulation of the spin relaxation and dephasing. Figure 76 shows the dependence of the spin relaxation time on the THz field strength at different impurity densities (a) and temperatures (b), together with the corresponding hot-electron temperatures (c). Two consequences of the THz field lead to the rich behaviors in the figure: (i) the total THz field-induced effective magnetic field $B\left[B=B_{\text {eff }}+B_{\text {av }}\right.$ with $B_{\text {av }}(t)=2 \alpha_{\mathrm{R}}\left\langle k_{x}\right\rangle /\left(|g| \mu_{B}\right)$ from the Rashba spin-orbit coupling] and (ii) the hot-electron effect. Effect (i) can give a magnetic field as large as several tesla [2.6 T per $1 \mathrm{kV} / \mathrm{cm} \mathrm{THz}$ field with $v \equiv \Omega /(2 \pi)=0.65 \mathrm{THz}$ ]. This effective magnetic field blocks the inhomogeneous broadening from the Rashba spin-orbit coupling and thus elongates the spin relaxation time. Effect (ii) leads to the enhancement of momentum scattering as well as the inhomogeneous broadening, while the enhancement of the inhomogeneous broadening tends to shorten the spin relaxation time, the boost of the scattering tends to increase (or decrease) the spin relaxation time in the strong (or weak) scattering limit as discussed in the previous sections. Similarly the spin relaxation time can also be manipulated by the THz frequency as shown in Fig. 77 


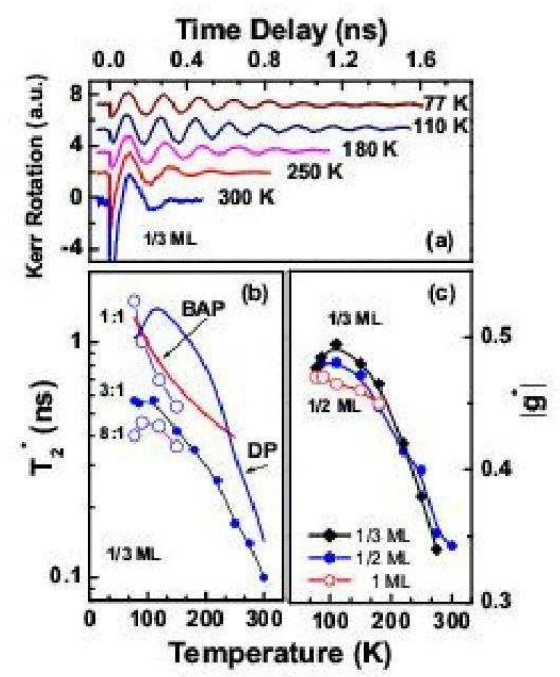

Figure 73: Kerr rotation of the $1 / 3$ monolayer InAs sample at different temperatures measured at $B=0.82 \mathrm{~T}$. The pumping density is about 1.5 $\times 10^{17} / \mathrm{cm}^{3}$. The temperature dependence of the electron spin decoherence time $T_{2}^{*}$ and the effective $g$-factor are shown in (b) and (c), respectively. The $T_{2}^{*}$ of 1/3 monolayer InAs sample with various pumping density at low temperature as specified is also shown for comparison. From Yang et al. [681].

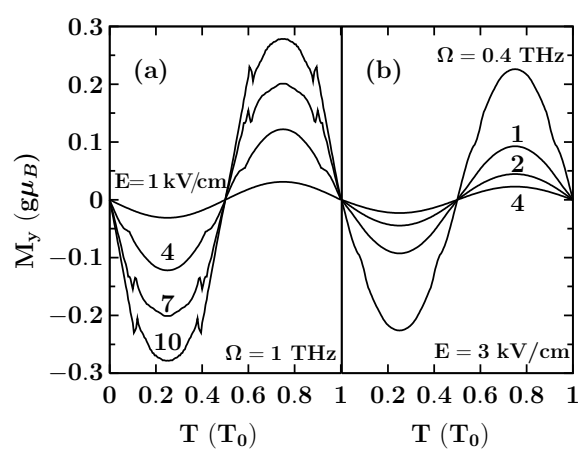

Figure 74: The average magnetic moment $M_{y} v s$. the time of InAs (001) quantum well at $E=1,4,7$ and $10 \mathrm{kV} / \mathrm{cm}$ for fixed $\Omega=1 \mathrm{THz}(\mathrm{a})$ at $\Omega=0.4,1,2$ and $4 \mathrm{THz}$ for fixed $E=3 \mathrm{kV} / \mathrm{cm}$ (b). The electron density is $N=10^{11} \mathrm{~cm}^{-2}$. From Cheng and Wu [841].

\subsubsection{Spin relaxation and dephasing in GaAs (110) quantum wells}

In symmetric GaAs (110) quantum wells, the D'yakonov-Perel' term mainly comes from the Dresselhaus term which reads

$$
\begin{aligned}
& g \mu_{B} \Omega_{x}^{n n^{\prime}}(\mathbf{k})=\gamma_{\mathrm{D}}\left[-\left(k_{x}^{2}+2 k_{y}^{2}\right)\left\langle n\left|k_{z}\right| n^{\prime}\right\rangle+\left\langle n\left|k_{z}^{3}\right| n^{\prime}\right\rangle\right], \\
& g \mu_{B} \Omega_{y}^{n n^{\prime}}(\mathbf{k})=4 \gamma_{\mathrm{D}} k_{x} k_{y}\left\langle n\left|k_{z}\right| n^{\prime}\right\rangle, \\
& g \mu_{B} \Omega_{z}^{n n^{\prime}}(\mathbf{k})=\gamma_{\mathrm{D}}\left(k_{x}^{2}-2 k_{y}^{2}-\left\langle n\left|k_{z}^{2}\right| n^{\prime}\right\rangle\right) \delta_{n n^{\prime}},
\end{aligned}
$$

where $\left\langle n\left|k_{z}^{m}\right| n^{\prime}\right\rangle=\int d z \phi_{n}^{*}(z)(-i \partial / \partial z)^{m} \phi_{n^{\prime}}(z)$ with $\phi_{n}(z)$ representing the envelope function of the electron in $n$-th subband. As $\left\langle n\left|k_{z}\right| n\right\rangle=\left\langle n\left|k_{z}^{3}\right| n\right\rangle=0$, when only the lowest subband is relevant, the effective magnetic field $\mathbf{\Omega}(\mathbf{k})$ is along the $z$-axis. Therefore, it was proposed both theoretically and experimentally [574, 577, 595, 662] that the D'yakonov-Perel' mechanism can not cause any spin relaxation if the spin polarization is along the $z$-axis.

Wu and Kuwata-Gonokami first pointed out that if a magnetic field in the Voigt configuration is applied in the system, there is spin relaxation and dephasing due to the D'yakonov-Perel' mechanism [675]. This can be seen from 


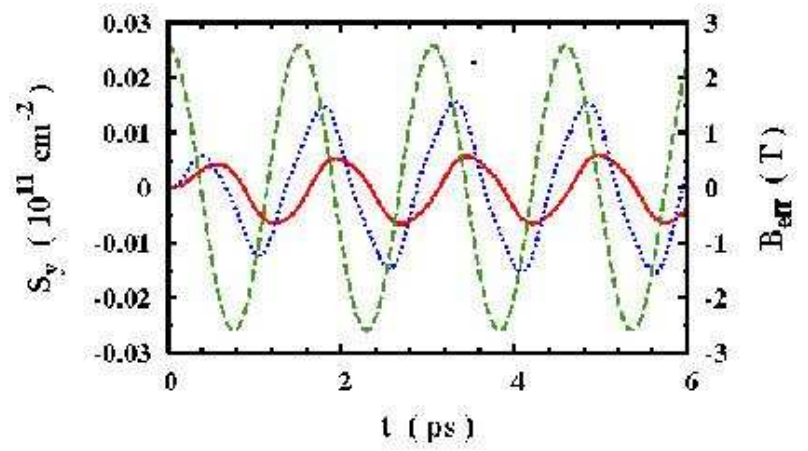

Figure 75: Spin polarization along $y$ axis, $S_{y}$, as function of time with zero initial spin polarization for $E=0.5 \mathrm{kV} / \mathrm{cm}$ (solid curve) and $1.0 \mathrm{kV} / \mathrm{cm}$ (dotted curve) in InAs (001) quantum well. $T=50 \mathrm{~K}$ and $N_{i}=0.05 N_{e}$. The dashed curve is the THz-field-induced effective magnetic field $B$ eff with $E=1.0 \mathrm{kV} / \mathrm{cm}$. Note that the scale of the dashed curve is on the right-hand side of the frame. From Jiang et al. [69].
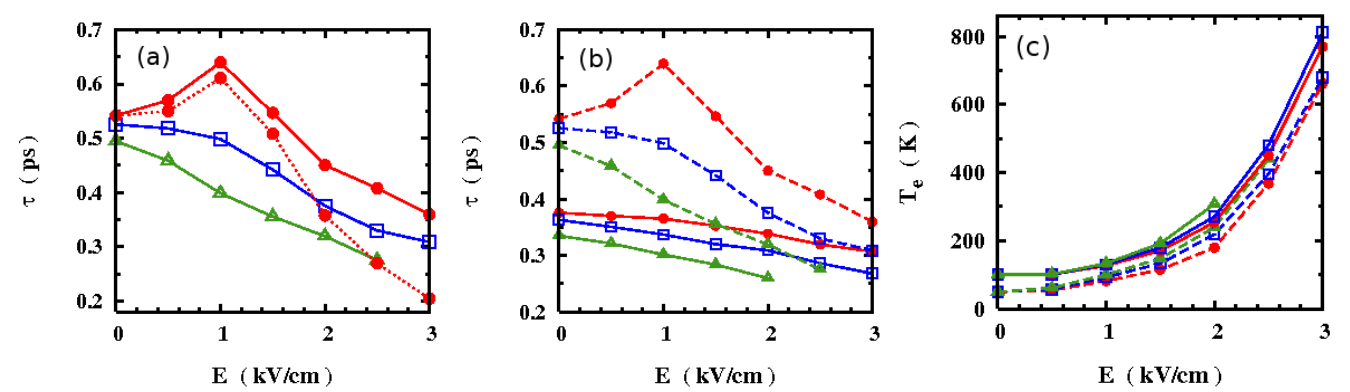

Figure 76: InAs (001) quantum wells: (a) Dependence of the spin relaxation time $\tau$ on $\mathrm{THz}$ field strength for impurity densities: $N_{i}=0(\bullet)$; $N_{i}=0.02 N_{e}(\square) ; N_{i}=0.05 N_{e}(\triangle)$. Solid curves: from full calculation; Dotted curve: from the calculation without the THz-field-induced effective magnetic field $B_{\text {eff }}$ (b) Dependence of the spin relaxation time $\tau$ on THz field strength for impurity densities: $N_{i}=0(\bullet) ; N_{i}=0.02 N_{e}(\square)$; and $N_{i}=0.05 N_{e}(\triangle) . T=100 \mathrm{~K}$ (solid curves) and $50 \mathrm{~K}$ (dashed curves). (c) Dependence of the hot-electron temperature $T_{e}$ on THz field strength for different impurity densities: $N_{i}=0(\bullet) ; N_{i}=0.02 N_{e}(\square) ; N_{i}=0.05 N_{e}(\triangle) . T=100 \mathrm{~K}$ (solid curves) and $T=50 \mathrm{~K}$ (dashed curves). From Jiang et al. [69].

the coherent term of the kinetic spin Bloch equations [675]:

$$
\begin{aligned}
\left.\frac{\partial f_{\mathbf{k} \sigma}}{\partial t}\right|_{\text {coh }} & =-g \mu_{B} B \operatorname{Im} \rho_{\mathbf{k}, \sigma-\sigma}+2 \operatorname{Im} \sum_{\mathbf{q}} V_{\mathbf{q}} \rho_{\mathbf{k}, \sigma-\sigma} \rho_{\mathbf{k}+\mathbf{q},-\sigma \sigma} \\
\left.\frac{\partial \rho_{\mathbf{k}, \sigma-\sigma}}{\partial t}\right|_{\text {coh }} & =\frac{i}{2} g \mu_{B} B\left(f_{\mathbf{k} \sigma}-f_{\mathbf{k}-\sigma}\right)-i \sigma \omega(\mathbf{k}) \rho_{\mathbf{k}, \sigma-\sigma}+i \sum_{\mathbf{q}} V_{\mathbf{q}}\left(f_{\mathbf{k}+\mathbf{q} \sigma}-f_{\mathbf{k}+\mathbf{q}-\sigma}\right) \rho_{\mathbf{k}, \sigma-\sigma}-i \sum_{\mathbf{q}} V_{\mathbf{q}}\left(f_{\mathbf{k} \sigma}-f_{\mathbf{k}-\sigma}\right) \rho_{\mathbf{k}+\mathbf{q}, \sigma-\sigma}
\end{aligned}
$$

where $B$ is along the $x$-axis and $\omega(\mathbf{k})=-g \mu_{B} \Omega_{z}^{11}(\mathbf{k})$. It is seen that in the presence of the magnetic field, the spin coherence $\rho_{\mathbf{k}, \sigma-\sigma}$ is involved and $\omega(\mathbf{k})$ in Eq. (221) provides the inhomogeneous broadening which leads to the spin relaxation and dephasing [675]. Experimentally Döhrmann et al. [388, 667] observed a "turn on" of the spin relaxation by switching on the magnetic field.

The spin relaxation in the absence of the magnetic field in symmetric GaAs (110) quantum wells with small well width is an interesting problem and has been studied extensively [183, 193, 388, 602, 660, 662, 663, 667]. In most of these works, the main reason limiting the spin relaxation time is attributed to the Bir-Aronov-Pikus mechanism. In the spin noise spectroscopy measurements by Müller et al. [660], the excitation of the semiconductor is negligible and hence the Bir-Aronov-Pikus mechanism is avoided. They reported spin relaxation about $24 \mathrm{~ns}$ and attributed it to the D'yakonov-Perel' mechanism due to the random Rashba fields caused by fluctuations in the donor density first proposed by Sherman [294, 657]. Zhou and Wu further investigated this effect using the kinetic spin Bloch equations, where the contribution of the Coulomb scattering originally missed in Refs. [294, 657] is included [670]. The method 

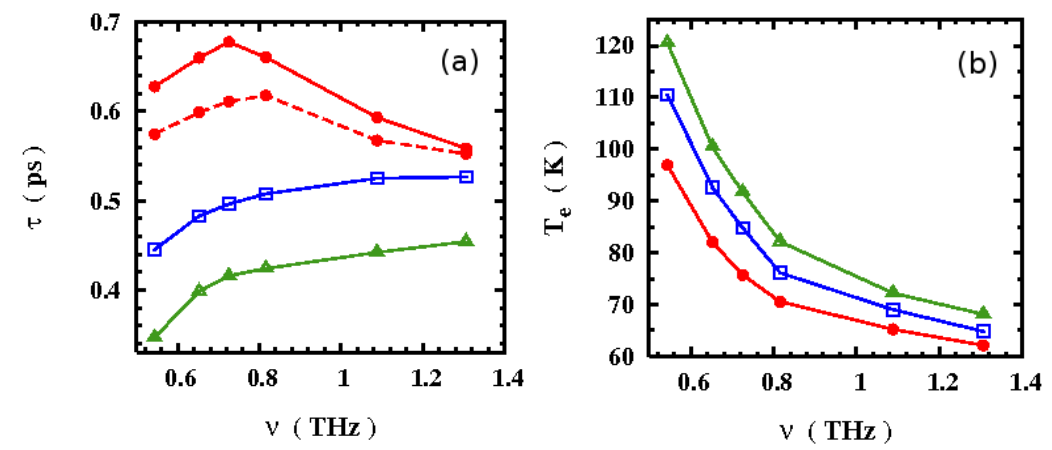

Figure 77: InAs (001) quantum wells: (a) Dependence of the spin relaxation time $\tau$ on THz frequency for impurity densities: $N_{i}=0(\bullet)$; $N_{i}=0.02 N_{e}(\square)$; and $N_{i}=0.05 N_{e}(\Delta)$. Solid curves: from full calculation; Dotted curve: from the calculation without the THz-field-induced effective magnetic field $B_{\text {eff. }} E=1 \mathrm{kV} / \mathrm{cm}$ and $T=50 \mathrm{~K}$. (b) Dependence of the hot-electron temperature $T_{e}$ on THz frequency for impurity densities: $N_{i}=0(\bullet) ; N_{i}=0.02 N_{e}(\square) ;$ and $N_{i}=0.05 N_{e}(\triangle) . E=1 \mathrm{kV} / \mathrm{cm}$ and $T=50 \mathrm{~K}$. From Jiang et al. [69].

to incorporate the effect of the Random Rashba spin-orbit coupling in the kinetic spin Bloch equation approach is to calculate the time evolutions of the kinetic spin Bloch equations under sufficient Rashba coefficients $\alpha_{\mathrm{R}}$, which are assumed to satisfy the Gaussian distribution $P\left(\alpha_{\mathrm{R}}\right)=\frac{1}{\sqrt{2 \pi} \Delta} e^{-\alpha_{\mathrm{R}}^{2} /\left(2 \Delta^{2}\right)}$. The spin relaxation time is obtained by the slope of the coherently summed spin polarization

$$
S_{z}=\int d \alpha_{\mathrm{R}} P\left(\alpha_{\mathrm{R}}\right) \sum_{\mathbf{k}} \frac{1}{2}\left(f_{\mathbf{k} \uparrow}^{\alpha_{\mathrm{R}}}-f_{\mathbf{k} \downarrow}^{\alpha_{\mathrm{R}}}\right) .
$$

A calculation based on the kinetic spin Bloch equations can nicely reproduce the experimental findings by Müller et al. [660] in Fig. 78 a). They also showed that at low impurity density, the Coulomb scattering has a strong influence to the spin relaxation, as shown in Fig. 78 b), in which a peak due to the Coulomb scattering in the temperature dependence of the spin relaxation time was predicted.

Besides the Random Rashba spin-orbit coupling field, Zhou and Wu proposed a virtual intersubband spin-flip spinorbit coupling induced spin relaxation in GaAs (110) quantum wells and showed this mechanism becomes important for samples with high impurity density [668].
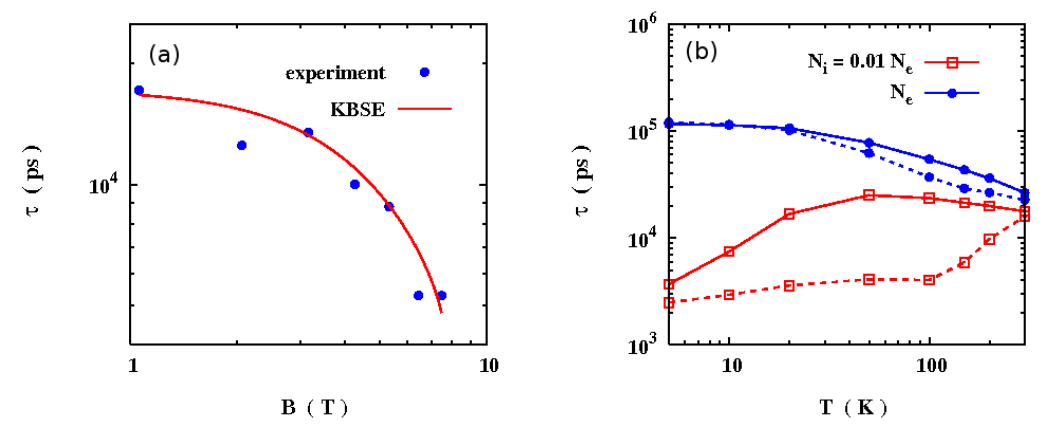

Figure 78: GaAs (110) quantum wells: (a) Spin relaxation times vs. the in-plane magnetic field strength from the KBSE approach and from the experimental data in Ref. [660] with temperature $T=20 \mathrm{~K}$, well width $a=16.8 \mathrm{~nm}$, electron density $N_{e}=1.8 \times 10^{11} \mathrm{~cm}^{-2}$ and impurity density $N_{i}=0.01 N_{e}$. The fitting parameters are $E_{w}=4.2 \mathrm{kV} / \mathrm{cm}, \gamma_{\mathrm{D}}=5.02 \mathrm{eV} \cdot \AA^{3}$. (b) Spin relaxation times due to the random Rashba spin-orbit coupling induced D'yakonov-Perel' mechanism vs. temperature $T$ for impurity densities $N_{i}=N_{e}$ and $0.01 N_{e}$ with $B=0, a=16.8 \mathrm{~nm}$ and $N_{e}=1.8 \times 10^{11} \mathrm{~cm}^{-2}$. The corresponding Fermi temperature is $75 \mathrm{~K}$. The solid (dashed) curves represent the results calculated with (without) the electron-electron Coulomb scattering. From Zhou and Wu [668]. 


\subsubsection{Spin dynamics in paramagnetic $G a(M n)$ As quantum wells}

Jiang et al. further extended the kinetic spin Bloch equations to study the electron spin relaxation in paramagnetic $\mathrm{Ga}(\mathrm{Mn})$ As quantum wells [111]. Besides the D'yakonov-Perel' [101, 102] and Bir-Aronov-Pikus [105, 106] mechanisms, they further included the spin relaxation mechanism due to the exchange coupling of the electrons and the localized Mn spins (the s-d exchange scattering mechanism) [114] and the Elliott-Yafet mechanism [104, 868] due to the presence of the heavily doped $\mathrm{Mn}$. In $\mathrm{Ga}(\mathrm{Mn}) \mathrm{As}$, the Mn dopants can be either substitutional or interstitial; the substitutional $\mathrm{Mn}$ accepts one electron whereas the interstitial Mn releases two. Direct doping in low-temperature molecular-beam epitaxy growth gives rise to more substitutional Mn ions than interstitial ones, which makes the $\mathrm{Ga}(\mathrm{Mn}) \mathrm{As}$ a $p$-type semiconductor [114, 233, 869, 870]. In GaAs quantum wells near $\mathrm{Ga}(\mathrm{Mn}) \mathrm{As}$ layer, the $\mathrm{Mn}$ dopants can diffuse into the GaAs quantum wells, where the Mn ions mainly take the interstitial positions, making the quantum well $n$-type [871-873].

The kinetic spin Bloch equations are the same as those in GaAs quantum wells, except the additional terms in the coherent and scattering terms associated with the $\mathrm{Mn} \operatorname{spin} \mathbf{S}$ and the Elliott-Yafet mechanism. The additional coherent term reads

$$
\left.\partial_{t} \hat{\rho}_{\mathbf{k}}\right|_{\mathrm{Mn}} ^{\mathrm{coh}}=-i\left[\hat{H}_{\mathrm{mf}}^{\mathrm{sd}}, \hat{\rho}_{\mathbf{k}}\right],
$$

with $\hat{H}_{\mathrm{sd}}^{\mathrm{mf}}=-N_{\mathrm{Mn}} \alpha\langle\mathbf{S}\rangle \cdot \frac{\sigma}{2}$. Here $\langle\mathbf{S}\rangle$ is the average spin polarization of Mn ions and $\alpha$ is the $s-d$ exchange coupling constant. For simplicity, Mn ions are assumed to be uniformly distributed within and around the quantum wells with a bulk density $N_{\mathrm{Mn}}$. The additional scattering terms are those from the $s-d$ exchange scattering and the Elliott-Yafet mechanism. The $s$ - $d$ exchange scattering term is given by

$$
\left.\partial_{t} \hat{\rho}_{\mathbf{k}}\right|_{s d} ^{\text {scat }}=-\pi N_{\mathrm{Mn}} \alpha^{2} I_{s} \sum_{\eta_{1} \eta_{2} \mathbf{k}^{\prime}} G_{\mathrm{Mn}}\left(-\eta_{1}-\eta_{2}\right) \delta\left(\varepsilon_{\mathbf{k}}-\varepsilon_{\mathbf{k}^{\prime}}\right)\left(\hat{s}^{\eta_{1}} \hat{\rho}_{\mathbf{k}^{\prime}}^{>} \hat{s}^{\eta_{2}} \hat{\rho}_{\mathbf{k}}^{<}-\hat{s}^{\eta_{2}} \hat{\rho}_{\mathbf{k}^{\prime}}^{<} \hat{s}^{\eta_{1}} \hat{\rho}_{\mathbf{k}}^{>}+\text {H.c. }\right)
$$

with $G_{\mathrm{Mn}}\left(\eta_{1} \eta_{2}\right)=\frac{1}{4} \operatorname{Tr}\left(\hat{S}^{\eta_{1}} \hat{S}^{\eta_{2}} \hat{\rho}_{\mathrm{Mn}}\right) . \hat{S}^{\eta}$ and $\hat{S}^{\eta}(\eta=0, \pm 1)$ are the spin ladder operators. The equation of motion for Mn spin density matrix consists of three parts $\partial_{t} \hat{\rho}_{\mathrm{Mn}}=\left.\partial_{t} \hat{\rho}_{\mathrm{Mn}}\right|_{\text {coh }}+\left.\partial_{t} \hat{\rho}_{\mathrm{Mn}}\right|_{\text {scat }}+\left.\partial_{t} \hat{\rho}_{\mathrm{Mn}}\right|_{\text {rel }}$. The first part describes the coherent precession around the external mangetic field and the $s-d$ exchange mean field, $\left.\partial_{t} \hat{\rho}_{\mathrm{Mn}}\right|_{\mathrm{coh}}=$ $-i\left[g_{\mathrm{Mn}} \mu_{\mathrm{B}} \mathbf{B} \cdot \hat{\mathbf{S}}-\alpha \sum_{\mathbf{k}} \operatorname{Tr}\left(\frac{\hat{\sigma}}{2} \hat{\rho}_{\mathbf{k}}\right) \cdot \hat{\mathbf{S}}, \hat{\rho}_{\mathrm{Mn}}\right]$. The second part represents the $s$ - $d$ exchange scattering with electrons $\left.\partial_{t} \hat{\rho}_{\mathrm{Mn}}\right|_{\text {scat }}=-\frac{\pi \alpha^{2}}{4} \sum_{\eta_{1} \eta_{2} \mathbf{k}} \delta\left(\varepsilon_{\mathbf{k}}-\varepsilon_{\mathbf{k}^{\prime}}\right) \operatorname{Tr}\left(\hat{S}^{-\eta_{2}} \hat{\rho}_{\mathbf{k}^{\prime}}^{<} \hat{s}^{-\eta_{1}} \hat{\rho}_{\mathbf{k}}^{>}\right)\left[\left(\hat{S}^{\eta_{1}} \hat{S}^{\eta_{2}} \hat{\rho}_{\mathrm{Mn}}-\hat{S}^{\eta_{1}} \hat{\rho}_{\mathrm{Mn}} \hat{S}^{\eta_{2}}\right)+\right.$ H.c. $]$. The third part characterizes the Mn spin relaxation due to other mechanisms, such as the $p$ - $d$ exchange interaction with holes or Mn-spin-lattice interaction, with a relaxation time approximation, $\left.\partial_{t} \hat{\rho}_{\mathrm{Mn}}\right|_{\mathrm{rel}}=-\left(\hat{\rho}_{\mathrm{Mn}}-\hat{\rho}_{\mathrm{Mn}}^{0}\right) / \tau_{\mathrm{Mn}}$. Here $\hat{\rho}_{\mathrm{Mn}}^{0}$ represents the equilibrium Mn spin density matrix. $\tau_{\mathrm{Mn}}$ is the Mn spin relaxation time, which is typically $0.1 \sim 10 \mathrm{~ns}$ [98].

After incorporating the Elliott-Yafet mechanism, besides the ordinary spin-conserving terms, there are spin-flip terms. For electron-impurity scattering these additional terms are

$$
\begin{aligned}
\left.\partial_{t} \hat{\rho}_{\mathbf{k}}\right|_{\mathrm{ei}} ^{\mathrm{EY}}=- & \pi n_{i} \sum_{\mathbf{k}^{\prime}} \delta\left(\varepsilon_{\mathbf{k}}-\varepsilon_{\mathbf{k}^{\prime}}\right)\left[U_{\mathbf{k}-\mathbf{k}^{\prime}}^{(1)}\left(\hat{\Lambda}_{\mathbf{k}, \mathbf{k}^{\prime}}^{(1)} \hat{\rho}_{\mathbf{k}^{\prime}}^{>} \hat{\Lambda}_{\mathbf{k}^{\prime}, \mathbf{k}}^{(1)} \hat{\rho}_{\mathbf{k}}^{<}-\hat{\Lambda}_{\mathbf{k}, \mathbf{k}^{\prime}}^{(1)} \hat{\rho}_{\mathbf{k}^{\prime}}^{<} \hat{\Lambda}_{\mathbf{k}^{\prime}, \mathbf{k}}^{(1)} \hat{\rho}_{\mathbf{k}}^{>}\right)\right. \\
& \left.+U_{\mathbf{k}-\mathbf{k}^{\prime}}^{(2)}\left(\hat{\Lambda}_{\mathbf{k}, \mathbf{k}^{\prime}}^{(2)} \hat{\rho}_{\mathbf{k}^{\prime}}^{>} \hat{\Lambda}_{\mathbf{k}^{\prime}, \mathbf{k}}^{(2)} \hat{\rho}_{\mathbf{k}}^{<}-\hat{\Lambda}_{\mathbf{k}, \mathbf{k}^{\prime}}^{(2)} \hat{\rho}_{\mathbf{k}^{\prime}}^{<} \hat{\Lambda}_{\mathbf{k}^{\prime}, \mathbf{k}}^{(2)} \hat{\rho}_{\mathbf{k}}^{>}\right)+\text {H.c. }\right],
\end{aligned}
$$

where $n_{i}=N_{\mathrm{Mn}}^{\mathrm{S}}+4 N_{\mathrm{Mn}}^{\mathrm{I}}+n_{i 0}$ with $N_{\mathrm{Mn}}^{\mathrm{S}}, N_{\mathrm{Mn}}^{\mathrm{I}}$ and $n_{i 0}$ representing the densities of substitutional Mn, interstitial Mn and non-magnetic impurities, respectively. $U_{\mathbf{k}-\mathbf{k}^{\prime}}^{(1)}=\frac{\lambda_{c}^{2}}{4} \sum_{q_{z}} V_{\mathbf{k}-\mathbf{k}^{\prime}, q_{z}}^{2}\left|I\left(i q_{z}\right)\right|^{2} q_{z}^{2}$ and $U_{\mathbf{k}-\mathbf{k}^{\prime}}^{(2)}=-\lambda_{c}^{2} \sum_{q_{z}} V_{\mathbf{k}-\mathbf{k}^{\prime}, q_{z}}^{2}\left|I\left(i q_{z}\right)\right|^{2}$. Here $\lambda_{c}=\frac{\eta(1-\eta / 2)}{3 m_{c} E_{g}(1-\eta / 3)}$ with $\eta=\frac{\Delta_{\mathrm{sO}}}{\Delta_{\mathrm{so}}+E_{g}} . E_{g}$ and $\Delta_{\mathrm{SO}}$ are the band-gap and the spin-orbit splitting of the valence band, respectively [3]. The spin-flip matrices are given by $\hat{\Lambda}_{\mathbf{k}^{\prime}, \mathbf{k}}^{(1)}=\left[\left(\mathbf{k}+\mathbf{k}^{\prime}, 0\right) \times \hat{\boldsymbol{\sigma}}\right]_{z}$ and $\hat{\Lambda}_{\mathbf{k}, \mathbf{k}^{\prime}}^{(2)}=\left[(\mathbf{k}, 0) \times\left(\mathbf{k}^{\prime}, 0\right)\right] \cdot \hat{\boldsymbol{\sigma}}$. It is noted that $\hat{\Lambda}_{\mathbf{k}^{\prime} \mathbf{k}}^{(1)}$ and $\hat{\Lambda}_{\mathbf{k}^{\prime}, \mathbf{k}}^{(2)}$ contribute to the out-of-plane and in-plane spin relaxations, respectively. They are generally different and therefore the spin relaxation due to the Elliott-Yafet mechanism in quantum wells is anisotropic. The Elliott-Yafet mechanism can be incorporated into other scatterings similarly [110].

By solving the kinetic spin Bloch equations, Jiang et al. [111] studied the spin relaxation in both $n$ - and $p$-type $\mathrm{Ga}(\mathrm{Mn})$ As quantum wells. For $n$-type sample, the total electron density is given by $N_{e}=N_{e}^{i}+N_{e}^{\mathrm{Mn}}+N_{\mathrm{ex}}$ with $N_{e}^{\mathrm{Mn}}$, $N_{e}^{i}$ and $N_{\text {ex }}$ representing the densities from Mn donors, other donors and photo excitation, respectively. The calculated spin relaxation times due to various mechanisms are shown as function of Mn concentration in $n$-type samples with 
$N_{e}^{i}=0$ (a) and $N_{e}^{i}=10^{11} \mathrm{~cm}^{-2}$ (b) respectively (Fig. 79). It is interesting to see that even for the strong $s$ - $d$ exchange coupling taken in the calculation, the spin relaxation due to the $s$ - $d$ exchange coupling is still much weaker than that due to the D'yakonov-Perel' mechanism. Also the Bir-Aronov-Pikus and Elliott-Yafet mechanisms are irrelevant to the spin relaxation. The spin relaxatin is solely determined by the D'yakonov-Perel' mechanism. Moreover, they predicted a peak in the Mn concentration dependence of the spin relaxation time. The physics leading to the peak is the same as the carrier-density dependence of the spin relaxation time in $n$-type GaAs quantum wells addressed in Sec.5.4.2 with the peak being around the electron Fermi temperature $T_{F}^{e}$.
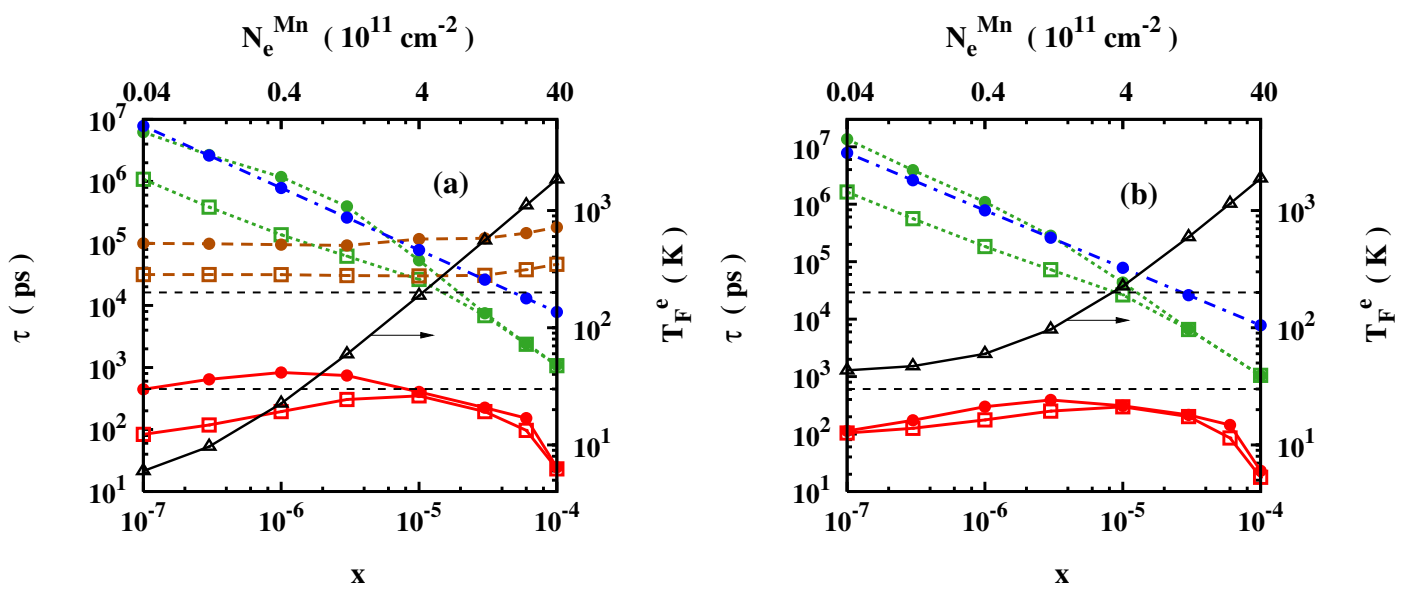

Figure 79: Spin relaxation time $\tau$ due to various mechanisms in $n$-type Ga(Mn)As quantum wells which are (a) undoped or (b) $n$-doped before Mn-doping as function of Mn concentration $x$ at $30 \mathrm{~K}(\bullet)$ and $200 \mathrm{~K}(\square)$. Red solid curves: the spin relaxation time due to the D'yakonov-Perel' mechanism $\tau_{\mathrm{DP}}$; Green dotted curves: the spin relaxation time due to the Elliott-Yafet mechanism $\tau_{\mathrm{EY}}$; Brown dashed curves: the spin relaxation time due to the Bir-Aronov-Pikus mechanism $\tau_{\mathrm{BAP}}$; Blue chain curve: the spin relaxation time due to the $s$ - $d$ exchange scattering mechanism $\tau_{\mathrm{sd}}$. The Fermi temperature of electrons $T_{\mathrm{F}}^{e}$ is ploted as black curve with $\Delta$ (the scale of $T_{\mathrm{F}}^{e}$ is on the right hand side of the frame) and $T_{\mathrm{F}}^{e}=T$ for both $T=30$ and $200 \mathrm{~K}$ cases are plotted as black dashed curves. The scale of the electron density from Mn donors $N_{e}^{\mathrm{Mn}}$ is also plotted on the top of the frame. From Jiang et al. [111].

For $p$-type $\mathrm{Ga}(\mathrm{Mn})$ As quantum wells, both substitutional and interstitial $\mathrm{Mn}$ ions exist in the system. For simplicity, all the holes are assumed free. Due to the presence of interstitial Mn ions, the ratio of the hole density $N_{h}$ to the Mn density $\mathrm{N}_{\mathrm{Mn}}$ was obtained by fitting the experimental data in Ref. [233], as shown in Fig. 80, The electron spin relaxations due to various mechanisms were calculated as function of Mn concentration at different temperatures by Jiang et al. [111], as shown in Fig. 81, Unlike the case of $n$-type samples, due to the presence of large hole density at high Mn concentration, the Bir-Aronov-Pikus and $s$ - $d$ exchange scattering mechanisms can be important. At very low temperature [Fig. 81 (a)], due to the Pauli blocking addressed in Sec. 5.4.6, the Bir-Aronov-Pikus mechanism is negligible. The spin relaxation is determined by the D'yakonov-Perel' mechanism at low Mn concentration and the $s$ - $d$ exchange scattering mechanism at high Mn concentration. At medium temperature [Fig. 81 (b)], both the Bir-Aronov-Pikus and $s$ - $d$ exchange scattering mechanisms determine the spin relaxation at high Mn concentrations. At high temperature [Fig. 81(c)], the spin relaxation is determined by the Bir-Aronov-Pikus mechanism. As the D'yakonov-Perel' mechanism determines the spin relaxation at low Mn concentrations, the spin relaxation time limited by it increases with increasing Mn concentration (increasing electron-impurity scattering). Moreover, both the Bir-Aronov-Pikus and $s-d$ exchange scattering mechanisms increase with increasing Mn density. As a result, there is a peak in the Mn density dependence of the electron spin relaxation time at any temperature (except for the extremely low temperature where the localization becomes important).

The temperature, photo-excitation density and magnetic field dependences of the spin relaxation in paramagnetic $\mathrm{Ga}(\mathrm{Mn})$ As quantum wells have also been investigated in detail in Ref. [111]. 


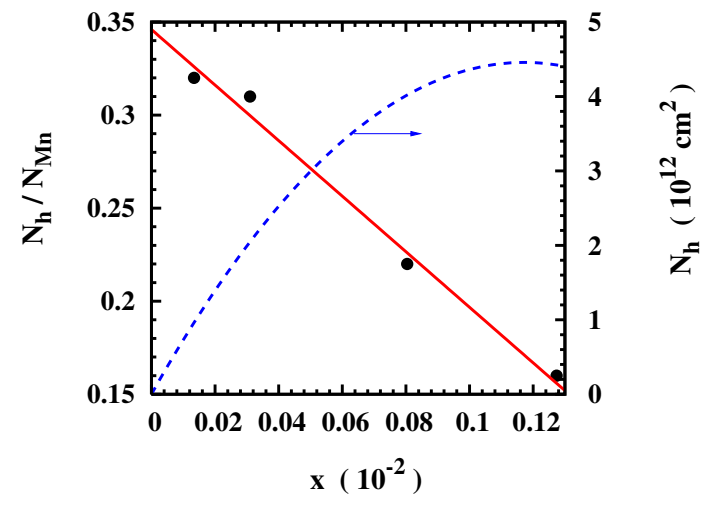

Figure 80: Ratio of the hole density to the Mn density $N_{h} / N_{\mathrm{Mn}}$ vs. the Mn concentration $x$ in $p$-type Ga(Mn)As quantum wells. The black dots represent the experimental data. The red solid curve is the fitted one. The hole density $N_{h}$ is also plotted (the blue dashed curve). Note that the scale of $N_{h}$ is on the right hand side of the frame. From Jiang et al. [111].
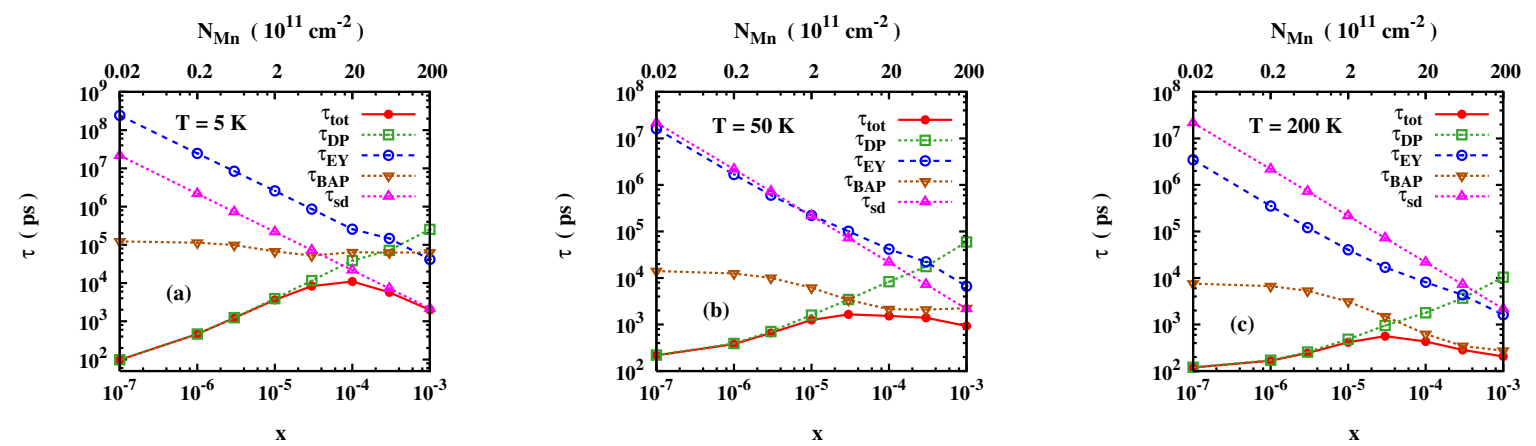

Figure 81: Spin relaxation time $\tau$ due to various mechanisms and the total spin relaxation time in $p$-type Ga(Mn)As quantum wells against the Mn concentration $x$ at (a) $T=5$, (b) 50 and (c) $200 \mathrm{~K}$. The scale of $N_{\mathrm{Mn}}$ is also plotted on the top of the frame. From Jiang et al. [111].

\subsubsection{Hole spin dynamics in (001) strained asymmetric Si/SiGe and Ge/SiGe quantum wells}

Among different kinds of hosts for spintronics devices, Silicon appears to be a particularly promising one, partly due to the high possibility of eliminating hyperfine couplings by isotropic purification and well developed microfabrication technology [874]. Many investigations have been carried out to understand the electron spin relaxation in bulk Silicon and its nanostructures [150, 290, 295, 763, 771, 776]. The study of hole spin relaxation in Silicon is very limited. Glavin and Kim presented a first calculation of the spin relaxation of two-dimensional holes in strained asymmetric $\mathrm{Si} / \mathrm{SiGe}(\mathrm{Ge} / \mathrm{SiGe})$ quantum wells [298] by means of the single-particle approximation where the important effect of the Coulomb scattering to the spin relaxation is absent. More seriously, as pointed out by Zhang and Wu [299], the nondegenerate perturbation method with only the lowest unperturbed subband of each hole state considered in the calculation of the subband energy spectrum and envelope functions by Glavin and Kim [298] is inadequate in converging the calculation. However, when more unperturbed subbands are included as basis functions, the nondegenerate perturbation method even fails. By applying the exact diagonalizing method to obtain the energy spectrum and envelope functions, Zhang and $\mathrm{Wu}$ studied the hole spin relaxation in (001) strained asymmetric $\mathrm{Si}_{/} / \mathrm{Si}_{0.7} \mathrm{Ge}_{0.3}$ $\left(\mathrm{Ge} / \mathrm{Si}_{0.3} \mathrm{Ge}_{0.7}\right)$ quantum wells in the situation with only the lowest hole subband being relevant by means of the kinetic spin Bloch equation approach [299].

The structures of $\mathrm{SiO}_{2} / \mathrm{Si}_{2} / \mathrm{Si}_{0.7} \mathrm{Ge}_{0.3}$ and $\mathrm{SiO}_{2} / \mathrm{Ge} / \mathrm{Si}_{0.3} \mathrm{Ge}_{0.7}$ (001) quantum wells are illustrated in Fig. 82, with the 
confining potential $V(z)$ approximated by the triangular potential due to the large gate voltage. The subband envelope functions are obtained by solving the eigen-equation of the $6 \times 6$ Luttinger Hamiltonian including the heavy hole, light hole and split-off hole states [122, 246, 875] under the confinement $V(z)$, with sufficient basis functions included [299]. Due to the biaxial strain [122, 246, 875, 876], the lowest subband in Si/SiGe quantum wells is light hole-like, which is an admixture of the light hole and split-off hole states, whereas that in Ge/SiGe quantum wells is a pure heavy hole state. By using the Löwdin partition method [113], the effective Hamiltonian of the lowest hole subband in $\mathrm{Si} / \mathrm{SiGe}(\mathrm{Ge} / \mathrm{SiGe})$ quantum wells can be written as [299]

$$
H_{\mathrm{eff}}^{(l, h)}=-\frac{\mathbf{k}^{2}}{2 m^{(l, h)}}-\frac{1}{2} \boldsymbol{\sigma} \cdot \mathbf{\Omega}^{(l, h)}\left(k_{x}, k_{y}\right)
$$

where $\mathbf{k}$ is the in-plane momentum, $m^{(l)}\left[m^{(h)}\right]$ is the in-plane effective mass of the lowest light (heavy) hole subband in $\mathrm{Si} / \mathrm{SiGe}(\mathrm{Ge} / \mathrm{SiGe})$ quantum wells, $\sigma$ are the Pauli matrices, and $\mathbf{\Omega}^{(l)}\left[\mathbf{\Omega}^{(h)}\right]$ is the Rashba term of the lowest light hole (heavy hole) subband in $\mathrm{Si} / \mathrm{SiGe}(\mathrm{Ge} / \mathrm{SiGe})$ quantum wells. $\boldsymbol{\Omega}^{(l)}$ has both the linear and cubic dependences on momentum, whereas $\mathbf{\Omega}^{(h)}$ has only the cubic dependence. For the lowest light hole subband in Si/SiGe quantum wells,

$$
\begin{aligned}
m^{(l)} & =m_{0}\left[A-B\left(\lambda_{00}^{(l 1 l 1)} / 2-\sqrt{2} \lambda_{00}^{(l 1 l 2)}\right)\right]^{-1}, \\
\mathbf{\Omega}^{(l)} & =\boldsymbol{\Omega}_{1}^{(l)}+\boldsymbol{\Omega}_{3}^{(l)}, \\
\Omega_{1 x, y}^{(l)} & =\Xi k_{x, y}, \\
\Omega_{3 x}^{(l)} & =\Pi B k_{x}\left(k_{x}^{2}+k_{y}^{2}\right)+\Theta\left[3 B k_{x}\left(k_{x}^{2}-k_{y}^{2}\right)+2 \sqrt{3\left(3 B^{2}+C^{2}\right)} k_{y}^{2} k_{x}\right], \\
\Omega_{3 y}^{(l)} & =\Pi B k_{y}\left(k_{x}^{2}+k_{y}^{2}\right)+\Theta\left[3 B k_{y}\left(k_{y}^{2}-k_{x}^{2}\right)+2 \sqrt{3\left(3 B^{2}+C^{2}\right)} k_{x}^{2} k_{y}\right],
\end{aligned}
$$

with

$$
\begin{aligned}
\Xi & =\frac{\hbar}{m_{0}} \sqrt{6\left(3 B^{2}+C^{2}\right)} \kappa_{00}^{(l 1 l 2)}, \\
\Pi & =-\frac{\hbar^{3}}{2 m_{0}^{2}} \sqrt{\frac{3\left(3 B^{2}+C^{2}\right)}{2}} \sum_{\alpha=l, s} \sum_{n=0}^{\infty}\left(1-\delta_{l \alpha} \delta_{0 n}\right) \frac{\kappa_{0 n}^{(l 1 \alpha 2)}-\kappa_{0 n}^{(l 2 \alpha 1)}}{E_{0}^{(l)}-E_{n}^{(\alpha)}}\left[\sqrt{2}\left(\lambda_{0 n}^{(l 1 \alpha 2)}+\lambda_{0 n}^{(l 2 \alpha 1)}\right)-\lambda_{0 n}^{(l 1 \alpha 1)}\right], \\
\Theta & =-\frac{\hbar^{3}}{2 m_{0}^{2}} \sqrt{\frac{3 B^{2}+C^{2}}{3}} \sum_{n=0}^{\infty} \frac{\kappa_{0 n}^{(l 1 h)}-\frac{1}{\sqrt{2}} \kappa_{0 n}^{(l 2 h)}}{E_{0}^{(l)}-E_{n}^{(h)}}\left(\sqrt{2} \lambda_{0 n}^{(l 2 h)}+\lambda_{0 n}^{(l 1 h)}\right) .
\end{aligned}
$$

For the lowest heavy hole subband in $\mathrm{Ge} / \mathrm{SiGe}$ quantum wells,

$$
\begin{aligned}
& m^{(h)}=m_{0}(A+B / 2)^{-1}, \\
& \mathbf{\Omega}^{(h)}=\mathbf{\Omega}_{3}^{(h)} \\
& \Omega_{3 x}^{(h)}=\Lambda\left[3 B k_{x}\left(k_{x}^{2}-k_{y}^{2}\right)-2 \sqrt{3\left(3 B^{2}+C^{2}\right)} k_{y}^{2} k_{x}\right], \\
& \Omega_{3 y}^{(h)}=\Lambda\left[3 B k_{y}\left(k_{x}^{2}-k_{y}^{2}\right)+2 \sqrt{3\left(3 B^{2}+C^{2}\right)} k_{x}^{2} k_{y}\right],
\end{aligned}
$$

with

$$
\Lambda=-\frac{\hbar^{3}}{2 m_{0}^{2}} \sqrt{\frac{3 B^{2}+C^{2}}{3}} \sum_{\alpha=l, s} \sum_{n=0}^{\infty} \frac{\frac{1}{\sqrt{2}} \kappa_{0 n}^{(h \alpha 2)}-\kappa_{0 n}^{(h \alpha 1)}}{E_{0}^{(h)}-E_{n}^{(\alpha)}}\left(\sqrt{2} \lambda_{0 n}^{(h \alpha 2)}+\lambda_{0 n}^{(h \alpha 1)}\right) .
$$

Here $A, B$ and $C$ are the valence band parameters, which relate to the Luttinger parameters $\gamma_{1}, \gamma_{2}$ and $\gamma_{3}$ through $A=\gamma_{1}, B=2 \gamma_{2}$ and $\sqrt{3 B^{2}+C^{2}}=2 \sqrt{3} \gamma_{3} . E_{n}^{(\alpha)}(\alpha=h, l, s)$ are the subband energy levels. $\lambda_{n n^{\prime}}^{(\alpha \beta)}$ and $\kappa_{n n^{\prime}}^{(\alpha \beta)}$ are defined as $\lambda_{n n^{\prime}}^{(\alpha \beta)}=\int_{-\infty}^{+\infty} d z \chi_{n}^{(\alpha)}(z) \chi_{n^{\prime}}^{(\beta)}(z)$ and $\kappa_{n n^{\prime}}^{(\alpha \beta)}=\int_{-\infty}^{+\infty} d z \chi_{n}^{(\alpha)}(z) \frac{d \chi_{n^{\prime}}^{(\beta)}(z)}{d z}$, with $\chi_{n}^{(\alpha)}$ the envelope functinos [299]. The calculated spin-orbit coupling coefficients of the lowest subband of $\mathrm{Si} / \mathrm{SiGe}$ quantum well $(\Xi, \Pi$ and $\Theta$ ) and Ge/SiGe quantum well $(\Lambda)$ are given in Fig. 83 


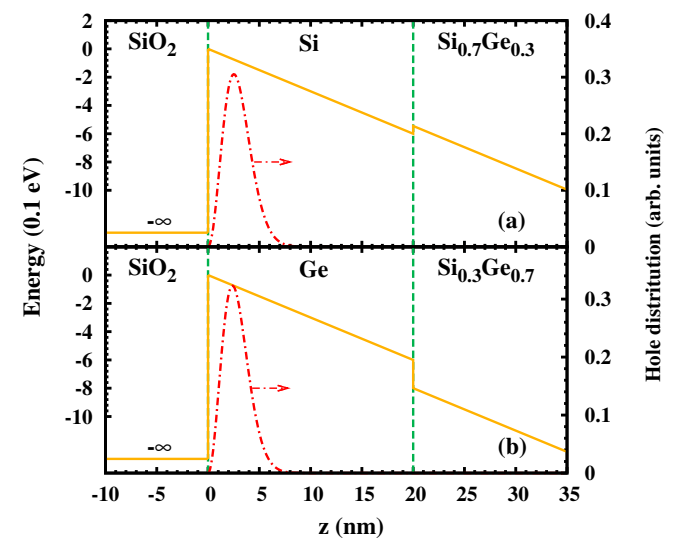

Figure 82: Schematics of the $\mathrm{SiO}_{2} / \mathrm{Si}_{/} / \mathrm{Si}_{0.7} \mathrm{Ge}_{0.3}$ quantum well structure (a) and $\mathrm{SiO}_{2} / \mathrm{Ge} / \mathrm{Si}_{0.3} \mathrm{Ge}_{0.7}$ quantum well structure (b). Two vertical dashed lines in each figure represent the two interfaces. The solid curves represent the confining potential $V(z)$ with electric field $E=300 \mathrm{kV} / \mathrm{cm}$. The valence band discontinuities at the $\mathrm{Si} / \mathrm{Si}_{0.7} \mathrm{Ge}_{0.3}$ and $\mathrm{Ge} / \mathrm{Si}_{0.3} \mathrm{Ge}_{0.7}$ interfaces are neglected in the triangular potential approximation. The chain curves with their scale on the right hand side of the frame respectively represent the lowest light hole and heavy hole distributions in $\mathrm{Si}_{/} \mathrm{Si}_{0.7} \mathrm{Ge}_{0.3}$ and $\mathrm{Ge} / \mathrm{Si}_{0.3} \mathrm{Ge}_{0.7}$ quantum wells along the $z$-axis. From Zhang and $\mathrm{Wu}$ [299].

The hole spin relaxation time is calculated by solving the kinetic spin Bloch equations, with the hole-deformation optical/acoustic phonon [877], hole-impurity and hole-hole Coulomb scatterings explicitly included [299]. The typical results are shown in Fig. 84 for $\mathrm{Si} / \mathrm{SiGe}$ quantum wells, where a peak appears in both the temperature dependence (located around the hole Fermi temperature) and the density dependence (located around the crossover from the degenerate to the nondegenerate regimes) of hole spin relaxation time. It is also shown that the hole-hole Coulomb scattering plays an essential role in the spin relaxation. As shown in Fig. 85, by switching off the hole-hole Coulomb scattering, the spin relaxation times differ dramatically. Similar behavior also happens in Ge/SiGe quantum wells, except that the peak in the temperature dependence of the hole spin relaxation time is located around half of the hole Fermi temperature. The shift of the peak to the lower temperature is suggested to be due to the cubic momentum dependence of the Rashba term [Eqs. (236, 238] ], in contrast to the linear one which is important in the case of $\mathrm{Si} / \mathrm{SiGe}$ quantum wells. The effects of impurity and gate voltage on the hole spin relaxation are discussed in detail in Ref. [299].

Finally, it is noted that unlike holes in GaAs quantum wells where the system is in the weak scattering limit [363], holes in $\mathrm{Si} / \mathrm{SiGe}$ quantum wells are generally in the strong scattering limit thanks to the strong hole-hole Coulomb scattering and weak Rashba spin-orbit coupling. However, holes in Ge/SiGe quantum wells can be in the weak scattering limit with high density at low temperature due to the larger Rashba term [299]. In any case, adding impurities can shift the system from weak scattering limit to the strong one [299, 363].

\subsubsection{Spin relaxation and dephasing in n-type wurtzite $\mathrm{ZnO}(0001)$ quantum wells}

While there are a lot of studies on the spin dynamics in cubic zinc-blende semiconductors, much attention has also been devoted to the spin properties of zinc oxide $(\mathrm{ZnO})$ with wurtzite structures [556, 559, 568, 878-882]. Harmon et al. calculated the spin relaxation time in bulk material in the framework of single particle approach [559]. Lü and Cheng [613] applied the kinetic spin Bloch equation approach and calculated the spin relaxation in $n$-type $\mathrm{ZnO}$ (0001) quantum wells under various conditions, including well width, impurity density and external electric field (hot-electron effect). In wurtzite structure, the D'yakonov-Perel' term comes from the Rashba term due to the intrinsic wurtzite structure inversion asymmetry $\boldsymbol{\Omega}_{\mathrm{R}}(\mathbf{k})$ and the Dresselhaus term $\boldsymbol{\Omega}_{\mathrm{D}}(\mathbf{k})$ which can be written as [245]:

$$
\begin{aligned}
& g \mu_{B} \boldsymbol{\Omega}_{\mathrm{R}}(\mathbf{k})=\alpha_{e}\left(k_{y},-k_{x}, 0\right), \\
& g \mu_{B} \boldsymbol{\Omega}_{\mathrm{D}}^{n}(\mathbf{k})=\gamma_{e}\left(b\left\langle k_{z}^{2}\right\rangle_{n}-k_{\|}^{2}\right)\left(k_{y},-k_{x}, 0\right),
\end{aligned}
$$




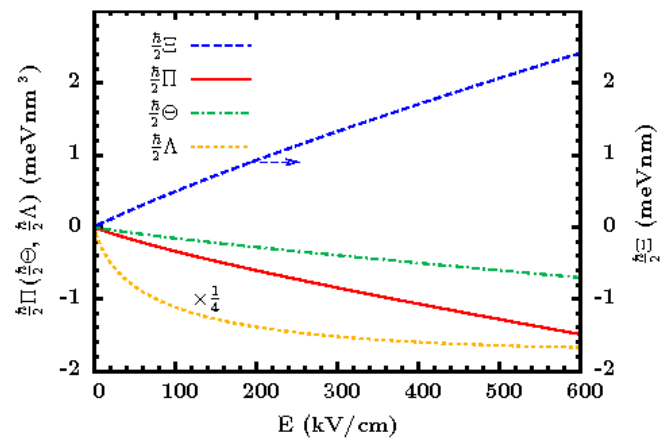

Figure 83: Spin-orbit coupling coefficients $\frac{\hbar}{2} \Xi, \frac{\hbar}{2} \Pi$ and $\frac{\hbar}{2} \Theta$ for the lowest light hole subband in Si/SiGe quantum wells and $\frac{\hbar}{2} \Lambda$ for the lowest heavy hole subband in $\mathrm{Ge} / \mathrm{SiGe}$ quantum wells against the electric field $E$. The scale of $\frac{\hbar}{2} \Xi$ is on the right hand side of the frame. From Zhang and Wu [299].
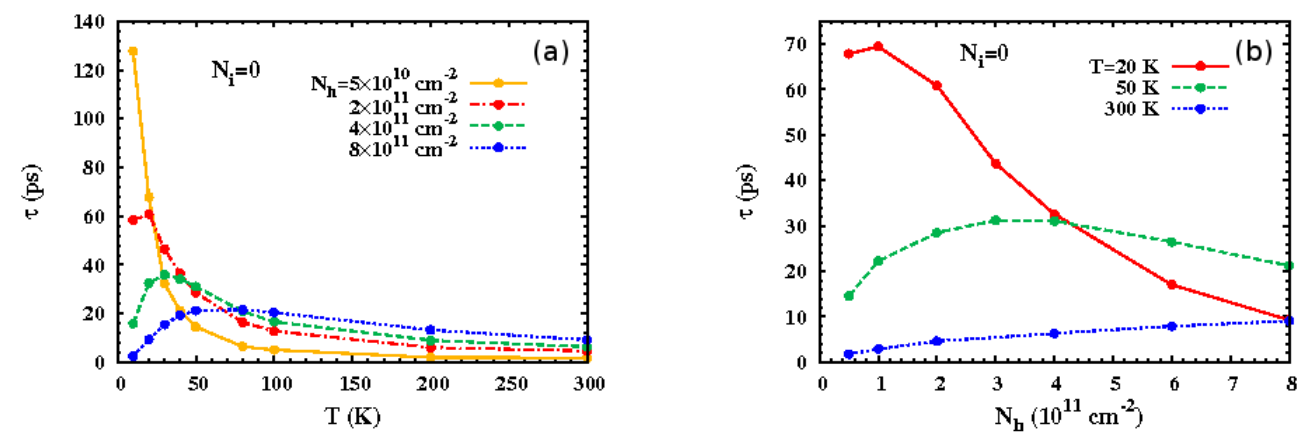

Figure 84: (a) Spin relaxation time $\tau$ against temperature $T$ with different hole densities. (b) Spin relaxation time $\tau$ against hole density $N_{h}$ with different temperatures. For both cases the impurity density $N_{i}=0$ and the electric field $E=300 \mathrm{kV} / \mathrm{cm}$. From Zhang and Wu [299].

with $\alpha_{e}, \gamma_{e}$ and $b$ standing for the spin-orbit coupling coefficients. $\left\langle k_{z}^{2}\right\rangle_{n}=n^{2} \pi^{2} / a^{2}$ is the subband energy in the hard wall potential approximation. By taking the lowest two subbands into account, Lü and Cheng solved the kinetic spin Bloch equations. The typical results are shown in Fig.86.

Basically the properties of the spin relaxation in $\mathrm{ZnO}$ quantum wells are all the same with those in GaAs ones. One still observes the peak in the temperature and density dependences (the former requires low impurity density) of the spin relaxation time. The difference is that for GaAs quantum wells, the temperature peak of the spin relaxation time can only be observed at low electron density (i.e., low transition temperature) and low impurity density [109, 372, 604], because the electron-phonon scattering becomes strong enough to destroy the nonmonotonic $T$ dependence of the scattering time induced by the electron-electron scattering. Such case can be avoided in $\mathrm{ZnO}$ quantum wells, in which the electron-phonon scattering is always pretty weak due to the large optical phonon energies $(\sim 800 \mathrm{~K})$. Thus the temperature peak can be found even for high electron density samples.

\subsection{Spin relaxation in quantum wires}

In recent years, progress in nanofabrication and growth techniques has made it possible to produce high-quality quantum wires and investigate physics in these nanostructures [883-890]. The energy spectrum of quantum wire systems with strong spin-orbit coupling has been studied both experimentally [239, 890, 891] and theoretically [191, $240,241,890,892-896]$. Unlike quantum wells discussed in Sec. [5.4, there is additional confinement in quantum wires and the remaining free degree of freedom is along the wire growth direction. Therefore, there are stronger 


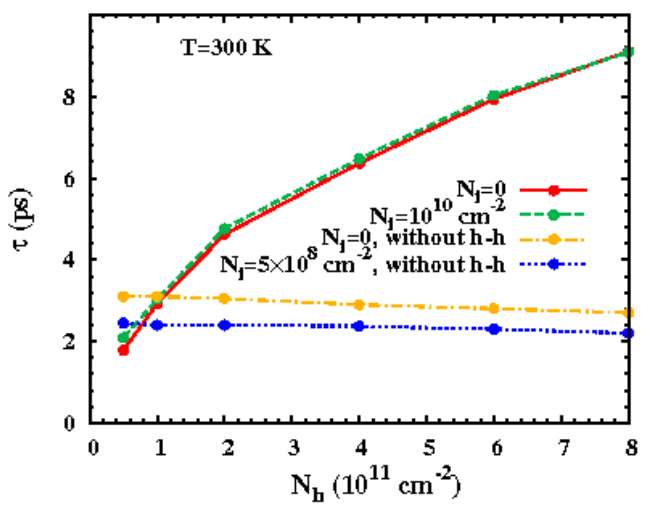

Figure 85: Spin relaxation time $\tau$ against hole density $N_{h}$ with different scatterings included in $\mathrm{Si} / \mathrm{SiGe}$ quantum well. $T=300 \mathrm{~K}$ and $E=$ $300 \mathrm{kV} / \mathrm{cm}$. Solid curve: with the hole-hole Coulomb (h-h), the hole-optical phonon (h-op) and the hole-acoustic phonon (h-ac) scatterings. Dashed curve: same as the solid curve with the additional hole-impurity scattering $\left(N_{i}=10^{10} \mathrm{~cm}^{-2}\right)$ included; Chain curve: with the h-op and h-ac scatterings; Dotted curve: same as the chain curve with the additional hole-impurity scattering $\left(N_{i}=5 \times 10^{8} \mathrm{~cm}^{-2}\right)$ included. From Zhang and Wu [299].
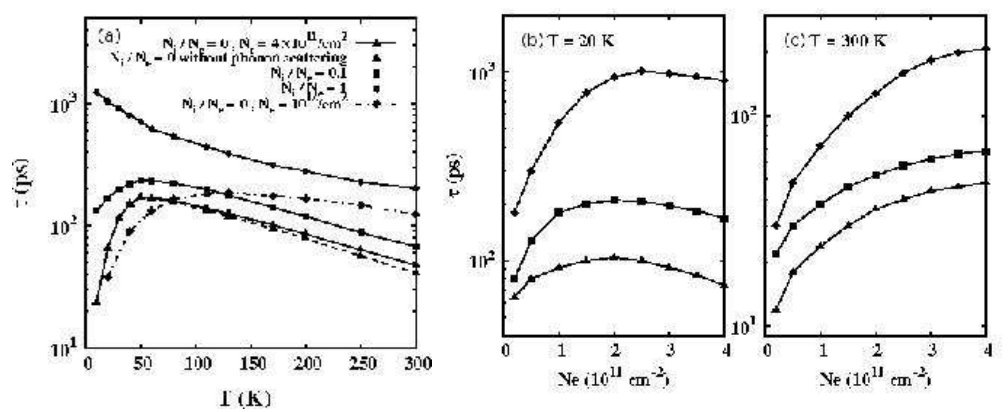

Figure 86: $\mathrm{ZnO}$ (0001) quantum wells: (a) Spin relaxation time $\tau$ vs. temperature $T$ at different impurity densities. The dashed curve is obtained from the calculation of excluding the electron-phonon scattering. (b) and (c): Spin relaxation time vs. the electron density with different impurity densities and temperatures $\left(20 \mathrm{~K}\right.$ and $300 \mathrm{~K}$ respectively). $\mathbf{\Delta}: N_{i} / N_{e}=0 ; \mathbf{\square}: N_{i} / N_{e}=0.1 ; \diamond: N_{i} / N_{e}=1$. From Lü and Cheng [613].

subband effect and also marked anisotropy along the growth directions of wires. Consequently this gives more choices for the manipulation of the spin degree of freedom.

For quantum wires, the spin relaxation was calculated in the framework of single particle approach [632, 698, 701, 897] and Monte Carlo simulations [651, 697, 701, 898]. Cheng et al. first applied the kinetic spin Bloch equations to study the spin dynamics in (001) oriented InAs quantum wires with only the lowest subband involved [899]. Lü et al. studied the influence of higher subbands and wire orientations on spin relaxation in $n$-type InAs quantum wires [696]. They reported that the intersubband Coulomb scattering can make an important contribution to the spin relaxation. Also due to intersubband scattering in connection with the spin-orbit coupling, spin relaxation in quantum wires can show different characteristics from those in bulk and in quantum wells. Hole spin relaxation in $p$-type GaAs quantum wires was also investigated by Lü et al. from the kinetic spin Bloch equation approach [705].

\subsubsection{Electron spin relaxation in n-type InAs quantum wires}

By modeling the InAs quantum wire by a rectangular confinement potential of infinite well depth along the $x-y$ plane $\left(|x| \leq a_{x},|y| \leq a_{y}\right)$, one can write the Rashba and Dresselhaus Hamiltonian by replacing $k_{x}, k_{x}^{2}, k_{y}$, and $k_{y}^{2}$ in the bulk Rashba and Dresselhaus Hamiltonian by $\left\langle\psi_{n x}|-i \partial / \partial x| \psi_{n^{\prime} x}\right\rangle,\left\langle\psi_{n x}\left|(-i \partial / \partial x)^{2}\right| \psi_{n^{\prime} x}\right\rangle,\left\langle\psi_{n y}|-i \partial / \partial y| \psi_{n^{\prime} y}\right\rangle$ and 
$\left\langle\psi_{n y}\left|(-i \partial / \partial y)^{2}\right| \psi_{n^{\prime} y}\right\rangle$, respectively. Here $\psi_{n x}=\sqrt{\frac{2}{a_{x}}} \sin \frac{n_{x} \pi x}{a_{x}}$ and $\psi_{n y}=\sqrt{\frac{2}{a_{y}}} \sin \frac{n_{n} \pi y}{a_{y}}$. The bulk Rashba term reads

$$
H_{\mathrm{R}}(\mathbf{k})=\gamma_{41}^{6 c 6 c} \boldsymbol{\sigma} \cdot \mathbf{k} \times \mathcal{E}=\gamma_{41}^{6 c 6 c}\left[\sigma_{x}\left(k_{y} \mathcal{E}_{z}-k_{z} \mathcal{E}_{y}\right)+\sigma_{y}\left(k_{z} \mathcal{E}_{x}-k_{x} \mathcal{E}_{z}\right)+\sigma_{z}\left(k_{x} \mathcal{E}_{y}-k_{y} \mathcal{E}_{x}\right)\right]
$$

For a (100) InAs quantum wire, the $x, y$ and $z$ axes correspond to the [100], [010] and [001] crystallographic directions, respectively, and the bulk Dresselhaus term can be written as [113]:

$$
H_{\mathrm{D}}^{100}=b_{41}^{6 c 6 c}\left\{\sigma_{x}\left[k_{x}\left(k_{y}^{2}-k_{z}^{2}\right)\right]+\sigma_{y}\left[k_{y}\left(k_{z}^{2}-k_{x}^{2}\right)\right]+\sigma_{z}\left[k_{z}\left(k_{x}^{2}-k_{y}^{2}\right)\right]\right\} .
$$

For a (110) quantum wire, the $x, y$ and $z$ directions correspond to the [110], [001] and [110] crystallographic directions, and one has

$$
H_{\mathrm{D}}^{110}=b_{41}^{6 c 6 c}\left\{\sigma_{x}\left[-\frac{1}{2} k_{z}\left(k_{x}^{2}-k_{z}^{2}+2 k_{y}^{2}\right)\right]+2 \sigma_{y} k_{x} k_{y} k_{z}+\sigma_{z}\left[\frac{1}{2} k_{x}\left(k_{x}^{2}-k_{z}^{2}-2 k_{y}^{2}\right)\right]\right\} .
$$

For a (111) quantum wire, the $x, y$ and $z$ directions correspond to the [112]], [1110], and [111] crystallographic directions, and one has

$$
\begin{aligned}
H_{\mathrm{D}}^{111}= & b_{41}^{6 c 6 c}\left\{\sigma_{x}\left[-\frac{\sqrt{2}}{\sqrt{3}} k_{x} k_{y} k_{z}-\frac{1}{2 \sqrt{3}} k_{y}^{3}-\frac{1}{2 \sqrt{3}} k_{y} k_{x}^{2}+\frac{2}{\sqrt{3}} k_{y} k_{z}^{2}-\frac{\sqrt{2}}{3} k_{y}^{2} k_{z}\right]\right. \\
& \left.+\sigma_{y}\left[\frac{1}{2 \sqrt{3}} k_{x}^{3}+\frac{1}{2 \sqrt{3}} k_{x} k_{y}^{2}-\frac{1}{\sqrt{6}} k_{x}^{2} k_{z}-\frac{1}{\sqrt{6}} k_{z}\left(k_{x}^{2}+k_{y}^{2}\right)\right]+\sigma_{z}\left[\frac{\sqrt{3}}{\sqrt{2}} k_{x}^{2} k_{y}-\frac{1}{\sqrt{6}} k_{y}^{3}-\frac{2}{3} k_{z} k_{y}^{2}\right]\right\} .
\end{aligned}
$$

The kinetic spin Bloch equations read $\dot{\rho}_{k}=\left.\dot{\rho}_{k}\right|_{\text {coh }}+\left.\dot{\rho}_{k}\right|_{\text {scat }}$, with the coherent terms being

$$
\left.\dot{\rho}_{k}\right|_{\mathrm{coh}}=i\left[\sum_{\mathbf{Q}} V_{\mathbf{Q}} I_{\mathbf{Q}} \rho_{k-q} I_{-\mathbf{Q}}, \rho_{k}\right]-i\left[H_{e}(k), \rho_{k}\right] .
$$

Here $\mathbf{Q} \equiv\left(q_{x}, q_{y}, q\right) . I_{\mathbf{Q}}$ is the form factor. In $\left(s, s^{\prime}\right)$ space $\left[s=\left(n_{x}, n_{y}, \sigma\right)\right]$, it reads

$$
I_{\mathbf{Q}, s_{1}, s_{2}}=\left\langle s_{1}\left|e^{i \mathbf{Q} \cdot \mathbf{r}}\right| s_{2}\right\rangle=\delta_{\sigma_{1}, \sigma_{2}} F\left(m_{1}, m_{2}, q_{y}, a_{y}\right) F\left(n_{1}, n_{2}, q_{x}, a_{x}\right),
$$

where

$$
F\left(m_{1}, m_{2}, q, a\right)=2 i a q\left[e^{i a q} \cos \pi\left(m_{1}-m_{2}\right)-1\right]\left[\frac{1}{\pi^{2}\left(m_{1}-m_{2}\right)^{2}-a^{2} q^{2}}-\frac{1}{\pi^{2}\left(m_{1}+m_{2}\right)^{2}-a^{2} q^{2}}\right] .
$$

The first term in Eq. (246) is the Coulomb Hartree-Fock term, and the second term comes from the single particle Hamiltonian $H_{e}=\frac{\mathbf{p}^{2}}{2 m^{*}}+H_{\mathrm{R}}+H_{\mathrm{D}}+V_{c}(\mathbf{r})$ in $\left(s, s^{\prime}\right)$ space. For small spin polarization, the contribution from the Hartree-Fock term in the coherent term is negligible [41, 42, 44] and the coherent spin dynamics is essentially due to the spin precession around the effective internal fields described by Eqs. (242)-(245).

The scattering contributions to the dynamic equation of the spin-density matrix include the electron-nonmagnetic impurity, electron-phonon and electron-electron scatterings:

$$
\begin{aligned}
\left.\frac{\partial \rho_{k}}{\partial t}\right|_{\mathrm{scat}}= & \left.\frac{\partial \rho_{k}}{\partial t}\right|_{\mathrm{im}}+\left.\frac{\partial \rho_{k}}{\partial t}\right|_{\mathrm{ph}}+\left.\frac{\partial \rho_{k}}{\partial t}\right|_{\mathrm{ee}}, \\
\left.\frac{\partial \rho_{k}}{\partial t}\right|_{\mathrm{im}}= & \pi N_{i} \sum_{\mathbf{Q}}^{s_{1}, s_{2}}\left|U_{\mathbf{Q}}^{i}\right|^{2} \delta\left(E_{s_{1}, k-q}-E_{s_{2}, k}\right) I_{\mathbf{Q}}\left[\left(1-\rho_{k-q}\right) T_{s_{1}} I_{-\mathbf{Q}} T_{s_{2}} \rho_{k}-\rho_{k-q} T_{s_{1}} I_{-\mathbf{Q}} T_{s_{2}}\left(1-\rho_{k}\right)\right]+\text { H.c., } \\
\left.\frac{\partial \rho_{k}}{\partial t}\right|_{\mathrm{ph}}= & \pi \sum_{\mathbf{Q}, \lambda}^{s_{1}, s_{2}}\left|M_{\mathbf{Q}, \lambda}\right|^{2} I_{\mathbf{Q}}\left\{\delta\left(E_{s_{1}, k-q}-E_{s_{2}, k}+\omega_{\mathbf{Q}, \lambda}\right)\left[\left(N_{\mathbf{Q}, \lambda}+1\right)\left(1-\rho_{k-q}\right) T_{s_{1}} I_{-\mathbf{Q}} T_{s_{2}} \rho_{k}-N_{\mathbf{Q}, \lambda} \rho_{k-q} T_{s_{1}} I_{-\mathbf{Q}} T_{s_{2}}\left(1-\rho_{k}\right)\right]\right. \\
& \left.\quad \delta\left(E_{s_{1}, k-q}-E_{s_{2}, k}-\omega_{\mathbf{Q}, \lambda}\right)\left[N_{\mathbf{Q}, \lambda}\left(1-\rho_{k-q}\right) T_{s_{1}} I_{-\mathbf{Q}} T_{s_{2}} \rho_{k}-\left(N_{\mathbf{Q}, \lambda}+1\right) \rho_{k-q} T_{s_{1}} I_{-\mathbf{Q}} T_{s_{2}}\left(1-\rho_{k}\right)\right]\right\}+ \text { H.c., } \\
\left.\frac{\partial \rho_{k}}{\partial t}\right|_{\mathrm{ee}}= & \pi \sum_{\mathbf{Q}, k^{\prime}}^{s_{1}, s_{2}, s_{3}, s_{4}} V_{\mathbf{Q}}^{2} \delta\left(E_{s_{1}, k-q}-E_{s_{2}, k}+E_{s_{3}, k^{\prime}}-E_{s_{4}, k^{\prime}-q}\right) I_{\mathbf{Q}}\left\{\left(1-\rho_{k-q}\right) T_{s_{1}} I_{-\mathbf{Q}} T_{s_{2}} \rho_{k} \operatorname{Tr}\left[\left(1-\rho_{k^{\prime}}\right) T_{s_{3}} I_{\mathbf{Q}} T_{s_{4}} \rho_{k^{\prime}-q} I_{-\mathbf{Q}}\right]\right. \\
& \left.-\rho_{\mathbf{k}-q} T_{s_{1}} I_{-\mathbf{Q}} T_{s_{2}}\left(1-\rho_{k}\right) \operatorname{Tr}\left[\rho_{k^{\prime}} T_{s_{3}} I_{\mathbf{Q}} T_{s_{4}}\left(1-\rho_{k^{\prime}-q}\right) I_{-\mathbf{Q}}\right]\right\}+ \text { H.c. },
\end{aligned}
$$


in which $T_{s_{1}, s, s^{\prime}}=\delta_{s_{1}, s} \delta_{s_{1}, s^{\prime}}$. The statically screened Coulomb potential in the random-phase approximation reads [833]

$$
V_{q}=\sum_{q_{x}, q_{y}} v_{Q}\left|I_{\mathbf{Q}}\right|^{2} / \kappa(q),
$$

with the bare Coulomb potential $v_{Q}=4 \pi e^{2} / Q^{2}$ and

$$
\kappa(q)=1-\sum_{q_{x}, q_{y}} v_{Q} \sum_{k s}\left|I_{\mathbf{Q}, s, s}\right|^{2} \frac{f_{k+q, s}-f_{k, s}}{E_{s, k+q}-E_{s, k}} .
$$

In Eq. (249), $N_{i}$ is the density of impurities, and $\left|U_{\mathbf{Q}}^{i}\right|^{2}$ is the impurity potential. Furthermore, $\left|M_{\mathbf{Q}, \lambda}\right|^{2}$ and $N_{\mathbf{Q}, \lambda}=$ $\left[\exp \left(\omega_{\mathbf{Q}, \lambda} / k_{B} T\right)-1\right]^{-1}$ are the matrix elements of the electron-phonon interaction and the Bose distribution function, respectively. The phonon energy spectrum for phonon with mode $\lambda$ and wavevector $\mathbf{Q}$ is denoted by $\omega_{\mathbf{Q}, \lambda}$.

By numerically solving the kinetic spin Bloch equations, Lü et al. [696] investigated the influence of the wire size, orientation, doping density, and temperature on the spin relaxation time. They also showed that the Coulomb scattering makes marked contribution to the spin relaxation. Some typical results that the spin relaxation time can be effectively manipulated in quantum wires with different orientations are summarized in Fig. 87. For (100) quantum wires [Fig. 87(a)], due to the competition of the longitudinal and transverse effective magnetic fields from the Dresselhaus and Rashba terms addressed above, the spin relaxation time can be efficiently manipulated by the wire size. Specifically, there is a minimum in the spin relaxation time when $a_{x}=a_{y}$, thanks to the cancellation of the longitudinal component from the Dresselhaus term $\left(\left\langle k_{x}^{2}\right\rangle-\left\langle k_{y}^{2}\right\rangle=0\right)$ when only the lowest subband is populated. For (110) quantum wire with $a_{x}=a_{y}=30 \mathrm{~nm}$, the electronic population is mainly in the lowest subband. In the presence of an electric field of the form $\left(E_{x}, E_{y}, 0\right)$, the relevant Rashba and Dresselhaus terms are

$$
\begin{aligned}
& H_{\mathrm{R}}^{110}=\gamma_{41}^{6 c 6 c}\left(-\sigma_{x} E_{y} k_{z}+\sigma_{y} E_{x} k_{z}\right), \\
& H_{\mathrm{D}}^{110}=-\frac{1}{2} b_{41}^{6 c 6 c} \sigma_{x} k_{z}\left(\left\langle k_{x}^{2}\right\rangle-k_{z}^{2}+2\left\langle k_{y}^{2}\right\rangle\right) .
\end{aligned}
$$

The effective magnetic field formed by the Dresselhaus term is along the $x$-direction, which corresponds to the [1110] crystallographic direction, and the effective magnetic field formed by the Rashba term is in the $x-y$ plane. If the direction of the total effective magnetic field formed by the spin-orbit coupling is tuned to be exactly the direction of the initial spin polarization, then one can expect an extremely long spin relaxation time as pointed out in Refs. [597, 697]. This is exactly the case as shown in Fig. 87 bb). However, for wider wire size, the spin relaxation time is much smaller due to the contribution of higher subbands. For (111) quantum wires, again in the case with only the lowest subband being relevant, the Rashba and Dresselhaus terms read

$$
\begin{aligned}
& H_{\mathrm{R}}^{111}=\gamma_{41}^{6 c 6 c}\left(-\sigma_{x} E_{y} k_{z}+\sigma_{y} E_{x} k_{z}\right), \\
& H_{\mathrm{D}}^{111}=b_{41}^{6 c c c}\left(-\frac{\sqrt{2}}{3} \sigma_{x} k_{z}\left\langle k_{y}^{2}\right\rangle-\frac{1}{\sqrt{6}} \sigma_{y} k_{z}\left(\left\langle k_{x}^{2}\right\rangle+\left\langle k_{y}^{2}\right\rangle\right)-\frac{2}{3} \sigma_{z} k_{z}\left\langle k_{y}^{2}\right\rangle\right) .
\end{aligned}
$$

Similar to the case of (110) quantum wires, one expects very long spin relaxation time if the total effective magnetic field points into the directions of initial spin polarization. For a numerical example of this effect, Lü et al. chose $E_{y}$ such that $\gamma_{41}^{6 c 6 c} E_{y}+(\sqrt{2} / 3) b_{41}^{6 c c c}\left\langle k_{y}^{2}\right\rangle=0$ for a small wire width $a_{x}=a_{y}=10 \mathrm{~nm}$, so that the $x$ component of the total effective magnetic field is zero. For an initial spin polarization along the $z$-direction, which corresponds to the [111] crystallographic direction, the spin relaxation time as a function of $E_{x}$ is shown in Fig. 87 (c). It is seen that when $a_{x}=a_{y}=10 \mathrm{~nm}$, there is a pronounced maximum of the spin relaxation time at $E_{x}=70 \mathrm{kV} / \mathrm{cm}$, which fulfills the relation $\gamma_{41}^{6 c 6 c} E_{x}+\frac{1}{\sqrt{6}} b_{41}^{6 c 6 c}\left(\left\langle k_{x}^{2}\right\rangle+\left\langle k_{y}^{2}\right\rangle\right) \approx 0$. Consequently, for this field strength, the direction of the total effective magnetic field is exactly along the direction of the initial spin polarization and this leads to a very long spin relaxation time.

\subsubsection{Hole spin relaxation in p-type (001) GaAs quantum wires}

Investigation on hole spin relaxation in quantum wires is very limited [698]. Unlike the hole spins in bulk III-V materials which relax very fast due to the mixture of the heavy-hole and light-hole states, in confined structures such 

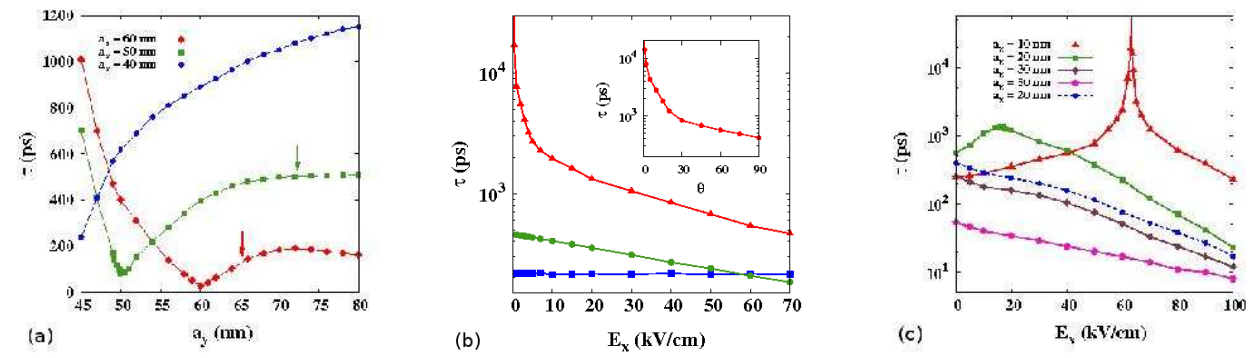

Figure 87: (a) Spin relaxation time $\tau v s$. the quantum wire width in $y$ direction $a_{y}$ for InAs (100) quantum wires at different $a_{x}$. The electron density is $N=4 \times 10^{5} \mathrm{~cm}^{-1}$ and $T=100 \mathrm{~K}$. The arrows mark the densities at which the electron populations in the second and higher subbands are approximately $30 \%$. (b) Spin relaxation time $\tau$ vs. $E_{x}$ for (110) quantum wires at $T=50 \mathrm{~K}$ and $N_{e}=4 \times 10^{5} \mathrm{~cm}^{-1} . \Delta: a_{x}=a_{y}=30 \mathrm{~nm}$ with an initial spin polarization along the $x$-direction; $\mathbf{\square}: a_{x}=a_{y}=30 \mathrm{~nm}$ with an initial spin polarization along the $y$-direction; $\bullet: a_{x}=a_{y}=50 \mathrm{~nm}$ with an initial spin polarization along the $x$-direction. (c) Spin relaxation time $\tau$ vs. $E_{x}$ for different wire sizes $a_{x}=a_{y}$ at $T=50 \mathrm{~K}$ and $N_{e}=4 \times 10^{5} \mathrm{~cm}^{-1}$. The growth direction of the quantum wire is along the [111] crystallographic direction. The solid curves are the results with the initial spin polarization along $z$-direction and the dashed curve is the result with the initial spin polarization along $x$-direction. From Lü et al. [696].

as quantum wells [363] and quantum dots [491], the degeneracy of the heavy and light hole bands is lifted and the mixture of these bands can be tuned by strain [491]. Therefore the spin lifetime of holes can be much longer than that in the bulk [363, 491]. In quantum wires, similar situation also happens. Lü et al. performed a systematic investigation on the spin relaxation of $p$-type GaAs quantum wires by numerically solving the kinetic spin Bloch equations [705]. They reported that the quantum wire size influences the spin relaxation time effectively by modulating the energy spectrum and the heavy-hole-light-hole mixing of wire states.

By considering a $p$-doped (001) GaAs quantum wire with rectangular confinement and hard wall potential, Lü et al. first obtained the subband structure by diagonalizing the hole Hamiltonian including the quantum confinement. Here the light-hole admixture is dominant in the lowest spin-split subband, but the heavy-hole admixture becomes also important in higher subbands due to the heavy-hole-light-hole mixing. Then they investigated the time evolution of holes by numerically solving the fully microscopic kinetic spin Bloch equations in the obtained subbands, with all the scatterings, particularly the Coulomb scattering, explicitly included. They found that the quantum wire size influences the spin relaxation time effectively because the spin-orbit coupling and the subband structure in quantum wires depend strongly on the confinement. When the quantum wire size increases, the lowest spin-split subband and the second-lowest spin-split subband can be very close to each other at an anticrossing point. If the anticrossing is close to the Fermi surface, the contribution from the spin-flip scattering reaches a maximum and correspondingly the spin relaxation time reaches a minimum. Moreover, they showed that the dependence of the spin relaxation time on confinement size in quantum wires behaves oppositely to the trend found in quantum wells. It was also found that, when the quantum wire size is very small, the spin relaxation time can either increase or decrease with increasing hole density, depending on the spin mixing of the subbands. However, the behavior of holes in quantum wires where the spin relaxation time increases or decreases with hole density is quite different from the one of light holes in quantum wells with small well width [363]. These features originate from the subband structure of the quantum wires and the spin mixing which give rise to the spin-flip scattering. The spin mixing and inter-subband scattering are modulated more dramatically in quantum wires by changing the hole distribution in different subbands. They also investigated the effects of temperature and initial spin polarization, showing that the inter-subband scattering and the Coulomb Hartree-Fock contribution can make a marked contribution to the spin relaxation.

By assuming the growth direction of the quantum wire along the $z$-axis ([001] crystallographic direction), the Hamiltonian of holes in the basis of spin-3/2 projection $\left(J_{z}\right)$ eigenstates with quantum numbers $+\frac{3}{2},+\frac{1}{2},-\frac{1}{2}$ and $-\frac{3}{2}$ can be written as [113]

$$
H_{h}=\left(\begin{array}{cccc}
H_{h h} & S & R & 0 \\
S^{\dagger} & H_{l h} & 0 & R \\
R^{\dagger} & 0 & H_{l h} & -S \\
0 & R^{\dagger} & -S^{\dagger} & H_{h h}
\end{array}\right)+H_{8 v 8 v}^{r}+H_{8 v 8 v}^{b}+V_{c}(\mathbf{r}),
$$


where $V_{c}(\mathbf{r})$ is the hard-wall confinement potential in $x$ and $y$ directions and

$$
\begin{aligned}
& H_{h h}=\frac{1}{2 m_{0}}\left[\left(\gamma_{1}+\gamma_{2}\right)\left[P_{x}^{2}+P_{y}^{2}\right]+\left(\gamma_{1}-2 \gamma_{2}\right) P_{z}^{2},\right. \\
& H_{l h}=\frac{1}{2 m_{0}}\left[\left(\gamma_{1}-\gamma_{2}\right)\left[P_{x}^{2}+P_{y}^{2}\right]+\left(\gamma_{1}+2 \gamma_{2}\right) P_{z}^{2},\right. \\
& S=-\frac{\sqrt{3} \gamma_{3}}{m_{0}} P_{z}\left[P_{x}-i P_{y}\right], R=-\frac{\sqrt{3}}{2 m_{0}}\left\{\gamma_{2}\left[P_{x}^{2}-P_{y}^{2}\right]-2 i \gamma_{3} P_{x} P_{y}\right\}, \\
& H_{8 v 8 v}^{r}=\frac{\gamma_{41}^{8 v 8 v}}{\hbar}\left[J_{x}\left(P_{y} \mathcal{E}_{z}-P_{z} \mathcal{E}_{y}\right)+J_{y}\left(P_{z} \mathcal{E}_{x}-P_{x} \mathcal{E}_{z}\right)+J_{z}\left(P_{x} \mathcal{E}_{y}-P_{y} \mathcal{E}_{x}\right)\right], \\
& H_{8 v 8 v}^{b}=\frac{b_{41}^{8 v 8 v}}{\hbar^{3}}\left\{J_{x}\left[P_{x}\left(P_{y}^{2}-P_{z}^{2}\right)\right]+J_{y}\left[P_{y}\left(P_{z}^{2}-P_{x}^{2}\right)\right]+J_{z}\left[P_{z}\left(P_{x}^{2}-P_{y}^{2}\right)\right]\right\} .
\end{aligned}
$$

In these equations, $m_{0}$ denotes the free electron mass, $\gamma_{1}, \gamma_{2}$ and $\gamma_{3}$ are the Luttinger parameters, $\mathcal{E}$ is the electric field and $J_{i}$ are spin-3/2 angular momentum matrices [122]. $H_{8 v 8 v}^{r}$ is the spin-orbit coupling arising from the structure inversion asymmetry and $H_{8 v 8 v}^{b}$ is the spin-orbit coupling from the bulk inversion asymmetry. As shown in Ref. [705], these two terms turn out to be one or two orders of magnitude smaller than the intrinsic spin-orbit coupling from the four-band Luttinger-Kohn Hamiltonian [the first term in Eq. (256)]. Moreover, from the first term in Eq. (256), one can see that the light hole spin-up states can be directly mixed with the heavy hole states by $S$ and $R$, but the mixing between light hole spin-up states and light hole spin-down states has to be mediated by the heavy hole states. All the mixing is related to the confinement. When the confinement decreases, the mixing increases due to the decrease of the energy gap between the light hole and heavy hole states.

The kinetic spin Bloch equations $\dot{\rho}_{k}+\left.\dot{\rho}_{k}\right|_{\text {coh }}+\left.\dot{\rho}_{k}\right|_{\text {scat }}=0$ can be written in either the collinear spin space which is constructed by basis $\{s\}$, with $\{s\}$ obtained from the eigenfunctions of the diagonal part of $H_{h}(k)$. $|s\rangle=|m, n\rangle|\sigma\rangle$ with $\langle r \mid m, n\rangle=\frac{2}{\sqrt{a_{x} a_{y}}} \sin \left(\frac{m \pi y}{a_{y}}\right) \sin \left(\frac{n \pi x}{a_{x}}\right) e^{i k z}$ and $|\sigma\rangle$ standing for the eigenstates of $J_{z}$. Then the matrix elements in the collinear spin space $\rho_{k, s_{1}, s_{2}}^{c}$ is written as $\rho_{k, s_{1}, s_{2}}^{c}=\left\langle s_{1}\left|\rho_{k}\right| s_{2}\right\rangle$. Here the superscript " $c$ " denotes the quantum number distinguishing states in the collinear spin space. One can also project $\rho_{k}$ in the "helix" spin space which is constructed by basis $\{\eta\}$ with $\eta$ being the eigenfunctions of $H_{h}(k)$ :

$$
H_{h}(k)|\eta\rangle=E_{\eta, k}|\eta\rangle
$$

This basis function is a mixture of light-hole and heavy-hole states and is $k$ dependent. Then the matrix elements in the helix spin space $\rho_{k, \eta, \eta^{\prime}}^{h}$ can be written as $\rho_{k, \eta, \eta^{\prime}}^{h}=\left\langle\eta\left|\rho_{k}\right| \eta^{\prime}\right\rangle$, with the superscript " $h$ " denoting the helix spin space. The density matrix in the helix spin space can be transformed from that in the collinear one by a unitary transformation: $\rho_{k}^{h}=U_{k}^{\dagger} \rho_{k}^{c} U_{k}$, where $U_{k}(i, \alpha)=\eta_{\alpha}^{i}(k)$ with $\eta_{\alpha}^{i}(k)$ being the $i$ th element of the $\alpha$ th eigenvector after the diagonalization of $H_{h}(k)$.

In helix spin space, the coherent terms read

$$
\left.\dot{\rho}_{k}^{h}\right|_{\mathrm{coh}}=i\left[\sum_{\mathbf{Q}} V_{\mathbf{Q}} U_{k}^{\dagger} I_{\mathbf{Q}} U_{k-q} \rho_{k-q}^{h} U_{k-q}^{\dagger} I_{-\mathbf{Q}} U_{k}, \rho_{k}^{h}\right]-i\left[U_{k}^{\dagger} H_{h}(k) U_{k}, \rho_{k}^{h}\right],
$$

where $I_{\mathbf{Q}}$ is the form factor in the collinear spin space with wave vector $\mathbf{Q} \equiv\left(q_{x}, q_{y}, q\right)$. The first term in Eq. (263) is the Coulomb Hartree-Fock term and the second term is the contribution from the intrinsic spin-orbit coupling from the Luttinger-Kohn Hamiltonian. $I_{\mathbf{Q}}$ can be written as $I_{\mathbf{Q}, s_{1}, s_{2}}=\left\langle s_{1}\left|e^{i \mathbf{Q} \cdot \mathbf{r}}\right| s_{2}\right\rangle=\delta_{\sigma_{1}, \sigma_{2}} F\left(m_{1}, m_{2}, q_{y}, a_{y}\right) F\left(n_{1}, n_{2}, q_{x}, a_{x}\right)$, with $F\left(m_{1}, m_{2}, q, a\right)$ being expressed as Eq. (248). For small spin polarization, the contribution from the Hartree-Fock term in the coherent term is negligible [41, 42, 44] and the spin precession is determined by the spin-orbit coupling, $\left.\dot{\rho}_{k, \eta, \eta^{\prime}}^{h}\right|_{\text {coh }}=-i \rho_{k, \eta, \eta^{\prime}}^{h}\left(E_{\eta, k}-E_{\eta^{\prime}, k}\right)$, which is proportional to the energy gap between $\eta$ and $\eta^{\prime}$ subbands.

The scattering terms include the hole-nonmagnetic-impurity, hole-phonon and hole-hole Coulomb scatterings. In the helix spin space, the scattering terms are given by 


$$
\begin{aligned}
\left.\dot{\rho}_{k}^{h}\right|_{\text {scat }}= & \pi N_{i} \sum_{\mathbf{Q}, \eta_{1}, \eta_{2}}\left|U_{\mathbf{Q}}^{i}\right|^{2} \delta\left(E_{\eta_{1}, k-q}-E_{\eta_{2}, k}\right) U_{k}^{\dagger} I_{\mathbf{Q}} U_{k-q}\left[\left(1-\rho_{k-q}^{h}\right) T_{k-q, \eta_{1}} U_{k-q}^{\dagger} I_{-\mathbf{Q}} U_{k} T_{k, \eta_{2}} \rho_{k}^{h}\right. \\
& \left.-\rho_{k-q}^{h} T_{k-q, \eta_{1}} U_{k-q}^{\dagger} I_{-\mathbf{Q}} U_{k} T_{k, \eta_{2}}\left(1-\rho_{k}^{h}\right)\right] \\
+ & \pi \sum_{\mathbf{Q}, \eta_{1}, \eta_{2}, \lambda}\left|M_{\mathbf{Q}, \lambda}\right|^{2} U_{k}^{\dagger} I_{\mathbf{Q}} U_{k-q}\left\{\delta ( E _ { \eta _ { 1 } , k - q } - E _ { \eta _ { 2 } , k } + \omega _ { \mathbf { Q } , \lambda } ) \left[\left(N_{\mathbf{Q}, \lambda}+1\right)\left(1-\rho_{k-q}^{h}\right) T_{k-q, \eta_{1}} U_{k-q}^{\dagger} I_{-\mathbf{Q}} U_{k} T_{k, \eta_{2}} \rho_{k}^{h}\right.\right. \\
& \left.-N_{\mathbf{Q}, \lambda} \rho_{k-q}^{h} T_{k-q, \eta_{1}} U_{\mathbf{k}-\mathbf{q}}^{\dagger} I_{-\mathbf{Q}} U_{k} T_{k, \eta_{2}}\left(1-\rho_{k}^{h}\right)\right]+\delta\left(E_{\eta_{1}, k-q}-E_{\eta_{2}, k}-\omega_{\mathbf{Q}, \lambda}\right)\left[N_{\mathbf{Q}, \lambda}\left(1-\rho_{k-q}^{h}\right) T_{k-q, \eta_{1}}\right. \\
& \left.\left.\times U_{k-q}^{\dagger} I_{-\mathbf{Q}} U_{k} T_{k, \eta_{2}} \rho_{k}^{h}-\left(N_{\mathbf{Q}, \lambda}+1\right) \rho_{k-q}^{h} T_{k-q, \eta_{1}} U_{k-q}^{\dagger} I_{-\mathbf{Q}} U_{k} T_{k, \eta_{2}}\left(1-\rho_{k}^{h}\right)\right]\right\} \\
+ & \pi \sum_{\mathbf{Q}, k^{\prime}} \sum_{\eta_{1}, \eta_{2}, \eta_{3}, \eta_{4}} V_{\mathbf{Q}}^{2} U_{k}^{\dagger} I_{\mathbf{Q}} U_{k-q} \delta\left(E_{\eta_{1}, k-q}-E_{\eta_{2}, k}+E_{\eta_{3}, k^{\prime}}-E_{\eta_{4}, k^{\prime}-q}\right)\left\{\left(1-\rho_{k-q}^{h}\right) T_{k-q, \eta_{1}} U_{k-q}^{\dagger} I_{-\mathbf{Q}} U_{k} T_{k, \eta_{2}} \rho_{k}^{h}\right. \\
& \times \operatorname{Tr}\left[\left(1-\rho_{k^{\prime}}^{h}\right) T_{\eta_{3}, k^{\prime}} U_{k}^{\dagger} I_{\mathbf{Q}} U_{k-q} T_{k^{\prime}-q, \eta_{4}} \rho_{k^{\prime}-q}^{h} U_{k-q}^{\dagger} I_{-\mathbf{Q}} U_{k}\right]-\rho_{\mathbf{k}-q}^{h} T_{k-q, \eta_{1}} U_{k-q}^{\dagger} I_{-\mathbf{Q}} U_{k} T_{k, \eta_{2}}\left(1-\rho_{k}^{h}\right) \\
& \left.\times \operatorname{Tr}\left[\rho_{k^{\prime}}^{h} T_{\eta_{3}, k^{\prime}} U_{k}^{\dagger} I_{\mathbf{Q}} U_{k-q} T_{k^{\prime}-q, \eta_{4}}\left(1-\rho_{k^{\prime}-q}^{h}\right) U_{k-q}^{\dagger} I_{-\mathbf{Q}} U_{k}\right]\right\}+ \text { H.c., }
\end{aligned}
$$

in which $T_{k, \eta}(i, j)=\delta_{\eta i} \delta_{\eta j}$. $V_{\mathbf{Q}}$ in Eq. (264) reads $V_{\mathbf{Q}}=4 \pi e^{2} /\left[\kappa_{0}\left(q^{2}+q_{\|}^{2}+\kappa^{2}\right)\right]$, with $\kappa_{0}$ representing the static dielectric constant and $\kappa^{2}=4 \pi e^{2} N_{h} /\left(k_{B} T \kappa_{0} a^{2}\right)$ standing for the Debye screening constant. $N_{i}$ in Eq. (264) is the impurity density and $\left|U_{\mathbf{Q}}^{i}\right|^{2}=\left\{4 \pi Z_{i} e^{2} /\left[\kappa_{0}\left(q^{2}+q_{\|}^{2}+\kappa^{2}\right)\right]\right\}^{2}$ is the impurity potential with $Z_{i}$ standing for the charge number of the impurity. $\left|M_{\mathbf{Q}, \lambda}\right|^{2}$ and $N_{\mathbf{Q}, \lambda}=\left[\exp \left(\omega_{\mathbf{Q}, \lambda} / k_{B} T\right)-1\right]^{-1}$ are the matrix element of the hole-phonon interaction and the Bose distribution function with phonon energy spectrum $\omega_{\mathbf{Q}, \lambda}$ at phonon mode $\lambda$ and wave vector $\mathbf{Q}$, respectively. Here the hole-phonon scattering includes the hole-longitudinal optical-phonon and hole-acoustic-phonon scatterings with the explicit expressions of $\left|M_{\mathbf{Q}, \lambda}\right|^{2}$ can be found in Refs. [44, 372, 844]. It is noted that the energy spectrum $E_{\eta, k}$ in the scattering term contains the spin-orbit coupling which cannot be ignored due to the strong coupling. By solving the kinetic spin Bloch equations, one obtains hole spin relaxation.
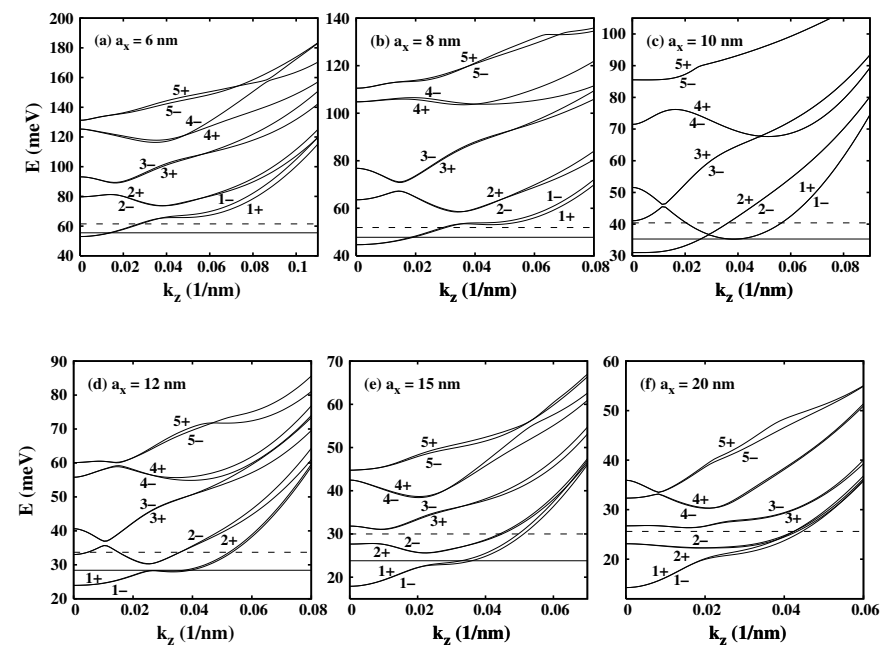

Figure 88: Typical energy spectra of holes in GaAs (001) quantum wires for (a) $a_{x}=6 \mathrm{~nm}$; (b) $a_{x}=8 \mathrm{~nm}$; (c) $a_{x}=10 \mathrm{~nm}$; (d) $a_{x}=12 \mathrm{~nm}$; (e) $a_{x}=15 \mathrm{~nm}$; and (f) $a_{x}=20 \mathrm{~nm}$. $a_{y}=10 \mathrm{~nm} .\langle E\rangle$ at $T=20 \mathrm{~K}$ is also plotted: solid line for $N_{h}=4 \times 10^{5} \mathrm{~cm}^{-1}$ and dashed line for $N_{h}=2 \times 10^{6} \mathrm{~cm}^{-1}$. From Lü et al. [705].

The typical subband structure is shown in Fig. 88 for different confinements. Each subband is denoted as $l+(l-)$ if the dominant spin component is the spin-up (-down) state. One can see from Fig. 88 that 1+ and 1- subbands are very close to each other, so are the subbands $2 \pm$. The spin-splitting between them is mainly caused by the spinorbit coupling arising from the bulk inversion asymmetry, because that the spin-splitting caused by the spin-orbit 
coupling arising from the structure inversion asymmetry is three orders of magnitude smaller than the diagonal terms in Eq. (256) and can not be seen in Fig. 88. The spin-splitting caused by the bulk inversion asymmetry is proportional to $\left(P_{x}^{2}-P_{y}^{2}\right)$, which disappears when the confinement in $x$ and $y$ directions are symmetrical. Therefore, $l \pm$ are almost degenerate when $a_{x}=a_{y}=10 \mathrm{~nm}$. If one excludes the spin-orbit coupling from the bulk inversion asymmetry and structure inversion asymmetry, $l \pm$ are always degenerate because of the Kramers degeneracy. One also observes that when $a_{x}$ gets larger, the subbands are closer to each other. Especially, in the case of $a_{x}=a_{y}=10 \mathrm{~nm}$, there are anticrossing points due to the heavy-hole-light-hole mixing in the Luttinger Hamiltonian. When $a_{x}$ keeps on increasing, the anticrossing point at small $k$ between the $1 \pm$ and $2 \pm$ gradually disappears. However, at large $k$ region, the lowest two subbands become very close to each other. These will lead to significant effect on spin relaxation time. In Fig. 88, a quantity $\langle E\rangle$, with

$$
\langle E\rangle=\frac{\sum_{l} \int_{-\infty}^{+\infty} d k\left(\rho_{k, l+, l+}^{h}-\rho_{k, l-, l-}^{h}\right)\left(E_{l+, k}+E_{l-, k}\right)}{2 \sum_{l} \int_{-\infty}^{+\infty} d k\left(\rho_{k, l+, l+}^{h}-\rho_{k, l-, l-}^{h}\right)},
$$

is introduced to represent the energy region where spin precession and relaxation between the + and - bands mainly take place. It is seen from the figure that for $N_{h}=4 \times 10^{5} \mathrm{~cm}^{-1}$ and $2 \times 10^{6} \mathrm{~cm}^{-1},\langle E\rangle$ only intersects with $1 \pm$ and $2 \pm$ subbands. It is also seen that the dominant spin component in $1+(1-)$ state is the spin-up (spin-down) light-hole state.

There are three mechanisms leading to spin relaxation. First, the spin-flip scattering, which includes the scattering between $l+$ and $l$ - subbands and the scattering between $l+$ and $l^{\prime}-$ subbands $\left(l \neq l^{\prime}\right)$, can cause spin relaxation. The spin relaxation time decreases with the spin-flip scattering, with the scattering strength being proportional to the spin mixing of the helix subbands. Second, because of the coherent term $\left.\dot{\rho}_{k}^{h}\right|_{\text {coh }}$, there is a spin precession between different subbands. The frequency of this spin precession depends on $k$ and this dependence serves as inhomogeneous broadening. As shown in Refs. [44, 332, 334, 350, 569, 844], in the presence of the inhomogeneous broadening, even the spin-conserving scattering can cause irreversible spin relaxation. As a result, the spin-conserving scattering, i.e., the scattering between $l+$ and $l^{\prime}+$ and the scattering between $l-$ and $l^{\prime}-$, can cause spin relaxation along with the inhomogeneous broadening. At last, the spin-flip scattering along with the inhomogeneous broadening can also cause an additional spin relaxation.

It is seen from Fig. 88 (a) that when $N_{h}=4 \times 10^{5} \mathrm{~cm}^{-1}$ and $a_{x}=6 \mathrm{~nm},\langle E\rangle$ only intersects with the $1 \pm$ subbands and is far away from the $2 \pm$ subbands. Therefore, holes populate the $1 \pm$ subbands only. As pointed out before, the coherent term $\left.\dot{\rho}_{k, 1+, 1-}^{h}\right|_{\text {coh }}$ is proportional to $\left(E_{1+, k}-E_{1-, k}\right)$. As holes are only populating states in the small $k$ region where the spin splitting between $1 \pm$ is negligible, the spin precession between these two states, and thus the inhomogeneous broadening, is very small. Consequently the main spin-relaxation mechanism is due to the spin-flip scattering, i.e., the scattering between $1 \pm$ subbands.

In the case of larger $a_{x}$ and $N_{h}$ as shown in Fig. 88(c) -88 (f), where $\langle E\rangle$ is close to or intersects with the $2 \pm$ subbands, holes populate both the $1 \pm$ and $2 \pm$ subbands. The spin-flip scattering here includes the scattering between $1 \pm$ states, the scattering between $2 \pm$ states and the spin-flip scattering between $1 \pm$ and $2 \pm$ subbands. This spin-flip scattering is still found to be the main spin relaxation mechanism. Besides, differing from the case of Fig. 2(a), the coherent term $\left.\dot{\rho}_{k, 1 \pm 2 \pm}^{h}\right|_{\text {coh }}$ is proportional to the energy gap between $1 \pm$ and $2 \pm$, and it is much larger than $\left.\dot{\rho}_{k, 1+, 1-}^{h}\right|_{\text {coh. }}$. As a result, there is a much stronger spin precession between $1 \pm$ and $2 \pm$ subbands with a frequency depending on $k$, and the inhomogeneous broadening caused by this precession along with both the spin-conserving scattering and the spin-flip scattering can make a considerable contribution to the spin relaxation.

A typical hole spin relaxation time as a function of wire width is given in Fig. 89 at different temperatures and hole densities. The underlying physics can be well understood from the corresponding subband mixing in Fig. 88 . Similarly the temperature, hole density, and spin polarization dependences of spin relaxation were also discussed in detail in Ref. [705].

\subsection{Spin relaxation in bulk III-V semiconductors}

The study of spin dynamics in bulk III-V semiconductors has a long history. The theoretical study of spin relaxation and the systematic experimental investigation started as early as 1970s [3]. The early studies have been reviewed comprehensively in the book "Optical Orientation" [3]. In the past decade, the topic gained renewed interest in the 

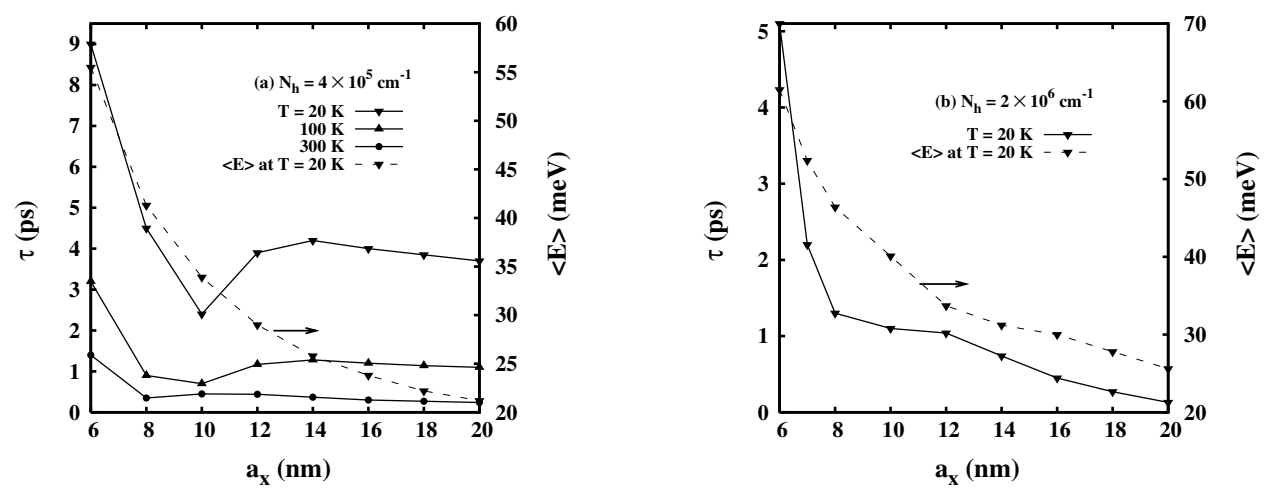

Figure 89: Spin relaxation time $\tau$ vs. the quantum wire width in $x$ direction $a_{x}$ for (a) $N_{h}=4 \times 10^{5} \mathrm{~cm}^{-1}$ at different temperatures and (b) $N_{h}=2 \times 10^{6} \mathrm{~cm}^{-1}$ at $T=20 \mathrm{~K} . a_{y}=10 \mathrm{~nm}$. From Lü et al. [705].

context of semiconductor spintronics [576]. Differing from early experimental studies which mainly focus on electron spin relaxation in $p$-type bulk III-V semiconductors, experimental investigations in the past decade mainly focus on $n$ type and intrinsic bulk III-V semiconductors [21]. Theoretically, Song and Kim systematically calculated the density and temperature dependences of electron spin relaxation time in bulk $n$-type and $p$-type GaAs, InAs, GaSb and InSb by including the D'yakonov-Perel', Elliott-Yafet and Bir-Aronov-Pikus mechanisms [108]. However, their approach was based on the approximate formulae in the book "Optical Orientation" [3] where the momentum scattering time is calculated via the approximate formulae for mobility [108]. A key mistake is that they used the formulae that can only be used in the nondegenerate regime. This makes their results in the low-temperature and/or high-density regime questionable. Moreover, the single-particle approach they used limits the validity of their results. Other theoretical investigations have similar problems [209, 390, 559, 577, 578, 583]. Therefore a systematic many-body investigation from the fully microscopic kinetic spin Bloch equation approach is needed.

In this subsection, we review the comprehensive study on the topic via the fully microscopic kinetic spin Bloch equation approach by Jiang and $\mathrm{Wu}[110]$ where many important predictions and results that can not be achieved via the single-particle approach were obtained 78

The system considered is the bulk III-V semiconductors, where the spin interactions have been introduced in Sec. 2. Specifically, the spin-orbit coupling consists of the Dresselhaus term,

$$
\mathbf{\Omega}(\mathbf{k})=2 \gamma_{D}\left[k_{x}\left(k_{y}^{2}-k_{z}^{2}\right), k_{y}\left(k_{z}^{2}-k_{x}^{2}\right), k_{z}\left(k_{x}^{2}-k_{y}^{2}\right)\right]
$$

and the strain-induced term which is linear in $\mathbf{k}$. The electron-hole exchange interaction is given by Eq. 37) which consists of both the short-range interaction [see Eq. (33)] and the long-range one [see Eq. (34)]. The Elliott-Yafet mechanism is included in the electron-impurity, electron-phonon, electron-electron and electron-hole scatterings in the kinetic spin Bloch equations [110].

\subsubsection{Comparison with experiments}

Jiang and $\mathrm{Wu}$ compared their calculation via the kinetic spin Bloch equation approach [110] with experimental results measured in GaAs in Refs. [4, 586, 900]. The results are presented in Fig. 90 a)-(c) where the calculated spin lifetimes are plotted as solid curves and the experimental results as red dots. It is seen that the calculation agrees quite well with experimental data for both $n$ - and $p$-type GaAs in a wide temperature and density regimes. The deviation in the low-temperature regime $(T<20 \mathrm{~K})$ in Fig. 90 a) is due to the rising of electron localization. Good quantitative agreement with experimental data for intrinsic GaAs at room temperature was also achieved by

\footnotetext{
${ }^{78}$ Review of the experimental studies and the single-particle theories on electron spin relaxation in bulk $n$-type and intrinsic III-V semiconductors in metallic regime is presented in Sec. 4.2.1 and 4.2.2, respectively. Studies on electron spin relaxation in bulk p-type III-V semiconductors was reviewed in the book "Optical Orientation" [117].
} 

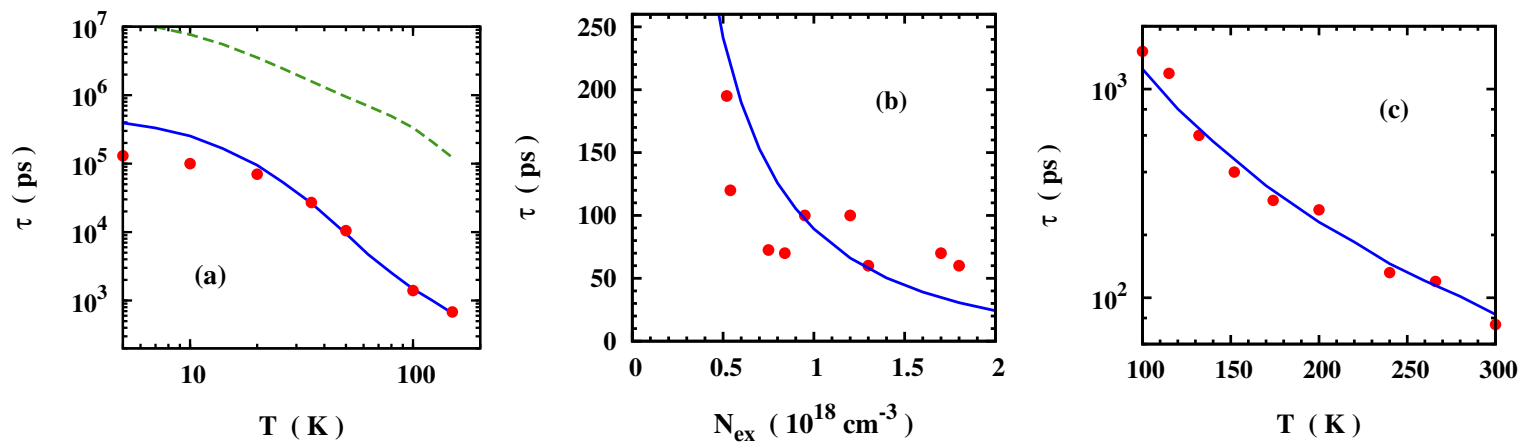

Figure 90: (a) $n$-GaAs. Spin relaxation times $\tau$ from the experiment in Ref. [4] (•) and from the calculation via the kinetic spin Bloch equation approach with only the D'yakonov-Perel' mechanism (solid curve) as well as that with only the Elliott-Yafet mechanism (dashed curve). $n_{e}=$ $10^{16} \mathrm{~cm}^{-3}, n_{i}=n_{e}$ and $N_{\mathrm{ex}}=10^{14} \mathrm{~cm}^{-3} \cdot \gamma_{D}=8.2 \mathrm{eV} \cdot \AA^{3}$. (b) $p$-GaAs. Spin relaxation times $\tau$ from the experiment in Ref. [586] ( $\bullet$ ) and from the calculation via the kinetic spin Bloch equation approach (solid curve). $n_{h}=6 \times 10^{16} \mathrm{~cm}^{-3}, n_{i}=n_{h}$ and $T=100 \mathrm{~K}$. $\gamma_{D}=8.2 \mathrm{eV} \cdot \AA^{3}$. (c) $p$-GaAs. Spin relaxation times $\tau$ from the experiment in Ref. [900] (•) and from the calculation via the kinetic spin Bloch equation approach (solid curve). $n_{h}=1.6 \times 10^{16} \mathrm{~cm}^{-3}, n_{i}=n_{h}$ and $N_{\mathrm{ex}}=10^{14} \mathrm{~cm}^{-3} \cdot \gamma_{D}=10 \mathrm{eV} \cdot \AA^{3}$. From Jiang and Wu [110].

Jiang and $\mathrm{Wu}$ in Ref. [589]. In those calculations, all the material parameters are taken from the standard handbook of Landolt-Börnstein [204]. The only free parameter is the Dresselhaus spin-orbit coupling constant $\gamma_{D}$ which has not been unambiguously determined by experiment or theory. Nevertheless the parameter $\gamma_{D}$ for GaAs used in the calculation is close to the value from recent $a b$ initio calculation with $\mathrm{GW}$ approximation $\left(\gamma_{D}=8.5 \mathrm{eV} \cdot \AA^{3}\right)[139]$ and that from the recent fitting of the magnetotransport in chaotic GaAs quantum dots $\left(\gamma_{D}=9 \mathrm{eV} \cdot \AA^{3}\right)$ [140]. The good agreement with experimental data indicates that the calculation has achieved quantitative accuracy.

\subsubsection{Electron-spin relaxation in n-type bulk III-V semiconductors}
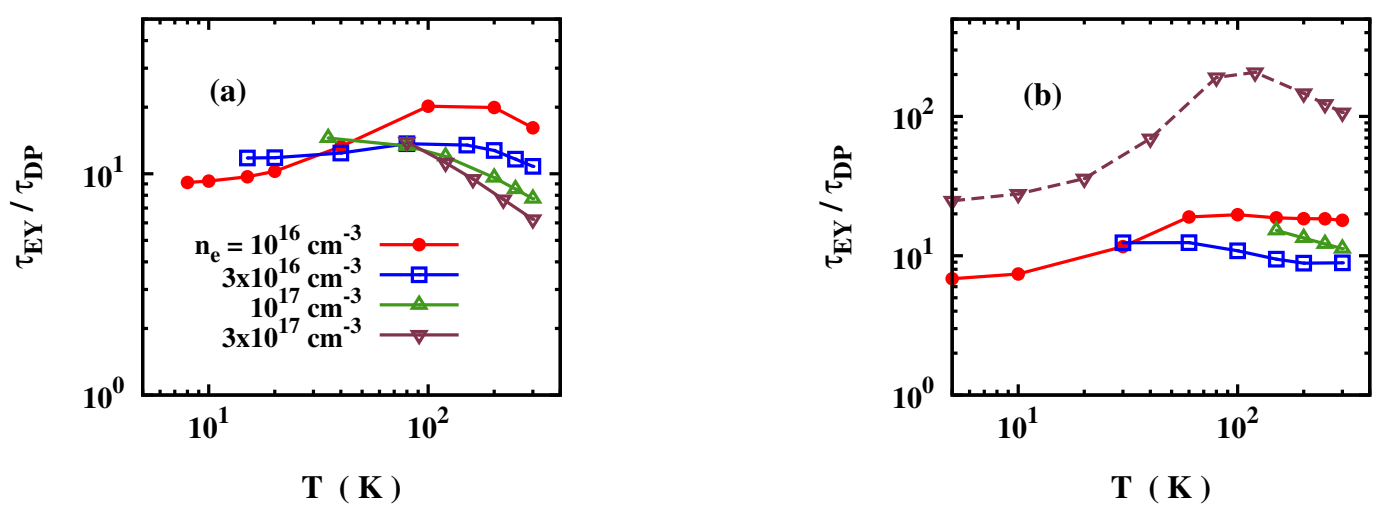

Figure 91: Ratio of the Elliott-Yafet spin relaxation time $\tau_{\mathrm{EY}}$ to the D'yakonov-Perel' one $\tau_{\mathrm{DP}}$ for (a) $n$-InSb (b) $n$-InAs and $n$-GaAs as function of temperature for various electron densities. In Fig.(b): $n_{e}=10^{16} \mathrm{~cm}^{-3}$ (curve with $\bullet$ ), $2 \times 10^{17} \mathrm{~cm}^{-3}$ (curve with $\square$ ), $10^{18} \mathrm{~cm}^{-3}$ (curve with $\triangle$ ) for InAs, and $n_{e}=10^{16} \mathrm{~cm}^{-3}$ (curve with $\nabla$ ) for GaAs. From Jiang and Wu 110 ].

Comparison of different spin relaxation mechanisms. In $n$-type bulk III-V semiconductors at low photoexcitation density, the Bir-Aronov-Pikus mechanism is irrelevant as the hole density is low. The mechanisms left are then the Elliott-Yafet and D'yakonov-Perel' mechanisms. Previously, it was believed that the Elliott-Yafet mechanism is important in narrow bandgap semiconductors, such as InSb and InAs. Jiang and Wu compared the relative efficiency of the two mechanisms at various conditions for InSb and InAs [110]. The results are shown in Fig. 91. In contrast to previous understanding, they found that the Elliott-Yafet mechanism is much less efficient than the 
D'yakonov-Perel' one in both InSb and InAs. Although the low-temperature results for the high density cases are absent in Fig. 91, it was found that in such regime the ratio $\tau_{\mathrm{EY}} / \tau_{\mathrm{DP}}$ varies slowly with temperature [110]. For GaAs, the Elliott-Yafet mechanism is still less important as indicated by Fig. 91(b).

To give a qualitative picture of the relative importance of the Elliott-Yafet mechanism in other III-V semiconductors, Jiang and $\mathrm{Wu}$ analyzed the problem by using the approximate formulae for the D'yakonov-Perel' and ElliottYafet spin relaxation time [Eqs. (53) and (62)]. From those equations,

$$
\frac{\tau_{\mathrm{EY}}}{\tau_{\mathrm{DP}}}=\frac{2 Q}{3 A}\left\langle\varepsilon_{\mathbf{k}}\right\rangle \tau_{p}^{2} \Theta
$$

Here $\Theta=8 \gamma_{D}^{2} m_{e}^{3} E_{g}^{2}(1-\eta / 3)^{2} /\left[(1-\eta / 2)^{2} \eta^{2}\right] . Q$ and $A$ are numerical factors around unity. The factor $\Theta$, which is solely determined by the material parameters, is listed in Table 2 for various III-V semiconductors. The spin-orbit coupling parameter $\gamma_{D}$ is fitted from experiments (except that $\gamma_{D}$ 's for InP and GaSb are from the $\mathbf{k} \cdot \mathbf{p}$ calculation in Ref. [138]), whereas other parameters are from Landolt-Börnstein [204]. One notices from the table that the factor $\Theta$ is much smaller for InAs and InSb than other III-V semiconductors. According to this, the Elliott-Yafet mechanism should be much less efficient than the D'yakonov-Perel' one in GaSb and InP. Actually, one notices that $\Theta \propto E_{g}^{2}$ which decreases rapidly with decreasing bandgap. In commonly used III-V semiconductors, InSb has smallest bandgap. However, even for InSb the Elliott-Yafet mechanism is much less important than the D'yakonov-Perel' mechanism in the metallic regime. Therefore, in other III-V semiconductors, the Elliott-Yafet mechanism is also unimportant 79

Table 2: The $\Theta$ factor for III-V semiconductors. From Jiang and Wu 110].

\begin{tabular}{llllll}
\hline \hline & GaAs & GaSb & InAs & InSb & InP \\
\hline$\Theta(\mathrm{eV})$ & $2.7 \times 10^{-2}$ & 0.12 & $2.0 \times 10^{-3}$ & $9.2 \times 10^{-4}$ & 0.27 \\
\hline \hline
\end{tabular}

Very recently, Litvinenko et al. studied the magnetic field dependence of spin lifetime in $n$-type InSb and InAs experimentally [901]. They found in $n$-InSb that the spin lifetime increases significantly with increasing magnetic field in the Faraday configuration (magnetic field parallel to spin polarization) [see Fig. 92]. As the Elliott-Yafet spin relaxation has little magnetic field dependence, this result implies that the dominant spin relaxation mechanism in $n$ InSb is the D'yakonov-Perel' mechanism at low magnetic field. At high magnetic field the Elliot-Yafet spin relaxation dominates as the D'yakonov-Perel' mechanism is strongly suppressed by the longitudinal magnetic field. They found similar results in $n$-InAs. These results confirmed the previous prediction by Jiang and Wu that the Elliott-Yafet mechanism is less efficient than the D'yakonov-Perel' one in $n$-type InSb and InAs [110].

The D'yakonov-Perel' spin relaxation. As both the Bir-Aronov-Pikus and Elliott-Yafet mechanisms are unimportant in bulk $n$-type III-V semiconductors in metallic regime, the only relevant one is the D'yakonov-Perel' mechanism. Although the D'yakonov-Perel' mechanism has been studied for about forty years, the understanding on it in bulk III-V semiconductors is yet adequate. For example, in the previous literature, the electron-electron scattering has long been believed to be irrelevant in bulk III-V semiconductors. Jiang and Wu showed that the electron-electron scattering is important for spin relaxation in $n$-GaAs in the nondegenerate regime except when the electron-longitudinaloptical-phonon dominates momentum scattering. The same conclusion should also hold for other bulk $n$-type III-V semiconductors.

The D'yakonov-Perel' spin relaxation: density dependence. In Fig. 93 (a), spin relaxation time as function of electron density is plotted for $n$-GaAs at $40 \mathrm{~K}$. The density of impurity is taken as the same as that of electron, $n_{i}=n_{e}$. Remarkably, one notices that the density dependence is nonmonotonic with a peak around $n_{e}=10^{16} \mathrm{~cm}^{-3}$. Previously, the nonmonotonic density dependence of spin lifetime was observed in low-temperature $(T \lesssim 5 \mathrm{~K})$ experiments, where the localized electrons play a crucial role and the electron system is in the insulating regime or around the metalinsulator transition point. Jiang and $\mathrm{Wu}$ found, for the first time, that the spin lifetime in metallic regime is also

\footnotetext{
${ }^{79}$ This is also true for intrinsic III-V semiconductors and in most cases for $p$-type semiconductors as well as for some II-VI semiconductors (such as CdTe, see Ref. [590]).
} 


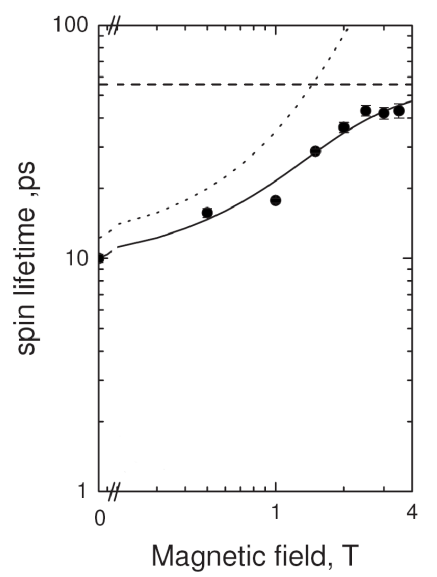

Figure 92: Spin lifetime in $n$-InSb as function of magnetic field at $100 \mathrm{~K}$. The magnetic field is parallel to the spin polarization direction (Faraday configuration). Theoretical dependences of the Elliott-Yafet (dashed curve), D'yakonov-Perel' and the total (solid curve) spin lifetimes are also shown. Reproduced from Litvinenko et al. [901].

nonmonotonic. Moreover, they pointed out that it is a universal behavior for all bulk III-V semiconductors at all temperature where the peak is located at $T_{F} \sim T$ with $T_{F}$ being the electron Fermi temperature. From the piont of view of spintronic device application, as devices are more favorable to operate in the metallic regime, this prediction gives the important information that the longest spin lifetime in metallic regime is at $T_{F} \sim T$. The underlying physics for the nonmonotonic density dependence in metallic regime is elucidated below.

To understand the D'yakonov-Perel' spin relaxation qualitatively, let us first recall the widely used approximate formulae [117], $\tau_{\mathrm{DP}} \simeq 1 /\left[\left\langle|\boldsymbol{\Omega}(\mathbf{k})|^{2}-\Omega_{z}^{2}(\mathbf{k})\right\rangle \tau_{p}^{*}\right]$ where $\langle\ldots\rangle$ denotes the ensemble average 80 The expression contains two key factors of the D'yakonov-Perel' spin relaxation: (i) the inhomogeneous broadening from the k-dependent transverse spin-orbit field $\sim\left\langle|\boldsymbol{\Omega}(\mathbf{k})|^{2}-\Omega_{z}^{2}(\mathbf{k})\right\rangle$; (ii) the momentum scattering time $\tau_{p}^{*}$ [including the contributions of the electron-impurity, electron-phonon, electron-electron and electron-hole (whenever holes exist) scatterings]. The D'yakonov-Perel' spin relaxation time increases with increasing momentum scattering rate, but decreases with increasing inhomogeneous broadening.

To elucidate the underlying physics, Jiang and $\mathrm{Wu}$ plotted the spin relaxation times calculated with only the electron-impurity, electron-electron and electron-phonon scatterings in Fig. 93 (a) respectively. It is seen that the spin relaxation time with only one kind of scattering is smaller than that with all scatterings, which is a consequence of the motional narrowing nature of the D'yakonov-Perel' mechanism $\tau_{s} \propto 1 / \tau_{p}$. One notices that the electron-electron scattering gives important contribution to spin relaxation in the nondegenerate (low density) regime. Interestingly, both the electron-electron and electron-impurity scatterings lead to nonmonotonic density dependence of spin relaxation time. One notices that the electron-phonon scattering is much weaker (the corresponding spin relaxation time is much shorter as $\tau_{s} \propto 1 / \tau_{p}$ ) as the temperature is low.

Let us first look at the density dependence of the electron-electron scattering time. In fact, the density and temperature dependences of the electron-electron scattering time have been widely investigated in spin-unrelated problems (see, e.g., Ref. [848]). From the previous works [843, 848], after some approximation, the asymptotic density and temperature dependences of the electron-electron scattering time $\tau_{p}^{e e}$ in the degenerate and nondegenerate regimes are given by,

$$
\begin{array}{ll}
\tau_{p}^{e e} \propto n_{e}^{\frac{2}{3}} / T^{2} & \text { for } \quad T \ll T_{F}, \\
\tau_{p}^{e e} \propto T^{\frac{3}{2}} / n_{e} & \text { for } \quad T \gg T_{F} .
\end{array}
$$

$$
{ }^{80}\langle\ldots\rangle=\frac{\int d \mathbf{k}\left(f_{\mathbf{k}}^{\uparrow}-f_{\mathbf{k}}^{\downarrow}\right) \ldots}{\int d \mathbf{k}\left(f_{\mathbf{k}}^{\uparrow}-f_{\mathbf{k}}^{\downarrow}\right)}
$$


From the above equations, one notices that the electron-electron scattering time in the nondegenerate and degenerate regimes has different density dependence. In the nondegenerate (low density) regime, the electron-electron scattering time decreases with electron density [see Eq. [269] ], where the inhomogeneous broadening $\sim\left\langle|\boldsymbol{\Omega}(\mathbf{k})|^{2}-\Omega_{z}^{2}(\mathbf{k})\right\rangle \propto$ $\left\langle\varepsilon_{\mathbf{k}}^{3}\right\rangle$ ( $\varepsilon_{\mathbf{k}}$ is electron kinetic energy) varies slowly with density as the electron distribution is close to the Boltzmann distribution in nondegenerate regime. The spin relaxation time thus increases with electron density. In degenerate (high density) regime, both the electron-electron scattering time [see Eq. (268)] and the inhomogeneous broadening increase with electron density. Therefore, the spin relaxation time $\tau_{s} \simeq 1 /\left[\left\langle|\boldsymbol{\Omega}(\mathbf{k})|^{2}-\Omega_{z}^{2}(\mathbf{k})\right\rangle \tau_{p}^{e e}\right]$ decreases with electron density.

For spin relaxation associated with the electron-impurity scattering, the scenario is similar: In nondegenerate regime, the decrease of the electron-impurity scattering time with electron density $\left(1 / \tau_{p}^{e i} \propto n_{i}=n_{e}\right)$ leads to the increase of the spin relaxation time with increasing electron density. In the degenerate regime, the inhomogeneous broadening increases with increasing electron density, as $\left\langle|\mathbf{\Omega}(\mathbf{k})|^{2}-\Omega_{z}^{2}(\mathbf{k})\right\rangle \propto k_{\mathrm{F}}^{6} \propto n_{e}^{2}$. On the other hand, the electronimpurity scattering time varies slowly with electron density because $1 / \tau_{p}^{e i} \sim n_{i} V_{k_{F}}^{2} k_{F} \sim n_{e} k_{F} / k_{F}^{4} \propto n_{e}^{0}$. Consequently, the spin relaxation time decreases with increasing electron density. For spin relaxation related to the electron-phonon scattering, the situation, however, is different: In nondegenrate regime both the inhomogeneous broadening and the electron-phonon scattering rate vary slowly with electron density as electron distribution is close to the Boltzmann distribution. In degenerate regime, the increase of the inhomogeneous broadening is faster than the variation of the electron-phonon scattering, which hence leads to the decrease of spin relaxation time with increasing electron density.

In summary, the electron density depdence of spin relaxation time is nonmonotonic as the momentum scattering time and the inhomogeneous broadening have different qualitative density dependences in the nondegenrate and degenerate regimes. The spin relaxation time increases (decreases) in the nondegenrate (degenerate) regime with increasing electron density. A peak hence locates in the crossover regime, where $T_{F}$ is around $T$. Such a scenario should hold for all the III-V semiconductors at all temperature and the nonmonotonic density dependence is thus a universal behavior 81 Furthermore, in Fig. 93 b), Jiang and Wu showed that the nonmonotonic density dependence of spin relaxation time also exists even when the spin-orbit coupling is dominated by the linear-k term 82 Subsequently, similar behavior was found in two-dimensional system [111, 299, 613], where the underlying physics is similar. The predicted peak was later observed by Krauss et al. [563] (see, also [564]).
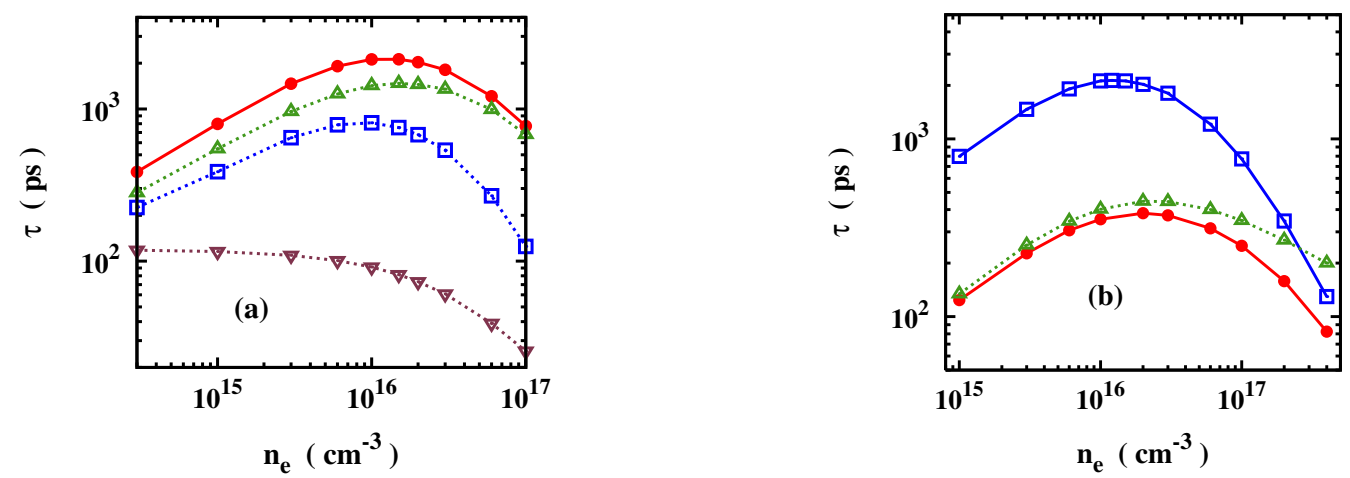

Figure 93: $n$-GaAs at $T=40 \mathrm{~K}$. (a) spin relaxation time $\tau$ as function of electron density $n_{e}\left(n_{i}=n_{e}\right)$ from full calculation (curve with $\bullet$ ), from calculation with only the electron-electron scattering (curve with $\square$ ), with only the electron-impurity scattering (curve with $\Delta$ ), and with only the electron-phonon scattering (curve with $\nabla$ ); (b) spin relaxation time $\tau$ as function of electron density $n_{e}\left(n_{i}=n_{e}\right)$ for the case with strain-induced spin-orbit coupling (curve with $\bullet$ : with both the linear- and the cubic-k spin-orbit coupling; curve with $\triangle$ : with only the linear-k spin-orbit coupling) and the case without strain (curve with $\square$ ). From Jiang and Wu [110].

\footnotetext{
${ }^{81}$ The nonmonotonic density dependence should also exist in bulk II-VI semiconductors with zinc-blende structures which has similar band structure with III-VI semiconductors.

${ }^{82}$ From this point, the nonmonotonic density dependence should also exist in bulk wurtzite semiconductors with bulk inversion asymmetry, such as GaN, AlN and $\mathrm{ZnO}$.
} 


\subsubsection{Electron-spin relaxation in intrinsic bulk III-V semiconductors}

In intrinsic semiconductors, the carriers are generated by photo-excitation where the electron density is equal to the hole density $n_{e}=n_{h}=N_{\mathrm{ex}}$ ( $N_{\mathrm{ex}}$ denotes the excitation density). As the impurity density is very low (one can take $n_{i}=0$ ), the carrier-carrier scattering is dominant unless at high temperature where the electron-longitudinal-opticalphonon scattering becomes more important. Intrinsic bulk semiconductors thus offer a good platform to study the many-body effect to electron spin relaxation.

Comparison of different mechanisms. As both the Bir-Aronov-Pikus and D'yakonov-Perel' mechanisms contribute to electron spin relaxation, the relative efficiency of the Bir-Aronov-Pikus and D'yakonov-Perel' mechanisms should be compared. In Fig. 94(a) the Bir-Aronov-Pikus and D'yakonov-Perel' spin relaxation times as function of temperature are plotted. It is noted that the Bir-Aronov-Pikus spin relaxation time is over one order of magnitude larger than the D'yakonov-Perel' one, which indicates that the Bir-Aronov-Pikus mechanism is much less efficient than the D'yakonov-Perel' one in intrinsic bulk III-V semiconductors. Systematic calculation for various temperatures and excitation densities confirms that the Bir-Aronov-Pikus mechanism is irrelevant in intrinsic bulk GaAs in the metallic regime. Such conclusion also holds for GaSb. Recent experiments arrived at the same conclusion for intrinsic InSb [553]. Therefore, the Bir-Aronov-Pikus mechanism is unimportant in intrinsic GaAs, GaSb and InSb.
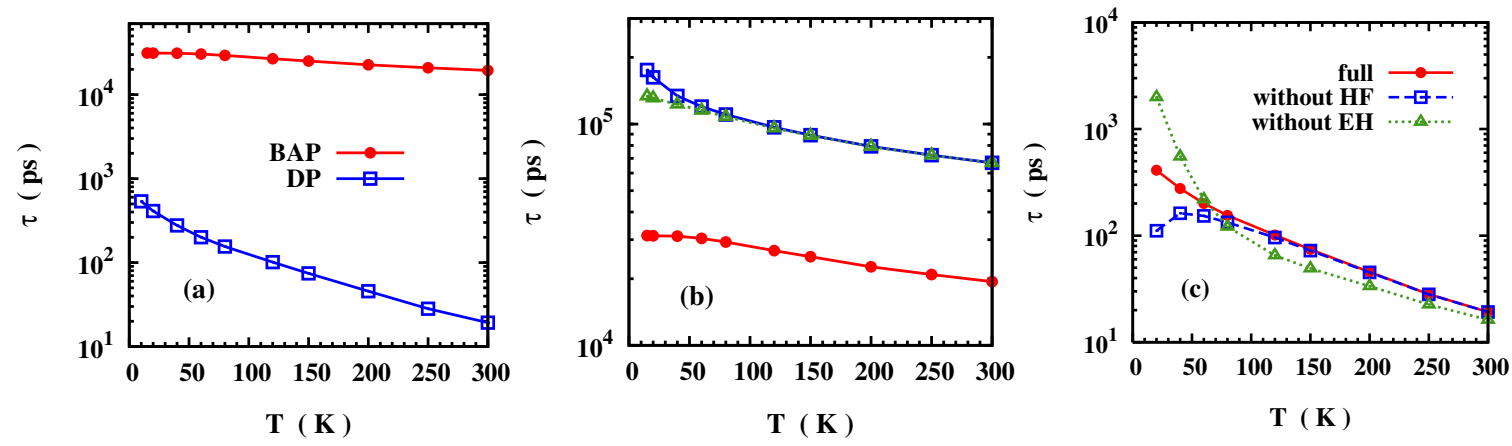

Figure 94: Spin relaxation time $\tau$ for intrinsic GaAs with excitation density $N_{\mathrm{ex}}=10^{17} \mathrm{~cm}^{-3}$. The initial spin polarization is $P=50 \%$. (a) spin relaxation time $\tau$ due to the Bir-Aronov-Pikus (BAP) and D'yakonov-Perel' (DP) mechanisms as function of temperature. (b) The Bir-AronovPikus spin relaxation time calculated from Eq. (270) (dotted curve with $\triangle$ ), from the kinetic spin Bloch equation approach with both long-range and short-range exchange scatterings (solid curve with $\bullet$ ) as well as from the kinetic spin Bloch equation approach with only the short-range exchange scattering (solid curve with $\square$ ). (c) The D'yakonov-Perel' spin relaxation time from full calculation (solid curve with •), from the calculation without the Coulomb Hartree-Fock term (dashed curve with $\square$ ), and from the calculation without the electron-hole Coulomb scattering (dotted curve with $\Delta$ ). From Jiang and Wu [110].

The Bir-Aronov-Pikus spin relaxation: short-range vs. long-range interaction and the Pauli blocking. As to the Bir-Aronov-Pikus spin relaxation, it should be mentioned that the long-range part of the electron-hole exchange interaction [see Eq. [34] ] has always been ignored in the literature [3, 21, 108, 436]. Jiang and Wu examined the relative contribution of the long-range and short-range interactions to the Bir-Aronov-Pikus spin relaxation. In Fig. 944b) the Bir-Aronov-Pikus spin relaxation time calculated with both the long-range and short-range exchange interactions as well as that without the long-range exchange interaction are plotted. It is seen that the spin relaxation time increases by about three times when the long-range exchange interaction is removed. This indicates that the long-range interaction is more important than the short-range one in GaAs and hence can not be neglected. Moreover, in the previous literature, the Bir-Aronov-Pikus spin relaxation time was calculated via the Fermi Golden rule [3, 390],

$$
\frac{1}{\tau_{\mathrm{BAP}}(\mathbf{k})}=4 \pi \sum_{\substack{\mathbf{q}, \mathbf{k}^{\prime} \\ m, m^{\prime}}} \delta\left(\varepsilon_{\mathbf{k}}+\varepsilon_{\mathbf{k}^{\prime} m^{\prime}}^{h}-\varepsilon_{\mathbf{k}-\mathbf{q}}-\varepsilon_{\mathbf{k}^{\prime}+\mathbf{q} m}^{h}\right)\left|\mathcal{J}_{\mathbf{k}^{\prime}+\mathbf{q} m}^{(+) \mathbf{k}^{\prime} m^{\prime}}\right|^{2} f_{\mathbf{k}^{\prime} m^{\prime}}^{h}\left(1-f_{\mathbf{k}^{\prime}+\mathbf{q} m}^{h}\right) .
$$

As pointed out by Zhou and Wu in the two-dimensional system [109] (see Sec. 5.4.6), such approach, which ignores the Pauli-blocking effect of electron distribution, fails at low temperature (in degenerate regime). The spin relaxation time calculated from Eq. (270) with only the short-range exchange interaction is plotted in Fig. 94(b). One notices 
that the results from Eq. (270) indeed deviate from the exact results via the kinetic spin Bloch equation approach at low temperature.

The D'yakonov-Perel' spin relaxation: temperature dependence and the effects of the Coulomb HartreeFock term and the electron-hole scattering. In Fig. 94(c) the D'yakonov-Perel' spin relaxation time as function of temperature is plotted. The initial spin polarization is $P=50 \%$, i.e., ideal circularly polarized light excitation. To elucidate the role of the Coulomb Hartree-Fock term, the spin relaxation time calculated without the Coulomb Hartree-Fock term is also plotted. It is seen that the Coulomb Hartree-Fock term largely affects the spin relaxation at low temperature, whereas at high temperature it is ineffective. The underlying physics is as follows: The spin relaxation time under the Hartree-Fock effective magnetic field is estimated as [similar to Eq. (64)]

$$
\tau_{s}(P)=\tau_{s}(P=0)\left[1+\left(g \mu_{\mathrm{B}} B_{\mathrm{HF}} \tau_{p}^{*}\right)^{2}\right]
$$

where $B_{\mathrm{HF}}$ is the averaged effective magnetic field and $\tau_{p}^{*}$ stands for the momentum scattering including the carriercarrier scattering. At low temperature, the carrier-carrier [see Eq. (268)] and electron-phonon scatterings are suppressed. Therefore, $\tau_{p}^{*}$ is large and the Hartree-Fock magnetic field has strong effect on spin relaxation time. However, at high temperature the momentum scattering is strong and $\tau_{p}^{*}$ is small. Consequently, the Hartree-Fock magnetic field has little effect on spin relaxation.

It is noted that without the Coulomb Hartree-Fock term the spin relaxation time has nonmonotonic temperature dependence. Usually, the spin relaxation time without the Coulomb Hartree-Fock term is close to the spin relaxation time at small initial spin polarization. To confirm that, Jiang and $\mathrm{Wu}$ plotted the spin relaxation time at the same condition but for $P=2 \%$ in Fig. 95. It is seen that the spin relaxation time is indeed nonmonotonic in temperature dependence 83 In contrast, the spin relaxation time decreases with increasing temperature in $n$-type III-V semiconductors as the electron-impurity scattering dominates at low temperature. The nonmonotonic temperature dependence of the spin relaxation time originates from the nonmonotonic temperature dependence of the electron-electron and electron-hole scattering times as noted from Eqs. (268) and (269) 84 The peak is then located in the crossover regime. Systematic calculation indicates that the peak temperature is around $T_{F} / 3$ and lies in the range of $\left(T_{F} / 4, T_{F} / 2\right)$ for various carrier density in both GaAs and InAs [110].

The spin relaxation time calculated without the electron-hole scattering is also plotted in Fig. 96 (c) to indicate the contribution of the electron-hole scattering. It is seen that at high temperature $(T>60 \mathrm{~K})$, the spin relaxation time becomes smaller without the electron-hole scattering. However, at low temperature $(T<60 \mathrm{~K})$, the spin relaxation time becomes larger. The decrease of the spin relaxation time at high temperature indicates the importance of the electron-hole scattering according to the motional narrowing $\tau_{s} \propto 1 / \tau_{p}$. However, at low temperature, the Coulomb Hartree-Fock term complicates the behavior. According to Eq. 271), if the Coulomb Hartree-Fock term plays significant role [i.e., $\left.\left(g \mu_{\mathrm{B}} B_{\mathrm{HF}} \tau_{p}^{*}\right)^{2}>1\right]$, then $\tau_{s} \sim\left[\left(|\boldsymbol{\Omega}|^{2}-\Omega_{z}^{2}\right) \tau_{p}^{*}\right]^{-1}\left(g \mu_{\mathrm{B}} B_{\mathrm{HF}} \tau_{p}^{*}\right)^{2} \sim \tau_{p}^{*}$. Therefore removing the electron-hole scattering leads to longer spin relaxation time. These results demonstrate that the electron-hole scattering plays an important role in both low and high temperature regimes.

The D'yakonov-Perel' spin relaxation: initial spin polarization dependence. The effect of the Coulomb Hartree-Fock term is also reflected in the initial polarization dependence of the spin relaxation time [41, 42, 44, 326], which is plotted in Fig. 95. It is seen that the spin relaxation time increases by about one order of magnitude when the initial spin polarization increases from $2 \%$ to $50 \%$. Without the Coulomb Hartree-Fock term the increment of the spin relaxation time is negligible (not shown). It was also found that, in contrast to the two-dimensional case [41, 42, 44, 326], the initial spin polarization dependence in $n$-type III-V semiconductors is very weak. This is because the momentum scattering in $n$-type III-V semiconductors is strong even at low temperature as the impurity density is high $\left(n_{i}=n_{e}\right)$ [110].

The D'yakonov-Perel' spin relaxation: density dependence. In Fig. 96(a), the density dependence of the spin relaxation time is plotted. It is seen again that the Bir-Aronov-Pikus mechanism is much less efficient then the D'yakonov-Perel' mechanism. Remarkably, the density dependence is nonmonotonic and a peak exhibits. The underlying physics is similar with that for the $n$-type case except that the electron-impurity scattering is substituted by

\footnotetext{
${ }^{83}$ The nonmonotonic temperature dependence of spin relaxation time has been observed in bulk intrinsic GaAs recently [902], which confirms the prediction by Jiang and $\mathrm{Wu}[110]$.

${ }^{84}$ The electron-hole scattering time has similar temperature dependence as that of the electron-electron scattering.
} 


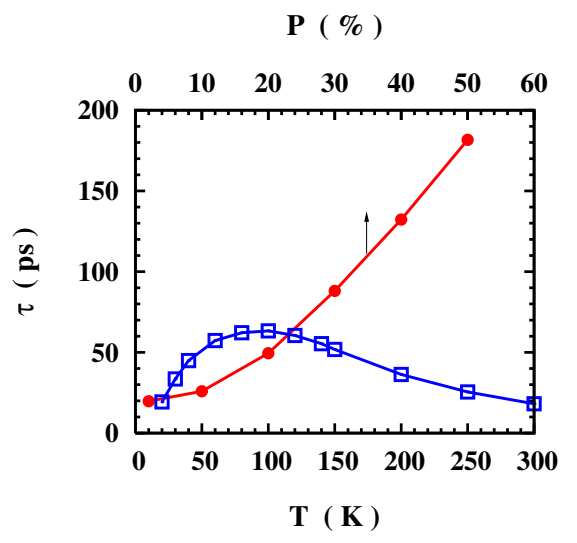

Figure 95: Intrinsic GaAs with $N_{\mathrm{ex}}=2 \times 10^{17} \mathrm{~cm}^{-3}$. Spin relaxation time $\tau$ as function of temperature for $P=2 \%$ (curve with $\square$ ) and the spin relaxation time as function of initial spin polarization $P$ for $T=20 \mathrm{~K}$ (curve with $\bullet$ ) (note that the scale of $P$ is on the top of the frame). From Jiang and $\mathrm{Wu}[110]$.

the electron-hole scattering. As the electron-hole scattering has similar density dependence with that of the electronelectron scattering, the density dependence of the spin relaxation time is then nonmonotonic. The peak is also located in the crossover regime, where $T_{F}$ is around $T$. Such behavior is also universal for all bulk intrinsic III-V semiconductors at all temperature 85

To elucidate the role of the electron-hole scattering and the Coulomb Hartree-Fock term in spin relaxation, the spin relaxation times calculated without these terms are plotted together with the spin relaxation time from the full calculation in Fig. 96 b). The importance of the electron-hole scattering is obvious in a wide density range. The Coulomb Hartree-Fock term is shown to be important only in the high density regime 86
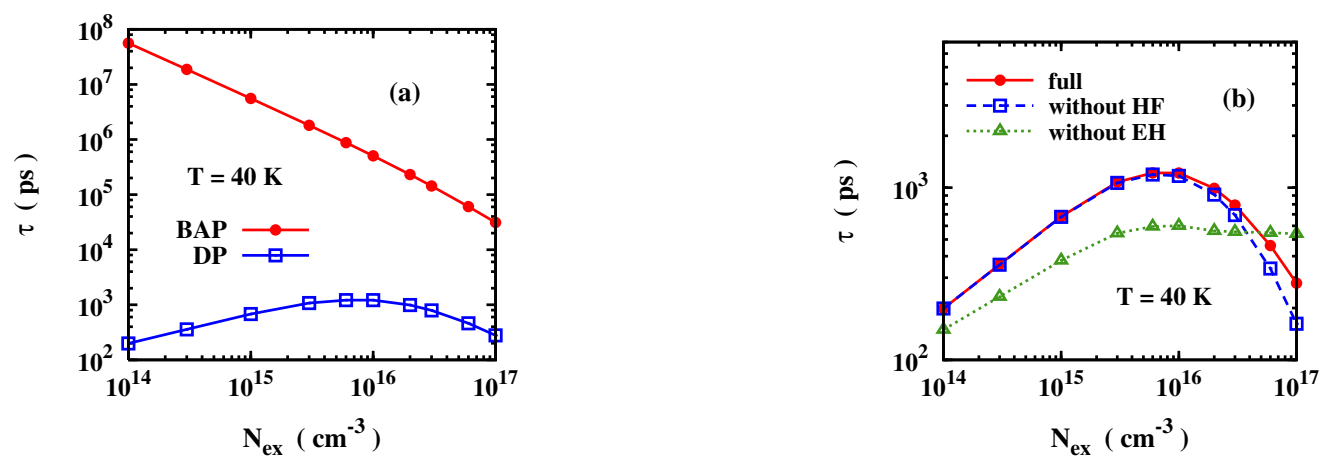

Figure 96: Intrinsic GaAs with $P=50 \%$ and $T=40 \mathrm{~K}$. (a) The Bir-Aronov-Pikus (BAP) and D'yakonov-Perel' (DP) spin relaxation times $\tau$ as function of photo-excitation density $N_{\mathrm{ex}}$. (b) The D'yakonov-Perel' spin relaxation time from the full calculation (curve with $\bullet$ ), from the calculation without the Coulomb Hartree-Fock term (curve with $\square$ ), and from the calculation without the electron-hole Coulomb scattering (curve with $\triangle$ ). From Jiang and $\mathrm{Wu}[110]$.

\subsubsection{Electron-spin relaxation in p-type bulk III-V semiconductors}

Comparison of the D'yakonov-Perel' and Bir-Aronov-Pikus mechanisms in GaAs. The main sources of spin relaxation have been recognized as the Bir-Aronov-Pikus and D'yakonov-Perel' mechanisms [3] 87 An important

\footnotetext{
${ }^{85}$ Such density dependence should also hold for strained III-V semiconductors, wurtzite semiconductors with bulk inversion asymmetry and bulk II-VI semiconductors.

${ }^{86}$ The Hartree-Fock effective magnetic field can be estimated as $B_{\mathrm{HF}}=\tilde{V}_{q} n_{e} P /\left(g \mu_{B}\right)$ where $\tilde{V}_{q}$ describes the average Coulomb interaction. Hence the Hartree-Fock effective magnetic field is strong at high electron density.

${ }^{87}$ The Elliott-Yafet mechanism was checked to be unimportant in metallic regime for both $p$-GaAs and $p$-GaSb by Jiang and Wu [110].
} 
issue is the relative efficiency of the two mechanisms under various conditions. This was studied comprehensively by Jiang and $\mathrm{Wu}$ in Ref. [110]. Below we review their results.

Low photo-excitation case. Jiang and Wu first discussed the low photo-excitation case. In this case, the electron density is low and the electron system is nondegenerate. The ratio of the Bir-Aronov-Pikus spin relaxation time to the D'yakonov-Perel' one is plotted in Fig. 97 (a) for various hole densities as function of temperature. It is seen that the D'yakonov-Perel' mechanism dominates at high temperature, whereas the Bir-Aronov-Pikus mechanism dominates at low temperature. This is consistent with the common belief in the literature [3, 21, 22, 106, 108, 436].

The temperature dependences of the Bir-Aronov-Pikus and D'yakonov-Perel' spin relaxation times are plotted in Fig. 97 (b) for a typical case with $n_{h}=3 \times 10^{18} \mathrm{~cm}^{-3}$. It is seen that both the D'yakonov-Perel' spin relaxation time and the Bir-Aronov-Pikus one decrease with temperature. As electron system is nondegenerate, the inhomogeneous broadening varies as $\sim\left\langle|\boldsymbol{\Omega}|^{2}-\Omega_{z}^{2}\right\rangle \propto T^{3}$, which leads to the rapid decrease of the D'yakonov-Perel' spin relaxation time. The Bir-Aronov-Pikus spin relaxation time decreases with temperature partly because of the Pauli blocking of holes. To elucidate this, the Bir-Aronov-Pikus spin relaxation time without the Pauli blocking of holes is plotted as dotted curve in Fig. 97(b). The results indicate that the Pauli blocking of holes effectively suppresses the BirAronov-Pikus spin relaxation at low temperature and makes the temperature dependence of the Bir-Aronov-Pikus spin relaxation time stronger.
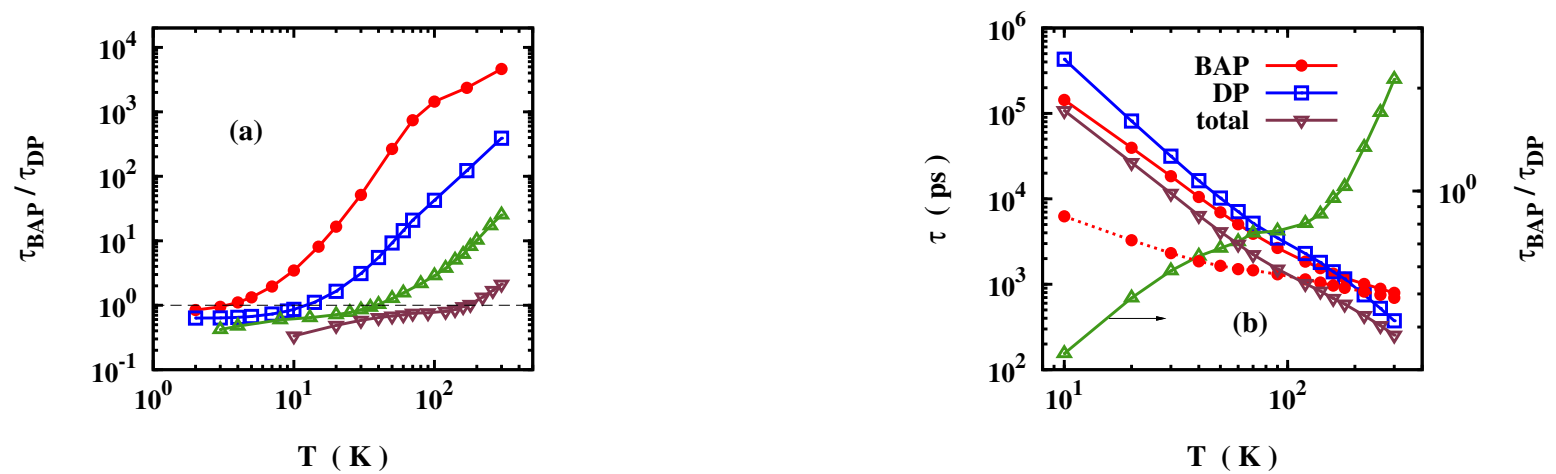

Figure 97: $p$-GaAs. Ratio of the Bir-Aronov-Pikus spin relaxation time to the D'yakonov-Perel' one $\tau_{\mathrm{BAP}} / \tau_{\mathrm{DP}}$ as function of temperature for various hole densities with $N_{\mathrm{ex}}=10^{14} \mathrm{~cm}^{-3}$ and $n_{i}=n_{h}$. (a): $n_{h}=3 \times 10^{15} \mathrm{~cm}^{-3}$ (curve with $\bullet$ ), $3 \times 10^{16} \mathrm{~cm}^{-3}$ (curve with $\square$ ), $3 \times 10^{17} \mathrm{~cm}^{-3}$ (curve with $\triangle$ ), and $3 \times 10^{18} \mathrm{~cm}^{-3}$ (curve with $\nabla$ ). (b): The spin relaxation times due to the Bir-Aronov-Pikus (BAP) and D'yakonov-Perel' (DP) mechanisms, the total spin relaxation time and the ratio $\tau_{\mathrm{BAP}} / \tau_{\mathrm{DP}}$ (curve with $\triangle$ ) vs. the temperature for $n_{h}=3 \times 10^{18} \mathrm{~cm}^{-3}$. The dotted curve represents the Bir-Aronov-Pikus spin relaxation time calculated without the Pauli blocking of holes. Note the scale of $\tau_{\mathrm{BAP}} / \tau_{\mathrm{DP}}$ is on the right hand side of the frame. From Jiang and $\mathrm{Wu}[110]$.

High photo-excitation case. The high photo-excitation case was also discussed by Jiang and Wu. The excitation density was taken as $N_{\mathrm{ex}}=0.1 n_{h}$. The ratio of the Bir-Aronov-Pikus spin relaxation time to the D'yakonov-Perel' one as function of temperature for various hole densities is plotted in Fig. 98 (a). Interestingly, the ratio is nonmonotonic and has a minimum roughly around the Fermi temperature of electrons, $T \sim T_{\mathrm{F}}$. In contrast to the low photoexcitation case, the Bir-Aronov-Pikus mechanism no longer dominates the low temperature regime. To understand such behavior, the spin relaxation times due to the two mechanisms and their ratio are plotted in Fig. 98 (b) for a typical case $n_{h}=3 \times 10^{18} \mathrm{~cm}^{-3}$. Before looking at the figure, one should note that the only difference for the two cases it that the electron density is much larger in the high excitation density. In the figure, one notices that, quite differently, the D'yakonov-Perel' spin relaxation time saturates at low temperature, which is the reason why the ratio of the two spin relaxation times increases at decreasing temperature. The underlying physics for the saturation of the D'yakonov-Perel' spin relaxation time at low temperature is as follows: As the electron density is high, at low temperature the electron system enters into the degenerate regime, where the inhomogeneous broadening and the momentum scattering (dominated by the electron-impurity scattering) varies slowly with temperature. This thus leads to the saturation of the D'yakonov-Perel' spin relaxation time at low temperature.

Role of screening on D'yakonov-Perel' spin relaxation. One may find from Fig. 98 (b) that the D'yakonov-Perel' spin relaxation time has nonmonotonic temperature dependence. Unlike the nonmonotonic temperature dependence in intrinsic semiconductors, here the behavior is not caused by the carrier-carrier scattering. The underlying physics is a 

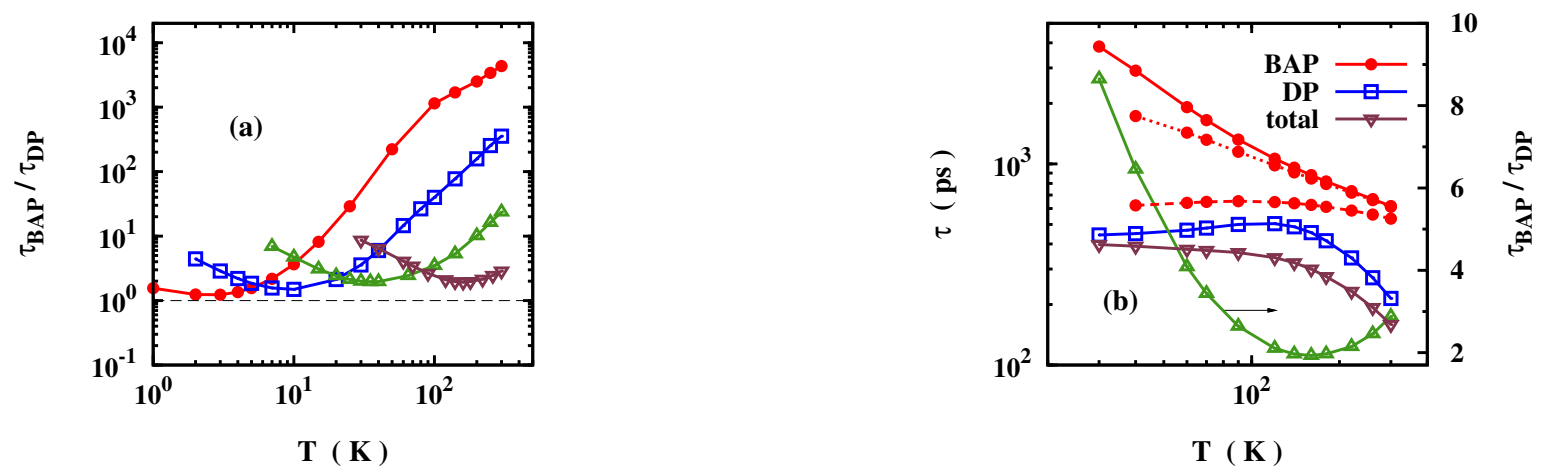

Figure 98: $p$-GaAs. Ratio of the Bir-Aronov-Pikus spin relaxation time to the D'yakonov-Perel' one $\tau_{\mathrm{BAP}} / \tau_{\mathrm{DP}}$ as function of temperature for various hole densities with $N_{\mathrm{ex}}=0.1 n_{h}$ and $n_{i}=n_{h}$. (a): $n_{h}=3 \times 10^{15} \mathrm{~cm}^{-3}$ (curve with $\bullet$ ), $3 \times 10^{16} \mathrm{~cm}^{-3}\left(\mathrm{curve}^{-}\right.$with $\square$ ), $3 \times 10^{17} \mathrm{~cm}^{-3}$ (curve with $\triangle$ ), and $3 \times 10^{18} \mathrm{~cm}^{-3}$ (curve with $\nabla$ ). The hole Fermi temperatures for these densities are $T_{\mathrm{F}}^{h}=1.7,7.7,36$, and $167 \mathrm{~K}$, respectively. The electron Fermi temperatures are $T_{\mathrm{F}}=2.8,13,61$, and $283 \mathrm{~K}$, respectively. (b): The spin relaxation times due to the Bir-Aronov-Pikus (BAP) and D'yakonov-Perel' (DP) mechanisms, the total spin relaxation time and the ratio $\tau_{\mathrm{BAP}} / \tau_{\mathrm{DP}}$ (curve with $\Delta$ ) vs. the temperature for $n_{h}=3 \times 10^{18} \mathrm{~cm}^{-3}$. The dotted (dashed) curve represents the Bir-Aronov-Pikus spin relaxation time calculated without the Pauli blocking of electrons (holes). Note the scale of $\tau_{\mathrm{BAP}} / \tau_{\mathrm{DP}}$ is on the right hand side of the frame. From Jiang and Wu [110].

little bit more complex: it is related to the temperature dependence of screening. To elucidate the underlying physics, Jiang and Wu plotted the D'yakonov-Perel' spin relaxation times calculated with the Thomas-Fermi screening [346] [which applies in the degenerate (low temperature) regime], the Debye-Huckle screening [346] [which applies in the nondegenerate (high temperature) regime] and the screening with the random-phase approximation [346] (which applies in the whole temperature regime) in Fig. 99 (a). It is noted that with the Thomas-Fermi screening (which is temperature-independent) the peak disappears, whereas with the Debye-Huckle screening the peak remains. This indicates that the increase of screening with decreasing temperature is crucial for the appearance of the peak. The scenario is as follows: With decreasing temperature, the electron system gradually enters into the degenerate regime and the temperature dependence of the inhomogeneous broadening $\sim\left\langle|\boldsymbol{\Omega}|^{2}-\Omega_{z}^{2}\right\rangle$ becomes mild. However, as the hole Fermi energy is smaller than the electron one (due to its large effective mass), there is a temperature interval where the hole system is still in the nondegenerate regime. The screening, which mainly comes from holes (again, due to its large effective mass), still increases with decreasing temperature significantly, $\kappa^{2} \sim 1 / T$. Therefore, the electron-impurity scattering (the dominant one at such temperatures) time, $\tau_{p}^{e i} \propto 1 /\left[\left\langle V_{q}^{2}\right\rangle \propto\left\langle\left(q^{2}+\kappa^{2}\right)^{-2}\right\rangle\right]$, increases with decreasing temperature. Thus the spin relaxation time, $\tau_{s} \sim 1 /\left(\left\langle|\boldsymbol{\Omega}|^{2}-\Omega_{z}^{2}\right\rangle \tau_{p}^{e i}\right)$, decreases with decreasing temperature at such temperature interval. When the temperature is further lowered down, both the screening and the inhomogeneous broadening vary little with temperature and the spin relaxation time saturates. These behaviors, together with the decrease of the spin relaxation time with increasing temperature at high temperature (mainly due to the increase of inhomogeneous broadening in nondegenerate regime), lead to the nonmonotonic temperature dependence. The peak is roughly around the electron Fermi temperature, $T \sim T_{F}$.

It is noted from Fig. 98 b) that the temperature dependence of the total spin relaxation time is still monotonic: it decreases with increasing temperature. This is due to the contribution of the Bir-Aronov-Pikus mechanism. It is expected that in other materials where the Bir-Aronov-Pikus mechanism is less important than that in GaAs, such as $\mathrm{GaSb}$, the temperature dependence of the total spin relaxation time is nonmonotonic.

The question arises that whether there is a nonmonotonic temperature dependence of the spin relaxation time in $n$-type semiconductors due to screening. A simple estimation may help to illustrate the problem: the electronimpurity scattering 88 rate varies as $1 / \tau_{p}^{e i} \propto\left\langle k V_{q}^{2}\right\rangle \propto\left\langle k\left(q^{2}+\kappa^{2}\right)^{-2}\right\rangle \sim\langle k\rangle\left(\kappa^{2}\right)^{-2} \sim T^{2.5} 89$ whereas the inhomogeneous broadening varies as $\sim\left\langle|\mathbf{\Omega}|^{2}-\Omega_{z}^{2}\right\rangle \sim\left\langle\varepsilon_{\mathbf{k}}^{3}\right\rangle \sim T^{3}$. Therefore, the spin relaxation time $\tau_{s} \sim 1 /\left[\left\langle|\boldsymbol{\Omega}|^{2}-\Omega_{z}^{2}\right\rangle \tau_{p}^{e i}\right] \sim T^{-0.5}$, still decreases with increasing temperature. Such estimation applies for nondegenerate regime. For other regimes, the

\footnotetext{
${ }^{88}$ The electron-impurity scattering is the cause of the monotonic temperature dependence of the spin lifetime in $n$-type semiconductors.

${ }^{89}$ The factor $\langle k\rangle$ comes from the density of states. This factor varies little in the $p$-type case in the above discussion at the relevant temperature, as electron system is in the degenerate regime.
} 
inhomogeneous broadening still varies with temperature faster than the scattering rate does. Therefore the the spin relaxation time always decreases with increasing temperature in $n$-type III-V semiconductors in metallic regime.

However, the situation may change when the strain-induced spin-orbit coupling dominates. In such case, the inhomogeneous broadening $\sim\left\langle|\boldsymbol{\Omega}|^{2}-\Omega_{z}^{2}\right\rangle \sim\left\langle\varepsilon_{\mathbf{k}}\right\rangle \sim T$ varies with temperature slower than the electron-impurity scattering does. One readily obtains that $\tau_{s} \sim T^{1.5}$ in nondegenerate regime. As shown in Fig. $99 \mathrm{~b}$ b), the temperature dependence is indeed nonmonotonic in strained $n$-GaAs with $n_{e}=2 \times 10^{15} \mathrm{~cm}^{-3}$. However, the screening effect is only important for low electron density $n_{e} \lesssim 10^{16} \mathrm{~cm}^{-3}$. At high electron density, the screening plays a marginal role in the electron-impurity scattering even at low temperature. This is because $1 / \tau_{p}^{e i} \propto\left\langle k\left(q^{2}+\kappa^{2}\right)^{-2}\right\rangle$. When electron density is high, the $q^{2}$ factor becomes larger than $\kappa^{2}$ on average.
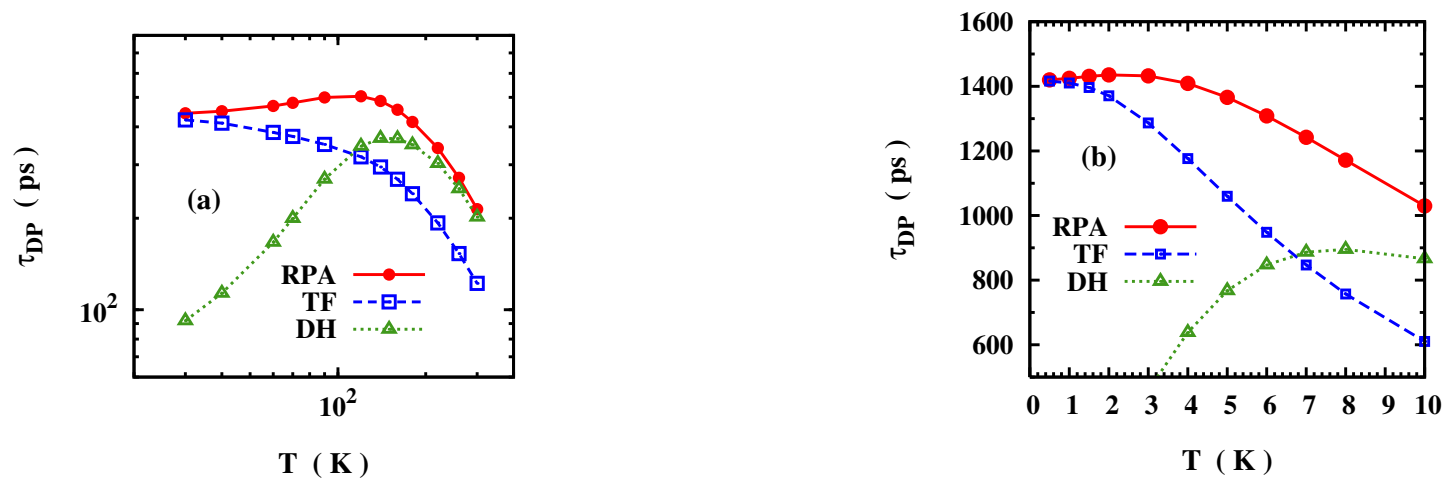

Figure 99: (a) $p$-GaAs with hole density $n_{h}=3 \times 10^{18} \mathrm{~cm}^{-3}, n_{i}=n_{h}$ and $N_{\mathrm{ex}}=0.1 n_{h}$. Temperature dependence of the D'yakonov-Perel' spin relaxation times calculated with the Thomas-Fermi (TF) (curve with $\square$ ) and Debye-Huckle (DH) (curve with $\triangle$ ) screenings as well as the screening with the random-phase-approximation (RPA) (curve with $\bullet$ ). (b) $n$-GaAs with electron density $n_{e}=2 \times 10^{15} \mathrm{~cm}^{-3}, n_{i}=n_{e}$ and $N_{\mathrm{ex}}=4 \times 10^{13} \mathrm{~cm}^{-3}$. The spin relaxation times calculated with the Thomas-Fermi and Debye-Huckle screenings as well as the screening with the random-phase-approximation vs. temperature. From Jiang and $\mathrm{Wu}$ [110].

Photo-excitation density dependence. After analyzing the relative efficiency of the D'yakonov-Perel' and BirAronov-Pikus mechanisms in the two limiting cases of low and high photo-excitation, one would be eager to see the crossover between the two limits. In Fig. 100 the photo-excitation density dependence of the Bir-Aronov-Pikus and D'yakonov-Perel' spin relaxation times as well as their ratio are plotted for two hole densities at $50 \mathrm{~K}$. In the low excitation limit, the D'yakonov-Perel' (Bir-Aronov-Pikus) mechanism is more important for the case in Fig. 100 (a) [(b)]. For both cases, the Bir-Aronov-Pikus and D'yakonov-Perel' spin relaxation times first decrease slowly then rapidly with increasing photo-excitation density. Moreover, the D'yakonov-Perel' spin relaxation time decreases faster than the Bir-Aronov-Pikus one. Hence the importance of the Bir-Aronov-Pikus mechanism decreases with photo-excitation density.

To understand such behavior, one notices that only the electron density varies significantly with the photoexcitation density. The photo-excitation density dependence of the Bir-Aronov-Pikus spin relaxation time mainly comes from the fact that $1 / \tau_{\text {BAP }} \propto\left\langle v_{\mathbf{k}}\right\rangle \propto\left\langle\varepsilon_{\mathbf{k}}^{1 / 2}\right\rangle$ [see Eq. [69] ], whereas that of the D'yakonov-Perel' spin relaxation time originates from the inhomogeneous broadening $1 / \tau_{\mathrm{DP}} \propto\left\langle|\boldsymbol{\Omega}|^{2}-\Omega_{z}^{2}\right\rangle \propto\left\langle\varepsilon_{\mathrm{k}}^{3}\right\rangle$. At low photo-excitation density, the electron system is nondegenerate, thus both the Bir-Aronov-Pikus and D'yakonov-Perel' spin relaxation times vary slowly. At higher photo-excitation density, both the Bir-Aronov-Pikus and D'yakonov-Perel' spin relaxation times decrease rapidly with photo-excitation density but the D'yakonov-Perel' spin relaxation time decreases faster.

Hole density dependence. The hole density dependence of both the D'yakonov-Perel' and Bir-Aronov-Pikus spin relaxation times together with their ratio are plotted in Fig. 101] It is noted that the Bir-Aronov-Pikus spin relaxation time decreases as $1 / n_{h}$ at low hole density, which is consistent with $\tau_{\text {BAP }} \propto 1 / n_{h}$ [see Eq. [67)]. At high hole density, $\tau_{\text {BAP }}$ decreases slower than $1 / n_{h}$ due to the Pauli blocking of holes. The density dependence of the D'yakonov-Perel' spin relaxation time is not so obvious: the spin relaxation time first increases, then decreases and again increases with the hole density. As the electron distribution (hence the inhomogeneous broadening) does not change with the hole density, the variation of the D'yakonov-Perel' spin relaxation time comes solely from the momentum scattering (dominated by the electron-impurity scattering). Jiang and $\mathrm{Wu}$ found that the nonmonotonic hole density dependence 

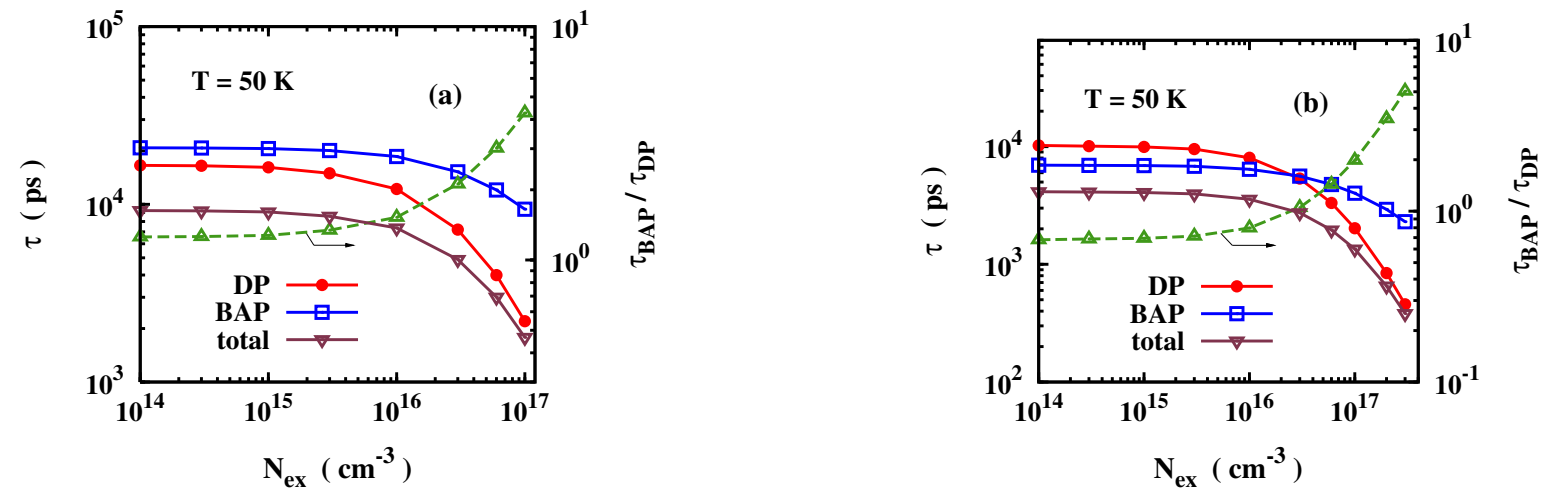

Figure 100: $p$-GaAs. Spin relaxation times $\tau$ due to the Bir-Aronov-Pikus (BAP) and D'yakonov-Perel' (DP) mechanisms together with the total spin relaxation time vs. the photo-excitation density $N_{\mathrm{ex}}$. The ratio of the two is plotted as dashed curve (note that the scale is on the right hand side of the frame). (a): $n_{i}=n_{h}=3 \times 10^{17} \mathrm{~cm}^{-3}$. (b): $n_{i}=n_{h}=3 \times 10^{18} \mathrm{~cm}^{-3} \cdot T=50 \mathrm{~K}$. From Jiang and Wu [110].

of the D'yakonov-Perel' spin relaxation time is again related to the screening.
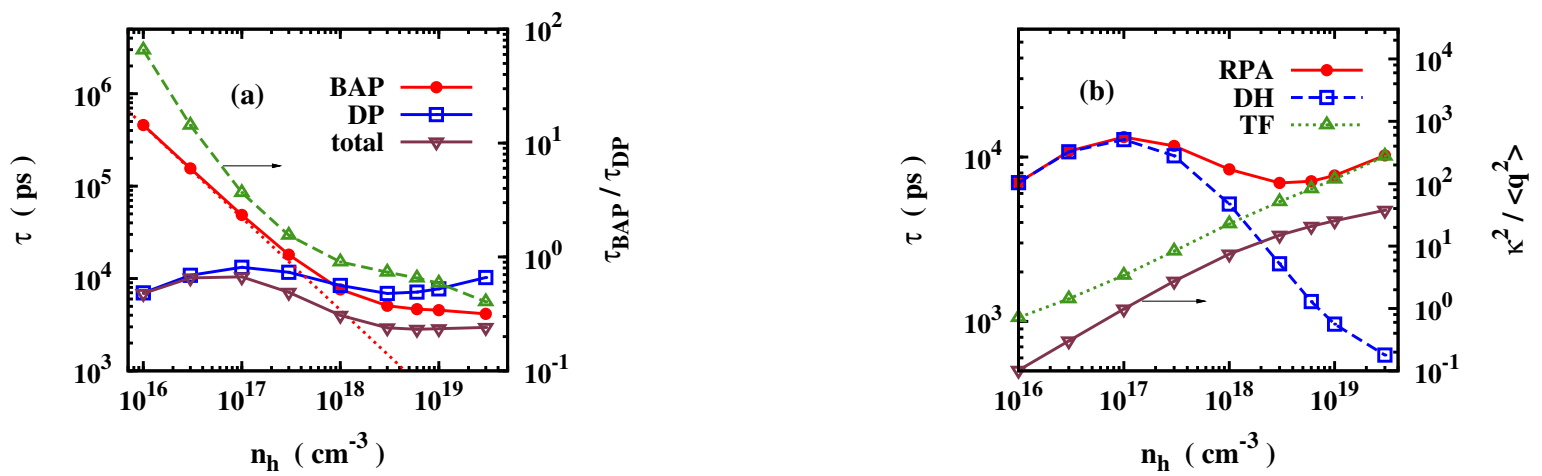

Figure 101: p-GaAs. (a): spin relaxation times $\tau$ due to the Bir-Aronov-Pikus (BAP) and D'yakonov-Perel' (DP) mechanisms together with the total spin relaxation time against hole density $n_{h} . N_{\mathrm{ex}}=10^{14} \mathrm{~cm}^{-3}, n_{i}=n_{h}$, and $T=60 \mathrm{~K}$. The dotted curve denotes a fitting of the curve with - using $1 / n_{h}$ scale. The curve with $\Delta$ denotes the ratio $\tau_{\mathrm{BAP}} / \tau_{\mathrm{DP}}$ (note that the scale is on the right hand side of the frame). (b): spin relaxation times due to the D'yakonov-Perel' mechanism with the Debye-Huckle (DH) (curve with $\square$ ), Thomas-Fermi (TF) (curve with $\triangle$ ), and the randomphase-approximation (RPA) (curve with $\bullet$ ) screenings. The ratio $\kappa^{2} /\left\langle q^{2}\right\rangle$ is plotted as curve with $\nabla$ (note that the scale is on the right hand side of the frame). From Jiang and $\mathrm{Wu}[110]$.

To elucidate the underlying physics, the D'yakonov-Perel' spin relaxation time calculated with the random-phaseapproximation screening together with those calculated with the Thomas-Fermi screening [346] and the Debye-Huckle screening [346] are plotted in Fig. 101 b). From the figure it is seen that the first increase and the decrease is related to the Debye-Huckle screening, whereas the second increase is connected with the Thomas-Fermi screening. The underlying physics is understood as follows: In the low hole density regime, the screening (mainly from holes) is small and the Coulomb potential, $V_{q} \propto 1 /\left(\kappa^{2}+q^{2}\right)$, changes slowly with the screening constant $\kappa$ as well as hole density. Hence the electron-impurity scattering increases with $n_{h}$ as $1 / \tau_{p}^{e i} \propto n_{i}=n_{h}$. For higher hole density $\left(n_{h}>10^{17} \mathrm{~cm}^{-3}\right)$, the screening constant $\kappa$ becomes larger than the transfered momentum $q$. Hence the electronimpurity scattering decreases with $n_{h}$ because $1 / \tau_{p}^{e i} \propto n_{i}\left\langle\left(\kappa^{2}+q^{2}\right)^{-2}\right\rangle \sim n_{h} \kappa^{-4} \propto n_{h}^{-1}$ as $\kappa^{2} \propto n_{h}$ for the Debye-Huckle screening. As the hole density increases, the hole system gradually enters into the degenerate regime, where the Thomas-Fermi screening applies and $\kappa^{2} \propto n_{h}^{1 / 3}$. Hence, the electron-impurity scattering increases with the hole density as $1 / \tau_{p}^{e i} \propto n_{h} \kappa^{-4} \propto n_{h}^{1 / 3}$. Consequently, the D'yakonov-Perel' spin relaxation time first increases, then decreases and again increases with increasing hole density. 


\section{Spin diffusion and transport in semiconductors}

\subsection{Introduction}

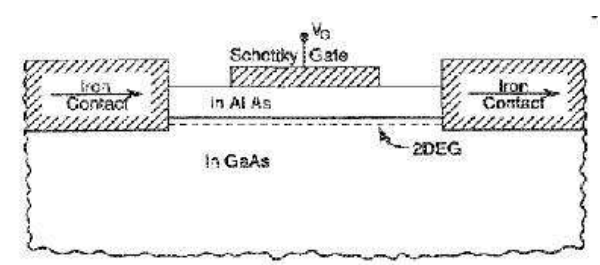

Figure 102: Schematic of Datta-Das transistor. From Datta and Das [54].

The implementation of spintronic devices also relies on the understanding of spin resolved transport phenomena. The first prototype of the spin field-effect-transistor, proposed by Datta and Das [54] and named after them, utilizes the Rashba spin-orbit coupling for operation. As shown in Fig. 102, the so called Datta-Das transistor consists of a three-layer structure with magnetic source and collector connected by a semiconductor channel, and a voltage gate. The spin polarized carriers are injected into the conducting channel from the source and driven towards and detected by the collector. The carriers can flow freely through the drain ("on" state) if their spins are parallel to that of drain or are blocked ("off" state) if anti-parallel. Similar to the traditional field-effect-transistor, the on/off states of spin field-effect-transistor are switched by the gate voltage which controls the spin direction of the passing carriers via the Rashba spin-orbit coupling acting as an effective magnetic field, with its strength controlled by the gate voltage. It is believed that spin field-effect-transistor has the advantages of low energy consumption and fast switching speed since it does not involve creating or eliminating the electrical conducting channel during the switching like the traditional field effect transistor [576, 903$] 90$

As one can see from the operation of the spin field-effect-transistor prototype, the topics of spin transport include the generation of nonequilibrium spin polarized carriers in conductors, the transfer of these carriers from one place to another insider the conductor and/or across the interfaces into other conductors, as well as the detection of the spin signal of these carriers.

Generation of the spin polarization can be achieved by optical orientation of electron spin through transfer of angular momentum of the circularly polarized photons to the carriers [3] and electric injection of spin polarized carriers from ferromagnetic conductor into non-magnetic conductor [904, 905]. When electrons transfer from spin polarized regime to unpolarized one, say from ferromagnetic metal or semiconductors across the interface into non-magnetic conductors, nonequilibrium spin polarization accumulates near the interface [23, 906]. Due to spin relaxation, the spin polarization of the carriers in the nonmagnetic conductor is not spacial uniform but decays as carriers move away from the interface, characterized by the spin injection length. The spacial inhomogeneity of the spin polarization also results in the spin diffusion, whose rate is described by the spin diffusion coefficient. In the presence of the electric field, spins are dragged by the electric field along with the carriers. The response to the electric field is characterized by the conductivity or mobility. An important task is to reveal the relations among these characteristic parameters and how these parameters change under different conditions. Spin detection is to extract the information of spin polarization by sensing the change of the magnetic, optical and/or electrical signals due to the nonequilibrium spin polarization in the non-magnetic conductor. In experiments, optical methods are usually more powerful as one can generate high spin polarization in semiconductor and detect the spin signals with spacial resolution optically. However, it is more desirable to generate and detect the spin signal electrically in real spintronic devices.

Even though the Datta-Das transistor is conceptually simple, there are some crucial obstacles which make it hard to be realized: The low spin injection/detection efficiency between the semiconductor and ferromagnetic source and

\footnotetext{
${ }^{90}$ Although operations of the spin field-effect-transistor do not create or eliminate conducting channel, the electrons have to travel between the source and drain when the transistor switches on/off states. Therefore, the switching time of spin field-effect-transistor is limited by the mobility of the channel. The energy dissipation and the switching time of traditional and spin field-effect-transistors were compared theoretically by Hall and Flatté [222]. They pointed out that the energy dissipation of a typical spin field-effect-transistor can be two orders of magnitude smaller than that of a typical traditional transistor, but the switching time is usually longer [222].
} 
drain due to the conductance mismatch [907-910] (see Sec.6.2.4 for details) and spin-flip scattering at the interfaces [911-914], spin relaxation in the semiconductor channel due to the joint effect of the spin-orbit coupling and (spin conversing) scattering [101, 102, 915] (see Sec. 5 for more details), and the precise control of the gate controlled spin-orbit coupling. Due to these difficulties the Datta-Das transistor has not been realized until Koo et al. claimed so recently [55], although there are still controversies on their claim [916-918].

To overcome these obstacles one must first understand spin transport inside the semiconductor channel and across the interfaces. A lot of understandings on spin transport, to be reviewed in following sections, have been gained in the past decade. Based on these understandings, some variants of the Datta-Das transistor have been proposed. Schliemann et al. first proposed a nonballistic spin field-effect-transistor based on the spin-orbit coupling of both the Rashba and the Dresselhaus types [339]. Due to the interplay of these two types of spin-orbit coupling, the spin diffusion length in the semiconductor channel becomes larger or even infinity for spin polarized along some special directions [194, 196, 203, 919, 920] or for spin polarized current flowing along some special directions [18, 20, 29, 37, 339, 921] (see Sec. 7.5 for details). This type of spin field-effect-transistor is robust against the spin conversing scattering and is a more realistic alternative to the Datta-Das spin field-effect-transistor.

\subsection{Theory of spin injection based on drift-diffusion model}

Although spin transport in semiconductor is a relatively new problem, study of the spin transport in ferromagnetic material has much longer history. It is thus natural to borrow the concepts and methods of spin transport in ferromagnetic metal to study the spin transport in semiconductor. In ferromagnetic conductors, the carriers and current are spontaneously spin polarized below the Curie temperature. The basic transport properties of ferromagnetic metal can be understood by two-current model, where majority (say, spin-up) and minority (spin-down) carriers contribute unequally and independently to the total conductance [922 926]. In two-current model, the spin-up and -down electrons transport independently except for weak spin-flip scattering that flips carriers of one spin to the other [907, 908, 925, 927]. The carriers of different spins usually have different diffusion coefficients $D_{\uparrow / \downarrow}$ and mobilities $\mu_{\uparrow / \downarrow}$. The electric currents of the spin-up and -down electrons are determined by the drift caused by the electric field and diffusion due to the spacial inhomogeneity of the carrier densities. Under linear approximation and in the diffusive limit, the currents are [22, 907, 908, 927]

$$
\mathbf{j}_{\eta}=-e n_{\eta}\left\langle\mathbf{v}_{\eta}\right\rangle=e \mu_{\eta} n_{\eta} \mathbf{E}+e D_{\eta} \boldsymbol{\nabla} n_{\eta}=\sigma_{\eta} \mathbf{E}+e D_{\eta} \boldsymbol{\nabla} n_{\eta}, \quad(\eta=\uparrow, \downarrow)
$$

where $n_{\eta}$ and $\sigma_{\eta}=e \mu_{\eta} n_{\eta}$ are the electron density and conductance of spin $\eta$, respectively. The electric field $\mathbf{E}$ is determined by the Poisson equation

$$
\boldsymbol{\nabla} \cdot \mathbf{E}=-\nabla^{2} \phi=e\left(n_{\uparrow}+n_{\downarrow}-n_{0}\right) / \varepsilon,
$$

with $\phi,-e, \varepsilon$ and $n_{0}$ being the electric potential, electron charge, dielectric constant and the background positive charge density respectively. Since the total electron number is conservative, one can write down the continuity equations for these two kinds of electrons:

$$
\frac{\partial n_{\eta}}{\partial t}=\frac{1}{e} \boldsymbol{\nabla} \cdot \mathbf{j}_{\eta}-\frac{\delta n_{\eta}}{\tau_{\eta \bar{\eta}}}+\frac{\delta n_{\bar{\eta}}}{\tau_{\bar{\eta} \eta}}
$$

with $\delta n_{\eta}=n_{\eta}-n_{\eta}^{0}$ and $\tau_{\eta \bar{\eta}}$ standing for deviation of the nonequilibrium charger density $n_{\eta}$ from the equilibrium one $n_{\eta}^{0}$ and the average carrier spin-flip time, respectively.

Combined with different initial and boundary conditions, the above drift-diffusion equation or its equivalence is widely used in the study of spin-related transport in semiconductors, including in the understanding of the existing experimental spin injection/extraction results and in the proposing of new schemes of spintronic devices. However, it should be pointed out that although these equations give qualitatively correct results in many cases, the validity of the drift-diffusion model should be carefully examined when applied to spin transport in semiconductors. This is because the spin transport in ferromagnetic metal and semiconductor can be quite different: First, the applied electric field and spacial gradient of electron density in metal are usually small due to large conductance and high carrier density, hence electrons are usually not far away from the equilibrium. Therefore Eq. (272) describes the current in metals accurately in most cases. In semiconductors, however, both the applied electric field and the gradient of carrier density can be very large, and electrons can be easily driven to states far away from the equilibrium. As a result, Eq. (272) may no 
longer hold. More importantly, the spin-up and -down branches in ferromagnetic metal are well separated and the coherence between these two branches is very small. While in nonmagnetic semiconductor the spin-up and -down electrons are usually degenerate, an applied or effective (from the spin-orbit coupling) magnetic field can cause spins to precess and result in large spin coherence.

Therefore quantitative calculation based on the drift-diffusion model is questionable when the spin coherence is essential to the spin kinetics or when the electrons involve in the transport are far away from the equilibrium. It has been shown that in the presence of the magnetic field, either the external applied one or the intrinsic effective one from the spin-orbit coupling, the drift-diffusion model is inadequate in accounting for the spin transport in semiconductors [25, 27, 28, 32, 336]. Nevertheless, the drift-diffusion model is still useful in qualitative study of spin transport since its simplicity and flexibility to add new factors of physics.

\subsubsection{Spin transport in nonmagnetic semiconductor using drift-diffusion model}

One can easily obtain some basic properties of the spin transport using drift-diffusion model to study the transport property of the magnetic momentum inside nonmagnetic semiconductors in the simplest case. Assuming that the charge density in nonmagnetic semiconductor is uniform and the electronic transport coefficients are not affected by the spin polarization, one can then write down the transport equation for the magnetic momentum $\mathbf{S}$ in a uniform electric field $\mathbf{E}$ and magnetic field $\mathbf{B}$ [915],

$$
\frac{\partial \mathbf{S}}{\partial t}=D \nabla^{2} \mathbf{S}-e \mu \mathbf{E} \cdot \nabla \mathbf{S}+g \mu_{B} \mathbf{B} \times \mathbf{S}-\frac{\mathbf{S}}{\tau_{s}}
$$

with $\mu, D$, and $1 / \tau_{s}$ being the charge mobility, diffusion coefficient and spin relaxation time, respectively. Note that the drift-diffusion equation here has been modified to include the Larmor precession of the spin around the applied magnetic field $\mathbf{B}$.

Spin accumulation in the steady state. When a semiconductor is in contact with a spin polarization source at $x=0, \mathbf{B}=0$ and the electric field is along the $x$-direction, the drift-diffusion model predicts spin accumulation with exponential decay in the semiconductor $S(x)=S_{0} \exp \left[-x / L_{S}(E)\right]$ in which the electric-field-dependent spin injection length reads [904, 928, 929]

$$
L_{s}^{-1}(E)=\frac{1}{L_{s}} \sqrt{1+\frac{L_{d}^{2}}{4 L_{s}^{2}}}-\frac{E}{|E|} \frac{L_{d}}{2 L_{s}^{2}},
$$

where $L_{s}=\sqrt{D \tau_{s}}$ is the diffusion length, $L_{d}=|e \mu E| \tau_{s}=\left|v_{d}\right| \tau_{s}$ is the drifting length over spin relaxation time with drift velocity $v_{d}$ determined by the electric field. Without the electric field, the spin injection length is the diffusion length $L_{s}$. The applied electric field can significantly change the injection length by dragging or pulling the electron [928, 929]: For large downstream electric field, the electron spin injection is the distance that the electrons move with drift velocity within the spin lifetime time, $L_{s}(E)=L_{d}$; For large upstream field, $L_{s}(E)=L_{s}^{2} / L_{d}$.

Evolution of the spin polarized electron package. The shape of a spin polarized carrier packet changes with time due to the drift and diffusion. The temporal evolution of a $\delta$ spin polarized packet of height $S_{0}$ at $x=0$ is

$$
S(x, t)=\frac{S_{0}}{\sqrt{2 \pi D t}} \exp \left[-\frac{t}{\tau_{s}}-\frac{\left(x-v_{d} t\right)^{2}}{4 D t}\right],
$$

which has the form of Gaussian function whose center is determined by the drifting, $v_{d} t$, and width determined by the diffusion, $\sqrt{D t}$.

\subsubsection{Hanle effect in spin transport}

In the spacial homogeneous system with a spin excitation source, such as circularly polarized light [2, 311, 930] or spin resonant microwave radiation [931, 932], the total spin is the accumulation of survival spin from the past. When a perpendicular magnetic field is presented, spin undergoes Larmor precession. Since spins excited at different time have different precession phases and tend to cancel each other, the perpendicular magnetic field reduces the total accumulated spin. This is known as the Hanle effect [915, 933]. In spin transport, the Hanle effect also appears [150]: Spin accumulation at position $x$ is the sum of the electron spins travel from different places. Since different electrons have different transit time when they reach $x$, their phases are different and tend to cancel each other. As shown in 
Fig. 103. for a constant spin pumping/injection starting from $t=0$ at $x=0$, when a constant magnetic field $B$ is applied along the $\hat{y}$ direction, the spin accumulation at time $t$ and position $x$ is

$$
\begin{aligned}
& S_{z}(x, t)=\int_{0}^{t} \frac{S_{0}}{\sqrt{2 \pi t^{\prime}}} e^{-t^{\prime} / \tau_{s}-\left(x-e \mu E t^{\prime}\right)^{2} /\left(4 D t^{\prime}\right)} \cos \omega t^{\prime} d t^{\prime}, \\
& S_{x}(x, t)=\int_{0}^{t} \frac{S_{0}}{\sqrt{2 \pi t^{\prime}}} e^{-t^{\prime} / \tau_{s}-\left(x-e \mu E t^{\prime}\right)^{2} /\left(4 D t^{\prime}\right)} \sin \omega t^{\prime} d t^{\prime},
\end{aligned}
$$

with $\omega=g \mu_{B} B$ being the Larmor frequency. For the steady state spin injection under a magnetic field, the spin accumulation can be calculated by letting time in Eqs. (278) and (279) to be infinity or can be solved directly from Eq. (275). The results are

$$
\begin{aligned}
& S_{z}(x)=S_{0} e^{-x / L_{s}(E, B)} \cos \left[x / L_{0}(E, B)\right] \\
& S_{x}(x)=S_{0} e^{-x / L_{s}(E, B)} \sin \left[x / L_{0}(E, B)\right]
\end{aligned}
$$

where

$$
\begin{aligned}
L_{s}^{-1}(E, B) & =\frac{E}{|E|} \frac{L_{d}}{2 L_{s}^{2}}+\frac{1}{L_{s}} \sqrt{\left(1+\frac{L_{d}^{2}}{4 L_{s}^{2}}\right)^{2}+\left(\omega \tau_{s}\right)^{2}} \cos \frac{\theta}{2}, \\
L_{0}^{-1}(E, B) & =\frac{1}{L_{s}} \sqrt{\left(1+\frac{L_{d}^{2}}{4 L_{s}^{2}}\right)^{2}+\left(\omega \tau_{s}\right)^{2} \sin \frac{\theta}{2},} \\
\tan \theta & =\frac{\omega \tau_{s}}{1+L_{d}^{2} /\left(4 L_{s}^{2}\right)} .
\end{aligned}
$$

One can see that the spin polarizations change with the position as a damped oscillation with the electric and magnetic field dependent injection length $L_{s}(E, B)$ and the spacial oscillation "period" $L_{0}(E, B)$ defined by Eqs. (282, 284). Even when there is no spin relaxation mechanism, i.e., $\tau_{s}=\infty$, the spin injection length is still finite in the presence of a perpendicular magnetic field, $L_{s}^{-1}(0, B)=L_{0}^{-1}(0, B)=\sqrt{\omega / 2 D} 91$ Without the driving of the electric field, the oscillation "period" is larger or close to the injection length, which means that the oscillation may not be easy to detect. Under a strong downstream electric field, $L_{s}(E, B) \simeq L_{d}$, and $L_{0}(E, B) \simeq L_{d} / \omega \tau_{s}$, it is then possible to observe many oscillations in spin accumulation.

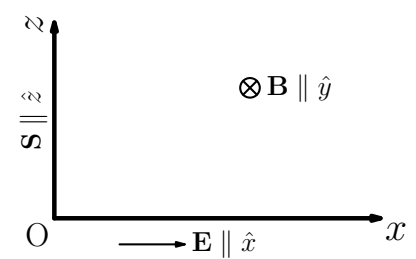

Figure 103: Orientations of spin transport and electric/magnetic fields.

\subsubsection{Spin injection theory}

To study the spin injection from ferromagnetic electrode to semiconductor, it is usually more convenient to use the drift-diffusion equation in the form of electrochemical potential [22, 907, 908, 927]. For the state near the equilibrium, $\delta n_{\eta}(\mathbf{r})=g_{\eta} \delta \tilde{\zeta}_{\eta}(\mathbf{r})$, with $\delta \tilde{\zeta}_{\eta}(\mathbf{r})=\tilde{\zeta}_{\eta}(\mathbf{r})-\tilde{\zeta}_{\eta}^{0}(\mathbf{r})$ standing for the deviation in the chemical potential $\tilde{\zeta}_{\eta}(\mathbf{r})$ away from the equilibrium one $\tilde{\zeta}_{\eta}^{0}(\mathbf{r})$ of the electron with spin $\eta$ at position $\mathbf{r} . g_{\eta}=\left(\partial n_{\eta} / \partial \tilde{\zeta}_{\eta}\right)$ is the temperature dependent density of states. The current can be rewritten as [22, 907, 908, 927]

$$
\mathbf{j}_{\eta}=-\sigma_{\eta} \boldsymbol{\nabla} \phi(\mathbf{r})+e D_{\eta} g_{\eta} \boldsymbol{\nabla} \delta \tilde{\zeta}=\frac{\sigma_{\eta}}{e} \boldsymbol{\nabla} \zeta_{\eta}(\mathbf{r})
$$

\footnotetext{
${ }^{91}$ This effect was first predicted by Weng and Wu microscopically from the kinetic spin Bloch equation approach [336].
} 
in which we have used the Einstein relation, $\sigma_{\eta}=e^{2} D_{\eta} g_{\eta}$, and introduced the electrochemical potential, $\zeta(\mathbf{r})=$ $\tilde{\zeta}(\mathbf{r})-e \phi(\mathbf{r})$.

Introducing the notations, $g=g_{\uparrow}+g_{\downarrow}, g_{s}=g_{\uparrow}-g_{\downarrow}, \sigma=\sigma_{\uparrow}+\sigma_{\downarrow}, \sigma_{s}=\sigma_{\uparrow}-\sigma_{\downarrow}, \zeta=\left(\zeta_{\uparrow}+\zeta_{\downarrow}\right) / 2, \zeta_{s}=\left(\zeta_{\uparrow}-\zeta_{\downarrow}\right) / 2$, $D=\left(D_{\uparrow}+D_{\downarrow}\right) / 2$ and $D_{s}=\left(D_{\uparrow}-D_{\downarrow}\right) / 2$, and enforcing the charge neutrality, i.e. $n_{\uparrow}+n_{\downarrow} \equiv n_{\uparrow}^{0}+n_{\downarrow}^{0}$, one obtains

$$
\zeta(x)+e \phi(x)=-g_{s} \zeta_{s}(x) / g .
$$

The non-equilibrium spin accumulation is expressed as

$$
\delta S=\frac{4 g_{\uparrow} g_{\downarrow}}{g} \zeta_{s} .
$$

From Eq. (285) one can further write down the charge and spin currents expressed in form of the electrochemical potential,

$$
\begin{aligned}
\mathbf{j}_{e} & =\mathbf{j}_{\uparrow}+\mathbf{j}_{\downarrow}=\frac{\sigma}{e} \nabla \zeta+\frac{\sigma_{s}}{e} \nabla \zeta_{s}, \\
\mathbf{j}_{s} & =\mathbf{j}_{\uparrow}-\mathbf{j}_{\downarrow}=\frac{\sigma_{s}}{e} \nabla \zeta+\frac{\sigma}{e} \nabla \zeta_{s}, \\
\frac{\partial \zeta_{s}}{\partial t} & =\bar{D} \nabla^{2} \zeta_{s}-\frac{\zeta_{s}}{\tau_{s}},
\end{aligned}
$$

with $\bar{D}=g\left(g_{\uparrow} / D_{\downarrow}+g_{\downarrow} / D_{\uparrow}\right)^{-1}$ being the effective spin diffusion coefficient.

From these equations, one can obtain the spin transport properties in conductors. For nonmagnetic conductor, the spin-up and -down bands are degenerate, one recovers the results obtained in Sec. 6.2.1 for the spin transport in nonmagnetic conductor under weak electric field. In ferromagnetic conductor, the dynamics of nonequilibrium spin accumulation is similar to that in nonmagnetic conductor, i.e., it exponentially decays with the position, with the spin diffusion length being $L_{s}=\sqrt{\bar{D} \tau_{s}}$. For a uniform ferromagnetic conductor without nonequilibrium spin accumulation, the current polarization is determined by the difference of the conductivities of the two spin branches,

$$
P_{\sigma}=j_{s} / j_{e}=\sigma_{s} / \sigma .
$$

In the presence of the spin accumulation, the current polarization is modified as

$$
P_{j}=P_{\sigma}+L_{s} \nabla \zeta_{s} /\left(j_{e} R\right)
$$

where $R=\sigma L_{s} /\left(4 \sigma_{\uparrow} \sigma_{\downarrow}\right)$ is the effective resistance of the conductor with unit surface area over a distance of the diffusion length.

\subsubsection{Spin injection efficiency of ferromagnetic conductor/nonmagnetic conductor junction}

To discuss the spin injection from ferromagnetic conductor into nonmagnetic conductor, one needs to study the transport through their interface. In drift-diffusion model, the effect of interface is phenomenally described by spin selective interface conductance. Assuming that there is no strong spin-flip scattering at the interface, the current of each spin branch is conservative. However, the electrochemical potential can be discontinuous if the contact is not ohmic. Introducing the contact conductance $\Sigma_{\eta}$ for spin $\eta$, the boundary condition at the interface $(\mathrm{x}=0)$ is then written as [904, 905, 907, 908, 910]

$$
j_{\eta}(0)=\Sigma_{\eta}\left[\zeta_{\eta F}(0)-\zeta_{\eta N}(0)\right] .
$$

Similarly, one can introduce $\Sigma=\Sigma_{\uparrow}+\Sigma_{\downarrow}, \Sigma_{s}=\Sigma_{\uparrow}-\Sigma_{\downarrow}$ and rewrite the boundary conditions for charge current and spin current.

Using the above drift-diffusion equation, the matching condition of interface at $x=0$, and the boundary conditions $\zeta_{S F}(-\infty)=0$ and $\zeta_{S N}(\infty)=0$ at the far left end of ferromagnetic electrode and the far right end of nonmagnetic electrode, one is able to write down the spin injection efficiency across the ferromagnet/nonmagnetic conductor junction. The current polarization at the interface is [907, 908]

$$
P_{j}(0)=\frac{j_{s}(0)}{j_{e}(0)}=\frac{R_{F} P_{\sigma F}+R_{c} P_{c}}{R_{F}+R_{c}+R_{N}}
$$


where $R_{F}, R_{N}$ and $R_{c}=\Sigma /\left(4 \Sigma_{\uparrow} \Sigma_{\downarrow}\right)$, are the effective resistances of ferromagnetic conductor, nonmagnetic conductor and contact, respectively. $P_{\sigma F}$ and $P_{c}=\left(\Sigma_{\uparrow}-\Sigma_{\downarrow}\right) / \Sigma$ are the conductance polarizations of the ferromagnetic conductor and contact respectively. Since the ferromagnetic conductor usually has much larger conductance and shorter spin diffusion length, $R_{F} \ll R_{N}$. Therefore, for transparent contact, the polarization of the injected current $P_{j}(0)=P_{\sigma F} R_{F} /\left(R_{F}+R_{N}\right)$ is much smaller than $P_{\sigma F}$, the current polarization in ferromagnetic conductor, due to the conductance mismatch [907-910]. For large contact resistance, the current polarization is determined by the contact polarization $P_{j}(0) \simeq P_{c}$. Therefore the conduction mismatch can be reduced by inserting an spin selective layer with large resistance [22, 907-910, 927, 934]. One can also avoid the conductance mismatch by using spin filter [935-937] to replace the ferromagnetic metal as spin injector.

\subsubsection{Silsbee-Johnson spin-charge coupling}

In electrical spin injection, the spin polarized current flows from a ferromagnetic electrode into nonmagnetic conductor and produces a non-equilibrium spin accumulation near the interface in the nonmagnetic conductor. The inverse of the above effect also holds: The presence of non-equilibrium spin accumulation in nonmagnetic conductor near the interface will produce an electromotive force in the circuit which drives a charge current to flow in a close circuit or results in a voltage drop in an open circuit. This effect is called Silsbee-Johnson spin charge coupling [905, 938]. This spin-charge coupling can affect the spin transport properties in return. In the spin injection, the electromotive force caused by the spin accumulation impedes the charge/spin current and reduces the overall conductivity, which is called the spin bottleneck effect [905, 938-941].

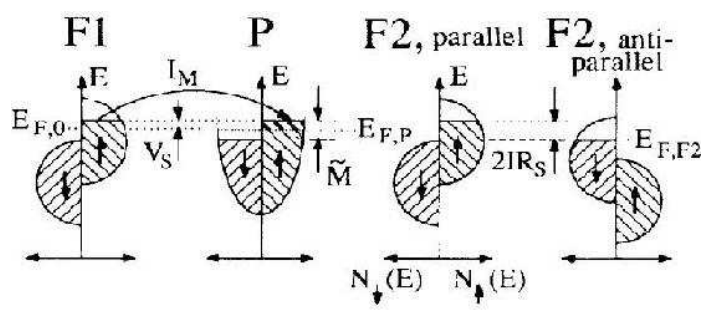

Figure 104: Schema of Silsbee-Johnson effect and all electrical spin detection. From Johnson [940].

Physically speaking, this spin-charge coupling can be understood by the simplified model shown in Fig. 104, proposed by Silsbee [938]. In this model, it is assumed that the ferromagnetic electrode is a half-metal so that only one spin branch (say, spin-up) electrons carry the current and Fermi levels in ferromagnetic and nonmagnetic conductors are aligned before they are connected. After they are connected, the spin accumulation near the interface raises the (electro)chemical potential of spin-up electrons in nonmagnetic electrode, and lowers that of spin-down electrons to ensure the neutrality of charge, as shown in the left part of Fig. 104. As a result of this electrochemical potential shifting in nonmagnetic electrode, an electromotive force must be produced to raise the electrochemical potential in ferromagnetic electrode so that it aligns with that of spin-up electrons in nonmagnetic electrode. Quantitative calculation of Silsbee-Johnson coupling based on drift-diffusion model can be obtained from Eqs. (286, 293). In close circuit the change in the resistance caused by the spin bottleneck effect is

$$
\delta R=\frac{R_{N}\left(R_{c} P_{c}^{2}+R_{F} P_{F}^{2}\right)+R_{F} R_{c}\left(P_{F}-P_{c}\right)^{2}}{R_{F}+R_{c}+R_{N}} .
$$

For more complicated structures like ferromagnetic/nonmagnetic/ferromagnetic conductors, the resistance change due to the spin accumulation depends on the length of nonmagnetic conductor and the spin polarizations of ferromagnetic electrodes. When the length of nonmagnetic conductor is much larger than the spin diffusion length, the resistance change is simply the sum of that of two ferromagnetic/nonmagnetic contacts. When the length of nonmagnetic conductor is smaller than or of the order of spin diffusion length, the change in resistance depends on the alignment of the magnetic momentum of the two electrodes, as shown in the right part of Fig. 104. This is known as spin valve effect. It can be quantitatively calculated by matching the boundary conditions on these two contacts [908]. 
In open circuit, for a spin accumulation at $x=0$, the voltage drop caused by the electromotive force over a distance far longer than the spin diffusion length is given by

$$
\Delta \phi=\left(g_{s} / g-\sigma_{s} / \sigma\right) \zeta_{s}(0) / e
$$

If a second ferromagnetic electrode is attached to the nonmagnetic electrode, it can be used to detect the spin accumulation at the interface of the nonmagnetic electrode and the second ferromagnetic electrode. If there is spin accumulation, the voltage drop between the nonmagnetic and the second ferromagnetic electrodes depends on the relative alignments of the spin momentums in these two electrodes, as shown in the right part of Fig. 104 SilsbeeJohnson spin-orbital coupling is particular useful in the non-local spin detection [281, 940-946].

\subsection{Spin injection through Schottky contacts}

In the drift-diffusion model, the contact of ferromagnetic conductor and semiconductor is usually described as a sheet layer with finite or zero resistance. Studies of the spin injection through Schottky barrier, which appears naturally at the interface of ferromagnetic metal and semiconductor, have also been carried out using drift-diffusion model [947, 948] and Monte Carlo simulation [949-954].

In the framework of the drift-diffusion model, the tunneling through the Schottky barrier is still described by (spin selective) interface resistance. However, the conductivity inside the semiconductor is no longer a constant, since it is proportional to carrier concentration which varies dramatically with position near the Schottky barrier. Taking the position dependence of the conductivity into account, it is shown that the existence of a depletion region of the Schottky barrier greatly reduces the spin accumulation. For a ferromagnetic metal/semiconductor contact with significant depletion region, the spin accumulation is reduced almost to zero in a distance less than $100 \mathrm{~nm}$ [947, 948]. Outside the depletion region, the spin accumulation decays with the distance at a slower rate of spin injection length (at the order of $1 \mu \mathrm{m}$ ). The existence of the Schottky barrier also greatly reduces the spin polarization of the injected current. Although the spin polarization of injected current decays at the rate of spin injection length throughout the semiconductor side, it drops rapidly inside the ferromagnetic conductor near the ferromagnetic metal/semiconductor surface when a high barrier is presented.

In the Monte Carlo simulations, studies focus on the transport inside the semiconductor. The ferromagnetic conductor is treated as source that provides spin polarized carriers and is not affected by the semiconductor. The current is injected into the semiconductor through the barrier by both thermionic emission and direct tunneling 955 , 956]. In this model, the tunneling probability through the barrier, proportional to the density of states for spin-up and -down carriers in the ferromagnetic conductor, is naturally spin dependent. Therefore, this model is beyond the drift-diffusion model. In the simulation, the spacial profile of the Schottky barrier is determined by solving the charge motion and the Poisson equation self-consistently [957]. The transport in the semiconductor is based on semiclassical approximation that includes "drifting" and "scattering" processes. The electron spin is subjected to the influent of the Rashba and/or Dresselhaus spin-obit interaction during the "drifting" process. The Monte Carlo simulations of the spin injection from Fe contact into GaAs quantum well showed that, although injected spin polarization is large right next to the Schottky barrier, similar to the results of the drift-diffusion model, the total spin polarization drops to nearly zero in a few tens of nanometers, a distance of the order of depletion region, due to large unpolarized carriers in the semiconductor. Moreover, the average magnetic momentum of the injected electrons also decays dramatically in the depletion region but much more slowly beyond that. This is because the Rashba and Dresselhaus spin-orbit couplings depend on the electron momentum, the larger the kinetic energy, the larger the effective magnetic field. In the depletion region, electrons have much larger kinetic energy, therefore suffer much faster decay rate. Outside the depletion region, the decay rate is much slower as electrons lose their kinetic energy to overcome the electrical potential barrier as well as due to the inelastic scattering [949-954]. Usually, the Rashba effect considered in the spin kinetics in quantum wells is determined by the electric field perpendicular to the quantum well plane (due to the built-in electric field or the gate voltage). In the presence of the Schottky barrier, there is a strong in-plane electric field in the depletion region induced by the barrier. This barrier induced electric field also leads to the Rashba effect and further reduces the magnetic momentum of the injected electrons [952].

As one can see from the above studies that, a high Schottky barrier with significant depletion region is highly undesirable for spin injection. In order to increase the spin injection efficiency, one needs to modify the Schottky barrier. A thin heavily doped $n^{+}$layer, which sharply reduces the height and thickness of the barrier, was proposed 
to be inserted between the ferromagnetic electrode and semiconductor to increase the injection efficiency [958-965]. Using this setup, highly efficient electrical spin injection can been achieved. It has been reported that in a wide range of temperature, up to $30 \%$ of the spin polarization is injected from Fe into $n$-type AlGaAs and GaAs and survives over a distance of $100 \mathrm{~nm}$ [958, 959, 964]. Up to 50\% spin polarization has been reported to survive over a distance of $300 \mathrm{~nm}$ in the experiments of spin injection from Fe into $n$-type ZnSe when the temperature is around or less than $100 \mathrm{~K}[966]$.

Theoretical studies of spin injection through the interface of ferromagnetic conductor and semiconductor with a $\delta$ doped layer near the interface were carried out in Refs. [960-963]. In these studies, it was assumed that the depletion region is only a few nanometers and the tunneling electrons do not lose their spin polarization over this region. By assuming that the depletion region is of triangular shape, a direct tunnel current was calculated and the transport outside the depletion region was performed using the drift-diffusion model. Unlike the traditional drift-diffusion model, the spin polarization of the tunneling carriers depends on the band structure of ferromagnetic conductor, but is independent on the transport properties of the ferromagnetic metal. Hence, there is no conductance mismatch in this model, and it is possible to achieve high spin polarization at the interface. Moreover, it was shown that the spin injection efficient is not a constant but strongly depends on the injection current, the larger the current amplitude, the higher the injection efficiency. In order to achieve high efficiency, a high electric field is required and therefore injection length also increases with the injection current as predicted by Eq. 276 [960-963]. However, the applied electric field also enhances the Rashba spin-orbit coupling and reduces the injected spin polarization as pointed out by Wang and $\mathrm{Wu}$ [952]. Recent experiment by Kum et al. showed that the competing effects of the electric field cancel each other, leading to an almost negligible decrease of magnetoresistance with bias current [967].

\subsection{Spin detection and experimental study of spin transport}

Experimental study of spin transport also requires an effective method to extract the spin information from semiconductors. In some senses, spin detection and extraction are reverse of the spin generation and injection. Each spin generation/injection mechanism can also be reversely used to extract the spin information. Generally speaking, there are two categories of spin detection: optical and electrical spin detections. Optical spin detection, such as the Faraday/Kerr rotation and circular polarization of electroluminescence, provides reliable information of spin polarization in semiconductor and has been proven to be a powerful experimental tool in the study of the spin transport. While high efficiency electrical spin detection is essential to the spin transistor.

\subsubsection{Spin detection using electroluminescence}

The electroluminescence is the reverse of the optical orientation. In the usual electroluminescence experiment setup, the electrons (holes) to be detected are driven to a structure like a light emitting diode where they recombine with unpolarized holes (electrons) and emit photons. Due to the selection rule, the emitted photons are circularly polarized if the carriers to be detected are spin polarized. By measuring the polarization of the electroluminescence, one obtains the spin polarization. This technique was employed in the first experimental observation of spin injection from ferromagnetic nickel tip of a scanning tunneling microscope into nonmagnetic $p$-type GaAs, and the injected spin polarization of about $40 \%$ at the surface was reported [968]. However, the early attempts on high spin injection efficiency in the real devices were not so successful due to spin-flip scattering at the interface and the conductance mismatch [907, 908]. Spin polarization injected from traditional ferromagnetic metals, such as Fe, Ni, Co and their alloys, into GaAs or InAs was usually only a few percents [969-971]. By various means, the spin injection efficiency has been improved over the years. Here we list some important results in the following, loosely cataloged by the structures used in the experiments.

- Spin injection from ferromagnetic or diluted magnetic semiconductors into GaAs: Ferromagnetic or diluted magnetic and nonmagnetic semiconductors have similar conductance, therefore spin injection in these structures surfers less conductance mismatch. However, since ferromagnetic semiconductor has low Curie temperature, the experiments are usually performed at low temperature. Spin injection from $n$-type II-V diluted magnetic semiconductor into $n$-GaAs under applied magnetic field is very efficient. Injected spin polarization from $\mathrm{Be}_{x} \mathrm{Mn}_{y} \mathrm{Zn}_{1-x-y} \mathrm{Se}$ into GaAs achieves $90 \%$ at low temperature $(<5 \mathrm{~K})$, but drops to $20 \%$ when the temperature rises to $35 \mathrm{~K}$ [522, 972]. Spin injection from $p$-type GaMnAs or MnAs into $n$-type GaAs is more difficult. The efficiency of direct spin injection from GaMnAs or MnAs into $n$-type GaAs usually is only a few 
percent even at low temperature [973-976]. Injection from GaMnAs or MnAs via interband tunneling in Esaki diode is much more efficient [977-984]. 80\% injected spin polarization in Esaki diode has been realized when temperature is $10 \mathrm{~K}$ [983]. Molenkamp group has proposed and demonstrated to use a magnetic double barrier resonant tunneling diode as spin injector [985-989]. A spin polarization up to $80 \%$ has been injected from $\mathrm{BeTe} / \mathrm{Zn}_{1-x} \mathrm{Se} / \mathrm{BeTe}$ into GaAs under 1.6 K temperature and 7.5 T magnetic field [985] 92

- Spin injection from traditional ferromagnetic metal through a Schottky barrier into GaAs or AlGaAs: By constructing a Schottky barrier with narrow depletion regime at the interface of the ferromagnetic metal and semiconductor, spin injection efficiency is greatly improved [974, 990-994]. Injected spin polarization of 30\% from Fe into GaAs at room temperature was reported [993].

- Spin injection from traditional ferromagnetic metal through an insulator layer into GaAs or AlGaAs: By inserting an insulator tunneling layer, the injected spin polarization can be further increased. The tunneling layer improves the surface condition by preventing the magnetic atoms from diffusing into semiconductor and thus reduces the spin-flip scattering at the interface. Moreover, this tunneling layer can be regarded as a spinselective contact with low conductance and hence reduces the conductance mismatch [22, 907, 908, 927, 934]. Typical tunneling layers are composed of $\mathrm{AlO}_{x}$ [995-998] and $\mathrm{MgO}$ [999-1007], although $\mathrm{GaO}_{x}$ has also been used to improve the charge injection efficiency [1008]. Using $\mathrm{AlO}_{x}$ as tunneling layer [995-998], the injection efficiency can achieve $40 \%$ when the temperature is lower than $180 \mathrm{~K}$ [997, 998]. $\mathrm{MgO}$ tunneling layer is even more efficient in improving the spin injection. Due to the band structures and symmetry, tunneling from $\mathrm{Fe}$, $\mathrm{Co}$ and their alloy through $\mathrm{MgO}$ barrier is strongly spin selective. The conductance of majority spin is orders of magnitude higher than that of minority spin $[1000,1009-1012]$. In this case, the injected spin polarization is determined by the tunneling spin polarization. Injected spin polarization of 70-80\% from CoFe electrode through $\mathrm{MgO}$ layer into GaAs at room temperature was reported [999, 1002].

- Spin injection from Heusler alloys into GaAs or AlGaAs: In additional to the spin injection from traditional ferromagnetic metal, there are also experiments on using Heusler alloys (some of them have been predicted to be half-metals [1013, 1014]) as spin injector in hoping that both high spin and charge injection efficiencies can be realized [1015-1019]. Over 50\% injection efficiency from Heusler alloy $\mathrm{Co}_{2} \mathrm{FeSi}$ into GaAs up to $100 \mathrm{~K}$ has been demonstrated [1018].

The electroluminescence technique has also been used to demonstrate that spin polarized carriers can be injected and sustain the polarization over a microscopic distance by constructing the light emitting diode structure away from the surface of ferromagnetic and nonmagnetic conductors [522, 523, 946, 958, 970, 975, 977, 1020, 1021] or away from optical injection position [1022]. Considering the optical absorption of the semiconductor, the photon emitted at position away from the surface should be weighted as $\exp [-\alpha(\lambda) x]$. By further combined with the fact that absorption coefficient is wave-length dependent, experimental measurement of wave-length dependence of the electroluminescence polarization was used to estimate the spin injection length [1023].

\subsubsection{Spin imaging}

The spin detection using the Faraday/Kerr effect measures the Faraday/Kerr rotation angle of transmitted/reflected linearly polarized photons. These angles are proportional to the spin component (magnetization) along the direction defined by the propagation of probe light beam [748], and thus provide direct information of the magnetization along this direction. Usually the probe light is nearly normal to the sample surface, therefore only the magnetization normal to the surface can be measured by this technique directly. The information of spin coherence can be obtained by applying an in-plane magnetic field to rotate the in-plane spin component to the normal direction. Recently developed tomographic Kerr rotation technique further enables the measurement of the spin coherence at any direction $[500$, 1024.

\footnotetext{
92 It is noted that the direction of the spin polarization through magnetic double barrier resonant diode can be changed by an external voltage which selects the spin dependent resonant tunneling level [985-989]. This is quite different from the spin injection from ferromagnetic material where the injected spin polarization is the same as the majority spin of the injector. To change the spin direction injected from the ferromagnetic injector, a magnetic field should be applied to change its spin direction. While using magnetic double barrier resonant diode, it can be achieved electrically.
} 

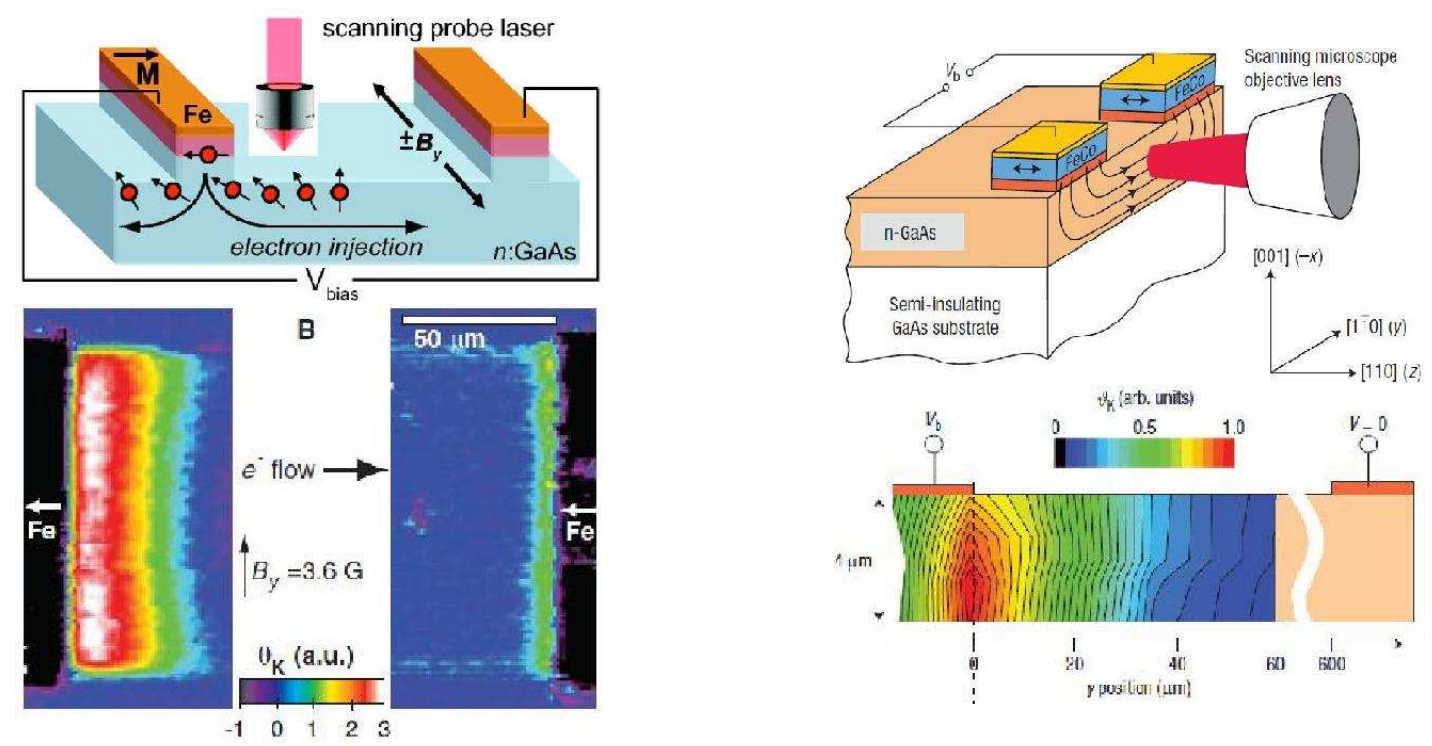

Figure 105: Schematic experiment setup for spin image of electrical spin injection. Left: Surface scanning, an in-plane magnetic field is applied to rotate the injected spin to out of plane for Kerr rotation measurement. From Crooker et al. [1025] ; Right: Side-surface scanning. From Kotissek et al. [1026].

By moving the focus of the probe light and performing the measurement at different time, one obtains the space and temporal resolved information of the spin polarization, and thus images the transport of spin polarization. Typical schemes of experiment setups and results for spin imaging are shown in Fig. 105 In the experiments, spin polarized carriers are usually injected from ferromagnetic films into the semiconductor. Depending on the experiment setup, the probe laser can be focused on the top surface of the semiconductor (left side of Fig. 105] [5, 30, 31, 527, 910, 1025, 1027] or on the side-surface (right side of Fig. 105] [1026]. Since the easy axis of the ferromagnetic film is usually parallel to the film plane, an in-plane magnetic field is required to rotate the injected spin to the normal of the surface [30, 31, 527, 910, 1025, 1027]. In the side surface scanning experiments, the easy axis of the ferromagnetic film can be arranged to be parallel to the probe laser and no magnetic field is required [1026]. The spin polarized electrons in ferromagnetic electrode are driven through interface into the semiconductors at low temperature. The polarization of the injected spin is estimated to be a few percents. Spin transport over macroscopic distance $(>1 \mu \mathrm{m})$ has been clearly demonstrated by these experiments $[30,31,527,910,1025-1027]$. The spin image technique has also been adopted to study the spin accumulations caused by the extrinsic spin Hall effect [7-10, 1028-1036].

\subsubsection{Electrical spin detection}

There are also many works on all electrical spin injection/transport/detection. The simplest electrical spin detection method is to utilize the magnetoresistive effect by driving the spin polarized carriers from one ferromagnetic electrode on one side across the interface to semiconductor and extract the spin using another ferromagnetic electrode on the other side as the Datta-Das transistor. Due to spin-valve effect, the resistances should be different when the spin momentum of left electrode is parallel and anti-parallel to that of the right electrode if the spin polarization injected from the left electrode survives at the right electrode. This effect is quantitatively characterized by magnetoresistance, the relative change of the resistance when the magnetic momentums change from parallel to anti-parallel alignments. Early works using this simple ferromagnetic/nonmagnetic/ferromagnetic sandwich structure indeed showed that the resistance does have the hysteresis loop when an magnetic field is applied to change the direction of the magnetic momentum on the right electrode [969, 1037, 1038]. Due to conductance mismatch, magnetoresistance of the ferromagnetic/semiconductor/ferromagnetic structure is not very large, usually less than $1 \%$ for ohmic ferromagnetic metal/semiconductor contact [969, 1037, 1038]. For the system suffered less from the conductance mismatch, magnetoresistance of $8.2 \%$ in $\mathrm{MnAs} / \mathrm{GaAs} / \mathrm{Ga}: \mathrm{MnAs}$ structure has been archived at low temperature [1039]. However, since magnetoresistance in this structure is usually low and the spin polarized current flows through ferromagnetic 

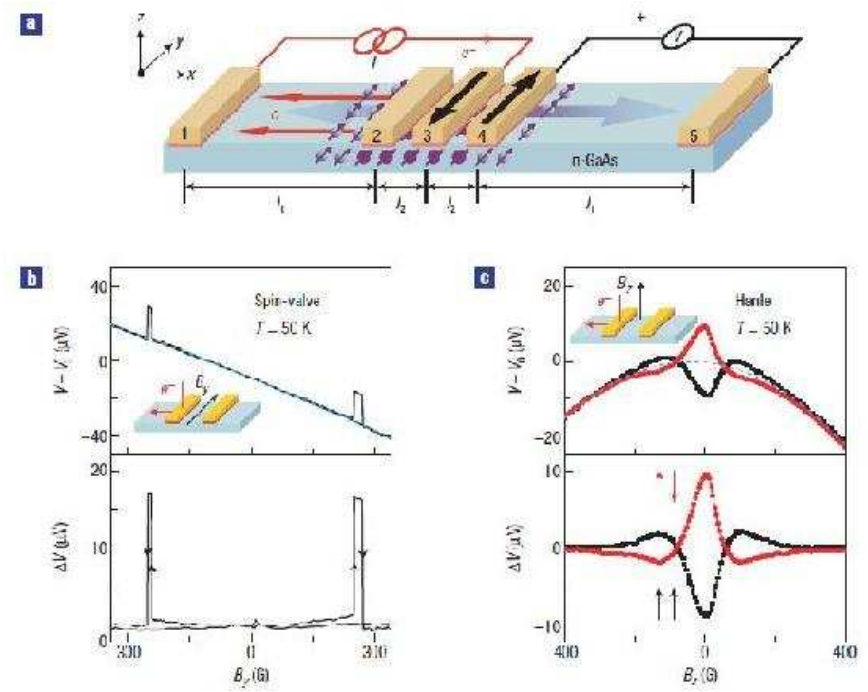

Figure 106: (a) A schematic diagram of the non-local experiment (not to scale). The five contacts have magnetic easy axes along $y$-axis. The large arrows indicate the magnetizations of the source and detector. Electrons are injected along the path shown in red. The injected spins (purple) diffuse in either direction from contact 3. The non-local voltage is detected at contact 4 . (b) Non-local voltage, $V_{4,5}$, versus in-plane magnetic field, $B_{y}$, (swept in both directions) for sample A at a current $I_{1,3}=1.0 \mathrm{~mA}$ at $\mathrm{T}=50 \mathrm{~K}$. The raw data are shown in the upper panel, the lower panel shows the data with this background subtracted. (c) Non-local voltage, $V_{4,5}$, versus perpendicular magnetic field, $B_{z}$, for the same contacts and bias conditions as in (b). The data in the lower panel have the background subtracted. The data shown in black are obtained with the magnetizations of contacts 3 and 4 parallel, and the data shown in red are obtained in the antiparallel configuration. From Lou et al. [944].

electrode, the spin accumulation signal can be masked by other magnetoresistance effects such as the anisotropic magnetoresistance of the magnetic electrode and local Hall effect caused by the fringe magnetic field near the contact [1040-1044]. These direct measurements of spin valve effect in the sandwich structure are not decisive.

A more sophisticated all electrical spin detection is to use non-local spin-valve effect [281, 940-946], first proposed by Johnson based on the Silsbee-Johnson spin charge coupling [905, 938, 940, 941]. The schematic of non-local spin-valve setup used in the experiment by Lou et al. is represented in Fig. 106 In the experiment different contacts are grown on top of a GaAs layer. The spin polarized electrons are driven to form the spin polarized current flow between contacts 1 and 3, while non-local voltage between contacts 4 and 5 is measured. Due to Silsbee-Johnson spin charge coupling, if there is a spin accumulation at the interface of GaAs layer and contact 4, a change in the non-local voltage can be detected when the direction of magnetization in contact 4 changes. This non-local geometry separates the injection and detection paths and thus avoids or reduces the spurious effects caused by the other magnetoresistance effects. Using this technique, clearer signals of spin accumulation were demonstrated through hysteresis loop behavior in the resistance change with the magnetic field [281, 942 946, 1045]. More recently, Koo et al. employed this method to demonstrate the oscillatory channel conductance due to gate voltage controlled spin precession [55]. This experiment is of particular interest since it is claimed to be the first experimental realization of electric spin injection, spin detection and coherent spin manipulation in one device, although there are some controversies on the claim $[916-918]$.

\subsubsection{Spin transport in silicon}

Spin related phenomenons in silicon has also attracted much attention recently because of the long spin life time in silicon and the compatibility to the current industrial semiconductor technologies $[149,150,279,280,282,290,291$, 295, 776, 781, 1046-1058]. A major difficulty for studying spin related properties in silicon is the spin generation and detection. Optical spin generation/detection is not effective since silicon is an indirect-band semiconductor [21, 1046]. Early studies on the Faraday/Kerr effects in silicon were limited in very narrow frequency ranges in microwave or infrared region [1059-1061]. Although the limitation was lifted by using terahertz time domain spectroscopy [1062, 1063 , which covers a wider frequency range, spin detection using the Faraday/Kerr rotation is yet to be conducted. There are a few experiments using electroluminescence to obtain the information of the spin polarization in silicon 


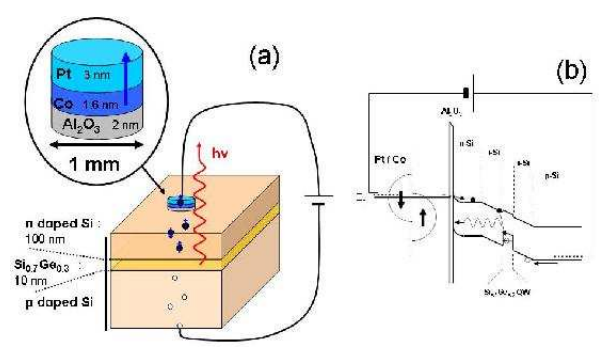

Figure 107: (a) Schematic device structure of the SiGe spin light-emitting diode with the $\mathrm{Co} / \mathrm{Pt}$ ferromagnetic top electrode and (b) associated simplified band diagram under bias in the injection regime. From Grenet et al. [279].
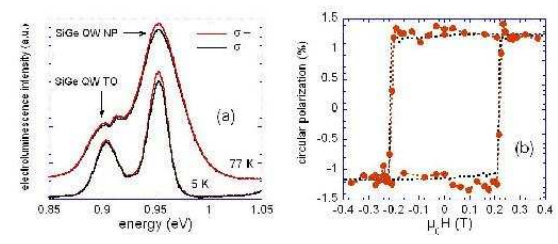

Figure 108: (a) Electroluminescence spectra recorded at $5 \mathrm{~K}$ and $77 \mathrm{~K}$ for a saturated remanent state of the ferromagnetic $\mathrm{Co} / \mathrm{Pt}$ contact. The applied voltage and current are respectively around $10 \mathrm{~V}$ and $10 \mathrm{~mA}$. The no-phonon (NP) peak is clearly circularly polarized indicating an efficient spin injection into the silicon top layer. The transverse optical (TO) phonon replica is also slightly circularly polarized. (b) Electroluminescence circular polarization recorded at the maximum of the non-phonon line for different remanent states of the $\mathrm{Co} / \mathrm{Pt}$ layer (red dots) From Grenet et al. [279].

[279, 282]. By constructing surface-emitting light emitting diodes with n-i-p silicon layers, Jonker et al. demonstrated the electrical spin injection from $\mathrm{Fe}$ through an $\mathrm{Al}_{2} \mathrm{O}_{3}$ layer into $n$-type silicon. The injected spin polarization under $3 \mathrm{~T}$ magnetic field was estimated to be $30 \%$ at $5 \mathrm{~K}$, with significant spin polarization extending to at least $125 \mathrm{~K}$. [282]. Using a fully strained $\mathrm{Si}_{0.7} \mathrm{Ge}_{0.3}$ quantum well to replace the intrinsic silicon layer, Grenet et al. demonstrated the electrical spin injection from CoFe into $n$-type silicon at zero magnetic field [279]. Their experiment setup and results are shown in Figs. 107 and 108 The advantage of the strained SiGe quantum well over bulk silicon is the appearance of the direct optical transition through the so called non-phonon transition [1064-1066]. Due to the nonphonon transition and its phonon replica [1066], radiative recombination in SiGe quantum well is much faster than that in silicon. The radiative relaxation time in SiGe quantum well is about tens of nanoseconds [279] compared to hundreds of microseconds in silicon [282, 1067, 1068]. Moreover the presence of germanium and stress also enhances the spin-orbit coupling and raises the circular polarization of the emitted light from the recombination of the spin polarized electrons with holes [1069-1071]. These factors improve the efficiency of the electroluminescence. Using this structure to detect the electrical spin injection from CoFe into silicon, as shown in Fig. 108, circular polarization of $3 \%$ was achieved at $5 \mathrm{~K}$ and remained almost constant up to $200 \mathrm{~K}$ at zero magnetic field [279].

Most spin related experiments in silicon use electrical injection/detection [149, 150, 280, 1048-1058, 1072]. Robust electric spin injection through a hot-electron spin valve [149, 150, 279, 280, 282, 1048-1054] or Schottky tunneling barrier contact [281, 1058, 1073, 1074] into silicon has been proposed and realized. The schematic electronic band diagram and experimental setup of spin injection and detection using the hot-electron spin valve are shown in Fig. 109. Driven by the voltage $V_{E}$, unpolarized electrons are injected from nonmagnetic emitter (Al electrode in the original experiment) into ferromagnetic base (FM1) through a tunneling barrier. FM1 base acts as a spin filter in which electrons with majority spin pass through the Schottky barrier into silicon ballistically, but electrons with minority spin can not pass as they quickly lose energy in the FM1 base due to the spin selective scattering. Under the voltage $V_{c 1}$, electrons transport inside the silicon and arrive at the second ferromagnetic layer (FM2). FM2 serves as spin detector and makes the current $I_{c 2}$ depend on the current spin polarization by allowing only electrons with majority spin of FM2 pass through into the second collector. The alignment of magnetic momentum of FM1 and FM2 can be changed by an in-plane magnetic field. The success of spin injection is demonstrated by spin valve effect and Hanle effect, shown in Fig. 110 Spin valve effect, that is the change in the spin momentum alignments of FM1 and FM2 results in the change in $I_{c 2}$, is quantitatively described by magnetocurrent, $\left(I_{c 2}^{P}-I_{c 2}^{A P}\right) / I_{c 2}^{A P}$, the relative change in the current when the alignment of magnetizations in FM1 and FM2 changes. 2\% magnetocurrent was observed in the original experiment [150]. By relocating the ferromagnetic bases away from silicon interfaces to eliminate the "magnetically-dead" silicide layer [1075-1077], the magnitude of magnetocurrent was substantially increased by more than one order of magnitude [280, 1050]. To further confirm that the change in $I_{c 2}$ is indeed caused by spin valve effect instead of other spurious effects, non-local spin detection was also used [281]. More conclusive results showing the spin precession and spin dephasing were obtained: A perpendicular magnetic field, which rotates the 
injected spin, changes the relative alignment of the injected spin and spin in FM2. As a result $I_{c 2}$ oscillates with the strength of the perpendicular magnetic field [149, 150, 280-282, 1048-1054].
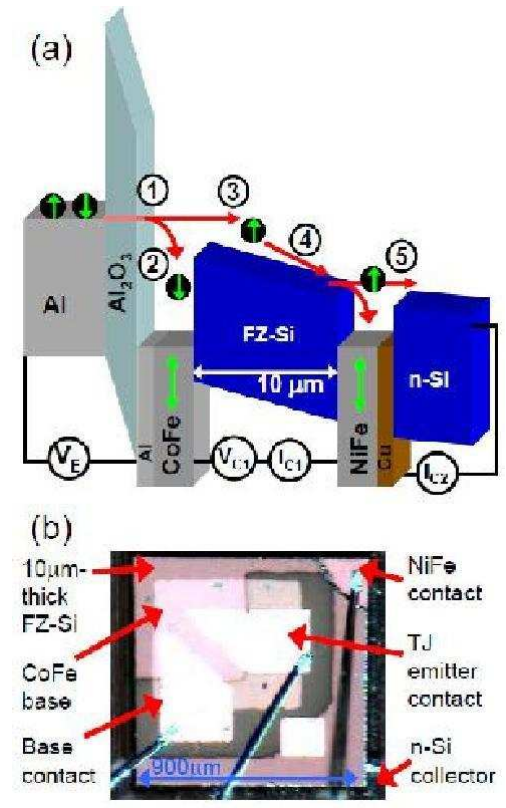

Figure 109: Schematic electronic band diagram (a) and experimental setup (b) of the hot-electron spin valve. From Appelbaum et al. [150].
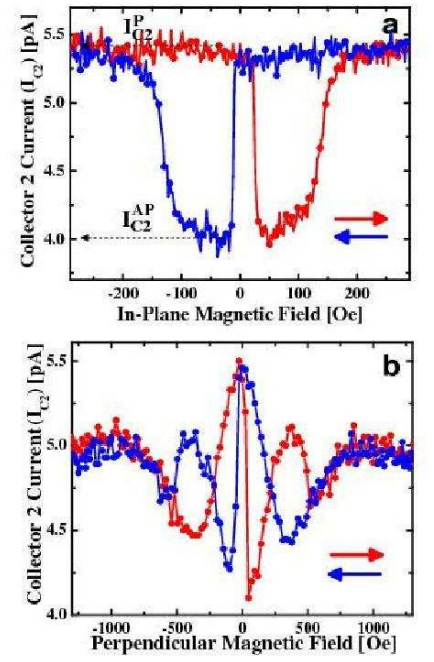

Figure 110: (a) In-plane spin-valve effect for the silicon spin transport device with emitter tunnel junction bias $V_{E}=-1.6 \mathrm{~V}$ and $V_{C 1}=0 \mathrm{~V}$ at $85 \mathrm{~K}$. (b) Spin precession and dephasing (Hanle effect), measured by applying a perpendicular magnetic field. From Huang et al. [1050].

Due to the spin relaxation and the interference of the spin precession around the magnetic field [25, 336, 846, 1052], the injected spin polarization decays with the distance. The spin injection length decreases with the increase of the magnetic field, and was shown to become very small when the magnetic field becomes slightly strong [150]. Since $I_{c 2}$ is proportional to the spin polarization at FM2, $\mathrm{I}_{c 2}$ also decreases with the magnetic field. This decay, first predicted by Weng and Wu [336] fully microscopically, was explained by the Hanle effect in Ref. [150].

\section{Microscopic theory of spin transport}

In Section 6 , the phenomenal theory of spin transport based on the drift-diffusion model is reviewed. In that model, all characteristic parameters such as the mobility $\mu$, diffusion coefficient $D$ in spin transport, and spin relaxation time $\tau_{s}$ can not be determined within the framework of model, but rather need to be experimentally measured or calculated from other theoretical model. It is usually assumed that $\mu$ and $D$ are simply the charge mobility and diffusion coefficient. However, there is no reason that these assumptions should be universally true aside from the naïve intuition, and the relations between the characteristic parameters of charge transport and spin transport remain yet to be revealed. In the presence of the spin-orbit coupling, the charge transport and spin transport are coupled. A fully microscopic theory is essential to understand this coupled transport. In this section, we will review spin transport inside the semiconductor from the fully microscopic point of view. The main results reviewed in this section are also based on the kinetic spin Bloch equation approach. The equations have been laid out in Sec. 5, but in Sec. 5 we focuses on reviewing the results of the spacial uniform system. The results reviewed in this section are focused on the spacial nonuniform system. There are several related researches based on the linear response theory [35, 1078] and kinetic theory 93 [1079-1086], whose results will also be briefly addressed.

\footnotetext{
${ }^{93}$ Equations of this theory are the same as the kinetic spin Bloch equations but without electron-electron Coulomb interaction.
} 


\subsection{Kinetic spin Bloch equations with spacial gradient}

The kinetic spin Bloch equations for the spin kinetics in the presence of spacial inhomogeneity are given by Eqs. (155 162). The electric field $\mathbf{E}$ in these equations is determined from the Poisson equation [Eq. [273] ]. As pointed out in Sec. 5.3, the kinetic spin Bloch equations include all the factors important to the spin dynamics, including the drifting driven by an electric field, diffusion due to the spacial inhomogeneity, spin precession around the total magnetic field as well as all the relevant scattering. By solving these equations, all the measurable quantities, such as mobility, charge diffusion length, spin diffusion length, can be obtained self-consistently without any fitting parameter.

More importantly, from this fully microscopic approach, some important issues overlooked by the phenomenological drift-diffusion model are recovered. Weng and $\mathrm{Wu}$ [336] performed the first fully microscopic investigation on spin diffusion and transport by setting up and solving the kinetic spin Bloch equations. Unlike the previous spin transport theories [907, 908, 927- 929, 1087-1089] where only the diagonal elements of the density matrix $\rho_{\sigma \sigma}(\mathbf{r}, \mathbf{k}, t)$ are included in the theory, they showed that it is of crucial importance to include the off-diagonal terms $\rho_{\sigma-\sigma}(\mathbf{r}, \mathbf{k}, t)$ in studying the spin diffusion and transport [25, 336]. They predicted spin oscillations in GaAs quantum well along the spin diffusion in the absence of any applied magnetic field [24, 25]. These oscillations were later observed in experiments [31, 567]. The spin oscillations without any applied magnetic field are beyond the two-current drift-diffusion model widely used in the spin transport study [22, 907, 908, 927-929, 1087-1089]. Moreover, by introducing the off-diagonal terms, Weng and $\mathrm{Wu}$ showed that there is an additional inhomogeneous broadening associated with the spacial gradient of the spin polarization [25, 28, 336]. As shown in Eq. [157), the coefficient of the spacial gradient is proportional to $\nabla_{\mathbf{k}} \bar{\varepsilon}_{\mathbf{k}}(\mathbf{r}, t)$, which is momentum dependent. It does not lead to any significant results for charge transport aside from the diffusion in space. However, when there is spin precession this momentum-dependent coefficient introduces an additional inhomogeneous broadening since the spacial spin precession is momentum dependent $[25,28,336]$. This inhomogeneous broadening, combined with any spin-conserving scattering, leads to an irreversible spin relaxation and dephasing in the spin transport.

In the following subsections, we will first present some simplified cases where analytical results can be obtained. Then we review the numerical results from the kinetic spin Bloch equations.

\subsection{Longitudinal spin decoherence in spin diffusion}

The importance of the off-diagonal terms of the density matrix in the study of spin transport can be qualitatively understood by studying the spin diffusion in GaAs quantum well in a much simplified case where there is no electric field, no scattering and no Hartree-Fock self-energy. Assuming that the transport is along the $x$-direction, in the steady state, the kinetic spin Bloch equations [Eqs. [155, [162)] can be simplified as

$$
\frac{k_{x}}{m^{*}} \frac{\partial \rho(x, \mathbf{k}, t)}{\partial x}+i\left[\mathbf{h}(\mathbf{k}) \cdot \frac{\boldsymbol{\sigma}}{2}, \rho(x, \mathbf{k}, t)\right]=0 .
$$

In order to avoid the complexity of the spin injection, it is assumed that the electron at $x=0$ is polarized along the $z$-axis, and the boundary conditions are written as

$$
\rho(0, \mathbf{k})=\left(\begin{array}{cc}
f_{\uparrow}^{0}(\mathbf{k}) & 0 \\
0 & f_{\downarrow}^{0}(\mathbf{k})
\end{array}\right),
$$

where $f_{\sigma}(0, \mathbf{k})=f_{\sigma}^{0}(\mathbf{k})=\left\{\exp \left[\left(\varepsilon_{k}-\mu_{\sigma}\right) / T\right]+1\right\}^{-1}$ is the Fermi distribution function with chemical potential $\mu_{\sigma}$. The solution to this equation in the steady state reads [28]

$$
\rho(x, \mathbf{k})=e^{-i\left(m^{*} / 2 k_{x}\right) \mathbf{h}(\mathbf{k}) \cdot \boldsymbol{\sigma} x} \rho(0, \mathbf{k}) e^{i\left(m^{*} / 2 k_{x}\right) \mathbf{h}(\mathbf{k}) \cdot \boldsymbol{\sigma} x} .
$$

This equation describes the spacial spin precession around the total magnetic field $\mathbf{h}(\mathbf{k})$. For electron with momentum $\mathbf{k}$, the precession period is characterized by

$$
\omega(\mathbf{k})=\mathbf{h}(\mathbf{k}) m^{*} / k_{x} .
$$

$\mathbf{h}(\mathbf{k})=g \mu_{B} \mathbf{B}+\boldsymbol{\Omega}(\mathbf{k})$ with $\boldsymbol{\Omega}(\mathbf{k})$ being the D'yakonov-Perel' term. Eq. (299) clearly shows the effect of the inhomogeneous broadening in the spacial spin precession on the spin transport. For each electron moving along the $x$-direction 
with fixed velocity $v_{k_{x}}=k_{x} / m^{*}$, its spin precesses with fixed spacial period without any decay. However, electrons with different momentums have different spacial precession periods and can have different precession axes if the Dresselhaus and/or Rashba terms are present. As a result, spins of different electrons eventually become out of phase and cancel each other as they moving along the transport direction. Due to this interference among electrons of different $\mathbf{k}$, the total spin momentum decays along the diffusion distance. Comparing with the spin kinetics in the time domain, where the inhomogeneous broadening is determined by inhomogeneity in the precession frequencies and direction determined by $\mathbf{h}(\mathbf{k})$ [44, 332, 334, 363, 372], in spin transport it is determined by the inhomogeneous in the spacial oscillation "frequency" and direction given by $\omega(\mathbf{k})$ in Eq. (300) [25, 28, 336]. Even for a uniform magnetic field in the Voigt configuration, which does not induce any inhomogeneous broadening in the time domain, the spacial precession "frequencies" $\omega(\mathbf{k})$ are still different for different electrons. Therefore, a spacial uniform magnetic field alone can provide an inhomogeneous broadening in the spin transport as first pointed out by Weng and Wu back to 2002 [25, 336]. This effect is illustrated in Fig. 111 where the electron densities $N_{\sigma}$ in a GaAs quantum well subject to a uniform in-plane magnetic field are plotted as functions of position $x$. In order to demonstrate the effect of this new inhomogeneous broadening, the spin-orbit coupling is assumed to be zero. One can clearly see the decay in spin polarization due to the inhomogeneous broadening caused by the difference in spacial precession periods. The stronger the magnetic field is, the quicker the spin polarization decays [25, 336]. It is noted that although the inhomogeneous broadening alone can reduce the total spin momentum, it is just an interference effect. There is no irreversible loss of spin coherence since there is no scattering (hence no true dissipation) in system. This can be seen from the fact that the incoherently summed spin coherence $\rho$, also plotted in Fig. 111 does not decay with the position. It should be pointed out that this additional spin decoherence is beyond the Hanle effect since for the latter the spin signal does not decay when there is no scattering according to Eq. (282).

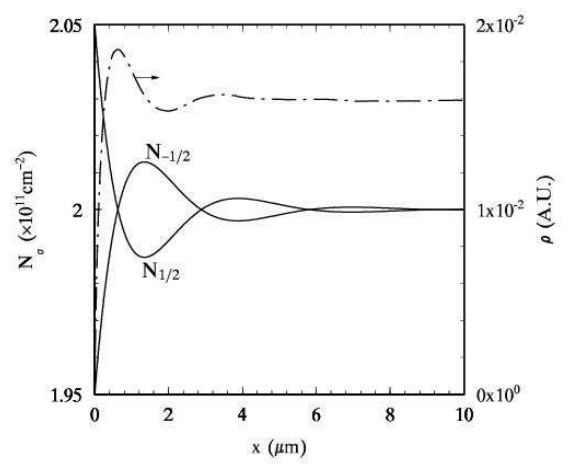

Figure 111: Electron densities of up spin and down spin (solid curves) and incoherently summed spin coherence $\rho$ (dashed curve) versus the diffusion length $x$ in $n$-type GaAs quantum well without spin-orbit coupling but with $B=1 \mathrm{~T}$. Note the scale of the spin coherence is on the right side of the figure. From Weng and Wu [336].

When the scattering is turned on, it provides a channel which combined with the inhomogeneous broadening leads to irreversible spin dephasing [44, 332, 334, 350, 569, 844]. As a result both spin polarization and spin coherence decay with the distance [24, 25, 27, 28, 336, 846]. Similar to the spin evolution in the time domain, the scattering also has counter effect that suppresses the inhomogeneous broadening in $\omega(\mathbf{k})$ given by Eq. (300) [225, 226, 334, 837840]. The spin dephasing/relaxation mechanism in spin transport has been realized experimentally by Appelbaum et al. [149, 150, 280, 1048-1053] in bulk silicon, where there is no D'yakonov-Perel' spin-orbit coupling due to the center inversion symmetry. For spin transport in bulk silicon in the presence of a magnetic field, the decay of the spin polarization should be mainly caused by the above mentioned inhomogeneous broadening if other spin-relaxation mechanisms are ignored. Moreover, the inhomogeneous broadening in this situation is particularly simple since all electrons have same precession axis, the only difference is the magnitude of the spacial precession frequencies $\omega_{\mathbf{k}}$ given by Eq. (300). Therefore bulk silicon provides an ideal platform to study this spin relaxation/decoherence due to the additional inhomogeneous broadening. As shown in Fig. 110 indeed the spin injection length decreases with the magnetic field, consistent with the theoretical prediction [25, 28, 32, 846].

Although the full kinetic spin Bloch equations with scattering are complicated, one can still obtain some analytical 
results under some approximations $[25,28,32,846,915]$. Assuming that there are no applied electric field, inelastic scattering and the Hartree-Fock term, the kinetic spin Bloch equations for spin transport along the $x$-axis can be written as [25, 28, 32, 846]

$$
\frac{\partial \rho^{l}(x, k, t)}{\partial t}+\frac{v_{k}}{2} \frac{\partial}{\partial x}\left[\rho^{l+1}(x, k, t)+\rho_{k}^{l-1}(x, k, t)\right]=-i \sum_{m}\left[\mathbf{h}^{l-m}(k) \cdot \boldsymbol{\sigma} / 2, \rho^{m}(x, k, t)\right]-\rho^{l}(x, k, t) / \tau_{k}^{l},
$$

where $v_{k}=k / m^{*}$ is the velocity of the electron with momentum $\mathbf{k} . \rho^{l}(x, k, t)$ and $\mathbf{h}^{l}(k)$ are the $l$-th order of the Fourier components of $\rho(x, \mathbf{k}, t)$ and $\mathbf{h}(\mathbf{k})$ with respect to the angle of $\mathbf{k}$ and the $x$-axis, respectively. $\tau_{k}^{l}$ is the $l$-th order momentum relaxation time due to the electron-impurity scattering. Noted that $1 / \tau_{k}^{0}=0$ and $\tau_{k}^{l}=\tau_{k}^{-l}$.

In the diffusive limit where the momentum relaxation time is small, the higher order components $\rho^{l}(x, k, t)$ with $|l|>1$ are small and can be neglected. If there is no spin-orbit coupling and the applied magnetic field is along the $y$-axis, by neglecting higher order Fourier components with $|l|>1$, in the steady state the kinetic spin Bloch equations can be simplified as [846]

$$
\frac{\partial^{2} \rho^{0}(x, k)}{\partial x^{2}}=-2\left(\frac{\omega}{2 v_{k}}\right)^{2}\left[\sigma_{y},\left[\sigma_{y}, \rho^{0}(x, k)\right]\right]+i \frac{\omega}{v_{k}^{2} \tau_{k}^{1}}\left[\sigma_{y}, \rho^{0}(x, k)\right],
$$

where $\omega=g \mu_{B} B$ is spin precession frequency in time domain under the uniform magnetic field $\mathbf{B}$. In term of the spin momentum, the solution to this equation for the spin injection at $x=0$ is a damped oscillation

$$
S_{z}(x, k)=S_{z}(0, k) \cos \left(\frac{x}{v_{k} \tau_{k}^{1} \Delta}\right) e^{-x \omega \Delta / v_{k}}
$$

with

$$
\Delta=\left(\sqrt{1+\frac{1}{\left(\omega \tau_{k}^{1}\right)^{2}}}-1\right)^{-1 / 2} .
$$

At low temperature, when the injected spin only polarized at the Fermi level, the spin injection length $L_{d}^{-1}=$ $\omega \Delta / v_{f}$, with $v_{f}$ standing for the Fermi velocity. The injection length obtained here decreases monotonely with the increase of magnetic field and scattering strength. Therefore, the scattering enhances the spin dephasing/relaxation in spin diffusion. The counter effect of the electron-impurity scattering on the inhomogeneous broadening here is not significant [846].

\subsubsection{Spin diffusion in Si/SiGe quantum wells}

In order to gain a deeper insight into the spin relaxation along the spin diffusion caused by the additional inhomogeneous broadening addressed in the previous subsection, Zhang and $\mathrm{Wu}$ studied the spin transport in a symmetric silicon quantum well with even number of monoatomic silicon layers through the kinetic spin Bloch equation approach [846]. There is no D'yakonov-Perel' spin-orbital coupling in this kind of quantum well [291]. Since the Rashba term is very small in the asymmetric $\mathrm{Si} / \mathrm{SiGe}$ quantum well [124, 290, 291, 295, 776, 781], it was argued that their results also hold for the asymmetric Si/SiGe quantum wells as long as the D'yakonov-Perel' term is weak enough [846].

The kinetic spin Bloch equations in (001) Si/SiGe quantum well are similar to those in GaAs quantum well [846]. In the equations the drifting under the electric field, diffusion due to the spacial inhomogeneity and precession around the magnetic field (including the contribution of the Hartree-Fock term), as well as the spin-conserving scattering are all addressed. Numerical solutions of full kinetic spin Bloch equations for spin transport in silicon quantum well at $T=80 \mathrm{~K}$ with different scattering mechanisms and under different magnetic fields are shown in Figs. [112] and 113

Figure 112 clearly shows that the spin injection length decreases with increasing magnetic field, in accordance with the prediction by Weng and $\mathrm{Wu}[25,336]$. This result is easy to understand since $\omega_{\mathbf{k}}=m^{*} g \mu_{B} \mathbf{B} / k_{x}$ is proportional to the magnetic field. Increase of the applied magnetic field results in the increase of inhomogeneous broadening.

Figure 113 shows the effect of scattering on the spin diffusion. It is seen from the figure that adding any new scattering leads to a shorter spin diffusion length. This is quite different to the results in the system with the D'yakonov-Perel' spin-orbit coupling. When the D'yakonov-Perel' spin-orbit coupling is present, the scattering not only opens a spin 
dephasing/relaxation channel, but also has a counter effect on the inhomogeneous broadening [25, 28, 29, 363, 1090]. In the strong scattering limit, adding a new scattering suppresses the inhomogeneous broadening and prolongs the spin diffusion length [25, 28]. However, for the system without the D'yakonov-Perel' spin-orbit coupling, the counter effect of the scattering on the inhomogeneous broadening is marginal. The scattering affects the spin diffusion only through the momentum relaxation time and hence only reduces the spin injection length as shown by Eqs. (303) and (304) [846].

The electron density dependence of the spin diffusion was also investigated at different temperatures. It was found that at relatively high temperature or low density, when the electrons are nondegenerate, the spin diffusion length and spacial precession period are insensitive to the electron density. At low temperate or high density when the electrons are degenerate, the spin diffusion length and spacial precession period increase with increasing density. This is understood from the Fermi wavevector dependence of the damping rate and precession period as shown in Eqs. (303) and (304).

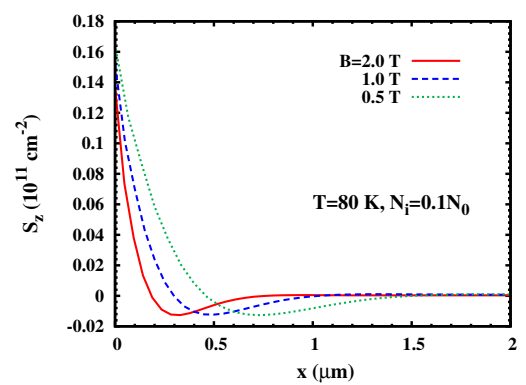

Figure 112: Spin polarization $S_{z} v s$. position $x$ in the steady state for intrinsic silicon quantum well under different magnetic field strengths. Solid curve: $B=2 \mathrm{~T}$; Dashed curve: $B=1 \mathrm{~T}$; Dotted curve: $B=0.5$ T. $T=80 \mathrm{~K}$ and $N_{i}=0.1 N_{0}$. From Zhang and $\mathrm{Wu}$ [846].

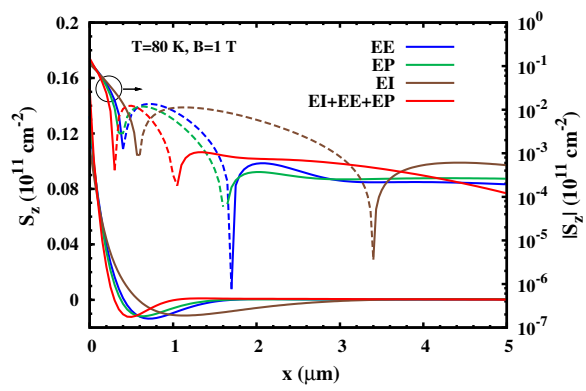

Figure 113: The steady-state spacial distributions of spin signal $S_{z}$ in silicon quantum well with different scatterings included. The curves labeled with "EE", "EP" or "EI" stand for the calculations with the electron-electron, electron-phonon or electronimpurity scattering, respectively, while the curve labeled with "EI+EE+EP" stands for the calculation with all the scatterings. In order to get a clear view of the decay and precession of $S_{z}$, the corresponding absolute value of $S_{z}$ against $x$ is also plotted on a log-scale (Note the scale is on the right hand of the frame). The dashed curves correspond to the part with $S_{z}<0$. From Zhang and $\mathrm{Wu}[846]$.

\subsection{Spin oscillations in the absence of magnetic field along the diffusion}

When the D'yakonov-Perel' term is present, the problem becomes more complicated since in this case both the spacial precession frequencies and axis are momentum dependent. Studies of spin transport in the system with the spin-orbit coupling from the kinetic spin Bloch equation approach have predicted many novel results [25, 27, 28, 32, 336, 846]. Some of them have been verified experimentally [31, 567].

One interesting result is the spin oscillation in the absence of any applied magnetic field along the spin diffusion when the Dresselhaus and/or Rashba terms are present. In the spacial homogeneous system, spin oscillation without any applied magnetic field can only be observed in time domain at very low temperature $(T<2 \mathrm{~K})$, i.e., in the weak scattering limit [368]. In high temperature regime where scattering is strong enough, the Dresselhaus and/or Rashba terms result in a monotonically decay of spin polarization versus time in the spacial uniform system. If one adopts simple relaxation approximation to describe the effect of the spin-orbit coupling, one should not expect any spin oscillation in the diffusive limit where the scattering is strong enough. Indeed, from the drift-diffusion equation Eq. (275), there is no spin oscillation either in spin injection in the steady state or in transient spin transport if the applied magnetic field is absent.

However according to the kinetic spin Bloch equation approach, in the spin transport the spacial spin precession is characterized by $\omega_{\mathbf{k}}$ [Eq. (300)]. If one only considers the Dresselhaus effective magnetic field, the average of $\omega_{\mathbf{k}}$ is $\left\langle\omega_{\mathbf{k}}\right\rangle=m^{*} \gamma\left(\left\langle k_{y}^{2}\right\rangle-\left\langle k_{z}^{2}\right\rangle, 0,0\right)$. For electrons in quantum well, this value is not zero. Therefore, the spacial spin 

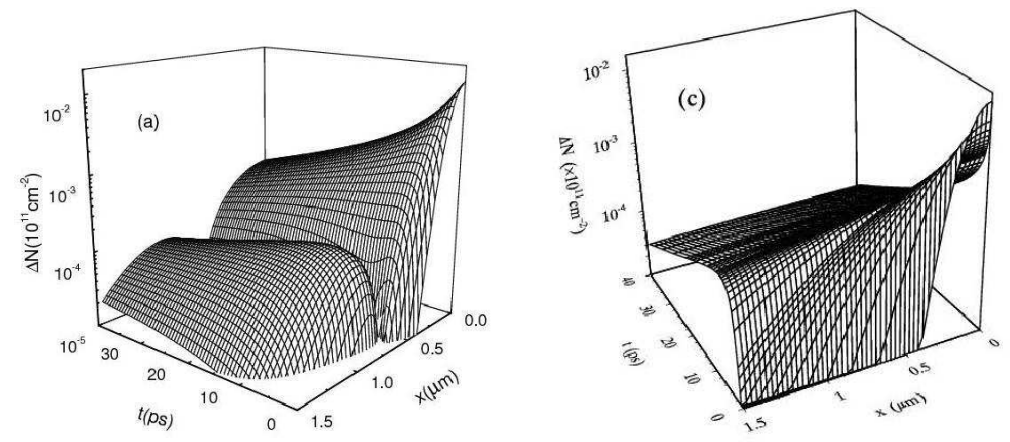

Figure 114: Theoretical predictions of the absolute value of the spin imbalance $|\Delta N|$ vs. the position $x$ and the time $t$ for a spin pulse in $n$-type GaAs (001) quantum well based on the kinetic spin Bloch equation approach (left) and drift-diffusion model (right). The initial pulse width $\delta x=0.15 \mu$ m. From Weng and $\mathrm{Wu}[25]$.

oscillation due to the Dresselhaus effective magnetic field survives even at high temperature when the scattering is strong enough.

By solving the kinetic spin Bloch equations, the spin oscillation without any applied magnetic field was first predicted in the transient diffusion of a spin pulse in GaAs quantum wells [24, 25, 27]. A typical time evolution of spin pulse is shown in Fig. 114 where the absolute value of the spin imbalance $\left|\Delta N_{\sigma}(x, t)\right|$ is plotted as a function of the position $x$ along the diffusion direction and time $t$. The initial state is a spin pulse of Gaussian spacial profile, $\Delta N(x, 0)=\Delta N_{0} \exp \left(-x^{2} / \delta x^{2}\right)$ with $\delta x=0.15 \mu \mathrm{m}$. For comparison, the result based on the drift-diffusion model is also shown. One can see that the kinetic spin Bloch equation approach and the drift-diffusion model give qualitatively similar results for the evolution at the center of the pulse, where it decays monotonically with time as a result of spin diffusion and spin dephasing. However, the results from these two theories are quite different for the evolution outside the original spin pulse. The result from the drift-diffusion model shows that spin polarization at large $x$ first increases with time and then decays to zero. The sign of the spin polarization does not change throughout the space and time. On the contrary, the kinetic spin Bloch equation theory predicts that spin polarization oscillates with time. The oscillation is the combined result of the spin precession caused by the Dresselhaus effective magnetic field and the spin diffusion.
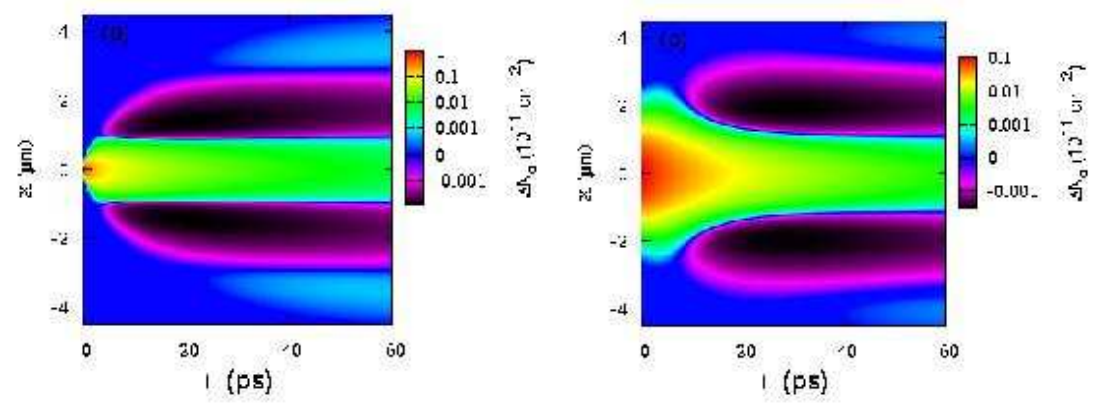

Figure 115: The contour plots of spin imbalance $\Delta N$ vs. the position $x$ and the time $t$ for different pulse widths in $n$-type GaAs (001) quantum well. $(a): \delta x=0.1 \mu \mathrm{m} ;(b): \delta x=1.0 \mu \mathrm{m} . T=200 \mathrm{~K}$. From Jiang et al. [27].

The evolutions of spin pulse with different pulse widths are shown in Fig. 115. Since the oscillation is a result of diffusion and spin precession around the effective magnetic field, the system with narrower spin pulse shows stronger spin oscillation in the transient spin transport. For the narrower pulse, the peak of the reversed spin polarization appears in shorter time. Moreover, more oscillation can be observed with narrower pulse [27].

The effects of the scattering and the electric field on the oscillation without any magnetic field were also studied. 
It was shown that the oscillation is robust against the scattering. Adding the electron-impurity scattering slows down the diffusion but does not eliminate the oscillation. In some regime, the electron-impurity scattering even boosts the oscillation as it helps to sustain the spin coherence [24, 27]. The Coulomb scattering has a similar effect on the spin diffusion and oscillation [27]. Without any electric field, the spin signals diffuse symmetrically in the space around the original pulse center. When an electric field is applied along the diffusion direction, the diffusion is no longer symmetrical. The pulse is dragged against the electric field and stronger opposite spin polarization appears at the side against the electric field [27].

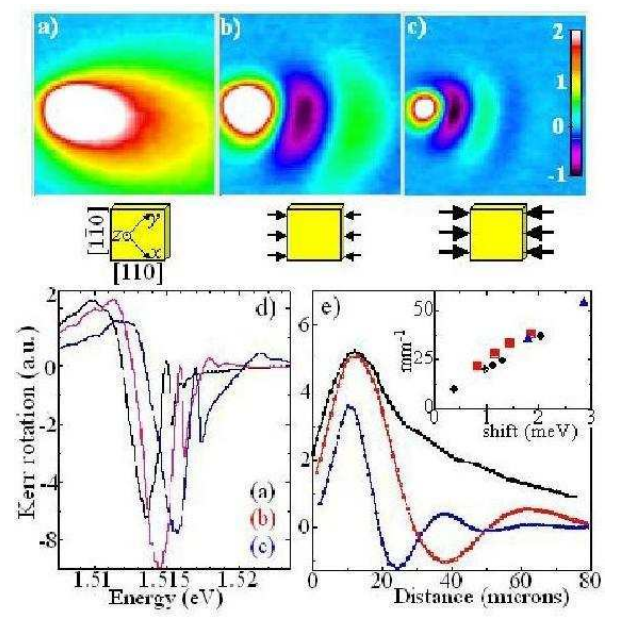

Figure 116: Experimental result of spin precession in bulk GaAs without magnetic field. (a)-(c) images of two-dimensional spin flow (E=10 V/cm) at $4 \mathrm{~K}$, showing induced spin precession with increasing [110] uniaxial stress. (d) Kerr rotation vs. probe photon energy for the images, showing blueshift of GaAs band edge with stress. (e) Line cuts through the images. Inset: Spacial frequency of spin precession vs. band edge shift. From Crooker and Smith [31].

The spin oscillation without any applied magnetic field in the transient spin transport was later observed experimentally by Crooker and Smith in strained bulk system [31], which is shown in Fig. 116. Unlike two-dimensional case, in bulk the average of $\omega_{\mathbf{k}}$ from the Dresselhaus term is zero, since $\left\langle\omega_{\mathbf{k}}\right\rangle=m^{*} \gamma\left(\left\langle k_{y}^{2}\right\rangle-\left\langle k_{z}^{2}\right\rangle, 0,0\right)=0$ due to the symmetry in the $y$ - and $z$-directions. This is consistent with the experimental result that there is no spin oscillation for the system without stress. However, when the stress is applied, an additional spin-orbit coupling, namely the coupling of electron spins to the strain tensor, appears [3, 31, 130, 246, 570, 1091, 1092]. This additional spin-orbit coupling also acts as an effective magnetic field. In the experiment setup, the stress applied is along the [110] axis, the spacial oscillation caused by this additional effective magnetic field is characterized by

$$
\left\langle\omega_{\mathbf{k}}\right\rangle=-c_{3} \epsilon_{y x}(0,1,0),
$$

where $c_{3}$ is a constant depends on the interband deformation potential and $\epsilon_{y x}$ is in-plane shear [3, 31, 130, 246, 1091, 1092]. Therefore, once the stress is applied, one can observe spacial spin oscillation as shown in Fig. 116, even when there is no applied magnetic field.

The spin oscillation without any applied magnetic field was later shown theoretically to survive even in the steady state spin injection [28, 35, 949-954].

The oscillation without an applied magnetic field can also be understood from the simplified kinetic spin Bloch equations Eq. (301). In the diffusive limit, by neglecting high order of the momentum relaxation time, Eq. (301) can be further simplified as

$$
\frac{\partial \mathbf{S}(x, t)}{\partial t}=D \frac{\partial^{2} \mathbf{S}(x, t)}{\partial x^{2}}-\overline{\mathbf{h}} \times \frac{\partial \mathbf{S}(x, t)}{\partial x}-\overleftrightarrow{\Gamma} \mathbf{S}(x, t)
$$

in (001) GaAs quantum well where the dominant spin-orbit coupling is the Dresselhaus term. Here $\mathbf{S}(x, k, t)=$ $\sum_{\mathbf{k}} \operatorname{Tr}\left\{\sigma \rho^{0}(x, k, t)\right\}$ is the spin momentum. $D=\left\langle k^{2} \tau_{k}^{1} / 2 m^{* 2}\right\rangle$ is the diffusion coefficient.

$$
\overline{\mathbf{h}}=\left\langle k^{2} \tau_{k}^{1} / m^{*}\right\rangle(\alpha, 0,0),
$$


is the net Dresselhaus effective magnetic field in the spin diffusion. The last term in Eq. (306) is the spin relaxation caused by the Dresselhaus term together with the spin conserving scattering. The relaxation matrix reads

$$
\overleftrightarrow{\Gamma}=\left(\begin{array}{ccc}
\frac{1}{\tau_{\|}} & 0 & 0 \\
0 & \frac{1}{\tau_{\|}} & 0 \\
0 & 0 & \frac{1}{\tau_{\perp}}
\end{array}\right)
$$

where $1 / \tau_{\|}=\alpha^{2}\left\langle k^{2} \tau_{k}^{1}\right\rangle / 2+\left\langle\left(\gamma k^{3}\right)^{2} \tau_{k}^{3}\right\rangle / 32$ and $1 / \tau_{\perp}=2 / \tau_{\|}$are the spin relaxation times of in-plane and out-of-plane components respectively. Similar equations have also been obtained from linear response theory [35, 1078]. One can clearly see the spin precession around the net effective magnetic field $\overline{\mathbf{h}}$ from Eq. (306). In the transient spin transport, the precession around the effective magnetic field results in spin oscillations in both time and space domains even when there is no applied magnetic field [25, 27, 28, 32, 336]. Moreover, due to the precession, the steady-state spin injection is no longer a simple exponential decay but a damped oscillation [28, 32, 35, 1078].

\subsection{Steady-state spin transport in GaAs quantum well}

Due to spin oscillation, in the steady state the polarization of injected spin in GaAs quantum well is a damped oscillation $\Delta N(x) \propto \exp \left(-x / L_{d}\right) \cos \left(x / L_{0}+\phi\right)$. Here $L_{d}$ and $L_{0}$ stand for the diffusion length and the spacial oscillation period, respectively. How these two parameters change with the scattering mechanism, temperature, applied magnetic or electric field are important to the realization of spintronic devices.

\subsubsection{Effect of the scattering on the spin diffusion}

Similar to the spin kinetics in the spacial uniform system, the scattering also affects the spin transport in complicated ways. On one hand, each scattering mechanism provides additional channel of spin relaxation/dephasing in the presence of inhomogeneous broadening. Adding new scattering mechanism tends to increase spin relaxation/dephasing. However, scattering also affects the charge and spin transport parameters such as diffusion coefficient and mobility. Electron-impurity and electron-phonon scattering can directly change the electron momentum and reduce the spin and charge diffusion coefficients and mobilities. For electron-electron Coulomb scattering, although it does not directly change the charge diffusion coefficient and mobility, it reduces the spin diffusion coefficient through the inhomogeneous broadening $\omega_{\mathbf{k}}$ and the Coulomb drag effect [13-15, 27, 1093-1101]. All of these effects result in shorter spin injection length $L_{d}$. On the other hand, the scattering tends to suppress the inhomogeneous broadening in time domain as well as in space domain. This helps to prolong the diffusion length $L_{d}$. These competing effects on spin diffusion and spin transport were investigated using the kinetic spin Bloch equation approach [24, 25, 27, 28, 32, 336]. The main results are reviewed in the following.
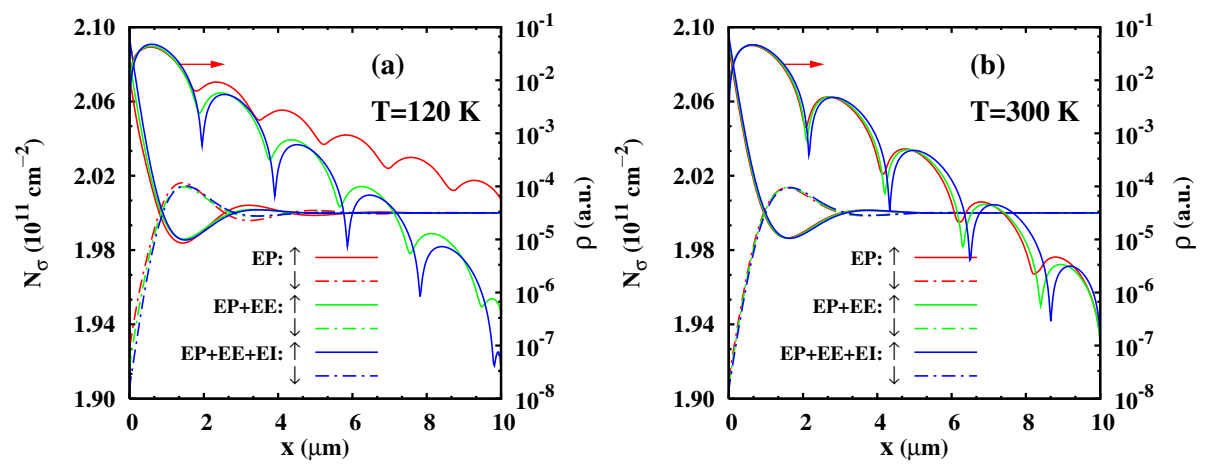

Figure 117: Effect of the scattering on spin diffusion in the steady state at (a) $T=120 \mathrm{~K}$ and (b) $T=300 \mathrm{~K}$ in GaAs quantum well with $a=7.5$ nm. Red curves: with only the electron-longitudinal-optical-phonon (EP) scattering; Green curves: with both the electron-electron (EE) and electronlongitudinal-optical-phonon scattering; Blue curves: with all the scattering, i.e., the electron-electron, electron-longitudinal-optical-phonon and electron-impurity (EI) scattering. The impurity density $N_{i}=N_{e}$. Note the scale of the incoherently summed spin coherence is on the right hand side of the figure. From Cheng and Wu [28]. 


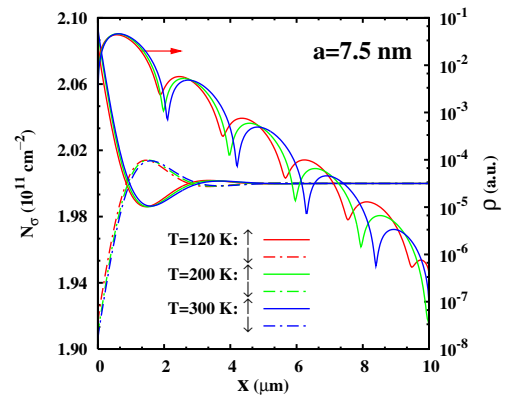

Figure 118: $N_{\sigma}$ and $\rho$ vs. the position $x$ at $T=120,200$ and $300 \mathrm{~K}$ in $n$-type GaAs (001) quantum well with width $a=7.5 \mathrm{~nm}$ and $L=10 \mu \mathrm{m} . N_{i}=0$. Note the scale of the incoherently summed spin coherence is on the right hand side of the figure. From Cheng and $\mathrm{Wu}$ [28].

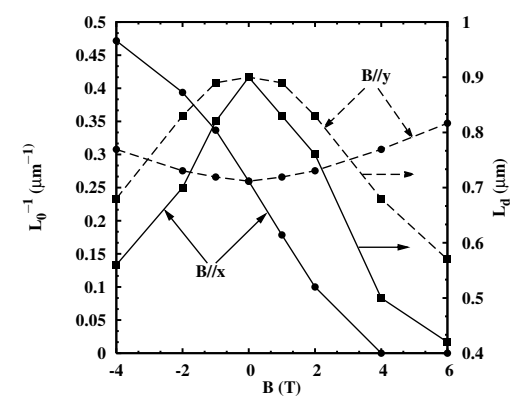

Figure 119: Inverse of the period of the spin oscillation $L_{0}^{-1}$ (curves with $\bullet$ ) and the spin diffusion length $L_{d}$ (curves with $\mathbf{\square}$ ) for $n$-type GaAs (001) quantum well vs. the external magnetic field $B$ which is applied either vertical ( $\| y$, dashed curves) or parallel ( $\| x$, solid curves) to the diffusion direction. $T=120$ $\mathrm{K}$ and $N_{i}=0$. The dashed curves are for the vertical magnetic field and the solid curves are for the parallel one. Note the scale of the diffusion length $L_{d}$ is on the right hand side of the figure. From Cheng and $\mathrm{Wu}[28]$.

In Fig. 117 the spin-resolved electron density $N_{\sigma}$ and the incoherently summed spin coherence $\rho$ in the steady state are plotted against the position $x$ by first including only the electron-longitudinal-optical-phonon scattering (red curves), then adding the electron-electron scattering (green curves) and finally adding the electron-impurity scattering (blue curves) with $N_{i}=N_{e}$ at $T=120 \mathrm{~K}$ (a) and $300 \mathrm{~K}$ (b). In the calculation there is no applied magnetic field. One can see that the scattering affects the transport in a complex way: At $T=120 \mathrm{~K}$, the spin injection length $L_{d}$ always decreases when a new scattering mechanism is added: $L_{d}$ is significantly reduced when the Coulomb scattering is added; adding electron-impurity scattering further reduces $L_{d}$, but only slightly. However, when $T=300 \mathrm{~K}, L_{d}$ becomes slightly shorter when the Coulomb scattering is first added, but becomes slightly longer when electronimpurity scattering is further added. These results show that for GaAs quantum well with width $a=7.5 \mathrm{~nm}$, all scattering mechanisms are not strong enough at $T=120 \mathrm{~K}$ and the system falls into weak scattering limit. The counter effect of the scattering to the inhomogeneous broadening is weak. Therefore adding a new scattering reduces the injection length. At $T=300 \mathrm{~K}$, the scattering becomes stronger. As a result the competing effects of the scatterings cancel each other and result in marginal change in diffusion length when a new scattering is added.

In Fig. 118, the results of steady-state spin injection at different temperatures are shown. It is seen that the spin injection length $L_{d}$ slightly decreases as the temperature rises. The change in $L_{d}$ is mild due to the cancellation of the opposite effects in scattering. The spacial period $L_{0}$ on the other hand systematically increases with the temperature. This is because when there is only the Dresselhaus term, $L_{0}^{-1} \simeq\left|\left\langle\omega_{\mathbf{k}}\right\rangle\right|=\left|m^{*} \gamma\left(\left\langle k_{y}^{2}\right\rangle-\left\langle k_{z}^{2}\right\rangle\right)\right|$. In small quantum well, $\left\langle k_{z}^{2}\right\rangle$ is larger than $\left\langle k_{y}^{2}\right\rangle$. As the temperature increases, $\left\langle k_{y}^{2}\right\rangle$ becomes larger and consequently $L_{0}$ becomes larger.

\subsubsection{Spin transport in the presence of magnetic and electric fields}

The magnetic field effect on the steady-state spin diffusion is shown in Fig. 119 where the spin diffusion length $L_{d}$ and the spin oscillation period $L_{0}$ as functions of the applied magnetic field at $T=120 \mathrm{~K}$ are plotted. The direction of the magnetic field is either vertical (along the $y$-axis) or parallel (along the $x$-axis) to the diffusion direction. As the magnetic field increases the inhomogeneous broadening in spin diffusion, it leads to additional spin relaxation/decoherence and therefore the spin diffusion length decreases with the magnetic field, regardless of the direction of the magnetic field. However, it is interesting to see that the spin diffusion length has different symmetries when the direction of the magnetic field changes: When $\mathbf{B}$ is parallel to the $y$-axis, $L_{d}(B)=L_{d}(-B)$; However, when $\mathbf{B}$ is parallel to the $x$-axis, $L_{d}(B) \neq L_{d}(-B)$. This can be understood from the fact that the density matrices from the kinetic spin Bloch equations have the symmetry $\rho_{k_{x}, k_{y}, k z}(B)=\rho_{k_{x},-k_{y}, k_{z}}(-B)$ when $\mathbf{B}$ is along the $y$-axis. This symmetry is broken if $\mathbf{B}$ is along the $x$-axis.

Differing from the magnetic field dependence of the spin diffusion length, the spin oscillation period $L_{0}$ decreases monotonically with the vertical magnetic field, regardless the sign of the field. However $L_{0}$ increases with 


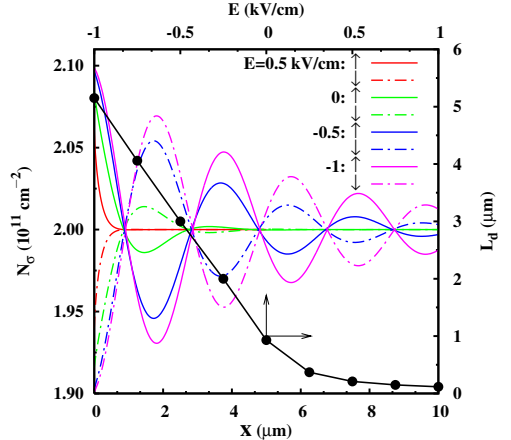

Figure 120: Spin-resolved electron density $N_{\sigma}$ vs. position $x$ at different electric field $E=0.5,0,-0.5$ and $-1 \mathrm{kV} / \mathrm{cm}$ and the spin diffusion length $L_{d}$ for $n$-type GaAs (001) quantum well against the electric field $E$ at $T=120 \mathrm{~K} . N_{i}=0$. It is noted that although $x$ is plotted up to $10 \mu \mathrm{m}, L=25 \mu \mathrm{m}$ when $E=-1.0$ and $-0.75 \mathrm{kV} / \mathrm{cm} ; 20 \mu \mathrm{m}$ when $E=-0.5$ and -0.25 $\mathrm{kV} / \mathrm{cm} ; 10 \mu \mathrm{m}$ when $E=0$; and $5 \mu \mathrm{m}$ when $E=0.5,0.75$ and $1.0 \mathrm{kV} / \mathrm{cm}$. Note the scales of the spin diffusion length are on the top and the right hand side of the figure. From Cheng and Wu [28].
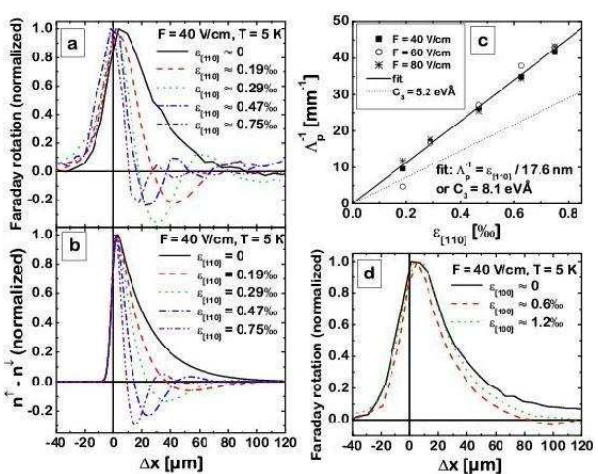

Figure 121: Spin transport at $F=40 \mathrm{~V} / \mathrm{cm}$ and $N_{D}=1.4 \times$ $10^{16} \mathrm{~cm}^{-3}$ in strained bulk GaAs. (a) measured Faraday rotation for drift along [1ํㅣㄹ and strain along [110]; (b) calculated spin polarization with the parameters of (a) (using $C_{3}=5.2 \mathrm{eV \AA}$ ); (c) Spacial oscillation "frequency" $\Lambda_{p}^{-1}$ versus strain $\epsilon_{[110]}$, linear fit corresponding to $C_{3}=8.1 \mathrm{eV \AA}$, and expected result for $C_{3}=5.2 \mathrm{eVA}$ (dotted line); (d) measured Faraday rotation for drift and strain along [100]. From Beck et al. [567].

the parallel magnetic field when the field is along the diffusion direction and less than $4 \mathrm{~T}$ (the spin precession disappears when the field is larger than $4 \mathrm{~T}$ ) but $L_{0}$ increases with the magnetic field when it is anti-parallel to the diffusion direction. In the presence of the Dresselhaus term and the applied magnetic field $\mathbf{B}$, the spacial period is determined by $m^{*} \gamma\left(\left\langle k_{y}^{2}\right\rangle-\left\langle k_{z}^{2}\right\rangle, 0,0\right)+m^{*} g \mu_{B} \mathbf{B}\left\langle 1 / k_{x}\right\rangle$. $\left\langle 1 / k_{x}\right\rangle$ represents the average of $1 / k_{x}$. Due to the spin transport it does not vanish and can be roughly estimated by $\left\langle 1 / k_{x}\right\rangle^{-1} \approx\left\langle k_{x}\right\rangle=\sum_{\mathbf{k}} k_{x} \Delta f_{\mathbf{k}} / \sum_{\mathbf{k}} \Delta f_{\mathbf{k}}$, which is a positive value for spin transport along $x$-direction. For the vertical magnetic field, the average of the Dresselhaus effective magnetic field and the applied one are perpendicular to each other, so the magnitude of the spin oscillation period is determined by $\left[\left(m^{*} g \mu_{B} B\right)^{2} /\left\langle k_{x}\right\rangle^{2}+m^{* 2} \gamma^{2}\left(\left\langle k_{y}^{2}\right\rangle-\left\langle k_{z}^{2}\right\rangle\right)^{2}\right]^{-1 / 2}$ which always decreases with the magnetic field. However, for the parallel field, these two vectors are in the same direction and the period is determined by $\left|m^{*} \gamma\left(\left\langle k_{y}^{2}\right\rangle-\left\langle k_{z}^{2}\right\rangle\right)+m^{*} g \mu_{\mathrm{B}} B /\left\langle k_{x}\right\rangle\right|^{-1}$. These two vectors can enhance or cancel each other depending on their relative strength and sign. For the particular case in Fig. 119, the the inverse of period $L_{0}^{-1}$ decreases with the magnetic field, until $B \sim 4$ T when $\left|\gamma\left(\left\langle k_{y}^{2}\right\rangle-\left\langle k_{z}^{2}\right\rangle\right)+g \mu_{\mathrm{B}} B /\left\langle k_{x}\right\rangle\right| \sim 0$. Further increase of the magnetic field does not lead to clear spin oscillation since the inhomogeneous broadening caused by the magnetic field becomes too large.

The effect of electric field on the spin transport is shown in Fig. 120 where the spin densities in the steady state are plotted against the position $x$ at different electric fields $E=0.5,0,-0.5$ and $-1 \mathrm{kV} / \mathrm{cm}$. In the calculations, the temperature is $120 \mathrm{~K}$ and only the intrinsic scattering mechanisms, i.e. the electron-phonon and electron-electron Coulomb scatterings, are included. It is seen from the figure that the spin oscillation period almost does not change when the electric field varies from $-1 \mathrm{kV} / \mathrm{cm}$ to $0.5 \mathrm{kV} / \mathrm{cm}$. The null effect of the electric field on spacial precession is quite different from the effect of electric field on the precession in time domain [569, 570]. In the spacial uniform system, due to the drifting of the electrons driven by the electric field, the Dresselhaus or Rashba term gives an effective magnetic field proportional to the electric field. This effective magnetic field in turn gives a precession frequency proportional to the electric field [569, 570]. However, in the spin transport, the average of the inhomogeneous broadening $\left\langle\omega_{\mathbf{k}}\right\rangle=m^{*} \gamma\left(\left\langle k_{y}^{2}\right\rangle-\left\langle k_{z}^{2}\right\rangle, 0,0\right)$ does not depend on the drifting velocity, therefore is not affected by the electric field. This is consistent with the previous experimental observation in the strained bulk system [567], which is shown in Fig. 121. In the experiment, the oscillation is induced by the additional spin-orbit coupling caused by the strain. As shown in Eq. (305), the spacial oscillation frequency [denoted as $\Gamma_{p}^{-1}$ in Fig. 121 (c)] is $C_{3} \epsilon_{y x}$, which is proportional to the strain but does not depend on the drifting velocity or electric field.

It is further noted from the figure that the spin diffusion length is markedly affected by the electric field. To reveal this effect, the spin diffusion length is plotted as function of electric field in the same figure. One finds when the electric field varies from $-1 \mathrm{kV} / \mathrm{cm}$ to $+1 \mathrm{kV} / \mathrm{cm}$, the spin diffusion length decreases monotonically, which is 


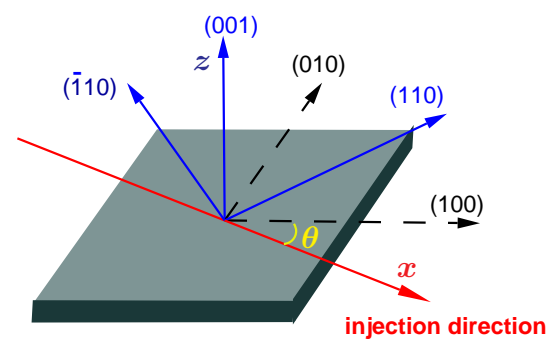

Figure 122: Schematic of the different directions considered for the spin polarizations ([110], [110] and [001]-axes and spin diffusion/injection ( $x$-axis). From Cheng et al. [29].

consistent with the prediction of the drift-diffusion model [928, 929]. From inhomogeneous broadening point of view, this can be easily understood from the fact that when an electric field is applied along $-x$-direction $(+x$-direction), the drift velocity caused by the electric field is along the $x$-direction ( $-x$-direction), which enhances (cancels) the velocity driven by the spin gradient. Therefore, $\left\langle k_{x}\right\rangle$ is increased (reduced) and the inhomogeneous broadening is decreased (enhanced). A longer (shorter) spin diffusion length is then observed [28].

\subsection{Anisotropy of spin transport in the presence of competing Rashba and Dresselhaus fields}

When the Dresselhaus and Rashba terms are both important in semiconductor quantum well, the total effective magnetic field can be highly anisotropic and spin kinetics is also highly anisotropic in regards to the direction of the spin [194]. For some special polarization direction, spin relaxation time is extremely large [194, 196, 203, 339, 919, 920]. For example, if the coefficients of the linear Dresselhaus and Rashba terms are equal to each other in (001) quantum well of small well width and the cubic Dresselhaus term is not important, the effective magnetic field is along the [110] direction for all electrons. For the spin components perpendicular to the [110] direction, this effective magnetic field flips the spin and leads to a finite spin dephasing time. For spin along the [110] direction, this effective magnetic field can not flip it. Therefore, when the spin polarization is along the [110] direction, the Dresselhaus and Rashba terms can not cause any spin dephasing. When the cubic Dresselhaus term is taken into account, the spin dephasing time for spin polarization along the [110] direction is finite but still much larger than other directions [597].

The anisotropy in the spin direction is also expected in spin transport. When the Dresselhaus and Rashba terms are comparable, the spin injection length $L_{d}$ for the spin polarization perpendicular to [110] direction is usually much shorter than that for the spin polarization along [110] direction. In the ideal case when there are only the linear Dresselhaus and Rashba terms with identical strengths, spin injection length for spin polarization parallel to the [110] direction becomes infinity [196, 339, 920]. This effect has promoted Schliemann et al. to propose the nonballistic spin-field-effect transistor [339]. In such a transistor, a gate voltage is used to tune the strength of the Rashba term and therefore control the spin injection length.

However, spin transport actually involves both the spin polarization and spin transport directions. The latter has long been overlooked in the literature of spin transport. In the kinetic spin Bloch equation approach, this direction corresponds to the spacial gradient in the diffusion term [Eq. (157)] and the electric field in the drifting term [Eq. (156)]. The importance of the spin transport direction has not been realized until Cheng et al. pointed out that the spin transport is highly anisotropic not only in the sense of the spin polarization direction but also in the spin transport direction when the Dresselhaus and Rashba effective magnetic fields are comparable [29]. They even predicted that in (001) GaAs quantum well with identical linear Dresselhaus and Rashba coupling strengths, the spin injection along [110] or [110] can be infinite regardless of the direction of the spin polarization [29]. In the following we review the results on the anisotropic spin transport from the kinetic spin Bloch equation approach.

The schematic of spin transport in (001) GaAs quantum well is shown in Fig. 122. The transport direction is chosen to be along the $x$-axis, the angle between $x$-axis and [100] crystal direction is $\theta$. In this coordinate system, the

\footnotetext{
${ }^{94}[\overline{1} 10]$ or [110] depends on the relative signs of the Dresselhaus and Rashba coupling strengths.
} 
Dresselhaus effective magnetic field is

$$
\mathbf{h}_{D}(\mathbf{k})=\beta\left(-k_{x} \cos 2 \theta+k_{y} \sin 2 \theta, k_{x} \sin 2 \theta+k_{y} \cos 2 \theta, 0\right)+\gamma\left(\frac{k_{x}^{2}-k_{y}^{2}}{2} \sin 2 \theta+k_{x} k_{y} \cos 2 \theta\right)\left(k_{y},-k_{x}, 0\right),
$$

while the Rashba field reads

$$
\mathbf{h}_{R}(\mathbf{k})=\alpha\left(k_{y},-k_{x}, 0\right),
$$

with $\beta=\gamma\left\langle k_{z}^{2}\right\rangle$. For the special case with $\alpha=\beta$, the total magnetic field can be written as

$$
\mathbf{h}(\mathbf{k})=2 \beta\left[\sin \left(\theta-\frac{\pi}{4}\right) k_{x}+\cos \left(\theta-\frac{\pi}{4}\right) k_{y}\right] \hat{\mathbf{n}}_{0}+\gamma\left[\frac{k_{x}^{2}-k_{y}^{2}}{2} \sin 2 \theta+k_{x} k_{y} \cos 2 \theta\right]\left(k_{y},-k_{x}, 0\right),
$$

with the special direction $\hat{\mathbf{n}}_{0}=(\cos (\pi / 4-\theta), \sin (\pi / 4-\theta), 0)$ representing the crystal direction [110]. As given by Eq. (300), the spin precession around the effective field magnetic field is characterized by $\omega_{\mathbf{k}}=m^{*} \mathbf{h}(\mathbf{k}) / k_{x}$ for spin transport along the $x$-axis.

\subsubsection{Spin injection under the identical Dresselhaus and Rashba strengths $\alpha=\beta$}

When $\alpha=\beta$,

$$
\omega_{\mathbf{k}}=m^{*}\left\{2 \beta\left(\sin \left(\theta-\frac{\pi}{4}\right)+\cos \left(\theta-\frac{\pi}{4}\right) \frac{k_{y}}{k_{x}}\right) \hat{\mathbf{n}}_{0}+\gamma\left(\frac{k_{x}^{2}-k_{y}^{2}}{2} \sin 2 \theta+k_{x} k_{y} \cos 2 \theta\right)\left(k_{y} / k_{x},-1,0\right)\right\} .
$$

It can be splitted into two parts: the zeroth-order term (on $k$ ) which is always along the same direction of $\hat{\mathbf{n}}_{0}$ and the second-order term which comes from the cubic Dresselhaus term. If the cubic Dresselhaus term is omitted, the effective magnetic fields for all $\mathbf{k}$ states align along $\hat{\mathbf{n}}_{0}$ (crystal [110]) direction. Therefore, if the spin polarization is along $\hat{\mathbf{n}}_{0}$, there is no spin relaxation even in the presence of scattering since there is no spin precession. Nevertheless, it is interesting to see from Eq. (312) that when $\theta=3 \pi / 4$, i.e., the spin transport is along the [110] direction, $\omega_{\mathbf{k}}=$ $2 m^{*} \beta \hat{\mathbf{n}}_{0}$ is independent on $\mathbf{k}$ if the cubic Dresselhaus term is neglected. Therefore, in this special spin transport direction, there is no inhomogeneous broadening in the spin transport for any spin polarization. The spin injection length is therefore infinite regardless of the direction of spin polarization. This result is highly counterintuitive, considering that the spin relaxation times for the spin components perpendicular to the effective magnetic field are finite in the spacial uniform system. The surprisingly contradictory results, i.e., the finite spin relaxation/dephasing time versus the infinite spin injection length, are due to the difference in the inhomogeneous broadening in spacial uniform and non-uniform systems. In spacial uniform system, the inhomogeneous broadening is determined by the D'yakonov-Perel' term, i.e., $\mathbf{h}(\mathbf{k})$ [Eq. (311)], which is momentum dependent. However in the spin transport problem the inhomogeneous broadening is determined by $\omega_{\mathbf{k}}$, which is momentum-independent when the transport direction is along [110] here. This prediction has not yet been realized experimentally. However, very recent experimental findings on spin helix [18, 20, 37, 921] have provide strong evidence to support this prediction.

\subsubsection{Spin-diffusion/injection-direction and spin-polarization-direction dependence at $\alpha=\beta$}

When the cubic Dresselhaus term is taken into account, $\omega_{\mathbf{k}}$ becomes momentum dependent again for all spin transport directions. This inhomogeneous broadening results in a finite but still highly anisotropic diffusion length for both spin polarization and spin transport directions. The anisotropy is shown in Fig. 123, where the spin diffusion length $L_{d}$ and the inverse of the spin oscillation period $L_{0}^{-1}$ are plotted against the spin diffusion/injection angle $\theta$ for spin polarizations along $\hat{\mathbf{n}}_{0}, \hat{\mathbf{z}}$ and $\hat{\mathbf{n}}_{1}=\hat{\mathbf{z}} \times \hat{\mathbf{n}}_{0}$ (crystal direction [110]), respectively.

It is seen that the spin injection length becomes finite but independent on the direction of the spin diffusion/injection if the spin polarization is along [110] $\left(=\hat{\mathbf{n}}_{0}\right)$. Meanwhile, the spin polarization along this direction has an oscillation period approaching infinity. This can be understood because the spin polarization is in the same direction as the effective magnetic field given by the zeroth-order term in $\omega_{\mathbf{k}}$ and, thus, results in no oscillation. It is noted that the effective magnetic field from the second-order term of $\omega_{\mathbf{k}}$ is $\mathbf{k}$-dependent and the average of the second-order term over $\mathbf{k}$ states is nearly zero. Therefore the cubic term does not lead to any oscillation at high temperature due to the scattering [363, 368, 597]. However, the inhomogeneous broadening due to the second-order term leads to a finite 
spin diffusion length. By rewriting the second-order term of $\omega_{\mathbf{k}}$ into $\frac{m^{*} \gamma}{2 \hbar^{2}} k^{2} \sin \left(2 \theta_{\mathbf{k}}+2 \theta\right)\left(k_{y} / k_{x},-1,0\right)$ with $k$ and $\theta_{\mathbf{k}}$ denoting the magnitude and the direction of the wavevector $\mathbf{k}$ respectively, one finds that the magnitude of the inhomogeneous broadening does not change with the spin diffusion/injection direction $\theta$. Consequently the spin diffusion length does not change with the spin diffusion/injection direction.

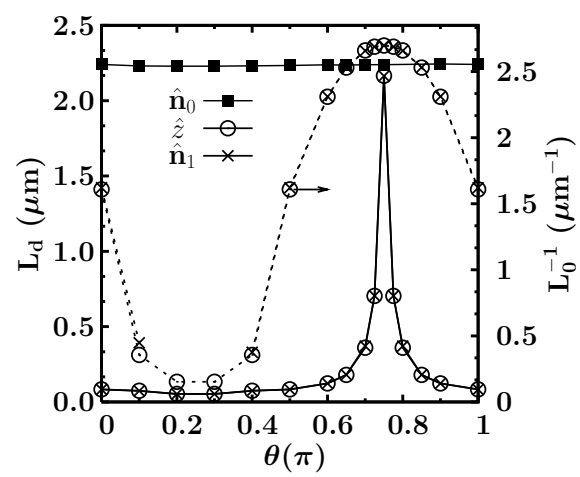

Figure 123: Spin diffusion length $L_{d}$ (solid curves) and the inverse of the spin oscillation period $L_{0}^{-1}$ (dashed curves) for $n$-type GaAs (001) quantum well with $\alpha=\beta$ as functions of the injection direction for different spin polarization directions $\hat{\mathbf{n}}_{0}, \hat{\mathbf{z}}$ and $\hat{\mathbf{n}}_{1}$ at $T=200 \mathrm{~K}$. It is noted that the scale of the spin oscillation period is on the right hand side of the frame. From Cheng et al. [29].

When the injected spin momentum is perpendicular to $\hat{\mathbf{n}}_{0}, L_{0}$ and $L_{d}$ become anisotropic in regard to the spin transport direction. The spin injection length is small except around $\theta=3 \pi / 4 . L_{0}$ and $L_{d}$ are almost identical for two spin polarization directions $\hat{z}$ and $\hat{\mathbf{n}}_{1}$. Because in this case, as $\gamma\left\langle k^{2}\right\rangle \ll \beta$, the kinetics of systems are determined by the zeroth order terms of $\omega_{\mathbf{k}}$ which are the same for these two directions. The oscillation period in the diffusion $L_{0}$ is determined by the k-independent component of $\omega_{\mathbf{k}}$ [29], i.e., $2 m^{*} \beta \sin (\theta-\pi / 4) / \hbar^{2}$ which is in good agreement with the results shown in Fig. 123 Moreover, when the injection direction is along [110] $(\theta=3 \pi / 4)$, the spin diffusion lengths for all of the three spin polarization directions are almost identical. The anisotropy in the direction of the spin polarization disappears. This is because when the spin diffusion/injection direction is $\theta=3 \pi / 4$, the k-dependent component in the zeroth order term of $\omega_{\mathbf{k}}$ disappears. The spin injection length is determined by the inhomogeneous broadening in the quadratic terms in $\omega_{\mathbf{k}}$, which are identical for any spin polarization directions.

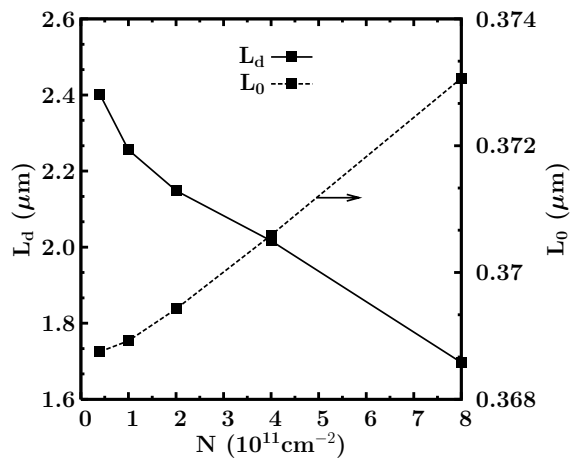

Figure 124: Spin diffusion length $L_{d}$ and spin oscillation period $L_{0}$ at $T=200 \mathrm{~K}$ for spin transport in $n$-type GaAs (001) quantum well along $\theta=3 \pi / 4$ direction as functions of the electron density. Note the scale of the oscillation period is on the right hand side of the frame. From Cheng et al. [29].

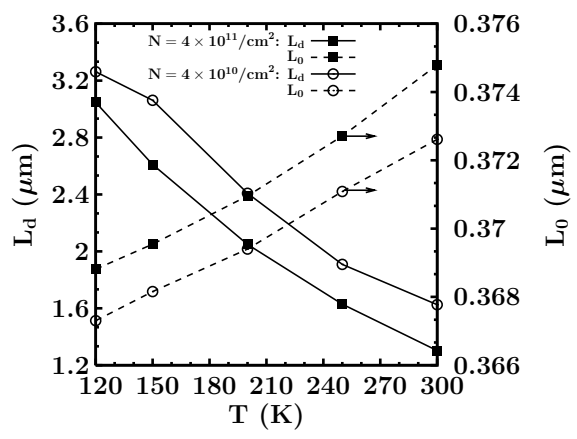

Figure 125: Spin diffusive length $L_{d}$ and spin oscillation period $L_{0}$ in $n$-type GaAs (001) quantum well at two different electron densities, $4 \times 10^{11} \mathrm{~cm}^{-2}$ and $4 \times 10^{10} \mathrm{~cm}^{-2}$ vs. the temperature. The spin diffusion/injection direction $\theta=3 \pi / 4$. Note the scale of the oscillation period is on the right hand side of the frame. From Cheng et al. [29]. 


\subsubsection{Temperature and electron-density dependence with injection along [1110] and $\alpha=\beta$}

The electron-density and temperature dependences of spin injection along [110] in the case $\alpha=\beta$ are shown in Figs. 124 and 125 respectively. It is seen that the diffusion length $L_{d}$ decreases with electron density and temperature, whereas the oscillation period $L_{0}$ increases with them. However, the change observed in the oscillation period is almost negligible (1\%) in contrast to the pronounced changes observed in the diffusion length (more than $40 \%$ ). This is quite different from the spin injection in GaAs quantum well when there is only the Dresselhaus term, where $L_{d}$ and $L_{0}$ only change slightly with the electron density and temperature [25, 28].

This is understood by the difference in the behavior of the inhomogeneous broadening $\omega_{\mathbf{k}}$. Unlike case of $\alpha=0$ where the zeroth order term dominates the inhomogeneous broadening, when $\alpha=\beta$ and $\theta=3 \pi / 4$, the secondorder term alone determines the inhomogeneous broadening. This term increases effectively with the temperature and electron density due to the increase of the average value of $k^{2}$. This is the reason for the obtained marked decrease of the spin diffusion length. The oscillation period $L_{0}$ is determined by the $\mathbf{k}$-independent zeroth-order term of $\omega_{\mathbf{k}}$, which does not change with the electron density and the temperature. Therefore one observes only a slight change in the oscillation period, originating from the second-order term and the scattering.

\subsubsection{Gate voltage dependence with injection direction along [1]10]}

Spin transport along [1110] under different gate voltages, i.e., different Rashba coupling strengths $\alpha$ since it can be controlled by the gate voltage, was also studied [29]. It was pointed out that when the cubic Dresselhaus term is present, the longest spin injection length no longer takes place at $\alpha=\beta[29]$ 95] This can also be understood from the inhomogeneous broadening. For spin transport along [110] with Dresselhaus and Rashba terms, $\omega_{\mathbf{k}}$ can be written as

$$
\omega_{\mathbf{k}}=2\left[\left(-\beta+\alpha+\frac{\gamma k^{2}}{2}\right) \frac{k_{y}}{k_{x}}-\gamma k_{x} k_{y}\right] \hat{\mathbf{n}}_{1}-2\left[\beta+\alpha-\frac{\gamma}{2}\left(k_{x}^{2}-k_{y}^{2}\right)\right] \hat{\mathbf{n}}_{0} .
$$

It is seen that the effect of $\alpha$ on the inhomogeneous broadening is determined by the first term. In order to get the longest spin injection length, $\alpha$ should be tuned to make the first term become minimal. Due to the cubic Dresselhaus term, the optimized condition is $\alpha=\hat{\beta}=\beta-\gamma\left\langle k^{2}\right\rangle / 2$ instead of $\alpha=\beta$ [29, 34]]. This prediction was later confirmed by the experiment [37].

\subsection{Transient spin grating}

The drift-diffusion model tells that the spin signal exponentially decays with the distance in spin injection and the injection length $L_{d}$ is related with the spin diffusion coefficient $D_{s}$ and spin relaxation time $\tau_{s}$ by the formula $L_{d}=\sqrt{D_{s} \tau_{s}}$ [Eq. [276]]. However, as pointed out by Weng and $\mathrm{Wu}$ [336], the drift-diffusion model oversimplifies spin transport in semiconductors by neglecting the off-diagonal terms of the density matrix. When these terms are included, the spin signal in the spin injection was shown to be a damped oscillation, characterized by spin injection length $L_{d}$ and spacial spin oscillation period $L_{0}$, instead of simple exponential decay [25, 28, 32]. In the extreme case of spin transport along the [110]-axis in (001) GaAs quantum well under the identical linear Dresselhaus and Rashba spin orbit coupling strengths, the spin injection length becomes infinite even when the spin diffusion coefficient and the spin relaxation time are finite [29]. This result is totally beyond the drift-diffusion model as both $D_{s}$ and $\tau_{s}$ are finite. Therefore, the relations among the characteristic parameters given by the drift-diffusion model need modification [29, 32, 34, 35]. Weng et al. presented the correct relations among the characteristic parameters, which hold even under the extreme case like the case in Sec.7.5, by studying the transient spin grating [32].

Transient spin grating, whose spin polarization varies periodically in real space, is excited optically by two noncollinear coherent light beams with orthogonal linear polarization [13, 33, 37, 495, 661]. Transient spin grating technique is well suited to study the spin transport since it can directly probe the decay rate of nonuniform spin distributions. Spin diffusion coefficient $D_{s}$ can be obtained from the transient spin grating experiments [13, 33, 495, 661]. In the literature, the drift-diffusion model was employed to extract $D_{s}$ from the experimental data. With the drift-diffusion model, the transient spin grating was predicted to decay exponentially with time with a decay rate of $\Gamma_{q}=D_{s} q^{2}+1 / \tau_{s}$, where $q$ is the wavevector of the spin grating [13, 495]. However, this result is not accurate since

\footnotetext{
${ }^{95}$ Similar conclusion has also been given by Stanescu and Galitski in Ref. [34].
} 
it neglects the spin precession which plays an important role in spin transport. Indeed, experimental results show that the decay of transient spin grating takes a double-exponential form instead of single exponential one [13, 33, 37]. Therefore, it is worthy to study transient spin grating using the kinetic spin Bloch equation approach.

\subsubsection{Simplified solution for transient spin grating}

The transient spin grating can be qualitatively understood in a simplified case where analytical solution can be obtained. By neglecting the Hartree-Fock term, the inelastic scattering such as the electron-phonon and the electronelectron Coulomb scatterings and the coupling to the Poisson equation, in the diffusive limit, one is able to write the kinetic spin Bloch equations as Eq. (306), with $\overline{\mathbf{h}}$ and $\overleftrightarrow{\Gamma}$ being

$$
\overline{\mathbf{h}}=\left\langle k^{2} \tau_{k}^{1} / m\right\rangle(-\hat{\beta} \cos 2 \theta, \hat{\beta} \sin 2 \theta-\alpha, 0),
$$

and

$$
\overleftrightarrow{\Gamma}=\frac{1}{2}\left(\begin{array}{ccc}
\left(\alpha^{2}+\hat{\beta}^{2}-2 \alpha \hat{\beta} \sin 2 \theta\right)\left\langle k^{2} \tau_{k}^{1}\right\rangle & 2 \alpha \hat{\beta}\left\langle k^{2} \tau_{k}^{1}\right\rangle \cos 2 \theta & 0 \\
+\left\langle\left(\gamma k^{3}\right)^{2} \tau_{k}^{3}\right\rangle / 16 & \left(\alpha^{2}+\hat{\beta}^{2}+2 \alpha \hat{\beta} \sin 2 \theta\right)\left\langle k^{2} \tau_{k}^{1}\right\rangle & 0 \\
2 \alpha \hat{\beta}\left\langle k^{2} \tau_{k}^{1}\right\rangle \cos 2 \theta & +\left\langle\left(\gamma k^{3}\right)^{2} \tau_{k}^{3}\right\rangle / 16 & 2\left(\alpha^{2}+\hat{\beta}^{2}\right)\left\langle k^{2} \tau_{k}^{1}\right\rangle \\
0 & 0 & +\left\langle\left(k^{3}\right)^{2} \tau_{k}^{3}\right\rangle / 8
\end{array}\right)
$$

for the system with both the Dresselhaus and Rashba terms. One can obtain the analytical solution of the above kinetic spin Bloch equations for transient spin grating as following [32]

$$
S_{z}(q, t)=S_{z}(q, 0)\left(\lambda_{+} e^{-t / \tau_{+}}+\lambda_{-} e^{-t / \tau_{-}}\right),
$$

with relaxation rates

$$
\Gamma_{ \pm}=\frac{1}{\tau_{ \pm}}=D q^{2}+\frac{1}{2}\left(\frac{1}{\tau_{s 1}}+\frac{1}{\tau_{s}}\right) \pm \frac{1}{2 \tau_{s 2}} \sqrt{1+\frac{16 D q^{2} \tau_{s 2}^{2}}{\tau_{s 1}^{\prime}}},
$$

in which

$$
\lambda_{ \pm}=\frac{1}{2}\left(1 \pm \frac{1}{\sqrt{1+16 D q^{2} \tau_{s 2}^{2} / \tau_{s 1}^{\prime}}}\right) .
$$

Here $\tau_{s 1}\left(\tau_{s 2}\right)$ is the spin relaxation time of the in-plane spin which mixes (does not mix) with the out-of-plane spin due to the net effective magnetic field. For spin injection/diffusion along the [110] axis, $\tau_{s 1}=\left\langle(\alpha-\hat{\beta})^{2} k^{2} \tau_{k}^{1}\right\rangle / 2+\gamma^{2}\left\langle k^{6} \tau_{k}^{3}\right\rangle / 32$ and $\tau_{s 1}^{\prime}=\left\langle(\alpha-\hat{\beta})^{2} k^{2} \tau_{k}^{1}\right\rangle / 2$. For spin injection/diffusion along the [110] axis, $\tau_{s 1}=\left\langle(\alpha+\hat{\beta})^{2} k^{2} \tau_{k}^{1}\right\rangle / 2+\gamma^{2}\left\langle k^{6} \tau_{k}^{3}\right\rangle / 32$ and $\tau_{s 1}^{\prime}=\left\langle(\alpha+\hat{\beta})^{2} k^{2} \tau_{k}^{1}\right\rangle / 2$. In the long wavelength limit $(q \ll 1), \Gamma_{+} \simeq 1 / \tau_{s}+\left(1+4 \tau_{s 2} / \tau_{s 1}^{\prime}\right) D q^{2}$ and $\Gamma_{-} \simeq$ $1 / \tau_{s 1}+\left(1-4 \tau_{s 2} / \tau_{s 1}^{\prime}\right) D q^{2}$ become quadratic functions of $q$, roughly correspond to the out-of-plane and the in-plane relaxation rates respectively. In general cases both of these two decay rates [Eq. (317)] are not simple quadratic functions of $q$. If one uses the quadratic fitting to yield the spin diffusion coefficient, one gets a value that is either larger (for $\Gamma_{+}$) or smaller (for $\Gamma_{-}$) than the true one. The accurate way to get the information of spin diffusion coefficient should be from the average of these two rates

$$
\Gamma=\left(\Gamma_{+}+\Gamma_{-}\right) / 2=D q^{2}+1 / \tau_{s}^{\prime}
$$

with $1 / \tau_{s}^{\prime}=\left(1 / \tau_{s}+1 / \tau_{s 1}\right) / 2$, which differs from the current widely used formula by replacing the spin decay rate by the average of the in- and out-of-plane relaxation rates. The difference of these two decay rates is a linear function of the wavevector $q$ when $q$ is relatively large:

$$
\Delta \Gamma=c q+d .
$$

For the simplified solution $c=2 \sqrt{D_{s} / \tau_{s 1}^{\prime}}$ and $d$ is a value close to zero.

The steady-state spin injection can be extracted from the transient spin grating signal by integrating the transient spin grating signal Eq. (316) over the time from 0 to $\infty$ and the wave-vector from $-\infty$ to $\infty$. From the simplified 
solution [Eqs. (316), (319) and (320)], the integrated transient spin grating reads $S_{z}(x)=S_{z}(0) e^{-x / L_{s}} \cos \left(x / L_{0}+\psi\right)$ with

$$
\begin{aligned}
& L_{s}=2 D_{s} / \sqrt{\left|c^{2}-4 D_{s}\left(1 / \tau_{s}^{\prime}-d\right)\right|}, \\
& L_{0}=2 D_{s} / c .
\end{aligned}
$$

It is noted that if one only considers the Rashba term or the linear Dresselhaus term one can recover the result $L_{s}=2 \sqrt{D_{s} \tau_{s}}$ from linear response theory [35]. It is seen that the spin precession actually prolongs the out-of-plane spin injection length by mixing the fast decaying out-of-plane spin with the slow decaying in-plane spin. Equations (321) and (322) give the right spin injection length and the spin oscillation period in the presence of the spinorbit coupling. From the experiment point of view, one can monitor the time evolutions of transient spin grating with different wavevectors $q$ and obtain the corresponding decay rates $\Gamma_{ \pm}$. By fitting $\Gamma_{ \pm}$with Eqs. (319) and (320), one can then calculate the spin injection length and spin oscillation period accurately from Eqs. (321) and (322), instead of using the inaccurate formula from the drift-diffusion model. Weng et al. demonstrated that the relations defined by Eqs. (321) and (322) are true even in the extreme case when the drift-diffusion model totally fails [32].

\subsubsection{Kinetic spin Bloch equation solution for transient spin grating}

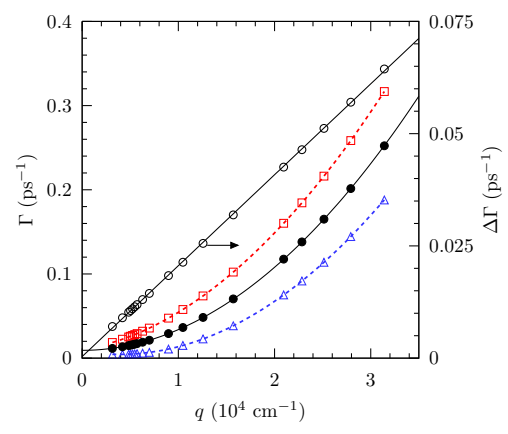

Figure 126: $\quad \Gamma=\left(\Gamma_{+}+\Gamma_{-}\right) / 2$ and $\Delta \Gamma=\left(\Gamma_{+}-\Gamma_{-}\right) / 2 v s$. $q$ at $T=295 \mathrm{~K}$ in $n$-type GaAs (001) quantum well. Open boxes/triangles are the relaxation rates $\Gamma_{+/-}$calculated from the full kinetic spin Bloch equations. Filled/open circles represent $\Gamma$ and $\Delta \Gamma$ respectively. Noted that the scale for $\Delta \Gamma$ is on the right hand side of the frame. The solid curves are the fitting to $\Gamma$ and $\Delta \Gamma$ respectively. The dashed curves are guide to eyes. From Weng et al. [32].

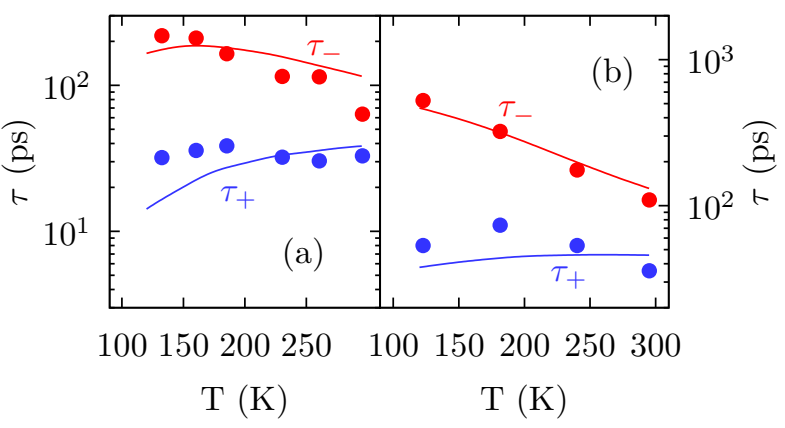

Figure 127: Spin relaxation times $\tau_{ \pm}$vs. temperature in $n$-type GaAs (001) quantum well for (a) high-mobility sample with $q=$ $0.58 \times 10^{4} \mathrm{~cm}^{-1}$ and (b) low-mobility sample with $q=0.69 \times 10^{4}$ $\mathrm{cm}^{-1}$. The dots are the experiment data from Ref. [33]. From Weng et al. [32].

The spin diffusion coefficient together with the relations Eqs. (321) and (322) obtained by the above simplified kinetic spin Bloch equations are derived with only the elastic scattering approximation. It does not take into account of the inelastic electron-phonon and electron-electron Coulomb scattering. Therefore the diffusion coefficient obtained from Eq. (319) does not include the contribution of the spin Coulomb drag. Moreover, this approach oversimplifies the complex effect of the scattering on the spin transport. Whether these relations remain valid in the genuine situation remains yet to be checked. To answer this, Weng et al. numerically solved the full kinetic spin Bloch equations with all the scattering explicitly included [32]. Their numerical results showed that all of the important qualitative results presented in Sec. 7.6.1 are still valid. Especially, the temporal evolution of the transient spin grating with all the scattering included still shows a double-exponential decay, which agrees with the experimental result [13, 33, 37] but is contrary to the result from the drift-diffusion model. Moreover the two decay rates can still be fitted with Eqs. (319) and (320) pretty well [32]. The fitting is shown in Fig. 126, where the relaxation rates $\Gamma_{ \pm}=1 / \tau_{ \pm}$, their average $\Gamma$ and difference $\Delta \Gamma$ of a typical transient spin grating are plotted as functions of $q$. The numerical results suggested that the two decay rates $\Gamma_{ \pm}=1 / \tau_{ \pm}$are not quadratic functions of $q$, but their average decay rate $\Gamma$ is. Using quadratic function to fit the decay rates $\Gamma_{ \pm}(q)$, one gets poor result. In contrast, the average decay rate $\Gamma$ fits pretty well by a quadratic function $\Gamma=D_{s} q^{2}+1 / \tau_{s}^{\prime}$. The resident error of the quadratic fitting for $\Gamma$ is two orders of magnitude smaller than those of $\Gamma_{ \pm}$. Moreover, for $\Gamma$ the constant term $\tau_{s}^{\prime}$ is very close to $4 \tau_{s} / 3$, inverse of the average of the in-plane and 
out-of-plane spin relaxation rates. Inspired by Eq. 319), the coefficient of the quadratic term $D_{s}$ can be reasonably assumed to be the spin diffusion coefficient. In this way one can calculate the spin diffusion coefficient with the effect of spin Coulomb drag included. For $\Delta \Gamma$, it can be accurately fitted by a linear function of $q$. It is therefore concluded that even with all the scatterings included, one can still extract the spin diffusion coefficient $D_{s}$ and obtain the injection length and oscillation period using Eqs. (321) and (322) from the transient spin grating experiments.

From Eqs. (319) and (320) one can see that $\tau_{+}$decreases monotonically as $q$ increases while $\tau_{-}$has a peak at some wavevector $q_{0}$. Earlier theoretical works, which only considered linear Dresselhaus term, predicted that the ratio of these two relaxation times $\tau_{-} / \tau_{+}$and the position of the peak $q_{0}$ do not vary with temperature [18, 35]. However, Weng et al. showed that once the cubic Dresselhaus term is included, both $\tau_{-} / \tau_{+}$and $q_{0}$ depend on temperature. With the cubic Dresselhaus term, the peak position $q_{0}$ moves from $q_{0}=\sqrt{15} \mathrm{~m}^{*} \beta / 2$ (which is independent of temperature) to $q_{0}=\sqrt{15} m^{*} \hat{\beta} / 2=\sqrt{15} m^{*}\left(\beta-\gamma k^{2} / 2\right) / 2$. Since $\left\langle k^{2}\right\rangle$ increases with increasing temperature, thus $q_{0}$ decreases with it. The temperature dependence of $\tau_{+} / \tau_{-}$also originates from the contribution of the cubic Dresselhaus term. These theoretical results qualitatively agree with experiment ones [33]. In additional to the correct qualitative agreement between the theoretical and experimental results, the quantitative accuracy of results from the kinetic spin Bloch equation approach is also good. The results are shown in Fig. 127, in which spin relaxation times are plotted as function of temperature together with the experimental data from Ref. [33]. In the calculation the finite square well assumption [372] was used, and all the parameters were chosen to be the experimental values if available. The only adjustable parameters were the spin-orbit coupling coefficients $\gamma$ and $\alpha$. In the calculation, $\gamma$ was chosen to be $11.4 \mathrm{eV}^{3}$ and $13.8 \mathrm{eV}^{3}$ for the high [Fig. 127(a)] and low mobility [Fig. 127(b)] samples respectively and $\alpha$ was set to be $0.3 \beta$, close to the choice in the experimental work [33].

\subsubsection{Spin Coulomb drag}

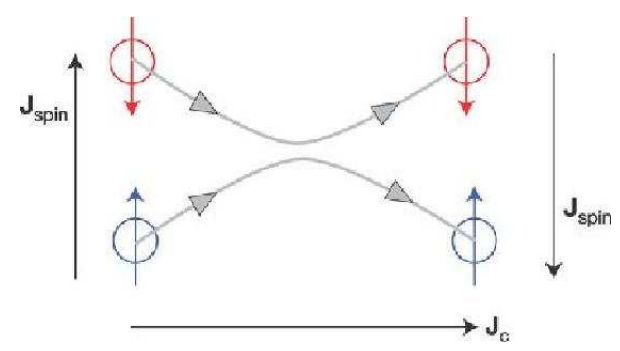

Figure 128: A representation of electron-electron Coulomb scattering that does not conserve spin-current. Before the collision the spin-current, $\mathbf{J}_{\text {spin }}$, is positive; after, it is negative. The charge current, $\mathbf{J}_{c}$, does not change. Colors correspond to spin states. From Weber et al. [13].

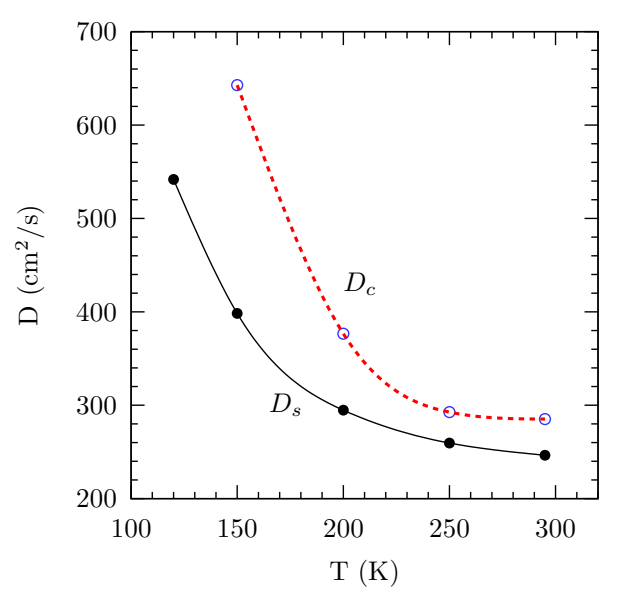

Figure 129: Diffusion coefficient as a function of temperature in $n$-type GaAs (001) quantum well. Solid circles: Spin diffusion constant $D_{s}$ with Coulomb drag; Open circles: Charge diffusion constant $D_{c}$. From Weng et al. [32].

From the evolution of the transient spin grating one can observe the Coulomb drag effect on the spin diffusion directly. The effect of electron-electron Coulomb scattering on the spin diffusion coefficient is illustrated in Fig. 128 , For spin-unpolarized charge diffusion, the electrons move along the same direction and the Coulomb scattering does not change the center-of-mass motion, therefore it does not change the charge diffusion coefficient directly. However, for the spin transport, spin-up and -down electrons move against each other. Therefore the Coulomb scattering between them slows down the relative motion of these two spin species and thus reduces spin diffusion coefficient. This is the so-called spin drag or spin Coulomb drag effect [13-15, 27, 1093-1101], which was first proposed by D'Amico and Vignale [14, 15, 1094-1098].

In Fig. 129 the spin diffusion coefficient calculated in the above mentioned method as a function of temperature was presented. For comparison, the charge diffusion coefficient, which is calculated by solving the kinetic spin Bloch 
equations with the initial condition being the charge gradient instead of the spin gradient, is also included. It is clearly seen from the figure that $D_{s}<D_{c}$.

From the figure one can tell that in the relative high temperature regime, as the temperature increases, the spin and charge diffusion coefficients and their difference all decrease. Therefore the Coulomb drag is stronger in the low temperature regime. However, even at room temperature the Coulomb drag is still strong enough to reduce the diffusion coefficient by 30\%. These results quantitatively agree with those of Refs. [1094, 1095]. It should be pointed out that the reduction of spin diffusion coefficient mostly comes from the Coulomb drag. The spin-orbit coupling only has small effect on the diffusion coefficient since the spin-orbit coupling is very small compared to the Fermi energy. The numerical result shows that the removal of the spin-orbit coupling only changes spin diffusion coefficient by one tenth percent for the system studied, which is consistent with the result from the linear response theory [1099].

Phenomenologically, the spin Coulomb drag modifies Ohm's law as $\mathbf{E}_{\eta}=\sum_{\eta^{\prime}} \rho_{\eta \eta^{\prime}} \mathbf{j}_{\eta^{\prime}}$, in which $\mathbf{E}_{\eta}$ is the "electric" field on electrons with spin $\eta$. The field also includes the contribution from the gradient of the local chemical potential, which can be spin dependent. The off-diagonal terms of the resistivity $\rho_{\uparrow \downarrow}$ correspond to the spin drag resistivity, defined by $\rho_{\uparrow \downarrow}=E_{\uparrow} / j_{\downarrow}$ when $j_{\uparrow}=0$. Using the generalized Einstein relations with the spin Coulomb drag effect taken into account, the spin diffusion coefficient reads [13-15, 1094]

$$
\frac{D_{s}}{D_{c}}=\frac{\chi_{0}}{\chi_{s}} \frac{1}{1+\left|\rho_{\uparrow \downarrow}\right| / \rho},
$$

where $D_{c}$ and $\rho$ are the charge diffusion coefficient and mobility, respectively and $\chi_{0} / \chi_{s}$ is the many-body enhancement of spin susceptibility of the electron gas. In normal electron density and temperature, $\chi_{0} / \chi_{s}$ is close to 1 [13, 848, 1102, 1103]. Spin drag resistivity can be calculated from the linear response theory. In two dimension, it takes the form of [14, 15, 1094, 1101]:

$$
\rho_{\uparrow \downarrow}=\frac{-1}{2 e^{2} n_{\uparrow} n_{\downarrow} k_{B} T} \int \frac{d^{2} q}{(2 \pi)^{2}} q^{2} \int_{0}^{\infty} \frac{d \omega}{2 \pi}\left|V_{\uparrow \downarrow}(\mathbf{q}, \omega)\right|^{2} \frac{\operatorname{Im} \Pi_{0 \uparrow}(\mathbf{q}, \omega) \operatorname{Im} \Pi_{0 \downarrow}(\mathbf{q}, \omega)}{\sinh ^{2}(\omega / 2 T)},
$$

in which $V_{\uparrow \downarrow}(\mathbf{q}, \omega)$ is the dynamically screened effective Coulomb interaction between spin $\uparrow$ and $\downarrow$ electrons. $\Pi_{0 \eta}$ is the non-interacting spin-resolved polarization function. Spin-orbit coupling is shown to have small enhancement to spin drag resistivity [1099]. $\rho_{\uparrow \downarrow}$ increases with temperature as $\left(T / T_{F}\right)^{2}$ in low temperature regime $\left(T \ll T_{F}\right)$ but decreases with temperature as $1 / T$ in high temperature regime $\left(T \gg T_{F}\right)$ [1093, 1095]. The quantitative comparison of $\rho_{\uparrow \downarrow}$ between theoretical calculation and experiment results is shown in Fig. 130. With the modification of finite quan-

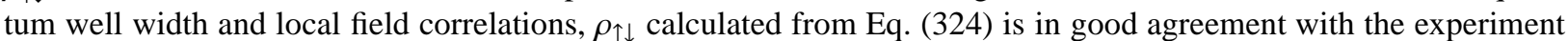
data extracted from Ref. [13] using Eq. [323] [1101].

Jiang et al. showed that the spin Coulomb drag also plays an important role in the transient spin transport [27]. In Fig. 131 the spacial profiles of the spin imbalance at different times are plotted for a Gaussian spin pulse with different scattering. It is clearly seen that when the Coulomb scattering is included, both the diffusion of the spin pulse and the decay of the spin polarization at the center of the pulse become much slower. When there is only electron-phonon scattering, a third peak in $\Delta N_{\sigma}$ appears after about 15 picosecond diffusion. The Coulomb scattering delays the appearance of the third peak to 35 picoseconds. Moreover, since the Coulomb scattering reduces the spin dephasing, the magnitudes of the spin polarization and the peaks with the Coulomb scattering are higher than those without.

\subsubsection{Infinite spin injection length in Sec. 7.5 revisited from the point of view of transient spin grating}

For most of the cases, $L_{s}$ determined by Eq. (321) is in the same order of $\sqrt{D_{s} \tau_{s}}$, although the former is usually larger. However, there are some special cases, say the denominator in Eq. (321) approaches zero, where $L_{s}$ can be orders of magnitude larger than $\sqrt{D_{s} \tau_{s}}$. Specifically, according to Cheng et al. [29], reviewed in Sec. 7.5, when the spin injection/transport direction is along [110] in (001) quantum well, $L_{s}$ becomes larger and larger as $\alpha$ approaches $\beta$, regardless of the direction of spin polarization. In the limit of $\alpha=\beta$, the spin injection length tends to infinity when the cubic Dresselhaus term is ignored and the spin polarization oscillates with a spacial period of $2 \pi /\left(2 m^{*} \beta\right)$ without any decay [29].

The non-decay spin oscillation model can also be understood from the point of view of transient spin grating. From the simplified solution in Sec.7.6.1 one obtains that the fitting parameters for $\Delta \Gamma$ are $d \rightarrow 0$ and $c \rightarrow 2 \sqrt{D_{s} / \tau_{s}^{\prime}}$ 


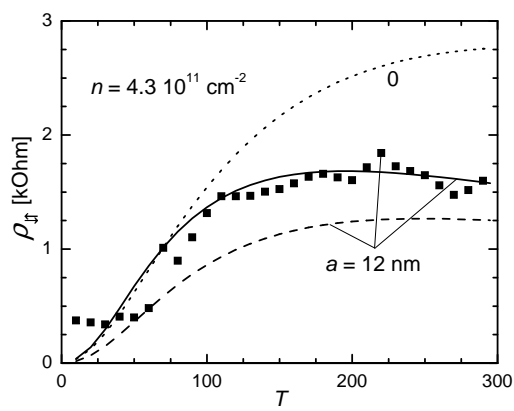

Figure 130: Spin drag resistivity $\rho_{\uparrow \downarrow}$ as a function of temperature for in $n$-type GaAs (001) quantum well with the electron density $n=4.3 \times 10^{11} \mathrm{~cm}^{-2}$. The dashed and dotted curves correspond to $\rho_{\uparrow \downarrow}$, calculated within the random phase approximation for quantum well width $a=0$ and $12 \mathrm{~nm}$, respectively. The solid curve correspond to $\rho_{\uparrow \downarrow}$, calculated beyond the random phase approximation for $a=12 \mathrm{~nm}$. The symbols represent $\rho_{\uparrow \downarrow}$, deduced from the experimental data for $a=12 \mathrm{~nm}$ of Ref. [13]. From Badalyan et al. [1101].

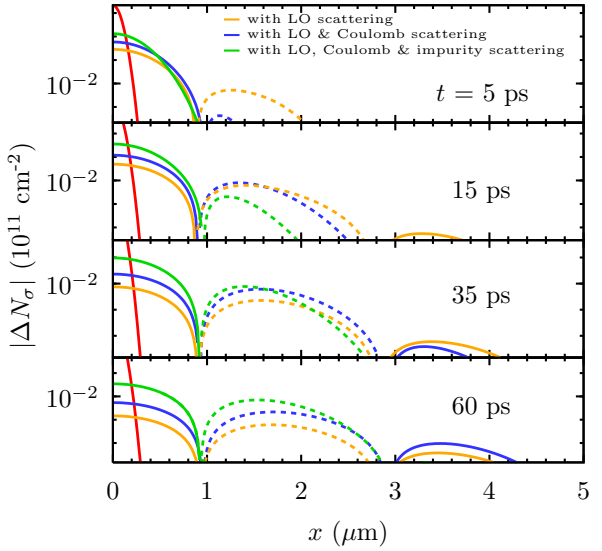

Figure 131: The absolute values of the spin imbalance $\left|\Delta N_{\sigma}\right|$ $v s$. the position $x$ at certain times $t$ in $n$-type GaAs (001) quantum well for the cases with only the electron-phonon scattering (orange); the electron-phonon and electron-electron scattering (blue); and the electron-phonon, electron-electron and electronimpurity scattering (green). The red curve shows the initial spin pulse. $\Delta N_{\sigma}(0)=10^{11} \mathrm{~cm}^{-2}, \delta x=0.1 \mu \mathrm{m}$ and $N_{i}=0.5 N_{e}$. Solid curves: $\Delta N>0$; Dashed curves: $\Delta N<0$. From Jiang et al. [27].

when $\alpha \rightarrow \beta$ in the system without the cubic Dresselhaus term. Consequently $\tau_{ \pm}=\left(\sqrt{D} q \pm 1 / \sqrt{\tau_{s}}\right)^{-2}$ when $\alpha=\beta$. It is then straightforward to see that $\tau_{-}$becomes infinite provided $q=q_{0}=1 / \sqrt{D \tau_{s}}=2 m^{*} \beta$. Therefore the steady-state spin injection along [110] axis is dominated by this non-decay transient spin grating mode which is responsible for the infinite spin injection length and the spacial oscillation period $2 \pi / q_{0}=2 \pi /\left(2 m^{*} \beta\right)$ [32].

\subsubsection{Persistent spin helix state}

The spin transport model with infinite spin injection length in the Sec.7.5.1 [29], or the non-decay transient spin grating with wavevector $\mathbf{q}_{\mathbf{0}}=\left(q_{0}, 0,0\right)$ in the previous subsection [32], is related to the persistent spin helix, which was first proposed by Bernevig et al. [18, 20, 37, 921]. Persistent spin helix has an SU(2) symmetry which is robust against the spin-conserving scattering. The $\mathrm{SU}(2)$ symmetry of the persistent spin helix is generated by the following operators [18]:

$$
S_{\mathbf{q}_{0}}^{-}=\sum_{\mathbf{k}} c_{\mathbf{k} ;-}^{\dagger} c_{\mathbf{k}+\mathbf{q}_{0} ;+}, \quad S_{\mathbf{q}_{0}}^{+}=\sum_{\mathbf{k}} c_{\mathbf{k}+\mathbf{q}_{0} ;+}^{\dagger} c_{\mathbf{k} ;-}, \quad S_{0}^{z}=\sum_{\mathbf{k}}\left(c_{\mathbf{k} ;+}^{\dagger} c_{\mathbf{k} ;+}-c_{\mathbf{k} ;-}^{\dagger} c_{\mathbf{k} ;-}\right) \text {. }
$$

Here $c_{\mathbf{k} ; \lambda}^{\dagger}\left(c_{\mathbf{k} ; \lambda}\right)$ is the creation (annihilation) operator of electron with momentum $\mathbf{k}$ and spin $\lambda . \lambda= \pm$ are the index of spin eigenstates with spin-orbit coupling. It is noted that nonzero $\left\langle S_{0}^{z}\right\rangle$ corresponds to spin polarization along [110] direction. While nonzero $\left\langle S_{\mathbf{q}_{0}}^{ \pm}\right\rangle$correspond to spin grating with wave-vector $\mathbf{q}_{0}$. The spin profile of nonzero $\left\langle S_{\mathbf{q}_{0}}^{ \pm}\right\rangle$in the real space is helical wave. The out-of-plane mode of the spin helix is illustrated in Fig. 132k.

The generating operators of persistent spin helix obey the communication relations for angular momentum,

$$
\left[S_{0}^{z}, S_{\mathbf{q}_{0}}^{ \pm}\right]= \pm 2 S_{\mathbf{q}_{0}}^{ \pm}, \quad\left[S_{\mathbf{q}_{0}}^{+}, S_{\mathbf{q}_{0}}^{-}\right]=S_{0}^{z} .
$$

Moreover, since $|\mathbf{k} ;+\rangle$ and $\left|\mathbf{k}+\mathbf{q}_{0} ;-\right\rangle$ are degenerate, these operators also commute with the electron Hamiltonian. This symmetry is robust against spin conserving scattering, such as electron-impurity, electron-phonon and electronelectron Coulomb scatterings, since these three operators also commute with the finite wave-vector particle densities $\rho_{\mathbf{q}}=\sum_{\sigma} c_{\mathbf{k}+\mathbf{q} \sigma}^{\dagger} c_{\mathbf{k} \sigma}$, which are the components that appear in the scattering Hamiltonian. This SU(2) symmetry means that the life time of expectation values of $S_{0}^{z}, S_{\mathbf{q}_{0}}^{ \pm}$are infinite, and hence the transient spin grating with $\mathbf{q}_{0}$ does not decay with time even when there is spin conserving scattering. In spin transport, this corresponds to the infinite spin injection length proposed by Cheng et al. [20, 29, 32]. When the cubic Dresselhaus term is present, the SU(2) 

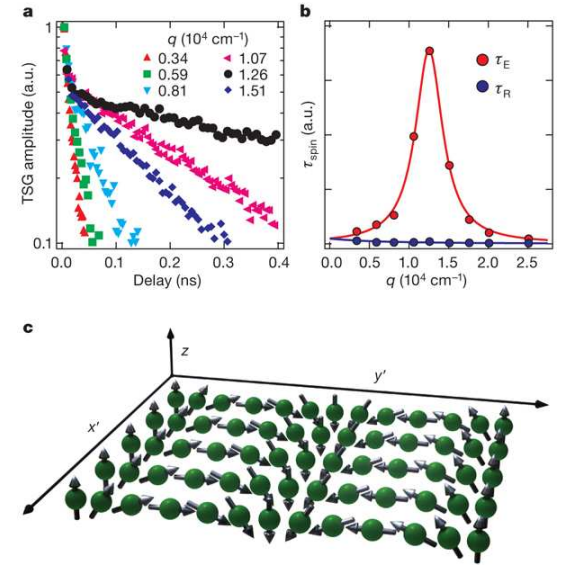

Figure 132: a, Transient spin grating decay curves at various wavevectors, q, for an asymmetrically doped GaAs quantum well with a mixture of Rashba and Dresselhaus spin-orbit couplings. $b$, Lifetimes for the spin-orbit-enhanced $\left[\tau_{\mathrm{E}}\right.$, corresponding to $\tau_{-}$in Eq. 317] ] and -reduced [ $\tau_{R}$, corresponding to $\tau_{+}$in Eq [317] ] helix modes extracted from double-exponential fits to the data in a. The solid lines are a theoretical fit (see text) using a single set of spin-orbit parameters for both helix modes. Error bars (s.d.) are the size of the data points. c, Illustration of a helical spin wave, which is one of the normal modes. In this picture, $z$ is the growth direction [001], and the axes $x^{\prime}$ and $y^{\prime}$ respectively refer to the [110] and [110] directions in the plane of the quantum well. The green spheres represent electrons whose spin directions are given by the arrows. From Koralek et al. [37].
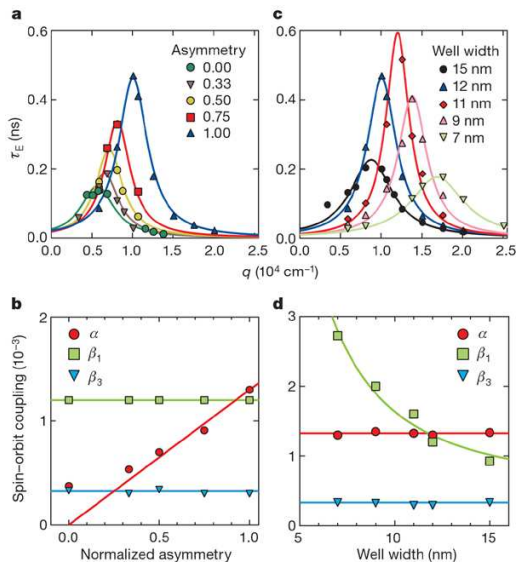

Figure 133: a, c, Lifetimes of the enhanced helix mode are shown for GaAs quantum wells with varying degrees of doping asymmetry (a) and well width (c). The normalized asymmetry is the difference between the concentrations of dopants on either side of the well, divided by the total dopant concentration. b, d, Plots summarizing the spin-orbit parameters from these fits in a and c. In this figure, the spin-orbit coupling strengths are expressed as dimensionless quantities by normalizing to the Fermi velocity. $\beta_{1}$ and $\beta_{3}$ are the coefficients of the linear and cubic Dresselhaus terms, corresponding to $\beta$ and $\gamma$ in the text, respectively. From Koralek et al. [37].

symmetry is broken, the lifetimes of the spin helix become finite, which corresponds to the finite spin injection length in the presence of the cubic Dresselhaus term [20, 29, 32].

Recently, Koralek et al. have observed the emergence of the persistent spin helix experimentally in the asymmetrically modulation-doped GaAs quantum wells, where both the Dresselhaus and Rashba spin-orbit couplings are important, by using the transient spin-grating spectroscopy [37]. Their results are shown in Figs. 132] and 133] The experiment results clearly show that the transient spin grating signal is in a double exponential decay form and can be fitted by two lifetimes, $\tau_{\mathrm{E}}$ and $\tau_{\mathrm{R}}$ (corresponding to $\tau_{-}$and $\tau_{+}$in the previous subsections). For some special wavevectors, $\tau_{\mathrm{E}}$ is much larger than $\tau_{\mathrm{R}}$ when the Dresselhaus and the Rashba terms are comparable. All of these experimental results consist with the theoretical ones [32, 33]. By constructing a series of quantum wells with different well widths (to tune the linear Dresselhaus spin-orbit coupling) and doping asymmetries (to change the Rashba spin-orbit coupling), Koralek et al. demonstrated how the lifetimes of spin helix depend on the Dresselhaus and Rashba terms as well as temperature. They found that the optimized condition to observe the peak spin helix lifetime is $\alpha=\hat{\beta}=\beta-\gamma k^{2} / 2$ instead of $\alpha=\beta$ due to the existence of the cubic Dresselhaus term. This finding is consistent with the previous theoretical predictions $[29,32,34]$.

\section{Summary}

The main concern of this review was to give an overview of the latest developments of the spin dynamics of semiconductors. We focused on two main issues: (i) spin relaxation and dephasing and (ii) spin diffusion and transport. We reviewed both experimental and theoretical progresses in these two directions. In theoretical part, besides the results based on the single-particle approach, we reviewed our systematic investigation based on fully microscopic many-body kinetic spin Bloch equation approach. Many novel effects predicted by this approach have been realized experimentally. Also many widely adopted common beliefs based on the single-particle theory in the literature were shown to be incorrect. We provided a comprehensive understanding of the spin dynamics of semiconductors and their 
confined structures from a fully microscopic many-body point of view.

\section{Acknowledgements}

This work was supported by the National Natural Science Foundation of China under Grant No. 10725417, the National Basic Research Program of China under Grant No. 2006CB922005 and the Knowledge Innovation Project of Chinese Academy of Sciences. One of the authors (MWW) would like to thank L. J. Sham and J. M. Kikkawa who first brought this exciting field into his attention back to 1999. He would also like to thank H. Haug, H. Metiu, J. Fabian, C. Schüller, T. Korn, X. Marie and E. L. Ivchenko for many discussions and/or collaborations. Many interesting discussions with H. Z. Zheng, T. S. Lai, Y. Ji and X. D. Cui on the experimental findings are also acknowledged. Last but not the least, he would like to thank his former and current students M. Q. Weng, J. L. Cheng, J. H. Jiang, K. Shen, P. Zhang and Y. Zhou for their excellent collaborations and P. Zhang for the typesetting of part of this manuscript and the proofreading reading of the whole manuscript.

\section{References}

[1] G. Lampel, Nuclear dynamic polarization by optical electronic saturation and optical pumping in semiconductors, Phys. Rev. Lett. 20 (1968) 491-493.

[2] R. R. Parsons, Band-to-band optical pumping in solids and polarized photoluminescence, Phys. Rev. Lett. 23 (1969) 1152-1154.

[3] F. Meier, B. P. Zakharchenya (Eds.), Optical Orientation, North-Holland, Amsterdam, 1984.

[4] J. M. Kikkawa, D. D. Awschalom, Resonant spin amplification in $n$-type gaas, Phys. Rev. Lett. 80 (1998) $4313-4316$.

[5] J. M. Kikkawa, D. D. Awschalom, Lateral drag of spin coherence in gallium arsenide, Nature 397 (1999) $139-141$.

[6] M. I. D'yakonov, V. I. Perel', Possibility of orienting electron spins with current, JETP Lett. 13 (1971) 467.

[7] M. I. D’yakonov, V. I. Perel', Current-induced spin orientation of electrons in semiconductors, Phys. Lett. A 35 (1971) 459-460.

[8] J. E. Hirsch, Spin hall effect, Phys. Rev. Lett. 83 (1999) 1834-1837.

[9] S. Zhang, Spin hall effect in the presence of spin diffusion, Phys. Rev. Lett. 85 (2000) 393-396.

[10] Y. K. Kato, R. C. Myers, A. C. Gossard, D. D. Awschalom, Observation of the Spin Hall Effect in Semiconductors, Science 306 (2004) 1910-1913.

[11] J. Wunderlich, B. Kaestner, J. Sinova, T. Jungwirth, Experimental observation of the spin-hall effect in a two-dimensional spin-orbit coupled semiconductor system, Phys. Rev. Lett. 94 (2005) 047204.

[12] S. O. Valenzuela, M. Tinkham, Direct electronic measurement of the spin hall effect, Nature 442 (2006) 176-179.

[13] C. P. Weber, N. Gedik, J. E. Moore, J. Orenstein, J. Stephens, D. D. Awschalom, Observation of spin coulomb drag in a two-dimensional electron gas, Nature 437 (2005) 1330-1333.

[14] I. D'Amico, G. Vignale, Theory of spin coulomb drag in spin-polarized transport, Phys. Rev. B 62 (2000) $4853-4857$.

[15] I. D'Amico, G. Vignale, Spin diffusion in doped semiconductors: The role of coulomb interactions, Europhys. Lett. 55 (2001) $566-572$.

[16] S. D. Ganichev, E. L. Ivchenko, V. V. Bel'kov, S. A. Tarasenko, M. Sollinger, D. Weiss, W. Wegscheider, W. Prettl, Spin-galvanic effect, Nature 417 (2002) 153-156.

[17] S. D. Ganichev, H. Ketterl, W. Prettl, E. L. Ivchenko, L. E. Vorobjev, Circular photogalvanic effect induced by monopolar spin orientation in p-gaas/algaas multiple-quantum wells, Appl. Phys. Lett. 77 (2000) 3146-3148.

[18] B. A. Bernevig, J. Orenstein, S.-C. Zhang, Exact su(2) symmetry and persistent spin helix in a spin-orbit coupled system, Phys. Rev. Lett. 97 (2006) 236601.

[19] J. D. Koralek, C. P. Weber, J. Orenstein, B. A. Bernevig, S.-C. Zhang, S. Mack, D. D. Awschalom, Emergence of the persistent spin helix in semiconductor quantum wells, Nature 458 (2009) 610-613.

[20] K. Shen, M. W. Wu, Infinite spin diffusion length of any spin polarization along direction perpendicular to effective magnetic field from dresselhaus and rashba spin-orbit couplings with identical strengths in (001) gaas quantum wells, J. Supercond. Nov. Magn. 22 (2009) 715-717.

[21] I. Žutić, J. Fabian, S. Das Sarma, Spintronics: Fundamentals and applications, Rev. Mod. Phys. 76 (2004) $323-410$.

[22] J. Fabian, A. Matos-Abiague, C. Ertler, P. Stano, I. Žutić, Semiconductor spintronics, Acta Phys. Slov. 57 (2007) $565-907$.

[23] M. I. Dyakonov (Ed.), Spin Physics in Semiconductors, Springer, Berlin/Heidelberg, 2008.

[24] M. Q. Weng, M. W. Wu, Q. W. Shi, Spin oscillations in transient diffusion of a spin pulse in $n$-type semiconductor quantum wells, Phys. Rev. B 69 (2004) 125310.

[25] M. Q. Weng, M. W. Wu, Kinetic theory of spin transport in $n$-type semiconductor quantum wells, J. Appl. Phys. 93 (2003) $410-420$.

[26] Y. Qi, S. Zhang, Spin diffusion at finite electric and magnetic fields, Phys. Rev. B 67 (2003) 052407.

[27] L. Jiang, M. Q. Weng, M. W. Wu, J. L. Cheng, Diffusion and transport of spin pulses in an $n$-type semiconductor quantum well, J. Appl. Phys. 98 (2005) 113702.

[28] J. L. Cheng, M. W. Wu, Spin diffusion/transport in n-type gaas quantum wells, J. Appl. Phys. 101 (2007) 073702.

[29] J. L. Cheng, M. W. Wu, I. C. da Cunha Lima, Anisotropic spin transport in gaas quantum wells in the presence of competing dresselhaus and rashba spin-orbit coupling, Phys. Rev. B 75 (2007) 205328.

[30] M. Hruška, Š. Kos, S. A. Crooker, A. Saxena, D. L. Smith, Effects of strain, electric, and magnetic fields on lateral electron-spin transport in semiconductor epilayers, Phys. Rev. B 73 (2006) 075306. 
[31] S. A. Crooker, D. L. Smith, Imaging spin flows in semiconductors subject to electric, magnetic, and strain fields, Phys. Rev. Lett. 94 (2005) 236601.

[32] M. Q. Weng, M. W. Wu, H. L. Cui, Spin relaxation in $n$-type gaas quantum wells with transient spin grating, J. Appl. Phys. 103 (2008) 063714.

[33] C. P. Weber, J. Orenstein, B. A. Bernevig, S.-C. Zhang, J. Stephens, D. D. Awschalom, Nondiffusive spin dynamics in a two-dimensional electron gas, Phys. Rev. Lett. 98 (2007) 076604.

[34] T. D. Stanescu, V. Galitski, Spin relaxation in a generic two-dimensional spin-orbit coupled system, Phys. Rev. B 75 (2007) 125307.

[35] A. A. Burkov, A. S. Núñez, A. H. MacDonald, Theory of spin-charge-coupled transport in a two-dimensional electron gas with rashba spin-orbit interactions, Phys. Rev. B 70 (2004) 155308.

[36] Y. V. Pershin, Long-lived spin coherence states in semiconductor heterostructures, Phys. Rev. B 71 (2005) 155317.

[37] J. D. Koralek, C. P. Weber, J. Orenstein, B. A. Bernevig, S.-C. Zhang, S. Mack, D. D. Awschalom, Emergence of the persistent spin helix in semiconductor quantum wells, Nature 458 (2009) 610-613.

[38] J. R. Petta, A. C. Johnson, J. M. Taylor, E. A. Laird, A. Yacoby, M. D. Lukin, C. M. Marcus, M. P. Hanson, A. C. Gossard, Coherent Manipulation of Coupled Electron Spins in Semiconductor Quantum Dots, Science 309 (2005) 2180-2184.

[39] R. I. Dzhioev, K. V. Kavokin, V. L. Korenev, M. V. Lazarev, B. Y. Meltser, M. N. Stepanova, B. P. Zakharchenya, D. Gammon, D. S. Katzer, Low-temperature spin relaxation in $n$-type gaas, Phys. Rev. B 66 (2002) 245204.

[40] K. V. Kavokin, Spin relaxation of localized electrons in $n$-type semiconductors, Semicond. Sci. Technol. 23 (2008) 114009 (13pp).

[41] D. Stich, J. Zhou, T. Korn, R. Schulz, D. Schuh, W. Wegscheider, M. W. Wu, C. Schüller, Effect of initial spin polarization on spin dephasing and the electron $g$ factor in a high-mobility two-dimensional electron system, Phys. Rev. Lett. 98 (2007) 176401.

[42] D. Stich, J. Zhou, T. Korn, R. Schulz, D. Schuh, W. Wegscheider, M. W. Wu, C. Schüller, Dependence of spin dephasing on initial spin polarization in a high-mobility two-dimensional electron system, Phys. Rev. B 76 (2007) 205301.

[43] H. Saarikoski, G. E. W. Bauer, Spin accumulation with spin-orbit interaction, Phys. Rev. Lett. 102 (2009) 097204.

[44] M. Q. Weng, M. W. Wu, Spin dephasing in $n$-type gaas quantum wells, Phys. Rev. B 68 (2003) 075312.

[45] D. P. DiVincenzo, Quantum Computation, Science 270 (1995) 255-261.

[46] D. Loss, D. P. DiVincenzo, Quantum computation with quantum dots, Phys. Rev. A 57 (1998) 120-126.

[47] J. M. Taylor, H.-A. Engel, W. Dur, A. Yacoby, C. M. Marcus, P. Zoller, M. D. Lukin, Fault-tolerant architecture for quantum computation using electrically controlled semiconductor spins, Nat. Phys. 1 (2005) 177-183.

[48] B. Trauzettel, D. V. Bulaev, D. Loss, G. Burkard, Spin qubits in graphene quantum dots, Nat. Phys. 3 (2007) 192-196.

[49] B. Trauzettel, M. Borhani, M. Trif, D. Loss, Theory of spin qubits in nanostructures, J. Phys. Soc. Jpn. 77 (2008) 031012.

[50] H.-A. Engel, L. P. Kouwenhoven, D. Loss, C. M. Marcus, Controlling spin qubits in quantum dots, Quant. Inf. Proc. 3 (2004) $115-132$.

[51] R. Hanson, L. P. Kouwenhoven, J. R. Petta, S. Tarucha, L. M. K. Vandersypen, Spins in few-electron quantum dots, Rev. Mod. Phys. 79 (2007) 1217.

[52] R. Hanson, D. D. Awschalom, Coherent manipulation of single spins in semiconductors, Nature 453 (2008) $1043-1049$.

[53] C. Flindt, A. S. Sørensen, K. Flensberg, Spin-orbit mediated control of spin qubits, Phys. Rev. Lett. 97 (2006) 240501.

[54] S. Datta, B. Das, Electronic analog of the electro-optic modulator, Appl. Phys. Lett. 56 (1990) 665-667.

[55] H. C. Koo, J. H. Kwon, J. Eom, J. Chang, S. H. Han, M. Johnson, Control of Spin Precession in a Spin-Injected Field Effect Transistor, Science 325 (2009) 1515-1518.

[56] S. Bandyopadhyay, M. Cahay, Alternate spintronic analog of the electro-optic modulator, Applied Physics Letters 85 (2004) $1814-1816$.

[57] E. I. Rashba, V. I. Sheka, Landau level spectroscopy, North-Holland, Amsterdam, 1991.

[58] E. I. Rashba, A. L. Efros, Efficient electron spin manipulation in a quantum well by an in-plane electric field, Appl. Phys. Lett. 83 (2003) 5295-5297.

[59] E. I. Rashba, A. L. Efros, Orbital mechanisms of electron-spin manipulation by an electric field, Phys. Rev. Lett. 91 (2003) 126405.

[60] Y. Kato, R. C. Myers, A. C. Gossard, D. D. Awschalom, Coherent spin manipulation without magnetic fields in strained semiconductors, Nature 427 (2004) 50-53.

[61] A. L. Efros, E. I. Rashba, Theory of electric dipole spin resonance in a parabolic quantum well, Phys. Rev. B 73 (2006) 165325.

[62] M. Duckheim, D. Loss, Electric-dipole-induced spin resonance in disordered semiconductors, Nat. Phys. 2 (2006) $195-199$.

[63] M. Duckheim, D. Loss, Resonant spin polarization and spin current in a two-dimensional electron gas, Phys. Rev. B 75 (2007) 201305.

[64] L. Meier, G. Salis, I. Shorubalko, E. Gini, S. Schon, K. Ensslin, Measurement of rashba and dresselhaus spin-orbit magnetic fields, Nat. Phys. 3 (2007) 650-654.

[65] L. Meier, G. Salis, E. Gini, I. Shorubalko, K. Ensslin, Two-dimensional imaging of the spin-orbit effective magnetic field, Phys. Rev. B 77 (2008) 035305.

[66] M. Studer, S. Schön, K. Ensslin, G. Salis, Spin-orbit interaction and spin relaxation in a two-dimensional electron gas, Phys. Rev. B 79 (2009) 045302.

[67] M. Studer, G. Salis, K. Ensslin, D. C. Driscoll, A. C. Gossard, Gate-controlled spin-orbit interaction in a parabolic gaas/algaas quantum well, Phys. Rev. Lett. 103 (2009) 027201.

[68] Z. Wilamowski, W. Ungier, W. Jantsch, Electron spin resonance in a two-dimensional electron gas induced by current or by electric field, Phys. Rev. B 78 (2008) 174423.

[69] J. H. Jiang, M. W. Wu, Y. Zhou, Kinetics of spin coherence of electrons in $n$-type inas quantum wells under intense terahertz laser fields, Phys. Rev. B 78 (2008) 125309.

[70] E. I. Rashba, Theory of electric dipole spin resonance in quantum dots: Mean field theory with gaussian fluctuations and beyond, Phys. Rev. B 78 (2008) 195302.

[71] V. N. Golovach, M. Borhani, D. Loss, Electric-dipole-induced spin resonance in quantum dots, Phys. Rev. B 74 (2006) 165319.

[72] D. V. Bulaev, D. Loss, Electric dipole spin resonance for heavy holes in quantum dots, Phys. Rev. Lett. 98 (2007) 097202.

[73] J. D. Walls, Parametric spin excitations in lateral quantum dots, Phys. Rev. B 76 (2007) 195307.

[74] P. Stano, J. Fabian, Control of electron spin and orbital resonances in quantum dots through spin-orbit interactions, Phys. Rev. B 77 (2008) 
045310.

[75] K. C. Nowack, F. H. L. Koppens, Y. V. Nazarov, L. M. K. Vandersypen, Coherent Control of a Single Electron Spin with Electric Fields, Science 318 (2007) 1430-1433.

[76] S. Debald, C. Emary, Spin-orbit-driven coherent oscillations in a few-electron quantum dot, Phys. Rev. Lett. 94 (2005) 226803.

[77] Y. Kato, R. C. Myers, D. C. Driscoll, A. C. Gossard, J. Levy, D. D. Awschalom, Gigahertz Electron Spin Manipulation Using VoltageControlled g-Tensor Modulation, Science 299 (2003) 1201-1204.

[78] G. Salis, Y. Kato, K. Ensslin, D. C. Driscoll, A. C. Gossard, D. D. Awschalom, Electrical control of spin coherence in semiconductor nanostructures, Nature 414 (2001) 619-622.

[79] T. Andlauer, P. Vogl, Electrically controllable $g$ tensors in quantum dot molecules, Phys. Rev. B 79 (2009) 045307.

[80] A. De, C. E. Pryor, M. E. Flatté, Electric-field control of a hydrogenic donor's spin in a semiconductor, Phys. Rev. Lett. 102 (2009) 017603.

[81] M. F. Doty, M. Scheibner, I. V. Ponomarev, E. A. Stinaff, A. S. Bracker, V. L. Korenev, T. L. Reinecke, D. Gammon, Electrically tunable $g$ factors in quantum dot molecular spin states, Phys. Rev. Lett. 97 (2006) 197202.

[82] Y. Tokura, W. G. van der Wiel, T. Obata, S. Tarucha, Coherent single electron spin control in a slanting zeeman field, Phys. Rev. Lett. 96 (2006) 047202

[83] M. Pioro-Ladriere, T. Obata, Y. Tokura, Y.-S. Shin, T. Kubo, K. Yoshida, T. Taniyama, S. Tarucha, Electrically driven single-electron spin resonance in a slanting zeeman field, Nat. Phys. 4 (2008) 776-779.

[84] E. A. Laird, C. Barthel, E. I. Rashba, C. M. Marcus, M. P. Hanson, A. C. Gossard, Hyperfine-mediated gate-driven electron spin resonance, Phys. Rev. Lett. 99 (2007) 246601.

[85] J. R. Petta, H. Lu, A. C. Gossard, A Coherent Beam Splitter for Electronic Spin States, Science 327 (2010) 669-672.

[86] J. R. Petta, A. C. Johnson, A. Yacoby, C. M. Marcus, M. P. Hanson, A. C. Gossard, Pulsed-gate measurements of the singlet-triplet relaxation time in a two-electron double quantum dot, Phys. Rev. B 72 (2005) 161301.

[87] J. A. Gupta, R. Knobel, N. Samarth, D. D. Awschalom, Ultrafast Manipulation of Electron Spin Coherence, Science 292 (2001) $2458-2461$.

[88] J. Berezovsky, M. H. Mikkelsen, N. G. Stoltz, L. A. Coldren, D. D. Awschalom, Picosecond Coherent Optical Manipulation of a Single Electron Spin in a Quantum Dot, Science 320 (2008) 349-352.

[89] S. G. Carter, Z. Chen, S. T. Cundiff, Ultrafast below-resonance raman rotation of electron spins in gaas quantum wells, Phys. Rev. B 76 (2007) 201308

[90] C. E. Pryor, M. E. Flatté, Predicted ultrafast single-qubit operations in semiconductor quantum dots, Appl. Phys. Lett. 88 (2006) 233108.

[91] D. Press, T. D. Ladd, B. Zhang, Y. Yamamoto, Complete quantum control of a single quantum dot spin using ultrafast optical pulses, Nature 456 (2008) 218-221.

[92] X. Xu, B. Sun, P. R. Berman, D. G. Steel, A. S. Bracker, D. Gammon, L. J. Sham, Coherent population trapping of an electron spin in a single negatively charged quantum dot, Nat. Phys. 4 (2008) 692-695.

[93] A. Greilich, S. E. Economou, S. Spatzek, D. R. Yakovlev, D. Reuter, A. D. Wieck, T. L. Reinecke, M. Bayer, Ultrafast optical rotations of electron spins in quantum dots, Nat. Phys. 5 (2009) 262-266.

[94] E. D. Kim, K. Smirl, Y. Wu, A. Amo, X. Xu, D. G. Steel, A. S. Bracker, D. Gammon, L. J. Sham, All-Optical Ultrafast Control and Read-Out of a Single Negatively Charged Self-Assembled InAs Quantum Dot, 2009. ArXiv:0905.0297.

[95] E. A. Zhukov, D. R. Yakovlev, M. M. Glazov, L. Fokina, G. Karczewski, T. Wojtowicz, J. Kossut, M. Bayer, Optical control of electron spin coherence in $\mathrm{CdTe} /(\mathrm{Cd}, \mathrm{Mg}) \mathrm{Te}$ quantum wells, 2009. ArXiv:0912.2589.

[96] J.-M. Tang, J. Levy, M. E. Flatté, All-electrical control of single ion spins in a semiconductor, Phys. Rev. Lett. 97 (2006) 106803.

[97] Y. Léger, L. Besombes, J. Fernández-Rossier, L. Maingault, H. Mariette, Electrical control of a single mn atom in a quantum dot, Phys. Rev. Lett. 97 (2006) 107401.

[98] R. C. Myers, M. H. Mikkelsen, J.-M. Tang, A. C. Gossard, M. E. Flatté, D. D. Awschalom, Zero-field optical manipulation of magnetic ions in semiconductors, Nat. Mater. 7 (2008) 203-208.

[99] J. Chovan, I. E. Perakis, Femtosecond control of the magnetization in ferromagnetic semiconductors, Phys. Rev. B 77 (2008) 085321.

[100] J. Chovan, E. G. Kavousanaki, I. E. Perakis, Ultrafast light-induced magnetization dynamics of ferromagnetic semiconductors, Phys. Rev. Lett. 96 (2006) 057402.

[101] M. I. D'yakonov, V. I. Perel', On spin orientation of electrons in interband absorption of light in semiconductors, Zh. Eksp. Teor. Fiz. 60 (1971) 1954-1965.

[102] M. I. D'yakonov, V. I. Perel', Spin relaxation of conduction electrons in noncentrosymmetric semiconductors, Fiz. Tverd. Tela (Leningrad) 13 (1971) 3581-3585.

[103] Y. Yafet, Solid State Physics, volume 14, Academic Press, New York, 1963.

[104] R. J. Elliott, Theory of the effect of spin-orbit coupling on magnetic resonance in some semiconductors, Phys. Rev. 96 (1954) $266-279$.

[105] G. L. Bir, A. G. Aronov, G. E. Pikus, Spin relaxation of electrons scattered by holes, Zh. Eksp. Teor. Fiz. 69 (1975) 1382-1397.

[106] A. G. Aronov, G. E. Pikus, A. N. Titkov, Spin relaxation of conduction electrons in p-type iii-v compounds, Zh. Eksp. Teor. Fiz. 84 (1983) 1170.

[107] G. E. Pikus, G. L. Bir, Exchange interaction in excitons in semiconductors, Sov. Phys. JETP 33 (1971) 108.

[108] P. H. Song, K. W. Kim, Spin relaxation of conduction electrons in bulk iii-v semiconductors, Phys. Rev. B 66 (2002) 035207.

[109] J. Zhou, M. W. Wu, Spin relaxation due to the bir-aronov-pikus mechanism in intrinsic and $p$-type gaas quantum wells from a fully microscopic approach, Phys. Rev. B 77 (2008) 075318

[110] J. H. Jiang, M. W. Wu, Electron-spin relaxation in bulk iii-v semiconductors from a fully microscopic kinetic spin bloch equation approach, Phys. Rev. B 79 (2009) 125206

[111] J. H. Jiang, Y. Zhou, T. Korn, C. Schüller, M. W. Wu, Electron spin relaxation in paramagnetic ga(mn)as quantum wells, Phys. Rev. B 79 (2009) 155201.

[112] Y. Zhou, J. H. Jiang, M. W. Wu, Electron spin relaxation in p-type gaas quantum wells, New J. Phys. 11 (2009) 113039 (16pp).

[113] R. Winkler, Spin-orbit Coupling Effects in Two-Dimensional Electron and Hole Systems, Springer-Verlag, Berlin/Heidelberg, 2003. 
[114] T. Jungwirth, J. Sinova, J. Mašek, J. Kučera, A. H. MacDonald, Theory of ferromagnetic (iii,mn)v semiconductors, Rev. Mod. Phys. 78 (2006) 809.

[115] J. K. Furdyna, Diluted magnetic semiconductors, J. Appl. Phys. 64 (1988) R29-R64.

[116] J. Fischer, M. Trif, W. A. Coish, D. Loss, Spin interactions, relaxation and decoherence in quantum dots, Solid State Commun. 149 (2009) 1443-1450.

[117] G. E. Pikus, A. N. Titkov, Spin relaxation under optical orientation in semiconductors, in: [3], pp. 73-132.

[118] M. Z. Maialle, E. A. de Andrada e Silva, L. J. Sham, Exciton spin dynamics in quantum wells, Phys. Rev. B 47 (1993) $15776-15788$.

[119] Y. Chen, B. Gil, P. Lefebvre, H. Mathieu, Exchange effects on excitons in quantum wells, Phys. Rev. B 37 (1988) 6429-6432.

[120] E. Bernardes, J. Schliemann, M. Lee, J. C. Egues, D. Loss, Spin-orbit interaction in symmetric wells with two subbands, Phys. Rev. Lett. 99 (2007) 076603.

[121] R. S. Calsaverini, E. Bernardes, J. C. Egues, D. Loss, Intersubband-induced spin-orbit interaction in quantum wells, Phys. Rev. B 78 (2008) 155313.

[122] J. M. Luttinger, Quantum theory of cyclotron resonance in semiconductors: General theory, Phys. Rev. 102 (1956) 1030-1041.

[123] G. Dresselhaus, Spin-orbit coupling effects in zinc blende structures, Phys. Rev. 100 (1955) 580-586.

[124] Y. A. Bychkov, E. I. Rashba, Oscillatory effects and the magnetic susceptibility of carriers in inversion layers, J. Phys. C 17 (1984) 6039-6045.

[125] Y. A. Bychkov, E. I. Rashba, Properties of a 2d electron gas with lifted spectral degeneracy, JETP Lett. 39 (1984) $78-81$.

[126] O. Krebs, P. Voisin, Giant optical anisotropy of semiconductor heterostructures with no common atom and the quantum-confined pockels effect, Phys. Rev. Lett. 77 (1996) 1829-1832.

[127] E. L. Ivchenko, A. Y. Kaminski, U. Rössler, Heavy-light hole mixing at zinc-blende (001) interfaces under normal incidence, Phys. Rev. B 54 (1996) 5852-5859.

[128] E. O. Kane, Band structure of indium antimonide, J. Phys. Chem. Solids 1 (1957) 249-261.

[129] P.-O. Löwdin, A note on the quantum-mechanical perturbation theory, J. Chem. Phys. 19 (1951) $1396-1401$.

[130] M. Cardona, N. E. Christensen, G. Fasol, Relativistic band structure and spin-orbit splitting of zinc-blende-type semiconductors, Phys. Rev. B 38 (1988) 1806-1827.

[131] P. D. Dresselhaus, C. M. A. Papavassiliou, R. G. Wheeler, R. N. Sacks, Observation of spin precession in gaas inversion layers using antilocalization, Phys. Rev. Lett. 68 (1992) 106-109.

[132] D. Richards, B. Jusserand, H. Peric, B. Etienne, Intrasubband excitations and spin-splitting anisotropy in gaas modulation-doped quantum wells, Phys. Rev. B 47 (1993) 16028-16031.

[133] B. Jusserand, D. Richards, G. Allan, C. Priester, B. Etienne, Spin orientation at semiconductor heterointerfaces, Phys. Rev. B 51 (1995) 4707-4710.

[134] P. V. Santos, M. Willatzen, M. Cardona, A. Cantarero, Tight-binding calculation of spin splittings in semiconductor superlattices, Phys. Rev. B 51 (1995) 5121-5129.

[135] W. Knap, C. Skierbiszewski, A. Zduniak, E. Litwin-Staszewska, D. Bertho, F. Kobbi, J. L. Robert, G. E. Pikus, F. G. Pikus, S. V. Iordanskii, V. Mosser, K. Zekentes, Y. B. Lyanda-Geller, Weak antilocalization and spin precession in quantum wells, Phys. Rev. B 53 (1996) $3912-$ 3924.

[136] P. Pfeffer, W. Zawadzki, Five-level $k \cdot p$ model for the conduction and valence bands of gaas and inp, Phys. Rev. B 53 (1996) $12813-12828$.

[137] J. B. Miller, D. M. Zumbühl, C. M. Marcus, Y. B. Lyanda-Geller, D. Goldhaber-Gordon, K. Campman, A. C. Gossard, Gate-controlled spin-orbit quantum interference effects in lateral transport, Phys. Rev. Lett. 90 (2003) 076807.

[138] J.-M. Jancu, R. Scholz, E. A. de Andrada e Silva, G. C. La Rocca, Atomistic spin-orbit coupling and $k \cdot p$ parameters in iii-v semiconductors, Phys. Rev. B 72 (2005) 193201.

[139] A. N. Chantis, M. van Schilfgaarde, T. Kotani, Ab initio prediction of conduction band spin splitting in zinc blende semiconductors, Phys. Rev. Lett. 96 (2006) 086405.

[140] J. J. Krich, B. I. Halperin, Cubic dresselhaus spin-orbit coupling in 2d electron quantum dots, Phys. Rev. Lett. 98 (2007) 226802.

[141] J.-W. Luo, G. Bester, A. Zunger, Full-zone spin splitting for electrons and holes in bulk gaas and gasb, Phys. Rev. Lett. 102 (2009) 056405.

[142] J. Y. Fu, M. Q. Weng, M. W. Wu, Spin-orbit coupling in bulk gaas, Physica E 40 (2008) 2890-2893.

[143] J.-W. Luo, A. N. Chantis, M. van Schilfgaarde, G. Bester, A. Zunger, Discovery of a novel linear-in- $k$ spin splitting for holes in the 2d gaas/alas system, Phys. Rev. Lett. 104 (2010) 066405.

[144] H. Knotz, A. W. Holleitner, J. Stephens, R. C. Myers, D. D. Awschalom, Spatial imaging and mechanical control of spin coherence in strained gaas epilayers, Appl. Phys. Lett. 88 (2006) 241918.

[145] A. N. Chantis, M. Cardona, N. E. Christensen, D. L. Smith, M. van Schilfgaarde, T. Kotani, A. Svane, R. C. Albers, Strain-induced conduction band spin splitting in gaas from first principles calculations, Phys. Rev. B 78 (2008) 075208.

[146] W. H. Lau, M. E. Flatté, Electric field dependence of spin coherence in (001) gaas/al ga $_{1-x}$ as quantum wells, Phys. Rev. B 72 (2005) 161311.

[147] Y. S. Gui, C. R. Becker, N. Dai, J. Liu, Z. J. Qiu, E. G. Novik, M. Schäfer, X. Z. Shu, J. H. Chu, H. Buhmann, L. W. Molenkamp, Giant spin-orbit splitting in a hgte quantum well, Phys. Rev. B 70 (2004) 115328.

[148] R. Lassnig, $k \cdot p$ theory, effective-mass approach, and spin splitting for two-dimensional electrons in gaas-gaalas heterostructures, Phys. Rev. B 31 (1985) 8076-8086.

[149] I. Appelbaum, D. J. Monsma, Transit-time spin field-effect transistor, Appl. Phys. Lett. 90 (2007) 262501.

[150] I. Appelbaum, B. Huang, D. J. Monsma, Electronic measurement and control of spin transport in silicon, Nature 447 (2007) $295-298$.

[151] G. Lommer, F. Malcher, U. Rossler, Spin splitting in semiconductor heterostructures for b $\rightarrow$ 0, Phys. Rev. Lett. 60 (1988) $728-731$.

[152] E. A. de Andrada e Silva, G. C. La Rocca, F. Bassani, Spin-orbit splitting of electronic states in semiconductor asymmetric quantum wells, Phys. Rev. B 55 (1997) 16293-16299.

[153] P. Pfeffer, Effect of inversion asymmetry on the conduction subbands in gaas-ga ${ }_{1-x} \mathrm{al}_{x}$ as heterostructures, Phys. Rev. B 59 (1999) 1590215909. 
[154] S. Lamari, Effect of the doping concentration on the zero-field spin splitting and rashba parameter in a $p$-inas mosfet, Phys. Rev. B 67 (2003) 165329

[155] W. Yang, K. Chang, Rashba spin splitting in biased semiconductor quantum wells, Phys. Rev. B 73 (2006) 113303.

[156] W. Yang, K. Chang, Nonlinear rashba model and spin relaxation in quantum wells, Phys. Rev. B 74 (2006) 193314.

[157] J. Li, K. Chang, G. Q. Hai, K. S. Chan, Anomalous rashba spin-orbit interaction in inas/gasb quantum wells, Appl. Phys. Lett. 92 (2008) 152107.

[158] G. Engels, J. Lange, T. Schäpers, H. Lüth, Experimental and theoretical approach to spin splitting in modulation-doped in ga $_{1-x}$ as/inp quantum wells for $b \rightarrow$ 0, Phys. Rev. B 55 (1997) R1958-R1961.

[159] J. P. Heida, B. J. van Wees, J. J. Kuipers, T. M. Klapwijk, G. Borghs, Spin-orbit interaction in a two-dimensional electron gas in a inas/alsb quantum well with gate-controlled electron density, Phys. Rev. B 57 (1998) 11911-11914.

[160] C. M. Hu, J. Nitta, T. Akazaki, H. Takayanagi, J. Osaka, P. Pfeffer, W. Zawadzki, Zero-field spin splitting in an inverted $\mathrm{in}_{0.53} \mathrm{ga}_{0.47} \mathrm{as}_{\mathrm{in}} \mathrm{in}_{0.52} \mathrm{al}_{0.48}$ as heterostructure: Band nonparabolicity influence and the subband dependence, Phys. Rev. B 60 (1999) 77367739.

[161] T. Matsuyama, R. Kürsten, C. Meißner, U. Merkt, Rashba spin splitting in inversion layers on p-type bulk inas, Phys. Rev. B 61 (2000) $15588-15591$.

[162] T. Schäpers, G. Engels, J. Lange, T. Klocke, M. Hollfelder, H. Lüth, Effect of the heterointerface on the spin splitting in modulation doped $\mathrm{in}_{x} \mathrm{ga}_{1-x}$ as/inp quantum wells for $\mathrm{b} \rightarrow 0$, J. Appl. Phys. 83 (1998) 4324-4333.

[163] Y. Sato, T. Kita, S. Gozu, S. Yamada, Large spontaneous spin splitting in gate-controlled two-dimensional electron gases at normal $\mathrm{in}_{0.75} \mathrm{ga}_{0.25} \mathrm{as}_{\mathrm{in}} 0.75 \mathrm{al}_{0.25}$ as heterojunctions, J. Appl. Phys. 89 (2001) 8017-8021.

[164] J. Nitta, T. Akazaki, H. Takayanagi, T. Enoki, Gate control of spin-orbit interaction in an inverted in $0.53 \mathrm{~g} a 0.47 \mathrm{as} / \mathrm{in} 0.52 \mathrm{a} / 0.48 \mathrm{as}$ heterostructure, Phys. Rev. Lett. 78 (1997) 1335-1338.

[165] D. Grundler, Large rashba splitting in inas quantum wells due to electron wave function penetration into the barrier layers, Phys. Rev. Lett. 84 (2000) 6074-6077.

[166] V. A. Guzenko, T. Schäpers, H. Hardtdegen, Weak antilocalization in high mobility ga $_{x}$ in $_{1-x}$ as/inp two-dimensional electron gases with strong spin-orbit coupling, Phys. Rev. B 76 (2007) 165301.

[167] J. H. Kwon, H. C. Koo, J. Chang, S.-H. Han, J. Eom, Channel width effect on the spin-orbit interaction parameter in a two-dimensional electron gas, Appl. Phys. Lett. 90 (2007) 112505.

[168] P. J. Simmonds, S. N. Holmes, H. E. Beere, D. A. Ritchie, Spin-orbit coupling in an in $_{0.52} \mathrm{ga}_{0.48}$ as quantum well with two populated subbands, J. Appl. Phys. 103 (2008) 124506.

[169] T. Koga, J. Nitta, T. Akazaki, H. Takayanagi, Rashba spin-orbit coupling probed by the weak antilocalization analysis in inalas/ingaas/inalas quantum wells as a function of quantum well asymmetry, Phys. Rev. Lett. 89 (2002) 046801.

[170] C. Schierholz, R. Krsten, G. Meier, T. Matsuyama, U. Merkt, Weak localization and antilocalization in the two-dimensional electron system on p-type inas, Phys. Stat. Sol. (b) 233 (2002) 436-444.

[171] C. Schierholz, T. Matsuyama, U. Merkt, G. Meier, Weak localization and spin splitting in inversion layers on $p$-type inas, Phys. Rev. B 70 (2004) 233311

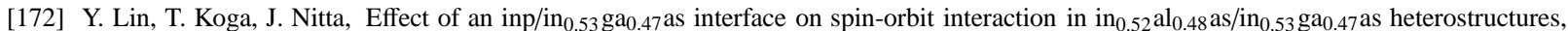
Phys. Rev. B 71 (2005) 045328.

[173] G. Yu, N. Dai, J. H. Chu, P. J. Poole, S. A. Studenikin, Experimental study of the spin-orbit quantum interference effect in a high-mobility in $_{x} \mathrm{ga}_{1-x}$ as/inp quantum well structure with strong spin-orbit interaction, Phys. Rev. B 78 (2008) 035304.

[174] Y. Kunihashi, T. Nihei, M. Kohda, J. Nitta, Rashba spin-orbit interaction of $\mathrm{in}_{0.53} \mathrm{ga}_{0.47} \mathrm{as}_{/} \mathrm{in}_{0.7} \mathrm{ga}_{0.3} \mathrm{as}_{\mathrm{in}} \mathrm{in}_{0.53} \mathrm{ga}_{0.47}$ as shallow twodimensional electron gas by surface etching, Phys. Stat. Sol. (c) 5 (2008) 322-325.

[175] A. M. Kreshchuk, S. V. Novikov, T. A. Polyanskaya, I. G. Savel'ev, Spin-orbit splitting and weak antilocalization in an asymmetric inas/inp quantum well, Semicond. Sci. Technol. 13 (1998) 384-388.

[176] S. A. Studenikin, P. T. Coleridge, N. Ahmed, P. J. Poole, A. Sachrajda, Experimental study of weak antilocalization effects in a highmobility in $_{x}$ ga $_{1-x}$ as/inp quantum well, Phys. Rev. B 68 (2003) 035317.

[177] P. S. Eldridge, W. J. H. Leyland, P. G. Lagoudakis, O. Z. Karimov, M. Henini, D. Taylor, R. T. Phillips, R. T. Harley, Temperature Dependence of Rashba Spin-orbit Coupling in Quantum Wells, 2007. ArXiv:0707.4493.

[178] P. S. Eldridge, W. J. H. Leyland, J. D. Mar, P. G. Lagoudakis, R. Winkler, O. Z. Karimov, M. Henini, D. Taylor, R. T. Phillips, R. T. Harley, Absence of the Rashba effect in undoped asymmetric quantum wells, 2008. ArXiv:0807.4845.

[179] P. S. Eldridge, W. J. H. Leyland, P. G. Lagoudakis, O. Z. Karimov, M. Henini, D. Taylor, R. T. Phillips, R. T. Harley, All-optical measurement of rashba coefficient in quantum wells, Phys. Rev. B 77 (2008) 125344.

[180] G. A. Khodaparast, R. E. Doezema, S. J. Chung, K. J. Goldammer, M. B. Santos, Spectroscopy of rashba spin splitting in insb quantum wells, Phys. Rev. B 70 (2004) 155322.

[181] B. Jusserand, D. Richards, H. Peric, B. Etienne, Zero-magnetic-field spin splitting in the gaas conduction band from raman scattering on modulation-doped quantum wells, Phys. Rev. Lett. 69 (1992) 848-851.

[182] R. G. Mani, J. H. Smet, K. von Klitzing, V. Narayanamurti, W. B. Johnson, V. Umansky, Radiation-induced oscillatory magnetoresistance as a sensitive probe of the zero-field spin-splitting in high-mobility gaas/al ${ }_{x}$ ga $_{1-x}$ as devices, Phys. Rev. B 69 (2004) 193304.

[183] V. V. Bel'kov, P. Olbrich, S. A. Tarasenko, D. Schuh, W. Wegscheider, T. Korn, C. Schüller, D. Weiss, W. Prettl, S. D. Ganichev, Symmetry and spin dephasing in (110)-grown quantum wells, Phys. Rev. Lett. 100 (2008) 176806.

[184] M. Nishioka, B. A. Gurney, E. E. Marinero, F. Mireles, Zero field spin splitting in AlSb/InAs/AlSb quantum wells induced by surface proximity effects, 2009. ArXiv:0912.2143.

[185] X. W. Zhang, J. B. Xia, Rashba spin-orbit coupling in insb nanowires under transverse electric field, Phys. Rev. B 74 (2006) 075304.

[186] E. A. de Andrada e Silva, G. C. La Rocca, Rashba spin splitting in semiconductor quantum wires, Phys. Rev. B 67 (2003) 165318.

[187] X. W. Zhang, J. B. Xia, Hole rashba effect and $g$-factor in inp nanowires, J. Phys. D 40 (2007) 541-546.

[188] X. W. Zhang, W. J. Fan, S. S. Li, J. B. Xia, Influence of n doping on the rashba coefficient, semiconductor-metal transition, and electron 
effective mass in insb ${ }_{1-x} \mathrm{n}_{x}$ nanowires: Ten-band $\mathbf{k} \cdot \mathbf{p}$ model, Phys. Rev. B 75 (2007) 205331.

[189] V. A. Guzenko, J. Knobbe, H. Hardtdegen, T. Schäpers, A. Bringer, Rashba effect in ingaas/inp parallel quantum wires, Appl. Phys. Lett. 88 (2006) 032102.

[190] V. A. Guzenko, A. Bringer, J. Knobbe, H. Hardtdegen, T. Schäpers, Rashba effect in ga $_{x}$ in $_{1-x}$ as/inp quantum wire structures, Appl. Phys. A 87 (2007) 577-584.

[191] S. Zhang, R. Liang, E. Zhang, L. Zhang, Y. Liu, Magnetosubbands of semiconductor quantum wires with rashba and dresselhaus spin-orbit coupling, Phys. Rev. B 73 (2006) 155316.

[192] M. A. Wilde, D. Reuter, C. Heyn, A. D. Wieck, D. Grundler, Inversion-asymmetry-induced spin splitting observed in the quantum oscillatory magnetization of a two-dimensional electron system, Phys. Rev. B 79 (2009) 125330.

[193] O. Z. Karimov, G. H. John, R. T. Harley, W. H. Lau, M. E. Flatté, M. Henini, R. Airey, High temperature gate control of quantum well spin memory, Phys. Rev. Lett. 91 (2003) 246601.

[194] N. S. Averkiev, L. E. Golub, Giant spin relaxation anisotropy in zinc-blende heterostructures, Phys. Rev. B 60 (1999) $15582-15584$.

[195] L. E. Golub, N. S. Averkiev, M. Willander, Electron spin relaxation in zinc-blende heterostructures, Nanotechnology 11 (2000) $215-217$.

[196] N. S. Averkiev, L. E. Golub, M. Willander, Spin relaxation anisotropy in two-dimensional semiconductor systems, J. Phys.: Condens. Matt. 14 (2002) R271-R283.

[197] J. Kainz, U. Rössler, R. Winkler, Anisotropic spin-splitting and spin-relaxation in asymmetric zinc blende semiconductor quantum structures, Phys. Rev. B 68 (2003) 075322.

[198] N. S. Averkiev, L. E. Golub, A. S. Gurevich, V. P. Evtikhiev, V. P. Kochereshko, A. V. Platonov, A. S. Shkolnik, Y. P. Efimov, Spinrelaxation anisotropy in asymmetrical (001) $\mathrm{al}_{x} \mathrm{ga}_{1-x}$ as quantum wells from hanle-effect measurements: Relative strengths of rashba and dresselhaus spin-orbit coupling, Phys. Rev. B 74 (2006) 033305.

[199] D. Stich, J. H. Jiang, T. Korn, R. Schulz, D. Schuh, W. Wegscheider, M. W. Wu, C. Schüller, Detection of large magnetoanisotropy of electron spin dephasing in a high-mobility two-dimensional electron system in a [001] gaas/al $\mathrm{gaa}_{1-x}$ as quantum well, Phys. Rev. B 76 (2007) 073309.

[200] S. D. Ganichev, V. V. Bel'kov, L. E. Golub, E. L. Ivchenko, P. Schneider, S. Giglberger, J. Eroms, J. De Boeck, G. Borghs, W. Wegscheider, D. Weiss, W. Prettl, Experimental separation of rashba and dresselhaus spin splittings in semiconductor quantum wells, Phys. Rev. Lett. 92 (2004) 256601.

[201] S. Giglberger, L. E. Golub, V. V. Bel'kov, S. N. Danilov, D. Schuh, C. Gerl, F. Rohlfing, J. Stahl, W. Wegscheider, D. Weiss, W. Prettl, S. D. Ganichev, Rashba and dresselhaus spin splittings in semiconductor quantum wells measured by spin photocurrents, Phys. Rev. B 75 (2007) 035327

[202] V. V. Bel'kov, S. D. Ganichev, Magneto-gyrotropic effects in semiconductor quantum wells, Semicond. Sci. Technol. 23 (2008) 114003 (11pp).

[203] R. Winkler, Spin orientation and spin precession in inversion-asymmetric quasi-two-dimensional electron systems, Phys. Rev. B 69 (2004) 045317.

[204] O. Madelung (Ed.), Semiconductors, Landolt-Börnstein, New Series, Group III-V, volume 17, Springer, Berlin, 1987.

[205] D. Culcer, C. Lechner, R. Winkler, Spin precession and alternating spin polarization in spin-3/2 hole systems, Phys. Rev. Lett. 97 (2006) 106601.

[206] R. Winkler, D. Culcer, S. J. Papadakis, B. Habib, M. Shayegan, Spin orientation of holes in quantum wells, Semicond. Sci. Technol. 23 (2008) 114017 (13pp).

[207] D. J. Hilton, C. L. Tang, Optical orientation and femtosecond relaxation of spin-polarized holes in gaas, Phys. Rev. Lett. 89 (2002) 146601.

[208] D. Culcer, R. Winkler, Spin polarization decay in spin-(1/2) and spin-(3/2) systems, Phys. Rev. B 76 (2007) 195204.

[209] Z. G. Yu, S. Krishnamurthy, M. van Schilfgaarde, N. Newman, Spin relaxation of electrons and holes in zinc-blende semiconductors, Phys. Rev. B 71 (2005) 245312

[210] M. Krauß, M. Aeschlimann, H. C. Schneider, Ultrafast spin dynamics including spin-orbit interaction in semiconductors, Phys. Rev. Lett. 100 (2008) 256601

[211] R. Winkler, Rashba spin splitting in two-dimensional electron and hole systems, Phys. Rev. B 62 (2000) $4245-4248$.

[212] B. Habib, E. Tutuc, S. Melinte, M. Shayegan, D. Wasserman, S. A. Lyon, R. Winkler, Negative differential rashba effect in two-dimensional hole systems, Appl. Phys. Lett. 85 (2004) 3151-3153.

[213] J. P. Lu, J. B. Yau, S. P. Shukla, M. Shayegan, L. Wissinger, U. Rössler, R. Winkler, Tunable spin-splitting and spin-resolved ballistic transport in gaas/algaas two-dimensional holes, Phys. Rev. Lett. 81 (1998) 1282-1285.

[214] S. J. Papadakis, E. P. De Poortere, H. C. Manoharan, M. Shayegan, R. Winkler, The Effect of Spin Splitting on the Metallic Behavior of a Two-Dimensional System, Science 283 (1999) 2056-2058.

[215] I. L. Aleiner, E. L. Ivchenko, Anisotropic exchange splitting in type-ii gaas/alas superlattices, JETP Lett. 55 (1992) $692-695$.

[216] L. Vervoort, R. Ferreira, P. Voisin, Effects of interface asymmetry on hole subband degeneracies and spin-relaxation rates in quantum wells, Phys. Rev. B 56 (1997) R12744-R12747.

[217] L. Vervoort, R. Ferreira, P. Voisin, Spin-splitting of the subbands of ingaas-inp and other 'no common atom' quantum wells, Semicond. Sci. Technol. 14 (1999) 227-230.

[218] U. Rössler, J. Kainz, Microscopic interface asymmetry and spin-splitting of electron subbands in semiconductor quantum structures, Solid State Commun. 121 (2002) 313-316.

[219] T. Guettler, A. L. C. Triques, L. Vervoort, R. Ferreira, P. Roussignol, P. Voisin, D. Rondi, J. C. Harmand, Optical polarization relaxation in $\mathrm{in}_{x} \mathrm{ga}_{1-x}$ as-based quantum wells: Evidence of the interface symmetry-reduction effect, Phys. Rev. B 58 (1998) R10179-R10182.

[220] C.-N. Chen, Y.-H. Wang, M.-P. Houng, J.-C. Chiang, Orientation dependence of interface inversion asymmetry effect on ingaas/inp quantum wells, Phys. Stat. Sol. (b) 231 (2002) 423-436.

[221] J. T. Olesberg, W. H. Lau, M. E. Flatté, C. Yu, E. Altunkaya, E. M. Shaw, T. C. Hasenberg, T. F. Boggess, Interface contributions to spin relaxation in a short-period inas/gasb superlattice, Phys. Rev. B 64 (2001) 201301.

[222] K. C. Hall, K. Gündoğdu, E. Altunkaya, W. H. Lau, M. E. Flatté, T. F. Boggess, J. J. Zinck, W. B. Barvosa-Carter, S. L. Skeith, Spin 
relaxation in (110) and (001) inas/gasb superlattices, Phys. Rev. B 68 (2003) 115311.

[223] N. R. Ogg, Conduction-band g factor anisotropy in indium antimonide, Proc. Phys. Soc. 89 (1966) $431-442$.

[224] V. G. Golubev, V. I. Ivanov-Omskii, I. G. Minervin, A. V. Osutin, D. G. Polyakov, Nonparabolicity and anisotropy of the electron energy spectrum in gaas, Sov. Phys. JETP 61 (1985) 1214-1219.

[225] M. J. Yang, R. J. Wagner, B. V. Shanabrook, J. R. Waterman, W. J. Moore, Spin-resolved cyclotron resonance in inas quantum wells: A study of the energy-dependent $g$ factor, Phys. Rev. B 47 (1993) 6807-6810.

[226] H. Mayer, U. Rössler, Spin splitting and anisotropy of cyclotron resonance in the conduction band of gaas, Phys. Rev. B 44 (1991) 9048-9051.

[227] M. A. Hopkins, R. J. Nicholas, P. Pfeffer, W. Zawadzki, D. Gauthier, J. C. Portal, M. A. DiForte-Poisson, A study of the conduction band non-parabolicity, anisotropy and spin splitting in gaas and inp, Semicond. Sci. Technol. 2 (1987) 568-577.

[228] M. Oestreich, W. W. Rühle, Temperature dependence of the electron landé $g$ factor in gaas, Phys. Rev. Lett. 74 (1995) $2315-2318$.

[229] T. Lai, X. Liu, H. Xu, Z. Jiao, J. Wen, W. Lin, Temperature dependence of electron-spin coherence in intrinsic bulk gaas, Appl. Phys. Lett. 88 (2006) 192106.

[230] J. Hübner, S. Döhrmann, D. Hägele, M. Oestreich, Temperature-dependent electron landé $g$ factor and the interband matrix element of gaas, Phys. Rev. B 79 (2009) 193307.

[231] K. L. Litvinenko, L. Nikzad, C. R. Pidgeon, J. Allam, L. F. Cohen, T. Ashley, M. Emeny, W. Zawadzki, B. N. Murdin, Temperature dependence of the electron landé $g$ factor in insb and gaas, Phys. Rev. B 77 (2008) 033204.

[232] W. Zawadzki, P. Pfeffer, R. Bratschitsch, Z. Chen, S. T. Cundiff, B. N. Murdin, C. R. Pidgeon, Temperature dependence of the electron spin $g$ factor in gaas, Phys. Rev. B 78 (2008) 245203.

[233] M. Poggio, R. C. Myers, N. P. Stern, A. C. Gossard, D. D. Awschalom, Structural, electrical, and magneto-optical characterization of paramagnetic gamnas quantum wells, Phys. Rev. B 72 (2005) 235313.

[234] P. Pfeffer, W. Zawadzki, Anisotropy of spin $g$ factor in gaas/ga ${ }_{1-x}$ al $_{x}$ as symmetric quantum wells, Phys. Rev. B 74 (2006) 233303.

[235] A. Malinowski, R. T. Harley, Anisotropy of the electron $g$ factor in lattice-matched and strained-layer iii-v quantum wells, Phys. Rev. B 62 (2000) 2051-2056.

[236] E. L. Ivchenko, A. A. Kiselev, Electron $g$ factor of quantum wells and superlattices, Sov. Phys. Semicond. 26 (1992) 827-831.

[237] H. W. Jiang, E. Yablonovitch, Gate-controlled electron spin resonance in gaas/al ga $_{1-x}$ as heterostructures, Phys. Rev. B 64 (2001) 041307.

[238] R. Winkler, S. J. Papadakis, E. P. De Poortere, M. Shayegan, Highly anisotropic g-factor of two-dimensional hole systems, Phys. Rev. Lett. 85 (2000) 4574-4577.

[239] R. Danneau, O. Klochan, W. R. Clarke, L. H. Ho, A. P. Micolich, M. Y. Simmons, A. R. Hamilton, M. Pepper, D. A. Ritchie, U. Zülicke, Zeeman splitting in ballistic hole quantum wires, Phys. Rev. Lett. 97 (2006) 026403

[240] D. Csontos, U. Zülicke, Large variations in the hole spin splitting of quantum-wire subband edges, Phys. Rev. B 76 (2007) 073313.

[241] D. Csontos, U. Zülicke, Tailoring hole spin splitting and polarization in nanowires, Appl. Phys. Lett. 92 (2008) 023108.

[242] J. C. H. Chen, O. Klochan, A. P. Micolich, A. R. Hamilton, T. P. Martin, L. H. Ho, U. Zuelicke, D. Reuter, A. D. Wieck, Observation of orientation- and \$k\$-dependent Zeeman spin-splitting in hole quantum wires on (100)-oriented AlGaAs/GaAs heterostructures, 2009. ArXiv:0909.5295.

[243] K.-M. Haendel, R. Winkler, U. Denker, O. G. Schmidt, R. J. Haug, Giant anisotropy of zeeman splitting of quantum confined acceptors in si/ge, Phys. Rev. Lett. 96 (2006) 086403.

[244] S. Roddaro, A. Fuhrer, P. Brusheim, C. Fasth, H. Q. Xu, L. Samuelson, J. Xiang, C. M. Lieber, Spin states of holes in ge/si nanowire quantum dots, Phys. Rev. Lett. 101 (2008) 186802.

[245] J. Y. Fu, M. W. Wu, Spin-orbit coupling in bulk zno and gan, J. Appl. Phys. 104 (2008) 093712.

[246] G. L. Bir, G. E. Pikus, Symmetry and Strain-Induced Effects in Semiconductors, John Wiley, New York, 1976.

[247] W. Weber, S. D. Ganichev, S. N. Danilov, D. Weiss, W. Prettl, Z. D. Kvon, V. V. Bel'kov, L. E. Golub, H.-I. Cho, J.-H. Lee, Demonstration of rashba spin splitting in gan-based heterostructures, Appl. Phys. Lett. 87 (2005) 262106.

[248] W.-T. Wang, C. L. Wu, S. F. Tsay, M. H. Gau, I. Lo, H. F. Kao, D. J. Jang, J.-C. Chiang, M.-E. Lee, Y.-C. Chang, C.-N. Chen, H. C. Hsueh, Dresselhaus effect in bulk wurtzite materials, Appl. Phys. Lett. 91 (2007) 082110.

[249] L. C. Lew Yan Voon, M. Willatzen, M. Cardona, N. E. Christensen, Terms linear in k in the band structure of wurtzite-type semiconductors, Phys. Rev. B 53 (1996) 10703-10714.

[250] J. A. Majewski, P. Vogl, Physics of Semiconductors: $27^{\text {th }}$ International Conference on the Physics of Semiconductors, American Institute of Physics, New York, 2005.

[251] Ç. Kurdak, N. Biyikli, U. Özgür, H. Morkoç, V. I. Litvinov, Weak antilocalization and zero-field electron spin splitting in al ${ }_{x}$ ga $_{1-x} \mathrm{n} / \mathrm{aln}_{\mathrm{g}} \mathrm{gan}$ heterostructures with a polarization-induced two-dimensional electron gas, Phys. Rev. B 74 (2006) 113308.

[252] N. Thillosen, T. Schäpers, N. Kaluza, H. Hardtdegen, V. A. Guzenko, Weak antilocalization in a polarization-doped $\mathrm{al}_{x}$ ga $_{1-x} \mathrm{n} / \mathrm{gan}_{\mathrm{ga}} \mathrm{het}_{-}$ erostructure with single subband occupation, Appl. Phys. Lett. 88 (2006) 022111.

[253] S. Schmult, M. J. Manfra, A. Punnoose, A. M. Sergent, K. W. Baldwin, R. J. Molnar, Large bychkov-rashba spin-orbit coupling in high-mobility gan/al $\mathrm{ga}_{1-x} \mathrm{n}$ heterostructures, Phys. Rev. B 74 (2006) 033302.

[254] A. E. Belyaev, V. G. Raicheva, A. M. Kurakin, N. Klein, S. A. Vitusevich, Investigation of spin-orbit interaction in algan/gan heterostructures with large electron density, Phys. Rev. B 77 (2008) 035311.

[255] J. H. Buß, J. Rudolph, F. Natali, F. Semond, D. Hägele, Anisotropic electron spin relaxation in bulk gan, Appl. Phys. Lett. 95 (2009) 192107.

[256] Y. Q. Tang, B. Shen, X. W. He, K. Han, N. Tang, W. H. Chen, Z. J. Yang, G. Y. Zhang, Y. H. Chen, C. G. Tang, Z. G. Wang, K. S. Cho, Y. F. Chen, Room-temperature spin-oriented photocurrent under near-infrared irradiation and comparison of optical means with shubnikov de-haas measurements in $\mathrm{al}_{x} \mathrm{ga}_{1-x} \mathrm{n} /$ gan heterostructures, Appl. Phys. Lett. 91 (2007) 071920.

[257] K. S. Cho, C.-T. Liang, Y. F. Chen, Y. Q. Tang, B. Shen, Spin-dependent photocurrent induced by rashba-type spin splitting in $\mathrm{al}_{0.25} \mathrm{ga}_{0.75} \mathrm{n} / \mathrm{gan}$ heterostructures, Phys. Rev. B 75 (2007) 085327.

[258] X. W. He, B. Shen, Y. Q. Tang, N. Tang, C. M. Yin, F. J. Xu, Z. J. Yang, G. Y. Zhang, Y. H. Chen, C. G. Tang, Z. G. Wang, Circular 
photogalvanic effect of the two-dimensional electron gas in $\mathrm{al}_{x} \mathrm{ga}_{1-x} \mathrm{n} / \mathrm{gan}$ heterostructures under uniaxial strain, Appl. Phys. Lett. 91 (2007) 071912.

[259] K. S. Cho, Y. F. Chen, Y. Q. Tang, B. Shen, Photogalvanic effects for interband absorption in algan/gan superlattices, Appl. Phys. Lett. 90 (2007) 041909.

[260] X. W. He, B. Shen, Y. H. Chen, Q. Zhang, K. Han, C. M. Yin, N. Tang, F. J. Xu, C. G. Tang, Z. J. Yang, Z. X. Qin, G. Y. Zhang, Z. G. Wang, Anomalous photogalvanic effect of circularly polarized light incident on the two-dimensional electron gas in al $\mathrm{ga}_{1-x} \mathrm{n} / \mathrm{gan} \mathrm{heterostructures}$ at room temperature, Phys. Rev. Lett. 101 (2008) 147402.

[261] K. Tsubaki, N. Maeda, T. Saitoh, N. Kobayashi, Spin splitting in modulation-doped algan/gan two-dimensional electron gas, Appl. Phys. Lett. 80 (2002) 3126-3128.

[262] N. Tang, B. Shen, K. Han, F.-C. Lu, Z.-X. Qin, G.-Y. Zhang, Abnormal shubnikov-de haas oscillations of the two-dimensional electron gas in $\mathrm{al}_{x} \mathrm{ga}_{1-x} \mathrm{n} /$ gan heterostructures in tilted magnetic fields, Phys. Rev. B 79 (2009) 073304.

[263] N. Tang, B. Shen, M. J. Wang, K. Han, Z. J. Yang, K. Xu, G. Y. Zhang, T. Lin, B. Zhu, W. Z. Zhou, J. H. Chu, Beating patterns in the oscillatory magnetoresistance originated from zero-field spin splitting in $\mathrm{al}_{x} \mathrm{ga}_{1-x} \mathrm{n} /$ gan heterostructures, Appl. Phys. Lett. 88 (2006) 172112.

[264] K. S. Cho, T.-Y. Huang, H.-S. Wang, M.-G. Lin, T.-M. Chen, C.-T. Liang, Y. F. Chen, I. Lo, Zero-field spin splitting in modulation-doped $\mathrm{al}_{x} \mathrm{ga}_{1-x} \mathrm{n}$ /gan two-dimensional electron systems, Appl. Phys. Lett. 86 (2005) 222102.

[265] W. Z. Zhou, T. Lin, L. Y. Shang, L. Sun, K. H. Gao, Y. M. Zhou, G. Yu, N. Tang, K. Han, B. Shen, S. L. Guo, Y. S. Gui, J. H. Chu, Weak antilocalization and beating pattern in high electron mobility $\mathrm{al}_{x} \mathrm{ga}_{1-x} \mathrm{n} / \mathrm{gan}$ two-dimensional electron gas with strong rashba spin-orbit coupling, J. Appl. Phys. 104 (2008) 053703.

[266] K. S. Cho, C.-T. Liang, Y. F. Chen, J. C. Fan, Demonstration of rashba spin splitting in an al0.25ga0.75n/gan heterostructure by microwavemodulated shubnikov-de haas oscillations, Semicond. Sci. Technol. 22 (2007) 870-874.

[267] W. Z. Zhou, T. Lin, L. Y. Shang, L. Sun, K. H. Gao, Y. M. Zhou, G. Yu, N. Tang, K. Han, B. Shen, S. L. Guo, Y. S. Gui, J. H. Chu, Influence of the illumination on weak antilocalization in an $\mathrm{al}_{x} \mathrm{ga}_{1-x} \mathrm{n} / \mathrm{gan}$ heterostructure with strong spin-orbit coupling, Appl. Phys. Lett. 93 (2008) 262104

[268] T. Schäpers, N. Thillosen, S. Cabañas, N. Kaluza, V. A. Guzenko, H. Hardtdegen, Spin-orbit coupling in gated algan/gan 2-dimensional electron gases, Phys. Stat. Sol. (c) 3 (2006) 4247-4250.

[269] M. Konig, S. Wiedmann, C. Brune, A. Roth, H. Buhmann, L. W. Molenkamp, X.-L. Qi, S.-C. Zhang, Quantum Spin Hall Insulator State in HgTe Quantum Wells, Science 318 (2007) 766-770.

[270] M. König, H. Buhmann, L. W. Molenkamp, T. Hughes, C.-X. Liu, X.-L. Qi, S.-C. Zhang, The quantum spin hall effect: Theory and experiment, J. Phys. Soc. Jpn. 77 (2008) 031007.

[271] A. Dargys, Spin properties of 2d charge carriers in semiconductors with inverted bands, Europhys. Lett. 81 (2008) 38003 (4pp).

[272] Y. S. Gui, J. Liu, V. Daumer, C. R. Becker, H. Buhmann, L. W. Molenkamp, Large rashba spin-orbit splitting in gate controlled n-type modulation doped hgte/hg0.3cd0.7-xmnxte quantum wells, Physica E 12 (2002) 416-419.

[273] M. Schultz, F. Heinrichs, U. Merkt, T. Colin, T. Skauli, S. Løvold, Rashba spin splitting in a gated hgte quantum well, Semicond. Sci. Technol. 11 (1996) 1168-1172.

[274] V. F. Radantsev, A. M. Yafyasov, Rashba splitting in mis structures hgcdte, JETP 95 (2002) 491-501.

[275] C. R. Becker, Y. S. Gui, J. Liu, V. Daumer, K. Ortner, V. Hock, A. Pfeuffer-Jeschke, H. Buhmann, L. W. Molenkamp, Growth and magneto-transport of gate controlled n-type hgte/ $\mathrm{hg}_{0.3} \mathrm{~cd}_{0.7}$ te quantum wells with the inclusion of mn, Phys. Stat. Sol. (b) 229 (2002) $775-779$.

[276] J. Hinz, H. Buhmann, M. Schafer, V. Hock, C. R. Becker, L. W. Molenkamp, Gate control of the giant rashba effect in hgte quantum wells, Semicond. Sci. Technol. 21 (2006) 501-506.

[277] X. C. Zhang, A. Pfeuffer-Jeschke, K. Ortner, V. Hock, H. Buhmann, C. R. Becker, G. Landwehr, Rashba splitting in n-type modulationdoped hgte quantum wells with an inverted band structure, Phys. Rev. B 63 (2001) 245305.

[278] V. F. Radantsev, A. M. Yafyasov, V. B. Bogevolnov, I. M. Ivankiv, O. Y. Shevchenko, Zero-field spin splitting in hgte surface quantum well, Surf. Sci. 482-485 (2001) 989-993.

[279] L. Grenet, M. Jamet, P. Noé, V. Calvo, J.-M. Hartmann, L. E. Nistor, B. Rodmacq, S. Auffret, P. Warin, Y. Samson, Spin injection in silicon at zero magnetic field, Appl. Phys. Lett. 94 (2009) 032502.

[280] B. Huang, D. J. Monsma, I. Appelbaum, Experimental realization of a silicon spin field-effect transistor, Appl. Phys. Lett. 91 (2007) 072501.

[281] O. M. J. van 't Erve, A. T. Hanbicki, M. Holub, C. H. Li, C. Awo-Affouda, P. E. Thompson, B. T. Jonker, Electrical injection and detection of spin-polarized carriers in silicon in a lateral transport geometry, Appl. Phys. Lett. 91 (2007) 212109.

[282] B. T. Jonker, G. Kioseoglou, A. T. Hanbicki, C. H. Li, P. E. Thompson, Electrical spin-injection into silicon from a ferromagnetic metal/tunnel barrier contact, Nat. Phys. 3 (2007) 542-546.

[283] H. W. Liu, T. Fujisawa, Y. Ono, H. Inokawa, A. Fujiwara, K. Takashina, Y. Hirayama, Pauli-spin-blockade transport through a silicon double quantum dot, Phys. Rev. B 77 (2008) 073310.

[284] N. Shaji, C. B. Simmons, M. Thalakulam, L. J. Klein, H. Qin, H. Luo, D. E. Savage, M. G. Lagally, A. J. Rimberg, R. Joynt, M. Friesen, R. H. Blick, S. N. Coppersmith, M. A. a. Eriksson, Spin blockade and lifetime-enhanced transport in a few-electron si/sige double quantum dot, Nat. Phys. 4 (2008) 540-544.

[285] Y. Hu, H. O. H. Churchill, D. J. Reilly, J. Xiang, C. M. Lieber, C. M. Marcus, A ge//si heterostructure nanowire-based double quantum dot with integrated charge sensor, Nat. Nano. 2 (2007) 622-625.

[286] M. Xiao, I. Martin, E. Yablonovitch, H. W. Jiang, Electrical detection of the spin resonance of a single electron in a silicon field-effect transistor, Nature 430 (2004) 435-439.

[287] C. Boehme, K. Lips, Electrical detection of spin coherence in silicon, Phys. Rev. Lett. 91 (2003) 246603.

[288] A. M. Tyryshkin, J. J. L. Morton, S. C. Benjamin, A. Ardavan, G. A. D. Briggs, J. W. Ager, S. A. Lyon, Coherence of spin qubits in silicon, J. Phys.: Condens. Matt. 18 (2006) S783-S794. 
[289] R. de Sousa, S. Das Sarma, Theory of nuclear-induced spectral diffusion: Spin decoherence of phosphorus donors in si and gaas quantum dots, Phys. Rev. B 68 (2003) 115322.

[290] C. Tahan, R. Joynt, Rashba spin-orbit coupling and spin relaxation in silicon quantum wells, Phys. Rev. B 71 (2005) 075315.

[291] L. E. Golub, E. L. Ivchenko, Spin splitting in symmetrical sige quantum wells, Phys. Rev. B 69 (2004) 115333.

[292] M. O. Nestoklon, L. E. Golub, E. L. Ivchenko, Spin and valley-orbit splittings in sige/si heterostructures, Phys. Rev. B 73 (2006) 235334.

[293] M. O. Nestoklon, E. L. Ivchenko, J.-M. Jancu, P. Voisin, Electric field effect on electron spin splitting in sige/si quantum wells, Phys. Rev. B 77 (2008) 155328.

[294] E. Y. Sherman, Random spin-orbit coupling and spin relaxation in symmetric quantum wells, Appl. Phys. Lett. 82 (2003) $209-211$.

[295] Z. Wilamowski, W. Jantsch, H. Malissa, U. Rössler, Evidence and evaluation of the bychkov-rashba effect in sige/si/sige quantum wells, Phys. Rev. B 66 (2002) 195315.

[296] Z. Wilamowski, W. Jantsch, Esr studies of the bychkov-rashba field in modulation doped si/sige quantum wells, Physica E 12 (2002) 439-442.

[297] Z. Wilamowski, W. Jantsch, N. Sandersfeld, M. Mühlberger, F. Schäffler, S. Lyon, Spin relaxation and $g$-factor of two-dimensional electrons in si/sige quantum wells, Physica E 16 (2003) 111-120.

[298] B. A. Glavin, K. W. Kim, Spin relaxation of two-dimensional holes in strained asymmetric sige quantum wells, Phys. Rev. B 71 (2005) 035321.

[299] P. Zhang, M. W. Wu, Hole spin relaxation in [001] strained asymmetric si/sige and ge/sige quantum wells, Phys. Rev. B 80 (2009) 155311.

[300] J. Schliemann, A. Khaetskii, D. Loss, Electron spin dynamics in quantum dots and related nanostructures due to hyperfine interaction with nuclei, J. Phys.: Condens. Matt. 15 (2003) R1809-R1833.

[301] J. Fischer, W. A. Coish, D. V. Bulaev, D. Loss, Spin decoherence of a heavy hole coupled to nuclear spins in a quantum dot, Phys. Rev. B 78 (2008) 155329.

[302] A. I. Ekimov, V. I. Safarov, Optical detection of dynamic of nuclei in semiconductors, JETP Lett. 15 (1972) 179.

[303] D. Paget, G. Lampel, B. Sapoval, V. I. Safarov, Low field electron-nuclear spin coupling in gallium arsenide under optical pumping conditions, Phys. Rev. B 15 (1977) 5780-5796.

[304] S. Tarucha, J. Baugh, Magnetic and electrical control of electron-nuclear spin coupling in gaas double quantum dots, J. Phys. Soc. Jpn. 77 (2008) 031011.

[305] J. Baugh, Y. Kitamura, K. Ono, S. Tarucha, Large nuclear overhauser fields detected in vertically coupled double quantum dots, Phys. Rev. Lett. 99 (2007) 096804

[306] J. R. Petta, J. M. Taylor, A. C. Johnson, A. Yacoby, M. D. Lukin, C. M. Marcus, M. P. Hanson, A. C. Gossard, Dynamic nuclear polarization with single electron spins, Phys. Rev. Lett. 100 (2008) 067601.

[307] O. Krebs, B. Eble, A. Lemaître, P. Voisin, B. Urbaszek, T. Amand, X. Marie, Hyperfine interaction in InAs/GaAs self-assembled quantum dots: dynamical nuclear polarization versus spin relaxation, Comptes Rendus Physique 9 (2008) 874-884.

[308] M. J. R. Hoch, J. Lu, P. L. Kuhns, W. G. Moulton, A. P. Reyes, Current-induced dynamic nuclear polarization in gaas, Phys. Rev. B 72 (2005) 233204

[309] J. Strand, B. D. Schultz, A. F. Isakovic, C. J. Palmstrøm, P. A. Crowell, Dynamic nuclear polarization by electrical spin injection in ferromagnet-semiconductor heterostructures, Phys. Rev. Lett. 91 (2003) 036602.

[310] B. Eble, O. Krebs, A. Lemaître, K. Kowalik, A. Kudelski, P. Voisin, B. Urbaszek, X. Marie, T. Amand, Dynamic nuclear polarization of a single charge-tunable inas/gaas quantum dot, Phys. Rev. B 74 (2006) 081306.

[311] J. M. Kikkawa, D. D. Awschalom, All-Optical Magnetic Resonance in Semiconductors, Science 287 (2000) $473-476$.

[312] J. M. Taylor, C. M. Marcus, M. D. Lukin, Long-lived memory for mesoscopic quantum bits, Phys. Rev. Lett. 90 (2003) 206803.

[313] W. M. Witzel, S. Das Sarma, Nuclear spins as quantum memory in semiconductor nanostructures, Phys. Rev. B 76 (2007) 045218.

[314] J. J. L. Morton, A. M. Tyryshkin, R. M. Brown, S. Shankar, B. W. Lovett, A. Ardavan, T. Schenkel, E. E. Haller, J. W. Ager, S. A. Lyon, Solid-state quantum memory using the 31p nuclear spin, Nature 455 (2008) 1085-1088.

[315] P. Kacman, Spin interactions in diluted magnetic semiconductors and magnetic semiconductor structures, Semicond. Sci. Technol. 16 (2001) R25-R39.

[316] P. W. Anderson, Localized magnetic states in metals, Phys. Rev. 124 (1961) 41-53.

[317] R. C. Myers, M. Poggio, N. P. Stern, A. C. Gossard, D. D. Awschalom, Antiferromagnetic $s-d$ exchange coupling in gamnas, Phys. Rev. Lett. 95 (2005) 017204

[318] G. Burkard, D. Loss, D. P. DiVincenzo, Coupled quantum dots as quantum gates, Phys. Rev. B 59 (1999) $2070-2078$.

[319] R. Hanson, B. Witkamp, L. M. K. Vandersypen, L. H. W. van Beveren, J. M. Elzerman, L. P. Kouwenhoven, Zeeman energy and spin relaxation in a one-electron quantum dot, Phys. Rev. Lett. 91 (2003) 196802.

[320] D. M. Zumbühl, C. M. Marcus, M. P. Hanson, A. C. Gossard, Cotunneling spectroscopy in few-electron quantum dots, Phys. Rev. Lett. 93 (2004) 256801.

[321] K. V. Kavokin, Optical manifestations of electron spin transport and relaxation in semiconductors, Phys. Stat. Sol. (a) 190 (2002) $221-227$.

[322] K. V. Kavokin, Symmetry of anisotropic exchange interactions in semiconductor nanostructures, Phys. Rev. B 69 (2004) 075302.

[323] K. V. Kavokin, Anisotropic exchange interaction of localized conduction-band electrons in semiconductors, Phys. Rev. B 64 (2001) 075305.

[324] W. O. Putikka, R. Joynt, Theory of optical orientation in $n$-type semiconductors, Phys. Rev. B 70 (2004) 113201

[325] L. P. Gor'kov, P. L. Krotkov, Spin relaxation and antisymmetric exchange in n-doped iii-v semiconductors, Phys. Rev. B 67 (2003) 033203.

[326] T. Korn, D. Stich, R. Schulz, D. Schuh, W. Wegscheider, C. Schüller, Spin dynamics in high-mobility two-dimensional electron systems, Adv. Solid State Phys. 48 (2009) 143.

[327] F. Zhang, H. Z. Zheng, Y. Ji, J. Liu, G. R. Li, Electrical control of dynamic spin splitting induced by exchange interaction as revealed by time-resolved kerr rotation in a degenerate spin-polarized electron gas, Europhys. Lett. 83 (2008) 47006 (6pp).

[328] U. Weiss, Quantum dissipative systems, World Scientific, Singapore, 3 edition, 2008.

[329] C. P. Slichter, Principles of magnetic resonance, Springer-Verlag, Berlin, 1980. 
[330] L. Allen, J. H. Eberly, Optical resonance and two-level atoms, Courier Dover Publications, New York, 1975.

[331] A. Greilich, D. R. Yakovlev, A. Shabaev, A. L. Efros, I. A. Yugova, R. Oulton, V. Stavarache, D. Reuter, A. Wieck, M. Bayer, Mode Locking of Electron Spin Coherences in Singly Charged Quantum Dots, Science 313 (2006) 341-345.

[332] M. W. Wu, Spin dephasing induced by inhomogeneous broadening in d'yakonov-perel' effect in a $n$-doped gaas quantum well, J. Phys. Soc. Jpn. 70 (2001) 2195-2198.

[333] A. D. Margulis, V. A. Margulis, Spin relaxation of conduction electrons in semiconductors in a strong magnetic field. the precession mechanism, Sov. Phys. Solid State 25 (1983) 918-921.

[334] M. W. Wu, C. Z. Ning, A novel mechanism for spin dephasing due to spin-conserving scatterings, Eur. Phys. J. B 18 (2000) $373-376$.

[335] C. F. Destefani, S. E. Ulloa, G. E. Marques, Spin-orbit coupling and intrinsic spin mixing in quantum dots, Phys. Rev. B 69 (2004) 125302.

[336] M. Q. Weng, M. W. Wu, Longitudinal spin decoherence in spin diffusion in semiconductors, Phys. Rev. B 66 (2002) 235109.

[337] P. Kleinert, V. V. Bryksin, Electric-field-induced long-lived spin excitations in two-dimensional spin-orbit coupled systems, Phys. Rev. B 79 (2009) 045317.

[338] B. A. Bernevig, J. Hu, Semiclassical theory of diffusive-ballistic crossover and the persistent spin helix, Phys. Rev. B 78 (2008) 245123.

[339] J. Schliemann, J. C. Egues, D. Loss, Nonballistic spin-field-effect transistor, Phys. Rev. Lett. 90 (2003) 146801.

[340] X. Cartoixà, D. Z.-Y. Ting, Y.-C. Chang, A resonant spin lifetime transistor, Appl. Phys. Lett. 83 (2003) $1462-1464$.

[341] C. W. Gardiner, P. Zoller, Quantum noise, Springer, Berlin, 2004.

[342] D. Pines, C. P. Slichter, Relaxation times in magnetic resonance, Phys. Rev. 100 (1955) 1014-1020.

[343] V. N. Golovach, A. Khaetskii, D. Loss, Phonon-induced decay of the electron spin in quantum dots, Phys. Rev. Lett. 93 (2004) 016601.

[344] J. H. Jiang, Y. Y. Wang, M. W. Wu, Reexamination of spin decoherence in semiconductor quantum dots from the equation-of-motion approach, Phys. Rev. B 77 (2008) 035323.

[345] J. Shah, Ultrafast spectroscopy of semiconductors and semiconductor nanostructures, Springer-Verlag, Berlin, 1996.

[346] H. Haug, S. W. Koch, Quantum Theory of the Optical and Electronic Properties of Semiconductors, World Scientific, Singapore, 1993.

[347] T. Kuhn, F. Rossi, Analysis of coherent and incoherent phenomena in photoexcited semiconductors: A monte carlo approach, Phys. Rev. Lett. 69 (1992) 977-980.

[348] Y. V. Pershin, Optically induced suppression of spin relaxation in two-dimensional electron systems with rashba interaction, Phys. Rev. B 75 (2007) 165320.

[349] M. W. Wu, C. Z. Ning, D’yakonov-perel' effect on spin dephasing in $n$-type gaas, Phys. Stat. Sol. (b) 222 (2000) 523-534

[350] M. W. Wu, H. Metiu, Kinetics of spin coherence of electrons in an undoped semiconductor quantum well, Phys. Rev. B 61 (2000) 2945-2956.

[351] X. R. Wang, Y. S. Zheng, S. Yin, Spin relaxation and decoherence of two-level systems, Phys. Rev. B 72 (2005) 121303.

[352] C. E. Pryor, M. E. Flatté, Accuracy of circular polarization as a measure of spin polarization in quantum dot qubits, Phys. Rev. Lett. 91 (2003) 257901

[353] Y. Masumoto, S. Oguchi, B. Pal, M. Ikezawa, Spin dephasing of doped electrons in charge-tunable inp quantum dots: Hanle-effect measurements, Phys. Rev. B 74 (2006) 205332.

[354] N. P. Stern, M. Poggio, M. H. Bartl, E. L. Hu, G. D. Stucky, D. D. Awschalom, Spin dynamics in electrochemically charged cdse quantum dots, Phys. Rev. B 72 (2005) 161303.

[355] F. Simon, B. Dóra, F. Murányi, A. Jánossy, S. Garaj, L. Forró, S. Bud'ko, C. Petrovic, P. C. Canfield, Generalized elliott-yafet theory of electron spin relaxation in metals: Origin of the anomalous electron spin lifetime in mgb 2 , Phys. Rev. Lett. 101 (2008) 177003.

[356] A. W. Overhauser, Paramagnetic relaxation in metals, Phys. Rev. 89 (1953) 689-700.

[357] F. Beuneu, P. Monod, The elliott relation in pure metals, Phys. Rev. B 18 (1978) 2422-2425.

[358] P. Monod, F. Beuneu, Conduction-electron spin flip by phonons in metals: Analysis of experimental data, Phys. Rev. B 19 (1979) $911-916$.

[359] J. Fabian, S. Das Sarma, Spin relaxation of conduction electrons in polyvalent metals: Theory and a realistic calculation, Phys. Rev. Lett. 81 (1998) 5624-5627.

[360] J. Fabian, S. Das Sarma, Phonon-induced spin relaxation of conduction electrons in aluminum, Phys. Rev. Lett. 83 (1999) $1211-1214$.

[361] J.-N. Chazalviel, Spin relaxation of conduction electrons in $n$-type indium antimonide at low temperature, Phys. Rev. B 11 (1975) $1555-1562$.

[362] J. L. Cheng, M. W. Wu, J. Fabian, Theory of the spin relaxation of conduction electrons in silicon, Phys. Rev. Lett. 104 (2010) 016601.

[363] C. Lü, J. L. Cheng, M. W. Wu, Hole spin dephasing in p-type semiconductor quantum wells, Phys. Rev. B 73 (2006) 125314.

[364] V. N. Gridnev, Theory of faraday rotation beats in quantum wells with large spin splitting, JETP Lett. 74 (2001) $380-383$.

[365] C. Grimaldi, Electron spin dynamics in impure quantum wells for arbitrary spin-orbit coupling, Phys. Rev. B 72 (2005) 075307.

[366] R. T. Harley, Spin dynamics of free carriers in quantum wells, in: [23], pp. 29-52.

[367] W. J. H. Leyland, R. T. Harley, M. Henini, A. J. Shields, I. Farrer, D. A. Ritchie, Oscillatory dyakonov-perel spin dynamics in twodimensional electron gases, Phys. Rev. B 76 (2007) 195305.

[368] M. A. Brand, A. Malinowski, O. Z. Karimov, P. A. Marsden, R. T. Harley, A. J. Shields, D. Sanvitto, D. A. Ritchie, M. Y. Simmons, Precession and motional slowing of spin evolution in a high mobility two-dimensional electron gas, Phys. Rev. Lett. 89 (2002) 236601.

[369] M. I. D’yakonov, V. Y. Kachorovskii, Spin relaxation of two-dimensional electrons in noncentrosymmetric semiconductors, Sov. Phys. Semicond. 20 (1986) 110-112.

[370] N. S. Averkiev, L. E. Golub, Spin relaxation anisotropy: microscopic mechanisms for 2d systems, Semicond. Sci. Technol. 23 (2008) 114002 (6pp).

[371] R. I. Dzhioev, K. V. Kavokin, V. L. Korenev, M. V. Lazarev, N. K. Poletaev, B. P. Zakharchenya, E. A. Stinaff, D. Gammon, A. S. Bracker, M. E. Ware, Suppression of dyakonov-perel spin relaxation in high-mobility $n$-gaas, Phys. Rev. Lett. 93 (2004) 216402.

[372] J. Zhou, J. L. Cheng, M. W. Wu, Spin relaxation in $n$-type gaas quantum wells from a fully microscopic approach, Phys. Rev. B 75 (2007) 045305 .

[373] E. L. Ivchenko, Spin relaxation of free carriers in a noncentrosymmetric semiconductor in a longitudinal magnetic field, Sov. Phys. Solid State 15 (1973) 1048 
[374] F. X. Bronold, I. Martin, A. Saxena, D. L. Smith, Magnetic-field dependence of electron spin relaxation in $n$-type semiconductors, Phys. Rev. B 66 (2002) 233206.

[375] V. I. Puller, L. G. Mourokh, N. J. M. Horing, A. Y. Smirnov, Electron spin relaxation in a semiconductor quantum well, Phys. Rev. B 67 (2003) 155309

[376] A. A. Burkov, L. Balents, Spin relaxation in a two-dimensional electron gas in a perpendicular magnetic field, Phys. Rev. B 69 (2004) 245312.

[377] M. M. Glazov, Magnetic field effects on spin relaxation in heterostructures, Phys. Rev. B 70 (2004) 195314.

[378] O. Bleibaum, Spin relaxation in the presence of crossed electric and magnetic fields: A quasiclassical approach, Phys. Rev. B 71 (2005) 235318.

[379] O. Bleibaum, Magnetic field effects in biased semiconductor heterostructures with rashba spin-orbit interaction, Phys. Rev. B 71 (2005) 195329.

[380] M. M. Glazov, Effect of structure anisotropy on low temperature spin dynamics in quantum wells, Solid State Commun. 142 (2007) 531-535.

[381] M. M. Glazov, E. Y. Sherman, Non-markovian spin relaxation in two-dimensional electron gas, Europhys. Lett. 76 (2006) $102-108$.

[382] D. M. Frenkel, Spin relaxation in gaas- $\mathrm{al}_{x} \mathrm{ga}_{1-x}$ as heterostructures in high magnetic fields, Phys. Rev. B 43 (1991) $14228-14231$.

[383] G. Bastard, Spin-flip relaxation time of quantum-well electrons in a strong magnetic field, Phys. Rev. B 46 (1992) $4253-4256$

[384] W. Apel, Y. A. Bychkov, Spin relaxation in quantum hall systems, Phys. Rev. Lett. 82 (1999) 3324-3327.

[385] S. Dickmann, Spin relaxation in the quantized hall regime in the presence of disorder, JETP Lett. 78 (2003) $452-456$.

[386] S. M. Dikman, S. V. Iordanskii, Spin relaxation of two-dimensional electrons with an odd landau-level filling factor in a strong magnetic field, Sov. Phys. JETP 83 (1996) 128-145.

[387] E. Y. Sherman, J. E. Sipe, Coherent spin dynamics in quantum wells in a quantizing magnetic field, Phys. Rev. B 73 (2006) 205335.

[388] S. Döhrmann, D. Hägele, J. Rudolph, M. Bichler, D. Schuh, M. Oestreich, Anomalous spin dephasing in (110) gaas quantum wells: Anisotropy and intersubband effects, Phys. Rev. Lett. 93 (2004) 147405.

[389] R. Winkler, Spin density matrix of spin-3/2 hole systems, Phys. Rev. B 70 (2004) 125301.

[390] M. Z. Maialle, Spin relaxation of electrons in p-doped quantum wells via the electron-hole exchange interaction, Phys. Rev. B 54 (1996) 1967-1974.

[391] M. Z. Maialle, M. H. Degani, Electron-spin relaxation in p-type quantum wells via the electron-hole exchange interaction:the effects of the valence-band spin mixing and of an applied longitudinal electric field, Phys. Rev. B 55 (1997) 13771-13777.

[392] A. Amo, L. Viña, P. Lugli, C. Tejedor, A. I. Toropov, K. S. Zhuravlev, Pauli blockade of the electron spin flip in bulk gaas, Phys. Rev. B 75 (2007) 085202.

[393] B. Baylac, T. Amand, X. Marie, B. Dareys, M. Brousseau, G. Bacquet, V. Thierry-Mieg, Hole spin relaxation in n-modulation doped quantum wells, Solid State Commun. 93 (1995) 57-60.

[394] P. Roussignol, P. Rolland, R. Ferreira, C. Delalande, G. Bastard, A. Vinattieri, J. Martinez-Pastor, L. Carraresi, M. Colocci, J. F. Palmier, B. Etienne, Hole polarization and slow hole-spin relaxation in an n-doped quantum-well structure, Phys. Rev. B 46 (1992) $7292-7295$.

[395] M. Z. Maialle, Spin polarization of holes on the valence-band edge of a semiconductor quantum well, Semicond. Sci. Technol. 13 (1998) $852-857$.

[396] J. A. Gupta, D. D. Awschalom, A. L. Efros, A. V. Rodina, Spin dynamics in semiconductor nanocrystals, Phys. Rev. B 66 (2002) 125307.

[397] J. A. Gupta, D. D. Awschalom, X. Peng, A. P. Alivisatos, Spin coherence in semiconductor quantum dots, Phys. Rev. B 59 (1999) R10421-R10424.

[398] Z. Chen, S. G. Carter, R. Bratschitsch, P. Dawson, S. T. Cundiff, Effects of disorder on electron spin dynamics in a semiconductor quantum well, Nat. Phys. 3 (2007) 265-269.

[399] Y. G. Semenov, K. N. Borysenko, K. W. Kim, Spin-phase relaxation of two-dimensional holes localized in a fluctuating potential, Phys. Rev. B 66 (2002) 113302

[400] M. Syperek, D. R. Yakovlev, A. Greilich, J. Misiewicz, M. Bayer, D. Reuter, A. D. Wieck, Spin coherence of holes in gaas/(al,ga)as quantum wells, Phys. Rev. Lett. 99 (2007) 187401.

[401] P.-F. Braun, X. Marie, L. Lombez, B. Urbaszek, T. Amand, P. Renucci, V. K. Kalevich, K. V. Kavokin, O. Krebs, P. Voisin, Y. Masumoto, Direct observation of the electron spin relaxation induced by nuclei in quantum dots, Phys. Rev. Lett. 94 (2005) 116601.

[402] F. H. L. Koppens, K. C. Nowack, L. M. K. Vandersypen, Spin echo of a single electron spin in a quantum dot, Phys. Rev. Lett. 100 (2008) 236802.

[403] I. A. Merkulov, A. L. Efros, M. Rosen, Electron spin relaxation by nuclei in semiconductor quantum dots, Phys. Rev. B 65 (2002) 205309.

[404] H. Hayashi, K. M. Itoh, L. S. Vlasenko, Nuclear magnetic resonance linewidth and spin diffusion in ${ }^{2} 9$ si isotopically controlled silicon, Phys. Rev. B 78 (2008) 153201.

[405] F. H. L. Koppens, D. Klauser, W. A. Coish, K. C. Nowack, L. P. Kouwenhoven, D. Loss, L. M. K. Vandersypen, Universal phase shift and nonexponential decay of driven single-spin oscillations, Phys. Rev. Lett. 99 (2007) 106803.

[406] A. Imamoglu, E. Knill, L. Tian, P. Zoller, Optical pumping of quantum-dot nuclear spins, Phys. Rev. Lett. 91 (2003) 017402.

[407] D. Stepanenko, G. Burkard, G. Giedke, A. Imamoglu, Enhancement of electron spin coherence by optical preparation of nuclear spins, Phys. Rev. Lett. 96 (2006) 136401.

[408] D. Klauser, W. A. Coish, D. Loss, Nuclear spin state narrowing via gate-controlled rabi oscillations in a double quantum dot, Phys. Rev. B 73 (2006) 205302

[409] D. J. Reilly, J. M. Taylor, J. R. Petta, C. M. Marcus, M. P. Hanson, A. C. Gossard, Suppressing Spin Qubit Dephasing by Nuclear State Preparation, Science 321 (2008) 817-821.

[410] A. Khaetskii, D. Loss, L. Glazman, Electron spin evolution induced by interaction with nuclei in a quantum dot, Phys. Rev. B 67 (2003) 195329.

[411] W. A. Coish, D. Loss, Hyperfine interaction in a quantum dot: Non-markovian electron spin dynamics, Phys. Rev. B 70 (2004) 195340.

[412] W. A. Coish, J. Fischer, D. Loss, Exponential decay in a spin bath, Phys. Rev. B 77 (2008) 125329. 
[413] C. Deng, X. Hu, Electron-spin dephasing via hyperfine interaction in a quantum dot: An equation-of-motion calculation of electron-spin correlation functions, Phys. Rev. B 78 (2008) 245301.

[414] A. V. Khaetskii, D. Loss, L. Glazman, Electron spin decoherence in quantum dots due to interaction with nuclei, Phys. Rev. Lett. 88 (2002) 186802.

[415] C. Deng, X. Hu, Analytical solution of electron spin decoherence through hyperfine interaction in a quantum dot, Phys. Rev. B 73 (2006) 241303.

[416] W. M. Witzel, R. de Sousa, S. Das Sarma, Quantum theory of spectral-diffusion-induced electron spin decoherence, Phys. Rev. B 72 (2005) 161306.

[417] W. M. Witzel, S. Das Sarma, Quantum theory for electron spin decoherence induced by nuclear spin dynamics in semiconductor quantum computer architectures: Spectral diffusion of localized electron spins in the nuclear solid-state environment, Phys. Rev. B 74 (2006) 035322.

[418] W. Yao, R.-B. Liu, L. J. Sham, Theory of electron spin decoherence by interacting nuclear spins in a quantum dot, Phys. Rev. B 74 (2006) 195301.

[419] S. K. Saikin, W. Yao, L. J. Sham, Single-electron spin decoherence by nuclear spin bath: Linked-cluster expansion approach, Phys. Rev. B 75 (2007) 125314.

[420] W. Yang, R.-B. Liu, Decoherence of coupled electron spins via nuclear spin dynamics in quantum dots, Phys. Rev. B 77 (2008) 085302.

[421] W. Yang, R.-B. Liu, Quantum many-body theory of qubit decoherence in a finite-size spin bath, Phys. Rev. B 78 (2008) 085315.

[422] Ł. Cywiński, W. M. Witzel, S. Das Sarma, Electron spin dephasing due to hyperfine interactions with a nuclear spin bath, Phys. Rev. Lett. 102 (2009) 057601

[423] R.-B. Liu, W. Yao, L. J. Sham, Control of electron spin decoherence caused by electron-nuclear spin dynamics in a quantum dot, New J. Phys. 9 (2007) 226

[424] W. Yang, R.-B. Liu, Universality of uhrig dynamical decoupling for suppressing qubit pure dephasing and relaxation, Phys. Rev. Lett. 101 (2008) 180403.

[425] W. M. Witzel, S. Das Sarma, Concatenated dynamical decoupling in a solid-state spin bath, Phys. Rev. B 76 (2007) 241303.

[426] R. de Sousa, N. Shenvi, K. B. Whaley, Qubit coherence control in a nuclear spin bath, Phys. Rev. B 72 (2005) 045330.

[427] B. Lee, W. M. Witzel, S. Das Sarma, Universal pulse sequence to minimize spin dephasing in the central spin decoherence problem, Phys. Rev. Lett. 100 (2008) 160505.

[428] W. M. Witzel, S. Das Sarma, Multiple-pulse coherence enhancement of solid state spin qubits, Phys. Rev. Lett. 98 (2007) 077601.

[429] W. Yao, R.-B. Liu, L. J. Sham, Restoring coherence lost to a slow interacting mesoscopic spin bath, Phys. Rev. Lett. 98 (2007) 077602.

[430] W. Zhang, V. V. Dobrovitski, L. F. Santos, L. Viola, B. N. Harmon, Dynamical control of electron spin coherence in a quantum dot: A theoretical study, Phys. Rev. B 75 (2007) 201302.

[431] Ł. Cywiński, W. M. Witzel, S. Das Sarma, Pure quantum dephasing of a solid-state electron spin qubit in a large nuclear spin bath coupled by long-range hyperfine-mediated interactions, Phys. Rev. B 79 (2009) 245314.

[432] J. Du, X. Rong, N. Zhao, Y. Wang, J. Yang, R. B. Liu, Preserving electron spin coherence in solids by optimal dynamical decoupling, Nature 461 (2009) 1265-1268.

[433] D. J. Reilly, J. M. Taylor, E. A. Laird, J. R. Petta, C. M. Marcus, M. P. Hanson, A. C. Gossard, Measurement of temporal correlations of the overhauser field in a double quantum dot, Phys. Rev. Lett. 101 (2008) 236803.

[434] F. G. G. Hernandez, A. Greilich, F. Brito, M. Wiemann, D. R. Yakovlev, D. Reuter, A. D. Wieck, M. Bayer, Temperature-induced spincoherence dissipation in quantum dots, Phys. Rev. B 78 (2008) 041303.

[435] M. I. D'yakonov, V. I. Perel', Optical orientation in a system of electrons and lattice-nuclei in semiconductors: Theory, Sov. Phys. JETP 65 (1973) 362-376

[436] G. Fishman, G. Lampel, Spin relaxation of photoelectrons in p-type gallium arsenide, Phys. Rev. B 16 (1977) 820-831.

[437] B. Eble, C. Testelin, P. Desfonds, F. Bernardot, A. Balocchi, T. Amand, A. Miard, A. Lemaître, X. Marie, M. Chamarro, Hole-nuclear spin interaction in quantum dots, Phys. Rev. Lett. 102 (2009) 146601.

[438] C. Testelin, F. Bernardot, B. Eble, M. Chamarro, Hole-spin dephasing time associated with hyperfine interaction in quantum dots, Phys. Rev. B 79 (2009) 195440.

[439] H. Kurtze, D. R. Yakovlev, D. Reuter, A. D. Wieck, M. Bayer, Hyperfine interaction of electron and hole spin with the nuclei in (in,ga)as/gaas quantum dots, 2009. ArXiv:0905.1586.

[440] W. A. Coish, J. Baugh, Nuclear Spins in Nanostructures, Phys. Stat. Sol. (b) 246 (2009) 2203-2215.

[441] D. Heiss, S. Schaeck, H. Huebl, M. Bichler, G. Abstreiter, J. J. Finley, D. V. Bulaev, D. Loss, Observation of extremely slow hole spin relaxation in self-assembled quantum dots, Phys. Rev. B 76 (2007) 241306.

[442] B. D. Gerardot, D. Brunner, P. A. Dalgarno, P. Ohberg, S. Seidl, M. Kroner, K. Karrai, N. G. Stoltz, P. M. Petroff, R. J. Warburton, Optical pumping of a single hole spin in a quantum dot, Nature 451 (2008) 441-444.

[443] P. I. Tamborenea, D. Weinmann, R. A. Jalabert, Relaxation mechanism for electron spin in the impurity band of n-doped semiconductors, Phys. Rev. B 76 (2007) 085209.

[444] F. Baruffa, P. Stano, J. Fabian, Theory of anisotropic exchange in laterally coupled quantum dots, 2009. ArXiv:0908.2961.

[445] I. A. Yugova, A. A. Sokolova, D. R. Yakovlev, A. Greilich, D. Reuter, A. D. Wieck, M. Bayer, Long-term hole spin memory in the resonantly amplified spin coherence of ingaas/gaas quantum well electrons, Phys. Rev. Lett. 102 (2009) 167402.

[446] T. Korn, M. Kugler, M. Griesbeck, R. Schulz, A. Wagner, M. Kubová, C. Gerl, D. Schuh, W. Wegscheider, C. Schüller, Engineering ultralong spin coherence in two-dimensional hole systems at sub-kelvin temperatures, 2009. ArXiv:0909.3711.

[447] S. A. Crooker, D. A. Tulchinsky, J. Levy, D. D. Awschalom, R. Garcia, N. Samarth, Enhanced spin interactions in digital magnetic heterostructures, Phys. Rev. Lett. 75 (1995) 505-508.

[448] G. Bastard, L. L. Chang, Spin-flip relaxation time of conduction electrons in $\mathrm{cd}_{1-x} \mathrm{mn}_{x}$ te quantum wells, Phys. Rev. B 41 (1990) $7899-$ 7902.

[449] C. Camilleri, F. Teppe, D. Scalbert, Y. G. Semenov, M. Nawrocki, M. D’yakonov, J. Cibert, S. Tatarenko, T. Wojtowicz, Electron and hole 
spin relaxation in modulation-doped cdmnte quantum wells, Phys. Rev. B 64 (2001) 085331.

[450] K. E. Rönnburg, E. Mohler, H. G. Roskos, K. Ortner, C. R. Becker, L. W. Molenkamp, Motional-narrowing-type dephasing of electron and hole spins of itinerant excitons in magnetically doped ii-vi bulk semiconductors, Phys. Rev. Lett. 96 (2006) 117203.

[451] J. Kossut, On the scattering of conduction electrons by magnetic impurities in semiconductors of insb-type and hgte-type band structure, Phys. Stat. Sol. (b) 72 (1975) 359-367.

[452] H. Krenn, K. Kaltenegger, T. Dietl, J. Spal/ek, G. Bauer, Photoinduced magnetization in dilute magnetic (semimagnetic) semiconductors, Phys. Rev. B 39 (1989) 10918-10934.

[453] L. M. Roth, $g$ factor and donor spin-lattice relaxation for electrons in germanium and silicon, Phys. Rev. 118 (1960) 1534-1540.

[454] A. V. Khaetskii, Y. V. Nazarov, Spin-flip transitions between zeeman sublevels in semiconductor quantum dots, Phys. Rev. B 64 (2001) 125316.

[455] S. I. Erlingsson, Y. V. Nazarov, Hyperfine-mediated transitions between a zeeman split doublet in gaas quantum dots: The role of the internal field, Phys. Rev. B 66 (2002) 155327.

[456] Y. G. Semenov, K. W. Kim, Correlation of phonon decay with localized electron spin-phase diffusion, Phys. Rev. B 70 (2004) 085305.

[457] C. L. Romano, G. E. Marques, L. Sanz, A. M. Alcalde, Phonon modulation of the spin-orbit interaction as a spin relaxation mechanism in quantum dots, Phys. Rev. B 77 (2008) 033301.

[458] C. Calero, E. M. Chudnovsky, D. A. Garanin, Field dependence of the electron spin relaxation in quantum dots, Phys. Rev. Lett. 95 (2005) 166603.

[459] A. V. Khaetskii, Y. V. Nazarov, Spin relaxation in semiconductor quantum dots, Phys. Rev. B 61 (2000) 12639-12642.

[460] C. Tahan, M. Friesen, R. Joynt, Decoherence of electron spin qubits in si-based quantum computers, Phys. Rev. B 66 (2002) 035314.

[461] T. G. Castner, Orbach spin-lattice relaxation of shallow donors in silicon, Phys. Rev. 155 (1967) 816-825.

[462] D. Mozyrsky, S. Kogan, V. N. Gorshkov, G. P. Berman, Time scales of phonon-induced decoherence of semiconductor spin qubits, Phys. Rev. B 65 (2002) 245213

[463] D. V. Guseinov, A. A. Ezhevskii, C. A. J. Ammerlaan, Epr line width and spin-relaxation rates of shallow and deep donors in isotopically controlled silicon, Physica B 395 (2007) 65-68.

[464] L. M. Woods, T. L. Reinecke, Y. Lyanda-Geller, Spin relaxation in quantum dots, Phys. Rev. B 66 (2002) 161318

[465] L. M. Woods, T. L. Reinecke, R. Kotlyar, Hole spin relaxation in quantum dots, Phys. Rev. B 69 (2004) 125330.

[466] L. Meza-Montes, C. F. Destefani, S. E. Ulloa, Spin-orbit coupling and the singlet-triplet transition in lateral double quantum dots, Phys. Rev. B 78 (2008) 205307.

[467] M. Chamarro, F. Bernardot, C. Testelin, Spin decoherence and relaxation processes in zero-dimensional semiconductor nanostructures, J. Phys.: Condens. Matt. 19 (2007) 445007 (18pp).

[468] K. Roszak, P. Machnikowski, Phonon-induced dephasing of singlet-triplet superpositions in double quantum dots without spin-orbit coupling, Phys. Rev. B 80 (2009) 195315.

[469] M. Q. Weng, Y. Y. Wang, M. W. Wu, Electron spin relaxation in cubic gan quantum dots: Perturbation theory and exact diagonalization study, Phys. Rev. B 79 (2009) 155309.

[470] Y. G. Semenov, K. W. Kim, Phonon-mediated electron-spin phase diffusion in a quantum dot, Phys. Rev. Lett. 92 (2004) 026601.

[471] V. A. Abalmassov, F. Marquardt, Electron-nuclei spin relaxation through phonon-assisted hyperfine interaction in a quantum dot, Phys. Rev. B 70 (2004) 075313.

[472] V. I. Fal'ko, B. L. Altshuler, O. Tsyplyatyev, Anisotropy of spin splitting and spin relaxation in lateral quantum dots, Phys. Rev. Lett. 95 (2005) 076603

[473] J. L. Cheng, M. W. Wu, C. Lü, Spin relaxation in gaas quantum dots, Phys. Rev. B 69 (2004) 115318.

[474] C. F. Destefani, S. E. Ulloa, Oscillatory spin relaxation rates in quantum dots, Phys. Rev. B 72 (2005) 115326.

[475] R. de Sousa, S. Das Sarma, Gate control of spin dynamics in iii-v semiconductor quantum dots, Phys. Rev. B 68 (2003) 155330.

[476] H. Westfahl, A. O. Caldeira, G. Medeiros-Ribeiro, M. Cerro, Dissipative dynamics of spins in quantum dots, Phys. Rev. B 70 (2004) 195320.

[477] D. V. Bulaev, D. Loss, Spin relaxation and anticrossing in quantum dots: Rashba versus dresselhaus spin-orbit coupling, Phys. Rev. B 71 (2005) 205324.

[478] P. Stano, J. Fabian, Orbital and spin relaxation in single and coupled quantum dots, Phys. Rev. B 74 (2006) 045320.

[479] A. V. Khaetskii, Y. V. Nazarov, Spin-dephasing processes in semiconductor quantum dots, Physica E 6 (2000) $470-473$.

[480] P. Stano, J. Fabian, Theory of phonon-induced spin relaxation in laterally coupled quantum dots, Phys. Rev. Lett. 96 (2006) 186602.

[481] Y. Y. Wang, M. W. Wu, Control of spin relaxation in semiconductor double quantum dots, Phys. Rev. B 74 (2006) 165312.

[482] J. H. Jiang, M. W. Wu, Spin relaxation in an inas quantum dot in the presence of terahertz driving fields, Phys. Rev. B 75 (2007) 035307.

[483] K. Shen, M. W. Wu, Triplet-singlet relaxation in semiconductor single and double quantum dots, Phys. Rev. B 76 (2007) 235313.

[484] Y. Y. Wang, M. W. Wu, Control of spin coherence in semiconductor double quantum dots, Phys. Rev. B 77 (2008) 125323.

[485] B. A. Glavin, K. W. Kim, Spin-lattice relaxation in si quantum dots, Phys. Rev. B 68 (2003) 045308.

[486] Y. G. Semenov, K. W. Kim, Elastic spin-relaxation processes in semiconductor quantum dots, Phys. Rev. B 75 (2007) 195342.

[487] M. Trif, V. N. Golovach, D. Loss, Spin dynamics in inas nanowire quantum dots coupled to a transmission line, Phys. Rev. B 77 (2008) 045434.

[488] V. N. Golovach, A. Khaetskii, D. Loss, Spin relaxation at the singlet-triplet crossing in a quantum dot, Phys. Rev. B 77 (2008) 045328.

[489] M. Borhani, V. N. Golovach, D. Loss, Spin decay in a quantum dot coupled to a quantum point contact, Phys. Rev. B 73 (2006) 155311.

[490] J. Lehmann, D. Loss, Cotunneling current through quantum dots with phonon-assisted spin-flip processes, Phys. Rev. B 73 (2006) 045328.

[491] C. Lü, J. L. Cheng, M. W. Wu, Hole spin relaxation in semiconductor quantum dots, Phys. Rev. B 71 (2005) 075308.

[492] D. V. Bulaev, D. Loss, Spin relaxation and decoherence of holes in quantum dots, Phys. Rev. Lett. 95 (2005) 076805.

[493] F. Marquardt, V. A. Abalmassov, Spin relaxation in a quantum dot due to nyquist noise, Phys. Rev. B 71 (2005) 165325.

[494] X. Hu, S. Das Sarma, Charge-fluctuation-induced dephasing of exchange-coupled spin qubits, Phys. Rev. Lett. 96 (2006) 100501.

[495] A. R. Cameron, P. Riblet, A. Miller, Spin gratings and the measurement of electron drift mobility in multiple quantum well semiconductors, 
Phys. Rev. Lett. 76 (1996) 4793-4796.

[496] S. A. Crooker, D. G. Rickel, A. V. Balatsky, D. L. Smith, Spectroscopy of spontaneous spin noise as a probe of spin dynamics and magnetic resonance, Nature 431 (2004) 49-52.

[497] M. Oestreich, M. Römer, R. J. Haug, D. Hägele, Spin noise spectroscopy in gaas, Phys. Rev. Lett. 95 (2005) 216603.

[498] M. Römer, J. Hübner, M. Oestreich, Spin noise spectroscopy in semiconductors, Rev. Sci. Ins. 78 (2007) 103903.

[499] S. A. Crooker, J. Brandt, C. Sandfort, A. Greilich, D. R. Yakovlev, D. Reuter, A. D. Wieck, M. Bayer, Spin noise of electrons and holes in self-assembled quantum dots, Phys. Rev. Lett. 104 (2010) 036601

[500] H. Kosaka, T. Inagaki, Y. Rikitake, H. Imamura, Y. Mitsumori, K. Edamatsu, Spin state tomography of optically injected electrons in a semiconductor, Nature 457 (2009) 702-705.

[501] R. I. Dzhioev, V. L. Korenev, I. A. Merkulov, B. P. Zakharchenya, D. Gammon, A. L. Efros, D. S. Katzer, Manipulation of the spin memory of electrons in $n$-gaas, Phys. Rev. Lett. 88 (2002) 256801.

[502] R. I. Dzhioev, B. P. Zakharchenya, V. L. Korenev, D. Gammon, S. Katzer, Long electron spin memory times in gallium arsenide, JETP Lett. 74 (2001) 182-185.

[503] J. S. Colton, T. A. Kennedy, A. S. Bracker, D. Gammon, Microsecond spin-flip times in $n$ - gaas measured by time-resolved polarization of photoluminescence, Phys. Rev. B 69 (2004) 121307.

[504] K.-M. C. Fu, W. Yeo, S. Clark, C. Santori, C. Stanley, M. C. Holland, Y. Yamamoto, Millisecond spin-flip times of donor-bound electrons in gaas, Phys. Rev. B 74 (2006) 121304.

[505] K.-M. C. Fu, C. Santori, C. Stanley, M. C. Holland, Y. Yamamoto, Coherent population trapping of electron spins in a high-purity $n$-type gaas semiconductor, Phys. Rev. Lett. 95 (2005) 187405.

[506] S. M. Clark, K.-M. C. Fu, Q. Zhang, T. D. Ladd, C. Stanley, Y. Yamamoto, Ultrafast optical spin echo for electron spins in semiconductors, Phys. Rev. Lett. 102 (2009) 247601.

[507] I. S. Lyubinskiy, A. P. Dmitriev, V. Y. Kachorovskii, Spin dynamics in the regime of hopping conductivity, JETP Lett. 85 (2007) 55-58.

[508] G. D. Mahan, R. Woodworth, Spin-exchange scattering in semiconductors, Phys. Rev. B 78 (2008) 075205.

[509] N. J. Harmon, W. O. Putikka, R. Joynt, Theory of Electron Spin Relaxation in n-Doped Quantum Wells, 2009. ArXiv:0911.2452.

[510] I. S. Lyubinskiy, Spin relaxation in the impurity band of a semiconductor in the external magnetic field, JETP Lett. 88 (2008) $814-818$.

[511] J. S. Colton, M. E. Heeb, P. Schroeder, A. Stokes, L. R. Wienkes, A. S. Bracker, Anomalous magnetic field dependence of the $t_{1}$ spin lifetime in a lightly doped gaas sample, Phys. Rev. B 75 (2007) 205201.

[512] B. I. Shklovskii, Dyakonov-perel spin relaxation near the metal-insulator transition and in hopping transport, Phys. Rev. B 73 (2006) 193201.

[513] K. S. Virk, J. E. Sipe, Conduction electrons and the decoherence of impurity-bound electrons in a semiconductor, Phys. Rev. B 72 (2005) 155312.

[514] M. Oestreich, M. Bender, J. Hübner, D. Hägele, W. W. Rühle, T. Hartmann, P. J. Klar, W. Heimbrodt, M. Lampalzer, K. Volz, W. Stolz, Spin injection, spin transport and spin coherence, Semicond. Sci. Technol. 17 (2002) 285-297.

[515] A. I. Ekimov, V. I. Safarov, Observation of optical orientation of equilibrium electrons in $n$-type semiconductors, JETP Lett. 13 (1971) 177.

[516] J. S. Colton, T. A. Kennedy, A. S. Bracker, D. Gammon, J. B. Miller, Optically oriented and detected electron spin resonance in a lightly doped n-gaas layer, Phys. Rev. B 67 (2003) 165315.

[517] P. E. Hohage, F. Seifert, T. Kümmell, G. Bacher, D. Reuter, A. D. Wieck, Magnetic field induced manipulation of the coherent electron spin dynamics in n-gaas, Phys. Stat. Sol. (c) 3 (2006) 4346-4349.

[518] S. A. Crooker, L. Cheng, D. L. Smith, Spin noise of conduction electrons in n-type bulk gaas, Phys. Rev. B 79 (2009) 035208

[519] L. Schreiber, M. Heidkamp, T. Rohleder, B. Beschoten, G. Güntherodt, Mapping of spin lifetimes to electronic states in n-type GaAs near the metal-insulator transition, 2007. ArXiv:0706.1884.

[520] M. Römer, H. Bernien, G. Müller, D. Schuh, J. Hübner, M. Oestreich, Electron spin relaxation in bulk GaAs for doping densities close to the metal-to-insulator transition, 2009. ArXiv:0911.4084.

[521] J. M. Kikkawa, J. A. Gupta, I. Malajovich, D. D. Awschalom, Spin coherence in semiconductors: storage, transport and reduced dimensionality, Physica E 9 (2001) 194-201.

[522] R. Fiederling, M. Keim, G. Reuscher, W. Ossau, G. Schmidt, A. Waag, L. W. Molenkamp, Injection and detection of a spin-polarized current in a light-emitting diode, Nature 402 (1999) 787-790.

[523] Y. Ohno, D. K. Young, B. Beschoten, F. Matsukura, H. Ohno, D. D. Awschalom, Electrical spin injection in a ferromagnetic semiconductor heterostructure, Nature 402 (1999) 790-792.

[524] D. Sprinzl, P. Horodyská, E. Belas, R. Grill, P. Malý, P. Němec, Systematic investigation of influence of n-type doping on electron spin dephasing in CdTe, 2010. ArXiv:1001.0869.

[525] I. Y. Gerlovin, Y. P. Efimov, Y. K. Dolgikh, S. A. Eliseev, V. V. Ovsyankin, V. V. Petrov, R. V. Cherbunin, I. V. Ignatiev, I. A. Yugova, L. V. Fokina, A. Greilich, D. R. Yakovlev, M. Bayer, Electron-spin dephasing in gaas/al $0_{0.34} \mathrm{ga}_{0.66}$ as quantum wells with a gate-controlled electron density, Phys. Rev. B 75 (2007) 115330.

[526] L. V. Fokina, I. A. Yugova, D. R. Yakovlev, M. M. Glazov, I. A. Akimov, A. Greilich, D. Reuter, A. D. Wieck, M. Bayer, Spin dynamics of electrons and holes in InGaAs/GaAs quantum wells at milliKelvin temperatures, 2010. ArXiv:1001.3228.

[527] M. Furis, D. L. Smith, S. A. Crooker, J. L. Reno, Bias-dependent electron spin lifetimes in n-gaas and the role of donor impact ionization, Appl. Phys. Lett. 89 (2006) 102102.

[528] J. S. Sandhu, A. P. Heberle, J. J. Baumberg, J. R. A. Cleaver, Gateable suppression of spin relaxation in semiconductors, Phys. Rev. Lett. 86 (2001) 2150-2153.

[529] J. Tribollet, E. Aubry, G. Karczewski, B. Sermage, F. Bernardot, C. Testelin, M. Chamarro, Enhancement of the electron spin memory by localization on donors in a cdte quantum well, Phys. Rev. B 75 (2007) 205304.

[530] E. A. Zhukov, D. R. Yakovlev, M. Bayer, G. Karczewski, T. Wojtowicz, J. Kossut, Spin coherence of two-dimensional electron gas in cdte/(cd,mg)te quantum wells, Phys. Stat. Sol. (b) 243 (2006) 878-881. 
[531] E. A. Zhukov, D. R. Yakovlev, M. Gerbracht, G. V. Mikhailov, G. Karczewski, T. Wojtowicz, J. Kossut, M. Bayer, Spin coherence of holes and electrons in undoped cdte/(cd,mg)te quantum wells, Phys. Rev. B 79 (2009) 155318.

[532] I. Malajovich, J. M. Kikkawa, D. D. Awschalom, J. J. Berry, N. Samarth, Coherent transfer of spin through a semiconductor heterointerface, Phys. Rev. Lett. 84 (2000) 1015-1018.

[533] G. V. Astakhov, T. Kiessling, D. R. Yakovlev, E. A. Zhukov, M. Bayer, W. Ossau, B. P. Zakharchenya, G. Karczewski, T. Wojtowicz, J. Kossut, Nanosecond spin memory of electrons in cdte/cdmgte quantum wells, Phys. Stat. Sol. (b) 243 (2006) $858-862$.

[534] H. Hoffmann, G. V. Astakhov, T. Kiessling, W. Ossau, G. Karczewski, T. Wojtowicz, J. Kossut, L. W. Molenkamp, Optical spin pumping of modulation-doped electrons probed by a two-color kerr rotation technique, Phys. Rev. B 74 (2006) 073407.

[535] E. A. Zhukov, D. R. Yakovlev, M. Bayer, M. M. Glazov, E. L. Ivchenko, G. Karczewski, T. Wojtowicz, J. Kossut, Spin coherence of a two-dimensional electron gas induced by resonant excitation of trions and excitons in cdte/(cd,mg)te quantum wells, Phys. Rev. B 76 (2007) 205310

[536] M. Kugler, T. Andlauer, T. Korn, A. Wagner, S. Fehringer, R. Schulz, M. Kubová, C. Gerl, D. Schuh, W. Wegscheider, P. Vogl, C. Schüller, Gate control of low-temperature spin dynamics in two-dimensional hole systems, Phys. Rev. B 80 (2009) 035325.

[537] W. A. J. A. van der Poel, A. L. G. J. Severens, H. W. van Kesteren, C. T. Foxon, Spin relaxation in type-ii gaas/alas quantum wells, Phys. Rev. B 39 (1989) 8552-8555.

[538] H. Mino, Y. Kouno, K. Oto, K. Muro, R. Akimoto, S. Takeyama, Optically induced long-lived electron spin coherence in znse/bete type-ii quantum wells, Appl. Phys. Lett. 92 (2008) 153101.

[539] A. Malinowski, R. T. Harley, Dynamic nuclear overhauser shifts in larmor beats from a quantum well, Solid State Commun. 114 (2000) 419-422.

[540] A. Malinowski, M. A. Brand, R. T. Harley, Nuclear effects in ultrafast quantum-well spin-dynamics, Physica E 10 (2001) 13-16.

[541] C. Testelin, B. Eble, F. Bernardot, G. Karczewski, M. Chamarro, Signature of the overhauser field on the coherent spin dynamics of donor-bound electrons in a single cdte quantum well, Phys. Rev. B 77 (2008) 235306.

[542] A. Dyson, B. K. Ridley, Spin relaxation in cubic iii-v semiconductors via interaction with polar optical phonons, Phys. Rev. B 69 (2004) 125211.

[543] P. E. Hohage, G. Bacher, D. Reuter, A. D. Wieck, Coherent spin oscillations in bulk gaas at room temperature, Appl. Phys. Lett. 89 (2006) 231101.

[544] C. Hautmann, F. Jaworeck, K. Kashani-Shirazi, M.-C. Amann, M. Betz, Temperature and doping dependence of electron spin relaxation in gasb, Semicond. Sci. Technol. 24 (2009) 025018 (6pp).

[545] B. Beschoten, E. Johnston-Halperin, D. K. Young, M. Poggio, J. E. Grimaldi, S. Keller, S. P. DenBaars, U. K. Mishra, E. L. Hu, D. D. Awschalom, Spin coherence and dephasing in gan, Phys. Rev. B 63 (2001) 121202.

[546] M. Fanciulli, T. Lei, T. D. Moustakas, Conduction-electron spin resonance in zinc-blende gan thin films, Phys. Rev. B 48 (1993) 15144 15147.

[547] W. E. Carlos, J. A. Freitas, M. A. Khan, D. T. Olson, J. N. Kuznia, Electron-spin-resonance studies of donors in wurtzite gan, Phys. Rev. B 48 (1993) 17878-17884.

[548] W. F. Koehl, M. H. Wong, C. Poblenz, B. Swenson, U. K. Mishra, J. S. Speck, D. D. Awschalom, Current-induced spin polarization in gallium nitride, Appl. Phys. Lett. 95 (2009) 072110.

[549] B. N. Murdin, K. Litvinenko, J. Allam, C. R. Pidgeon, M. Bird, K. Morrison, T. Zhang, S. K. Clowes, W. R. Branford, J. Harris, L. F. Cohen, Temperature and doping dependence of spin relaxation in $n$-inas, Phys. Rev. B 72 (2005) 085346.

[550] K. L. Litvinenko, B. N. Murdin, J. Allam, C. R. Pidgeon, T. Zhang, J. J. Harris, L. F. Cohen, D. A. Eustace, D. W. McComb, Spin lifetime in inas epitaxial layers grown on gaas, Phys. Rev. B 74 (2006) 075331.

[551] K. L. Litvinenko, L. Nikzad, J. Allam, B. N. Murdin, C. R. Pidgeon, J. J. Harris, L. F. Cohen, Spin dynamics in narrow-gap semiconductor epitaxial layers, J. Supercond. Novel Mag. 20 (2007) 461-465.

[552] R. N. Kini, K. Nontapot, G. A. Khodaparast, R. E. Welser, L. J. Guido, Time resolved measurements of spin and carrier dynamics in inas films, J. Appl. Phys. 103 (2008) 064318.

[553] B. N. Murdin, K. Litvinenko, D. G. Clarke, C. R. Pidgeon, P. Murzyn, P. J. Phillips, D. Carder, G. Berden, B. Redlich, A. F. G. van der Meer, S. Clowes, J. J. Harris, L. F. Cohen, T. Ashley, L. Buckle, Spin relaxation by transient monopolar and bipolar optical orientation, Phys. Rev. Lett. 96 (2006) 096603.

[554] B. Li, M. C. Tamargo, C. A. Meriles, Electron spin dynamics in fe-doped inp, Appl. Phys. Lett. 91 (2007) 222114.

[555] I. Malajovich, J. M. Kikkawa, D. D. Awschalom, J. J. Berry, N. Samarth, Resonant amplification of spin transferred across a gaas/znse interface, J. Appl. Phys. 87 (2000) 5073-5075.

[556] S. Ghosh, V. Sih, W. H. Lau, D. D. Awschalom, S.-Y. Bae, S. Wang, S. Vaidya, G. Chapline, Room-temperature spin coherence in zno, Appl. Phys. Lett. 86 (2005) 232507.

[557] P. Murzyn, C. R. Pidgeon, P. J. Phillips, J.-P. Wells, N. T. Gordon, T. Ashley, J. H. Jefferson, T. M. Burke, J. Giess, M. Merrick, B. N. Murdin, C. D. Maxey, Electron spin lifetimes in $\mathrm{hg}_{0.78} \mathrm{~cd}_{0.22}$ te and insb, Physica E 20 (2004) 220-223.

[558] P. Murzyn, C. R. Pidgeon, P. J. Phillips, J.-P. Wells, N. T. Gordon, T. Ashley, J. H. Jefferson, T. M. Burke, J. Giess, M. Merrick, B. N. Murdin, C. D. Maxey, Electron spin lifetimes in long-wavelength $\mathrm{hg}_{1-x} \mathrm{~cd}_{x}$ te and insb at elevated temperature, Phys. Rev. B 67 (2003) 235202.

[559] N. J. Harmon, W. O. Putikka, R. Joynt, Theory of electron spin relaxation in zno, Phys. Rev. B 79 (2009) 115204.

[560] R. L. Kallaher, J. J. Heremans, Spin and phase coherence measured by antilocalization in n-insb thin films, Phys. Rev. B 79 (2009) 075322.

[561] G. M. Müller, M. Römer, J. Hübner, M. Oestreich, Ghz spin noise spectroscopy in n-doped bulk gaas, 2009. ArXiv:0909.3406.

[562] R. Bratschitsch, Z. Chen, S. T. Cundiff, Ultrafast spin phenomena in highly excited n-doped gaas, Phys. Stat. Sol. (c) 0 (2003) $1506-1508$.

[563] M. Krauß, H. C. Schneider, R. Bratschitsch, Z. Chen, S. T. Cundiff, Ultrafast spin dynamics in optically excited bulk gaas at low temperatures, Phys. Rev. B 81 (2010) 035213.

[564] K. Shen, A peak in density dependence of electron spin relaxation time in $n$-type bulk gaas in the metallic regime, Chin. Phys. Lett. 26 (2009) 067201. 
[565] T. Lai, L. Teng, Z. Jiao, H. Xu, L. Lei, J. Wen, W. Lin, Evolution of spin coherence dynamics and $g$ factor with electron excess energy in bulk intrinsic gaas, Appl. Phys. Lett. 91 (2007) 062110.

[566] H. Ma, Z. Jin, G. Ma, W. Liu, S. H. Tang, Photon energy and carrier density dependence of spin dynamics in bulk cdte crystal at room temperature, Appl. Phys. Lett. 94 (2009) 241112.

[567] M. Beck, C. Metzner, S. Malzer, G. H. Dohler, Spin lifetimes and strain-controlled spin precession of drifting electrons in gaas, Europhys. Lett. 75 (2006) 597-603.

[568] S. Ghosh, D. W. Steuerman, B. Maertz, K. Ohtani, H. Xu, H. Ohno, D. D. Awschalom, Electrical control of spin coherence in zno, Appl. Phys. Lett. 92 (2008) 162109.

[569] M. Q. Weng, M. W. Wu, L. Jiang, Hot-electron effect in spin dephasing in $n$-type gaas quantum wells, Phys. Rev. B 69 (2004) 245320.

[570] L. Jiang, M. W. Wu, Control of spin coherence in $n$-type gaas quantum wells using strain, Phys. Rev. B 72 (2005) 033311.

[571] V. Sih, H. Knotz, J. Stephens, V. R. Horowitz, A. C. Gossard, D. D. Awschalom, Mechanical control of spin-orbit splitting in gaas and $\mathrm{in}_{0} .04 \mathrm{ga}_{0} .96 \mathrm{as}$ epilayers, Phys. Rev. B 73 (2006) 241316.

[572] M. I. D'yakonov, V. A. Marushchak, V. I. Perel', A. N. Titkov, The effect of strain on the spin relaxation of conduction electrons in iii-v semiconductors, Sov. Phys. JETP 63 (1986) 655-661.

[573] A. V. Kimel, F. Bentivegna, V. N. Gridnev, V. V. Pavlov, R. V. Pisarev, T. Rasing, Room-temperature ultrafast carrier and spin dynamics in gaas probed by the photoinduced magneto-optical kerr effect, Phys. Rev. B 63 (2001) 235201.

[574] T. F. Boggess, J. T. Olesberg, C. Yu, M. E. Flatté, W. H. Lau, Room-temperature electron spin relaxation in bulk inas, Appl. Phys. Lett. 77 (2000) 1333-1335.

[575] P. Murzyn, C. R. Pidgeon, P. J. Phillips, M. Merrick, K. L. Litvinenko, J. Allam, B. N. Murdin, T. Ashley, J. H. Jefferson, A. Miller, L. F. Cohen, Suppression of d'yakonov-perel spin relaxation in inas and insb by $n$-type doping at $300 \mathrm{k}$, Appl. Phys. Lett. 83 (2003) 5220-5222.

[576] S. A. Wolf, D. D. Awschalom, R. A. Buhrman, J. M. Daughton, S. von Molnar, M. L. Roukes, A. Y. Chtchelkanova, D. M. Treger, Spintronics: A Spin-Based Electronics Vision for the Future, Science 294 (2001) 1488-1495.

[577] W. H. Lau, J. T. Olesberg, M. E. Flatté, Electron-spin decoherence in bulk and quantum-well zinc-blende semiconductors, Phys. Rev. B 64 (2001) 161301.

[578] S. Krishnamurthy, M. van Schilfgaarde, N. Newman, Spin lifetimes of electrons injected into gaas and gan, Appl. Phys. Lett. 83 (2003) $1761-1763$.

[579] D. Jena, Spin scattering by dislocations in iii-v semiconductors, Phys. Rev. B 70 (2004) 245203.

[580] P. Boguslawski, Electron-electron spin-flip scattering and spin relaxation in iii-v and ii-vi semiconductors, Solid State Commun. 33 (1980) 389-391.

[581] P. I. Tamborenea, M. A. Kuroda, F. L. Bottesi, Spin relaxation in n-doped gaas due to impurity and electron-electron elliot-yafet scattering, Phys. Rev. B 68 (2003) 245205.

[582] S. Oertel, J. Hübner, M. Oestreich, High temperature electron spin relaxation in bulk gaas, Appl. Phys. Lett. 93 (2008) 132112.

[583] E. A. Barry, A. A. Kiselev, K. W. Kim, Electron spin relaxation under drift in gaas, Appl. Phys. Lett. 82 (2003) 3686-3688.

[584] J. M. Kikkawa, I. P. Smorchkova, N. Samarth, D. D. Awschalom, Room-Temperature Spin Memory in Two-Dimensional Electron Gases, Science 277 (1997) 1284-1287.

[585] L. H. Teng, K. Chen, J. H. Wen, W. Z. Lin, T. S. Lai, Density dependence of electron-spin polarization and relaxation in intrinsic gaas at room temperature, J. Phys. D: Appl. Phys. 42 (2009) 135111 (6pp).

[586] R. J. Seymour, M. R. Junnarkar, R. R. Alfano, Spin relaxation of photogenerated degenerate electron distributions in gaas, Phys. Rev. B 24 (1981) 3623-3625.

[587] A. V. Kimel, A. A. Tsvetkov, A. Kirilyuk, T. Rasing, V. N. Gridnev, Picosecond dynamics of bleaching and spin splitting in inp revealed by the photoinduced magneto-optical kerr effect near the spin-orbit split-off exciton transition, Phys. Rev. B 69 (2004) 165214.

[588] K. L. Litvinenko, L. Nikzad, J. Allam, B. N. Murdin, C. R. Pidgeon, J. J. Harris, T. Zhang, L. F. Cohen, Spin lifetime in high quality insb epitaxial layers grown on gaas, J. Appl. Phys. 101 (2007) 083105

[589] J. H. Jiang, M. W. Wu, Comment on "density dependence of electron-spin polarization and relaxation in intrinsic gaas at room temperature", J. Phys. D: Appl. Phys. 42 (2009) 238001 (2pp).

[590] J. H. Jiang, M. W. Wu, Comment on "Photon energy and carrier density dependence of spin dynamics in bulk CdTe crystal at room temperature", Appl. Phys. Lett. 96 (2010). In press.

[591] W. J. H. Leyland, G. H. John, R. T. Harley, M. M. Glazov, E. L. Ivchenko, D. A. Ritchie, I. Farrer, A. J. Shields, M. Henini, Enhanced spin-relaxation time due to electron-electron scattering in semiconductor quantum wells, Phys. Rev. B 75 (2007) 165309.

[592] A. Tackeuchi, T. Kuroda, S. Muto, Y. Nishikawa, O. Wada, Electron spin-relaxation dynamics in gaas/algaas quantum wells and ingaas/inp quantum wells, Jpn. J. Appl. Phys. 38 (1999) 4680-4687.

[593] K. L. Litvinenko, B. N. Murdin, J. Allam, C. R. Pidgeon, M. Bird, K. Morris, W. Branford, S. K. Clowes, L. F. Cohen, T. Ashley, L. Buckle, Spin relaxation in n-insb/alinsb quantum wells, New J. Phys. 8 (2006) 49.

[594] D. Hägele, M. Oestreich, W. W. Rühle, J. Hoffmann, S. Wachter, H. Kalt, K. Ohkawa, D. Hommel, Relation between spin and momentum relaxation in znse/znmgsse quantum wells, Physica B 272 (1999) 338-340.

[595] Y. Ohno, R. Terauchi, T. Adachi, F. Matsukura, H. Ohno, Spin relaxation in gaas(110) quantum wells, Phys. Rev. Lett. 83 (1999) 4196-4199.

[596] K. C. Hall, M. E. Flatté, Performance of a spin-based insulated gate field effect transistor, Appl. Phys. Lett. 88 (2006) 162503.

[597] J. L. Cheng, M. W. Wu, Spin relaxation under identical dresselhaus and rashba coupling strengths in gaas quantum wells, J. Appl. Phys. 99 (2006) 083704.

[598] B. Liu, H. Zhao, J. Wang, L. Liu, W. Wang, D. Chen, H. Zhu, Electron density dependence of in-plane spin relaxation anisotropy in gaas/algaas two-dimensional electron gas, Appl. Phys. Lett. 90 (2007) 112111.

[599] A. V. Larionov, L. E. Golub, Electric-field control of spin-orbit splittings in gaas $/ \mathrm{al}_{x} \mathrm{ga}_{1-x}$ as coupled quantum wells, Phys. Rev. B 78 (2008) 033302.

[600] V. Sih, D. D. Awschalom, Electrical manipulation of spin-orbit coupling in semiconductor heterostructures, J. Appl. Phys. 101 (2007) 
081710.

[601] R. C. Miller, D. A. Kleinman, W. A. Nordland, A. C. Gossard, Luminescence studies of optically pumped quantum wells in gaasalxga 1 - xas multilayer structures, Phys. Rev. B 22 (1980) 863-871.

[602] Y. Ohno, R. Terauchi, T. Adachi, F. Matsukura, H. Ohno, Electron spin relaxation beyond d'yakonov-perel' interaction in gaas/algaas quantum wells, Physica E 6 (2000) 817-820.

[603] A. Malinowski, R. S. Britton, T. Grevatt, R. T. Harley, D. A. Ritchie, M. Y. Simmons, Spin relaxation in gaas/al ${ }_{x} \mathrm{ga}_{1-x}$ as quantum wells, Phys. Rev. B 62 (2000) 13034-13039.

[604] X. Z. Ruan, H. H. Luo, Y. Ji, Z. Y. Xu, V. Umansky, Effect of electron-electron scattering on spin dephasing in a high-mobility low-density two-dimensional electron gas, Phys. Rev. B 77 (2008) 193307.

[605] W. J. H. Leyland, R. T. Harley, M. Henini, A. J. Shields, I. Farrer, D. A. Ritchie, Energy-dependent electron-electron scattering and spin dynamics in a two-dimensional electron gas, Phys. Rev. B 77 (2008) 205321

[606] M. J. Snelling, A. S. Plaut, G. P. Flinn, A. C. Tropper, R. T. Harley, T. M. Kerr, Spin relaxation in optically excited quantum wells, J. Lumin. 45 (1990) 208-210.

[607] R. Aleksiejunas, A. Kadys, K. Jarasiunas, F. Saas, U. Griebner, J. W. Tomm, All-optical analysis of carrier and spin relaxation in ingaas/gaas saturable-absorber structures, Appl. Phys. Lett. 90 (2007) 102105.

[608] A. Pugzlys, P. J. Rizo, K. Ivanin, A. Slachter, D. Reuter, A. D. Wieck, C. H. van der Wal, P. H. M. van Loosdrecht, Charge and spin dynamics in a two-dimensional electron gas, J. Phys.: Condens. Matt. 19 (2007) 295206 (20pp).

[609] L. H. Teng, P. Zhang, T. S. Lai, M. W. Wu, Density dependence of spin relaxation in gaas quantum well at room temperature, Europhys. Lett. 84 (2008) 27006 (5pp).

[610] V. Srinivas, Y. J. Chen, C. E. C. Wood, Spin relaxation of two-dimensional electrons in gaas quantum wells, Phys. Rev. B 47 (1993) 10907-10910.

[611] S. L. Lu, T. Ushiyama, A. Tackeuchi, S. Muto, Carrier spin relaxation in undoped gaas double quantum wells, Phys. Stat. Sol. (c) 5 (2008) 326-329.

[612] H. Zhao, M. Mower, G. Vignale, Ambipolar spin diffusion and d'yakonov-perel' spin relaxation in gaas quantum wells, Phys. Rev. B 79 (2009) 115321.

[613] C. Lü, J. L. Cheng, Spin relaxation in n-type zno quantum wells, Semicond. Sci. Technol. 24 (2009) 115010 (5pp).

[614] R. Terauchi, Y. Ohno, T. Adachi, A. Sato, F. Matsukura, A. Tackeuchi, H. Ohno, Carrier mobility dependence of electron spin relaxation in gaas quantum wells, Jpn. J. Appl. Phys. 38 (1999) 2549-2551.

[615] M. M. Glazov, E. L. Ivchenko, Precession spin relaxation mechanism caused by frequent electron-electron collisions, JETP Lett. 75 (2002) $403-405$.

[616] A. Tackeuchi, Y. Nishikawa, O. Wada, Room-temperature electron spin dynamics in gaas/algaas quantum wells, Appl. Phys. Lett. 68 (1996) 797-799.

[617] A. Tackeuchi, O. Wada, Y. Nishikawa, Electron spin relaxation in ingaas/inp multiple-quantum wells, Appl. Phys. Lett. 70 (1997) $1131-$ 1133

[618] R. S. Britton, T. Grevatt, A. Malinowski, R. T. Harley, P. Perozzo, A. R. Cameron, A. Miller, Room temperature spin relaxation in gaas/algaas multiple quantum wells, Appl. Phys. Lett. 73 (1998) 2140-2142.

[619] J. T. Hyland, G. T. Kennedy, A. Miller, C. C. Button, Spin relaxation and all optical polarization switching at 1.52 micrometres in ingaas(p)/ingaasp multiple quantum wells, Semicond. Sci. Technol. 14 (1999) 215-221.

[620] W. H. Lau, V. Sih, N. P. Stern, R. C. Myers, D. A. Buell, A. C. Gossard, D. D. Awschalom, Room temperature electron spin coherence in telecom-wavelength quaternary quantum wells, Appl. Phys. Lett. 89 (2006) 142104.

[621] V. Sih, W. H. Lau, R. C. Myers, A. C. Gossard, M. E. Flatté, D. D. Awschalom, Control of electron-spin coherence using landau level quantization in a two-dimensional electron gas, Phys. Rev. B 70 (2004) 161313.

[622] Z. Wilamowski, W. Jantsch, Suppression of spin relaxation of conduction electrons by cyclotron motion, Phys. Rev. B 69 (2004) 035328.

[623] K. Morita, H. Sanada, S. Matsuzaka, C. Y. Hu, Y. Ohno, H. Ohno, Electron spin dynamics in ingaas quantum wells, Physica E 21 (2004) $1007-1011$.

[624] I. G. Saveliev, D. D. Bykanov, S. V. Novikov, T. A. Polyanskaya, H. Ruda, Electron phase and spin decoherence in the vicinity of the second subband edge in an asymmetrical quantum well, J. Phys.: Condens. Matt. 16 (2004) 641-650.

[625] F. Zhang, H. Z. Zheng, Y. Ji, J. Liu, G. R. Li, Spin dynamics in the second subband of a quasi-two-dimensional system studied in a single-barrier heterostructure by time-resolved kerr rotation, Europhys. Lett. 83 (2008) 47007 (5pp).

[626] H. Luo, X. Qian, X. Ruan, Y. Ji, V. Umansky, Spin dynamics of electrons in the first excited subband of a high-mobility low-density two-dimensional electron system, Phys. Rev. B 80 (2009) 193301.

[627] H. Luo, X. Qian, X. Gu, Y. Ji, V. Umansky, Effect of ka-band microwave on the spin dynamics of electrons in a gaas/al $\mathrm{a}_{0.35} \mathrm{ga}_{0.65}$ as heterostructure, Appl. Phys. Lett. 94 (2009) 192107.

[628] A. Tackeuchi, T. Kuroda, S. Muto, O. Wada, Picosecond electron-spin relaxation in gaas/algaas quantum wells and ingaas/inp quantum wells, Physica B 272 (1999) 318-323.

[629] L. Lombez, P.-F. Braun, H. Carrère, B. Urbaszek, P. Renucci, T. Amand, X. Marie, J. C. Harmand, V. K. Kalevich, Spin dynamics in dilute nitride semiconductors at room temperature, Appl. Phys. Lett. 87 (2005) 252115.

[630] D. Lagarde, L. Lombez, X. Marie, A. Balocchi, T. Amand, V. K. Kalevich, A. Shiryaev, E. Ivchenko, A. Egorov, Electron spin dynamics in gaasn and ingaasn structures, Appl. Phys. Lett. 204 (2007) 208-220.

[631] C. Reith, S. J. White, M. Mazilu, A. Miller, J. Konttinen, M. Guina, M. Pessa, Room temperature electron spin relaxation in gainnas multiple quantum wells at $1.3 \mu \mathrm{m}$, Appl. Phys. Lett. 89 (2006) 211122.

[632] A. Dyson, B. K. Ridley, Spin relaxation in cubic iii-v semiconductor low-dimensional structures via the interaction with polar-optical phonons, Phys. Rev. B 72 (2005) 045326.

[633] W. H. Lau, J. T. Olesberg, M. E. Flatté, Electronic structures and electron spin decoherence in (001)-grown layered zincblende semiconductors, 2004. ArXiv:0406201. 
[634] Z. Wilamowski, H. Malissa, F. Schäffler, W. Jantsch, g-factor tuning and manipulation of spins by an electric current, Phys. Rev. Lett. 98 (2007) 187203

[635] N. S. Averkiev, L. E. Golub, M. Willander, Spin relaxation in asymmetrical heterostructures, Semiconductors 36 (2002) $91-97$.

[636] J. Kainz, U. Rössler, R. Winkler, Temperature dependence of dyakonov-perel spin relaxation in zinc-blende semiconductor quantum structures, Phys. Rev. B 70 (2004) 195322.

[637] A. M. Gilbertson, M. Fearn, J. H. Jefferson, B. N. Murdin, P. D. Buckle, L. F. Cohen, Zero-field spin splitting and spin lifetime in n-insb/in ${ }_{1-x} \mathrm{al}_{x} \mathrm{sb}$ asymmetric quantum well heterostructures, Phys. Rev. B 77 (2008) 165335.

[638] J. Li, K. Chang, F. M. Peeters, Dyakonov-perel spin relaxation in insb/al in $_{1-x}$ sb quantum wells, Phys. Rev. B 80 (2009) 153307.

[639] A. M. Gilbertson, W. R. Branford, M. Fearn, L. Buckle, P. D. Buckle, T. Ashley, L. F. Cohen, Zero-field spin splitting and spin-dependent broadening in high-mobility insb/in ${ }_{1-x} \mathrm{al}_{x} \mathrm{sb}$ asymmetric quantum well heterostructures, Phys. Rev. B 79 (2009) 235333.

[640] W. H. Lau, M. E. Flatté, Tunability of electron spin coherence in iii-v quantum wells, J. Appl. Phys. 91 (2002) $8682-8684$.

[641] M. Griesbeck, M. M. Glazov, T. Korn, E. Y. Sherman, D. Waller, C. Reichl, D. Schuh, W. Wegscheider, C. Schüller, Cyclotron effect on coherent spin precession of two-dimensional electrons, Phys. Rev. B 80 (2009) 241314.

[642] D. Fukuoka, T. Yamazaki, N. Tanaka, K. Oto, K. Muro, Y. Hirayama, N. Kumada, H. Yamaguchi, Spin dynamics of two-dimensional electrons in a quantum hall system probed by time-resolved kerr rotation spectroscopy, Phys. Rev. B 78 (2008) 041304.

[643] J. H. Smet, R. A. Deutschmann, F. Ertl, W. Wegscheider, G. Abstreiter, K. von Klitzing, Gate-voltage control of spin interactions between electrons and nuclei in a semiconductor, Nature 415 (2002) 281-286.

[644] A. G. Mal'shukov, K. A. Chao, M. Willander, Quantum localization effects on spin transport in semiconductor quantum wells with zinc-blende crystal structure, Phys. Rev. Lett. 76 (1996) 3794-3797.

[645] I. S. Lyubinskiy, V. Y. Kachorovskii, Slowing down of spin relaxation in two-dimensional systems by quantum interference effects, Phys. Rev. B 70 (2004) 205335

[646] I. S. Lyubinskiy, V. Y. Kachorovskii, Hanle effect driven by weak localization, Phys. Rev. Lett. 94 (2005) 076406.

[647] I. S. Lyubinskiy, V. Y. Kachorovskii, Classical memory effects on spin dynamics in two-dimensional systems, Phys. Rev. B 73 (2006) 041301.

[648] Y. V. Pershin, V. Privman, Slow spin relaxation in two-dimensional electron systems with antidots, Phys. Rev. B 69 (2004) 073310.

[649] M. Trushin, J. Schliemann, Spin dynamics in rolled-up two-dimensional electron gases, New J. Phys. 9 (2007) 346.

[650] A. G. Mal'shukov, K. A. Chao, Waveguide diffusion modes and slowdown of d'yakonov-perel' spin relaxation in narrow two-dimensional semiconductor channels, Phys. Rev. B 61 (2000) R2413-R2416.

[651] A. A. Kiselev, K. W. Kim, Progressive suppression of spin relaxation in two-dimensional channels of finite width, Phys. Rev. B 61 (2000) $13115-13120$.

[652] A. A. Kiselev, K. W. Kim, Suppression of dyakonov-perel spin relaxation in 2d channels of finite width, Phys. Stat. Sol. (b) 221 (2000) 491-494.

[653] S. Kettemann, Dimensional control of antilocalization and spin relaxation in quantum wires, Phys. Rev. Lett. 98 (2007) 176808.

[654] A. W. Holleitner, V. Sih, R. C. Myers, A. C. Gossard, D. D. Awschalom, Suppression of spin relaxation in submicron ingaas wires, Phys. Rev. Lett. 97 (2006) 036805.

[655] A. W. Holleitner, V. Sih, R. C. Myers, A. C. Gossard, D. D. Awschalom, Dimensionally constrained d'yakonov-perel' spin relaxation in n-ingaas channels: transition from 2d to 1d, New J. Phys. 9 (2007) 342.

[656] C.-H. Chang, J. Tsai, H.-F. Lo, A. G. Mal'shukov, Semiclassical path integral approach for spin relaxations in narrow wires, Phys. Rev. B 79 (2009) 125310.

[657] E. Y. Sherman, Minimum of spin-orbit coupling in two-dimensional structures, Phys. Rev. B 67 (2003) 161303.

[658] M. M. Glazov, E. Y. Sherman, Nonexponential spin relaxation in magnetic fields in quantum wells with random spin-orbit coupling, Phys. Rev. B 71 (2005) 241312.

[659] V. K. Dugaev, E. Y. Sherman, V. I. Ivanov, J. Barnaś, Spin relaxation and combined resonance in two-dimensional electron systems with spin-orbit disorder, Phys. Rev. B 80 (2009) 081301.

[660] G. M. Müller, M. Römer, D. Schuh, W. Wegscheider, J. Hübner, M. Oestreich, Spin noise spectroscopy in gaas (110) quantum wells: Access to intrinsic spin lifetimes and equilibrium electron dynamics, Phys. Rev. Lett. 101 (2008) 206601.

[661] S. G. Carter, Z. Chen, S. T. Cundiff, Optical measurement and control of spin diffusion in n-doped gaas quantum wells, Phys. Rev. Lett. 97 (2006) 136602.

[662] T. Adachi, Y. Ohno, F. Matsukura, H. Ohno, Spin relaxation in n-modulation doped gaas/algaas (1 10$)$ quantum wells, Physica E 10 (2001) 36-39.

[663] P. Olbrich, J. Allerdings, V. V. Bel'kov, S. A. Tarasenko, D. Schuh, W. Wegscheider, T. Korn, C. Schüller, D. Weiss, S. D. Ganichev, Magnetogyrotropic photogalvanic effect and spin dephasing in (110)-grown gaas/al $\mathrm{ga}_{1-x}$ as quantum well structures, Phys. Rev. B 79 (2009) 245329.

[664] L. Schreiber, D. Duda, B. Beschoten, G. Güntherodt, H.-P. Schönherr, J. Herfort, Spin lifetime of (in,ga)as/gaas (110) quantum wells, Phys. Stat. Sol. (b) 244 (2007) 2960-2970.

[665] K. Morita, H. Sanada, S. Matsuzaka, C. Y. Hu, Y. Ohno, H. Ohno, Strong anisotropic spin dynamics in narrow n-ingaas/algaas (110) quantum wells, Appl. Phys. Lett. 87 (2005) 171905.

[666] L. Schreiber, D. Duda, B. Beschoten, G. Güntherodt, H.-P. Schönherr, J. Herfort, Anisotropic electron spin lifetime in (in,ga)as/gaas (110) quantum wells, Phys. Rev. B 75 (2007) 193304.

[667] D. Hägele, S. Döhrmann, J. Rudolph, M. Oestreich, Electron spin relaxation in semiconductors, Adv. Solid State Phys. 45 (2005) 253.

[668] Y. Zhou, M. W. Wu, A virtual intersubband spin-flip spin-orbit coupling induced spin relaxation in gaas (110) quantum wells, Solid State Commun. 149 (2009) 2078.

[669] S. Starosielec, D. Hägele, Ultrafast spin noise spectroscopy, Appl. Phys. Lett. 93 (2008) 051116.

[670] Y. Zhou, M. W. Wu, Spin relaxation due to random Rashba spin-orbit coupling in GaAs (110) quantum wells, Europhys. Lett. 89 (2010) 57001 . 
[671] M. Henini, O. Z. Karimov, G. H. John, R. T. Harley, R. J. Airey, Gated spin relaxation in (110)-oriented quantum wells, Physica E 23 (2004) 309-314.

[672] K. C. Hall, K. Gündoğdu, J. L. Hicks, A. N. Kocbay, M. E. Flatté, T. F. Boggess, K. Holabird, A. Hunter, D. H. Chow, J. J. Zinck, Room-temperature electric-field controlled spin dynamics in (110) inas quantum wells, Appl. Phys. Lett. 86 (2005) 202114.

[673] O. D. D. Couto, Jr., F. Iikawa, J. Rudolph, R. Hey, P. V. Santos, Anisotropic spin transport in (110) gaas quantum wells, Phys. Rev. Lett. 98 (2007) 036603.

[674] O. D. D. Couto, Jr., R. Hey, P. V. Santos, Spin dynamics in (110) gaas quantum wells under surface acoustic waves, Phys. Rev. B 78 (2008) 153305 .

[675] M. W. Wu, M. Kuwata-Gonokami, Can d'yakonov-perel' effect cause spin dephasing in gaas(110) quantum wells?, Solid State Commun. 121 (2002) 509-512.

[676] S.-W. Chang, S.-L. Chuang, Strain-induced enhancement of spin relaxation times in [110] and [111] grown quantum wells, Phys. Rev. B 72 (2005) 115429.

[677] S. A. Tarasenko, Spin relaxation of conduction electrons in (110)-grown quantum wells: A microscopic theory, Phys. Rev. B 80 (2009) 165317.

[678] M. M. Glazov, M. A. Semina, E. Y. Sherman, Spin relaxation in multiple (110) quantum wells, 2009. ArXiv:0911.1444.

[679] X. Cartoixà, D. Z.-Y. Ting, Y.-C. Chang, Suppression of the d'yakonov-perel' spin-relaxation mechanism for all spin components in [111] zincblende quantum wells, Phys. Rev. B 71 (2005) 045313.

[680] I. Vurgaftman, J. R. Meyer, Spin-relaxation suppression by compensation of bulk and structural inversion asymmetries in [111]-oriented quantum wells, J. Appl. Phys. 97 (2005) 053707.

[681] C. Yang, X. Cui, S.-Q. Shen, Z. Xu, W. Ge, Spin relaxation in submonolayer and monolayer inas structures grown in a gaas matrix, Phys. Rev. B 80 (2009) 035313.

[682] Z. Sun, Z. Y. Xu, X. Z. Ruan, Y. Ji, B. Q. Sun, M. Wang, H. Q. Ni, Effect of electron-hole spatial correlation on spin relaxation dynamics in inas submonolayer, Solid State Communications 143 (2007) $158-160$.

[683] G. Salis, Y. Kato, K. Ensslin, D. C. Driscoll, A. C. Gossard, D. D. Awschalom, Electrical control of spin precession in semiconductor quantum wells, Physica E 16 (2003) 99-103.

[684] J. Tribollet, F. Bernardot, M. Menant, G. Karczewski, C. Testelin, M. Chamarro, Interplay of spin dynamics of trions and two-dimensional electron gas in a n-doped cdte single quantum well, Phys. Rev. B 68 (2003) 235316.

[685] K. C. Ku, S. H. Chun, W. H. Wang, W. Fadgen, D. A. Issadore, N. Samarth, R. J. Epstein, D. D. Awschalom, Fabrication and characterization of modulation-doped znse/(zn,cd)se (110) quantum wells: A new system for spin coherence studies, J. Supercond. 18 (2005).

[686] R. Bratschitsch, Z. Chen, S. T. Cundiff, D. R. Yakovlev, G. Karczewski, T. Wojtowicz, J. Kossut, Electron spin dephasing in n-doped cdte/(cd, mg)te quantum wells, Phys. Stat. Sol. (b) 243 (2006) 2290-2292.

[687] T. C. Damen, L. Viña, J. E. Cunningham, J. Shah, L. J. Sham, Subpicosecond spin relaxation dynamics of excitons and free carriers in gaas quantum wells, Phys. Rev. Lett. 67 (1991) 3432-3435.

[688] J. Wagner, H. Schneider, D. Richards, A. Fischer, K. Ploog, Observation of extremely long electron-spin-relaxation times in $p$-type $\delta$-doped gaas/al $\mathrm{ga}_{1-x}$ as double heterostructures, Phys. Rev. B 47 (1993) 4786-4789.

[689] H. Gotoh, H. Ando, T. Sogawa, H. Kamada, T. Kagawa, H. Iwamura, Effect of electron-hole interaction on electron spin relaxation in gaas/algaas quantum wells at room temperature, J. Appl. Phys. 87 (2000) 3394-3398.

[690] M. D. Martin, L. Viña, M. Potemski, K. H. Ploog, On the spin-flip mechanisms of electrons in semiconductor quantum wells, Phys. Stat. Sol. (b) 215 (1999) 229-233.

[691] M. Potemski, E. Pérez, D. Martin, L. Viña, L. Gravier, A. Fisher, K. Ploog, Spin polarization of an optically pumped electron gas, Solid State Commun. 110 (1999) 163-168.

[692] L. Viña, Spin relaxation in low-dimensional systems, J. Phys.: Condens. Matt. 11 (1999) 5929-5952.

[693] H. C. Schneider, J.-P. Wüstenberg, O. Andreyev, K. Hiebbner, L. Guo, J. Lange, L. Schreiber, B. Beschoten, M. Bauer, M. Aeschlimann, Energy-resolved electron spin dynamics at surfaces of p-doped gaas, Phys. Rev. B 73 (2006) 081302.

[694] T. Uenoyama, L. J. Sham, Carrier relaxation and luminescence polarization in quantum wells, Phys. Rev. B 42 (1990) $7114-7123$.

[695] T. Uenoyama, L. J. Sham, Hole relaxation and luminescence polarization in doped and undoped quantum wells, Phys. Rev. Lett. 64 (1990) 3070-3073.

[696] C. Lü, H. C. Schneider, M. W. Wu, Electron spin relaxation in n-type inas quantum wires, J. Appl. Phys. 106 (2009) 073703.

[697] J. Liu, T. Last, E. J. Koop, S. Denega, B. J. van Wees, C. H. van der Wal, Spin-dephasing anisotropy for electrons in a diffusive quasi-1d gaas wire, J. Supercond. Nov. Magn. (2009).

[698] T. Sogawa, H. Ando, S. Ando, H. Kanbe, Spin-polarization spectroscopy in rectangular gaas quantum wires, Phys. Rev. B 58 (1998) $15652-15659$.

[699] Y. Kunihashi, M. Kohda, J. Nitta, Enhancement of spin lifetime in gate-fitted ingaas narrow wires, Phys. Rev. Lett. 102 (2009) 226601.

[700] S. Z. Denega, T. Last, J. Liu, A. Slachter, P. J. Rizo, P. H. M. van Loosdrecht, B. J. van Wees, D. Reuter, A. D. Wieck, C. H. van der Wal, Crystal-orientation dependant spin dephasing for 2D and bulk electrons in GaAs wires, 2009. ArXiv:0910.2336.

[701] S. Pramanik, S. Bandyopadhyay, M. Cahay, Spin dephasing in quantum wires, Phys. Rev. B 68 (2003) 075313.

[702] R. Ferreira, G. Bastard, Spin-flip scattering of holes in semiconductor quantum wells, Phys. Rev. B 43 (1991) 9687-9691.

[703] G. Bastard, R. Ferreira, Spin-flip scattering times in semiconductor quantum wells, Surf. Sci. 267 (1992) 335-341.

[704] M. I. D'yakonov, A. V. Khaetskii, Relaxation of nonequilibrium carrier-density matrix in semiconductors with degenerate bands, Sov. Phys. JETP 59 (1984) 1072-1078.

[705] C. Lü, U. Zülicke, M. W. Wu, Hole spin relaxation in p-type gaas quantum wires investigated by numerically solving fully microscopic kinetic spin bloch equations, Phys. Rev. B 78 (2008) 165321.

[706] R. Ferreira, G. Bastard, Hole \&ldquo;spin\&rdquo; relaxation in semiconductor quantum wells, Europhys. Lett. 23 (1993) $439-444$.

[707] W. Kauschke, N. Mestres, M. Cardona, Spin relaxation of holes in the split-hole band of inp and gasb, Phys. Rev. B 35 (1987) $3843-3853$.

[708] Y. A. Serebrennikov, Single-hole spin dephasing in bulk crystals, Phys. Rev. B 71 (2005) 233202. 
[709] C. Y. Hu, K. Morita, H. Sanada, S. Matsuzaka, Y. Ohno, H. Ohno, Spin precession of holes in wurtzite gan studied using the time-resolved kerr rotation technique, Phys. Rev. B 72 (2005) 121203.

[710] M. I. D'yakonov, V. I. Perel', Influence of an electric field and deformation on the optical orientation in semiconductors, Sov. Phys. Semicond. 7 (1974) 1551-1553.

[711] S. D. Ganichev, S. N. Danilov, V. V. Bel'kov, E. L. Ivchenko, M. Bichler, W. Wegscheider, D. Weiss, W. Prettl, Spin-sensitive bleaching and monopolar spin orientation in quantum wells, Phys. Rev. Lett. 88 (2002) 057401.

[712] P. Schneider, J. Kainz, S. D. Ganichev, S. N. Danilov, U. Rössler, W. Wegscheider, D. Weiss, W. Prettl, V. V. Bel'kov, M. M. Glazov, L. E. Golub, D. Schuh, Spin relaxation times of two-dimensional holes from spin sensitive bleaching of intersubband absorption, J. Appl. Phys. 96 (2004) 420-424.

[713] G. M. Minkov, A. A. Sherstobitov, A. V. Germanenko, O. E. Rut, V. A. Larionova, B. N. Zvonkov, Antilocalization and spin-orbit coupling in hole strained gaas/ingaas/gaas quantum well heterostructures, Phys. Rev. B 71 (2005) 165312

[714] T. Li, Y.-G. Zhu, X.-H. Zhang, S.-S. Ma, P.-F. Wang, Z.-C. Niu, Hole spin relaxation in an ultrathin inas monolayer, Chin. Phys. Lett. 26 (2009) 057303.

[715] T. Li, X. H. Zhang, Y. G. Zhu, X. Huang, L. F. Han, X. J. Shang, H. Q. Ni, Z. C. Niu, Temperature dependence of hole spin relaxation in ultrathin inas monolayers, Physica E: Low-dimensional Systems and Nanostructures 42 (2010) 1597 - 1600.

[716] B. Baylac, X. Marie, T. Amand, M. Brousseau, J. Barrau, Y. Shekun, Hole spin relaxation in intrinsic quantum wells, Surf. Sci. 326 (1995) $161-166$.

[717] X. Marie, T. Amand, P. Le Jeune, M. Paillard, P. Renucci, L. E. Golub, V. D. Dymnikov, E. L. Ivchenko, Hole spin quantum beats in quantum-well structures, Phys. Rev. B 60 (1999) 5811-5817.

[718] P. Roussignol, R. Ferreira, C. Delalande, G. Bastard, A. Vinattieri, J. Martinez-Pastor, L. Carraresi, M. Colocci, J. F. Palmier, B. Etienne, Hole spin relaxation in a n-doped quantum well structure, Surf. Sci. 305 (1994) 263-266.

[719] T. Kawazoe, Y. Masumoto, T. Mishina, Spin-relaxation process of holes in type-ii al0.34ga0.66as/alas multiple quantum wells, Phys. Rev. B 47 (1993) 10452-10455.

[720] Z. Chen, R. Bratschitsch, S. G. Carter, S. T. Cundiff, D. R. Yakovlev, G. Karczewski, T. Wojtowicz, J. Kossut, Electron spin polarization through interactions between excitons, trions, and the two-dimensional electron gas, Phys. Rev. B 75 (2007) 115320.

[721] F. J. Teran, M. Potemski, D. K. Maude, D. Plantier, A. K. Hassan, A. Sachrajda, Z. Wilamowski, J. Jaroszynski, T. Wojtowicz, G. Karczewski, Collective character of spin excitations in a system of $m n 2+$ spins coupled to a two-dimensional electron gas, Phys. Rev. Lett. 91 (2003) 077201

[722] N. P. Stern, R. C. Myers, M. Poggio, A. C. Gossard, D. D. Awschalom, Confinement engineering of s-d exchange interactions in $\mathrm{ga}_{1-x} \mathrm{mn}_{x}$ as/al $\mathrm{ga}_{1-y}$ as quantum wells, Phys. Rev. B 75 (2007) 045329.

[723] M. Vladimirova, S. Cronenberger, P. Barate, D. Scalbert, F. J. Teran, A. P. Dmitriev, Dynamics of the localized spins interacting with two-dimensional electron gas: Coexistence of mixed and pure modes, Phys. Rev. B 78 (2008) 081305.

[724] S. A. Crooker, J. J. Baumberg, F. Flack, N. Samarth, D. D. Awschalom, Terahertz spin precession and coherent transfer of angular momenta in magnetic quantum wells, Phys. Rev. Lett. 77 (1996) 2814-2817.

[725] S. A. Crooker, D. D. Awschalom, J. J. Baumberg, F. Flack, N. Samarth, Optical spin resonance and transverse spin relaxation in magnetic semiconductor quantum wells, Phys. Rev. B 56 (1997) 7574-7588.

[726] M. G. Tyazhlov, V. D. Kulakovskii, A. I. Filin, D. R. Yakovlev, A. Waag, G. Landwehr, Mn spin domains in highly photoexcited (cd,mn)te/(cd,mg)te quantum wells, Phys. Rev. B 59 (1999) 2050-2056.

[727] D. Keller, D. R. Yakovlev, B. König, W. Ossau, T. Gruber, A. Waag, L. W. Molenkamp, A. V. Scherbakov, Heating of the magnetic ion system in (zn, mn)se/(zn, be)se semimagnetic quantum wells by means of photoexcitation, Phys. Rev. B 65 (2001) 035313.

[728] M. K. Kneip, D. R. Yakovlev, M. Bayer, A. A. Maksimov, I. I. Tartakovskii, D. Keller, W. Ossau, L. W. Molenkamp, A. Waag, Direct energy transfer from photocarriers to $\mathrm{mn}$-ion system in ii-vi diluted-magnetic-semiconductor quantum wells, Phys. Rev. B 73 (2006) 035306.

[729] M. K. Kneip, D. R. Yakovlev, M. Bayer, A. A. Maksimov, I. I. Tartakovskii, D. Keller, W. Ossau, L. W. Molenkamp, A. Waag, Spin-lattice relaxation of mn ions in znmnse/znbese quantum wells measured under pulsed photoexcitation, Phys. Rev. B 73 (2006) 045305.

[730] A. V. Akimov, A. V. Scherbakov, D. R. Yakovlev, I. A. Merkulov, M. Bayer, A. Waag, L. W. Molenkamp, Multiple transfer of angular momentum quanta from a spin-polarized hole to magnetic ions in $\mathrm{zn}_{1-x} \mathrm{mn}_{x} \mathrm{se} / \mathrm{zn}_{1-y}$ be $\mathrm{y}_{y} \mathrm{se}$ quantum wells, Phys. Rev. B 73 (2006) 165328.

[731] E. Tsitsishvili, H. Kalt, Spin-conserving scattering of holes by magnetic ions in semimagnetic quantum wells, Phys. Rev. B 77 (2008) 155305.

[732] N. Linder, L. J. Sham, Theory of the coherent spin dynamics in magnetic semiconductor quantum wells, Physica E 2 (1998) $412-416$.

[733] M. R. Freeman, D. D. Awschalom, J. M. Hong, L. L. Chang, Femtosecond spin-polarization spectroscopy in diluted-magneticsemiconductor quantum wells, Phys. Rev. Lett. 64 (1990) 2430-2433.

[734] J. F. Smyth, D. A. Tulchinsky, D. D. Awschalom, N. Samarth, H. Luo, J. K. Furdyna, Femtosecond scattering dynamics in magnetic semiconductor spin superlattices, Phys. Rev. Lett. 71 (1993) 601-604.

[735] R. C. Myers, K. C. Ku, X. Li, N. Samarth, D. D. Awschalom, Optoelectronic control of spin dynamics at near-terahertz frequencies in magnetically doped quantum wells, Phys. Rev. B 72 (2005) 041302.

[736] R. Akimoto, K. Ando, F. Sasaki, S. Kobayashi, T. Tani, Carrier spin dynamics in cdte/cd ${ }_{1-x} \mathrm{mn}_{x}$ te quantum wells, Phys. Rev. B 56 (1997) 9726-9733.

[737] M. R. Freeman, D. D. Awschalom, Femtosecond probes of magnetic phenomena: Spin-polarization spectroscopy in dilute-magneticsemiconductor quantum wells (invited), J. Appl. Phys. 67 (1990) 5102-5107.

[738] D. A. Tulchinsky, J. J. Baumberg, D. D. Awschalom, N. Samarth, H. Luo, J. K. Furdyna, Femtosecond spin spectroscopy in magnetically tunable heterostructures, Phys. Rev. B 50 (1994) 10851-10855.

[739] J. J. Baumberg, S. A. Crooker, D. D. Awschalom, N. Samarth, H. Luo, J. K. Furdyna, Ultrafast faraday spectroscopy in magnetic semiconductor quantum structures, Phys. Rev. B 50 (1994) 7689-7700.

[740] I. A. Buyanova, G. Y. Rudko, W. M. Chen, K. Kayanuma, A. Murayama, Y. Oka, A. A. Toropov, S. V. Sorokin, S. V. Ivanov, Effect of 
momentum relaxation on exciton spin dynamics in diluted magnetic semiconductor znmnse / cdse superlattices, Phys. Rev. B 71 (2005) 165203.

[741] S. A. Crooker, D. D. Awschalom, N. Samarth, Time-resolved faraday rotation spectroscopy of spin dynamics in digital magnetic heterostructures, Select. Top. Quant. Electron. 1 (1995) 1082-1092.

[742] J. J. Baumberg, D. D. Awschalom, N. Samarth, Femtosecond faraday rotation in spin-engineered heterostructures (invited), J. Appl. Phys. 75 (1994) 6199-6204.

[743] J. C. Egues, J. W. Wilkins, Spin-dependent phenomena in digital-magnetic heterostructures: Clustering and phase-space filling effects, Phys. Rev. B 58 (1998) R16012-R16015.

[744] Y. G. Semenov, Electron spin relaxation in semiconductors and semiconductor structures, Phys. Rev. B 67 (2003) 115319.

[745] E. Tsitsishvili, H. Kalt, Magnetic field effects on spin-flip processes in semimagnetic quantum wells, Phys. Rev. B 73 (2006) 195402.

[746] A. Murayama, K. Seo, K. Nishibayashi, I. Souma, Y. Oka, Spin dynamics in a diluted magnetic semiconductor quantum well studied by pump-probe absorption spectroscopy: Magnetic-field-induced suppression of electron-spin relaxation, Appl. Phys. Lett. 88 (2006) 261105.

[747] K. Seo, K. Kayanuma, I. Aoshima, K. Nishibayashi, I. Souma, A. Murayama, Y. Oka, Suppression of electron-spin relaxation induced by magnetic fields in $\mathrm{a} \mathrm{cd}_{1-x} \mathrm{mn}_{x}$ te quantum well, Phys. Stat. Sol. (c) 3 (2006) 1109-1113.

[748] J. J. Baumberg, D. D. Awschalom, N. Samarth, H. Luo, J. K. Furdyna, Spin beats and dynamical magnetization in quantum structures, Phys. Rev. Lett. 72 (1994) 717-720.

[749] C. J. P. Smits, S. C. A. van Driel, M. van Kampen, W. J. M. de Jonge, B. Koopmans, G. Karczewski, Excitonic enhancement of spin relaxation in diluted magnetic semiconductor quantum wells, Phys. Rev. B 70 (2004) 115307

[750] J. M. Kikkawa, I. P. Smorchkova, N. Samarth, D. D. Awschalom, Optical studies of spin precession in magnetic two-dimensional electron gases, Physica E 2 (1998) 394-398.

[751] Y. Zhu, X. Zhang, T. Li, L. Chen, J. Lu, J. Zhao, Spin relaxation and dephasing mechanism in (ga,mn)as studied by time-resolved kerr rotation, Appl. Phys. Lett. 94 (2009) 142109.

[752] R. Zhou, B. Q. Sun, X. Z. Ruan, H. H. Luo, Y. Ji, W. Z. Wang, F. Zhang, J. H. Zhao, Temperature dependence of effective g factor in diluted magnetic semiconductor (ga,mn)as, J. Appl. Phys. 103 (2008) 053901.

[753] S. Cronenberger, P. Barate, A. Brunetti, M. Vladimirova, D. Scalbert, F. J. Teran, G. Karzewski, T. Wojtowicz, Electron spin relaxation in very diluted cdmnte quantum wells, Superlatt. Micro. 43 (2008) 427-430.

[754] K. Saito, J. H. Park, I. Souma, Y. Oka, A. Murayama, Direct observation of relaxation dynamics of spin-polarized electrons excited at a higher-energy spin-split subband in a diluted magnetic semiconductor quantum well, Appl. Phys. Lett. 90 (2007) 262111.

[755] M. R. Junnarkar, R. R. Alfano, J. K. Furdyna, High-density photogenerated free-carrier spin relaxation processes in wurtzite semiconductors: Cdse and semimagnetic semiconductor $\mathrm{cd}_{1-x} \mathrm{mn}_{x}$ se, IEEE J. Quant. Electron. 24 (1988) 315-324.

[756] C. Śliwa, T. Dietl, Electron-hole contribution to the apparent $s$ - $d$ exchange interaction in iii-v dilute magnetic semiconductors, Phys. Rev. B 78 (2008) 165205.

[757] G. V. Astakhov, R. I. Dzhioev, K. V. Kavokin, V. L. Korenev, M. V. Lazarev, M. N. Tkachuk, Y. G. Kusrayev, T. Kiessling, W. Ossau, L. W. Molenkamp, Suppression of electron spin relaxation in mn-doped gaas, Phys. Rev. Lett. 101 (2008) 076602.

[758] I. A. Akimov, R. I. Dzhioev, V. L. Korenev, Y. G. Kusrayev, E. A. Zhukov, D. R. Yakovlev, M. Bayer, Electron-spin dynamics in mn-doped gaas using time-resolved magneto-optical techniques, Phys. Rev. B 80 (2009) 081203.

[759] E. Abrahams, Donor electron spin relaxation in silicon, Phys. Rev. 107 (1957) 491-496.

[760] J. W. Culvahouse, F. M. Pipkin, Experimental study of spin-lattice relaxation times in arsenic-doped silicon, Phys. Rev. 109 (1958) 319-327.

[761] G. Feher, E. A. Gere, Electron spin resonance experiments on donors in silicon. ii. electron spin relaxation effects, Phys. Rev. 114 (1959) 1245-1256.

[762] H. Hasegawa, Spin-lattice relaxation of shallow donor states in ge and si through a direct phonon process, Phys. Rev. 118 (1960) $1523-$ 1534.

[763] D. J. Lépine, Spin resonance of localized and delocalized electrons in phosphorus-doped silicon between 20 and $30{ }^{\circ} \mathrm{k}$, Phys. Rev. B 2 (1970) 2429-2439.

[764] D. K. Wilson, Electron spin resonance experiments on shallow donors in germanium, Phys. Rev. 134 (1964) A265-A286.

[765] A. M. Tyryshkin, S. A. Lyon, A. V. Astashkin, A. M. Raitsimring, Electron spin relaxation times of phosphorus donors in silicon, Phys. Rev. B 68 (2003) 193207.

[766] A. Ferretti, M. Fanciulli, A. Ponti, A. Schweiger, Electron spin-echo relaxation and envelope modulation of shallow phosphorus donors in silicon, Phys. Rev. B 72 (2005) 235201.

[767] V. Zarifis, T. G. Castner, Esr linewidth behavior for barely metallic $n$-type silicon, Phys. Rev. B 36 (1987) 6198-6201.

[768] J. D. Quirt, J. R. Marko, Absolute spin susceptibilities and other esr parameters of heavily doped $n$-type silicon. i. metallic samples, Phys. Rev. B 5 (1972) 1716-1728.

[769] G. P. Carver, D. F. Holcomb, J. A. Kaeck, p31 spin echoes in metallic phosphorus-doped silicon, Phys. Rev. B 3 (1971) $4285-4286$.

[770] H. Ue, S. Maekawa, Electron-spin-resonance studies of heavily phosphorus-doped silicon, Phys. Rev. B 3 (1971) $4232-4238$.

[771] G. Lancaster, J. A. van Wyk, E. E. Schneider, Spin-lattice relaxation of conduction electrons in silicon, Proc. Phys. Soc. 84 (1964) 19-24.

[772] M. Prada, G. Klimeck, R. Joynt, Spin-orbit splittings in Si/SiGe quantum wells, 2009. ArXiv:0908.2417.

[773] A. M. Tyryshkin, S. A. Lyon, W. Jantsch, F. Schäffler, Spin manipulation of free two-dimensional electrons in si/sige quantum wells, Phys. Rev. Lett. 94 (2005) 126802.

[774] N. Nestle, G. Denninger, M. Vidal, C. Weinzierl, K. Brunner, K. Eberl, K. von Klitzing, Electron spin resonance on a two-dimensional electron gas, Phys. Rev. B 56 (1997) R4359-R4362.

[775] N. Sandersfeld, W. Jantsch, Z. Wilamowski, F. Schäffler, Esr investigations of modulation-doped si/sige quantum wells, Thin Solid Films 369 (2000) 312-315.

[776] W. Jantsch, Z. Wilamowski, N. Sandersfeld, M. Mühlberger, F. Schäffler, Spin lifetimes and $g$-factor tuning in si/sige quantum wells, Physica E 13 (2002) 504-507. 
[777] W. Jantsch, Z. Wilamowski, Z. Wilamowski, N. Sandersfeld, F. Schäffler, Esr investigations of modulation-doped si/sige quantum wells, Phys. Stat. Sol. (b) 210 (1998) 643-648.

[778] H. Malissa, W. Jantsch, M. Mühlberger, F. Schäffler, Z. Wilamowski, M. Draxler, P. Bauer, Anisotropy of g-factor and electron spin resonance linewidth in modulation doped sige quantum wells, Appl. Phys. Lett. 85 (2004) 1739-1741.

[779] J. L. Truitt, K. A. Slinker, K. L. M. Lewis, D. E. Savage, C. Tahan, L. J. Klein, J. O. Chu, P. M. Mooney, A. M. Tyryshkin, D. W. van der Weide, R. Joynt, S. N. Coppersmith, M. Friesen, M. A. Eriksson, Electron Spin Resonance and Related Phenomena in Low-Dimensional Structures, Springer, Berlin, 2009.

[780] S. Shankar, A. M. Tyryshkin, J. He, S. A. Lyon, Spin relaxation and coherence times for electrons at the Si/SiO2 interface, 2009. ArXiv:0912.3037.

[781] C. F. O. Graeff, M. S. Brandt, M. Stutzmann, M. Holzmann, G. Abstreiter, F. Schäffler, Electrically detected magnetic resonance of two-dimensional electron gases in si/sige heterostructures, Phys. Rev. B 59 (1999) 13242-13250.

[782] J. Matsunami, M. Ooya, T. Okamoto, Electrically detected electron spin resonance in a high-mobility silicon quantum well, Phys. Rev. Lett. 97 (2006) 066602.

[783] M. M. Glazov, E. L. Ivchenko, Electron-electron scattering effect on spin relaxation in multi-valley nanostructures, Europhys. Lett. 87 (2009) 57005 (6pp).

[784] H. Ohno, Making Nonmagnetic Semiconductors Ferromagnetic, Science 281 (1998) 951-956.

[785] H. Akinaga, H. Ohno, Semiconductor spintronics, IEEE Trans. Nanotechnol. 1 (2002) 19-31.

[786] A. H. MacDonald, P. Schiffer, N. Samarth, Ferromagnetic semiconductors: moving beyond (ga,mn)as, Nat. Mater. 4 (2005) $195-202$.

[787] S. Das Sarma, Ferromagnetic semiconductors: A giant appears in spintronics, Nat. Mater. 2 (2003) $292-294$.

[788] J. Wang, C. Sun, J. Kono, A. Oiwa, H. Munekata, L. Cywinski, L. J. Sham, Ultrafast quenching of ferromagnetism in inmnas induced by intense laser irradiation, Phys. Rev. Lett. 95 (2005) 167401.

[789] J. Wang, I. Cotoros, K. M. Dani, X. Liu, J. K. Furdyna, D. S. Chemla, Ultrafast enhancement of ferromagnetism via photoexcited holes in gamnas, Phys. Rev. Lett. 98 (2007) 217401.

[790] J. Wang, L. Cywinski, C. Sun, J. Kono, H. Munekata, L. J. Sham, Femtosecond demagnetization and hot-hole relaxation in ferromagnetic ga $_{1-x} \mathrm{mn}_{x}$ as, Phys. Rev. B 77 (2008) 235308.

[791] J. Wang, I. Cotoros, D. S. Chemla, X. Liu, J. K. Furdyna, J. Chovan, I. E. Perakis, Memory effects in photoinduced femtosecond magnetization rotation in ferromagnetic gamnas, Appl. Phys. Lett. 94 (2009) 021101.

[792] K. C. Hall, J. P. Zahn, A. Gamouras, S. March, J. L. Robb, X. Liu, J. K. Furdyna, Ultrafast optical control of coercivity in gamnas, Appl. Phys. Lett. 93 (2008) 032504

[793] G. V. Astakhov, A. V. Kimel, G. M. Schott, A. A. Tsvetkov, A. Kirilyuk, D. R. Yakovlev, G. Karczewski, W. Ossau, G. Schmidt, L. W. Molenkamp, T. Rasing, Magnetization manipulation in (ga,mn)as by subpicosecond optical excitation, Appl. Phys. Lett. 86 (2005) 152506.

[794] T. Kondo, K. Nomura, G. Koizumi, H. Munekata, Photo-induced changes in magnetization orientation in (ga,mn)as observed directly by a scanning laser magneto-optical microscope, Phys. Stat. Sol. (c) 3 (2006) 4263-4266.

[795] A. Oiwa, Y. Mitsumori, R. Moriya, T. Słupinski, H. Munekata, Effect of optical spin injection on ferromagnetically coupled mn spins in the iii-v magnetic alloy semiconductor (ga,mn)as, Phys. Rev. Lett. 88 (2002) 137202.

[796] S. Koshihara, A. Oiwa, M. Hirasawa, S. Katsumoto, Y. Iye, C. Urano, H. Takagi, H. Munekata, Ferromagnetic order induced by photogenerated carriers in magnetic iii-v semiconductor heterostructures of (in,mn)as/gasb, Phys. Rev. Lett. 78 (1997) 4617-4620.

[797] J. Qi, Y. Xu, N. H. Tolk, X. Liu, J. K. Furdyna, I. E. Perakis, Coherent magnetization precession in gamnas induced by ultrafast optical excitation, Appl. Phys. Lett. 91 (2007) 112506.

[798] E. Rozkotova, P. Nemec, N. Tesarova, P. Maly, V. Novak, K. Olejnik, M. Cukr, T. Jungwirth, Coherent control of magnetization precession in ferromagnetic semiconductor (ga,mn)as, Appl. Phys. Lett. 93 (2008) 232505.

[799] Y. Hashimoto, H. Munekata, Coherent manipulation of magnetization precession in ferromagnetic semiconductor (ga,mn)as with successive optical pumping, Appl. Phys. Lett. 93 (2008) 202506

[800] E. Rozkotova, P. Nemec, P. Horodyska, D. Sprinzl, F. Trojanek, P. Maly, V. Novak, K. Olejnik, M. Cukr, T. Jungwirth, Light-induced magnetization precession in gamnas, Appl. Phys. Lett. 92 (2008) 122507.

[801] E. Rozkotova, P. Nemec, D. Sprinzl, P. Horodyska, F. Trojanek, P. Maly, V. Novak, K. Olejnik, M. Cukr, T. Jungwirth, Laser-induced precession of magnetization in gamnas, IEEE Trans. Magn. 44 (2008) 2674.

[802] S. Kobayashi, Y. Hashimoto, H. Munekata, Investigation of an effective anisotropy field involved in photoinduced precession of magnetization in (ga,mn)as, J. Appl. Phys. 105 (2009) 07C519.

[803] Y. Mitsumori, A. Oiwa, T. Słupinski, H. Maruki, Y. Kashimura, F. Minami, H. Munekata, Dynamics of photoinduced magnetization rotation in ferromagnetic semiconductor p-(ga,mn)as, Phys. Rev. B 69 (2004) 033203.

[804] J. Qi, Y. Xu, A. Steigerwald, X. Liu, J. K. Furdyna, I. E. Perakis, N. H. Tolk, Ultrafast laser-induced coherent spin dynamics in ferromagnetic $\mathrm{ga}_{1-x} \mathrm{mn}_{x}$ as/gaas structures, Phys. Rev. B 79 (2009) 085304.

[805] Y. Hashimoto, S. Kobayashi, H. Munekata, Photoinduced precession of magnetization in ferromagnetic (ga,mn)as, Phys. Rev. Lett. 100 (2008) 067202

[806] G. V. Astakhov, H. Hoffmann, V. L. Korenev, T. Kiessling, J. Schwittek, G. M. Schott, C. Gould, W. Ossau, K. Brunner, L. W. Molenkamp, Nonthermal photocoercivity effect in a low-doped (ga,mn)as ferromagnetic semiconductor, Phys. Rev. Lett. 102 (2009) 187401.

[807] E. Kojima, R. Shimano, Y. Hashimoto, S. Katsumoto, Y. Iye, M. Kuwata-Gonokami, Observation of the spin-charge thermal isolation of ferromagnetic ga0.94mn0.06as by time-resolved magneto-optical measurements, Phys. Rev. B 68 (2003) 193203.

[808] A. V. Kimel, G. V. Astakhov, G. M. Schott, A. Kirilyuk, D. R. Yakovlev, G. Karczewski, W. Ossau, G. Schmidt, L. W. Molenkamp, T. Rasing, Picosecond dynamics of the photoinduced spin polarization in epitaxial (ga,mn)as films, Phys. Rev. Lett. 92 (2004) 237203.

[809] A. Chernyshov, M. Overby, X. Liu, J. K. Furdyna, L. P. Rokhinson, Reversible control of magnetization in a ferromagnetic material via spin-orbit magnetic field, Nat. Phys. 5 (2009) 656-659.

[810] H. Ohno, D. Chiba, F. Matsukura, T. Omiya, E. Abe, T. Dietl, Y. Ohno, K. Ohtani, Electric-field control of ferromagnetism, Nature 408 (2000) 944-946. 
[811] Z. Li, J. A. Mol, L. Lagae, G. Borghs, R. Mertens, W. Van Roy, Pulsed field induced magnetization switching in (ga,mn)as, Appl. Phys. Lett. 92 (2008) 112513

[812] D. Chiba, M. Sawicki, Y. Nishitani, Y. Nakatani, F. Matsukura, H. Ohno, Magnetization vector manipulation by electric fields, Nature 455 (2008) 515-518.

[813] E. Beaurepaire, J.-C. Merle, A. Daunois, J.-Y. Bigot, Ultrafast spin dynamics in ferromagnetic nickel, Phys. Rev. Lett. 76 (1996) 42504253.

[814] B. Koopmans, M. van Kampen, J. T. Kohlhepp, W. J. M. de Jonge, Ultrafast magneto-optics in nickel: Magnetism or optics?, Phys. Rev. Lett. 85 (2000) 844-847.

[815] B. Koopmans, J. J. M. Ruigrok, F. D. Longa, W. J. M. de Jonge, Unifying ultrafast magnetization dynamics, Phys. Rev. Lett. 95 (2005) 267207.

[816] J. Hohlfeld, E. Matthias, R. Knorren, K. H. Bennemann, Nonequilibrium magnetization dynamics of nickel, Phys. Rev. Lett. 78 (1997) 4861-4864.

[817] M. Cinchetti, M. S. Albaneda, D. Hoffmann, T. Roth, J.-P. Wustenberg, M. Krauss, O. Andreyev, H. C. Schneider, M. Bauer, M. Aeschlimann, Spin-flip processes and ultrafast magnetization dynamics in co: Unifying the microscopic and macroscopic view of femtosecond magnetism, Phys. Rev. Lett. 97 (2006) 177201.

[818] C. Stamm, T. Kachel, N. Pontius, R. Mitzner, T. Quast, K. Holldack, S. Khan, C. Lupulescu, E. F. Aziz, M. Wietstruk, H. A. Durr, W. Eberhardt, Femtosecond modification of electron localization and transfer of angular momentum in nickel, Nat. Mater. 6 (2007) $740-743$.

[819] U. Atxitia, O. Chubykalo-Fesenko, J. Walowski, A. Mann, M. Munzenberg, Tracing the thermal mechanism in femtosecond spin dynamics, 2009. ArXiv:0904.4399.

[820] E. Carpene, E. Mancini, C. Dallera, M. Brenna, E. Puppin, S. De Silvestri, Dynamics of electron-magnon interaction and ultrafast demagnetization in thin iron films, Phys. Rev. B 78 (2008) 174422

[821] A. Vaterlaus, T. Beutler, D. Guarisco, M. Lutz, F. Meier, Spin-lattice relaxation in ferromagnets studied by time-resolved spin-polarized photoemission, Phys. Rev. B 46 (1992) 5280-5286.

[822] M. Aeschlimann, M. Bauer, S. Pawlik, W. Weber, R. Burgermeister, D. Oberli, H. C. Siegmann, Ultrafast spin-dependent electron dynamics in fcc co, Phys. Rev. Lett. 79 (1997) 5158-5161.

[823] G. P. Zhang, W. Hübner, Laser-induced ultrafast demagnetization in ferromagnetic metals, Phys. Rev. Lett. 85 (2000) $3025-3028$.

[824] U. Atxitia, O. Chubykalo-Fesenko, R. W. Chantrell, U. Nowak, A. Rebei, Ultrafast spin dynamics: The effect of colored noise, Phys. Rev. Lett. 102 (2009) 057203.

[825] M. Krauß, T. Roth, S. Alebrand, D. Steil, M. Cinchetti, M. Aeschlimann, H. C. Schneider, Ultrafast demagnetization of ferromagnetic transition metals: The role of the coulomb interaction, Phys. Rev. B 80 (2009) 180407.

[826] L. Cywinski, L. J. Sham, Ultrafast demagnetization in the sp-d model: A theoretical study, Phys. Rev. B 76 (2007) 045205.

[827] J. Sinova, T. Jungwirth, X. Liu, Y. Sasaki, J. K. Furdyna, W. A. Atkinson, A. H. MacDonald, Magnetization relaxation in (ga,mn)as ferromagnetic semiconductors, Phys. Rev. B 69 (2004) 085209.

[828] Y. Tserkovnyak, G. A. Fiete, B. I. Halperin, Mean-field magnetization relaxation in conducting ferromagnets, Appl. Phys. Lett. 84 (2004) 5234-5236.

[829] A. Oiwa, H. Takechi, H. Munekata, Photoinduced magnetization rotation and precessional motion of magnetization in ferromagnetic (ga,mn)as, J. Supercond. 18 (2005) 9-13.

[830] D. M. Wang, Y. H. Ren, X. Liu, J. K. Furdyna, M. Grimsditch, R. Merlin, Light-induced magnetic precession in (ga,mn)as slabs: Hybrid standing-wave damon-eshbach modes, Phys. Rev. B 75 (2007) 233308.

[831] M. D. Kapetanakis, I. E. Perakis, Spin dynamics in (iii,mn)v ferromagnetic semiconductors: The role of correlations, Phys. Rev. Lett. 101 (2008) 097201.

[832] M. D. Kapetanakis, I. E. Perakis, K. J. Wickey, C. Piermarocchi, J. Wang, Femtosecond coherent control of spins in (ga,mn)as ferromagnetic semiconductors using light, Phys. Rev. Lett. 103 (2009) 047404.

[833] H. Haug, A.-P. Jauho, Quantum Kinetics in Transport and Optics of Semiconductors, Springer, Berlin, 1996.

[834] T. Östreich, K. Schönhammer, L. J. Sham, Exciton-exciton correlation in the nonlinear optical regime, Phys. Rev. Lett. 74 (1995) 46984701.

[835] C. Lü, J. L. Cheng, M. W. Wu, I. C. da Cunha Lima, Spin relaxation time, spin dephasing time and ensemble spin dephasing time in $n$-type gaas quantum wells, Phys. Lett. A 365 (2007) 501-504.

[836] M. E. Flatté, J. M. Byers, W. H. Lau, Spin Dynamics in Semiconductors, Springer, Berlin, 2002.

[837] C. R. Pidgeon, D. L. Mitchell, R. N. Brown, Interband magnetoabsorption in inas and insb, Phys. Rev. 154 (1967) 737-742.

[838] B. D. McCombe, R. J. Wagner, Electric-dipole-excited electron spin resonance in insb, Phys. Rev. B 4 (1971) $1285-1288$.

[839] D. Stein, K. v. Klitzing, G. Weimann, Electron spin resonance on gaas-alxga1-xas heterostructures, Phys. Rev. Lett. 51 (1983) $130-133$.

[840] G. Lommer, F. Malcher, U. Rössler, Reduced g factor of subband landau levels in algaas/gaas heterostructures, Phys. Rev. B 32 (1985) 6965-6967.

[841] J. L. Cheng, M. W. Wu, Intense terahertz laser fields on a two-dimensional electron gas with rashba spin-orbit coupling, Appl. Phys. Lett. 86 (2005) 032107.

[842] K. Shen, G. Tatara, M. W. Wu, Effect of spin-conserving scattering on gilbert damping in ferromagnetic semiconductors, 2010. ArXiv:1001.4576.

[843] M. M. Glazov, E. L. Ivchenko, Effect of electron-electron interaction on spin relaxation of charge carriers in semiconductors, JETP 99 (2004) 1279-1290.

[844] M. Q. Weng, M. W. Wu, Multisubband effect in spin dephasing in semiconductor quantum wells, Phys. Rev. B 70 (2004) 195318.

[845] P. Zhang, J. Zhou, M. W. Wu, Multivalley spin relaxation in the presence of high in-plane electric fields in $n$-type gaas quantum wells, Phys. Rev. B 77 (2008) 235323.

[846] P. Zhang, M. W. Wu, Spin diffusion in si/sige quantum wells: Spin relaxation in the absence of d'yakonov-perel' relaxation mechanism, 
Phys. Rev. B 79 (2009) 075303.

[847] F. X. Bronold, A. Saxena, D. L. Smith, Semiclassical kinetic theory of electron spin relaxation in semiconductors, Phys. Rev. B 70 (2004) 245210.

[848] G. F. Giulianni, G. Vignale, Quantum Theory of the Electron Liquid, Cambridge University Press, Cambridge, England, 2005.

[849] A. P. Dmitriev, V. Y. Kachorovskii, M. S. Shur, M. Stroscio, Electron runaway and negative differential mobility in two-dimensional electron gas in elementary semiconductors, Solid State Commun. 113 (2000) 565-568.

[850] A. P. Dmitriev, V. Y. Kachorovskii, M. S. Shur, High-field transport in a dense two-dimensional electron gas in elementary semiconductors, J. Appl. Phys. 89 (2001) 3793-3797.

[851] E. M. Conwell, High Field Transport in Semiconductors, Pergamon, Oxford, 1972.

[852] E. L. Ivchenko, G. E. Pikus, Superlattices and Other Heterostructures: Symmetry and Optical Phenomena, Springer, Berlin, 1997.

[853] K. Shen, M. Q. Weng, M. W. Wu, L-valley electron g-factor in bulk gaas and alas, J. Appl. Phys. 104 (2008) 063719.

[854] P. Zhang, M. W. Wu, Non-markovian hole spin kinetics in p-type gaas quantum wells, Phys. Rev. B 76 (2007) 193312.

[855] L. Bányai, D. B. T. Thoai, E. Reitsamer, H. Haug, D. Steinbach, M. U. Wehner, M. Wegener, T. Marschner, W. Stolz, Exciton lo-phonon quantum kinetics: Evidence of memory effects in bulk gaas, Phys. Rev. Lett. 75 (1995) 2188-2191.

[856] S. Hallstein, J. D. Berger, M. Hilpert, H. C. Schneider, W. W. Rühle, F. Jahnke, S. W. Koch, H. M. Gibbs, G. Khitrova, M. Oestreich, Manifestation of coherent spin precession in stimulated semiconductor emission dynamics, Phys. Rev. B 56 (1997) R7076-R7079.

[857] P. Nemec, Y. Kerachian, H. M. van Driel, A. L. Smirl, Spin-dependent electron many-body effects in gaas, Phys. Rev. B 72 (2005) 245202.

[858] E. I. Rashba, Spin dynamics and spin transport, J. Supercond. 18 (2005) 137.

[859] K. Johnsen, Spin-dynamic field coupling in strongly terahertz-field-driven semiconductors: Local inversion symmetry breaking, Phys. Rev. B 62 (2000) 10978-10983.

[860] J. H. Jiang, M. Q. Weng, M. W. Wu, Intense terahertz laser fields on a quantum dot with rashba spin-orbit coupling, J. Appl. Phys. 100 (2006) 063709 .

[861] Y. Zhou, Intense terahertz laser fields on a two-dimensional hole gas with rashba spin-orbit coupling, Physica E 40 (2008) $2847-2850$.

[862] K. Johnsen, A. P. Jauho, Linear optical absorption spectra of mesoscopic structures in intense thz fields: Free-particle properties, Phys. Rev. B 57 (1998) 8860-8872.

[863] A. P. Jauho, K. Johnsen, Dynamical franz-keldysh effect, Phys. Rev. Lett. 76 (1996) 4576-4579.

[864] J. H. Shirley, Solution of the schrödinger equation with a hamiltonian periodic in time, Phys. Rev. 138 (1965) B979-B987.

[865] M. Grifoni, P. Hänggi, Driven quantum tunneling, Phys. Rep. 304 (1998) 229-354.

[866] S. Kohler, J. Lehmann, P. Hänggi, Driven quantum transport on the nanoscale, Phys. Rep. 406 (2005) 379-443.

[867] S. Kohler, T. Dittrich, P. Hänggi, Floquet-markovian description of the parametrically driven, dissipative harmonic quantum oscillator, Phys. Rev. E 55 (1997) 300-313.

[868] Y. Yafet, Calculation of the $g$ factor of metallic sodium, Phys. Rev. 85 (1952) 478.

[869] J. K. Furdyna, J. Kossut, Diluted Magnetic Semiconductors, Academic, New York, 1988.

[870] M. Averous, M. Balkanski, Semimagnetic semiconductors and diluted magnetic semiconductors, Plenum, New York, 1991.

[871] K. W. Edmonds, P. Bogusławski, K. Y. Wang, R. P. Campion, S. N. Novikov, N. R. S. Farley, B. L. Gallagher, C. T. Foxon, M. Sawicki, T. Dietl, M. Buongiorno Nardelli, J. Bernholc, Mn interstitial diffusion in (ga, mn)as, Phys. Rev. Lett. 92 (2004) 037201.

[872] R. Schulz, T. Korn, D. Stich, U. Wurstbauer, D. Schuh, W. Wegscheider, C. Schüller, Ultrafast optical studies of diffusion barriers between ferromagnetic ga(mn)as layers and non-magnetic quantum wells, Physica E 40 (2008) 2163-2165.

[873] T. Korn, R. Schulz, S. Fehringer, U. Wurstbauer, D. Schuh, W. Wegscheider, M. W. Wu, C. Schüller, Manganese-diffusion-induced ndoping in semiconductor structures containing $\mathrm{Ga}(\mathrm{Mn})$ As layers, 2008. ArXiv:0809.3654.

[874] F. Schäffler, High-mobility si and ge structures, Semicond. Sci. Technol. 12 (1997) 1515-1549.

[875] J. M. Luttinger, W. Kohn, Motion of electrons and holes in perturbed periodic fields, Phys. Rev. 97 (1955) 869-883.

[876] C. Y.-P. Chao, S. L. Chuang, Spin-orbit-coupling effects on the valence-band structure of strained semiconductor quantum wells, Phys. Rev. B 46 (1992) 4110-4122.

[877] F. M. Bufler, A. Schenk, W. Fichtner, Simplified model for inelastic acoustic phonon scattering of holes in si and ge, J. Appl. Phys. 90 (2001) 2626-2628.

[878] U. Özgür, Y. I. Alivov, C. Liu, A. Teke, M. A. Reshchikov, S. Doğan, V. Avrutin, S.-J. Cho, H. Morkoç, A comprehensive review of zno materials and devices, J. Appl. Phys. 98 (2005) 041301.

[879] W. K. Liu, K. M. Whitaker, A. L. Smith, K. R. Kittilstved, B. H. Robinson, D. R. Gamelin, Room-temperature electron spin dynamics in free-standing zno quantum dots, Phys. Rev. Lett. 98 (2007) 186804.

[880] D. Lagarde, A. Balocchi, P. Renucci, H. Carrère, T. Amand, X. Marie, Z. X. Mei, X. L. Du, Hole spin quantum beats in bulk zno, Phys. Rev. B 79 (2009) 045204.

[881] D. Lagarde, A. Balocchi, P. Renucci, H. Carrère, F. Zhao, T. Amand, X. Marie, Z. X. Mei, X. L. Du, Q. K. Xue, Exciton and hole spin dynamics in zno investigated by time-resolved photoluminescence experiments, Phys. Rev. B 78 (2008) 033203.

[882] T. Dietl, H. Ohno, F. Matsukura, J. Cibert, D. Ferrand, Zener Model Description of Ferromagnetism in Zinc-Blende Magnetic Semiconductors, Science 287 (2000) 1019-1022.

[883] L. N. Pfeiffer, R. de Picciotto, K. W. West, K. W. Baldwin, C. H. L. Quay, Ballistic hole transport in a quantum wire, Appl. Phys. Lett. 87 (2005) 073111.

[884] R. Danneau, W. R. Clarke, O. Klochan, A. P. Micolich, A. R. Hamilton, M. Y. Simmons, M. Pepper, D. A. Ritchie, Conductance quantization and the $0.7 \times 2 \mathrm{e}[\sup 2] / \mathrm{h}$ conductance anomaly in one-dimensional hole systems, Appl. Phys. Lett. 88 (2006) 012107.

[885] O. Klochan, W. R. Clarke, R. Danneau, A. P. Micolich, L. H. Ho, A. R. Hamilton, K. Muraki, Y. Hirayama, Ballistic transport in induced one-dimensional hole systems, Appl. Phys. Lett. 89 (2006) 092105.

[886] K.-D. Hof, C. Rossler, S. Manus, J. P. Kotthaus, A. W. Holleitner, D. Schuh, W. Wegscheider, Dynamic photoconductive gain effect in shallow-etched algaas/gaas quantum wires, Phys. Rev. B 78 (2008) 115325.

[887] S. Farhangfar, Quantum size effects in solitary wires of bismuth, Phys. Rev. B 76 (2007) 205437. 
[888] P. Lehnen, T. Schäpers, N. Kaluza, N. Thillosen, H. Hardtdegen, Enhanced spin-orbit scattering length in narrow $\mathrm{al}_{x} \mathrm{ga}_{1-x} \mathrm{n} / \mathrm{gan}$ wires, Phys. Rev. B 76 (2007) 205307.

[889] B. K. Agrawal, V. Singh, R. Srivastava, S. Agrawal, Ab initio study of the structural, electronic, and optical properties of ultrathin lead nanowires, Phys. Rev. B 74 (2006) 245405.

[890] D. Csontos, P. Brusheim, U. Zülicke, H. Q. Xu, Spin-(3/2) physics of semiconductor hole nanowires: Valence-band mixing and tunable interplay between bulk-material and orbital bound-state spin splittings, Phys. Rev. B 79 (2009) 155323.

[891] G. Goldoni, F. Rossi, E. Molinari, A. Fasolino, R. Rinaldi, R. Cingolani, Valence band spectroscopy in v-grooved quantum wires, Appl. Phys. Lett. 69 (1996) 2965-2967.

[892] S. Pramanik, S. Bandyopadhyay, M. Cahay, Energy dispersion relations of spin-split subbands in a quantum wire and electrostatic modulation of carrier spin polarization, Phys. Rev. B 76 (2007) 155325.

[893] Y. Arakawa, T. Yamauchi, J. N. Schulman, Tight-binding analysis of energy-band structures in quantum wires, Phys. Rev. B 43 (1991) $4732-4738$.

[894] D. S. Citrin, Y.-C. Chang, Valence-subband structures of gaas/alxga1 - xas quantum wires: The effect of split-off bands, Phys. Rev. B 40 (1989) 5507-5514.

[895] O. Stier, D. Bimberg, Modeling of strained quantum wires using eight-band k.p theory, Phys. Rev. B 55 (1997) 7726-7732.

[896] N. Shtinkov, P. Desjardins, R. A. Masut, S. J. Vlaev, Lateral confinement and band mixing in ultrathin semiconductor quantum wells with steplike interfaces, Phys. Rev. B 70 (2004) 155302.

[897] P. Schwab, M. Dzierzawa, C. Gorini, R. Raimondi, Spin relaxation in narrow wires of a two-dimensional electron gas, Phys. Rev. B 74 (2006) 155316.

[898] M. Ohno, K. Yoh, Vanishing of inhomogeneous spin relaxation in inas-based field-effect transistor structures, Phys. Rev. B 75 (2007) 241308.

[899] J. L. Cheng, M. Q. Weng, M. W. Wu, Manipulation of spin dephasing in inas quantum wires, Solid State Commun. 128 (2003) 365-368.

[900] K. Zerrouati, F. Fabre, G. Bacquet, J. Bandet, J. Frandon, G. Lampel, D. Paget, Spin-lattice relaxation in p-type gallium arsenide single crystals, Phys. Rev. B 37 (1988) 1334-1341.

[901] K. L. Litvinenko, M. A. Leontiadou, J. Li, S. K. Clowes, M. T. Emeny, T. Ashley, C. R. Pidgeon, L. F. Cohen, B. N. Murdin, Strong dependence of spin dynamics on the orientation of an external magnetic field for insb and inas, Appl. Phys. Lett. 96 (2010) 111107.

[902] P. J. Rizo, A. Pugzlys, A. Slachter, S. Z. Denega, D. Reuter, A. D. Wieck, P. H. M. van Loosdrecht, C. H. van der Wal, Optical probing of spin dynamics of two-dimensional and bulk electrons in a GaAs/AlGaAs heterojunction system, 2009. ArXiv:0910.1714.

[903] D. D. Awschalom, D. Loss, N. Samarth (Eds.), Semiconductor Spintronics and Quantum Computation, Springer-Verlag, Berlin, 2002.

[904] A. G. Aronov, Spin injection in metals and polarization of nuclei, Pis'ma Zh. Eksp. Teor. Fiz. 24 (1976) 37-39. [JETP Lett. 24, 32-34 (1976)].

[905] M. Johnson, R. H. Silsbee, Interfacial charge-spin coupling: Injection and detection of spin magnetization in metals, Phys. Rev. Lett. 55 (1985) 1790-1793.

[906] M. Johnson, Spin injection, in: [23], pp. 279-308.

[907] G. Schmidt, D. Ferrand, L. W. Molenkamp, A. T. Filip, B. J. van Wees, Fundamental obstacle for electrical spin injection from a ferromagnetic metal into a diffusive semiconductor, Phys. Rev. B 62 (2000) R4790-R4793.

[908] E. I. Rashba, Diffusion theory of spin injection through resistive contacts, Eur. Phys. J. B 29 (2002) 513-527.

[909] A. Khaetskii, J. C. Egues, D. Loss, C. Gould, G. Schmidt, L. W. Molenkamp, Spin injection across magnetic/nonmagnetic interfaces with finite magnetic layers, Phys. Rev. B 71 (2005) 235327.

[910] S. A. Crooker, M. Furis, X. Lou, C. Adelmann, D. L. Smith, C. J. Palmstrom, P. A. Crowell, Imaging Spin Transport in Lateral Ferromagnet/Semiconductor Structures, Science 309 (2005) 2191-2195.

[911] C. Galinon, K. Tewolde, R. Loloee, W.-C. Chiang, S. Olson, H. Kurt, J. W. P. Pratt, J. Bass, P. X. Xu, K. Xia, M. Talanana, Pd/ag and $\mathrm{pd} / \mathrm{au}$ interface specific resistances and interfacial spin flipping, Appl. Phys. Lett. 86 (2005) 182502.

[912] J. Bass, W. P. P. Jr, Spin-diffusion lengths in metals and alloys, and spin-flipping at metal/metal interfaces: an experimentalist\&rsquo;s critical review, J. Phys.: Condens. Matt. 19 (2007) 183201 (41pp).

[913] S. Garzon, I. Žutić, R. A. Webb, Temperature-dependent asymmetry of the nonlocal spin-injection resistance: Evidence for spin nonconserving interface scattering, Phys. Rev. Lett. 94 (2005) 176601.

[914] R. Godfrey, M. Johnson, Spin injection in mesoscopic silver wires: Experimental test of resistance mismatch, Phys. Rev. Lett. 96 (2006) 136601.

[915] M. I. D'yakonov, V. I. Perel', Theory of optical spin orientation of electrons and nuclei in semiconductors, in: [3], pp. 11-72.

[916] S. Bandyopadhyay, Comment on "control of spin precession in a spin-injected field effect transistor", 2009. ArXiv:0911.0210v1.

[917] A. N. M.. Zainuddin, S. Hong, L. Siddiqui, S. Datta, Voltage controlled spin precession, 2010. ArXiv:1001.1523v1.

[918] P. Agnihotri, S. Bandyopadhyay, Analysis of the two dimensional datta-das spin field effect transistor, Physica E 42 (2010) $1736-1740$.

[919] S. I. Erlingsson, J. Schliemann, D. Loss, Spin susceptibilities, spin densities, and their connection to spin currents, Phys. Rev. B 71 (2005) 035319.

[920] E. Shafir, M. Shen, S. Saikin, Modulation of spin dynamics in a channel of a nonballistic spin field effect transistor, Phys. Rev. B 70 (2004) 241302.

[921] J. Fabian, Solid-state physics: Spin's lifetime extended, Nature 458 (2009) 580-581.

[922] N. F. Mott, The electrical conductivity of transition metals, Proc. R. Soc. Lond. A 153 (1936) 699-717.

[923] N. F. Mott, The resistance and thermoelectric properties of the transition metals, Proc. R. Soc. Lond. A 156 (1936) 368-382.

[924] I. A. Campbell, A. Fert, A. R. Pomeroy, Evidence for two current conduction iron, Philos. Mag. 15 (1967) 977-983.

[925] A. Fert, I. A. Campbell, Two-current conduction in nickel, Phys. Rev. Lett. 21 (1968) 1190-1192.

[926] T. Valet, A. Fert, Theory of the perpendicular magnetoresistance in magnetic multilayers, Phys. Rev. B 48 (1993) 7099-7113.

[927] S. Hershfield, H. L. Zhao, Charge and spin transport through a metallic ferromagnetic-paramagnetic-ferromagnetic junction, Phys. Rev. B 56 (1997) 3296-3305. 
[928] Z. G. Yu, M. E. Flatté, Electric-field dependent spin diffusion and spin injection into semiconductors, Phys. Rev. B 66 (2002) 201202.

[929] Z. G. Yu, M. E. Flatté, Spin diffusion and injection in semiconductor structures: Electric field effects, Phys. Rev. B 66 (2002) 235302.

[930] V. L. Vekua, R. I. Dzhioev, B. P. Zakharchenya, V. G. Fleisher, Fiz. Tekh. Poluprovodn. 10 (1976) 354.

[931] M. Seck, M. Potemski, P. Wyder, High-field spin resonance of weakly bound electrons in gaas, Phys. Rev. B 56 (1997) $7422-7427$.

[932] C. Y. Hu, P. H. Tan, W. Ossau, T. Wojtowicz, G. Karczewski, J. Kossut, Experimental measurement of microwave-induced electron spin-flip time, Appl. Phys. Lett. 78 (2001) 204-206.

[933] W. Hanle, über magnetische beeinflussung der polarisation der resonanzfluoreszenz, Z. Phys. 30 (1924) 93-105.

[934] A. Fert, H. Jaffrès, Conditions for efficient spin injection from a ferromagnetic metal into a semiconductor, Phys. Rev. B 64 (2001) 184420.

[935] J. C. Egues, Spin-dependent perpendicular magnetotransport through a tunable znse/zn1 - xmnxse heterostructure: A possible spin filter?, Phys. Rev. Lett. 80 (1998) 4578-4581.

[936] J. C. Egues, C. Gould, G. Richter, L. W. Molenkamp, Spin filtering and magnetoresistance in ballistic tunnel junctions, Phys. Rev. B 64 (2001) 195319.

[937] P. Recher, E. V. Sukhorukov, D. Loss, Quantum dot as spin filter and spin memory, Phys. Rev. Lett. 85 (2000) $1962-1965$.

[938] R. H. Silsbee, Novel method for the study of spin transport in conductors, Bull. Magn. Reson. 2 (1980) $284-285$.

[939] P. C. van Son, H. van Kempen, P. Wyder, Boundary resistance of the ferromagnetic-nonferromagnetic metal interface, Phys. Rev. Lett. 58 (1987) 2271-2273.

[940] M. Johnson, Spin accumulation in gold films, Phys. Rev. Lett. 70 (1993) 2142-2145.

[941] M. Johnson, Bipolar spin switch, Science 260 (1993) 320-323.

[942] F. J. Jedema, A. T. Filip, B. J. van Wees, Electrical spin injection and accumulation at room temperature in an all-metal mesoscopic spin valve, Nature 410 (2001) 345-348.

[943] F. J. Jedema, M. S. Nijboer, A. T. Filip, B. J. van Wees, Spin injection and spin accumulation in all-metal mesoscopic spin valves, Phys. Rev. B 67 (2003) 085319.

[944] X. Lou, C. Adelmann, S. A. Crooker, E. S. Garlid, J. Zhang, K. S. M. Reddy, S. D. Flexner, C. J. Palmstrom, P. A. Crowell, Electrical detection of spin transport in lateral ferromagnet-semiconduct or devices, Nat. Phys. 3 (2007) 197-202.

[945] A. van Staa, J. Wulfhorst, A. Vogel, U. Merkt, G. Meier, Spin precession in lateral all-metal spin valves: Experimental observation and theoretical description, Phys. Rev. B 77 (2008) 214416.

[946] M. Ciorga, A. Einwanger, U. Wurstbauer, D. Schuh, W. Wegscheider, D. Weiss, Electrical spin injection and detection in lateral allsemiconductor devices, Phys. Rev. B 79 (2009) 165321.

[947] J. D. Albrecht, D. L. Smith, Electron spin injection at a schottky contact, Phys. Rev. B 66 (2002) 113303.

[948] J. D. Albrecht, D. L. Smith, Spin-polarized electron transport at ferromagnet/semiconductor schottky contacts, Phys. Rev. B 68 (2003) 035340 .

[949] M. Shen, S. Saikin, M.-C. Cheng, Monte carlo modeling of spin injection through a schottky barrier and spin transport in a semiconductor quantum well, J. Appl. Phys. 96 (2004) 4319-4325.

[950] S. Saikin, Y. V. Pershin, V. Privman, Modelling for semiconductor spintronics, IEE Proc. Circ., Dev. Syst. 152 (2005) $366-376$.

[951] S. Saikin, M. Shen, M.-C. Cheng, V. Privman, Semiclassical monte carlo model for in-plane transport of spin-polarized electrons in iii-V heterostructures, J. Appl. Phys. 94 (2003) 1769-1775.

[952] Y. Y. Wang, M. W. Wu, Schottky-barrier-induced spin relaxation in spin injection, Phys. Rev. B 72 (2005) 153301.

[953] S. Saikin, M. Shen, M.-C. Cheng, Spin dynamics in a compound semiconductor spintronic structure with a schottky barrier, J. Phys.: Condens. Matt. 18 (2006) 1535-1544.

[954] H. López, X. Oriols, J. Suñé, X. Cartoixà, Spin-dependent injection model for monte carlo device simulation, J. Appl. Phys. 104 (2008) 073702.

[955] S. M. Sze, Physics of Semiconductor Devices, Wiley, New York, 1981

[956] L. Sun, X. Y. Liu, M. Liu, G. Du, R. Q. Han, Monte carlo simulation of schottky contact with direct tunnelling model, Semicond. Sci. Technol. 18 (2003) 576-581.

[957] M. J. Martin, T. Gonzalez, D. Pardo, J. E. Velazquez, Monte carlo analysis of a schottky diode with an automatic space-variable charge algorithm, Semicond. Sci. Technol. 11 (1996) 380-387.

[958] A. T. Hanbicki, B. T. Jonker, G. Itskos, G. Kioseoglou, A. Petrou, Efficient electrical spin injection from a magnetic metal/tunnel barrier contact into a semiconductor, Appl. Phys. Lett. 80 (2002) 1240-1242.

[959] A. T. Hanbicki, O. M. J. van 't Erve, R. Magno, G. Kioseoglou, C. H. Li, B. T. Jonker, G. Itskos, R. Mallory, M. Yasar, A. Petrou, Analysis of the transport process providing spin injection through an fe/algaas schottky barrier, Appl. Phys. Lett. 82 (2003) $4092-4094$.

[960] V. V. Osipov, A. M. Bratkovsky, Efficient nonlinear room-temperature spin injection from ferromagnets into semiconductors through a modified schottky barrier, Phys. Rev. B 70 (2004) 205312.

[961] A. M. Bratkovsky, V. V. Osipov, Efficient spin extraction from nonmagnetic semiconductors near forward-biased ferromagneticsemiconductor modified junctions at low spin polarization of current, J. Appl. Phys. 96 (2004) 4525-4529.

[962] A. M. Bratkovsky, V. V. Osipov, Efficient polarized injection luminescence in forward-biased ferromagnetic-semiconductor junctions at low spin polarization of current, Appl. Phys. Lett. 86 (2005) 071120.

[963] V. V. Osipov, A. M. Bratkovsky, Spin accumulation in degenerate semiconductors near modified schottky contact with ferromagnets: Spin injection and extraction, Phys. Rev. B 72 (2005) 115322.

[964] C. Adelmann, X. Lou, J. Strand, C. J. Palmstrøm, P. A. Crowell, Spin injection and relaxation in ferromagnet-semiconductor heterostructures, Phys. Rev. B 71 (2005) 121301.

[965] D. Saha, M. Holub, P. Bhattacharya, Y. C. Liao, Epitaxially grown mnas/gaas lateral spin valves, Appl. Phys. Lett. 89 (2006) 142504.

[966] A. T. Hanbicki, G. Kioseoglou, M. A. Holub, O. M. J. van 't Erve, B. T. Jonker, Electrical spin injection from fe into znse(001), Appl. Phys. Lett. 94 (2009) 082507.

[967] H. Kum, D. Basu, P. Bhattacharya, W. Guo, Electric field control of magnetoresistance in a lateral inas quantum well spin valve, Appl. Phys. Lett. 95 (2009) 212503. 
[968] S. F. Alvarado, P. Renaud, Observation of spin-polarized-electron tunneling from a ferromagnet into gaas, Phys. Rev. Lett. 68 (1992) 1387-1390.

[969] A. T. Filip, B. H. Hoving, F. J. Jedema, B. J. van Wees, B. Dutta, S. Borghs, Experimental search for the electrical spin injection in a semiconductor, Phys. Rev. B 62 (2000) 9996-9999.

[970] H. J. Zhu, M. Ramsteiner, H. Kostial, M. Wassermeier, H.-P. Schönherr, K. H. Ploog, Room-temperature spin injection from fe into gaas, Phys. Rev. Lett. 87 (2001) 016601.

[971] H. Ohno, K. Yoh, K. Sueoka, K. Mukasa, A. Kawaharazuka, M. E. Ramsteiner, Spin-polarized electron injection through an fe/inas junction, Jpn. J. Appl. Phys. 42 (2003) L87-L89.

[972] G. Schmidt, L. W. Molenkamp, Electrical spin injection into semiconductors, Physica E 9 (2001) $202-208$.

[973] Y. Ohno, D. K. Young, B. Beschoten, F. Matsukura, H. Ohno, D. D. Awschalom, Electrical spin injection in a ferromagnetic semiconductor heterostructure, Nature 402 (1999) 790-792.

[974] K. H. Ploog, Spin injection in ferromagnet-semiconductor heterostructures at room temperature (invited), J. Appl. Phys. 91 (2002) 7256-7260.

[975] M. Ramsteiner, H. Y. Hao, A. Kawaharazuka, H. J. Zhu, M. Kästner, R. Hey, L. Däweritz, H. T. Grahn, K. H. Ploog, Electrical spin injection from ferromagnetic mnas metal layers into gaas, Phys. Rev. B 66 (2002) 081304.

[976] M. Ramsteiner, H. Y. Hao, A. Kawaharazuka, H. J. Zhu, M. Kästner, R. Hey, L. Däweritz, H. T. Grahn, K. H. Ploog, Electrical spin injection from ferromagnetic mnas metal layers into gaas, Phys. Rev. B 66 (2002) 081304.

[977] M. Kohda, Y. Ohno, K. Takamura, F. Matsukura, H. Ohno, A spin esaki diode, Jpn. J. Appl. Phys. 40 (2001) L1274-L1276.

[978] E. Johnston-Halperin, D. Lofgreen, R. K. Kawakami, D. K. Young, L. Coldren, A. C. Gossard, D. D. Awschalom, Spin-polarized zener tunneling in (ga,mn)as, Phys. Rev. B 65 (2002) 041306.

[979] S. E. Andresen, B. S. Sørensen, F. B. Rasmussen, P. E. Lindelof, J. Sadowski, C. M. Guertler, J. A. C. Bland, Spin-polarized electron tunneling across a si delta-doped gamnas/n-gaas interface, J. Appl. Phys. 94 (2003) 3990-3994.

[980] P. Van Dorpe, Z. Liu, W. Van Roy, V. F. Motsnyi, M. Sawicki, G. Borghs, J. De Boeck, Very high spin polarization in gaas by injection from a (ga,mn)as zener diode, Appl. Phys. Lett. 84 (2004) 3495-3497.

[981] T. Kita, M. Kohda, Y. Ohno, F. Matsukura, H. Ohno, Spin injection with three terminal device based on (ga,mn)as/n+-gaas tunnel junction, Phys. Stat. Sol. (c) 3 (2006) 4164-4167.

[982] M. Ciorga, A. Einwanger, J. Sadowski, W. Wegscheider, D. Weiss, Tunneling anisotropic magnetoresistance effect in a p+-(ga,mn)as/n+gaas esaki diode, Phys. Stat. Sol. (a) 204 (2006) 186-190.

[983] M. Kohda, T. Kita, Y. Ohno, F. Matsukura, H. Ohno, Bias voltage dependence of the electron spin injection studied in a three-terminal device based on a (ga,mn)as/n[sup +]-gaas esaki diode, Appl. Phys. Lett. 89 (2006) 012103.

[984] A. Einwanger, M. Ciorga, U. Wurstbauer, D. Schuh, W. Wegscheider, D. Weiss, Tunneling anisotropic spin polarization in lateral (ga,mn)as/gaas spin esaki diode devices, Appl. Phys. Lett. 95 (2009) 152101.

[985] T. Gruber, M. Keim, R. Fiederling, G. Reuscher, W. Ossau, G. Schmidt, L. W. Molenkamp, A. Waag, Electron spin manipulation using semimagnetic resonant tunneling diodes, Appl. Phys. Lett. 78 (2001) 1101-1103.

[986] A. Slobodskyy, C. Gould, T. Slobodskyy, C. R. Becker, G. Schmidt, L. W. Molenkamp, Voltage-controlled spin selection in a magnetic resonant tunneling diode, Phys. Rev. Lett. 90 (2003) 246601.

[987] S. Maximov, T. Slobodskyy, A. Groger, F. Lehmann, P. Grabs, L. Hansen, C. R. Becker, C. Gould, G. Schmidt, L. W. Molenkamp, Micro-patterned (zn,be)se/(zn,mn)se resonant tunnelling diodes, Semiconductor Science and Technology 19 (2004) $946-949$.

[988] A. V. Scherbakov, A. V. Akimov, D. R. Yakovlev, W. Ossau, L. Hansen, A. Waag, L. W. Molenkamp, Spin control in heteromagnetic nanostructures, Appl. Phys. Lett. 86 (2005) 162104.

[989] D. Supp, T. Slobodskyy, A. Slobodskyy, C. Gould, G. Schmidt, L. W. Molenkamp, All ii-vi magnetic resonant tunneling diodes as voltage controlled spin filters, Phys. Stat. Sol. (c) 4 (2007) 3390-3396.

[990] A. Kawaharazuka, M. Ramsteiner, J. Herfort, H.-P. Schönherr, H. Kostial, K. H. Ploog, Spin injection from fe 3 si into gaas, Appl. Phys. Lett. 85 (2004) 3492-3494.

[991] C. Adelmann, X. Lou, J. Strand, C. J. Palmstrøm, P. A. Crowell, Spin injection and relaxation in ferromagnet-semiconductor heterostructures, Phys. Rev. B 71 (2005) 121301.

[992] M. C. Hickey, S. N. Holmes, T. Meng, I. Farrer, G. A. C. Jones, D. A. Ritchie, M. Pepper, Strongly bias-dependent spin injection from fe into n-type gaas, Phys. Rev. B 75 (2007) 193204.

[993] A. T. Hanbicki, B. T. Jonker, G. Itskos, G. Kioseoglou, A. Petrou, Efficient electrical spin injection from a magnetic metal/tunnel barrier contact into a semiconductor, Appl. Phys. Lett. 80 (2002) 1240-1242.

[994] A. T. Hanbicki, G. Kioseoglou, M. A. Holub, O. M. J. van 't Erve, B. T. Jonker, Electrical spin injection from fe into znse(001), Appl. Phys. Lett. 94 (2009) 082507.

[995] T. Manago, H. Akinaga, Spin-polarized light-emitting diode using metal/insulator/semiconductor structures, Appl. Phys. Lett. 81 (2002) 694-696.

[996] V. F. Motsnyi, J. De Boeck, J. Das, W. Van Roy, G. Borghs, E. Goovaerts, V. I. Safarov, Electrical spin injection in a ferromagnet/tunnel barrier/semiconductor heterostructure, Appl. Phys. Lett. 81 (2002) 265-267.

[997] O. M. J. van 't Erve, G. Kioseoglou, A. T. Hanbicki, C. H. Li, B. T. Jonker, R. Mallory, M. Yasar, A. Petrou, Comparison of fe/schottky and fe/al $\mathrm{a}_{2} \mathrm{O}_{3}$ tunnel barrier contacts for electrical spin injection into gaas, Appl. Phys. Lett. 84 (2004) 4334-4336.

[998] E. Oh, T. K. Lee, J. H. Park, J. H. Choi, Y. J. Park, K. H. Shin, K. Y. Kim, Carrier lifetime and spin relaxation time study for electrical spin injection into gaas, J. Appl. Phys. 106 (2009) 043515.

[999] X. Jiang, R. Wang, R. M. Shelby, R. M. Macfarlane, S. R. Bank, J. S. Harris, S. S. P. Parkin, Highly spin-polarized room-temperature tunnel injector for semiconductor spintronics using mgo(100), Phys. Rev. Lett. 94 (2005) 056601.

[1000] S. S. P. Parkin, C. Kaiser, A. Panchula, P. M. Rice, B. Hughes, M. Samant, S.-H. Yang, Giant tunnelling magnetoresistance at room temperature with mgo (100) tunnel barriers, Nat. Mater. 3 (2004) 862-867.

[1001] R. Wang, X. Jiang, R. M. Shelby, R. M. Macfarlane, S. S. P. Parkin, S. R. Bank, J. S. Harris, Increase in spin injection efficiency of a 
cofe/mgo(100) tunnel spin injector with thermal annealing, Appl. Phys. Lett. 86 (2005) 052901.

[1002] G. Salis, R. Wang, X. Jiang, R. M. Shelby, S. S. P. Parkin, S. R. Bank, J. S. Harris, Temperature independence of the spin-injection efficiency of a mgo-based tunnel spin injector, Appl. Phys. Lett. 87 (2005) 262503.

[1003] A. Sinsarp, T. Manago, F. Takano, H. Akinaga, Electrical spin injection from out-of-plane magnetized fept/mgo tunneling junction into gaas at room temperature, Jpn. J. Appl. Phys. 46 (2007) L4-L6.

[1004] Y. Lu, V. G. Truong, P. Renucci, M. Tran, H. Jaffrès, C. Deranlot, J.-M. George, A. Lemaître, Y. Zheng, D. Demaille, P.-H. Binh, T. Amand, X. Marie, Mgo thickness dependence of spin injection efficiency in spin-light emitting diodes, Appl. Phys. Lett. 93 (2008) 152102.

[1005] V. G. Truong, P.-H. Binh, P. Renucci, M. Tran, Y. Lu, H. Jaffrès, J.-M. George, C. Deranlot, A. Lemaître, T. Amand, X. Marie, High speed pulsed electrical spin injection in spin-light emitting diode, Appl. Phys. Lett. 94 (2009) 141109.

[1006] S. Hövel, N. C. Gerhardt, M. R. Hofmann, F.-Y. Lo, A. Ludwig, D. Reuter, A. D. Wieck, E. Schuster, H. Wende, W. Keune, O. Petracic, K. Westerholt, Room temperature electrical spin injection in remanence, Appl. Phys. Lett. 93 (2008) 021117.

[1007] T. Inokuchi, T. Marukame, M. Ishikawa, H. Sugiyama, Y. Saito, Electrical spin injection into n-gaas channels and detection through mgo/cofeb electrodes, Appl. Phys. Express 2 (2009) 023006.

[1008] H. Saito, J. C. L. Breton, V. Zayets, S. Yuasa, K. Ando, Highly enhanced electron-injection efficiency in gaas-based light-emitting diodes using a fe/gao $x$ tunnel injector, Appl. Phys. Express 2 (2009) 083003.

[1009] P. Mavropoulos, N. Papanikolaou, P. H. Dederichs, Complex band structure and tunneling through ferromagnet/insulator/ferromagnet junctions, Phys. Rev. Lett. 85 (2000) 1088-1091.

[1010] W. H. Butler, X.-G. Zhang, T. C. Schulthess, J. M. MacLaren, Spin-dependent tunneling conductance of $f e|m g o| f e$ sandwiches, Phys. Rev. B 63 (2001) 054416.

[1011] J. Mathon, A. Umerski, Theory of tunneling magnetoresistance of an epitaxial fe/mgo/fe(001) junction, Phys. Rev. B 63 (2001) 220403.

[1012] X.-G. Zhang, W. H. Butler, Large magnetoresistance in bcc co/mgo/co and feco/mgo/feco tunnel junctions, Phys. Rev. B 70 (2004) 172407.

[1013] R. A. de Groot, F. M. Mueller, P. G. van Engen, K. H. J. Buschow, New class of materials: Half-metallic ferromagnets, Phys. Rev. Lett. 50 (1983) 2024-2027.

[1014] T. Block, M. J. Carey, B. A. Gurney, O. Jepsen, Band-structure calculations of the half-metallic ferromagnetism and structural stability of full- and half-heusler phases, Phys. Rev. B 70 (2004) 205114.

[1015] X. Y. Dong, C. Adelmann, J. Q. Xie, C. J. Palmstrøm, X. Lou, J. Strand, P. A. Crowell, J.-P. Barnes, A. K. Petford-Long, Spin injection from the heusler alloy $\mathrm{Co}_{2}$ mnge into $\mathrm{al}_{0.1} \mathrm{ga}_{0.9}$ as/gaas heterostructures, Appl. Phys. Lett. 86 (2005) 102107.

[1016] S. Kawagishi, T. Uemura, Y. Imai, K.-I. Matsuda, M. Yamamoto, Structural, magnetic, and electrical properties of co ${ }_{2} \mathrm{mnsi} / \mathrm{mgo} / n$-gaas tunnel junctions, J. Appl. Phys. 103 (2008) 07A703.

[1017] M. C. Hickey, C. D. Damsgaard, S. N. Holmes, I. Farrer, G. A. C. Jones, D. A. Ritchie, C. S. Jacobsen, J. B. Hansen, M. Pepper, Spin injection from $\mathrm{CO}_{2}$ mnga into an ingaas quantum well, Appl. Phys. Lett. 92 (2008) 232101.

[1018] M. Ramsteiner, O. Brandt, T. Flissikowski, H. T. Grahn, M. Hashimoto, J. Herfort, H. Kostial, Co 2 fesi/gaas/(al,ga)as spin light-emitting diodes: Competition between spin injection and ultrafast spin alignment, Phys. Rev. B 78 (2008) 121303.

[1019] C. D. Damsgaard, M. C. Hickey, S. N. Holmes, R. Feidenhans'l, S. O. Mariager, C. S. Jacobsen, J. B. Hansen, Interfacial, electrical, and spin-injection properties of epitaxial $\mathrm{co}_{2}$ mnga grown on gaas(100), J. Appl. Phys. 105 (2009) 124502.

[1020] V. F. Motsnyi, J. De Boeck, J. Das, W. Van Roy, G. Borghs, E. Goovaerts, V. I. Safarov, Electrical spin injection in a ferromagnet/tunnel barrier/semiconductor heterostructure, Appl. Phys. Lett. 81 (2002) 265-267.

[1021] M. Kohda, T. Kita, Y. Ohno, F. Matsukura, H. Ohno, Bias voltage dependence of the electron spin injection studied in a three-terminal device based on a (ga,mn)as/n[sup +]-gaas esaki diode, Appl. Phys. Lett. 89 (2006) 012103.

[1022] D. Hägele, M. Oestreich, W. W. Rühle, N. Nestle, K. Eberl, Spin transport in gaas, Applied Physics Letters 73 (1998) $1580-1582$.

[1023] R. Dzhioev, B. Zakharchenya, V. Korenev, M. Stepanova, Spin diffusion of optically oriented electrons and photon entrainment in n-gallium arsenide, Phys. Solid State 39 (1997) 1765-1768.

[1024] H. Kosaka, H. Shigyou, Y. Mitsumori, Y. Rikitake, H. Imamura, T. Kutsuwa, K. Arai, K. Edamatsu, Coherent transfer of light polarization to electron spins in a semiconductor, Phys. Rev. Lett. 100 (2008) 096602.

[1025] S. A. Crooker, M. Furis, X. Lou, P. A. Crowell, D. L. Smith, C. Adelmann, C. J. Palmstrøm, Optical and electrical spin injection and spin transport in hybrid fe/gaas devices, J. Appl. Phys. 101 (2007) 081716.

[1026] P. Kotissek, M. Bailleul, M. Sperl, A. Spitzer, D. Schuh, W. Wegscheider, C. H. Back, G. Bayreuther, Cross-sectional imaging of spin injection into a semiconductor, Nat. Phys. 3 (2007) 872-877.

[1027] M. Furis, D. L. Smith, S. Kos, E. S. Garlid, K. S. M. Reddy, C. J. Palmstrom, P. A. Crowell, S. A. Crooker, Local hanle-effect studies of spin drift and diffusion in n:gaas epilayers and spin-transport devices, New J. Phys. 9 (2007) 347.

[1028] M. I. D’yakonov, V. I. Perel', Possibility of orienting electron spins with current, Pis'ma Zh. Eksp. Ter. Fiz. 13 (1971) 657-660. [JETP Lett. 13, 467-469].

[1029] V. Sih, R. C. Myers, Y. K. Kato, W. H. Lau, A. C. Gossard, D. D. Awschalom, Spatial imaging of the spin hall effect and current-induced polarization in two-dimensional electron gases, Nat. Phys. 1 (2005) 31-35.

[1030] J. Schliemann, Spin hall effect, Int. J. Mod. Phys. B 20 (2006) 1015-1036

[1031] H.-A. Engel, E. I. Rashba, B. I. Halperin, Theory of spin hall effects in semiconductors, in: H. Kronmüller, S. Parkin (Eds.), Handbook of Magnetism and Advanced Magnetic Materials, volume 5, Wiley, New York, 2007, pp. 2858-2877.

[1032] J. L. Cheng, M. W. Wu, Kinetic investigation of the extrinsic spin hall effect induced by skew scattering, J. Phys.: Condens. Matt. 20 (2008) 085209 (5pp)

[1033] M. I. Dyakonov, A. V. Khaetskii, Spin physics in semiconductors, in: [23], pp. 211-243.

[1034] M. I. D’yakonov, Spin hall effect, volume 7036, SPIE, 2008, p. 70360R.

[1035] E. M. Hankiewicz, G. Vignale, Spin-hall effect and spin-coulomb drag in doped semiconductors, J. Phys.: Condens. Matt. 21 (2009) $253202(16 \mathrm{pp})$.

[1036] G. Vignale, Ten years of spin hall effect, J. Supercond. Nov. Magn. 23 (2009) 3-10.

[1037] W. Y. Lee, S. Gardelis, B.-C. Choi, Y. B. Xu, C. G. Smith, C. H. W. Barnes, D. A. Ritchie, E. H. Linfield, J. A. C. Bland, Magnetization 
reversal and magnetoresistance in a lateral spin-injection device, J. Appl. Phys. 85 (1999) 6682-6685.

[1038] P. R. Hammar, B. R. Bennett, M. J. Yang, M. Johnson, Observation of spin injection at a ferromagnet-semiconductor interface, Phys. Rev. Lett. 83 (1999) 203-206.

[1039] P. N. Hai, Y. Sakata, M. Yokoyama, S. Ohya, M. Tanaka, Spin-valve effect by ballistic transport in ferromagnetic metal (mnas)/semiconductor (gaas) hybrid heterostructures, Phys. Rev. B 77 (2008) 214435.

[1040] F. G. Monzon, M. Johnson, M. L. Roukes, Strong hall voltage modulation in hybrid ferromagnet/semiconductor microstructures, Appl. Phys. Lett. 71 (1997) 3087-3089.

[1041] F. G. Monzon, M. L. Roukes, Spin injection and the local hall effect in inas quantum wells, J. Magn. Magn. Mater. 198-199 (1999) 632-635.

[1042] F. G. Monzon, D. S. Patterson, M. L. Roukes, Characterization of individual nanomagnets by the local hall effect, J. Magn. Magn. Mater. 195 (1999) 19-25.

[1043] F. G. Monzon, H. X. Tang, M. L. Roukes, Magnetoelectronic phenomena at a ferromagnet-semiconductor interface, Phys. Rev. Lett. 84 (2000) 5022 .

[1044] H. X. Tang, F. G. Monzon, R. Lifshitz, M. C. Cross, M. L. Roukes, Ballistic spin transport in a two-dimensional electron gas, Phys. Rev. B 61 (2000) 4437-4440.

[1045] T. Yang, T. Kimura, Y. Otani, Giant spin-accumulation signal and pure spin-current-induced reversible magnetization switching, Nat. Phys. 4 (2008) 851-854.

[1046] I. Zutić, J. Fabian, S. C. Erwin, Spin injection and detection in silicon, Phys. Rev. Lett. 97 (2006) 026602.

[1047] I. Žutić, Spintronics: Gadolinium makes good spin contacts, Nat. Mater. 5 (2006) 771-772.

[1048] B. Huang, D. J. Monsma, I. Appelbaum, Spin lifetime in silicon in the presence of parasitic electronic effects, J. Appl. Phys. 102 (2007) 013901.

[1049] B. Huang, D. J. Monsma, I. Appelbaum, Coherent spin transport through a 350 micron thick silicon wafer, Phys. Rev. Lett. 99 (2007) 177209.

[1050] B. Huang, L. Zhao, D. J. Monsma, I. Appelbaum, 35\% magnetocurrent with spin transport through si, Appl. Phys. Lett. 91 (2007) 052501.

[1051] R. Jansen, B. C. Min, Detection of a spin accumulation in nondegenerate semiconductors, Phys. Rev. Lett. 99 (2007) 246604.

[1052] B. Huang, I. Appelbaum, Spin dephasing in drift-dominated semiconductor spintronics devices, Phys. Rev. B 77 (2008) 165331.

[1053] J. Li, B. Huang, I. Appelbaum, Oblique hanle effect in semiconductor spin transport devices, Appl. Phys. Lett. 92 (2008) 142507.

[1054] H.-J. Jang, J. Xu, J. Li, B. Huang, I. Appelbaum, Non-ohmic spin transport in n-type doped silicon, Phys. Rev. B 78 (2008) 165329.

[1055] H. Tashpour, M. A. Vesaghi, Spin dependent recombination in magnetic semiconductor, Appl. Phys. Lett. 94 (2009) 242103.

[1056] R. de Sousa, C. C. Lo, J. Bokor, Spin-dependent scattering in a silicon transistor, Phys. Rev. B 80 (2009) 045320.

[1057] H.-J. Jang, I. Appelbaum, Spin polarized electron transport near the si/sio 2 interface, Phys. Rev. Lett. 103 (2009) 117202.

[1058] Y. Ando, K. Hamaya, K. Kasahara, Y. Kishi, K. Ueda, K. Sawano, T. Sadoh, M. Miyao, Electrical injection and detection of spin-polarized electrons in silicon through an fe 3 si/si schottky tunnel barrier, Appl. Phys. Lett. 94 (2009) 182105.

[1059] J. K. Furdyna, S. Broersma, Microwave faraday effect in silicon and germanium, Phys. Rev. 120 (1960) 1995-2003.

[1060] J. K. Furdyna, M. E. Brodwin, Dependence of the free-carrier faraday ellipticity in semiconductors on scattering mechanisms, Phys. Rev. 124 (1961) 740-744.

[1061] E. D. Palik, J. K. Furdyna, Infrared and microwave magnetoplasma effects in semiconductors, Rep. Progr. Phys. 33 (1970) $1193-1322$.

[1062] O. Morikawa, A. Quema, S. Nashima, H. Sumikura, T. Nagashima, M. Hangyo, Faraday ellipticity and faraday rotation of a doped-silicon wafer studied by terahertz time-domain spectroscopy, J. Appl. Phys. 100 (2006) 033105.

[1063] Y. Ikebe, R. Shimano, Characterization of doped silicon in low carrier density region by terahertz frequency faraday effect, Appl. Phys. Lett. 92 (2008) 012111

[1064] T. P. Pearsall, J. Bevk, L. C. Feldman, J. M. Bonar, J. P. Mannaerts, A. Ourmazd, Structurally induced optical transitions in ge-si superlattices, Phys. Rev. Lett. 58 (1987) 729-732

[1065] K. Eberl, G. Krötz, R. Zachai, G. Abstreiter, Structural, compositional, and optical properties of ultrathin si/ge superlattices, J. Phys. Colloq. 48 (1987) C5-329-332.

[1066] J. Weber, M. I. Alonso, Near-band-gap photoluminescence of si-ge alloys, Phys. Rev. B 40 (1989) $5683-5693$.

[1067] M. L. W. Thewalt, S. P. Watkins, U. O. Ziemelis, E. C. Lightowlers, M. O. Henry, Photoluminescence lifetime, absorption and excitation spectroscopy measurements on isoelectronic bound excitons in beryllium-doped silicon, Solid State Commun. 44 (1982) $573-577$.

[1068] P. Y. Yu, M. Cardona, Fundamentals of Semiconductors: Physics and Materials Properties, Springer, Berlin, 2005.

[1069] L. D. Laude, F. H. Pollak, M. Cardona, Effects of uniaxial stress on the indirect exciton spectrum of silicon, Phys. Rev. B 3 (1971) 2623-2636.

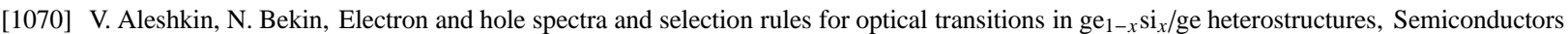
31 (1997) 132-138.

[1071] J. Olajos, J. Engvall, H. G. Grimmeiss, H. Kibbel, H. Presting, Optical anisotropies in strained si/sige systems, Appl. Surf. Sci. 102 (1996) $283-287$.

[1072] T. Sasaki, T. Oikawa, T. Suzuki, M. Shiraishi, Y. Suzuki, K. Tagami, Electrical spin injection into silicon using mgo tunnel barrier, Appl. Phys. Express 2 (2009) 053003.

[1073] M. A. I. Nahid, M. Oogane, H. Naganuma, Y. Ando, Structural and magnetic properties of co 2 mnsi heusler alloy thin films on si, Jpn. J. Appl. Phys. 48 (2009).

[1074] T. Uhrmann, T. Dimopoulos, A. Kovacs, A. Kohn, S. Weyers, U. Paschen, J. Smoliner, H. Bruckl, Evaluation of schottky and mgo-based tunnelling diodes with different ferromagnets for spin injection in n-si, J. Phys. D: Appl. Phys. 42 (2009) 145114 (9pp).

[1075] J. Y. Veuillen, J. Derrien, P. A. Badoz, E. Rosencher, C. d'Anterroches, Co/si(111) interface: Formation of an initial cosiz phase at room temperature, Appl. Phys. Lett. 51 (1987) 1448-1450.

[1076] J.-S. Tsay, Y.-D. Yao, Magnetic phase diagram of ultrathin co/si(111) film studied by surface magneto-optic kerr effect, Appl. Phys. Lett. 74 (1999) 1311-1313. 
[1077] J. S. Tsay, C. S. Yang, Y. Liou, Y. D. Yao, Magnetic properties of ultrathin co films on si(111) and cosiz surfaces, J. Appl. Phys. 85 (1999) $4967-4969$.

[1078] D. Culcer, J. Sinova, N. A. Sinitsyn, T. Jungwirth, A. H. MacDonald, Q. Niu, Semiclassical spin transport in spin-orbit-coupled bands, Phys. Rev. Lett. 93 (2004) 046602.

[1079] E. G. Mishchenko, A. V. Shytov, B. I. Halperin, Spin current and polarization in impure two-dimensional electron systems with spin-orbit coupling, Phys. Rev. Lett. 93 (2004) 226602.

[1080] O. Bleibaum, Spin diffusion equations for systems with rashba spin-orbit interaction in an electric field, Phys. Rev. B 73 (2006) 035322.

[1081] O. Bleibaum, Boundary conditions for spin-diffusion equations with rashba spin-orbit interaction, Phys. Rev. B 74 (2006) 113309.

[1082] O. Bleibaum, Spin-diffusion equations for semiconductor heterostructures with long-range impurity scattering, J. Phys.: Condens. Matt. 18 (2006) 6237-6247.

[1083] V. V. Bryksin, P. Kleinert, Theory of electric-field-induced spin accumulation and spin current in the two-dimensional rashba model, Phys. Rev. B 73 (2006) 165313

[1084] V. V. Bryksin, P. Kleinert, Coupled spin-charge drift-diffusion approach for a two-dimensional electron gas with rashba spin-orbit coupling, Phys. Rev. B 75 (2007) 205317.

[1085] V. V. Bryksin, P. Kleinert, Coupled spin-charge drift-diffusion equations for the rashba model subject to an in-plane electric field, Phys. Rev. B 76 (2007) 075340.

[1086] P. Kleinert, V. V. Bryksin, Spin accumulation and spin-coherent excitations in a strongly confined two-dimensional hole gas, Phys. Rev. B 76 (2007) 073314.

[1087] J. Fabian, I. Žutić, S. Das Sarma, Theory of spin-polarized bipolar transport in magnetic $p-n$ junctions, Phys. Rev. B 66 (2002) 165301.

[1088] I. Martin, Spin-drift transport and its applications, Phys. Rev. B 67 (2003) 014421.

[1089] M. E. Flatté, J. M. Byers, Spin diffusion in semiconductors, Phys. Rev. Lett. 84 (2000) 4220-4223.

[1090] M. W. Wu, M. Q. Weng, J. L. Cheng, in: V. E. Borisenko, V. S. Gurin, S. V. Gaponenko (Eds.), Physics, Chemistry and Application of Nanostructures: Reviews and Short Notes to Nanomeeting 2007, World Scientific, Singapore, 2007, p. 14

[1091] D. G. Seiler, B. D. Bajaj, A. E. Stephens, Inversion-asymmetry splitting of the conduction band in insb, Phys. Rev. B 16 (1977) $2822-2833$.

[1092] M. Cardona, V. A. Maruschak, A. N. Titkov, Stress-induced splitting of the conduction bands of gaas and gasb, Solid State Commun. 50 (1984) 701-703.

[1093] K. Flensberg, T. S. Jensen, N. A. Mortensen, Diffusion equation and spin drag in spin-polarized transport, Phys. Rev. B 64 (2001) 245308

[1094] I. D’Amico, G. Vignale, Coulomb interaction effects in spin-polarized transport, Phys. Rev. B 65 (2002) 085109.

[1095] I. D'Amico, G. Vignale, Spin coulomb drag in the two-dimensional electron liquid, Phys. Rev. B 68 (2003) 045307.

[1096] I. D'Amico, G. Vignale, High field and coulomb interaction effects on spin injection in degenerate semiconductors, Semicond. Sci. Technol. 19 (2004) S383-S385.

[1097] I. D'Amico, Spin injection and electric-field effect in degenerate semiconductors, Phys. Rev. B 69 (2004) 165305.

[1098] G. Vignale, Observing the spin coulomb drag in spin-valve devices, Phys. Rev. B 71 (2005) 125103.

[1099] W.-K. Tse, S. Das Sarma, Coulomb drag and spin drag in the presence of spin-orbit coupling, Phys. Rev. B 75 (2007) 045333.

[1100] Y. Takahashi, N. Inaba, F. Hirose, Temperature dependence of spin-polarized electron transport including electron-electron interaction, J. Appl. Phys. 104 (2008) 023714.

[1101] S. M. Badalyan, C. S. Kim, G. Vignale, Finite width and local field corrections to spin coulomb drag in a quasi-two-dimensional electron gas, Phys. Rev. Lett. 100 (2008) 016603.

[1102] S. Yarlagadda, G. F. Giuliani, Spin susceptibility in a two-dimensional electron gas, Phys. Rev. B 40 (1989) 5432-5440.

[1103] Y. Kwon, D. M. Ceperley, R. M. Martin, Quantum monte carlo calculation of the fermi-liquid parameters in the two-dimensional electron gas, Phys. Rev. B 50 (1994) 1684-1694. 


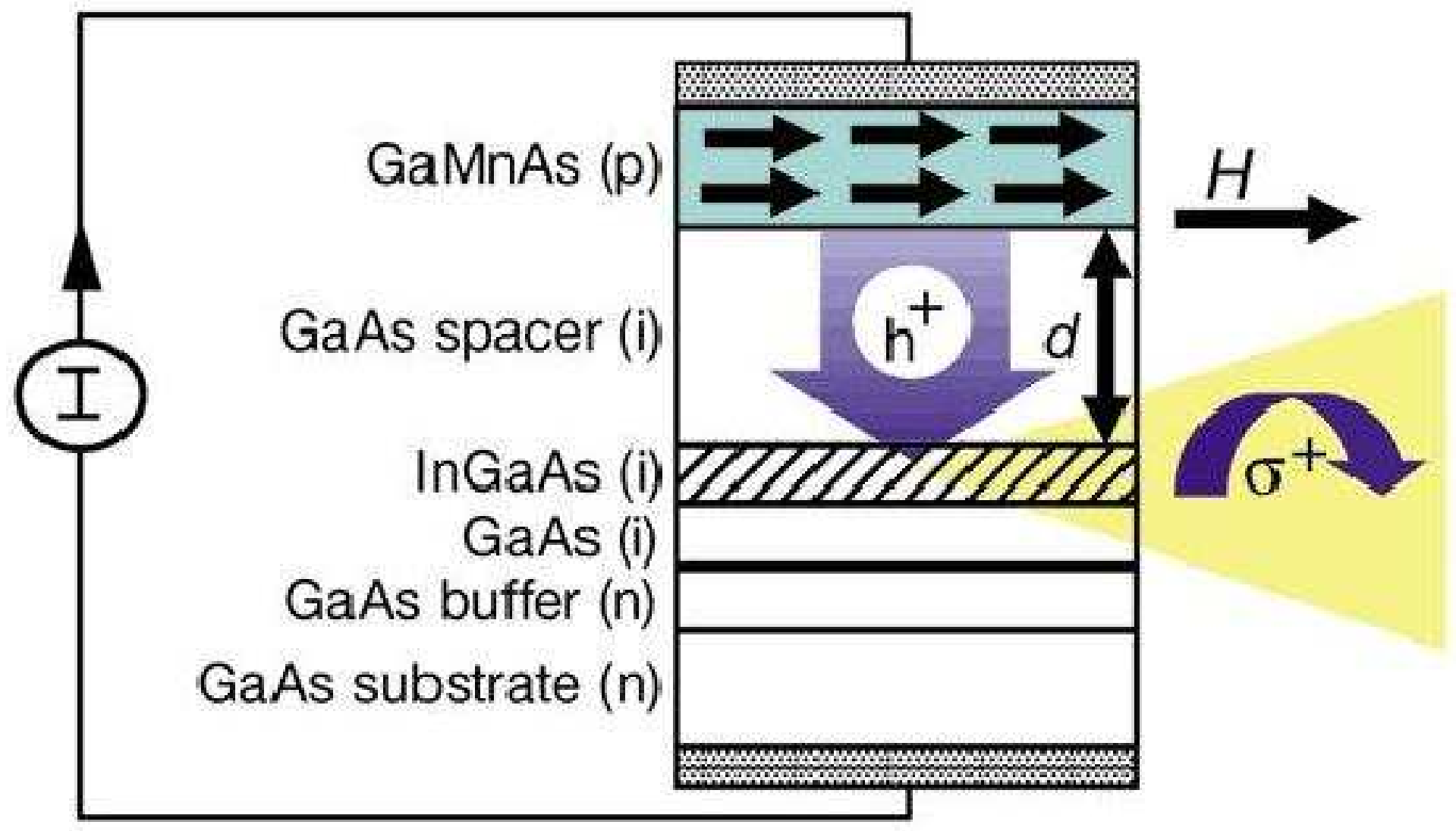

